
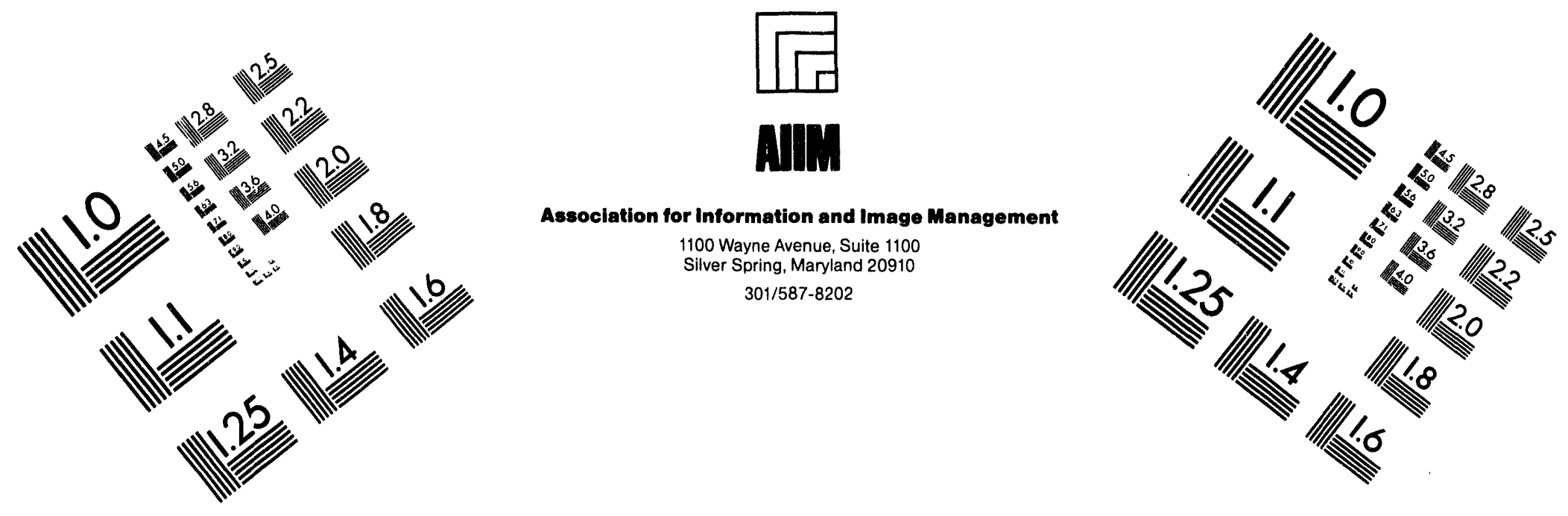

Centimeter

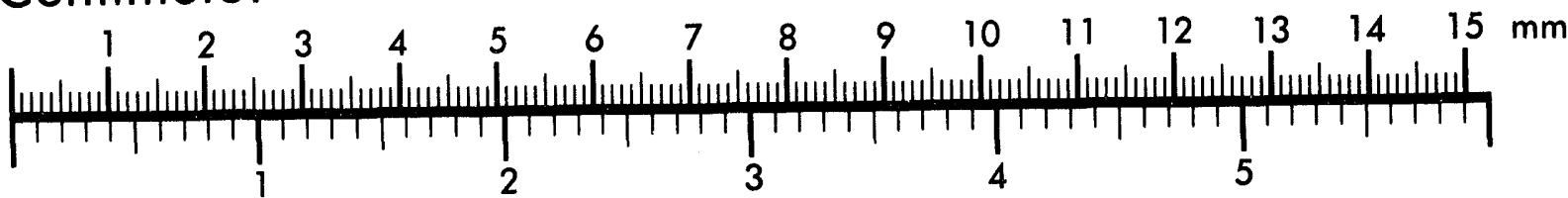

Inches
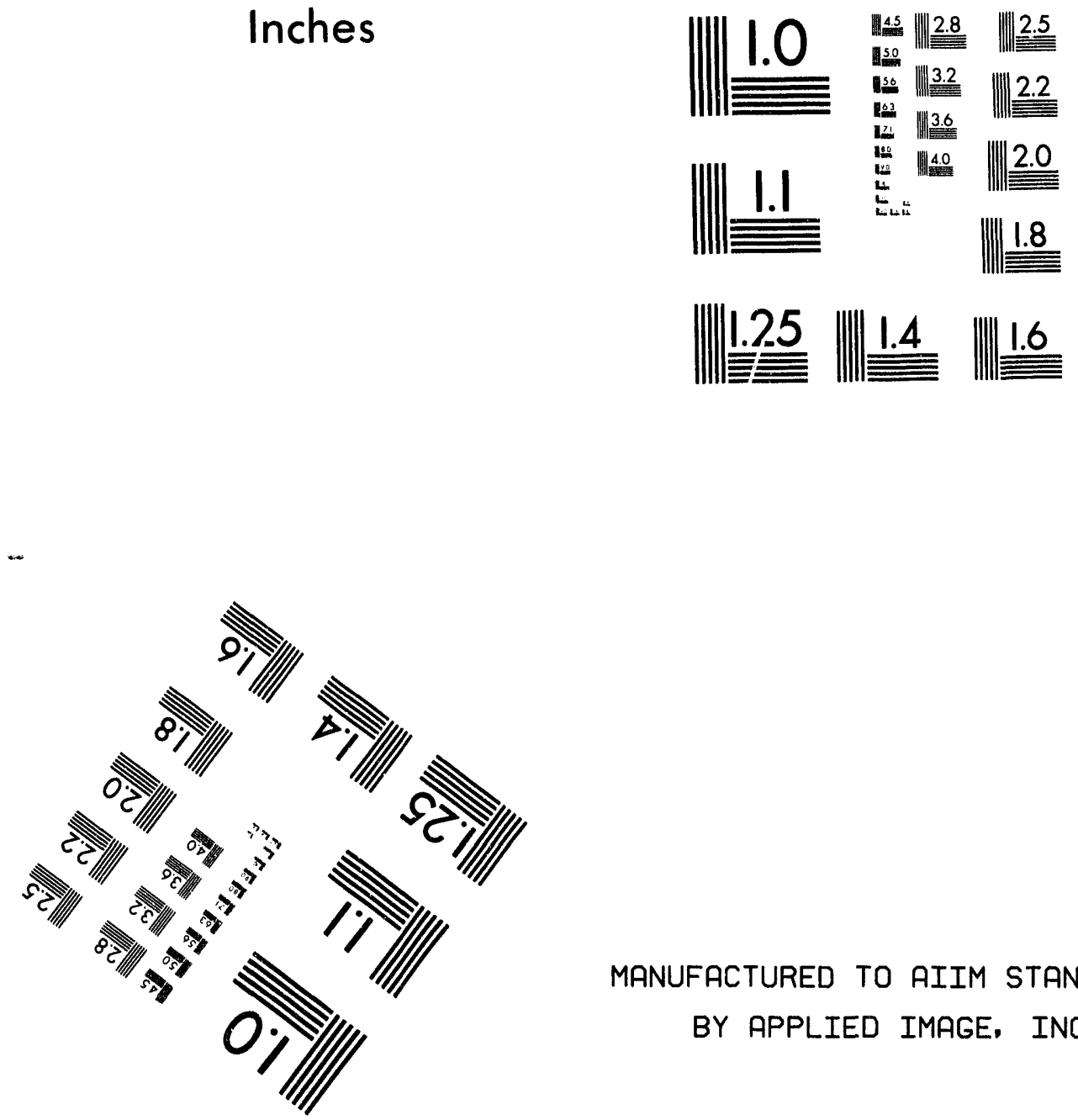

MANUFACTURED TO AIIM STANDARDS

BY APPLIED IMAGE, INC.

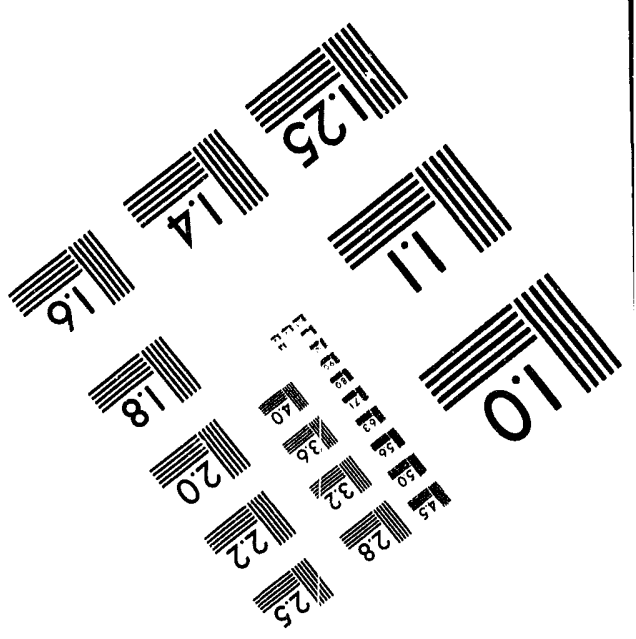



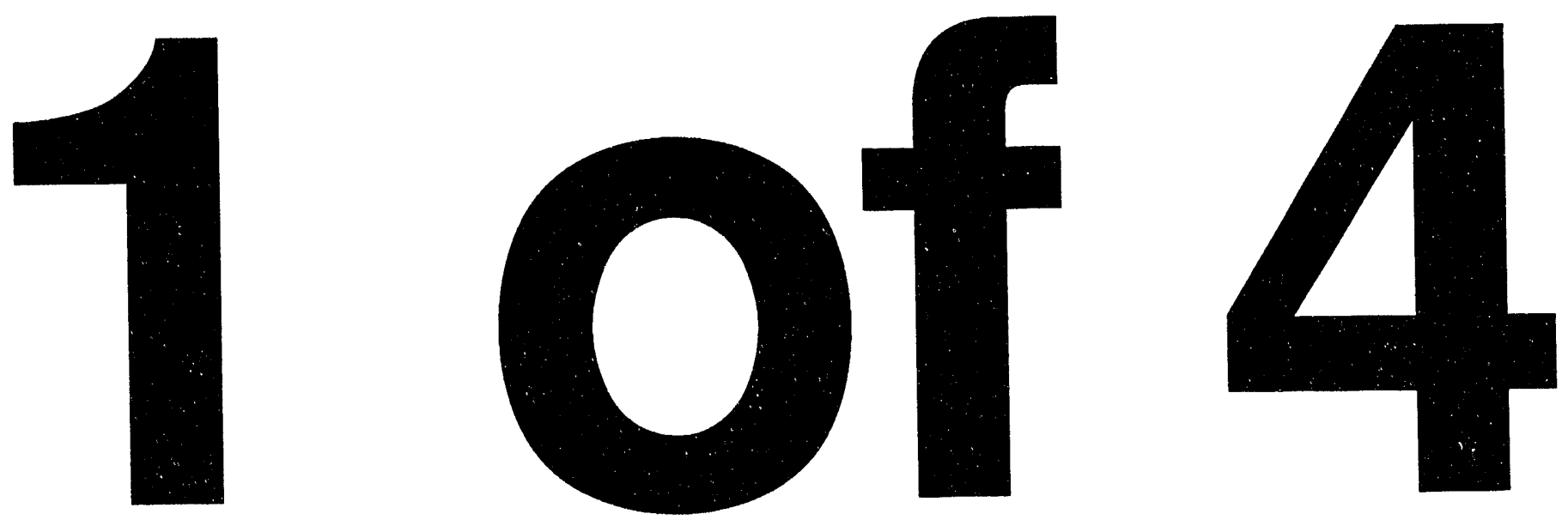


\section{Letter of Intent \\ for the \\ Study of $C P$ Violation and \\ Heavy Flavor Physics \\ at PEP-II}

The BABAR Collaboration

June 18, 1994

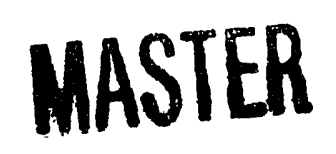


This document and the material and data contained therein, was developed under sponsorship of the United States Government. Neither the United States nor the Department of Energy, nor the Leland Stanford Junior University, nor their employees, nor their respective contractors, subcontractors, or their employees, makes any warrenty, express or implied, or assumes any liability or responsibility for accuracy, completeness or usefulness of any information, apparatus, product or process disclosed, or represents that its use will not infringe privately owned rights. Mention of any product, its manufacturer, or suppliers shall not, nor is intended to, imply approval, disapproval, or fitness for any particular use. A royalty-free, nonexclusive right to use and disseminate same for any purpose whatsoever, is expressly reserved to the United States and the University. 
Front Cover: A cutaway view of the BABAR detector showing from inside to outside: the support tube carrying beamline elements (purple) and the vertex detector (blue), the central tracking chamber (green), the particle identification system (blue), the CsI(Tl) crystal calorimeter (red), the superconducting coil (orange), and the instrumented flux return steel (blue). The proposed support system for the doors and the barrel, with cutaways for the routing of cables and services, is also shown. 
LETTER OF INTENT FOR THE BABAR Detector 


\section{The BABAR Collaboration}

LAPP Annecy, Annecy-le-Vieux, France

D. Boutigny, C. Goy, Y. Karyotakis, J.-P. Lees, S. Lees-Rosier

INFN, Sezione di Bari and Università di Bari, Bari, Italy

C. Evangelista, A. Palano

Beijing Glass Research Institute, Beijing, China

G. Chen, Y.T. Wang, O. Wen

Institute of High Energy Physics, Beijing, China

Y.N. Guo, H.B. Lan, H.S. Mao, N.D. Qi, W.G. Yan, C.C. Zhang, W.R. Zhao, Y.S. Zhu

University of Bristol, Bristol, UK

N. Dyce, B. Foster, R.S. Gilmore, C.J.S. Morgado.

University of British Columbia, Vancouver, British Columbia, Canada

C. Hearty, J. Heise, J. McKenna

Brunel University, London, UK

A.K. McKemey

Budker Institute of Nuclear Physics, Novosibirsk, Russia

M.N. Achasov, P.M. Beschasnov, A.V. Bozhenok, A.X. Buzykaev, K.G. Cherepanov, S.I. Dolinsky, V.P. Druzhinin, M.S. Dubrovin, V.N. Golubev, V.R. Groshev, V.N. Ivanchenko, G.M. Kolachev, S.G. Klimenko, A.L. Maslennikov, A.P. Onuchin, V.S. Panin, S.V. Peleganchuk, S.G. Pivovarov, A.A. Salnikov, S.I. Serednyakov, A.G. Shamov, V.A. Sidorov, Yu.I. Skovpen, V.A. Tayursky, V.I. Telnov, V.I. Tepligin, E.A. Undrus, Yu.V. Usov, V.I. Yurchenko

California Institute of Technology, Pasadena, California, USA

G. Eigen, D.G. Hitlin, J. Oyang, F.C. Porter, X. Shi, A.J. Weinstein, R. Zhu

University of California, Davis, Davis, California, USA

F. Rouse

University of California, IIRPA, La Jolla, California, USA

A.M. Eisner, M. Sullivan, W. Vernon, Y.-X. Wang

University of California, Irvine, Irvine, California, USA

K. Gollwitzer, A. Lankford, M. Mandelkern, G. McGrath, B. Schmid, J. Schultz,

D. Stoker, G. Zioulas 
University of California, Los Angeles, Los Angeles, California, USA

K. Arisaka, C. Buchanan, W. Slater, J. Park

University of California, Santa Barbara, Santa Barbara, California, USA

D. Bauer, D. Caldwell, A. Lu, H. Nelson, J. Richman, D. Roberts, M. Witherell, S. Yellin

University of California, Santa Cruz, Santa Cruz, California, USA

P. Burchat, D. Dorfan, A. Grillo, C. Heusch, R. Johnson, W. Lockman, K. O'Shaughnessy,

H. Sadrozinski, A. Seiden

Carleton University and CRPP ${ }^{\dagger}$, Ottawa, Ontario, Canada

K. Edwards, D. Karlen, M. O'Neill ${ }^{\dagger}$

University of Cincinnati, Cincinnati, Ohio, USA

B. Meadows, A. Santha, M. Sokoloff

University of Colorado, Boulder, Colorado, USA

E. Erdos, W. Ford, U. Nauenberg, P. Rankin, J. Smith

Colorado State University, Fort Collins, Colorado, USA

R. Malchow, M. Smy, H. Staengle, W. Toki, D. Warner, R. Wils $r n$

Technische Universität Dresden, Institut für Kern- und 'Teilchenphysik, Dresden, Germany P. Eckstein, K.R. Schubert, R. Schwierz, R. Waldi

Joint Institute for Nuclear Research, Dubna, Russia

A. Bannikov, G. Chelkov, Yu. Gornushkin, M. Ignatenko, Z. Krumstein, A. Nozdrin,

N. Rusakovich, A. Sissakian, Z. Silagadze, L. Vertogradov, Yu. Yatsunenko

University of Edinburgh, Edinburgh, UK

K. Peach, A. Walker

INFN, Sezione di Ferrara, Ferrara, Italy

L. Piemontese

Laboratori Nazionali di Frascati dell' INFN, Frascati, Italy

R. Baldini, A. Calcaterra, R. De Sangro, I. Peruzzi (also Univ. Perugia), M. Piccolo, A. Zallo

INFN, Sezione di Genova and Università di Genova, Genova, Italy

R. Contri, G. Crosetti, P. Fabbricatore, M. Macri, R. Parodi, S. Passaggio, C. Patrignani,

M.G. Pia, A. Santroni, S. Squarcia

University of Houston, Houston, Texas, USA

K. Lau, B. Mayes, G. Mo, L. Pinsky, J. Pyrlik, R. Weinstein 
University of Iowa, Iowa City, Iowa, USA

U. Mallik, E. Mccliment, M.-Z. Wang

Iowa State University, Ames, Iowa, USA

H.B. Crawley, A. Firestone, J.W. Lamsa, W.T. Meyer, E. I. Rosenberg

Universität Karlsruhe, Institut für Experimentelle Kernphysik, Karlsruhe, Germany

W. de Boer, G. Maehlum, S. Weseler

University of Lancaster, Lancaster, UK

C.J. Bowdery, A. Finch, F. Foster

Lawrence Berkeley Laboratory, Berkeley, California, USA

G.S. Abrams, H. Bingham, A. Ciocio, C. Day, S. Dow, G. Gidal, R. Jared, J. Kadyk, L. Kerth, J.F. Kral, M. Levi, G.R. Lynch, P. Oddone, M. Pripstein, D. Quarrie, J. Rasson, N. Roe, M. Ronan, H. Spieler, W. Wenzel, S. Wunduke

Lawrence Livermore National Laboratory, Livermore, California, USA

R.M. Bionta, F.S. Dietrich, O.D. Fackler, B.A. Fuchs, M.N. Kreisler, M.A. Libkind,

N. Martovetsky, G.J. Mauger, M.J. Mugge, W. Stoeff, L. Pedrotti, K. van Bibber, T.J. Wenaus,

D.M. Wright, C.R. Wuest, R.M. Yamamoto

University of Liverpool, Liverpool, UK

J.R. Fry, E. Gabathuler, P. Sanders

University of London, Imperial College of Science, Technology and Medicine, London, UK

M. Cattaneo, P. Dornan, J. Nash, A. Duane, D. Price

University of London, Queen Mary \& Westfield College, London, UK

D. Bugg, P.F. Harrison, B. Zou

University of London, Royal Holloway \& Bedford New College, Egham, Surrey, UK

Y. Gao, M.G. Green

McGill University Montréal, Quebec, Canada

D. Britton, R. Fernholz, D. MacFarlane, P. Patel, C. Smith, B. Spaan

University of Manchester, Manchester, $U K$

J. Allison, R. Barlow, G. Lafferty

University of Maryland, College Park, Maryland, USA

C. Dallapiccola, H. Jawahery, A. Skuja

University of Massachusetts, Amherst, Massachusetts, USA

J. Button-Shafer, J.J. Gomez-Cadenas, S.S. Hertzbach, R.R. Kofler, M.G. Strauss 
Massachusetts Institute of Technology, Cambridge, Massachusetts, USA

R.F. Cowan, M.J. Fero, R.K. Yamamoto

Max-Planck-Institute für Physik, Munich, Germany ${ }^{1}$

D. Brown, H. Kroha, E. Lorenz, H. Seywerd

INFN, Sezione di Milano and Università di Milano, Milano, Italy

R. Diaferia, F. Palombo, A. Sala

University of Mississippi, Oxford, Mississippi, USA

S. Bracker, L. Cremaldi, K. Gounder, J. Reidy, D. Summers

Université de Montréal, Montréal, Quebec, Canada

M. Beaulieu, J.P. Martin, P. Taras, V. Zacek

Mount Holyoke College, South Hadley, Massachusetts, USA

H. Nicholson, S. Sutton

INFN, Sezione di Napoli and Università di Napoli, Napoli, Italy

N. Cavallo, S. Lanzano, C. Sciacca

National Taiwan University, Taipei, Taiwan

W.-S. Hou

University of Notre Dame, Notre Dame, Indiana, USA

J. Bishop, N. Biswas, N. Cason, J. LoSecco, A. Sanjari, W. Shephard

Oak Ridge National Laboratory/Y-12, Oak Ridge, Tennessee, USA

F.S. Alsmiller, R.G. Alsmiller, Jr., T. A. Gabriel, J.L. Heck, Jr., R.A. Todd

LAL Orsay, Orsay, France

R. Cizeron, A.-M. Lutz, M.-H. Schune, E. Torassa, G. Wormser

INFN, Sezione di Padova and Università di Padova, Padova, Italy

F. Dalcorso, U. Dosselli, M. Posocco, R. Stroili, C. Voci

Ecole Polytechnique Palaiseau, LPNHE, Palaiseau, France

G. Bonneaud, J. M. Dieulot, G. Vasileiadis

LPNHE des Universités Paris 6 et Paris 7, Paris, France ${ }^{2}$

H. Briand, J. Chauveau, P. David, C. De La Vaissiere, L. Del Buono, J.F. Genat,

O. Hamon, J. Lory

\footnotetext{
${ }^{1}$ Participation in the preparation of the Letter of Intent

${ }^{2}$ Subject to approval by the Scientific Council of the LPNHE, Paris 6 (to be held end of June, 1994) 
Collége de France, Laboratoire de Physique Corpusculaire, Paris, France ${ }^{3}$

M. Benayoun, P. Leruste, J.-L. Narjoux

INFN, Sezione di Milano and Università di Pavia, Pavia, Italy

P.F. Manfredi, V. Re, V. Speziali

University of Pennsylvania, Philadelphia, Pennsylvania, USA

L. Gladney

INFN, Sezione di Pisa, Università di Pisa ${ }^{\dagger}$ and Scuola Normale Superiore ${ }^{\ddagger}$, Pisa, Italy G. Batignani ${ }^{\dagger}$, F. Bosi, U. Bottigli ${ }^{\dagger}$, M. Carpinelli, F. Costantini ${ }^{\dagger}$, F. Forti, M. Giorgi ${ }^{\dagger}$, A. Lusiani ${ }^{\ddagger}$, P.S. Marrocchesi, M. Morganti ${ }^{\dagger}$, G. Triggiani ${ }^{\dagger}$, J. Walsh

Princeton University, Princeton, New Jersey, USA

C. Bula, C. Lu, K.T. McDonald, M.V. Purohit

INFN, Instituto Superiore di Sạità, Roma, Italy

C. Bosio

Rutgers University, Rutgers, New Jersey, USA

P. Jacques, M. Kalelkar, R. Plano, P. 'Stamer

Rutherford Appleton Laboratory, Chilton, Didcot, UK

P.D. Dauncey, B. Franek, N.I. Geddes, G.P. Gopal

CEA, DAPNIA, CE-Saclay, Gif-sur-Yvette, France

R. Aleksan, A. Amadon, P. Besson, T. Bolognese, P. Bourgeois, A. De Lesquen, A. Gaidot,

G. Hamel de Monchenault, P. Jarry, G. London, M. Turluer, G. Vasseur, M. Zito

Shanghai Institute of Ceramics (SICCAS), Shanghai, China

J.R. Jing, P.J. Li, D.S. Yan, Z.W. Yin

Stanford Linear Accelerator Center, Stanford University, Stanford, California, USA

D. Aston, R. Becker-Szendy, E. Bloom, G. Bowden, A. Boyarski, D. Briggs, F. Bulos, W. Burgess,

D. Cords, D. Coward, D. Coupal, W. Craddock, H. DeStaebler, J. Dorfan, W. Dunwoodie,

D. Freytag, R. Gearhart, T. Glanzman, G. Godfrey, G. Haller, J. Hewitt, T. Himel, J. Hoeflich, W. Innes, C. Jessop, H. Kawahara, M.E. King, P. Kunz, E. Lee, D.W.G.S. Leith, V. Lüth, H. Lynch, H. Marsiske, T. Mattison, D. Muller, M. Perl, G. Oxoby, H. Quinn, B. Ratcliff, R. Reif, S. Schaffner, R.H. Schindler, S. Shapiro, C. Simopolous, A. Snyder, E. Soderstrom, P. Stiles, A. Tolstykh, J. Vav'ra, S. Wagner, R. Wang, W. Wisniewski

Academia Sinica, Taipei, Taiwan

H.-Y. Chau, M.-L. Chu, S.-C. Lee

\footnotetext{
${ }^{3}$ Subject to approval
} 
University of Texas at Dallas, Richardson, Texas, USA

J.M. Izen, X. Lou

INFN, Sezione di Torino and Università di Torino, Torino, Italy

P. Bianchi, D. Gamba, A. Romero

INFN, Sezione di Torino and Università di Torino, Torino, Italy

L. Bosisio

TRIUMF, Vancouver, British Columbia, Canada

R. Henderson, A. Trudel

Tsinghua University, Beijing, China

Y.P. Kuang, R.C. Shang, B.B. Shao, J.J. Wang

Vanderbilt University, Nashville, Tennessee, USA

R.S. Panvini, P. Sheldon, T. Reeves

University of Victoria, Victoria, British Columbia, Canada

C. Goodenough, M. McDougald, D. Pitman

University of Wisconsin, Madison, Wisconsin, USA

H.R. Band, J.R. Johnson, R. Prepost, G.H. Zapalac

York University, Toronto, Ontario, Canada

W. Frisken

Babar and the distinctive likeness are trademarks of

Laurent de Brunhoff and are used with his permission.

Copyright (C) Laurent de Brunhoff

All Rights Reserved 


\section{Table of Contents}

1 Introduction

2 Physics at PEP-II 5

$2.1 C P$ Violation in $B^{0}$ Decays . . . . . . . . . . 6

2.i.1 Decay Asynmetries . . . . . . . . . . 6

2.1.2 The Unitarity Triangle . . . . . . . . . 8

$2.2 \quad B$ Physics . . . . . . . . . . . . . 10

2.2.1 CKM Matrix Elements ........... 10

2.2.2 Beyond the Standard Model . . . . . . . . . 14

2.2.3 Penguin Modes . . . . . . . . . . . . 15

2.3 Other Physics Opportunities . . . . . . . . . 16

2.3.1 $B_{s}$ Physics . . . . . . . . . . 16

2.3.2 Charm Physics . . . . . . . . . . 16

$2.3 .3 \tau$ Physics ............... 17

2.3.4 Two-Photon Physics . . . . . . . . . . 18

3 Detector Overview 23

3.1 General Design Considerations . . . . . . . . . 24

3.1.1 Physics Constraints . . . . . . . . . . 24

3.1 .2 Acceptance . . . . . . . . . . . . 24

3.1.3 Resolution and Multiple Scattering . . . . . 27

3.1.4 Photon Efficiency and Resolution . . . . . . 27

3.1.5 Identification of Hadrons . . . . . . . . . . 28 
3.1.6 Identification of Leptons . . . . . . . . . 28

3.1.7 Interaction with the Accelerator . . . . . . 28

3.1.8 Considerations of Cost and Schedule . . . . . 29

3.2 The Baseline Design $\ldots \ldots$. . . . . . . . . 30

3.3 Other Geometry Options . . . . . . . . . . . . 35

3.3.1 Long-Barrel Designs . . . . . . . . . . . 36

3.3.2 Liquid Krypton Calorimeter Designs . . . 37

3.3.3 Designs with Lower Magnetic Field . . . . . 37

3.3.4 Reports on Experimental Options . . . . . . 38

3.4 Luector Performance and Sensitivity to $C P$ Asymmetries . . . . . . . . . . . . . . 38

4 PEP-II and the Interaction Region 43

4.1 Luminosity Optimization . . . . . . . . . . . . 4 43

4.1 .1 Tune-Shift Limits . . . . . . . . . . . . . . 45

4.1.2 Energy Transparency and Luminosity . . . . . 46

4.1 .3 Beam Separation . . . . . . . . . . . . 47

4.1 .4 PEP-II Design . . . . . . . . . . . . . 48

4.2 Components . . . . . . . . . . . . . . . . . . 49

$4.2 .1 \quad$ Lattice Design . . . . . . . . . . . . . . 51

4.2 .2 Vacuum System . . . . . . . . . . . . 51

4.2 .3 RF System . . . . . . . . . . . . . 52

4.3 Interaction Region $\ldots \ldots \ldots \ldots \ldots$

4.3.1 Beampipe Design . . . . . . . . . . . . 55

4.3 .2 Support Tube . . . . . . . . . . . . . . 55

4.4 Backgrounds . . . . . . . . . . . . . . . 56

4.4 .1 Overview .............. 56 
4.4.2 Synchrotron Radiation Backgrounds . . . . 58

4.4.3 Detector Backgrounds due to Lost Beam Particles ................ 62

4.4.4 Detector Backgrounds due to Radiative Bhabhas ................ 63

4.4 .5 Summary .............. 65

5 Vertex Detector $\quad 69$

5.1 Design Considerations ............ 70

5.2 Intrinsic Resolution of Microstrip Detectors . . . . 71

5.3 Silicon Strip Baseline Design . . . . . . . . . . 73

5.4 Resolution of the Baseline Design . . . . . . . . 76

5.5 Acceptance for Low-Momentum Particles . . . . . . . 78

5.6 Detector Specifications . . . . . . . . . . 80

5.7 Mechanical Support . . . . . . . . . . 81

5.8 Machine-Related Backgrounds and Radiation Hardness 82

5.9 Front-End Electronics and Data Transmission . . . . 84

5.10 Forward $\mu$ TPC Option . . . . . . . . . 86

$5.10 .1 \mu$ TPC Setup and Performance . . . . . . 86

5.10 .2 R\&D Issues and Prototype Schedule . . . . . 89

5.11 Pixel Detector Option . . . . . . . . . . 89

6 Main Tracking Chamber 93

6.1 Requirements ................ 94

6.2 Design Considerations . . . . . . . . . . 96

6.3 Conceptual Chamber Design . . . . . . . . . . . 98

6.3.1 Geometrical Shape and Dimensions . . . . . . 99

6.3.2 Inner and Outer Shells and the Endplates . . 100 
6.3.3 Cell Design . . . . . . . . . . . . . 102

6.3.4 Layer Arrangement . . . . . . . . . . . . . . 103

6.3.5 Wires and Feedthroughs .......... 105

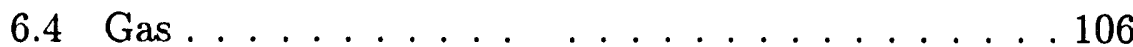

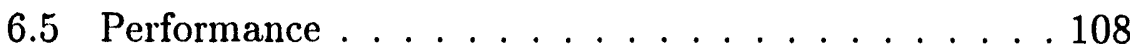

6.6 Effect of Backgrouinds . . . . . . . . . . 111

6.7 Front-End Electronics . . . . . . . . . . . . 113

6.8 High Voltage and Control System . . . . . . . . . 114

6.9 R\&D Program . . . . . . . . . . . . . . 115

7 Particle Identification $\quad 119$

7.1 Physics Requirements . . . . . . . . . . . . . 119

7.1.1 Inclusive Spectra and B-Flavor Tagging . . . 119

7.1.2 Exclusive $B$ Decays . . . . . . . . . . . . 121

7.1.3 Summary of Requirements . . . . . . . . . . . 121

7.2 Options for Particle Identification . . . . . . . . . 122

7.2.1 $d E / d x$ and Kinematic Separation . . . . . . 122

7.2.2 Time-of-Flight Counter System . . . . . . . 125

7.2.3 Dedicated Cherenkov Devices . . . . . . . 127

7.3 Aerogel Threshold Counters . . . . . . . . . . . . 128

7.3.1 Conceptual Design . . . . . . . . . . 130

7.3.2 Expected Physics Performance . . . . . . . . 134

7.3.3 R\&D Program . . . . . . . . . . . . . 137

7.4 DIRC . . . . . . . . . . . . . . 138

7.4.1 Conceptual Design . . . . . . . . . . . 139

7.4 .2 Geometric Layouts . . . . . . . . . . . . 141

7.4.3 Existing Experimental Measurements . . . . . 143

LETTER OF INTENT FOR THE BABAR DETECTOR 
7.4.4 Expected Physics Performance . . . . . . . 147

7.4.5 R\&D Program . . . . . . . . . . . . . 149

7.5 Fast RICH . . . . . . . . . . . . . . 151

7.5.1 Conceptual Design ... . . . . . . 152

7.5.2 Existing Experimental Measurements . . . . . 155

7.5.3 Expected Physics Performance . . . . . . 158

7.5.4 R \& D Plans . . . . . . . . . . . . 159

7.6 Strategy for choosing a Particle ID System . . . . . 161

7.6.1 Proof-of-principle Requirements for Cherenkov PID systems . . . . . . . . . . . . . 161

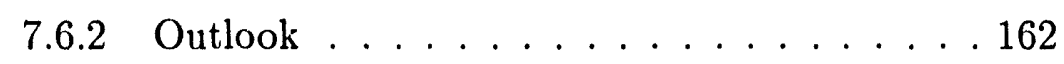

8 Electromagnetic Calorimeter $\quad 167$

8.1 Calorimeter Requirements . . . . . . . . . 167

8.1.1 Physics Requirements . . . . . . . . . 167

8.1.2 Radiation and Backgrounds ........ . 171

8.2 Choice of Technology . . . . . . . . . . . . 172

8.2.1 General Performance . . . . . . . . . . 172

8.2.2 Physics Considerations . . . . . . . . . 173

8.2.3 Technology Risk . . . . . . . . . . . . 173

8.2.4 Impact on the Detector . . . . . . . . . 174

8.2.5 Cost and Schedule Impact . . . . . . . . . 174

8.2 .6 Conclusion . . . . . . . . . . . 175

8.3 Calorimeter Description . . . . . . . . . 175

8.3.1 Cesium Iodide Properties . . . . . . . . . . 175

8.3.2 Geometry and Crystal Dimensions . . . . . 175

8.3.3 Light Collection and Readout . . . . . . . . 177 
8.3.4 Mechanical Support Structure . . . . . . . 179

8.3.5 Electronics................ . 182

8.4 Calorimeter Performance . . . . . . . . . . . 183

8.4.1 Factors Influencing Energy Resolution . . . 183

8.4.2 Expected Performance for Photons . . . . 184

8.4.3 Expected Performance for $\pi^{0}$ 's . . . . . . . 189

8.5 Calibration, Monitoring and Stability . . . . . . . 192

8.6 Procurement and Assembly . . . . . . . . . 193

8.7 Optimization Studies . . . . . . . . . . 195

8.7.1 Light Collection and Readout . . . . . . . 195

8.7.2 Radiation Hardness Studies . . . . . . . 196

8.7.3 A Prototype Calorimeter . . . . . . . . . 198

9 Muon and Neutral Hadron Detector 201

9.1 Design Considerations . . . . . . . . . . . 202

9.2 Muon Identification . . . . . . . . . . . . . 204

$9.3 K_{L}^{0}$ Detection . . . . . . . . . . . 206

9.4 The Active Detectors . . . . . . . . . . . . . 208

9.4.1 Resistive Plate Chambers . . . . . . . . 209

9.4.2 Plastic Streamer Tubes . . . . . . . . . . 211

9.5 Collaborating Institutions . . . . . . . . . . 213

10 Magnet Coil and Flux Return 215

10.1 Physics Motivation . . . . . . . . . . . . 216

10.2 Magnet System Requirements . . . . . . . . . 217

10.2.1 So enoid Requirements . . . . . . . . . . 218

10.2.2 Flux Return Requirements . . . . . . . . 219 
10.3 Trade Studies . . . . . . . . . . . . 221

10.4 Solenoid Description . . . . . . . . . . . 223

10.5 Cryogen Supply Systtm . . . . . . . . . . 227

10.6 Procurement Plan . . . . . . . . . . . . 230

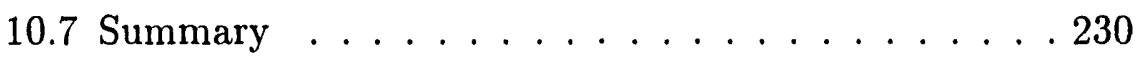

11 Electronics, Trigger, and Data Acquisition 235

11.1 Environment . . . . . . . . . . . . . 235

11.2 Trigger Requirements . . . . . . . . . . . . 236

11.3 System Organization . . . . . . . . . . . . 238

11.4 Trigger Rates . . . . . . . . . . . . . . . 239

11.4.1 Cosmic Rays . . . . . . . . . . . . 240

11.4.2 Lost Particles (Beam-Gas Scattering) . . . . . 241

11.4.3 Hadronic Interactions from Lost Particles . . . 242

11.4.4 Physics Triggers . . . . . . . . . . . . . . 243

11.4.5 Further Work on Trigger Rates . . . . . . . . 244

11.5 Implementation . . . . . . . . . . . . . . 245

11.5.1 Level 1 Electronics . . . . . . . . . . . . 245

11.5.2 Level 1 Trigger . . . . . . . . . . . . . 249

11.5.3 Connecting Level 1 to Level 2 . . . . . . . 250

11.5.4 Level 2 . . . . . . . . . . . . . . . 250

11.6 Research and Development . . . . . . . . . . 251

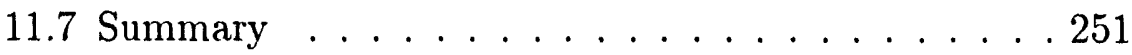

12 Computing $\quad 255$

12.1 Functional Requirements . . . . . . . . . . 255

12.1.1 CPU Requirements . . . . . . . . . . . 255 
12.1.2 Bandwidth. . . . . . . . . . . 257

12.1.3 Storage . . . . . . . . . . . . . . 259

12.2 Computing Model . . . . . . . . . . . . . 260

12.2.1 Computing Hardware and Operating System . 260

12.2.2 Networking . . . . . . . . . . . . 261

12.2.3 Integration of Remote Sites and Data Sharing 261

12.2.4 Event Processing Systems . . . . . . . . . . 262

12.2.5 Schematic Computing Model . . . . . . . . . 262

12.3 Software Environment . . . . . . . . . . 265

12.3.1 Development Environment . . . . . . . . . 266

12.3.2 Software Methodology . . . . . . . . . 267

12.3.3 Data Model . . . . . . . . . . . . 268

12.3.4 Code Management . . . . . . . . . . . . 269

12.4 Analysis Framework . . . . . . . . . . . . 270

12.5 On-line computing . . . . . . . . . . 270

12.5.1 General Requirements . . . . . . . . . 271

12.5.2 Monitor and Control . . . . . . . . . . 272

12.5.3 Partitioning and Configuration . . . . . . . 272

12.5.4 Accelerator/Detector Interface . . . . . . . 273

12.6 Computing Support . . . . . . . . . . . . . 274

13 CP Asymmetry Simulations 279

13.1 Simulation Tools . . . . . . . . . . . . . . 279

13.1 .1 ASLUND . . . . . . . . . . . . 279

13.1.2 GEANT Simulation - BBSIM . . . . . . . 280

13.2 CP Reach Calculations . . . . . . . . . . . . 281

13.2.1 Kaon Tags . . . . . . . . . . . . . 283 
13.2.2 Lepton Tags . . . . . . . . . . . . . . . . . 285

13.2.3 CP Asymmetry Channels . . . . . . . . 285

$13.2 .4 B^{0} \rightarrow J / \psi K^{0} \ldots \ldots \ldots 286$

$13.2 .5 B^{0} \rightarrow J / \psi K^{* 0} \ldots \ldots \ldots \ldots \ldots 286$

$13.2 .6 B^{0} \rightarrow D^{+} D^{-} \ldots \ldots \ldots \ldots \ldots 28$

13.2.7 $B^{0} \rightarrow D^{*+} D^{*-} \ldots \ldots \ldots \ldots . \ldots 288$

$13.2 .8 B^{0} \rightarrow \pi^{+} \pi^{-} \ldots \ldots \ldots \ldots \ldots$

$13.2 .9 B^{0} \rightarrow \rho^{ \pm} \pi^{\mp} \ldots \ldots \ldots \ldots \ldots 1$

13.3 Summary of $C P$ Reach Analysis . . . . . . . . . . 291

14 Collaboration Issues 295

14.1 Membership . . . . . . . . . . . . . . . 295

14.2 Collaboration Council . . . . . . . . . . . . . 296

14.3 Spokesperson . . . . . . . . . . . . . . 296

14.4 Executive and Technical Boards, Finance Review Committee . . . . . . . . . . . . . . . 297

15 Project Organization and Management 299

15.1 Project Organization . . . . . . . . . . . . . . . . 299

15.2 Management . . . . . . . . . . . . . . . . . 301

15.3 Hall Facilities and Services . . . . . . . . . . . . 301

15.3.1 Facility Integration . . . . . . . . . . . 301

15.3.2 Conventional Systems _ . . . . . . . . . 303

15.4 Mechanical Integration . . . . . . . . . . . . 304

15.4.1 Subsystem Dimension Control . . . . . . . . 304

15.4.2 Cables, Utilities Routing . . . . . . . . . 305

15.5 Detector Assembly . . . . . . . . . . . . . . . 305

15.6 Detector Interface with PEP-II _ . . . . . . . 308 
15.7 Environmental Safety and Health . . . . . . . 309

15.7.1 Safety Analysis Report . . . . . . . . . . 309

15.7.2 Seismic Protection . . . . . . . . . . 310

15.7.3 Hazard Protection . . . . . . . . . 310

15.8 Electrical Engineering Integration $\ldots \ldots . \ldots 311$

15.9 Software Engineering Integration . . . . . . . . . 311

16 Budget and Schedule 313

16.1 Vertex Detector . . . . . . . . . . . . 314

16.2 Main Tracking Chamber . . . . . . . . . . 315

16.3 Particle Identification . . . . . . . . . . . . . 315

16.3.1 Aerogel Threshold Cherenkov Detector . . . . 316

16.3.2 DIRC Detector . . . . . . . . . . . 316

16.3.3 Fast RICH Detector . . . . . . . . . . . 317

$16.4 \mathrm{CsI}(\mathrm{Tl})$ Calorimeter . . . . . . . . . . . . . . . 317

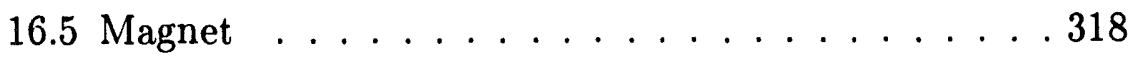

16.6 Flux Return Instrumentation . . . . . . . . . 318

16.7 Trigger and Data Acquisition . . . . . . . . . 318

16.8 Computing . . . . . . . . . . . . . . . 319

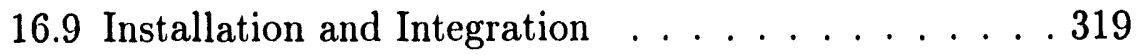




\section{Introduction}

$\mathbf{T}$ hirty years have elapsed since the discovery of $C P$ violation in $K_{L}^{0}$ decays. In those three decades, the Standard Model of elementary particle physics has evolved, providing us with a phenomenological explanation of a wide variety of experimental information. With three quark generations, this framework accommodates $C P$ violation in quark decays in an elegant and economical manner, through the imaginary parameter that, along with three rotation angles, serves to completely characterize the weak couplings of the six quarks as expressed in the Cabibbo-Kobayashi-Maskawa matrix. We do not know, however, whether this explanation is quantitatively correct, despite a staggering investment of experimental effort.

The study of $C P$-violating asymmetries in $B^{0}$ decays to $C P$ eigenstates promises, at last, to allow a test of the Standard Model explanation of $C P$ violation. More generally, the measurement of these $C P$ asymmetries can provide us with a series of unique, and uniquely stringent, consistency tests of the quark sector of the Standard Model. These tests, which will be carried out by the BABAR detector at PEP-II, will provide us with perhaps the best chance to challenge the Standard Model in new and quantitatively precise ways. This challenge has come about through the interplay of a series of experimental and theoretical events.

While it had been understood for several years that the measurement of $C P$-violating asymmetries in $B^{0}$ decay could lead to important tests of the CKM matrix, the experiments seemed beyond reach. The discovery of a surprisingly long $b$-quark lifetime by MAC [Fer83] and Mark II [Loc83] at PEP in 1983, together with that of unexpectedly large $B^{0}-\bar{B}^{0}$ mixing by UA1 [Alb87a] and ARGUS [Alb87b] in 1987, made it possible to conceive of making these measurements. It rapidly became clear [SNO89] that the richest and most straightforward approach to this problem involved the exploitation of a variety of $e^{+} e^{-}$machines, either in the $\Upsilon(4 S)$ re-
$C P$-violating asymmetries in $B^{0}$ meson decays can tell us whether the the CKM phase is the source of $C P$ violation

The long $b$ quark lifetime and the large $B^{0}$ mixing are essential to making $C P$ asymmetries measurable 
An asymmetric $e^{+} e^{-}$ $B$ Factory, such as PEP-II, provides an ideal tool for measuring $C P$ violation
SLAC has invited a single proposal for the single interaction region of PEP-II gion, in the continuum at PEP/PETRA, or at the $Z^{0}$ pole. Hadron machines, while they produce large numbers of $B$ mesons, have not, to this point, been convincingly shown to have equivalent capability. The most favorable $e^{+} e^{-}$experimental situation, that is the one producing the smallest statistical error with the least integrated luminosity is, by common consent, the asymmetric-energy storage ring first proposed by Oddone [Odd87]. By boosting the decaying $B^{0}$ mesons in the laboratory frame, existing vertex-measuring technology is able to cope with the short $B$ meson lifetime, an essential element to resolving the difference in the decay times of the $C P$ eigenstate and the tagging $B$ meson. However, even this machine requires event samples that are not obtainable without significant advances in storage ring luminosity.

PEP-II promises to provide the required luminosity [SLA91], initially $3 \times 10^{33} \mathrm{~cm}^{-2} \mathrm{~s}^{-1}$ and ultimately $10^{34}$, with asymmetric-energy $\Upsilon(4 S)$ production of $9 \mathrm{GeV}$ electrons on $3.1 \mathrm{GeV}$ positrons. The experimental challenge is then to provide high efficiency, high resolution exclusive state reconstruction in a situation new to the $e^{+} e^{-}$ world: a center-of-mass in motion in the laboratory. The boost is not extreme. At PEP-II, $90^{\circ}$ in the center-of-mass is at $65^{\circ}$ in the laboratory. Nevertheless, the challenges for the detector posed by the boost are novel, and not easily met.

Soon after the approval of PEP-II in October 1993, efforts began to form a major international collaboration to build and operate a new detector at PEP-II. The collider design has a single interaction region, time to build a detector before first collisions is short, and there has been a clear convergence of thinking [SLA93, BEL94, HEL92] about new detector designs over the past few years. Therefore, the SLAC directorate, with the approval of the Scientific Policy Committee, decided to invite a single group of experimentalists to submit a Letter of Intent and a Conceptual Design Report. The primary aim of this group is the detailed study of $C P$-violating asymmetries in $B^{0}$ meson decay, although a wide variety of other $B$, charm, $\tau$ and two-photon physics will also be accessible at new levels of precision. An inaugural meeting was held in December 1994. This has been followed by a series of collaboration meetings at regular intervals, leading to the writing of this Letter of Intent.

The group involved in this enterprise now numbers more than 400 physicists and engineers. The collaboration, guided by an Interim 
Steering Committee appointed by the Laboratory, has put in place a governance mechanism, has chosen a name, $B A B A R$, and has initiated the process of developing a colierent and detailed detector design. The detector described hesein is a conceptual design, which will be fleshed out in more detaii in the Conceptual Design Report that is to follow by the end of 1994. Efforts are nonetheiess sufficiently far advanced that it has been possible, in nearly all instances, to choose a technology for each detector subsystem. In the case of the particle identification system, there remains sufficient uncertainty as to the optimum choice that it seemed wiser to describe alternatives. The three alternative particle identification systems have been constrained to be compatible with a single overall detector geometry and set of dimensions. This allows detailed engineering of critical path systems such as the magnet and calorimeter to proceed without impediment.

The planning of the PEP-II project has been done in sufficient detail that the Laboratory is confident that it can be constructed in five years, starting in October 1993. We take it as a most important constraint that a detector capable of measuring $C P$ violating asymmetries in $B^{0}$ meson decay must be operational on a time scale comparable to that for building the accelerator.

The next stage beyond the Letter of Intent will be the creation of a management structure for the project, the assignment of responsibilities in detector construction and the development of the Conceptual Design Report. This must be accompanied by the conclusion of financial arrangements with the Laboratory and the funding agencies of the various countries represented, and by the establishment of Memoranda of Understanding that detail the specific responsibilities of each collaborating group.
The detector design will be described in more detail in the Conceptual Design Report 


\section{References}

[Alb87a] C. Albejar et al., (UA1 Collaboration), Phys. Lett. 186B, 247 (1987).

[Alb87b] H. Albrecht et al., (ARGUS Collaboration), Phys. Lett. 192B, 245 (1987).

[BEL94] Letter of Intent for A Study of $C P$ Violation in $B$ Meson Decays, The BELLE Collaboration, submitted to the TRISTAN Program Advisory Committee, February, 1994.

[Fer83] E. Fernandez et al., (MAC Collaboration), Phys. Rev. Lett. 51, 1022 (1983).

[HEL92] HELENA, A Beauty Factory at Hamburg, DESY Report DESY 92-41 (1992).

[Loc83] N. Lockyer et al., (Mark II Collaboration), Phys. Rev. Let.t. 51, 1316 (1983).

[Odd87] P. Oddone, in Proceedings of the UCLA Workshop: Linear Collider $B \bar{B}$ Factory Conceptual Design, D. Stork, ed., World Scientific, p. 243 (1987).

[SLA91] An Asymmetric B Factory Based on PEP: Conceptual Design Report, M. Zisman, ed., SLAC-372, LBL PUB5303 (1991).

[SLA93] Status Report on the Design of a Detector for the Study of CP Violation at PEP-II at SLAC, SLAC-419 (1993).

[SNO89] Report of the $B$ Physics Group: 1. Physics and Techniques, G. Feldman et al., in Proceedings of the 1988 DPF Summer Study, High Energy Physics in the 1990's (Snowmass, '88), 561 (1989). 


\section{Physics at PEP-II}

$\mathbf{C P}$ violation remains one of the most compelling issues in particle physics. In the Standard Model, the source of $C P$ violation is the Cabibbo-Kobayashi-Maskawa (CKM) matrix which describes the mixing of the quarks. We have no evidence that this is the full explanation of the observed $C P$ asymmetry, however. The central prediction of the Standard Model explanation of $C P$ violation is the existence of large, calculable asymmetries in the decays of the $B^{0}$ into $C P$ eigenstates.

These asymmetries are best measured at an asymmetric $e^{+} e^{-}$storage ring running at the $\Upsilon(4 S)$. This is the primary motivation for building PEP-II and the BABAR detector. The colliding $e^{+} e^{-}$beams produce an $\Upsilon(4 S)$ which decays approximately $50 \%$ of the time to $B^{+} B^{-}$and $50 \%$ to $B^{0} \bar{B}^{0}$. The two $B$ mesons are almost at rest in their center of mass with no additional particles making this an ideal situation for complete reconstruction of rare decay modes. The PEP-II electron beam energy is $9 \mathrm{GeV}$; the positron beam energy is $3.1 \mathrm{GeV}$, producing the $\Upsilon(4 S)$ state at a $\beta \gamma$ of 0.56 . This gives the $\Upsilon(4 S)$ system sufficient boost that the two $B^{0}$ decay vertices can be reconstructed and the distance between their positions can be used to determine the time ordering between the two decays. It is therefore possible to measure the dependence of the $C P$ asymmetry on the relative decay time of the two $B^{0}$ mesons, from which one can extract a precise measurement of the $C P$-violating parameters in the CKM matrix. The separated $B$ vertices also lead to improved pattern recognition and background rejection in the reconstruction of rare $B$ decays. This fact and the unprecedented luminosity of PEP-II thus provide an ideal facility for the study of these decays.
An asymmetric $e^{+} e^{-}$ storage ring provides the best experimental access to the study of $C P$ violation in $B^{0}$ decays 


\section{1 $C P$ Violation in $B^{0}$ Decays}

The primary physics goal of PEP-II is to overdetermine the parameters of the $C \bar{K} M$ matrix
The design of a detector for PEP-II must be governed by the primary physics objective of the facility [SLA89], which is to overdetermine the CKM parameters of the Standard Model and thereby- to seek evidence for physics beyond the Standard Model. This will be done by making many independent measurements of the magnitudes and relative phases of CKM matrix elements and in particular of $C P$-violating asymmetries in various channels of $B^{0}$ decays to $C P$ eigenstates [Nir93].

\subsubsection{Decay Asymmetries}

The two mass eigenstates of the neutral $B$ meson system can be written as:

$$
\begin{aligned}
& \left|B_{L}>=p\right| B^{0}>+q \mid \bar{B}^{0}> \\
& \left|B_{H}>=p\right| B^{0}>-q \mid \bar{B}^{0}>
\end{aligned}
$$

where $H$ and $L$ stand for Heavy and Light, respectively. Defining $M \equiv\left(M_{H}+M_{L}\right) / 2, \quad \Delta M \equiv M_{H}-M_{L}$, and neglecting the tiny difference in width between $B_{H}$ and $B_{L}$, the decay widths satisfy $\Gamma_{H}=\Gamma_{L} \equiv \Gamma$. In this approximation, mixing in the $B_{d}^{0}$ system is governed by a single phase:

$$
\left(\frac{q}{p}\right)_{B_{d}^{0}}=\frac{V_{t b}^{*} V_{t d}}{V_{t b} V_{t d}^{*}}=e^{2 i \phi_{M}}
$$

The amplitudes for decays into a $C P$ eigenstate $f$ are

$$
A \equiv<f|\mathcal{H}| B^{0}>, \quad \bar{A} \equiv<f|\mathcal{H}| \bar{B}^{0}>
$$

Let us define

$$
r(f) \equiv \frac{q}{p} \frac{\bar{A}}{A}
$$

The time-dependent rates for initially pure $B^{0}$ or $\bar{B}^{0}$ states to decay into a final $C P$ eigenstate at time $t$ can then be written as

$$
\Gamma\left(B_{\mathrm{phys}}^{0}(t) \rightarrow f\right)=|A|^{2} e^{-\Gamma t} \times\left(\frac{1+|r(f)|^{2}}{2}\right.
$$




$$
\begin{gathered}
\left.+\frac{1-|r(f)|^{2}}{2} \cos (\Delta M t)-\operatorname{Im} r(f) \sin (\Delta M t)\right) \\
\Gamma\left(\bar{B}_{\text {phys }}^{0}(t) \rightarrow f\right)=|A|^{2} e^{-\Gamma t} \times\left(\frac{1+|r(f)|^{2}}{2}\right. \\
\left.-\frac{1-|r(f)|^{2}}{2} \cos (\Delta M t)+\operatorname{Im} r(f) \sin (\Delta M t)\right)
\end{gathered}
$$

The time-dependent $C P$ asymmetry

$$
a_{f}(t)=\frac{\Gamma\left(B^{0}(t) \rightarrow f\right)-\Gamma\left(\bar{B}^{0}(t) \rightarrow f\right)}{\Gamma\left(B^{0}(t) \rightarrow f\right)+\Gamma\left(\bar{B}^{0}(t) \rightarrow f\right)}
$$

is given by

$$
a_{f}(t)=\frac{\left(1-|r(f)|^{2}\right) \cos (\Delta M t)-2 \operatorname{Im} r(f) \sin (\Delta M t)}{1+|r(f)|^{2}}
$$

This result holds for a $C P$-even final state, while for $C P$-odd states there is an additional minus sign in $r(f)$. When only a single amplitude with a given weak decay phase $\phi_{D}$ dominates the decay one has

$$
\bar{A}=e^{-2 i \phi_{D}}
$$

Since $\operatorname{Im} r(f)=\sin 2\left(\phi_{M}-\phi_{D}\right)$, equation 2.8 simplifies to

$$
a_{f}(t)=-\sin 2\left(\phi_{M}-\phi_{D}\right) \sin (\Delta M t)
$$

Thus, at $t \approx 2.3 \tau_{B^{0}}$, the asymmetry reaches its maximum absolute value of $\left|\sin 2\left(\phi_{M}-\phi_{D}\right)\right|$.

At PEP-II the initial $B^{0}$ and $\bar{B}^{0}$ are produced in a coherent state which remains a $B^{0} \bar{B}^{0}$ until such time as one of the particles decays. If one $B^{0}$ decays to a flavor-tagging mode while the other decays to a $C P$-eigenstate we have an event that can be used to reconstruct the time dependence of the asymmetry. The time that appears in the equations above is the time difference between the tagging decay and the $C P$-eigenstate decay. The tagging decay may be the later decay, in which case a negative time is assigned to the event. Note the essential nature of the measurement of time dependence, since The $B^{0}$ and $\bar{B}^{0}$ are produced in a coherent state, so the timedifference of their decays must be known the time-integrated $C P$ asymmetry vanishes if $|r(f)|=1$.

The consistency of these measurements with Standard Model predictions will provide information on whether the $C P$-violating phase 
Theories of the baryon asymmetry of the universe suggest sources of $C P$ violation beyond the Standard Model

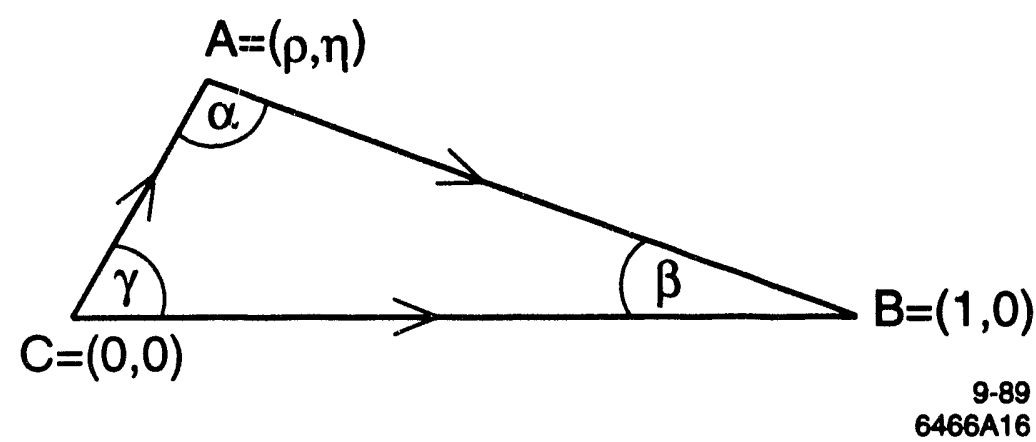

Figure 2-1. Representation in the complex plane of the rescaled unitarity triangle.

of the CKM matrix is the sole source of $C P$ violation in nature, or whether instead, as suggested by attempts to understand the baryon number asymmetry of the universe, there are additional sources of $C P$ violation beyond the Standard Model [Hue94]. Additional effects can show up as a failure of Standard Model predictions such as those that relate measured asymmetries to the angles of the unitarity triangle as discussed below.

\subsubsection{The Unitarity Triangle}

The relationship

$$
V_{t d} V_{t b}^{*}+V_{c d} V_{c b}^{*}+V_{u d} V_{u b}^{*}=0
$$

among CKM matrix elements follows from the assumption of unitarity of the three generations. In this event, the three products $V_{i d} V_{i b}^{*}$ must form a closed triangle in the complex plane, referred to as the unitarity triangle. The three angles of this triangle are labeled

$$
\begin{aligned}
& \alpha \equiv \arg \left(-\frac{V_{t d} V_{t b}^{*}}{V_{u d} V_{u b}^{*}}\right) \\
& \beta \equiv \arg \left(-\frac{V_{c d} V_{c b}^{*}}{V_{t d} V_{t b}^{*}}\right) \\
& \gamma \equiv \arg \left(-\frac{V_{u d} V_{u b}^{*}}{V_{c d} V_{c b}^{*}}\right)
\end{aligned}
$$


A $3 \times 3$ unitary matrix can be fully described by three real and one imaginary parameters. A parametrization of the CKM matrix due to Wolfenstein [Wol85] is particularly instructive for understanding the structure of the matrix:

$$
\left(\begin{array}{ccc}
1-\lambda^{2} / 2 & \lambda & \lambda^{3} A(\rho-i \eta) \\
-\lambda & 1-\lambda^{2} / 2 & \lambda^{2} A \\
\lambda^{3} A(1-\rho-i \eta) & -\lambda^{2} A & 1
\end{array}\right)
$$

Figure 2-1 shows the unitarity triangle, as it is usually drawn, rescaled by the side $V_{c d} V_{c b}^{*}$. This makes the base of the triangle real and of unit length, while the apex is then the point $(\rho, \eta)$ in the complex plane.

To overdetermine this triangle, experiments must fix as many as possible of the parameters that give the sides, $\left|V_{t d} V_{t b}^{*}\right| /\left|V_{c d} V_{c b}^{*}\right|$ and $\left|V_{u d} V_{u b}^{*}\right| /\left|V_{c d} V_{c b}^{*}\right|$, and the angles $\alpha, \beta$, and $\gamma$ of the unitarity triangle. The quantities $\left|V_{u d}\right|=\cos \theta_{\mathrm{C}}+O\left(\lambda^{4}\right)$ and $\left|V_{t b}\right|=1+O\left(\lambda^{4}\right)$, where $\lambda=\sin \theta_{\mathrm{C}}$, are alrealy well known; the value of $V_{c b}$ will be accurately determined by a study of semileptonic $B$ decays at CLEO-II. Thus the primary aim of PEP-II is precision measurements of the value of $V_{u b}$, and the $C P$-violating asymmetries in the decay modes $B^{0} \rightarrow$ $J / \psi K_{S}^{0}, B^{0} \rightarrow J / \psi K^{* 0}, B^{0} \rightarrow D^{+} D^{-}, B^{0} \rightarrow \pi^{+} \pi^{-}, B^{0} \rightarrow \rho \pi$.

\begin{tabular}{|l|c|c|}
\hline Quark process & $B_{d}^{0}$ Mode & $\phi_{M}-\phi_{D}$ \\
\hline \hline$b \rightarrow c \bar{c} s$ & $J / \psi K_{S}^{0}$ & $\beta$ \\
$b \rightarrow c \bar{c} s$ & $J / \psi K^{* 0}$ & $\beta$ \\
\hline$b \rightarrow c \bar{c} d$ & $D^{+} D^{-}$ & $\beta$ \\
$b \rightarrow c \bar{c} d$ & $D^{*+} D^{*-}$ & $\beta$ \\
\hline$b \rightarrow u \bar{u} d, d \bar{d} d$ & $\pi^{+} \pi^{-}$ & $\alpha$ \\
$b \rightarrow u \bar{u} d, d \bar{d} d$ & $\rho^{ \pm} \pi^{\mp}$ & $\alpha$ \\
\hline$b \rightarrow s \bar{s} d$ & $\phi \pi^{0}$ & 0 \\
$b \rightarrow s \bar{s} d$ & $K_{S}^{0} K_{S}^{0}$ & 0 \\
\hline
\end{tabular}

Table 2-1. CP modes and Standard Model asymmetry predictions.

Table 2-1 shows the Standard Model relationship between the angles of the unitarity triangle and the predicted value of $\arg (r(f))=$ $2\left(\phi_{M}-\phi_{D}\right)$ for these modes, assuming that a single weak decay amplitude dominates in each of these cases. Channels for which

The question of whether a single weak decay amplitude dominates can be tested experimentally 
the Standard Model predicts zero asymmetry because $\phi_{M}=\phi_{D}$, such as $B_{d}^{0} \rightarrow \phi \pi^{0}$ and $B_{d}^{0} \rightarrow K_{S}^{0} K_{S}^{0}$, can be studied to test this assumption.

\section{$2.2 \quad B$ Physics}

\subsubsection{CKM Matrix Elements}

The $B_{A} B A R$ detector is also designed for the study of a broad range of physics beyond the $C P$-violating asymmetries. Its vertex separation, good particle identification and excellent electromagnetic calorimetry make it an excellent tool for the study of rare $B$-decay modes. The charmless-strangeless modes that can be used to determine $\left|V_{u b}\right| /\left|V_{c b}\right|$ are of particular importance, as this measurement, in combination with a determination of the two angles $\alpha$ and $\beta$, will provicle an overconstrained test of the Standard Model $C P$ violation predictions.

Present measurements of $\left|V_{u b}\right|$ from CLEO-II come from the study of the momentum spectrum of the lepton emitted in $B$ semileptonic decays past the end-point of $b \rightarrow c$ transitions at $2.4 \mathrm{GeV} / \mathrm{c}[\mathrm{Bar} 93 b]$. This measurement is experimentally clean, with leptons from continuum events as the major background, but the extraction of $\left|V_{u b}\right|$ from this tiny part of the phase space $(2.4-2.6 \mathrm{GeV} / c)$ is a major theoretical challenge. The precision of the determination of $\left|V_{u b}\right| /\left|V_{c b}\right|$ is thus currently dominated by large model-dependent uncertainties.

Progress in determining $\left|V_{u b}\right|$ will come from the detailed study of exclusive semileptonic $b \rightarrow u$ and $c \rightarrow d$ transitions to help refine

Improved measurements of $\left|V_{u b}\right|$ can be made using exclusive semileptonic $B$ decay modes and constrain theoretical models. The first step is the measurement of the branching fractions for $B \rightarrow(\pi, \rho, \omega) \ell \nu$ and $D \rightarrow(\pi, \rho, \omega) \ell \nu$. It is likely that this program can be initiated before the start of the $B_{A} B_{A R}$ experiment by either CLEO-II or by LEP experiments. The present upper limit from CLEO-II for these branching fractions is around $2 \times 10^{-4}$ [Bea93]. The next step requires the measurement of the various form factors involved in these decays. BABAR will have the alsility and the high statistics needed to perform these delicate measurements. Along with high luminosity, vertex separation and $\pi / K$ iclentification capabilities are the keys to the success of such 
measurements. The isolation of the $B$ (or $D) \rightarrow \rho \ell \nu$ decay, for instance, requires the identification of a common $\pi \pi \ell$ vertex detached from the other $B$ (or $D$ ) vertex. With $100 \mathrm{fb}^{-1}$ we expect $600 \mathrm{re-}$ constructed decays with small background, for a branching fraction sensitivity of $10^{-4}$. The excellent $\pi^{0}$ reconstruction capabilities of the detector are vital to the observation of the $\omega \ell \nu$ decay.

Another important reaction in the $b \rightarrow u$ sector is the purely leptonic decay $B^{+} \rightarrow \tau^{+} \nu_{\tau}$. This branching ratio measures the product $\left|V_{u b}\right| \times f_{B}$ and thus provides a determination of $f_{B}$ once $\left|V_{u b}\right|$ is known. This will in turn give a significant reduction in the error on $\left|V_{t d}\right|$ as deduced from $B^{0}$ oscillations. According to a preliminary analysis, the sensitivity of this experiment is estimated to be around $6 \times 10^{-5}$ for this branching ratio, while the Standard Model prediction lies around $10^{-4}$.

Measurement of the angle $\gamma$ provides a further method of overconstraining the unitarity triangle. The CP asymmetry in the decay $B_{s}^{0} \rightarrow \rho K_{S}^{0}$ produced at the $\Upsilon(5 S)$ is sensitive to $\sin 2 \gamma$, but the small $B_{s}^{0}$ production cross section and the small branching fraction of this mode make for a difficult measurement. Another option for determining $\gamma$ is the study of the modes $D_{C P} K$ in either charged or neutral $B$ decays [Gro91b], where $D_{C P}$ denotes either a $D^{0}$ or a $\bar{D}^{0}$ which decays to a $C P$-eigenstate mode such as $K_{S}^{-0} \pi^{0}$. While this experiment also looks difficult given the predicted branching fractions, it is important to maintain a capability for study of this mode. The efficient detection of $\pi^{0}$ 's by the electromagnetic calorimeter will be crucial for maintaining the required high acceptance.

Further constraints on the unitarity triangle come from the value of $\epsilon$ in $K_{L}^{0}$ decays [Gib93] and from the measured rate for $B^{0}-\bar{B}^{0}$ mixing [Bar93a]. At present there are significant theoretical and experimental uncertainties in the relationship between these measured quantities and the parameters of the underlying theory. However, the trend of steadily improving lattice calculations of quantities such as the $f_{B}$ and $B_{B}$ parameters may holp this situation. The status of the constraints on the unitarity triangle from existing measurements, plus theoretical estimates of these parameters, is shown in Figure 2-2. The inclicated contours correspond to the experimentally determined values for the measured quantities, but the uncertainties are dominated by the likely range for theoretical inputs or by model dependence. The values of the parameters used
The measurement of $f_{B}$ from the branching fraction of $B^{+} \rightarrow \tau^{+} \nu_{\tau}$ is a difficult but important goal

Measurement of the angle $\sin 2 \gamma$ with the $C P$ asymmetry in $B_{s}^{0} \rightarrow \rho K_{S}^{0}$ is difficult 


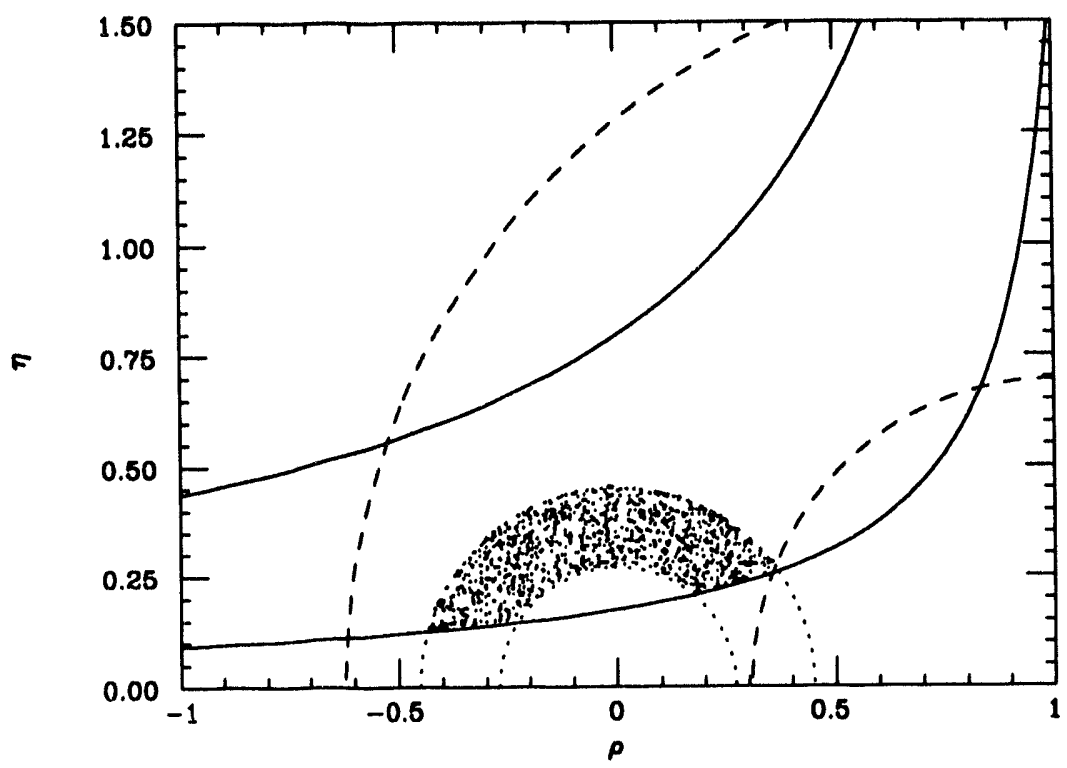

Figure 2-2. Constraints in the Standard Model on the rescaled unitarity triangle from $\left|V_{u b}\right| /\left|V_{c b}\right|$ (dotted circles), $B^{0}-\bar{B}^{0}$ mixing (dashed circles), and $\epsilon$ (solid hyperbolas). The shaded region is that allowed for the triangle apex $(\rho, \eta)$.

here are consistent with those given by Buras et al., [Bur94] and by lattice calculations. The value for the running top mass corresponds to the range claimed by CDF [Abe94], including statistical and systematic errors added in quadrature. This result translates into an allowed range for the three unitarity angles of:

$$
\begin{array}{r}
-0.89 \leq \sin 2 \alpha \leq 1.00 \\
0.18 \leq \sin 2 \beta \leq 0.81 \\
-1.00 \leq \sin 2 \gamma \leq 1.00
\end{array}
$$

Measurements in $B^{0}$ and $K$ decays pertinent to the precision of the unitarity triangle constraint will improve with time, as will theoretical calculations to some extent; the allowed region for $\eta$ and $\rho$ will shrink substantially as $C P$-violating asymmetries are measured [Bur94].

This picture can be altered if new physics is present, even if there are no new sources of additional $C P$-violating phases. Figure 23 shows the constraints in the $\rho-\eta$ plane for a two Higgs-doublet model, where one doublet generates a mass for the up-type quarks 


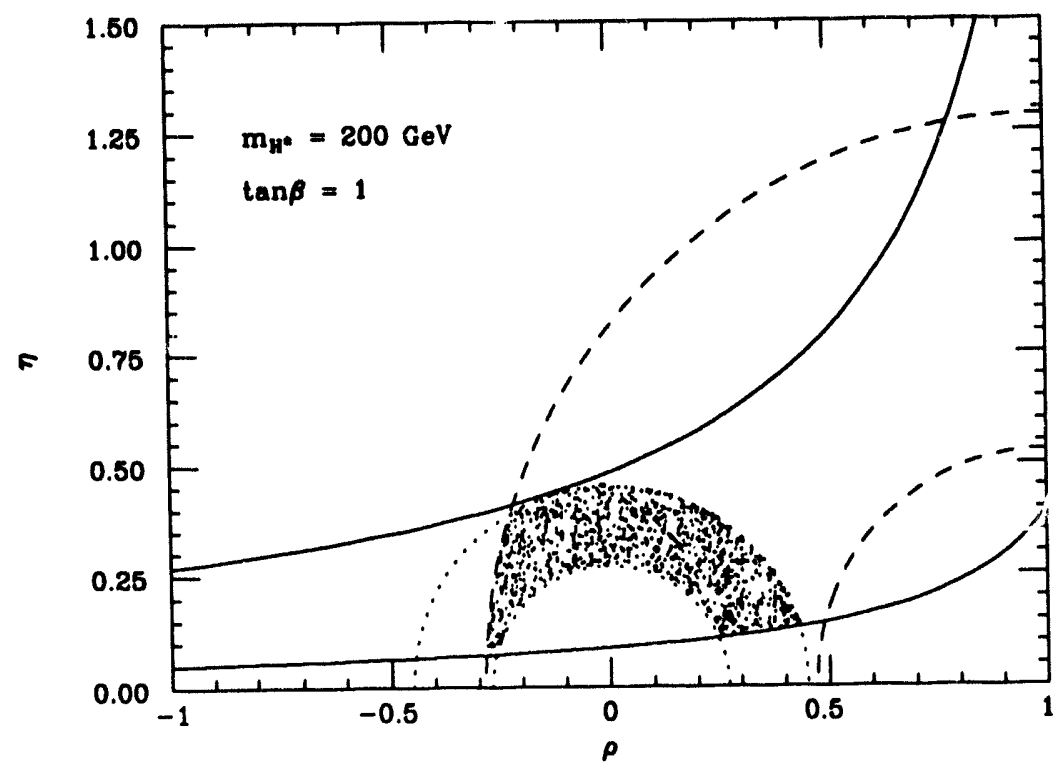

Figure 2-3. Constraints in the two Higgs-doublet model on the rescaled unitarity triangle from $\left|V_{u b}\right| /\left|V_{c b}\right|$ (dotted circles), $B^{0}-\bar{B}^{0}$ mixing (dashed circles), and $\epsilon$ (solid hyperbolas) with a charged Higgs mass of $200 \mathrm{GeV} / \mathrm{c}^{2}$ and the ratio of vacuum expectation values $\tan \beta=1$. The shaded region is that allowed for the triangle apex $(\rho, \eta)$.

and the down-type quarks reccive their masses from the second doublet. In this case, the presence of the extra Higgs doublet is felt by the virtual exchange of a charged Higgs boson in the box diagrams for the processes governing the values of $\epsilon$ and the rate for $B^{0}-\bar{B}^{0}$ mixing. For the values of the parameters shown here, the allowed ranges of the angles of the unitarity triangle become:

$$
\begin{array}{r}
-1.00 \leq \sin 2 \alpha \leq 1.00 \\
0.12 \leq \sin 2 \beta \leq 0.81 \\
-1.00 \leq \sin 2 \gamma \leq 1.00
\end{array}
$$

The interplay between future measurements of $\left|V_{u b}\right| /\left|V_{c b}\right|$, improved theoretical calculations of the bag and form-factor parameters, and $C P$-violating asymmetry measurements will further sharpen Standard Model tests and possibly allow us to explore the physics that lies beyond it. 


\subsubsection{Beyond the Standard Model}

Measuring $C P$ asymmetries in several $B^{0}$ decay modes that depend on the same unitarity angle tests specific Standard Model assumptions

The use of partial wave analysis can make a variety of vector-vector final states useful for the measurement of $C P$ asymmetries

Certain decays, such as $B_{d}^{0} \rightarrow \phi \pi^{0}$ and $B_{d}^{0} \rightarrow$ $K_{S}^{0} K_{S}^{0}$, are expected to have zero $C P$ asymmetries in the Standard Model
In probing $C P$-violating asymmetries for evidence of non-Standard Model contributions it is important to make as many cross checks as possible. One way to do this is by measuring quantities from many channels that are related in the Standard Model but may differ when non-Standard Model contributions are included. While designed to study $C P$ asymmetries in the $C P$ eigenstate modes discussed above, the $B_{A} B_{A R}$ detector can do much more. For example, there are many higher spin modes that can be analysed using decay angular analyses to separate the odd and even $C P$ components and thus provide additional independent tests of the Standard Model relationships between CKM parameters [Dun91]. These modes typically have higher final-state particle multiplicity than those discussed above; precision tracking and a highly segmented calorimeter are needed to reconstruct them. The separated vertices of the two $B^{0}$ decays at PEP-II and the resulting suppression of continuum events reduce the problem of backgrounds in these analyses.

In addition to the study of the unitarity triangle, there are a number of other checks on the Standard Model predictions for $C P$ asymmetries. Many models beyond the Standard Model introduce additional contributions to $B^{0}-\bar{B}^{0}$ mixing. Typically, these contributions have a different weak phase from the Standard Model contribution and thus destroy the relationship between the mixing phases and decay phases given in the Standard Model. This in turn produces a non-zero $C P$ asymmetry in channels such as $B_{d}^{0} \rightarrow$ $\phi \pi^{0}$ or $B_{d}^{0} \rightarrow K_{S}^{0} K_{S}^{0}$ which are predicted to have zero asymmetry in the Standard Model. A special case is the superweak model, which predicts that the $C P$ asymmetry arises solely from the mixing phase, and thus should be the same for all channels, including those having zero asymmetry in the Standard Model. Hence it is important to be able to study these channels well in order to search for non-vanishing asymmetries that violate Standard Model predictions. In models in which significant additional mixing effects occur there is no reason why the asymmetries in these channels should be significantly smaller than those in the $J / \psi K_{S}^{0}$ or $\pi^{+} \pi^{-}$ chamnels. 


\subsubsection{Penguin Modes}

A question of much importance for the measurement of the unitarity angle $\alpha$ is the size of the penguin contributions in the $B^{0} \rightarrow \pi^{+} \pi^{-}$ and $\rho^{ \pm} \pi^{\mp}$ channels. Only if these effects are small can the measured asymmetry be directly related to the CKM angle $\alpha$. Much will be learned by comparing $B\left(B^{0} \rightarrow K^{+} \pi^{-}\right)$to $B\left(B^{0} \rightarrow \pi^{+} \pi^{-}\right)$, both of which have penguin and spectator contributions. The penguin amplitudes are in the ratio $A_{p}\left(\pi^{+} \pi^{-}\right) / A_{p}\left(K^{+} \pi^{-}\right) \approx\left|V_{t d} / V_{t s}\right| \approx \lambda$; the spectator amplitudes are in the ratio $A_{s}\left(K^{+} \pi^{-}\right) / A_{s}\left(\pi^{+} \pi^{-}\right) \approx$ $\left|V_{u s} / V_{u d}\right| \approx \lambda$. Good measurements of both rates will allow a reasonable estimate of the relative importance of the two diagrams for each decay. This requires excellent particle identification.

Fortunately, if the penguin contributions turn out to be significant, their effects can be measured in some detail by analysis of a set of isospin-related channels, allowing the extraction of the angle $\alpha$. For $\pi \pi$, the isospin analysis requires a rate, but not an asymmetry measurement, for the channel $\pi^{0} \pi^{0}$ [Gro91]. This essential crosscheck of the accuracy of the value for $\alpha$ is one of the strong motivations for having excellent calorimetry in the $B_{A} B_{A}$ detector. For the mode $\rho^{ \pm} \pi^{\mp}$, a similar check can be made using an analysis of the interference between the lifferent charge channels of $\rho \pi$ in the $\pi^{+} \pi^{-} \pi^{0}$ time-dependent Dalit\% plot [Qui93]. Once again good $\pi^{0}$ reconstruction efficiency is essential. This analysis also provides a measurement of $\cos 2 \alpha$ in addition to $\sin 2 \alpha$ and hence resolves the otherwise troublesome ambiguity between $\alpha$ and $90^{\circ}-\alpha$. For this channel the largest problem is likely to be the size of background contamination. A full simulation of this problem has yet to be done, but it is clear that good resolution and accurate calorimetry will be important.

The question of direct $C P$ violation call also be probed by a search for $C P$-violating asymmetries in charged $B$ decays. The Standard Model predicts such effects at the few percent level due to the interference of tree and penguin contributions to the amplitude for these decays. While there are considerable uncertainties in the precise value of the preclicted asymmetries, measurements that showed an asymmetry at the level of ten percent or more would be a strong suggestion of the presence of effects from physics beyond the Standard Model. It will certainly be worthwhile to search for
Possible penguin contributions can be isolated by measuring a series of isospin-related channels

$C P$ asymmetries in $B^{ \pm}$ decays are difficult to interpret in terms of the unitarity angles, but can be useful to understand direct $C P$ violation 
such effects, given that about fifty percent of the events produced at the $\Upsilon(4 S)$ are $B^{+} B^{-}$pairs.

\subsection{Other Physics Opportunities}

PEP-II has excellent sensitivity for measuring $x_{s}$
Searches for $D^{0}-\bar{D}^{0}$ mixing and $C P$-violation in the $D$ sector will be of continuing interest
While the primary aim of the $B_{A} B_{A R}$ detector is specifically designed to address the program of $C P$-violation measurements described above, it is also well-suited to the investigation of a wide array of important topics in heavy flavor physics in general. Some of the possible areas of interest are briefly outlined in the following sections.

\subsection{1 $B_{s}$ Physics}

PEP-II can also operate at the $\Upsilon(5 S)$ resonance, with somewhat reduced luminosity but at a higher energy asymmetry [PEP93]. The $\Upsilon(5 S)$ is heavy enough to decay into $B_{s}^{0} \bar{B}_{s}^{0}$ pairs. While measurement of $\sin 2 \gamma$ in $B_{s}^{0}$ decay is difficult, PEP-II operating at a $\beta \gamma$ of 0.88 on the $\Upsilon(5 S)$ has excellent sensitivity for the the study of $B_{s}-\bar{B}_{s}$ mixing, up to $x_{s} \approx 20$.

\subsubsection{Charm Physics}

A number of interesting measurements in the charm sector will remain to be done with $B_{A} B_{A R}$, such as the measurement of $D^{0}-\bar{D}^{0}$ mixing and the search for direct $C P$ violation in the $D$ sector. Other topics to be pursued are precision measurements of the form factors involved in the $c \rightarrow d$ semileptonic decays $D \rightarrow \pi(\rho) \ell \nu$, the pseudoscalar coupling constants $f_{D}$ and $f_{D_{s}}$, and excited charmed-meson spectroscopy. The large statistics (around $10^{7}$ charm particles of each species produced), the decay vertex separation, and the superb particle identification capabilities will lead to excellent signal-tonoise ratios in the reconstruction of all charmed states.

In the $D$ sector, the mixing rate is expected to be quite small, so indirect $C P$ violation will be almost completely suppressed. On the 
other hand, direct $C P$ violation could give rise to asymmetries of a few $10^{-3}$ in decays to $C P$ eigenstates such as $\pi^{+} \pi^{-}$or $K^{+} K^{-}$, or in charged $D$ decays such as $K^{+} \bar{K}^{* 0}$ or $\pi^{+} \eta$. For $C P$ eigenstates, tagging can be provided by selecting $D^{0}$ 's coming from $D^{*+}$ decays, in which the sign of the slow pion unambiguously tags the $D^{0}$ flavor at $t=0$. In each mode, a precision around $1 \%$ will be reached after one year of running.

For the mixing measurement, the main background comes from doubly Cabibbo-suppressed decays (DCSD), which are expected to occur at a rate an order of magnitude larger than mixing effects. The flavor tagging at production is again done most effectively using the slow pion from $D^{*+} \rightarrow \pi^{+} D^{0}$ decay. Wrong-sign semileptonic decays are an unambiguous sign of mixing. For hadronic modes such as $K^{+} \pi^{-}$, the study of the $D^{0}$ time evolution is necessary to separate the non-exponential time distribution of mixing from DCSD decays. The expected sensitivity lies in the $10^{-4}$ to $10^{-5}$ range.

\subsection{3 $\tau$ Physics}

PEP-II is also a $\tau$ factory, with the potential to bring the precision of tau physics to a level comparable with that achieved for the muon [SLA89]. BABAR will collect a very large data sample, reaching $10^{8} \tau$ pairs after three years of running at design luminosity. A detector optimized for studying time-dependent $C P$ asymmetries in the $B \bar{B}$ system is also an excellent facility for $\tau$ physics.

Excellent vertex position resolution is needed for a high-precision measurement of the $\tau$ lifetime; similarly, mass resolution is the key to the measurement of the $\nu_{\tau}$ mass; efficient $\pi^{0}$ and $\gamma$ reconstruction is crucial for measurements of branching fractions, the detection of multi-pion modes and for searches for exotic decays including second class currents; efficient $\pi / \mathrm{e}, \pi / \mu$ and good $K / \pi$ separation aids measurements of small branching fractions and the study of QCD spectral functions, including Cabibbo-suppressed modes. While the trigger for $\tau$ pairs is more difficult than for $\Upsilon(4 S)$ events because of the smaller charged-particle multiplicity, the $B_{A} B_{A R}$ trigger algorithms are being designed to be highly effective for $\tau$ physics channels (see Chapter 11).
In searching for $D^{0}-\bar{D}^{0}$ mixing, it is vital to use time dependence to eliminate DCSD background 
An order-of-magnitude improvement in the limit on the $\nu_{\tau}$ mass can be expected

$\mu-e$ and $\mu-\tau$ universality tests can be brought to a level of precision equal to that currently available for $\mu$-e universality from $\pi$ and $\mu$ decay
Two-photon physics involving hadronic resonances can be brought to a new level of precision
If neutrinos are massive, then a hierarchy is expected among their masses similar to that in the charged sector. Improvements on the present $\nu_{\tau}$ mass experimental limit of $31 \mathrm{MeV} / c^{2}$ by ARGUS [Alb88] are thus important to constrain all neutrino masses. To obtain a limit on $m_{\nu_{r}}$, one studies the end point of the hadronic mass distribution $m_{\text {had }}$ for high-mass hadronic states such as $\tau \rightarrow 5 \pi^{ \pm}$, $\tau \rightarrow 3 \pi^{ \pm} 2 \pi^{0}$, and $\tau \rightarrow K K \pi$, where $m_{\nu_{r}}=m_{\tau}-m_{\text {had }}$. As outlined above, there are several advantages to measuring $m_{\nu_{r}}$ at PEP-II. In particular, the measurement benefits from excellent mass resolution together with good understanding of backgrounds stemming from hermeticity, particle identification, and the instrumented flux return (used as a $K_{L}^{0}$ veto). With a sample of $10^{8} \tau$ pairs, a limit of $2.5 \mathrm{MeV} / c^{2}$ on $m_{\nu_{r}}$ is attainable [Gom $94 \mathrm{a}$ ].

Very precise tests of $\mu-e$ and $\mu-\tau$ universality depend on accurate measurements of the tau leptonic branching ratios $B_{e}$ and $B_{\mu}$, and of the tau lifetime $\tau_{\tau}$. The current precision on the ratios of couplings $g_{\mu} / g_{c}$ and $g_{\tau} / g_{\mu}$ from tau decays is about $0.6 \%$, where systematic errors in present-day experiments limit much further improvement. PEP-II has the potential of measuring $B_{e}, B_{\mu}$ and $\tau_{\tau}$ to a relative precision of about $0.2 \%$ to $0.3 \%$, using the advantages listed above, i.e., hermeticity, efficient trigger, excellent particle identification and precise vertexing for unambiguous selection of $\tau$ decays and for measuring $\tau_{\tau}$. These would bring $\mu-e$ and $\mu-\tau$ universality tests using $\tau$ decays to a level of precision of $0.15 \%$ [Gom $94 \mathrm{~b}$ ], equal to that currently available for $\mu-e$ universality from $\pi$ and $\mu$ decays.

\subsubsection{Two-Photon Physics}

Monte Carlo studies [Bau91, Bau92] have shown that PEP-II can extend the study of exclusive two-photon physics from the present $2 \mathrm{GeV} / c^{2}$ up to at least $5 \mathrm{GeV} / c^{2}$ in mass. This could reveal many of the exotic bound-states now being sought, such as glueballs, $q \bar{q} g$ hybrids, and four-quark resonances. Measurements of the two-photon couplings of light-quark and charmonium resonances will continue to be important for understanding quark dynamics. Furthermore, a real challenge to perturbative QCD predictions of exclusive hadron production from two-photon interactions can be made by obtaining statistics in the $>3 \mathrm{GeV} / c^{2}$ mass range. Finally, there is a strong incentive to study single-tagged two-photon reactions (where one 
of the scattered $e^{ \pm}$is detected) where spin-1 resonances can be isolated (especially $J^{P C}=1^{-+} q \bar{q} g$ hybrid states) and unique QCD predictions can be tested.

\begin{tabular}{|c|c|c|}
\hline $\begin{array}{c}\text { Untagged } \\
\text { Reactions }\end{array}$ & $\begin{array}{c}\text { Published } \\
\text { Data }\end{array}$ & $\begin{array}{c}\text { PEP-II } \\
\left(100 \mathrm{fb}^{-1}\right)\end{array}$ \\
\hline \hline$\gamma \gamma \rightarrow \eta^{\prime} \rightarrow \pi^{+} \pi^{-} \gamma$ & 2000 & 300,000 \\
$\gamma \gamma \rightarrow \iota\left(1.42 \mathrm{GeV} / c^{2}\right) \rightarrow \bar{K} K \pi$ & 0 & 600 \\
$\gamma \gamma \rightarrow \eta_{c} \rightarrow$ all modes & 10 & 30000 \\
$\gamma \gamma \rightarrow \pi^{+} \pi^{-}\left(\mathrm{W}>3 \mathrm{GeV} / c^{2}\right)$ & 10 & 800 \\
\hline \hline Single-tagged & Published & PEP-II \\
Reactions & Data & $\left(100 \mathrm{fb}^{-1}\right)$ \\
\hline \hline$\gamma \gamma * \rightarrow \eta^{\prime} \rightarrow \pi^{+} \pi^{-} \gamma$ & 300 & 6000 \\
$\gamma \gamma * \rightarrow 1^{++}\left(1.42 \mathrm{GeV} / c^{2}\right) \rightarrow \bar{K} K \pi$ & 30 & 1100 \\
$\gamma \gamma * \rightarrow \eta_{c} \rightarrow$ all modes & 1 & 3000 \\
$\gamma \gamma * \rightarrow \pi^{+} \pi^{-}\left(\mathrm{W}>3 \mathrm{GeV} / c^{2}\right)$ & - & 100 \\
\hline
\end{tabular}

Table 2-2. Expected PEP-II two-photon yields compared to existing data.

Predictions for the number of events in $100 \mathrm{fb}^{-1}$ of data at PEP-II are summarized in Table 2-2, along with the present world data samples. Clearly, at least a factor of 30 improvement in statistics can be made in all of these exclusive processes. Furthermore, with a high-quality detector, new channels will be available which have not been explored in the past. Most of this can be accomplished with little impact on the optimal detector for studying $C P$ violation. The main requirement is a very flexible trigger to allow detection of low-mass and low-multiplicity final states with balanced transverse momenta. An additional need is for detection of low-angle scattered positron "tags" in the backwards direction down to as small a polar angle as possible. This does not require the full power of the detector, but only the CsI calorimetry and some modest ability to distinguish charged from neutral particles. 


\section{References}

[Abe94] F. Abe et al., Fermilab Report FERMILAB-PUB-94/116E, 1994 (to be published in Phys. Rev. Lett. ).

[Ala94] M.S. Alan, Cornell Report CLNS 94/1270, 1994.

[Alb88] H. Albrecht et al., Phys. Lett. B202, 149 (1988).

[Baro3a] J. Bartelt et al., Phys. Rev. Lett. 71, 1680 (1993).

[Bar93b] J. Bartelt et al., Phys. Rev. Lett. 71, 4111 (1993).

[Bat93] M. Battle et al., Phys. Rev. Lett. 71, 3922 (1993).

[Bau91] D. Bauer, "The Impact of Two-Photon Physics on a $B$ Factory Detector", BABAR Note \#77, Jan.1992.

[Bau92] D. Bauer, "Two Photon Physics at a B-Factory", BABAR Note \#21, Jan.1991.

[Bea93] A. Bean et al., Phys. Rev. Lett. 70, 2681 (1993).

[Bur94] A.J. Buras et al., Max Planck Institute Report MPIPh/94-14, TUM-T31-57/94, 1994.

[Dun91] I. Dunietz et al., Phys. Rev. D43, 2193 (1991).

[Gib93] L.K. Gibbons et al., Phys. Rev. Lett. 70, 1203 (1993); L. Iconomidau Fayard et al., Phys. Lett. B317, 233 (1993).

[Gom94a] J.J. Gomez-Cadenas, "Sensitivity of Future HighLuminosity $e^{+} e^{-}$Colliders to a Massive $\tau$ Neutrino", Proceedings of the Marbella Workshop on the Tau/Charm Factory, Marbella, Spain, 1993, CERN-PPE/94-12, 1994.

[Gom94b] J.J. Gomez-Cadenas, "Experimental Results on Tau Physics", CERN Report CERN-PPE/94-13, 1994 (submitted to Int. Jour. Mod. Phys. ).

[Gro91a] M. Gronau and D. London, Phys. Rev. Lett. 65, 3381 (1991).

[Gro91b] M. Gronau and D. Wyler, Phys. Lett. B265, 172 (1991); I. Dunietz, Phys. Lett. B211, 75 (1991). 
[Hue94] For recent reviews of "Electroweak Baryogenesis in the Standard Model", see P. Huet, SLAC-PUB-6492, 1994.

[Nir93] For a review of Standard Model predictions on $C P$ violation in $B$ decays see J. Nir and H.R. Quinn, Ann. Rev. Nucl. Part. Sci. 42, 211 (1992) and references therein.

[PEP93] PEP-II, An Asymmetric B Factory: Conceptual Design Report, LBL-PUB-5379, SLAC-418, CALT-68-1869, UCRL-ID-114055, UC-IIRPA-93-01, 1993.

[Qui93] H.R. Quinn and A. Snyder, Phys. Rev. D48, 2139 (1993).

[SLA89] For earlier studies see The Physics Program of a HighLuminosity Asymmetric B Factory at SLAC, SLAC-353, 1989; B Factories: The State of the Art in Accelerators, Detectors and Physics, SLAC-400, 1992; and Status Report on the Design of a Detector for the Study of CP Violation at PEP-II at SLAC, SLAC-419, 1993.

[Wol85] L. Wolfenstein, Plyys. Rev. D31, 2381 (1985). 
LETTER OF INTENT FOR THE BABAR Detector 


\section{Detector Overview}

$\mathbf{T}$ he primary physics goal of the $B_{A} B_{A R}$ experiment is the systematic study of $C P$ asymmetries in decays of the $B^{0}$ to $C P$ eigenstates, as discussed in the previous chapter. The secondary goals are to explore the wide range of other $B$ physics, charm physics, $\tau$ physics, two-photon physics, and $\Upsilon$ physics that becomes accessible with the high luminosity of PEP-II. The design of the detector is optimized for the $C P$ studies, but also serves well for the other physics opportunities.

The critical experimental objectives required to achieve the desired sensitivity for $C P$ measurements are:

- To observe the decays of $B^{0}$ mesons to a wide range of exclusive final states with high efficiency and low background;

- To tag the flavor of the other $B$ meson in the event with high efficiency and accuracy;

- To measure the relative decay time of the two $B$ mesons.

Studies carried out over the last several years at SLAC [SLA91, SLA93] and for other B-Factory proposals [Eic86, HEL92, CLE93, KEK92] have led to a fairly common picture of the performance requirements that the detector systems must meet. In this chapter, some general considerations which guide the process of choosing a detector design are outlined, and then the $B_{A} B A R$ baseline design is described.
Studies carried out for a number of B-Factory proposals have led to a consensus on basic detector performance requirements 


\subsection{General Design Considerations}

\subsubsection{Physics Constraints}

The production cross section for the $\Upsilon(4 S)$, which decays to either $B^{+} B^{-}$or $B^{0} \bar{B}^{0}$, is $1.2 \mathrm{nb}$. The $\Upsilon(4 S)$, however, sits on a $q \bar{q}$ continuum that is 3.5 times larger. $B$ mesons decay to many different final states with small branching fractions; the largest branching fractions are a few percent. To offset this small production cross section and the small exclusive branching fractions, a large detector acceptance is mandatory. Experimental sensitivity is further enhanced by providing background reduction from both $q \bar{q}$ continuum and from other less interesting $B$ decays. Such rejection is obtained by excellent $B$ mass resolution and by vertexing constraints. Mass resolution must be achieved for decay modes containing charged and neutral tracks. Neutral reconstruction requires particular care as the $B$ momenta are small at the $\Upsilon(4 S)$. Photon energy resolution and not angular resolution, contributes most significantly to $B$ meson mass resolution.

\subsubsection{Acceptance}

The CP violation measurements require reconstruction and tagging of $B^{0}$ decay modes with rather small branching fractions
The crucial $C P$ measurements at PEP-II are made using events in which a $B^{0}$ decay into a $C P$ eigenstate (having, in general, a branching ratio below $10^{-4}$ ) is completely reconstructed, and the other $B$ in the event is tagged as a $B^{0}$ or $\bar{B}^{0}$, using either a lepton or cliarged kaon. This requires observing anywhere from three to seven charged particles and often one or more $\pi^{0} \mathrm{~s}$. The charged particles must be detected with very good momentum resolution, precise vertex information, and clean particle identification. To achicve good efficiency for these rare events, it is important to have full performance of the detector over a very large solid angle. This is made more difficult by the energy asymmetry, which folds onehalf of the particles into the region $\cos \theta_{l a b}>0.5$. Figure 3-1 shows the relation between center-of-mass and laboratory polar angles for plotons at $\beta \gamma=0.56$. 


\section{Detector Protractor $-\gamma / s$}

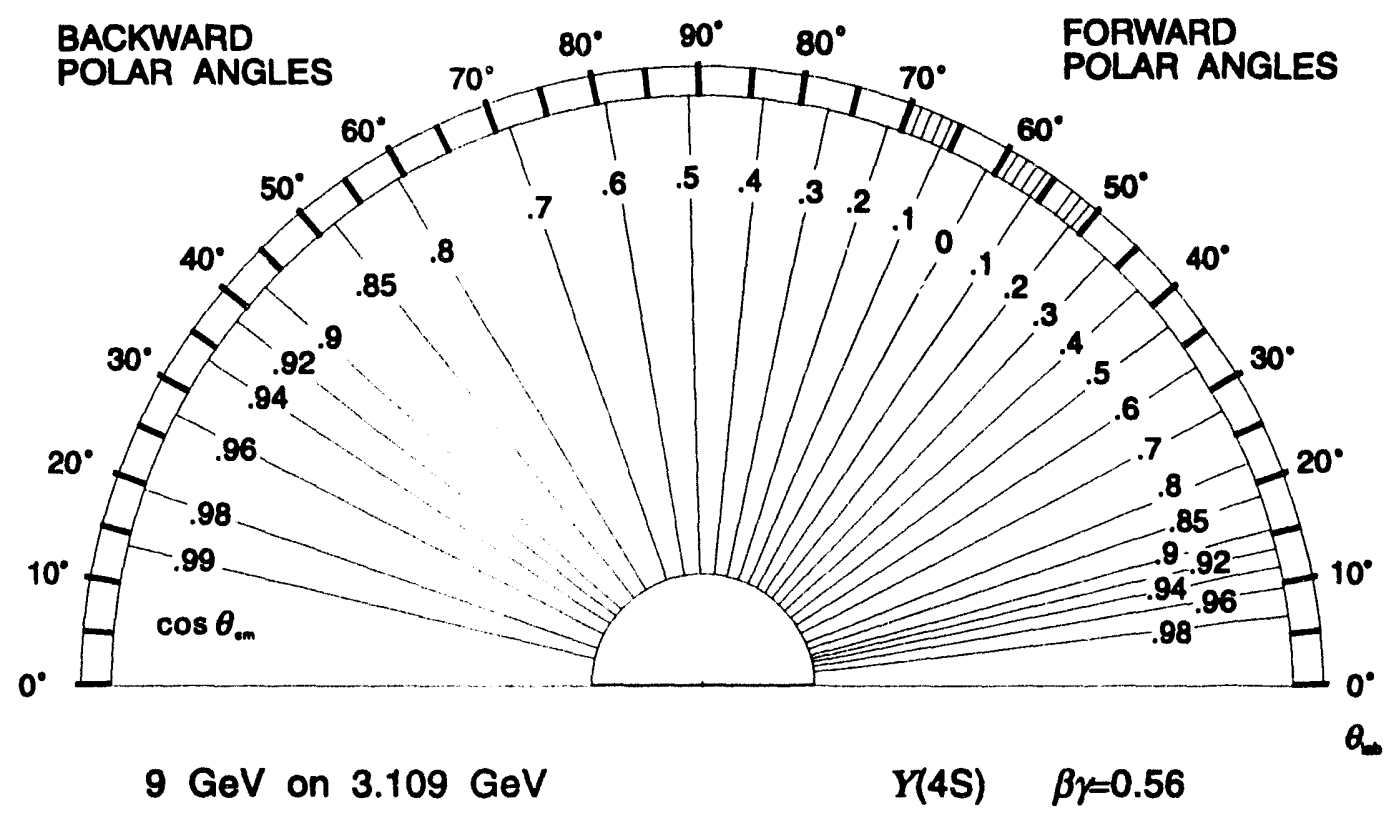

Figure 3-1. Protractor showing the relation between centerof-mass and laboratory polar angles for $\gamma$ 's at $\beta \gamma=0.56$.

Figure 3-2 shows the detection efficiency for the decay $B^{0} \rightarrow J / \psi K_{S}^{0}$, where $J / \psi \rightarrow e^{+} e^{-}$and $K_{S}^{-0} \rightarrow \pi^{+} \pi^{-}$, as a function of forward polar angle coverage, which is one critical parameter in achieving good acceptance. With acceptance down to $300 \mathrm{mr}$ in the forward direction, the efficiencies are about $75 \%$, falling rapidly as the forward acceptance is decreased. It is thus important to maintain good performance for all of the detector components down to the $300 \mathrm{mr}$ limit imposed by beamline components.

Another critical parameter is the minimum momentum cutoff in the acceptance for charged and neutral particles. The momentum distribution for the particles from $B$ decays at rest is quite soft: the average momentum for a pion from the process studied in Figure 3-2 is about $1.8 \mathrm{GeV} / \mathrm{c}$, but the momentum range for tagging kaons extends below $0.3 \mathrm{GeV} / c$. The pion from the process $B^{0} \rightarrow D^{*+} D^{*-}, D^{*+} \rightarrow D^{0} \pi^{+}$has a typical momentum of around $130 \mathrm{MeV} / c$. To maintain good efficiency and resolution for this

Achieving good coverage in the forward direction is complicated by the energy asymmetry

Good detection efficiency for low momentum charged particles and $\pi^{0}$ 's is required 


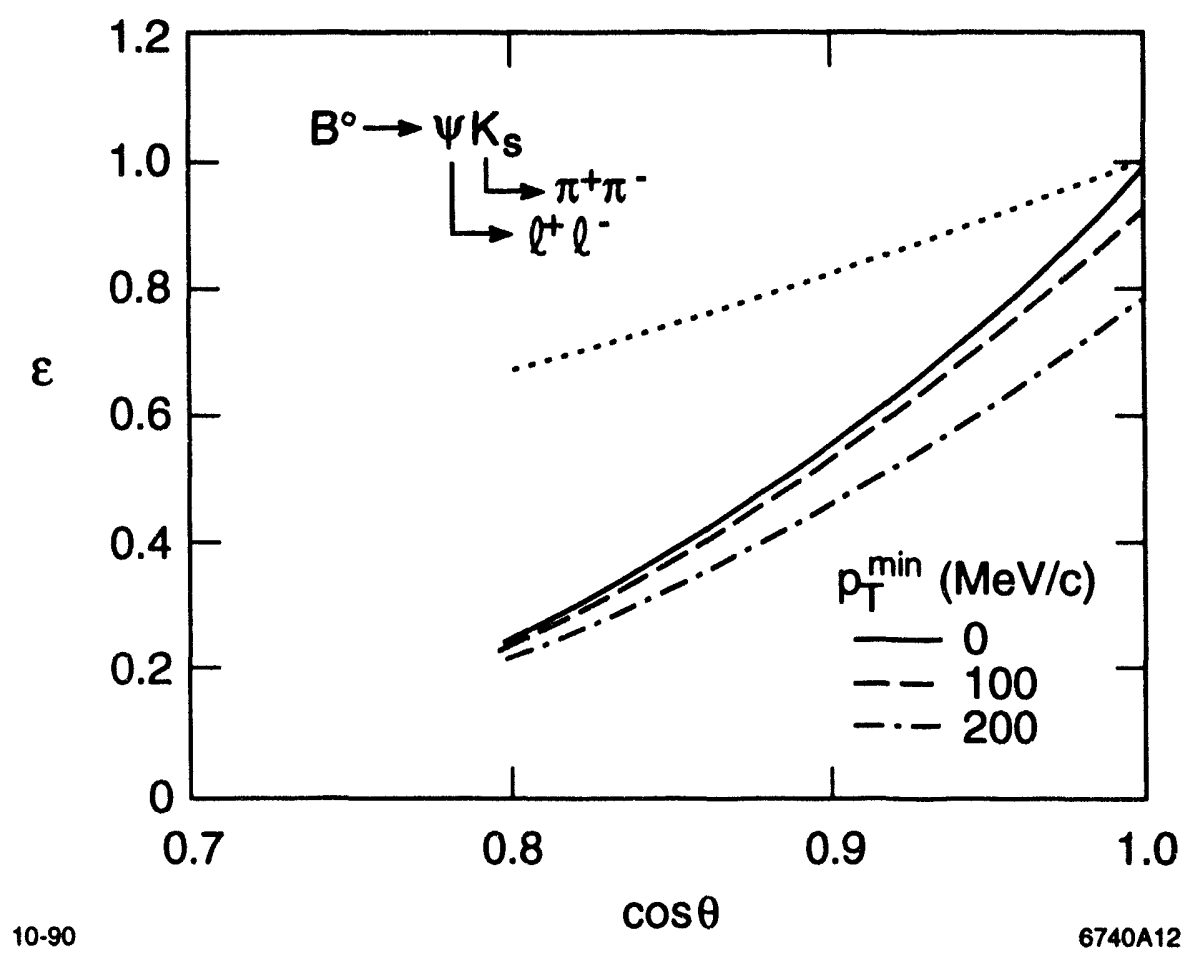

Figure 3-2. Detection efficiency for the decay $B^{0} \rightarrow J / \psi K_{S}^{0} \rightarrow$ $e^{+} e^{-} \pi^{+} \pi^{-}$as a function of forward polar angle coverage $\cos \eta$, for different cuts on the minimum transverse momentum accepted. The dotted line shows the efficiency as a function of backward polar angle coverage.

mode, charged tracks must be reconstructed down to $\sim 60 \mathrm{MeV} / c$ and for photons to $\sim 20 \mathrm{MeV} / c$. See Figures 6-1 and 8-3 for further details on the dependence of reconstruction efficiencies for exclusive channels on solid angle and low energy/momentum cutoffs.

It is particularly important to identify electrons and muons cleanly over as wide a range of angle and momentum as possible. The leptons useful for tagging the other $B$ in events used in $C P$ measurements have momentum as low as about $1 \mathrm{GeV} / c$. Alternative tagging techniques using secondary leptons could push this value considerably lower. Thus, the efficiency of the lepton tag is limited by the minimum momentum at which electrons and muons can be cleanly identified. 


\subsubsection{Resolution and Multiple Scattering}

As a result of the characteristically low momentum of particles produced in $B$ decay, the errors on charged particle track parameters are usually dominated by multiple Coulomb scattering rather than by the intrinsic spatial resolution of the tracking chamber. For example, the resolution in measuring the longitudinal $(z)$ position of the $B$ decay vertex, and therefore the decay time, is determined primarily by the amount of material before the first two measurements in the vertex detector and by the radius of the beam pipe. This leads to a strategy of maling the first $z$ measurement as close as possible to the beam pipe. It is also important to ensure that there is very little material other than the silicon and the beam pipe inside the second measuring layer.

Multiple Coulomb scattering also dominates both the angle and the momentum resolution. Good momentum resolution requires a long track length in a continuous tracking volume filled with a gas of long radiation length. The other way to improve momentum resolution is to increase the magnetic ficld, although one must be careful not to compromise the acceptance for low momentum particles. For a large part of the momentıum range, the angle measurements are made primarily in the vertex detector, before the original angle information is lost by multiple scattering.

\subsubsection{Photon Efficiency and Resolution}

As with charged particles, thr low momentum range determines the detector requirements for photon detection. Very good energy and angular resolution in the electromagnetic calorimeter for photons in the energy range $20 \mathrm{McV}$ to $3 \mathrm{GeV}$ are most important. To achieve the required performance, however, one must also keep the number of radiation lengths of material before the calorimeter to a minimum. The major sources of such material are the particle identification device, and, in the endcap region, the drift chamber endplate and associated hardware as well.
Multiple scattering tends to dominate charged particle momentum and direction measurements

Photon detection efficiency is degraded by material in front of the calorimeter 


\subsubsection{Identification of Hadrons}

$d E / d x$ and TOF techniques must be supplemented with Cherenkov devices to achieve particle identification over the required momentum range
The traditional tools for identifying hadrons in experiments at $e^{+} e^{-}$ colliclers are $d E / d x$ and time of flight (TOF), which work well out to momenta somewhat above $1 \mathrm{GeV} / c$. One essential requirement for the $C P$ physics is to identify kaons for tagging, which are mostly in this range. The other requirement is to separate pions from kaons in decays such as $B^{0} \rightarrow \pi^{+} \pi^{-}\left(K^{+} \pi^{-}\right)$, as well as in charmed meson and $\tau$ decays. Particles from $B^{0} \rightarrow \pi^{+} \pi^{-}$decay have a momentum of about $1.8 \mathrm{GeV} / c$ at the minimum backward angle and about $4 \mathrm{GeV} / c$ at the minimum forward angle covered. Although kinematic fitting provides some help in discriminating these twobody decay modes, to achieve complete separation it is clearly necessary to use either a threshold or an imaging Cherenkov detector for particle identification.

\subsubsection{Identification of Leptons}

The iclentification of leptons (electrons and muons) is of particular importance for the tagging of $B$ decays. Clean and efficient identification is however also essential for exclusive studies of semileptonic and pure leptonic charm and beauty meson reconstruction. Electrons are well identified by comparison of their momentum measured in the central tracker and their energy deposit in the calorimeter. Muons are identified by their energy deposit in the calorimeter and their range and scattering angle in the Instrumented Flux Return (IFR). The IFR also serves as a detector of neutral hadrons (particularly $K_{L}^{0}$ ), which will significantly reduce backgrounds in exclusive studies which involve missing neutrinos.

\subsubsection{Interaction with the Accelerator}

PEP-II represents a new type of $e^{+} e^{-}$collider and therefore is an ambitious project. Highest priority is being given to making the machine reliable and easy to operate with consistently high luminosity and low background. Design choices for the interaction region have impact on the detector. Thus, a support tube, containing the interaction region magnets, masks to reduce beam-related 
backgrounds, and the vertex detector, is envisioned to run through the interaction region at a radius of $\sim 20 \mathrm{~cm}$. This is the best method to achieve precise and reproducible relative alignment of the machine components within the detector. While the support tube introduces $\sim 0.5 \% X_{0}$ of extra material into the fiducial volume of the detector, the prospect of better machine performance outweighs the consequences for tracking resolution.

The accelerator magnets closest to the interaction point also have consequences for the machine design. A separation scheme with zero crossing angle, and the need to focus both beams to a very small size, leads to dipole magnets (B1) being placed only $22 \mathrm{~cm}$ from the collision point, and cyuadrupoles (Q1) which are also completely within the detector volume. The dipole magnets constrain the vertex detector mechanical design, although not in a way that compromises performance. The length of the detector solenoid magnet is also limited, because if it were to become too large, it would be necessary to shield a beamline quadrupole which contains iron (Q2), causing severe complications for detector and accelerator, as well as making access to inner detector components difficult.

Because of the unprecedented beam currents at PEP-II, a great deal of attention has been given to backgrounds in the various detectors. Detailed simulations of the synchrotron radiation and lost beam particle backgrounds have been refined continuously since the earliest days of the machine design, and have had substantial impact on the design of the interaction region. The anticipated background levels, inflated by a reasonable safety factor, are used to set detector requirements such as radiation hardness of silicon electronics and bandwidth of the data acquisition system.

\subsubsection{Considerations of Cost and Schedule}

Although the primary considerations in designing the detector are physics requirements and detector capabilities, the process should and does involve consideration of cost. The interplay between overall geometry and performance requirements on the one hand, and the capabilities and costs of particular subsystem technologies on the other, is often complex. As in many similar detectors, the calorimeter is the most expensive single system. Because the calo-
Detailed simulations of synchrotron radiation and lost particle backgrounds have been conservatively interpreted in arriving at radiation hardness and occupancy tolerances
The calorimeter is the single most expensive detector system 
rimeter cost increases rapidly with volume, a considerable effort has been made to minimize the size of this particular subsystem as much as possible within the constraints imposed by physics performance.

The schedule for PEP-II construction encompasses a six-month commissioning run starting in the fall of 1998 . Detector commissioning off the beamline should commence at the same time, so that the detector is ready for installation into the interaction region at the end of the machine commissioning period. This represents an extremely tight schedule on which to design, engineer, construct, and assemble an experimental apparatus as large and complex as $B_{A} B_{A R}$. In considering detector technologies and alternative experimental designs, schedule constraints have been used as an important criterion.

\subsection{The Baseline Design}

The vertex detector measures the position of $B^{0}$ decay vertices and also provides efficient pattern recognition for low momentum particles
The $B_{A} B_{A R}$ baseline design is shown in Figure 3-3 and 3-4. It consists of a silicon vertex detector, a drift chamber, a particle identification system, a CsI electromagnetic calorimeter, and a magnet with an instrumented flux return. The superconducting solenoid is designed for a field of $1.5 \mathrm{~T}$, and the magnet flux return is instrumented for muon identification and coarse hadron calorimetry. All of these detectors operate with good performance down to forward angles of $300 \mathrm{mr}$ and backward angles of $\sim 400 \mathrm{mr}$. The vertex detector is mounted inside a support tube, along with the first accelerator dipole and quadrupole magnets.

The vertex detector is critical to the BABAR detector, since it is used to meisure the difference in the decay time of the two $B^{0}$ mesons. It must have excellent spatial resolution, low multiple scattering, small segmentation and reasonably good resistance to radiation. A silicon microstrip system with five layers is the baseline design. The inner three layers are in a barrel geometry with detectors parallel to the beam pipe. The outer two layers combine barrel detectors in the central region with wedge detectors forward and backward. There is no other tracking inside a radius of about $22 \mathrm{~cm}$, so the vertex detector must provide not only precise vertex measurements, but also good measurements of track angles and good pattern recognition efficiency for low momentum tracks. The possibility of increasing charged particle coverage down to $200 \mathrm{mr}$ 


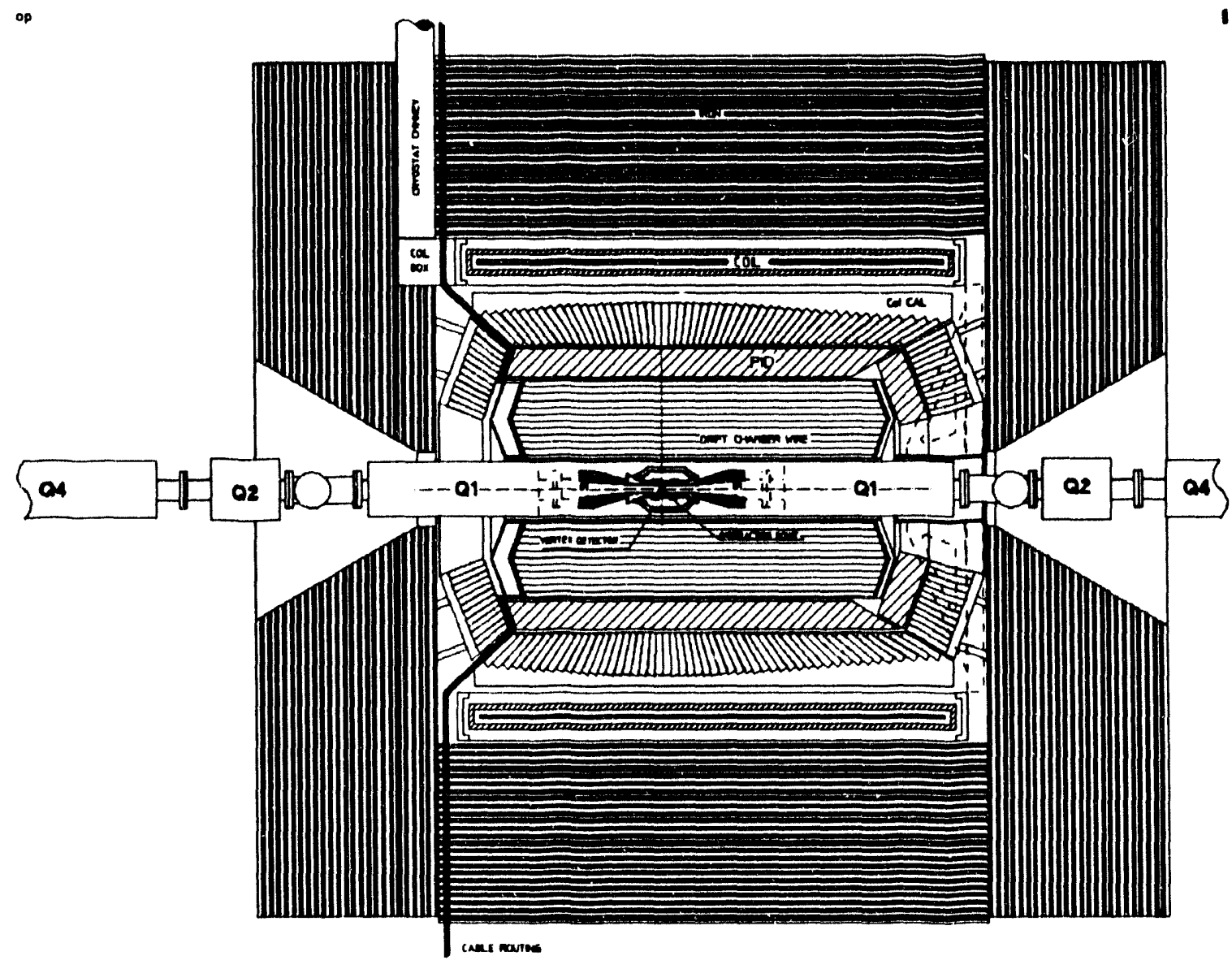

Figure 3-3. Cross-sectional view of the baseline detector showing from the beamline outward a silicon vertex detector, central tracking chamber, particle identification (PID) system, CsI calorimeter, superconducting coil, and instrumented flux return (IFR). The space allowed for the PID system can accommodate any of the three possible detector technologies: aerogel threshold counters, DIRC or Fast RICH. The detector is $6.687 \mathrm{~m}$ long and $3.196 \mathrm{~m}$ in radius at the back of the IFR, with the drift chamber displaced by $46 \mathrm{~cm}$ in the high-energy beam direction towards the right. 


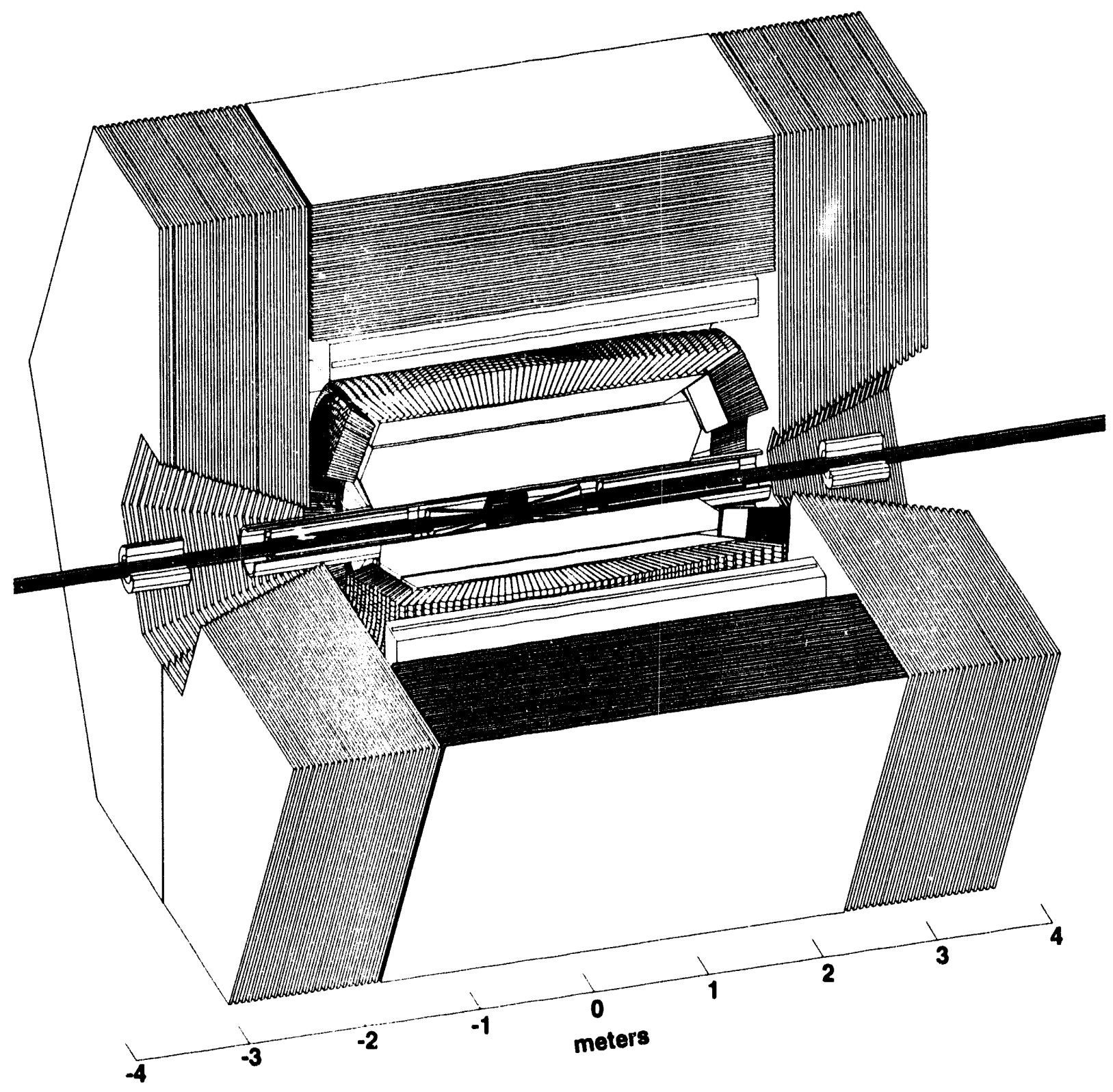

Figure 3-4. Three-dimensional view of the baseline detector. 
with a forward $\mu \mathrm{TPC}$ is also being considered in order to enhance the reconstruction efficiency for some exclusive final states.

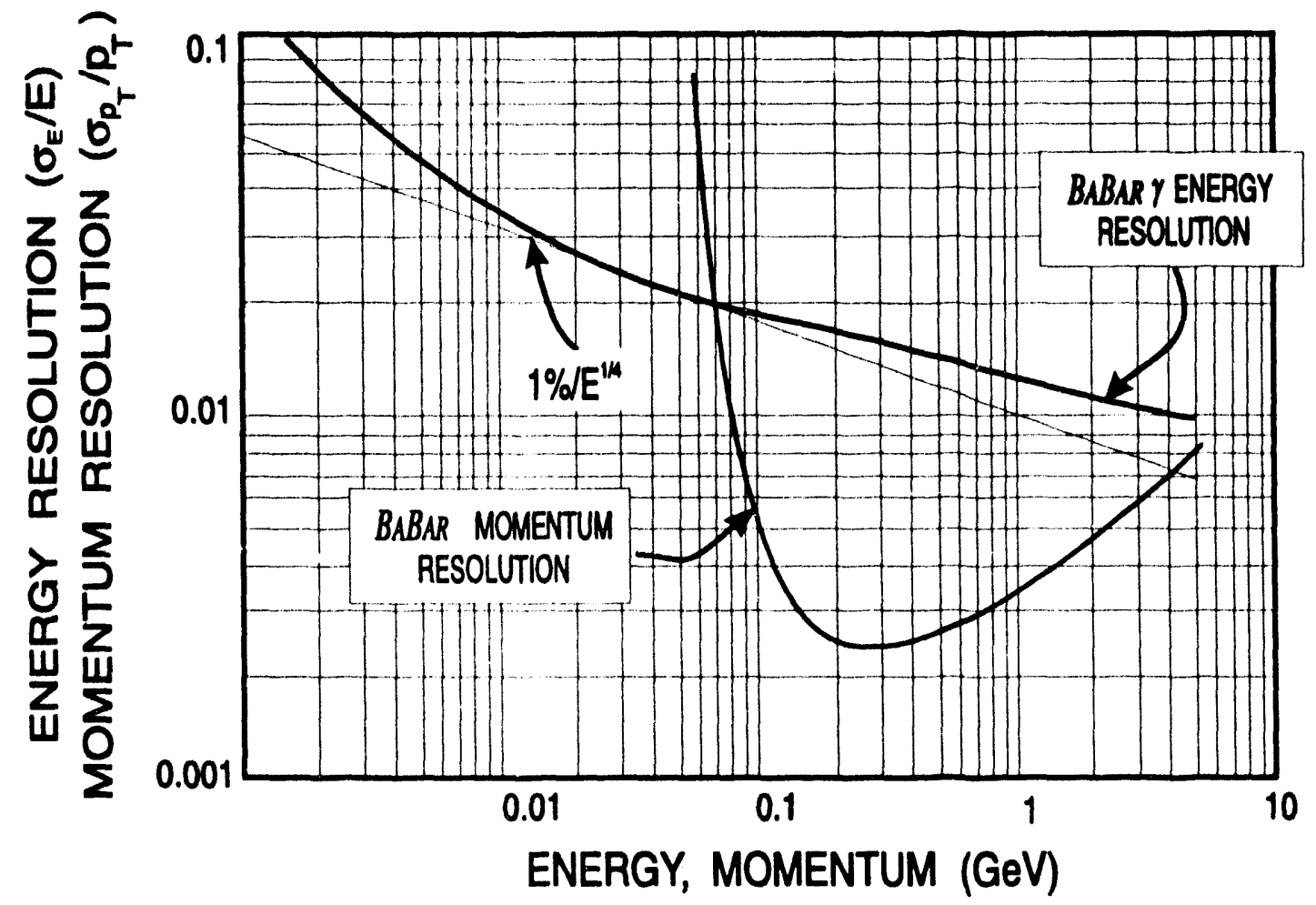

Figure 3-5. Energy and momentum resolution (at $\theta_{l a b}=90^{\circ}$ )

The main drift chamber is used primarily to provide excellent momentum resolution and pattern recognition for charged particles. It also supplies information for a charged track trigger, a measurement of $d E / d x$ for particle identification, and some improvement to the angular resolution. For most particles of interest at PEP-II, the momentum resolution is achieved by having a continuous tracking volume with a minimum of multiple scattering inside. The drift chamber extends from $22 . \bar{u} \cdot \mathrm{cm}$ to $80 \mathrm{~cm}$ in radius. By using a helium-based gas nixture with low-mass wires and a magnetic field of $1.5 \mathrm{~T}$, the momentum resollition shown in Figure 3-5 should be obtained. The readout electronics are mounted only on the backward end of the chamber, reducing the amount of material in front of the particle identification and calorimeter systems in the heavily
Low-mass construction and a helium-based gas reduce multiple scattering to a minimum in the main tracking chamber 
Three alternative particle identification technologies are considered, all of which fit into a single overall detector geometry

The CsI (TI) calorimeter provides excellent energy and angular resolution and retains high detection efficiency at the lowest relevant photon energies

The crystal length varies from $18 X_{0}$ in the forward endcap to $15 X_{0}$ in the backward endcap

A 1.5 T magnetic field is required to achieve adequate momentum resolution populated forward direction. The use of composite materials for the endplates is being considered to reduce their thickness further.

As stated above, an important goal for the PEP-II detector is to identify hadrons up to the kinematic limit for $B^{0} \rightarrow \pi^{+} \pi^{-}$ decay, which is above $4 \mathrm{GeV} / c$. This requires a dedicated particle identification device based on the Cherenkov technique to be placed outside the drift chamber. The material in the particle identification device should be kept as small as possible to minimize degradation of the performance of the electromagnetic calorimeter behind it, especially at low energy. This is the one system that requires a fundamentally new detector, and there has been a very active effort to develop a solution. There are three promising alternatives, but no single technology has been chosen for the baseline design. In order not to impede the design of the solenoid and calorimeter which are critical path items, particle identification designs have been chosen that can all fit into a single detector geometry with only small variations. Enough space is allowed to accommodate any of the three options in the barrel and forward endcap regions; in the backward endcap region the $d E / d x$ measurements in the main tracking chamber are sufficient. The final design choice will be based on results from ongoing prototype work, and may include different solutions in the forward endcap and barrel region.

The electromagnetic calorimeter should have the best possible energy resolution down to very low photon energies. This is provided by a fully projective $\mathrm{CsI}(\mathrm{Tl})$ crystal calorimeter, which has excellent energy and angular resolution and retains high detection efficiency at the lowest relevant photon energies. The crystal length varies from $18 X_{0}$ in the forward endcap to $15 X_{0}$ in the backward endcap; the typical crystal size is approximately rectangular $(\sim 4.5 \mathrm{~cm})$ on a side at the front face. The energy resolution expected for the calorimeter is shown in Figure 3-5. The rms energy resolution is $2.5 \%$ at $100 \mathrm{MeV}$, and $1.5 \%$ at $1 \mathrm{GeV}$. As mentioned above, minimizing the calorimeter volume was an important consideration in choosing the detector geometry; in the baseline design it is $\sim 8$ cubic meters.

To achicve very good momentum resolution, without increasing the tracking volume and therefore the calorimeter cost, it is necessary to have a magnetic field of $1.5 \mathrm{~T}$. The magnet is therefore of a 
superconducting design, with a radius of $1.5 \mathrm{~m}$ and a cryostat length of $4.0 \mathrm{~m}$.

The magnet yoke itself is lighly segmented and instrumented with chambers to provide muon and $K_{L}^{0}$ detection capability. This Instrumented Flux Return is designed to identify neutral hadrons and muons of momentum as low as $0.5 \mathrm{GeV} / c$ making it possible to use $B^{0} \rightarrow J / \psi K_{L}^{0}$ decays in addlition to $B^{0} \rightarrow J / \psi K_{S}^{0}$ for $C P$ studies. To achieve this, the flux return is divided into approximately 24 layers, with Resistive Platc Chambers or Plastic Streamer Tubes between the layers as the active elements. For the final design, we will chose between these two proven chamber technologies.

The high data rate at PEP-II requires a data acquisition system which is more elaborate than those used at present $e^{+} e^{-}$experiments. The bunch crossing period of $4.2 \mathrm{~ns}$ is so short that the interactions are effectively continuous, as in fixed-target experiments. The goal is to opcrate with negligible deadtime even if the backgrounds are much higher than present calculations estimate, an environment which might develop, especially early in the life of the experiment. The solution is to use pipelined information and an asynchronous trigger. The design uses commercial processors and data links for pattern recognition and data flow wherever practical, and minimizes custom harlware. The offline computing loads are also larger than previous experiments at $e^{+} e^{-}$colliders, but are within the capacity of the proposed system, which is based on existing technology.

\subsection{Other Geometry Options}

In the process of arriving at the baseline design for $B_{A} B_{A R}$, the collaboration considered and cliscarded a wide range of detector technologies and geometry options. These were not chosen because they failed to satisfy the criteria discussed above as well as the baseline design. It is worth reviewing some of these alternatives, however, to illustrate the process by which the final design was specified.
The flux return is highly segmented and instrumented to achieve efficient muon and neutral hadron detection capability below $1 \mathrm{GeV} / \mathrm{c}$

The data acquisition system is asynchronous and fully pipelined 
Long-barrel designs were considered in detail

The use of the TOPAZ coil was evaluated

The performance of the CsI calorimeter suffers in a long-barrel design

\subsubsection{Long-Barrel Designs}

One major family of detector geometries, known as "long-barrel" options, covers the entire polar angle range without endcap systems. Instead, the barrel extends along the beamline to the length needed to obtain the desired solid angle coverage. The main consequences of this design are a very long drift chamber (about four meters in length), a CsI barrel calorimeter with larger volume at a given radius, whose performance is compromised by having crystals at steep angles, and a longer magnet. The advantage is that a longbarrel design is a natural match for the DIRC particle identification system (see Figure 4.17 of reference [SLA93]).

The collaboration studied the possibility of using the TOPAZ magnet in a long-barrel geometry. The small radius and modest field of the magnet led to rather poor momentum resolution, and its length led to a very difficult problem of shielding the machine components. In particular, a compensating superconducting solenoid and iron flux return cone were needed to provide a field-free region for the Q2 magnet. This also adversely affected access to inner detector systems. For these reasons, the TOPAZ magnet was considered unacceptable.

Other long-barrel designs were considered which had a $1.5 \mathrm{~T}$ field and a calorimeter radius close to the standard value of $1 \mathrm{~m}$. The difficulty of shielding the Q2 magnet was a serious problem in these designs as well. The projective $\mathrm{CsI}(\mathrm{Tl})$ crystals mounted at $300 \mathrm{mr}$ to the beam direction have offsets in the position of the front face up to about $14 \mathrm{~cm}$ relative to a neighboring crystal, leading to severe leakage both at the front and the back of the crystals. This produces a calorimeter energy response that varies across the crystal face. Filling in this "sawtooth" pattern with additional CsI(Tl) can cure the energy leakage problem at the front of the crystals, but produces difficulties in light collection. Finally, the calorimeter volume is larger for the long-barrel geometry un! nss the radius is correspondingly smaller. This would be incompatible with the space needed for particle identification options other than the DIRC. For these reasons, and the need to proceed with the full engineering of the magnet and calorimeter, it was decided not to adopt the long-barrel design. 


\subsubsection{Liquid Krypton Calorimeter Designs}

The other experimental configuration considered was one based on a liquid krypton calorimeter. The motivation was the possibility that the cost would be significantly less than for $\mathrm{CsI}(\mathrm{Tl})$. The expected energy resolution of such a calorimeter was considered to be somewhat worse than for CsI, although the angular resolution would be somewhat better. The detection efficiency for low energy photons could be compromised by the material in the dewars. The cryostat needed for the krypton calorimeter increases the radius and length of the magnet, and leads to some of the same difficulties with enclosing the Q2 magnet as for the long-barrel design. In addition, the most natural calorimeter design would include a cryostat which wraps around the forward endcap region without a break, thereby making access to the inner detectors very difficult at that end.

A detailed study by an ad hoc calorimeter committee found that the cost benefit of the liquid krypton calorimeter was nearly canceled by the additional cost for the larger superconducting magnet. The initial funding requirements, including first-year commitments for the superconducting coil, were actually larger for the krypton case. In addition, the problems of shielding the machine components without restricting access to most of the detector were quite severe. Following the recommendations of the committee, the collaboration adopted the CsI calorimeter as the baseline design choice.

\subsubsection{Designs with Lower Magnetic Field}

If adequate momentum resolution could be obtained with a magnetic field of $1.0 \mathrm{~T}$, it would be possible to use a somewhat cheaper normal-conductor magnet. Therefore, the question of whether it was possible to achieve the necessary momentum resolution with such a field was examined. With a drift chamber extending to $80 \mathrm{~cm}$, a support tube as in the baseline design, and a $1 \mathrm{~T}$ field, the resolution on the mass of the $B$ for the process $B^{0} \rightarrow \pi^{+} \pi^{-}$is $33 \mathrm{MeV} / c^{2}$, which does not allow kinematic separation from $B^{0} \rightarrow$ $K^{+} \pi^{-}$. With a $1.5 \mathrm{~T}$ field, the mass resolution is $22 \mathrm{MeV} / c^{2}$, enough to reduce the probability of a false assignment between these two modes to about $20 \%$ on the basis of kinematics alone.
The LKr designs posed problems of difficult access to other detector systems and of integration with machine components

A $1 T$ field in a design with a support tube does not provide adequate twobody $B^{0}$ mass resolution 
$1 \mathrm{~T}$ designs with adequate resolution were either considered to be too risky or too expensive
There are two options which could obtain adequate momentum resolution using only a $1 \mathrm{~T}$ field. One involves removing the support tube and reducing the material at the inside of the drift chamber to a minimum. This would allow effective use of the outer silicon measurements to improve the momentum resolution for high momentum tracks and would lead to a resolution for $B^{0} \rightarrow \pi^{+} \pi^{-}$of $26 \mathrm{MeV} / c^{2}$. The risk of compromising accelerator reliability and performance was considered too great without the support tube, however. Another possibility is to extend the outer radius of the drift chamber to $95 \mathrm{~cm}$, which yields a resolution of $23 \mathrm{MeV} / c^{2}$ for $B^{0} \rightarrow \pi^{+} \pi^{-}$. This would increase the volume of the $\mathrm{CsI}(\mathrm{Tl})$ calorimeter dramatically, however, as well as the size of the magnet. The cost of this solution is significantly greater than that for a design based on a smaller magnet capable of $1.5 \mathrm{~T}$.

\subsubsection{Reports on Experimental Options}

Much of the detailed work done in considering these options is contained in three reports: one on the TOPAZ magnet design, one on calorimetry, and one which considered at the various particle identification options and led to the collaboration choosing to develop further three types of Cherenkov-based detectors [BAB94c]. These reports are part of the supporting documentation for this Letter of Intent [BAB94a, BAB94b].

\subsection{Detector Performance and Sensi- tivity to $C P$ Asymmetries}

The performance of the detector can best be measured by the expected resolutions for the $C P$-violating asymmetries $\sin 2 \beta$ and $\sin 2 \alpha$ in various decay modes. The ASLUND parametric Monte Carlo has been used to estimate these numbers for the baseline detector. This was checked with the full GEANT-based Monte Carlo, BBSIM, for a limited number of modes. For example, the $\pi^{+} \pi^{-}$mass resolution in the two simulations was found to agree to within $10 \%$. For further details on the simulations and the physics analysis can be found in Chapter 13. A summary of the main results is given here. 
For a nominal year of running with PEP-II, an integrated luminosity of $30 \mathrm{fb}^{-1}$ is assumed, accumulated on the $\Upsilon(4 S)$ resonance. Table 3-1 shows the efficiencies and the expected number of tagged events reconstructed for some of the major $C P$ modes. The backgrounds are negligible in all but one case. Also shown is the error in determining $\sin 2 \phi$, which is the ultimate measure of sensitivity for the experiment.

\begin{tabular}{|l|c|c|c|}
\hline Mode & $\begin{array}{c}\text { Events } \\
\text { Reconstructed }\end{array}$ & Background & $\delta[\sin 2 \phi]$ \\
\hline \hline$J / \psi K_{S}^{0}$ & 367 & 0 & 0.13 \\
$J / \psi K^{* 0}$ & 150 & 0 & 0.20 \\
$D^{+} D^{-}$ & 158 & 0 & 0.20 \\
$D^{*+} D^{*-}$ & 272 & 30 & 0.16 \\
\hline$\pi^{+} \pi^{-}$ & 106 & 9 & 0.24 \\
$\rho^{ \pm} \pi^{\mp}$ & 567 & 126 & 0.12 \\
\hline
\end{tabular}

Table 3-1. CP reach of the BABAR detector for some major decay modes in a data sample of $30 \mathrm{fb}^{-1}$ (one "Snowmass year") accumulated on the $\Upsilon(4 S)$ resonance.

A crucial parameter in obtaining this level of sensitivity is the effective tagging efficiency, $\epsilon_{e f f}=\epsilon_{\text {tag }}(1-2 w)^{2}$. In this expression $\epsilon_{\text {tag }}$ is the fraction of reconstructed $B$ decays in which the other $B$ is tagged, including possible wrong-sign tags, and $w$ is the fraction of those tags which are of the wrong sign. Table 3-2 gives a summary of the tagging efficiencies from the Montc Carlo simulation. The effective tagging efficiency is determined to be about $11 \%$ for observing the direct leptons from semileptonic $B$ decays. Tagging using charged kaons from the charm decay of the other $B$ adds $16 \%$ to the effective tagging efficiency, after subtracting events which are found using both methods.

Combining the modes shown in Table $3-1$, the errors on the $C P$ asymmetry parameters are $\delta[\sin 2 \beta]=0.09$ and $\delta[\sin 2 \alpha]=0.11$. Thus, the significance of the $\sin 2 \beta$ measurement would be anywhere from 2 to 9 standard deviations in one running year, depending on where its value lies within the presently allowed range. The parameter $\sin 2 \alpha$ has no minimum value, but the error can be compared with the allowed range which varies from -0.9 to +1.0 . 


\begin{tabular}{|l|c|c|c|c|}
\hline Tag Type & $\begin{array}{c}\text { Branching } \\
\text { Fraction }(b)\end{array}$ & $\begin{array}{c}\text { Efficiency } \\
(b \epsilon)\end{array}$ & $\begin{array}{c}\text { Wrong-sign } \\
\text { Fraction }(w)\end{array}$ & $\epsilon(1-2 w)^{2}$ \\
\hline \hline kaons & 37 & 30 & 10.0 & 19 \\
muons & 11 & 8.8 & 11.7 & 5.2 \\
electrons & 11 & 8.5 & 9.9 & 5.5 \\
\hline
\end{tabular}

Table 3-2. Summary of tagging efficiencies (\%).

With these sensitivities, available in about one year of operation at design luminosity, one would already have a powerful test of the Standard Model explanation of $C P$ violation. More running time would make possible increasingly precise tests of whether the CKM matrix is the sole source of the observed $C P$ asymmetries. 


\section{References}

[BAB94a] Report of the ad hoc Committee on using the TOPAZ Magnet, May 1994.

[BAB94b] Report of the ad hoc Committee on Calorimetry, May 1994.

[BAB94c] Report of the ad hoc Committee on Particle Identification, May 1994.

[CLE93] Detector for a B-Factory, CLEO collaboration, Cornell Report CLNS-91-1047-REV, 1993.

[Eic86] R. Eichler et al., Motivation and Design Study for a $B$ Meson Factory with High Luminosity, PSI Report SINPR-86-13, 1986;

R. Eichler et al., On the Use of the Proposed PSI $B$ Meson Factory as an Energy Asymmetric Electron Positron Collider for CP Violation Studies in $B$ Meson Decays, PSI Report PSI-PR-88-22, 1988.

[HEL92] HELENA, A Beauty Factory at Hamburg, DESY 92/41 (1992).

[KEK92] Progress Report on Physics and Detector of KEK Asymmetric $B$ Factory, $B$ Physics Task Force, KEK Report KEK-92-3, 1992.

[SLA91] Workshop on Physics and Detector Issues for a High- Luminosity Asymmetric B Factory at SLAC, SLAC Report SLAC-373, 1991.

[SLA93] Status Report on the Design of a Detector for the Study of CP Violation at PEP-II at SLAC, SLAC Report SLAC419, 1993. 
LETTER OF INTENT FOR THE BABAR DeteCtor 


\section{4}

\section{PEP-II and the Interaction Region}

$\mathbf{P}$ EP-II is an $e^{+} e^{-}$colliding beam storage ring complex designed to produce a luminosity of at least $3 \times 10^{33} \mathrm{~cm}^{-2} \mathrm{~s}^{-1}$ at $E_{c m}=$ $10.58 \mathrm{GeV}$, the mass of the $\Upsilon(4 S)$ resonance, with unequal energy beams of 3.1 and $9.0 \mathrm{GeV}$. Figure 4-1 shows a schematic view of PEP-II on the SLAC site. PEP-II is being built by a collaboration of the Stanford Linear Accelerator Center, Lawrence Berkeley Laboratory, and Lawrence Livermore Natioual Laboratory. Both the high luminosity and the energy asymmetry require significant advances in storage ring design and construction. A full description of the PEPII project may be found in the Conceptual Design Report [PEP93].

This chapter first reviews the limitations on luminosity in $e^{+} e^{-}$ machines, and the strategy for overcoming them in PEP-II. Next, the components of the PEP-II complex are described, with special emphasis on the interaction region, where the asymmetric energy beams are brought together from their separate machines and collided inside the detector. Finally, the machine-induced backgrounds in the detector are estimated.

\subsection{Luminosity Optimization}

The luminosity of a colliding beam machine is given by

$$
\mathcal{L}=\frac{N^{+} N^{-} f}{A}
$$

where $N^{+}$and $N^{-}$are the number of particles per bunch, $f$ is the collision frequency, and $A$ is the effective collision area [San70]. Increasing the luminosity requires some combination of increasing the number of particles per bunch, increasing the collision frequency, or decreasing the collision area. 


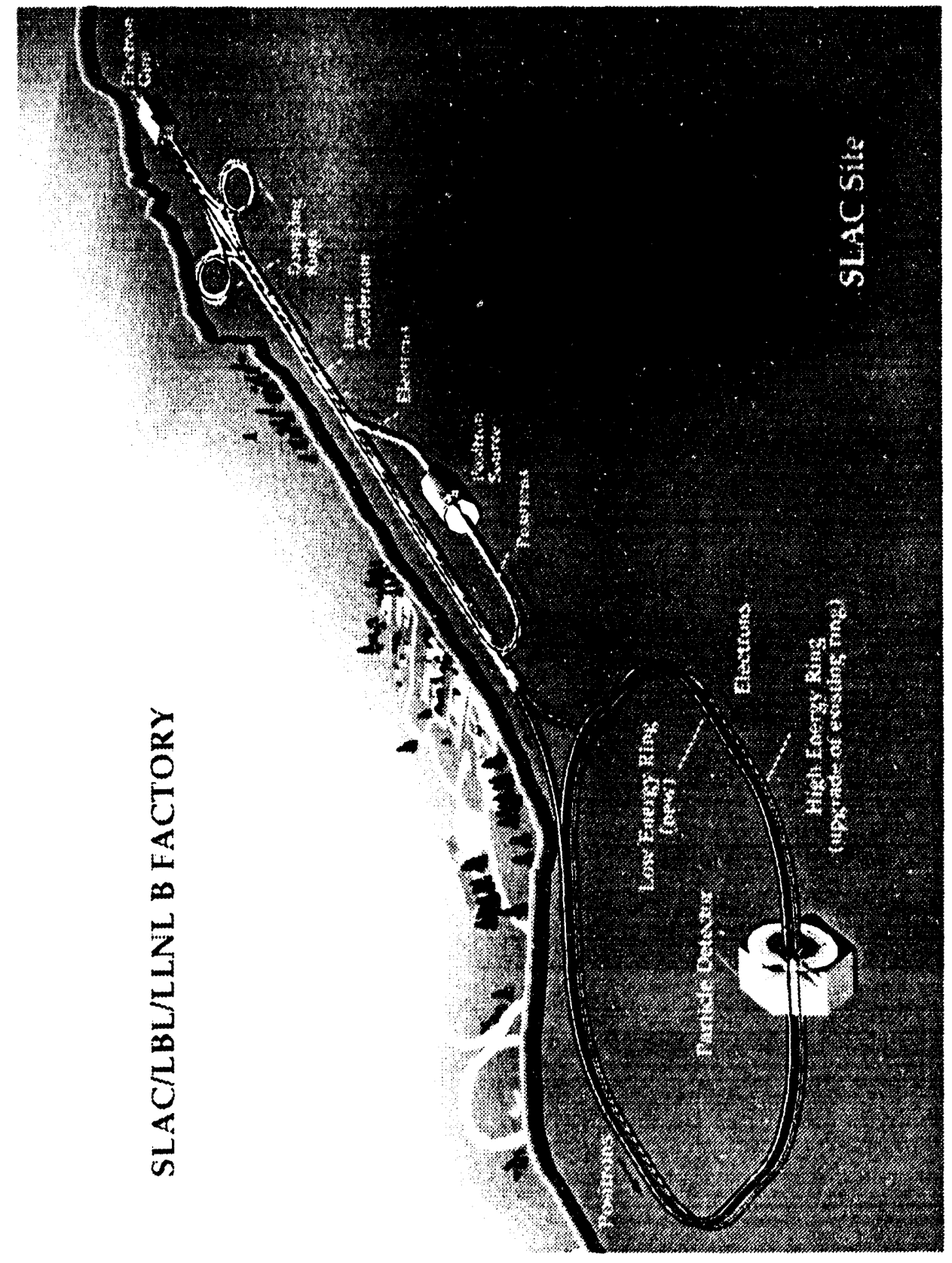

Figure 4-1. Schematic View of PEP-II Complex on SLAC site 
There are direct limits on $\mathrm{N}^{+}$and $\mathrm{N}^{-}$set by the interaction of the electromagnetic field of the bunch with its environment. (Hereinafter the superscript of $N$ is suppressed when not essential). The longitudinal microwave instability or bunch lengthening and transverse mode-coupling instability are driven by the broad band impedance of the vacuum chamber. The product $N \times f$, or equivalently the beam current, is limited by the narrow band impedance of the vacuum chamber, which is dominated by the RF accelerating cavity system. There are also limits on the beam current from synchrotron radiation and electromagnetic heating of the vacuum chamber, from gas desorption caused by synchrotron radiation, and from ion trapping.

\subsubsection{Tune-Shift Limits}

The electromagnetic field of one beam deflects the particles of the other beam. For particles passing near the center of the other beam, the deflection is proportional to the distance from the beam center, i.e. the other beam acts like a lens. This is quantified by the beambeam tune-shift $\xi$, the ratio of the optical beta function at the crossing point to the focal length of focusing applied by the other beam. The tune-shift is given by

$$
\xi_{i}=\frac{r_{e}}{2 \pi} \frac{N^{\text {other }}}{\gamma} \frac{\beta_{i}^{*}}{\sigma_{i}\left(\sigma_{x}+\sigma_{y}\right)}
$$

where $r_{e}$ is the classical electron radius, $N^{\text {other }}$ is the number of particles in a bunch of the other beam, $\gamma$ is the relativistic boost, $\sigma_{x}$ and $\sigma_{y}$ are the horizontal and vertical beam sizes at the collision point, and $i$ is $x$ for horizontal or $y$ for vertical. The beam-beam tune shift need not be equal in the horizontal and vertical planes.

Unfortunately, Gaussian bunches are not very linear lenses, and there is a limit on the tolerable strength of non-linear elements in a storage ring lattice. Increasing $N$ with fixed optics eventually results in such large non-linear beam-beam forces that the beam becomes unstable. The exact limit on $\xi$, and thus on $N$, depends on how rapidly beam perturbations damp out and on details of the ring lattice and its imperfections. Experience with many $\mathrm{e}^{+} \mathrm{e}^{-}$machines indicates that the PEP-II design value of $\xi \approx 0.03$ is conservative (the original PEP machine achieved $\xi \approx 0.05$ ).
The beam current is limited by the narrow band impedance of the vacuum chamber
Tune-shift limits depend on the details of the ring lattice and its imperfections 
The placement of the final focusing elements must balance reduced chromaticity with adequate space for the detector
There are several "energy transparency" conditions, aimed at making the beam-beam behavior of an asymmetric machine similar to that in a conventional storage ring
The tune-shift also imposes constraints on luminosity improvements from reducing the beam size. The beam sizes at the collision point are given by $\sigma_{i}=\sqrt{\epsilon_{i} \beta_{i}^{*}}$, where $\epsilon_{i}$ is the beam emittance, and $i$ is $x$ or $y$. For the common case $\sigma_{x} \gamma \gamma \sigma_{y}$, reductions in $\epsilon_{x}, \beta_{x}^{*}$, and $\epsilon_{y}$ are eventually limited by $\xi_{x}$ or $\xi_{y}$. On the other hand, a reduction in $\beta_{y}^{*}$ reduces the beam area thus increasing the luminosity, without changing $\xi_{x}$, and even decreases $\xi_{y}$.

While beam-beam effects do not limit reductions in $\beta_{y}^{*}$, there are other limits. Lowering $\beta^{*}$ by changing the strengths of focusing magnets at fixed locations inherently increases the chromaticity, or sensitivity of focusing to small energy variations. This reduces the aperture over which particle orbits are linear and stable, which in turn limits the storable beam intensity. The chromaticity can be reduced by moving the focusing magnets closer to the collision point, but this is limited by the free space needed for the detector. When $\beta^{*}$ approaches the bunch length, the colliding bunches become colliding hourglasses, and the beam-beam effects become stronger. Reducing the bunch length significantly requires very large increases in RF voltage.

\subsubsection{Energy Transparency and Luminosity}

In a single-ring symmetric machine, the two beam energies are identical, $\epsilon$ and $\beta$ for the two beams are normally the same, and optimum beam-beam performance is normally obtained with $N^{+}=$ $N^{-}$. An asymmetric energy machine necessarily has two different $\gamma$ factors and separate rings with different beam parameters, so the luminosity and beam-beam parameter space is much larger. It is prudent to design an asymmetric energy machine so its beam-beam behavior can be made as similar to a symmetric machine as possible. To this end, "energy-transparency" conditions have been proposed by several authors [Gar89, Chi90, Fur91, Kri90].

To achieve $\xi_{i}^{+}=\xi_{i}^{-}$and $\sigma_{i}^{+}=\sigma_{i}^{-}$in an asymmetric machine, it is necessary that $\left(E I / \beta_{y}^{*}\right)^{+}=\left(E I / \beta_{y}^{*}\right)^{-}$, where $E$ is the beam energy, and the bunch population $N$ and the collision frequency $f$ have been absorbed into the current $I$. In this case, the luminosity can 
be written

$$
\mathcal{L}=\left(2.17 \times 10^{34} \mathrm{~cm}^{-2} \mathrm{~s}^{-1}\right) \times(1+r) \xi_{y}\left(\frac{E I}{\beta_{y}^{*}}\right)^{ \pm}
$$

where $r=\sigma_{y} / \sigma_{x}, E$ is in $\mathrm{GeV}, I$ is in amperes, and $\beta_{y}^{*}$ is in $\mathrm{cm}$.

In this formulation, it appears advantageous to collide round beams, $r=1$, but this does not appear to be practical. With the energytransparency conditions assumed above, producing round beams requires $\beta_{x}^{*}=\beta_{y}^{*}$ and $\epsilon_{x}=\epsilon_{y}$. It is difficult to increase $\epsilon_{y}$ by wigglers, etc. to the natural value of $\epsilon_{x}$ from synchrotron radiation in the arcs, and introducing strong $x-y$ coupling would severely constrain the working point in tune space. Very strong focusing quadrupoles near the collision point would be required to achieve $\beta_{x}^{*}=\beta_{y}^{*}$, and it is not clear that the resulting chromaticity would be tolerable. Since at least one of the two beams must be off-axis in these quads, the synchrotron radiation would be intense, and it was not possible to find a solution to the resulting backgrounds in the detector.

\subsubsection{Beam Separation}

The collision frequency $f$ is set by the spacing of the bunches. It is desirable to prevent the bunches from colliding except inside detectors, to eliminate any contribution to the tune-shift from extra collisions. The effect of extra collisions is particularly damaging if they occur at points of high beta, or if the collisions are a few sigma away from being head-on. In an asymmetric energy machine, the beams are necessarily in separate rings, so additional collisions can only occur in the region where the rings are brought together. A limit to $f$ is then set by the ability to separate the beams.

One method of separating the beams is to collide them at a substantial angle. In this case, not every particle has a chance to collide with every particle in the other bunch, and the luminosity is decreased. An additional problem with large crossing angles is that

Parasitic collisions must be minimized

In PEP-II, the beams collide with zero crossing angle particles at the front and rear of the bunch, which are at opposite phases of their synchrotron motion in longitudinal-position-energy phase-space, receive opposite transverse kicks from the other beam. This synchrotron-betatron coupling greatly complicates the beam 
A "crab-crossing" scheme can eliminate the coupling of synchrotron and betatron motions that is endemic to a non-zero crossing angle. Such a scheme has never been implemented
In a head-on collison scheme, separation dipoles and quadrupoles must function inside the magnetic field of the detector

The PEP-II energy range extends over the $\Upsilon$ resonances and the nearby continuum dynamics and reduces the luminosity dramatically. The original two-ring DORIS machine at DESY with a $12 \mathrm{mr}$ crossing angle was limited to a very low luminosity by synchro-betatron resonances [Piw77]. In principle it is possible to avoid both the above problems by rotating the bunches by exactly the crossing angle before the collision point, and reversing the rotation afterward, the "crabcrossing" scheme of Palmer [Pal88]. This requires cavities with large transverse RF fields on both sides of the collision point. The "crabcrossing" technique has never been demonstrated in practice.

Since the beam energies are asymmetric, it is possible to collide the beams head-on, but separate them with a simple dipole magnet. To allow a high collision frequency without extra collisions, the separation magnet must be very close to the collision point. Additionally, the separation magnet will be a source of synchrotron radiation, and thus potentially a source of detector background.

\subsubsection{PEP-II Design}

The route to higher luminosity chosen for PEP-II is to reduce $\beta_{y}^{*}$ to the limit set by the bunch length, and increase $f$ by reducing the bunch spacing to the limit of practical beam-separation schemes. The parameters of PEP-II (see Table 4-1) satisfy the energy transparency conditions. The bunch populations do not require a vacuum chamber with a lower broad-band impedance than that achieved in PEP. The beams collide with zero crossing angle and are separated magnetically. This strategy does require separation dipoles and focusing quadrupoles that can function inside the detector solenoid magnetic field, a vacuum system that can tolerate intense synchrotron radiation, an RF system with excellent damping of narrow-band impedance, and a feedback system to stabilize the remaining multi-bunch instabilities. With a conservative tune-shift of $\xi=0.03$, a luminosity of $3 \times 10^{33} \mathrm{~cm}^{-2} \mathrm{~s}^{-1}$ can be achieved at $E_{c m}=10.58 \mathrm{GeV}$.

The permanent magnets used in the interaction region limit the easily available range of $E_{c m}$, but the full energy range of the $\Upsilon$ resonances and nearby continuum [PEP93, pages 101-102] can be covered. It will also be possible to study mixing and $C P$ violation in the $B_{s}^{0}$ system on the $\Upsilon(5 S)$ resonance at somewhat reduced lumi- 
nosity with no major changes to the machine. A dedicated $\Upsilon(5 S)$ run would probably be done with a re-optimized interaction region at a larger asymmetry, perhaps along with a significant luminosity upgrade.

\begin{tabular}{|l|c|c|c|}
\hline Parameter & HER & LER & Units \\
\hline \hline Center-of-mass energy & \multicolumn{2}{|c|}{10.580} & $\mathrm{GeV}$ \\
Peak Luminosity & \multicolumn{2}{|c|}{$3.0 \times 10^{33}$} & $\mathrm{~cm}^{-2} \mathrm{~s}^{-1}$ \\
Luminosity lifetime & \multicolumn{2}{|c|}{1.55} & $\mathrm{hr}$ \\
Bunch spacing & \multicolumn{2}{|c|}{1.26} & $\mathrm{~m}$ \\
Circumference & \multicolumn{2}{|c|}{2219} & $\mathrm{~m}$ \\
Beam energy & 9.000 & 3.109 & $\mathrm{GeV}$ \\
Bunch length & 1.0 & 1.0 & $\mathrm{~cm}$ \\
Number of bunches & 1658 & 1658 & \\
Particles per bunch & $2.73 \times 10^{10}$ & $5.91 \times 10^{10}$ & \\
Beam current & 0.986 & 2.140 & $\mathrm{Amps}$ \\
Transverse damping time & 36.8 & 40.4 & $\mathrm{msec}$ \\
Horizontal emittance & 48.2 & 64.3 & $\mathrm{~nm}-\mathrm{rad}$ \\
Horizontal IP beta & 50.0 & 37.5 & $\mathrm{~cm}$ \\
Horizontal IP spot size & 155 & 155 & $\mu \mathrm{m}$ \\
Horizontal tune-shift & 0.03 & 0.03 & \\
Vertical emittance & 1.93 & 2.57 & $\mathrm{~nm}-\mathrm{rad}$ \\
Vertical IP beta & 2.00 & 1.50 & $\mathrm{~cm}$ \\
Vertical IP spot size & 6.2 & 6.2 & $\mu \mathrm{m}$ \\
Vertical tune-shift & 0.03 & 0.03 & \\
\hline
\end{tabular}

Table 4-1. PEP-II Parameters

\subsection{Components}

Both the High Energy Ring (HER) and Low Energy Ring (LER) of PEP-II are housed in the original PEP tunnel, thus avoiding civil construction. The electrical and cooling-water utilities of PEP are sufficient for PEP-II with only minor modifications. The large $2.2 \mathrm{~km}$ circumference results in relatively low synchrotron radiation power losses, which reduce the requirements on the RF accelerating 
Two new injection bypass lines from the $S L C$ will be built for PEP-II

Injection in top-off mode (the normal mode of operation) will take 3 minutes. Injection from scratch will take 6 minutes system. It also spreads the SR power over a large area, reducing the thermal stresses on the vacuum system. The LER has the same circumference as the HER; asymmetric machines with unequal circumference rings suffer from beam-beam dynamics complications that are avoided by having identical circumferences. Any savings from a LER with a smaller circumference would, in any case, likely be absorbed by the cost of civil construction of a separate tunnel.

The PEP tunnel has six arcs, and six long straight sections with surface access (IR halls). The detector will be located in the IR2 hall, which is 22 meters wide along the beam and 35 meters long. A wall of movable shielding blocks divides the hall, so the detector can be assembled while PEP-II is being commissioned. The HER RF system will be located in IR8 and IR12; the LER RF system will be in IR4. The original PEP RF system was located in these IR's, so the electrical and cooling utilities have adequate capacity. Injection into the HER occurs at IR10, and injection into the LER is at IR8. The existing injection beam lines from the SLAC linac beam switchyard to PEP will be used with modest upgrades.

The SLAC Linear Collider (SLC) will be somewhat modified for PEP-II injection. The SLC $e^{+}$and $e^{-}$damping rings and highpower $e^{+}$production system will be retained, but two new extraction points from the linac will be introduced at locations where the beam energies will be adjusted to equal the PEP-II ring energies. These extraction points will be located at Sector 4 for the low-energy beam and Sector 10 for the high-energy beam. The beams will be transported in new bypass beam lines to the end of the existing linac tunnel where they will join the PEP injection beam lines. The bypass lines avoid the difficulties of transporting low-energy beam bunches through the wakefields and higher-energy focusing of the linac that impeded previous attempts to inject SLC beams into PEP. It will be possible to recover from complete loss of both beams in 6 minutes, and to top off both rings in 3 minutes. For an injection time of 6 minutes, the optimum time between fills is 30 minutes, yielding an average of $71 \%$ of peak luminosity. The average luminosity decreases to $65 \%$ of peak if the injection time increases to 10 minutes. 


\subsubsection{Lattice Design}

The original PEP arcs contained 192 half-cells, each of which contained a bend (dipole) magnet, a focusing or defocusing quadrupole, and a sextupole for chromaticity control. This scheme is being retained for both rings of PEP-II. The PEP magnets are being refurbished for the PEP-II HER, while new magnets are being constructed for the LER. The LER bend magnets are much shorter than the HER bends, since it is desirable to increase the LER damping rate by increasing the synchrotron radiation. New supports will carry the HER magnets somewhat closer to the floor than PEP, and the LER magnets will be mounted above the HER magnets on corresponding supports.

The FODO quadrupole lattice of the arcs will be maintained through most of the straight sections in both the HER and LER (this requires some additional HER quadrupoles to be constructed). In particular, the RF straight sections in PEP-II will have considerably lower beta values than in PEP, to minimize synchro-betatron coupling problems at the RF cavities. The injection straight sections have special optics with a central high-beta point. This makes the beam size larger compared to the septum size, to make the construction and operation of the injection components easier. Two of the LER straight sections have special optics to accommodate wiggler magnets. The wigglers can be used to increase the damping rate in the LER to be as large as in the HER. The optics of the interaction region is of course special, and is discussed in detail below.

\subsubsection{Vacuum System}

The vacuum chamber in the HER arcs will be made of copper, as is the HERA electron ring. Copper has a low gas photo-desorption coefficient from synchrotron radiation compared to aluminum. This allows the vacuum requirement of $10 \mathrm{nTorr}$ in the arcs to be achieved by the conventional means of distributed ion pumps integrated into magnets. The distributed pumps will be supplemented by conventional ion pumps between the bend magnets. In the LER, most of the synchrotron radiation is absorbed between the short bend
To increase the damping rate, the LER bends are shorter than the HER bends

There are wiggler magnets in the LER for control of the damping rate

The vacuum system uses discrete and distributed ion pumps 
To minimize impedance, the two PEP-II rings have a total of $30 R F$ cavity cells, compared to 120 for PEP

Longitudinal and transverse feedback systems are required to damp instabilities magnets rather than inside them. Conventional discrete ion pumps will be used in the region between the bends. In both rings, the straight section vacuum chambers will be primarily stainless steel, with one discrete ion pump per quadrupole.

\subsubsection{RF System}

Since PEP-II will have beam currents far larger than in past $e^{+} e^{-}$ machines, the beam currents will excite far larger fields in the RF system. It is desirable to apply the accelerating voltage required to overcome synchrotron radiation losses with as few RF cavities as possible, to minimize multibunch instabilities. Thus PEP-II has only 20 cavity cells for the HER and 10 for the LER, compared to 120 for PEP. The high beam currents also imply that more power must be transmitted to the beam for a given RF voltage, so the power absorbed by the cavity walls is less important than in lower-current machines. In fact, the minimum number of cavities is determined more by the power-handling capacity of waveguideto-cavity couplers than the cavities themselves. Thus, there is little advantage to superconducting cavities, and the PEP-II RF cavities are made of copper. The higher order modes (HOM) of the cavities are damped by three waveguides terminated by RF absorbers. These waveguides propagate the HOM power, but not the fundamental accelerating mode. Low-power tests of the cavity design show that the HOM driving the worst beam instabilities are reduced by more than three orders of magnitude.

Despite the damping of the RF cavity HOMs, one longitudinal and two transverse beam instability modes are expected to have growth rates greater than the damping provided by synchrotron radiation. PEP-II therefore has longitudinal and transverse feedback systems. The longitudinal system measures the phase of each bunch every time it passes a pickup, continuously analyzes the data in a digital processor farm, and applies the bunch energy corrections with a broadband accelerating-structure kicker. Prototypes have been constructed, and have been tested at SPEAR at SLAC and at the Advanced Light Source at LBL. The transverse feedback system is very similar, with many components derived from the longitudinal system. Information from beam position monitors approximately $90^{\circ}$ apart in betatron phase is used to calculate a transverse cor- 
rection for each bunch on each turn. The correction is applied by a transverse kicker on the next ring turn.

\subsection{Interaction Region}

The PEP-II interaction region (IR) must take beams from two separate storage rings with energies differing by a factor of three, combine them to a common axis, focus them to sub-pinhead sizes, collide them a few centimeters away from the closest detector elements, then separate them and return them to their respective rings. The layout of the PEP-II interaction region is seen in Figure 4-2. It is described fully in the PEP-II Conceptual Design Report [PEP93, pages 55-58 and 66-74].

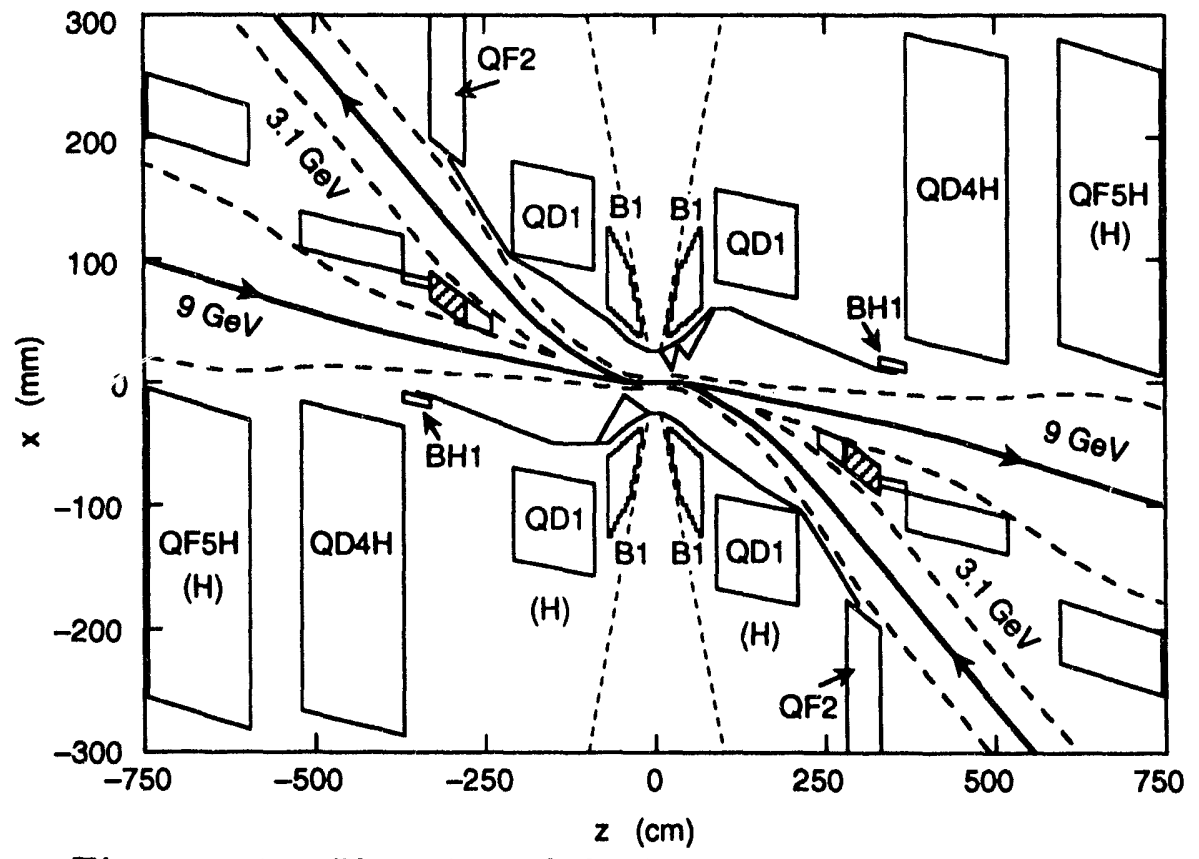

Figure 4-2. Plan view of the interaction region. The lowenergy beam enters from the lower right and exits from the upper left. The high-energy beam enters from the left and exits on the right. The vertical scale is highly exaggerated. The dashed lines represent the beam stay-clear envelopes, and the $300 \mathrm{mr}$ detector acceptance cutoff.

As noted above, if bunches collided or passed closely at any point except the intended low-beta collision point, the accumulated beam- 
The first bending magnet, $B 1$, is only $20 \mathrm{~cm}$ from the interaction point

The high energy beam is centered on the Q1 axis; the low energy beam is off-axis in the horizontal plane

$B 1$ and $Q 1$ are permanent (SmCo) magnets beam tune shift from the parasitic collisions would greatly limit the luminosity. Particle-tracking studies (see [Ten91], [PEP93, pages 200-208]) indicate that if the beams are separated by at least $7 \sigma_{x}$ parasitic collisions are unimportant. The IR design achieves $12 \sigma_{x}$ separation in the horizontal plane at the first parasitic collision at $63 \mathrm{~cm}$ by placing the inner end of the $\mathrm{B} 1$ dipole magnet at a distance of only $20 \mathrm{~cm}$ from the interaction point (IP). The Q1 quadrupole, the primary vertical focusing element for the low energy beam (LEB), starts at $90 \mathrm{~cm}$ from the IP. The high energy beam (HEB) is centered on the Q1 axis, but the LEB is off-axis in the horizontal plane. The orbit of the LEB is thus deflected further away from the HEB. At a distance of $280 \mathrm{~cm}$ from the IP, the Q2 quadrupole provides the primary horizontal focusing for the LEB. Q2 is located on the LEB axis, and the separation of the two beams is sufficient for the HEB to traverse a field-free hole in Q2. The Q4 and Q5 quadrupoles, at 370 and $595 \mathrm{~cm}$, are the final doublet for the $\mathrm{HEB}$, while the LEB travels through the field-free region outside them.

The B1 and Q1 magnets are inside the $1.5 \mathrm{~T}$ detector solenoid field. Conventional iron magnets do not function in this environment, so the only possibilities are superconducting or permanent magnets. There is not enough room between the beampipe and the $300 \mathrm{mr}$ detector acceptance envelope at the location of B1 to allow a cryostat, so the only option is a permanent magnet. In the PEP-II baseline design, Q1 is also a permanent magnet. (A superconducting Q1 design is also being considered. It would not change the IR optics in a fundamental way, nor would it change the luminosity, but it would allow greater operational flexibility, at a significantly higher cost.)

$\mathrm{B} 1$ and $\mathrm{Q} 1$ are constructed of rings of samarium-cobalt (SmCo) permanent magnet material. Each ring is constructed of SmCo blocks, with their magrietic moments normal to the beam direction and varying in azimuth around the beam in the manner described by Halbach [Hal81]. Permanent quadrupole magnets very similar to Q1 have been used at the Cornell Electron Storage Ring for many years [Her87a], [Her87b].

The Q2 quadrupole is outside the detector solenoid, so it is a conventional iron and copper magnet. However, the field must change from a high-quality quadrupole for the LEB to a field-free region for 
the HEB over a distance of $3 \mathrm{~cm}$. This is accomplished by squeezing the conductor of one quadrant of the quadrupole into the small space between the pole tips, leaving the normal coil position open for the HEB to travel through it. The Q4 and Q5 quadrupoles are iron and copper magnets with similar but less significant perturbations to allow the LEB to travel in field-free regions.

\subsubsection{Beampipe Design}

The beam pipe at the interaction point is a double-wall structure, with an inner tube $50 \mathrm{~mm}$ in cliameter and $800 \mu \mathrm{m}$ thick, separated by $2 \mathrm{~mm}$ from an outer tube of $400 \mu \mathrm{m}$ thickness, with helium gas flowing between the tubes to remove the $200 \mathrm{~W}$ of beam induced heating. It is made of beryllium to minimize multiple scattering, totalling $0.34 \%$ of a radiation length. It is brazed to stainless steel beam pipe beyond the $300 \mathrm{mr}$ acceptance of the detector. The helium flow is $22 \mathrm{~g} / \mathrm{sec}$ at $2 \mathrm{~atm}$, giving a film temperature drop of $2^{\circ} \mathrm{C}$ and a temperature rise of $1.1^{\circ} \mathrm{C}$. The structure is designed to withstand $50 \mathrm{~atm}$ of pressure.

There are water-cooled masks inside the B1 magnet to prevent synchrotron radiation from striking the beam pipe. There are additional masks to protect the scptum region of the Q2 quads from synchrotron radiation. The IP beampipe and synchrotron radiation masks are described in the PEP-II Conceptual Design Report [PEP93, pages 357-371].

\subsubsection{Support Tube}

The two Q1 magnets and the B1 magnets, the IP beam pipe, and the vertex detector are assembled into a single rigid support barrel of $43 \mathrm{~cm}$ outside diameter, shown in Figure 4-3. The desig $n$ s for B1, Q1, Q2, and the support tube are described in [PEP93, pages 276-301].

The support barrel is assembled and internally aligned outside the detector and installed by sliding it through the drift chamber. The two end sections of the support barrel, which carry the Q1 magnet assemblies, are made from stainless steel. The middle barrel section
Q2, a conventional iron magnet, is a quadrupole for the $L E B$ and has a field-free region for the HEB

The beam pipe is a doublewalled Be structure, cooled with helium gas at $2 \mathrm{~atm}$

The synchrotron radiation masks are water-cooled

The beam pipe, $B 1$ and $Q 1$ magnets and the vertex detector are assembled in a rigid support barrel

The support barrel is less than $0.5 \%$ of a radiation length thick 


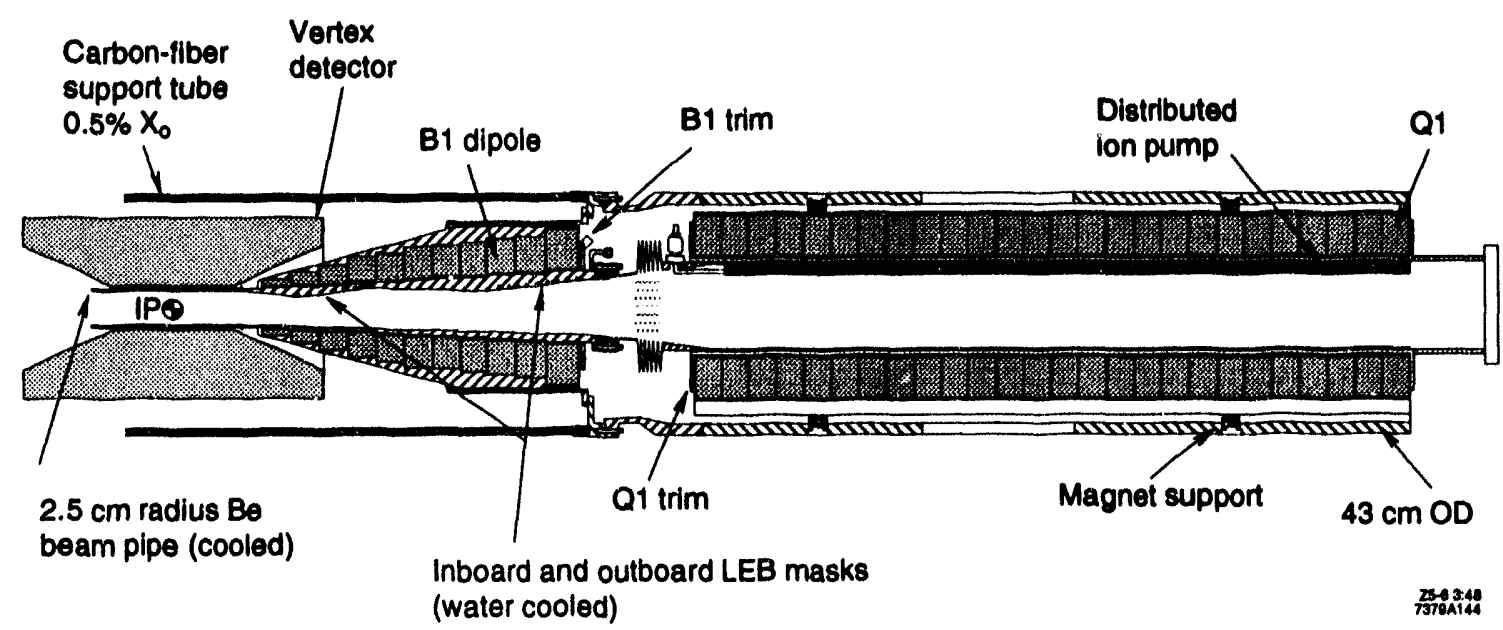

Figure 4-3. Support barrel for interaction region components inside the detector. Only one end is shown.

One method of holding the support tube and drift chanber is using vertical columns at the two ends of the coil is carbon fiber composite of $0.5 \%$ radiation length thickness. The total assembled weight is approximately $2500 \mathrm{~kg}$, concentrated in the end sections.

During installation, when the barrel is supported by its ends, the highest stress levels occur in the carbon fiber section. This section is designed with a safety factor of 3. The support barrel is mounted at each end near its quarter-point balance locations in a vertical support column. These structures are fixed across the vertical diameter of the magnet coil and provide support for the drift chamber as well as the support barrel. There are some failure scenarios for the superconducting Q1 option that could apply larger torques than occur during installation. These abnormal loads would be carried by the barrel support mounts and not the central carbon fiber section.

\subsection{Backgrounds}

\subsubsection{Overview}

The problem of machine-related backgrounds is one of the leading challenges in the PEP-II project. The detector must be sufficiently well protected to prevent excessive component occupancies or deterioration from radiation damage. In effect, what is required is 
to achieve background rates similar to those of existing colliders, but at beam currents an order of magnitude higher. Three primary sources of detector backgrounds have been considered:

- Synchrotron radiation photons produced in the machine magnetic elements,

- Interactions of beam particles with background gas molecules (Bremsstrahlung and Coulomb scattering), and

- Beam-beam Bremsstrahlung at the interaction point, in which beam particles radiate enough energy to be bent into machine elements by $\mathrm{B} 1$ or $\mathrm{Q} 1$.

These background sources can give rise to primary particles that can either enter the detector directly or generate secondary debris that ultimately reaches the detector.

A primary design strategy of the machine elements near the detector was to choose the placement and apertures of these elements in such a way as to ensure that most (about $90 \%$ ) of the synchrotron radiation power produced close to the collision point is absorbed on downstream surfaces far from the detector. This is a key ingredient of the design, as it ensures that local sources of scattered synchrotron radiation are greatly limited. In addition to the careful placement of magnetic elements, it is also important to provide low pressure (below $1 \mathrm{nTorr}$ ) in the section of beam pipe in each ring that immediately precedes the detector to reduce the rate of beamgas interactions.

The following sections describe the current state of the background calculations. Further discussion of these simulations can be found in [PEP93, pages 103-143]. Background studies are continuing along several directions. Tools are being developed to interface the current background generation code to a full GEANT detector simulation, which will allow realistic trigger rate studies. Work is also in progress towards estimating the rate of inelastic beam-gas interactions and the resulting trigger rate.
Background rates similar

to those at existng $e^{+} e^{-}$machines must be achieved with an order-ofmagnitude more current

Most of the synchrotron radiation power generated close to the collision region is absorbed far from the detector

A pressure below 1 nTorr is required near the detector

The current background generation code is being merged with GEANT for detailed trigger studies 


\subsubsection{Synchrotron Radiation Backgrounds}

There are several sources of synchrotron radiation backgrounds:

- Direct synchrotron radiation,

- Photons that scatter through a mask tip,

- Synchrotron radiation from elements far upstream of the interaction point, and

- Sources of backscattered photons from downstream surfaces.

Separating the unequal-energy beams by the use of bending magnets and offset quadrupoles generates several fans of synchrotron radiation. The geometry of the interaction region optics, however, is designed to minimize the amount of synchrotron radiation that strikes nearby surfaces. In particular, the "S-bend" geometry of the beamlines allows most of the synchrotron radiation generated by magnetic elements upstream of the interaction region to pass through the detector region without hitting local surfaces. Primary masks near the collision point are used to prevent direct radiation from hitting the detector beam pipe, while at the same time keeping the number of photons that strike the mask tips to an acceptable level. The synchrotron radiation fans from both beams are shown in Figure 4-4.

The Low Energy Beam (LEB) generates synchrotron radiation fans as it passes through the Q1 and B1 magnets on its way to the collision point. The LEB mask is designed to prevent the synchrotron radiation (either fan or quadrupole) generated by the upstream magnets from directly striking the detector beam pipe. The surfaces of the LEB mask are sloped such that scattered photons cannot travel directly to the detector beam pipe. The LEB mask absorbs

The LEB mask absorbs $3.6 \mathrm{~kW}$, the septum mask $3.5 \mathrm{~kW}$ about $3.6 \mathrm{~kW}$ of synchrotron radiation power. Fans generated by the LEB in the two B1 magnets and in the downstream Q1 magnet pass through the interaction region without striking nearby surfaces. The first surface that intercepts the B1 fans is the septum mask in front of the Q2 septum quadrupole, located $2.8 \mathrm{~m}$ from the IP. This septum mask absorbs $3.5 \mathrm{~kW}$ of power.

Synchrotron radiation fans generated by the High Energy Beam (HEB) in upstream bend magnets and offset quadrupole magnets 


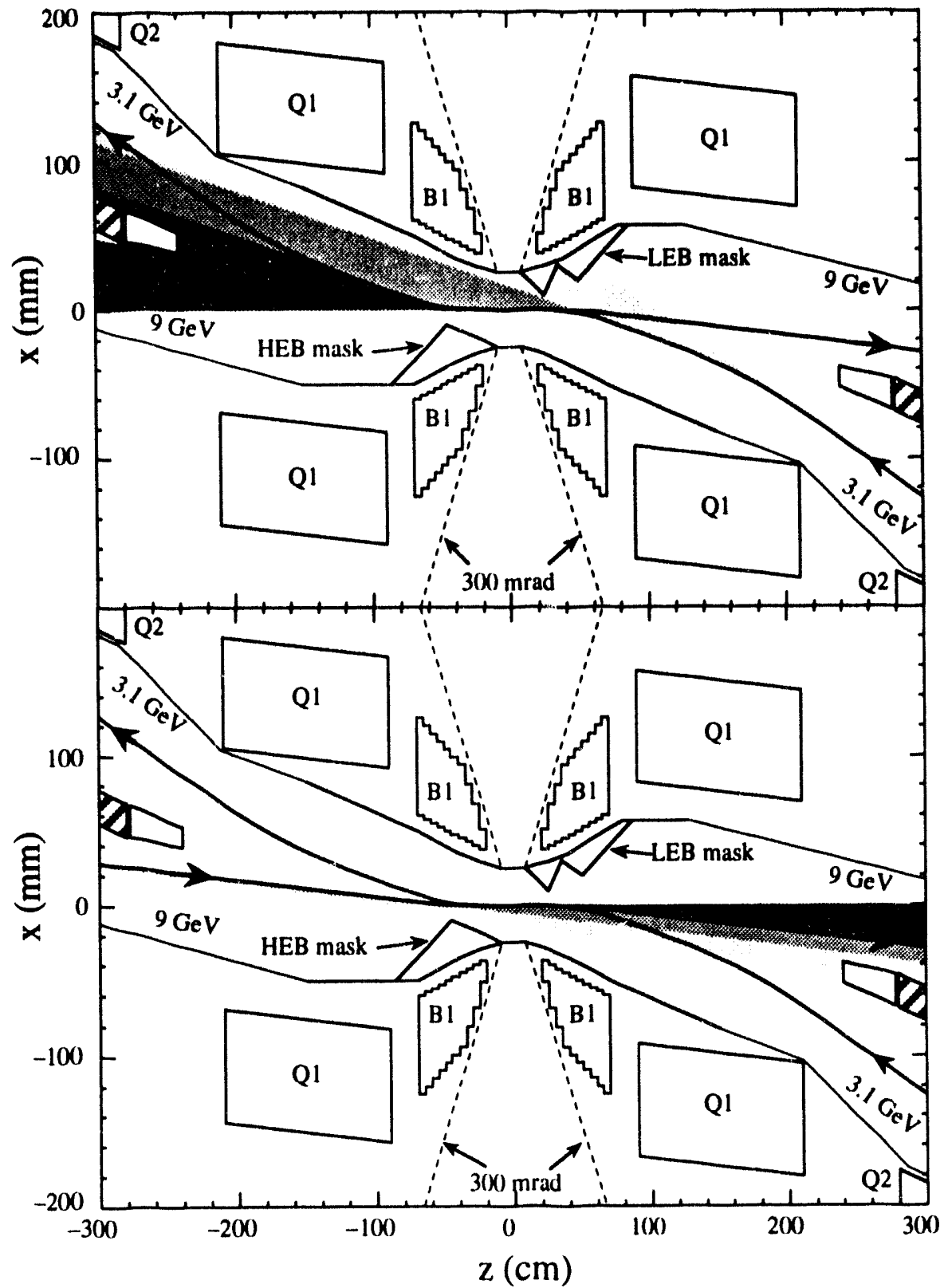

Figure 4-4. Synchrotron radiation fans from the low-energy (top) and high-energy (bottom) beams. The density of shading gives an indication of the relative photon intensity from the various radiation fans. 
PEP-II and the Interaction Region

\begin{tabular}{|c|c|c|c|c|c|}
\hline & $\begin{array}{c}\text { Incident } \\
\text { on Be } \\
\text { pipe }\end{array}$ & $\begin{array}{c}\text { Absorbed } \\
\text { in first } \\
\text { Si layer }\end{array}$ & $\begin{array}{c}\text { Absorbed } \\
\text { in second } \\
\text { Si layer }\end{array}$ & $\begin{array}{c}\text { Absorbed } \\
\text { in third } \\
\text { Si layer }\end{array}$ & $\begin{array}{c}\text { Incident } \\
\text { on Drift } \\
\text { Chamber }\end{array}$ \\
\hline $\begin{array}{c}4<E_{\gamma}<100 \mathrm{keV} \\
\text { \# of photons }\end{array}$ & 1.24 & .028 & $6.9 \times 10^{-4}$ & $5.6 \times 10^{-4}$ & $2.4 \times 10^{-5}$ \\
Energy (keV) & 17.6 & 0.43 & $2.2 \times 10^{-2}$ & $2.0 \times 10^{-2}$ & $3.2 \times 10^{-3}$ \\
\hline $\begin{array}{c}4<E_{\gamma}<20 \mathrm{keV} \\
\text { \# of photons }\end{array}$ & 0.96 & .022 & $3.8 \times 10^{-5}$ & $8.6 \times 10^{-5}$ & $2.1 \times 10^{-7}$ \\
Energy (keV) & 9.34 & 0.17 & $9.2 \times 10^{-4}$ & $1.3 \times 10^{-4}$ & $3.7 \times 10^{-6}$ \\
\hline $\begin{array}{c}\text { Detector Limits } \\
\text { \# of photons } \\
\text { Energy (keV) }\end{array}$ & - & 2.3 & $>2.3$ & $>2.3$ & 125 \\
\hline
\end{tabular}

Table 4-2. Syachrotron radiation background simulation results. The numbers are for each crossing. Multiply by $2.38 \times 10^{8}$ to get photons per second. In the simulation, the beam pipe consists of $25 \mu \mathrm{m}$ of $\mathrm{Cu}$ and $1 \mathrm{~mm}$ of $\mathrm{Be}$. The beam pipe inner radius is $2.5 \mathrm{~cm}$. The Si layers are $300 \mu \mathrm{m}$ thick.

The HEB mask absorbs nearly $1 \mathrm{~kW}$

Synchrotron radiation cannot hit the beam pipe directly deposit nearly $1 \mathrm{~kW}$ of power on the HEB mask. These upstream HEB elements are positioned to ensure that radiation generated by beam particles even $10 \sigma$ off-axis in these elements does not strike the LEB mask. The fans of radiation generated by the HEB as it passes through the two B1 magnets do not strike any surfaces in the detector region and are absorbed in a dump about $17 \mathrm{~m}$ downstream of the detector.

The geometry of the masking near the IP is such that no synchrotron radiation can hit the IP beam pipe directly, nor can it scatter off the face of a mask onto the IP beam pipe. Scattering through the tips of tiie LEB and HEB masks is the dominant source of photons striking the detector beam pipe. This process has been simulated in great detail. The individual photons that strike the LEB and HEB mask tips are generated from an energy spectrum given by a program that traces the photons from sources to surfaces of interest. The photons that end up striking the detector beam pipe are followed through the detector components by the EGS Monte Carlo program [Nel85]. Further details of this simulation package can be found in [PEP93, pages 108-112]. The results of the simulations are the photon spectra found in Figure 4-5; backgrounds resulting from synchrotron radiation are shown in Table 4-2. 


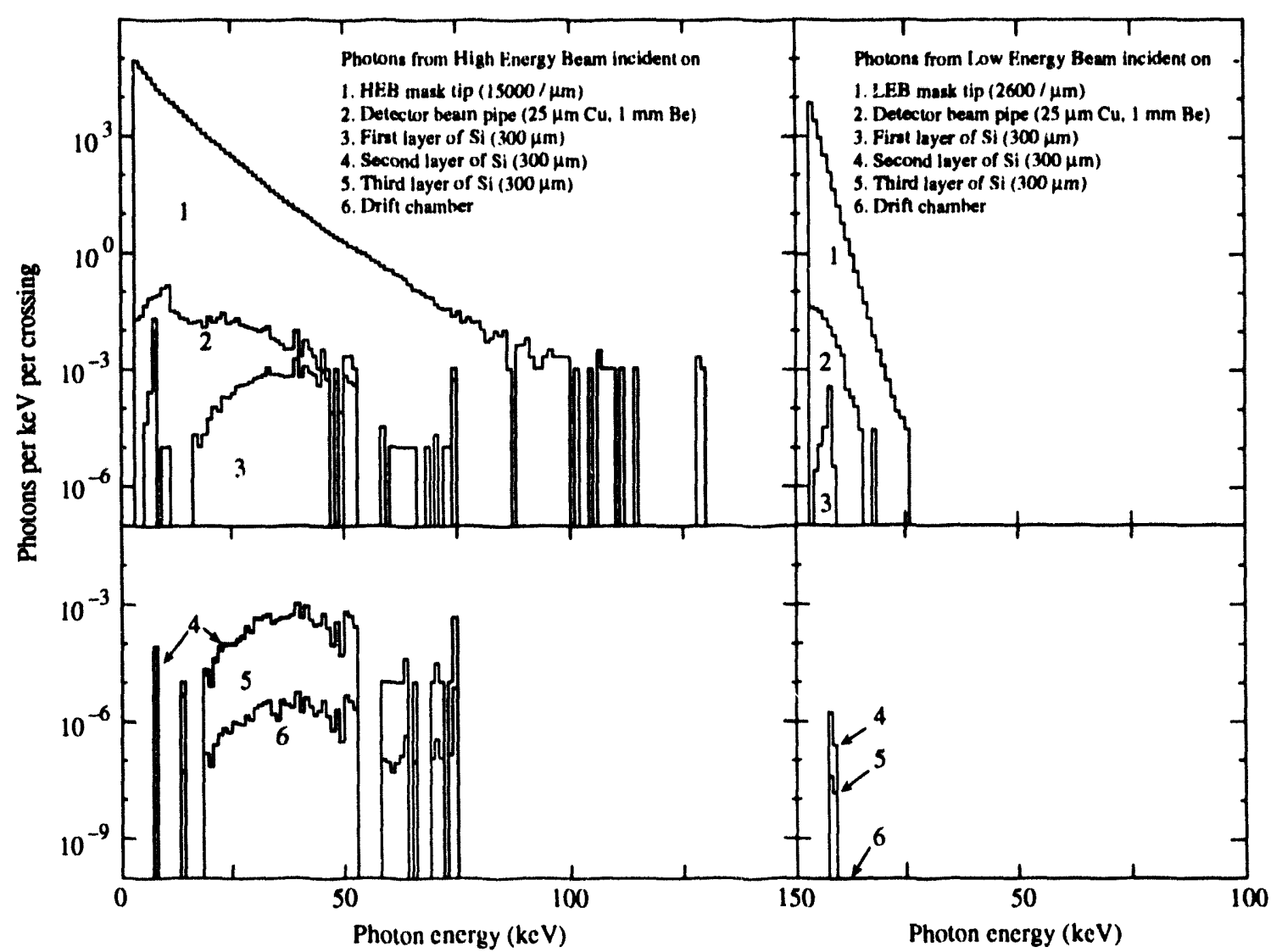

Figure 4-5. Photon spectra from synchrotron radiation. Left plot is for high energy bean, right plot is for low energy beam.

Other sources of synchrotron radiation, such as magnets farther upstream, and back-scatter from the Q2 septum masks, produce negligible contributions to the detector background compared to primary mask tip scattering. While a high photon flux strikes the dump for HEB radiation from the B1 magnets, the small solid angle: of the detector beam pipe as seen from this source, the small angle of incidence photons from this source have on the beam pipe, and the fact that most of the beam pipe is shielded by the LEB mask, together reduce the background the $17 \mathrm{~m}$ dump to a low level.

The effect of magnet misalignments on detector backgrounds has also been evaluated. There is a threefold increase in the background rate in one direction for a 5-mm displacement in $x$ of Q1, Q4 or Q5. The rest of the misalignment checks produced small $(<50 \%)$ increases in backgrounds, with some configurations producing rates that are actually below the nominal background rate. None of
The effect of misalignment of the interaction region magnets on detector backgrounds has been evaluated 
these misalignment checks resulted in synchrotron radiation photons directly striking the detector beam pipe.

Lost beam particles are a source of detector occupancy and radiation damage

Upstream masks are arranged to form a graded aperture

\subsubsection{Detector Backgrounds due to Lost Beam Particles}

Bremsstrahlung and Coulomb scattering of beam particles from residual gas molecules in the beam pipe can lead to high-energy electrons and photons striking masks and the beam pipe near the IP. The resulting electromagnetic showers can cause excessive detector occupancy and/or lead to radiation damage.

In calculating the rates at which particles strike near the IP due to bremsstrahlung and Coulomb scattering, both the HER and LER lattices have been simulated for a distance of $185 \mathrm{~m}$ upstream of the IP (halfway around the adjacent arc). The beam particles that have interacted with the residual gas (lost beam particles) are transported through the ring optics to the IP region. Particles striking the beam pipe near the detector start electromagnetic showers that are simulated with EGS.

The rate estimates are based upon a nominal beamline pressure of 1 nTorr ( $\mathrm{N}_{2}$ equivalent) for a distance of $35-60 \mathrm{~m}$ upstream of the IP. For the HER, a lower pressure ( 0.2 nTorr) is assumed from 35 to $3 \mathrm{~m}$ upstream of the IP, and for the LER, the region from 15 to $2 \mathrm{~m}$ upstream of the IP is kept at 0.2 nTorr. For both rings, the pressure close to the IP is taken as $1 \mathrm{n}$ Torr. In the discussion below, the background rates are quoted per microsecond, since $1 \mu \mathrm{s}$ is a typical integration time for detector elements. One microsecond corresponds to 238 beam crossings.

The number of rays striking near the IP is reduced by suitably placed upstream masks. A graded aperture is employed in this region of the machine lattice ( $\pm 60 \mathrm{~m}$ from the IP), that is, progressively larger apertures are used as one approaches the IP. This masking scheme essentially eliminates rays from the higher-pressure (about 5 nTorr) upstream arc regions that start $60 \mathrm{~m}$ from the IP in each ring. Some of the remaining rays, from soft bremsstrahlung interactions, remain inside the beam stay-clear envelope until they are well inside the detector region. The rate of these lost particles is 


\begin{tabular}{|c|c|c|c|c|c|}
\hline & $\begin{array}{c}\text { First } \\
\text { Si layer }\end{array}$ & $\begin{array}{l}\text { Second } \\
\text { Si layer }\end{array}$ & $\begin{array}{l}\text { Third } \\
\text { Si layer }\end{array}$ & $\begin{array}{c}\text { Drift } \\
\text { Chamber }\end{array}$ & $\begin{array}{c}\text { CsI } \\
\text { Calorimeter }\end{array}$ \\
\hline$\overline{\text { Hits per } \mu \mathrm{sec}}$ & 20 & $\overline{14}$ & 7 & $0.4 /$ layer & $\overline{0.8}$ \\
\hline Dose $(\mathrm{krad} / \mathrm{yr})$ & 10 & 3.3 & 0.8 & $0.001 \mathrm{C} / \mathrm{cm} / \mathrm{yr}$ & 0.009 \\
\hline $\begin{array}{l}\text { Detector Limits } \\
\text { Hits per } \mu \mathrm{s}\end{array}$ & 140 & 260 & 360 & 7-30/layer & 10 \\
\hline Dose (krad/yr) & 200 & 200 & 200 & $0.1 \mathrm{C} / \mathrm{cm} / \mathrm{yr}$ & 10 \\
\hline
\end{tabular}

Table 4-3. Summary of predicted average occupancy and radiation dosage for the silicon detector, drift chamber and CsI calorimeter for lost beam particle backgrounds. Tolerable levels for each subsystm are indicated under detector limits. The rate in the calorimeter is for photons of greater than $10 \mathrm{MeV}$ in energy, averaged over the entire calorimeter above $45 \mathrm{~cm}$ radius.

controlled by maintaining a low pressure in the region immediately upstream of the detector.

Using the EGS Monte Carlo code with an interface for complex geometries [Hea91], the hits per $\mu \mathrm{sec}$ in the various detector components have been determined. The simulation includes the geometry and material type of the beam pipe, masks, magnets, and detector components as well as the IR optics and detector magnetic fields. The occupancy and radiation dosage for each detector component are listed in Table 4-3. Figure 4-6 shows the energy and azimuthal distribution of elecirons and photons incident on the inner layers of the silicon, the drift chamber, and the inner face of the calorimeter.

Based on the results in the Table 4-3, occupancy and radiation dose are well within acceptable limits for the silicon detector, the drift chamber, and the CsI calorimeter.

\subsubsection{Detector Backgrounds due to Radiative Bhabhas}

Radiative Bhabhas produced at the collision point generate a spectrum of off-energy beam particles that can be swept into the detector by the downstream B1 and Q1 magnets. This process is similar to beam-gas bremsstrahlung except that it occurs solely at the IP
The OBJEGS package is used to estimate the number of lost particle hits per microsecond

Occupancy and radiation doses are within acceptable limits for all detector systems 

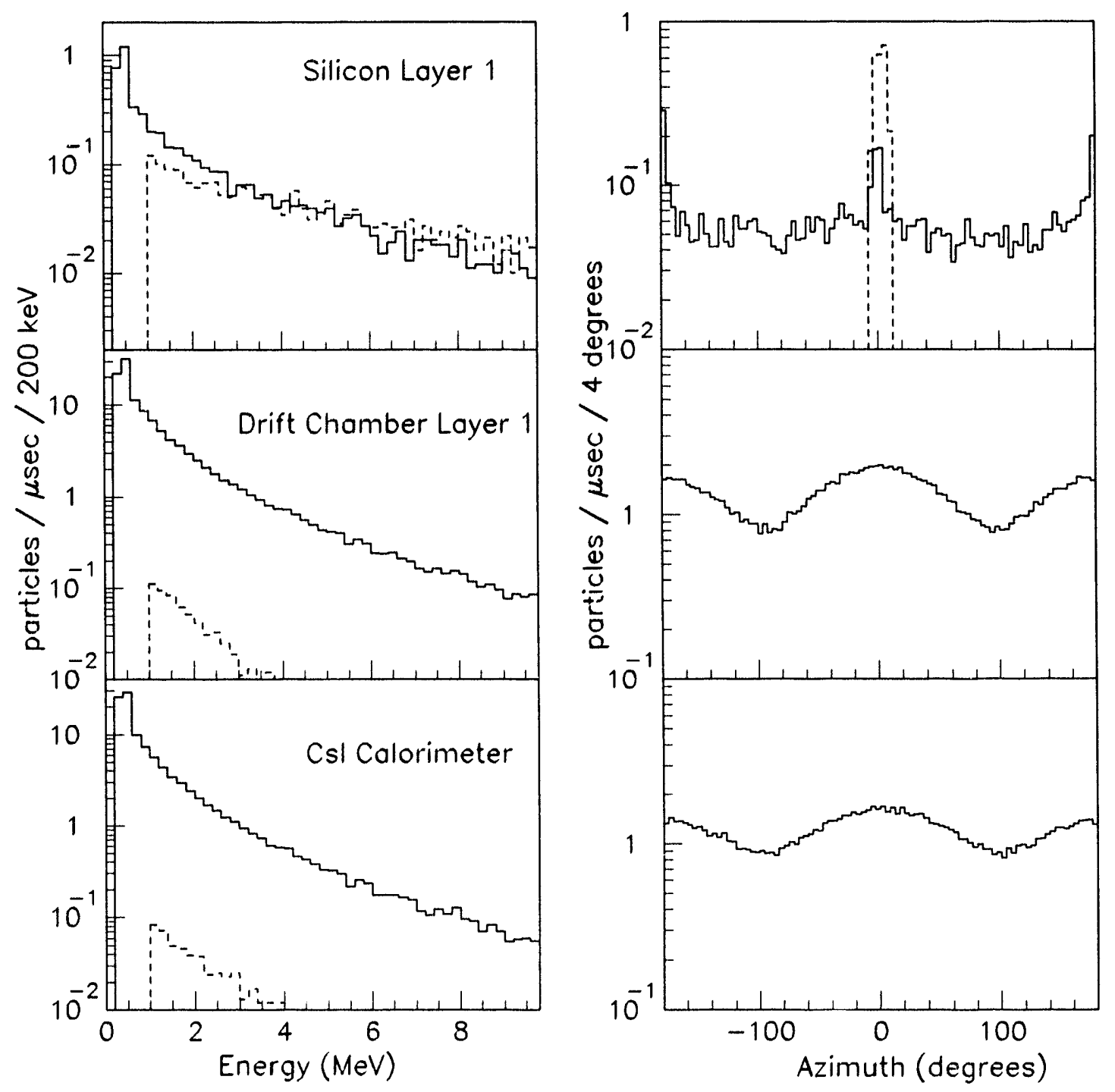

Figure 4-6. Energy and azimuthal distributions of photons (solid) and electrons (dashed) incident on the inner face of the silicon detector, drift chamber and CsI calorimeter for lost beam particle backgrounds. Rates correspond to the number of incident particles per microsecond. 
rather than being distributed throughout the beamline. Because of this similarity, the same analysis procedure that was used for beamgas bremsstrahlung can be employed here to study this process as well.

The main source of detector background comes from photons with energies near the minimum absorption cross section (1-2 MeV). These photons are remnants of showers that develop when off energy beam particles hit the beam pipe and machine components inside the detector. These photons are not uniformly distributed but are concentrated in azimuth on the side where the degraded beam particles hit, and in $z$ toward the ends of the drift chamber. The photons Compton scatter in the chamber producing spurious hits and increased radiation dosage. Estimating the peaking factor due to the nonuniform $\phi$ and $z$ distributions to be about 5 , the maximum charge deposition in the drift chamber is found to be $0.009 \mathrm{C} / \mathrm{cm} / \mathrm{yr}$, which is 11 times below our maximum acceptable level of $0.5 \mathrm{C} / \mathrm{cm}$ over a five-year operating lifetime.

\subsubsection{Summary}

The masking design for synchrotron radiation produces detector backgrounds that are $\sim 80$ times below the specified limits for radiation damage and detector occupancy. A detailed study of lostparticle backgrounds in the detector found that all devices are well within acceptable limits for both radiation damage and average occupancy, with typical safety factors relative to conservative limits of more than 20 for a five-year operating life. The effect of radiative Bhabhas on the detector has also been studied, indicating a safety factor of at least 11 in the regions of the central drift chamber where this source deposits excess energy.
The main source of detector backgrounds is few $\mathrm{MeV}$ photons from showers produced by lost beam particles

Synchrotron radiation and lost-particle induced backgrounds are well within acceptable limits 


\section{References}

[Chi90] Y.H. Chin, "Symmetrization of the Beam-Beam Interaction in an Asymmetric Collider", in Beam Dynamics Issues of High Luminosity Asymmetric Collider Rings, ed. by A. Sessler, American Institute of Physics (1990).

[Fur91] M.A. Furman, "Luminosity Formulas for Asymmetric Colliders with Beam Symmetries", SLAC Report SLAC ABC-25, 1991.

[Gar89] A. Garren et al.,"An Asymmetric B-Meson Factory at PEP", in Proceedings of the 1989 Particle Accelerator Conference, (Chicago IL, 1989) ed. by F. Bennet and J. Kopta, p. 1847.

[Hal81] K. Halbach, "Design of Permanent Multipole Magnets with Oriented Rare Earth Material", Nucl. Instr. Meth. 169, 1 (1980).

[Hea91] C. Hearty, "OBJEGS Users' Manual”, BABAR Note \#73, Oct. 1991.

[Her87a] S.W. Herb, "Construction of Large Permanent Magnet Quadrupoles", Cornell Report CLNS-87/61, 1987.

[Her87b] S.W. Herb and J. Kirchgessner, "Operation of CESR with Permanent Interaction Region Quadrupoles", Cornell Report CLNS-87/64, 1987.

[Kri90] S. Krishnagopal and R. Siemann, "Beam-energy inequality in the beam-beam interaction", Phys. Rev. D41, 1741 (1990).

[Nel85] W. Nelson, H. Hirayama, and D. Rogers, "The EGS4 Code System", SLAC Report SLAC-PUB-265, 1985.

[Pal88] R. Palmer, "Energy Scaling, Crab Crossing, and the Pair Problem", SLAC Report SLAC-PUB-4707, 1988.

[PEP93] PEP-II, An Asymmetric B Factory: Conceptual Design Report, LBL-PUB-5379, SLAC-PUB-418, CALT-68-1869, UCRL-ID-114055, UC-IIRPA-93-01, 1993. 
[Piw77] A. Piwinski, "Satellite Resonances due to Beam-Beam Interaction", IEEE Trans. on Nucl. Sci., NS-24, 1408 (1977).

[San70] M. Sands, "The Physics of Electron Storage Rings", SLAC Report SLAC-PUB-121, 1970.

[Ten91] J.L. Tennyson, "Parasitic Crossings in APIARY 6.3d", SLAC Report SLAC ABC-29, 1991. 
LETTER OF INTENT FOR THE BABAR Detector 


\section{Vertex Detector}

$\mathbf{T}$ he purpose of the vertex detector is to provide precise information on both the position and direction of charged particle trajectories close to the interaction point. When combined with tracking information from the drift chamber, the precision of the vertex detector dominates the measurement of the particle's position near the interaction point both along the beam axis and perpendicular to it. It also contributes significantly to the measurement of azimuthal and polar angles. For very low momentum charged particles, the track parameters must be completely determined in the vertex detector.

These track parameters (especially slope and position near the interaction point) are used to measure the location of $B, D$ and $\tau$ decays. For $C P$ violation studies, it is particularly important to measure the location of the $B$ decay along the beam direction (the $z$ axis). The mean distance between $B$ decays at PEP-II (with $\beta \gamma=0.56$ at the $\Upsilon(4 S)$ ) is about $250 \mu \mathrm{m}$. Both analytical calculations and Monte Carlo simulations show that the reduction in precision of $C P$-violating asymmetries due to imperfect vertex resolution is less than $10 \%$ if the distance between the reconstructed $B$ vertices is measured with a resolution equal to half the mean separation [LeD90a, LeD90b]. Therefore, our goal is to measure the $z$ position of a single $B$ decay with a precision of $125 \mu \mathrm{m} / \sqrt{2} \approx 90 \mu \mathrm{m}$ or better. However, pattern recognition, vertex-finding efficiency and background rejection all benefit from greater precision.

In this chapter, the required vertex resolution is shown to be achievable with double-sided silicon microstrip detectors. A conceptual design is presented based on five layers of silicon sensors with a readout pitch of $100 \mu \mathrm{m}$ in the inner layers and $200 \mu \mathrm{m}$ in the outer layers. Given the material in the beampipe and the first layer of silicon, the track parameter resolution is dominated by multiplescattering errors, and not by intrinsic position resolution, except for the highest momentum tracks from $B$ decays. Mechanical support
$A$ measurement of the $z$ position of the $B$ decay vertex to a precision of $\sim 90 \mu \mathrm{m}$ is adequate, but better precision improves background rejection and vertex finding efficiency

The conceptual design is a five-layer device, allowing efficient trackfinding within the vertex detector itself 
The required precision of the vertex detector is set by the limitations of multiple Coulomb scattering in the material upstream of the measurement issues and the electronic readout requirements for such a system are discussed.

$R \& D$ is described for a small high-precision Time Projection Chamber based on microstrip gas chamber readout. The potential for such a system to provide increased acceptance in the forward region is being explored.

Finally, an outline is given of the features of a pixel-based silicon detector that make it attractive as a potential future upgrade. Also identified are the challenges that must be overcome to reap the benefits of the improved intrinsic spatial resolution of a pixel system.

\subsection{Design Considerations}

The measurement errors on each of the charged-track parameters depend on both the intrinsic detector resolution and multiple scattering. The precision of the measurement device should be good enough so that its contribution to the track error is not significantly larger than that from multiple scattering. The multiple scattering contribution to the impact parameter resolution is dominated by the material upstream of the measurement system (in this case the beam pipe and the first detector layer). It varies as the square root of the number of radiation lengths multiplied by the radial position of this material. Section 5.4 (curve (a) in Figure 5-5) shows that the multiple-scattering contributions to the uncertainty on the track position and angle for the highest momentum particles from $B$ decays are approximately $12 \mu \mathrm{m}$ and $0.5 \mathrm{mr}$, respectively, at normal incidence for realistic choices of detector radii and material thickness. Thus the precision of the measurement device need not be significantly better than these values.

For the simple case of two measurements $z_{1}$ and $z_{2}$, the errors on the impact parameter along the beam direction, $z_{0}$, and the tangent of the dip angle, $\tan \lambda$, from the detector resolution only (i.e. no multiple scattering) are

$$
\sigma_{z_{0}}^{2}=\frac{r_{2}^{2} \sigma_{z_{1}}^{2}+r_{1}^{2} \sigma_{z_{2}}^{2}}{\left(r_{2}-r_{1}\right)^{2}} \text { and } \sigma_{\tan \lambda}^{2}=\frac{\sigma_{z_{1}}^{2}+\sigma_{z_{2}}^{2}}{\left(r_{2}-r_{1}\right)^{2}}
$$

where $r_{1}, r_{2}$ are the fixed radii of the measurements and $\sigma_{z_{1}}, \sigma_{z_{2}}$ are the errors on the $z$ measurements. From the first equation, it 
can be seen that $\sigma_{z_{0}}$ is approximately equal to $\sigma_{z_{1}}$ if the lever arm $\left(r_{2}-r_{1}\right)$ is large compared to $r_{1}$. Therefore, the error on the first measurement should be as small as possible, but does not have to be much smaller than the contribution from multiple scattering $(\approx$ $12 \mu \mathrm{m})$. The resolution for the second measurement should satisfy $\sigma_{z_{2}}<r_{2} \sigma_{z_{1}} / r_{1}$; the benefit of reducing it much below this value is marginal. From the second equation, it can be seen that for normal incidence an angular precision of $0.5 \mathrm{mr}$ can be achieved with a $10 \mathrm{~cm}$ lever arm and an intrinsic resolution on $z_{2}$ of about $50 \mu \mathrm{m}$. The same conclusions apply to the $x-y$ plane if the momentum is assumed to be determined with the drift chamber.

These considerations lead to the following design guidelines:

1. The product $r_{1} \sqrt{X_{0}}$ should be minimized, where $r_{1}$ is the radius of the first measurement and $X_{0}$ is the amount of material traversed by the narticle through the first measurement layer.

2. The resolution in $z$ and $r \phi$ for the first measurement on the track should not be much larger than $\approx 12 \mu \mathrm{m}$ at normal incidence.

3. The resolution in $z$ and $r \phi$ for the outer measurement(s) should be $\approx 50 \mu \mathrm{m}$ or less.

A design concept based on double-sided silicon strip detectors has been developed that satisfies these guidelines and meets the goals for the vertex detector. The achievable intrinsic resolution for silicon strip detectors is first discussed and then a particular layout presented.

\subsection{Intrinsic Resolution of Microstrip Detectors}

With silicon strip detectors, a resolution of $15 \mu \mathrm{m}$ is readily achievcd with $50 \mu \mathrm{m}$ pitch (center-to-center spacing of strips) for tracks close to normal incidence in the plane perpendicular to the strips. However, as the angle of incidence increases, the resolution degrades due to inefficiencies in the readout for strips with small signals at the
The precision of the first measurement of the decay vertex position should be as high as is practical 
The degradation of the resolution at larger angles of incidence has been studied with a detailed Monte Carlo calculation

Digital and analog centroid-finding algorithms have been compared

$100 \mu \mathrm{m}$ readout pitch with floating strips provides excellent resolution at small angles with only very modest degradation at large incident angles ends of the cluster of hit strips. A Monte Carlo-based simulation has been used to study this effect, taking into account fluctuations in energy loss, diffusion, channel-to-channel gain variations $(5 \%)$ and noise. This simulation was used to study the effects of strip pitch, noise levels, threshold levels and readout schemes on intrinsic position resolution.

The position of the charged track can be determined using several different algorithms. The simplest is a digital algorithm that calculates the average of the center positions of the first and last strips with a signal larger than a particular threshold. The second method uses analog readoc information to correct the digital centroid by the quantity $(P / 2)\left(S_{N}-S_{1}\right) /\left(S_{N}+S_{1}\right)$ where $P$ is the strip pitch, and $S_{1}$ and $S_{N}$ are the pulse heights in the first and last strips in the cluster, respectively. The final method assumes that every second strip is floating so that charge is capacitively partitioned to adjacent strips. Analog pulse height information is read out for the strips between the floating strips and the centroid of the charge distribution is calculated.

The intrinsic resolution for $300 \mu \mathrm{m}$-thick silicon is shown as a function of incident angle $(\lambda)$ in Figure 5-1 for 50 and $100 \mu \mathrm{m}$ pitch digital readout with a noise level of $1200 e^{-}$, corresponding to a signal-to-noise ratio of about 20 for perpendicular tracks. The readout threshold is set to 4 times the noise. For $50 \mu \mathrm{m}$ pitch, the resolution is significantly degraded for large angles of incidence and becomes worse than that for $100 \mu \mathrm{m}$ pitch at about $45^{\circ}$. Further studies have shown that this degradation can be reduced by lowering the threshold. However, the resolution is then worse for small angles of incidence for $50 \mu \mathrm{m}$ pitch and for all angles of incidence for $100 \mu \mathrm{m}$ pitch. For these pitches, the analog algorithm does not improve the resolution significantly. However, the analog centroid method, combined with floating strips, results in improved resolution for small angles of incidence. In particular, $100 \mu \mathrm{m}$ readout pitch with floating strips gives a resolution equivalent to $50 \mu \mathrm{m}$ readout pitch without floating strips for angles less than $45^{\circ}$. Above $45^{\circ}$, it is equivalent to $100 \mu \mathrm{m}$ readout pitch. Floating strips also improve the resolution at small incident angles for digital readout.

The resolution is shown in Figure 5-2 for the same noise and threshold assumptions but for 150 and $200 \mu \mathrm{m}$ pitch for the digital and analog algorithms. For these larger pitches, there is no degradation 


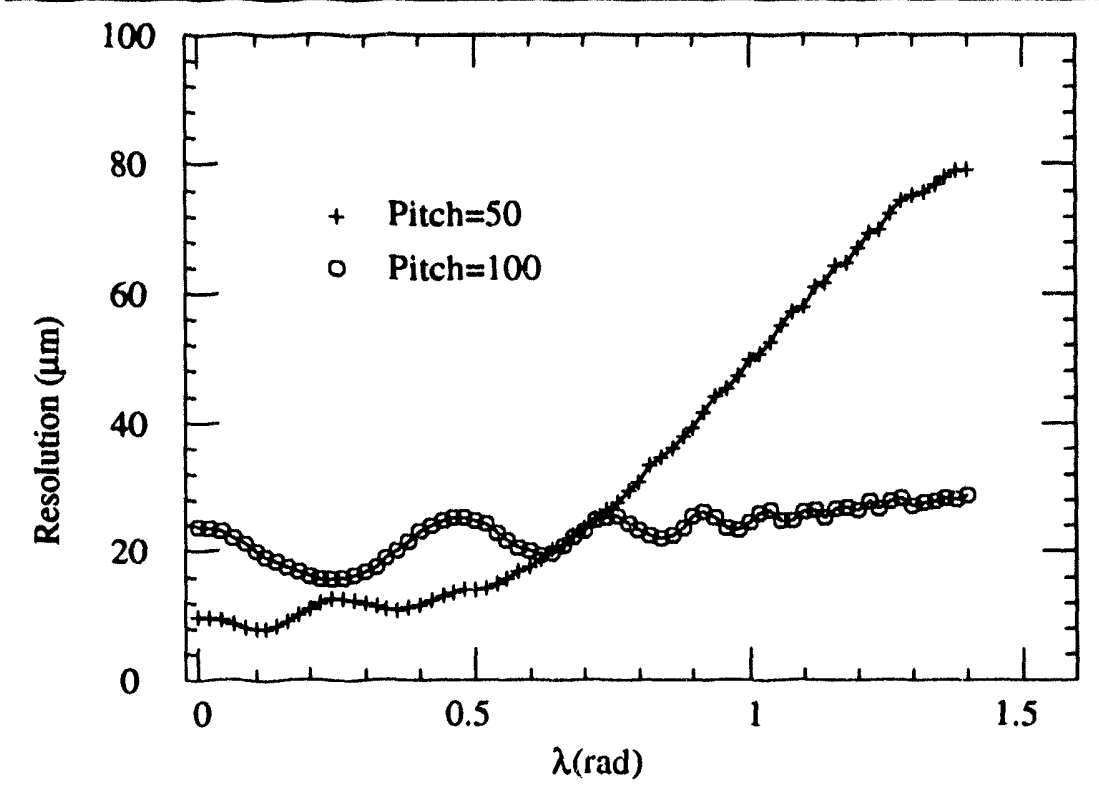

Figure 5-1. Calculated intrinsic resolution for a $300 \mu \mathrm{m}$-thick silicon vertex detector as a function of the angle of incidence of the charged track in the plane perpendicular to the strips for 50 and $100 \mu \mathrm{m}$ pitch, for digital readout. The noise level is $1200 e^{-}$ and the readout threshold is 4 times the noise. The waves in the curves are due to the absence of position smearing for the particle origin in the Monte Carlo.

of the resolution with angle and the analog centroid improves the resolution significantly at large angles of incidence. The same readout pitch with floating strips improves the resolution by another factor of two at small angles of incidence.

\subsection{Silicon Strip Baseline Design}

Based on the guidelines and the predictions for intrinsic resolution discussed in the previous sections, a conceptual design has been developed based on double-sided silicon strip detectors with $90^{\circ}$ stereo angle. This design has the following elements:

1. Two high-precision measurements as close to the beam pipe as possible. Since the first measurement is crucial for $z_{0}$, two layers of silicon are needed to deal with possible inefficiencies in the first layer. A readout pitch of $100 \mu \mathrm{m}$ with floating strips 


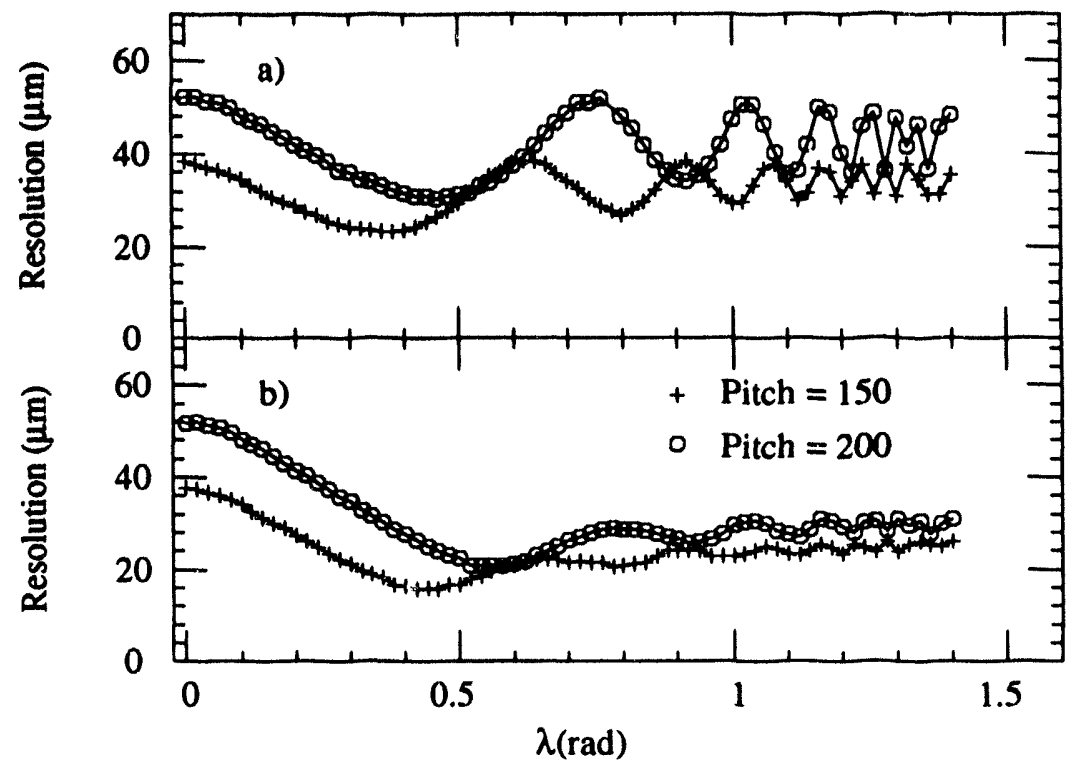

Figure 5-2. Calculated resolution for a $300 \mu \mathrm{m}$-thick silicon vertex detector as a function of the angle of incidence $(\lambda)$ of the charged track in the plane perpendicular to the strips for 150 and $200 \mu \mathrm{m}$ pitch for (a) a digital readout scheme and (b) an analog readout scheme.

provides adequate intrinsic resolution $(\approx 12 \mu \mathrm{m}$ for normal incidence). For $r \phi$ readout, one could use $50 \mu \mathrm{m}$ readout with or without floating strips.

2. Two measurements (for redundancy) at larger radii with somewhat reduced precision ( $200 \mu \mathrm{m}$ pitch with floating strips), mainly for the angle determination and for linking tracks to the drift chamber.

3. One measurement at an intermediate radius for purposes of pattern recognition, especially for low-momentum tracks.

The reference design has three barrel-only inner layers and two outer layers with a barrel section and inclined endcaps ("lampshades")
A layout consistent with these design guidelines is shown in Figure 5-3. The first three layers of detectors are organized in barreltype structures while the outer two layers have a barrel section in the central region and inclined sections ("lampshades") in the forward and backward regions. These lampshade detectors are inclined to minimize both the amount of material traversed by particles and the area of silicon. They are mechanically and electrically connected to the barrel detectors. 


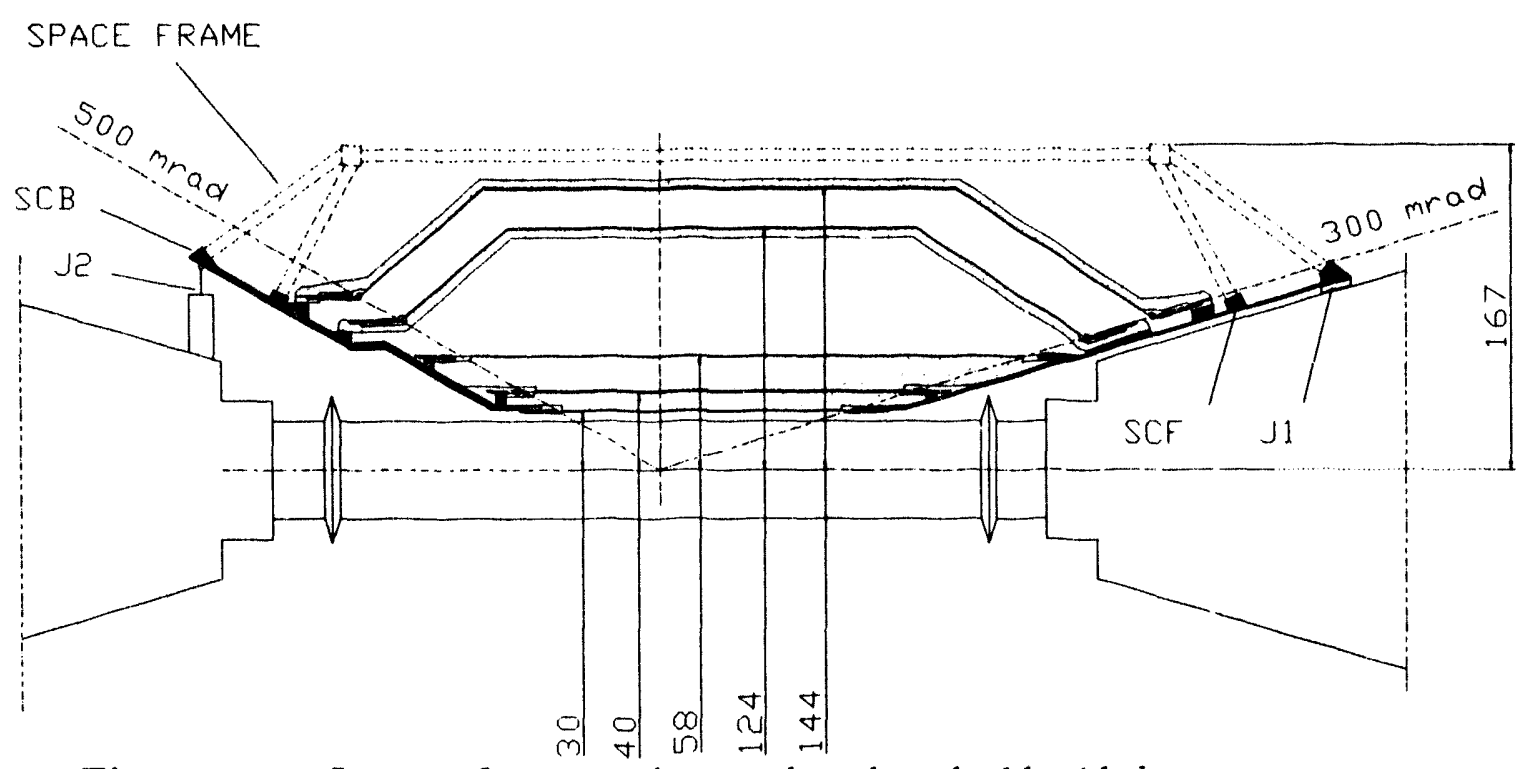

Figure 5-3. Layout of a vertex detector based on double-sided silicon strip detectors. The radii of five layers in the barrel region are given in $\mathrm{mm}$.

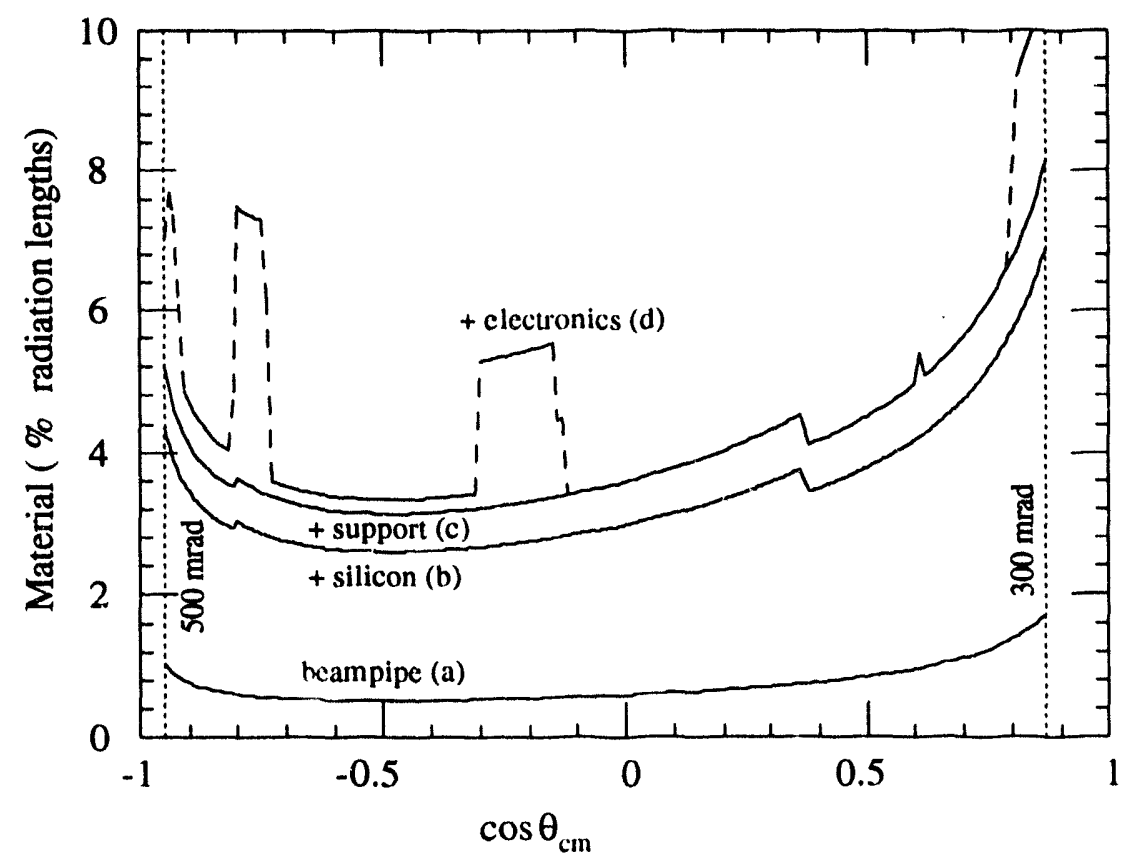

Figure 5-4. Material traversed by a particle inside the support tube, for the layout shown in Figure 5-3, with (curve d) or without (curve c) some of the electronics inside the active volume. 

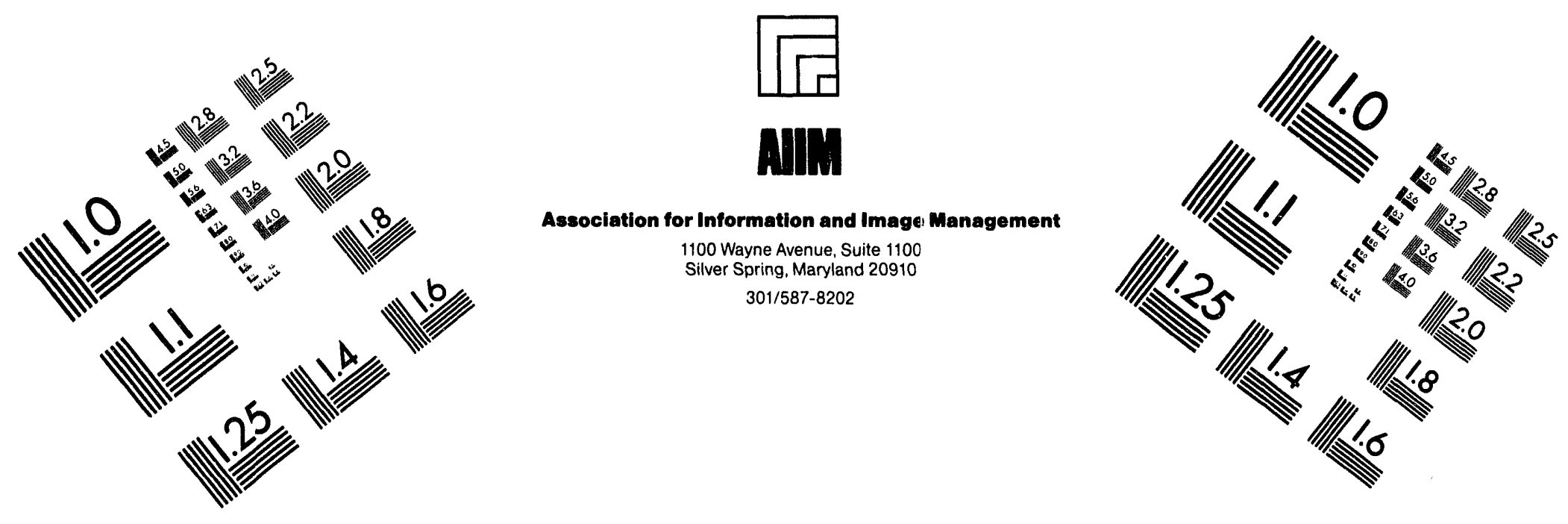

\section{Centimeter}

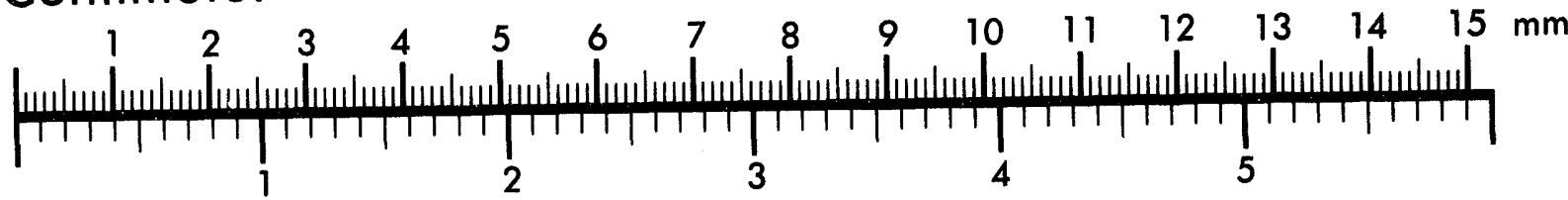
Inches
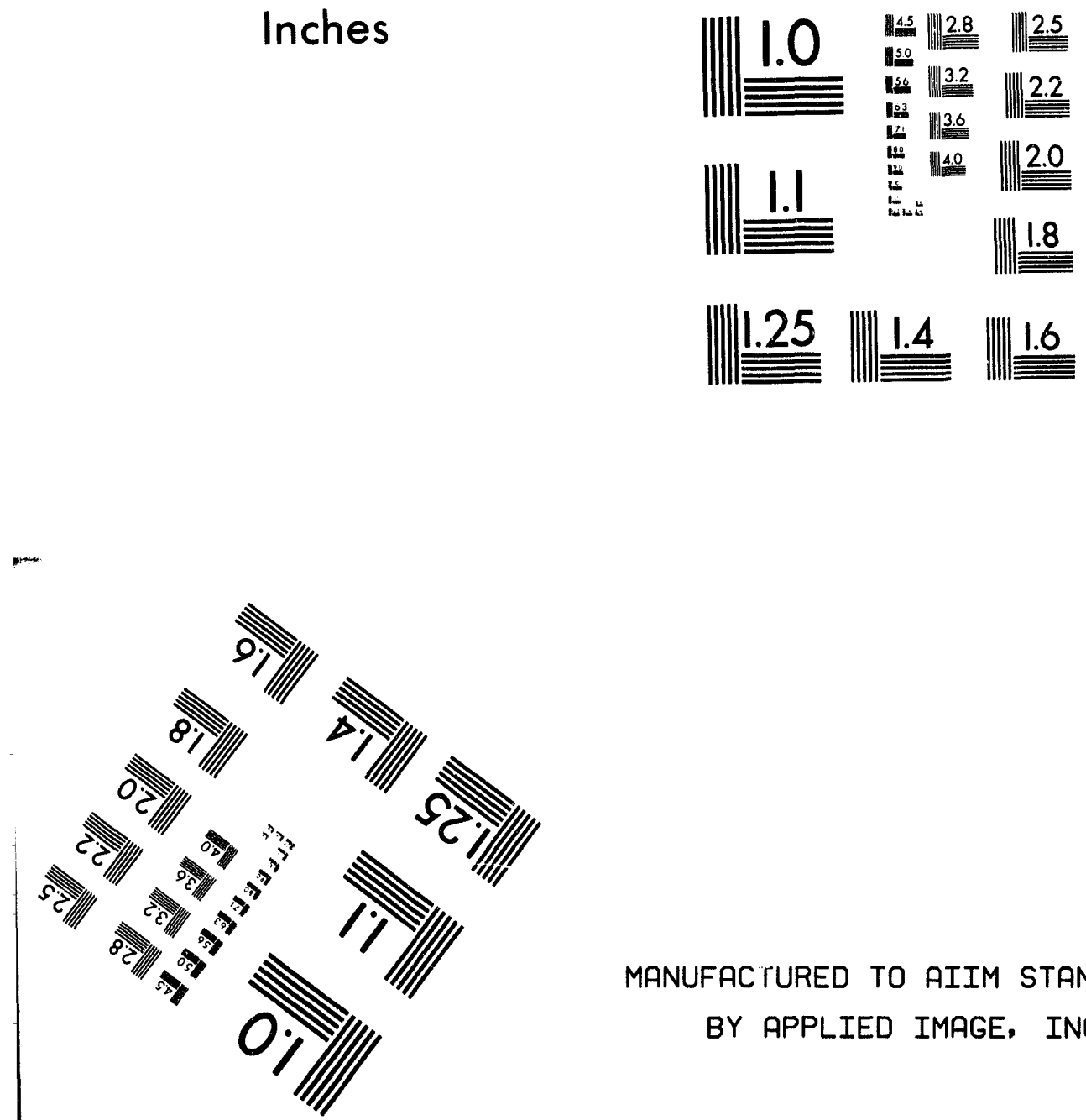

MANUFACTURED TO AIIM STANDARDS

BY APPLIED IMAGE, INC.

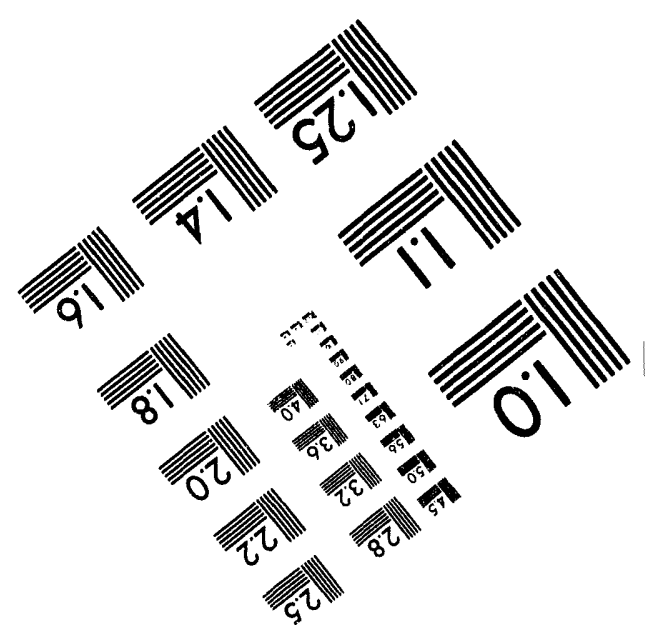



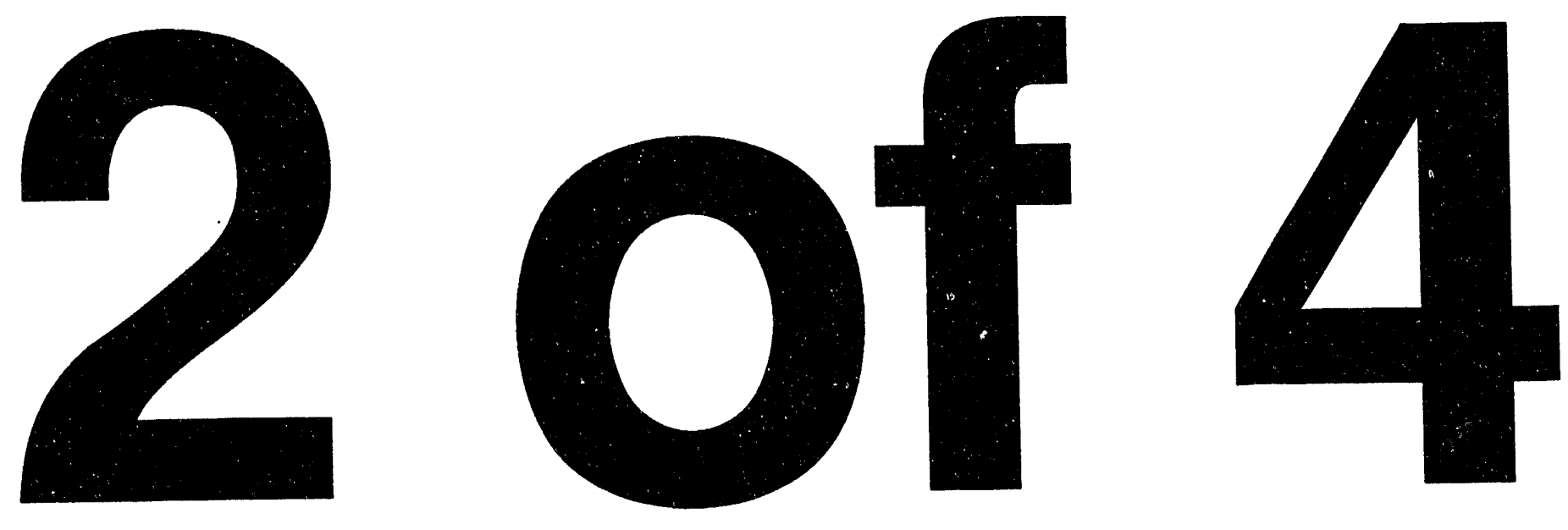
This layout permits all electronics to be located outside the fiducial volume

$z$ signals are routed on traces mounted on a bonded kapton layer
In this layout, all of the electronic readout is located outside the fiducial volume (i.e. below $300 \mathrm{mr}$ in the forward region and $500 \mathrm{mr}$ in the backward region). The total amount of material traversed by a track between the interaction point and the outside of the vertex detector is shown as curve (c) in Figure 5-4. The material is dominated by the silicon but also has contributions from the beampipe (about $0.5 \%$ of a radiation length at normal incidence) and the support structures.

To bring the signals from the $z$ strips to the readout electronics outside the tracking volume, a kapton insulator is glued to the detectors and metal traces on the kapton are wirebonded to the readout strips. To read out all the strips at the narrow end of the wedge-shaped lampshade detectors, up to three strips are ganged together and routed to the same preamplifier channel. This scheme results in $\sim 150 \mathrm{k}$ readout channels for the entire detector.

Curve (d) in Figure 5-4 shows the increase in material in the case that detailed engineering of the forward region just outside the active volume forces us to move the electronics into the fiducial region. The effect of this extra material on the vertex detector performance is negligible because it is located after each measurement plane, but it has some impact, which is under study, on the overall tracking performance.

\subsection{Resolution of the Baseline Design}

The track parameter resolution for the vertex detector design shown in Figure 5-3 plus the drift chamber described in Chapter 6 was calculated with a program that takes into account intrinsic resolution, multiple scattering, energy loss and energy-loss fluctuations [Inn93]. The results for $z$ and $\tan \lambda$ are shown in Figure 5-5 as a function of polar angle for a range of momenta, calculated in the center-ofmass frame. The position resolution of the detectors is assumed to vary with track incident angle in a manner consistent with the resolution expected for $100 \mu \mathrm{m}$ readout pitch in the first three layers and $200 \mu \mathrm{m}$ readout pitch in the outer layers with intermediate floating strips. 


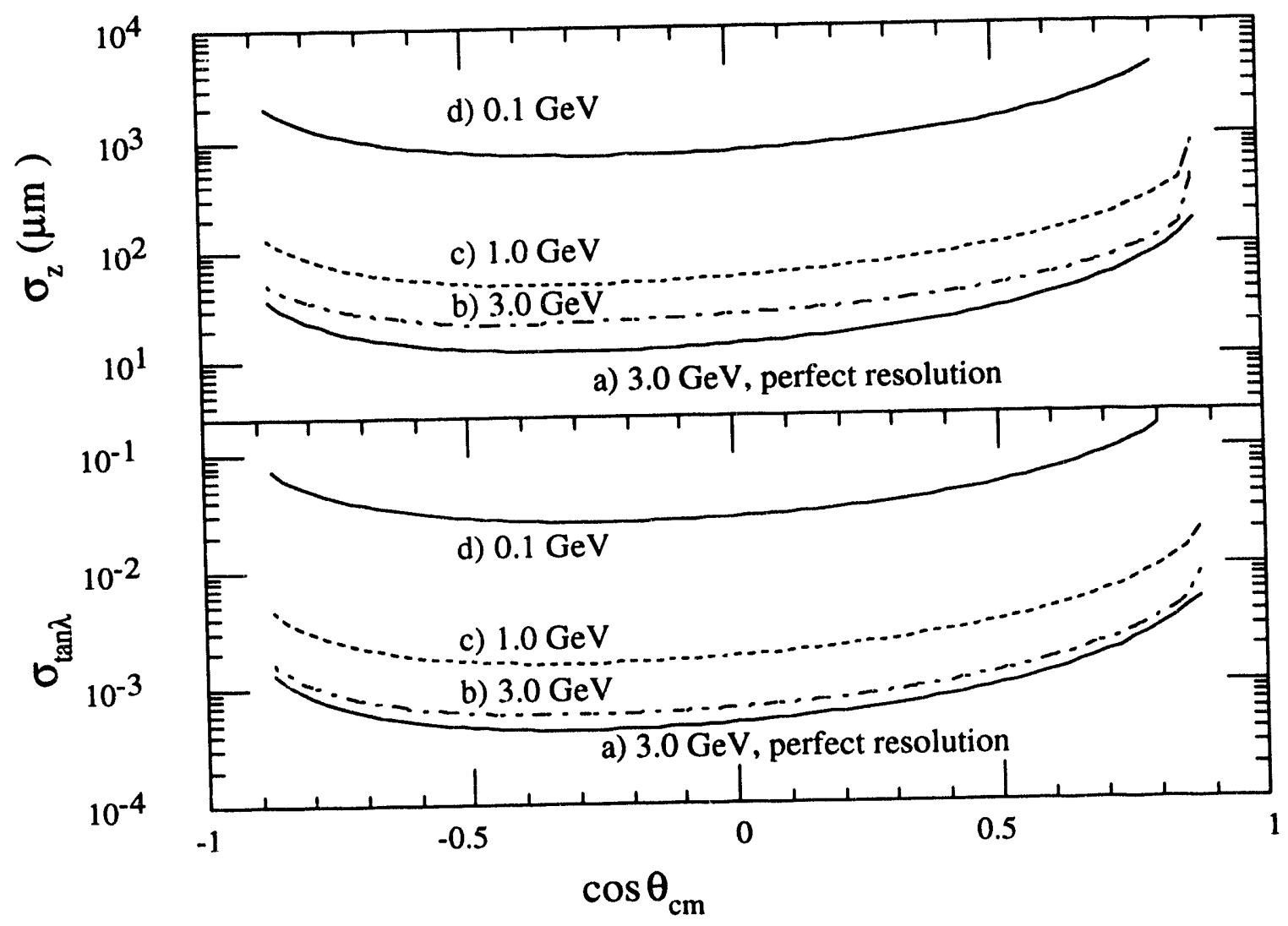

Figure 5-5. Single-track resolution in $z$ and $\tan \lambda$ as a function of the polar angle in the center-of-mass frame for the design shown in Figure 5-3. The curves correspond to various particle momenta. For curve (a), the intrinsic resolution is assumed to be perfect.

The $z$ resolution for a $3 \mathrm{GeV} / c$ track has been calculated, including multiple scattering but assuming perfect intrinsic resolution. This is shown as curve (a) in Figure 5-5. The results show that the resolution is dominated by multiple scattering, and not by the intrinsic position resolution of the silicon detectors, except for the highest momentum tracks from $B$ decays.

Except for the highest momenta, $z$ resolution is dominated by multiple scattering

Using the track parameter resolutions shown in Figure 5-5, the vertex resolution in $z$ has been determined for three $B$ decay modes: $B^{0} \rightarrow \pi^{+} \pi^{-}, B^{0} \rightarrow J / \psi K_{S}^{0}$ and $B^{0} \rightarrow D^{+} D^{-}$. The difference between the reconstructed and generated $B^{0}$ vertex position in the $z$ direction is shown in Figure 5-6 for the three final states. In each case, the distribution is fit with a sum of two Gaussian func- 
The $z$ vertex resolution for three representative $B$ decay modes to $C P$ eigenstate ranges between $22 \mu \mathrm{m}$ and $41 \mu \mathrm{m}$

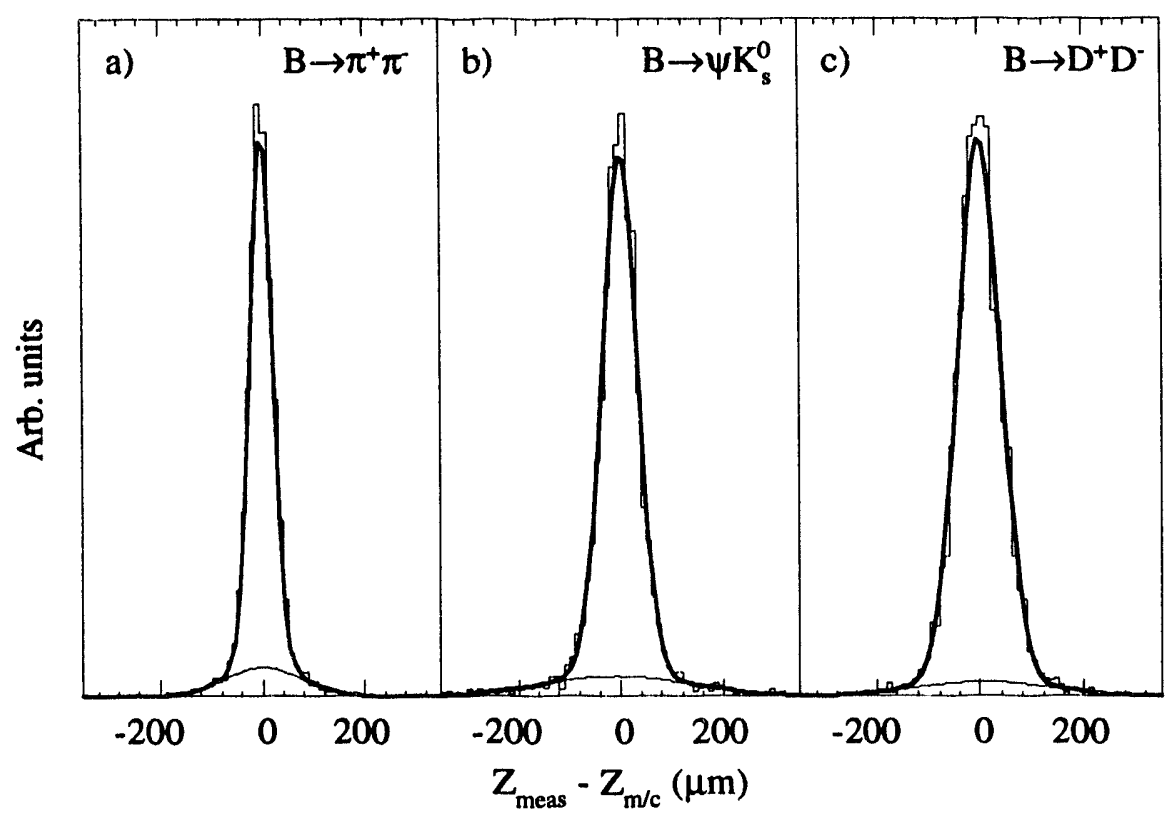

Figure 5-6. Distribution of the difference between the reconstructed and generated $B^{0}$ vertex position in $z$ for three decay modes. Fits to these distributions with a sum of two Gaussian functions (dark curve) yields resolutions for the narrower Gaussian of (a) $22 \mu \mathrm{m}$, (b) $34 \mu \mathrm{m}$ and (c) $41 \mu \mathrm{m}$. The wider Gaussian alone is shown as a light curve.

tions, shown as the dark curve in the figures. The standard deviation of the narrower functicn is $22 \mu \mathrm{m}$ for $B^{0} \rightarrow \pi^{+} \pi^{-}, 34 \mu \mathrm{m}$ for $B^{0} \rightarrow J / \psi K_{S}^{0}$, and $41 \mu \mathrm{m}$ for $B^{0} \rightarrow D^{+} D^{-}$. The contribution of the wider Gaussian, shown as a light curve in the figures, is not very large, indicating that the effect of poorly measured tracks at small polar angles is not significant.

\subsection{Acceptance for Low-Momentum Particles}

In PEP-II, a large number of low-momentum pions are produced from $B$ decays, $\tau$ decays, and direct charm production. The soft pions produced in $D^{*+}$ decays represent one particular source. The reconstruction of $D^{*+}$ mesons is important for $C P$ violation studies 


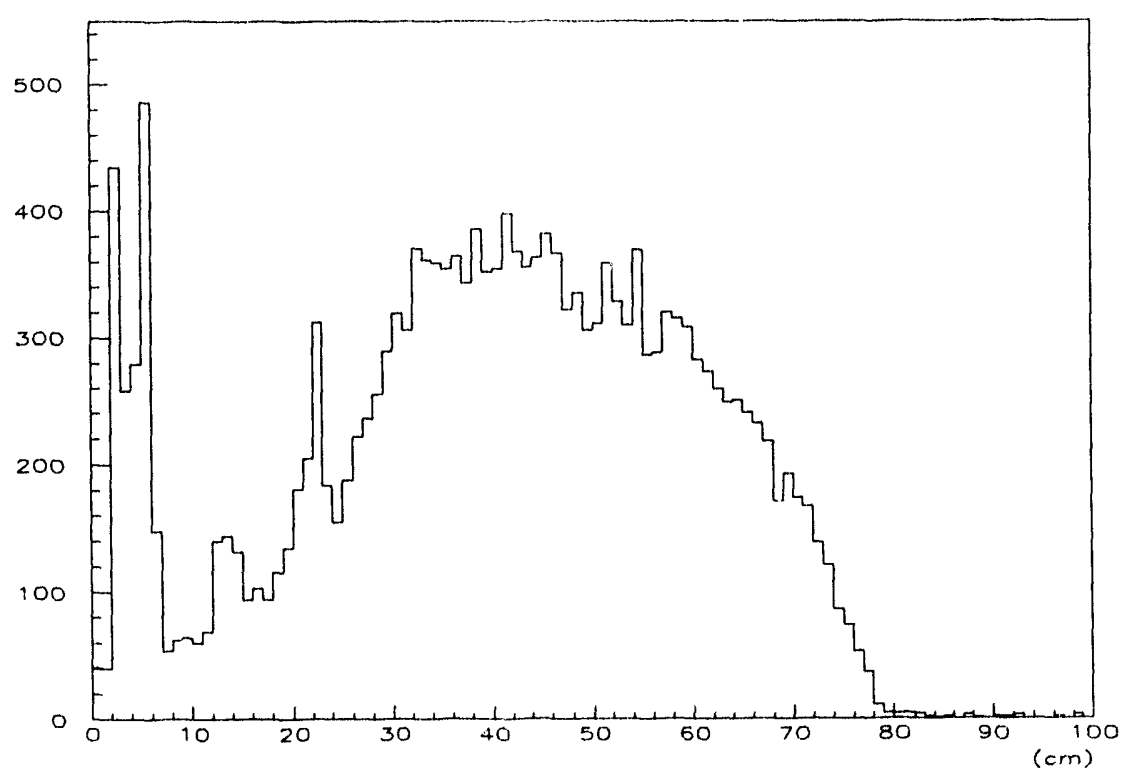

Figure 5-7. Distribution of the maximum radius reached by the soft pions in $D^{*+}$ decay from $B$ meson decays in a $1.5 \mathrm{~T}$ magnetic field.

in $B$ decays such as $B^{0} \rightarrow D^{*+} D^{*-}$, and for charm studies such as $D^{0} \bar{D}^{0}$ mixing.

With a $1.5 \mathrm{~T}$ magnetic field, many low-momentum particles will not travel very far into the central drift chamber. Figure 5-7 shows the maximum radius reached by the soft pions produced in $D^{*+}$ decays from the decay $B^{0} \rightarrow D^{*+} D^{*-}$. This study was done usin; a GEANT simulation in order to incorporate the curvature change due to energy loss in materials. Figure 5-7 shows that $30 \%$ of the pions do not reach a radius of $30 \mathrm{~cm}$, the minimum radius for at least four drift chamber measurements. Some of these particles can be reconstructed if the vertex detector independently has the capability to find and fully reconstruct low-momentum tracks. Of the pions that do not reach a radius of $30 \mathrm{~cm}$, about $60 \%$ traverse all five layers of silicon. This represents a sizable potential increase in reconstruction efficiency, particularly for $B^{0} \rightarrow D^{*+} D^{*-}$ where the $D^{*+}$ efficiency enters twice. The desire for stand-alone track reconstruction in the silicon is one of the motivating factors for a five-layer detector.

$A$ vertex detector capable of stand-alone pattern recognition improves the reconstruction efficiency for low momentum tracks in a $1.5 \mathrm{~T}$ field 
The detectors are fabricated using $300 \mu \mathrm{m}$ thick high-resistivity ntype silicon

The junction side is used for $z$ strips and the ohmic side for the orthogonal $\phi$ strips

Both poly-silicon resistor and punch-through biasing are under consideration

A second metallized layer is an alternative to kapton routing of the $z$ strip signals

\subsection{Detector Specifications}

Double-sided silicon strip detectors provide two-dimensional position information with a minimum amount of scattering material. They provide a conservative choice for the vertex detector for $B A B A R$, as the technology is now well established [Bat91]. The detectors are fabricated on $300 \mu \mathrm{m}$-thick high-resistivity $(4-8 \mathrm{k} \Omega \mathrm{cm}) \mathrm{n}$-type silicon substrate. The readout electrodes consist of p-doped strips on the junction side and n-doped strips on the ohmic side. On both sides, a thin insulating layer $\left(\mathrm{SiO}_{2}\right.$ and/or $\left.\mathrm{Si}_{3} \mathrm{~N}_{4}\right)$ covered with aluminum is deposited on top of the strip to provide $\mathrm{AC}$ coupling to the preamplifier input $(\mathrm{C}>20 \mathrm{pF} / \mathrm{cm})$ and to ensure sufficiently low strip resistance $(\mathrm{R}<50 \Omega / \mathrm{cm})$.

On the ohmic side, p-doped strips are placed between the n-doped readout strips to increase the otherwise low interstrip resistance. This leads to higher capacitance on the ohmic side than on the junction side. Therefore the junction side will be used for the $z$ strips and the ohmic side for the orthogonal $\phi$ strips. This reflects the greater importance of the $z$ measurements compared to the $\phi$ measurements and also partially compensates for the extra capacitance of the kapton fanout used to bring the signals from the $z$ strips to the preamplifiers.

Each strip is individually biased from a ring surrounding the active region, either with a poly-silicon resistor or with the punch-through mechanism. Poly-silicon resistors are straightforward to implement but require extra processing steps. Their resistance can be of the order of $10 \mathrm{M} \Omega$. Punch-through biasing, obtained by carefully adjusting the distance (a few $\mu \mathrm{m}$ ) between the detector strip implant and the biasing ring, offers a very high dynamic resistance $(>1 \mathrm{G} \Omega)$ without extra processing steps in the detector production [Kem93]. Both methods are being studied and should be sufficiently radiation hard.

An alternative approach to kapton fanouts for $z$ strip readout (described in Section 5.3) is a second layer of metal strips separated from the first layer by a $2-5 \mu \mathrm{m}$-thick silicon oxide or polyimide deposit. The implications of the two solutions in terms of yield, cost, mechanical design and performance are being investigated. 
To reduce the amount of scattering material, detectors as thin as $150 \mu \mathrm{m}$ are also being considered. In this case, a different substrate crystal orientation [100] would increase mechanical stability.

The detector layout described in Section 5.3 requires both rectangular and wedge-shaped detectors. The exact geometry of the strips on the wedge detectors will require further studies. In the detailed design of the vertex detector, the detector dimensions will be chosen to make efficient use of the area on a silicon wafer.

\subsection{Mechanical Support}

The silicon detectors and the readout hybrids are assembled in a single mechanical unit, called a module, whose rigidity is provided by a light-weight support structure, such as Kevlar beams, glued to the detectors. At each end of a module, a beryllium oxide or aluminum block, referenced to the strips on the detector, provides the required mechanical precision and acts as a heat sink for the electronics.

In the first three layers, the modules are stacked in hextants, each containing one module from each layer. The hextants and the outer layer lampshades are mounted on two support cones (SCF and SCB in Figure 5-3) that run along and are supported by the B1 dipole magnets. The support cones are divided into halves to allow the vertex detector to be assembled in two halves and then mounted on the B1 magnets. The stiffness of the overall structure is provided by a very low-mass space frame, constructed out of carbon fiber tubes, connecting the forward and backward support cones. The motivation for this space frame stems mainly from the possible relative motion of the two B.l magnets during the assembly procedure.

The assembly and alignment of the beam pipe and the magnets take place in a staging area outside the interaction hall. After the vertex detector is installed on the B1 magnets, the support tube (see Chapter 4) is slid over the entire assembly and mated to the Q1 quadrupoles. The assembly is now sufficiently rigid to be moved to the interaction hall, but during transportation the B1 magnets can move with respect to each other by as much as $1 \mathrm{~mm}$, although they
Fabrication on thinner substrates could reduce multiple scattering

Rectangular and wedgeshaped detectors must be fabricated

A structure, such as Kevlar beams, glued to the detectors provides the mechanical support

The vertex detector structure is supported by the inner permanent magnet dipoles of the accelerator

Overall stiffness is provided by a low-mass space frame

Assembly and alignment are done before the vertex detector is installed in the interaction region 
Utilities are routed along along the $B 1$ magnets to points outside the active region
The design goal is radiation hardness of ten times the expected worst-case yearly dose will return to the original position once installed in the accelerator. The support of the detector from the magnets must allow for this relative motion without placing stress on the silicon wafers. The forward support cone is therefore fixed to the B1 magnet by a mechanical joint ( $\mathrm{J} 1$ in Figure 5-3) that constrains all but one of the six degrees of freedom of the vertex detector assembly. It allows the detector to rotate about a horizontal axis perpendicular to the beam (that is, it hinges up and down). The mounting of the backward cone (J2 in Figure 5-3), on the other hand, constrains only the vertical position of the vertex detector with respect to the magnet. This allows the B1 magnets to twist with respect to each other. The proposed mounting scheme provides reproducible and accurate positioning of one end of the vertex detector with respect to one of the B1 magnets. The space frame and support cones form a rigid structure. The connections to the $\mathrm{B} 1$ magnets isolate the detectors from stresses when the magnets shift. Cooling water, power and signals are routed along the B1 magnets to points outside the active region where manifolds for the cooling water and drivers for the electronics are located.

\subsection{Machine-Related Backgrounds and Radiation Hardness}

The dominant machine-related backgrounds originate from beambeam interactions and from bremsstrahlung or Coulomb scattering of the beam particles (see Chapter 4). The background rate has a $1 / r^{2}$ dependence and, as shown in Figure $5-8$, is strongly peaked in a small region in the horizontal plane spanning a few degrees in azimuth, with a ratio between peak and average of about 10 . Background considerations influence several aspects of the design: readout segmentation, electronics shaping time, data transmission rate, triggering and radiation hardness. The readout issues will be discussed in secion 5.9 ; this section is mainly concerned with radiation damage.

The expected radiation dose in the worst region of the detector (first layer, near the horizontal plane) is about $150 \mathrm{krad} /$ year. The design goal is set at 10 times this number, $1.5 \mathrm{Mrad}$, corresponding to an equivalent proton fluence of about $2.5 \times 10^{12}$ protons $/ \mathrm{cm}^{2}$ 


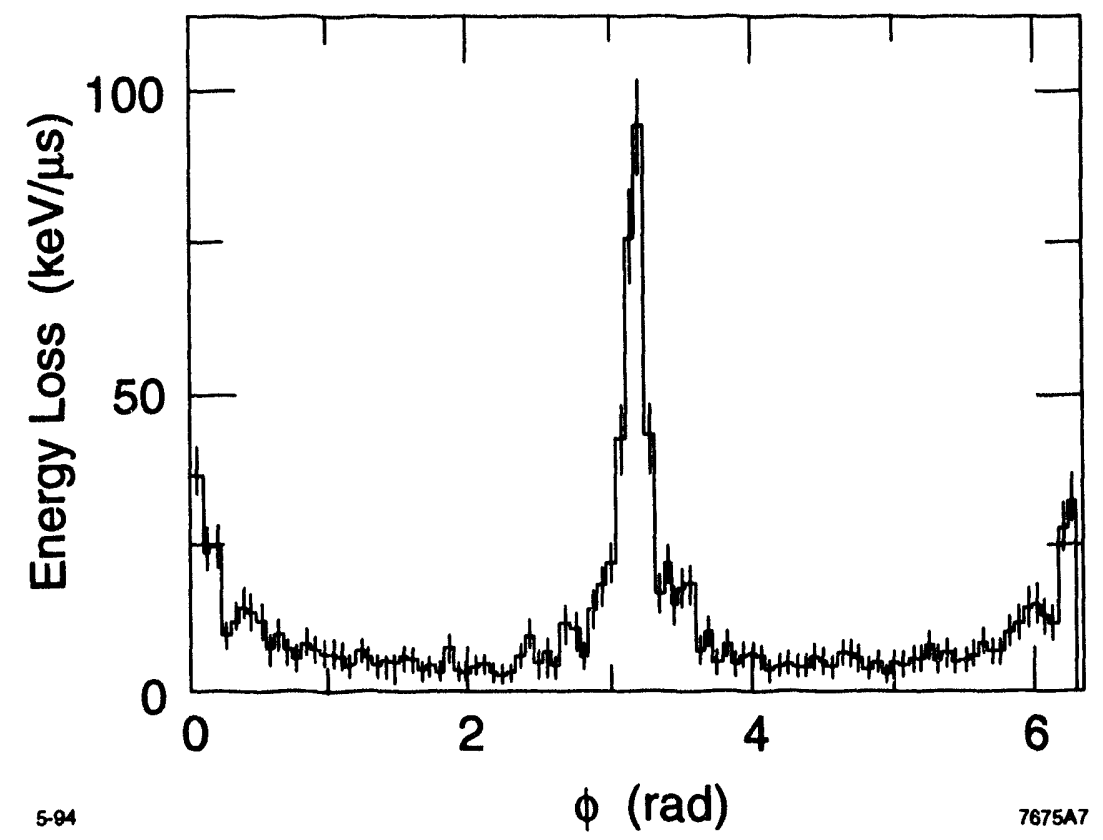

Figure 5-8. Energy deposited in the first layer of silicon due to machine-related backgrounds as a function of the azimuthal angle

(equivalent proton fluence is used because proton-induced radiation damage has been extensively studied [Zio94]). All components located close to the interaction region (sensors, electronics, glues, hybrids, cables) must be radiation resistant at this level.

Radiation damage to bulk silicon can result in (i) an increased leakage current and (ii) changes in the depletion voltage of the silicon sensors. For a $7 \mathrm{~cm}$-long detector with $50 \mu \mathrm{m}$ pitch, the increase in the leakage current per strip in the worst region of the detector is $65 \mathrm{nA}$, corresponding to a noise of about 285 electrons for $100 \mathrm{~ns}$ shaping time, to be added in quadrature to an expected overall noise level of about 490 electrons (see Section 5.9). The change in depletion voltage has been estimated to be at most a few volts after ten years of PEP-II operation at room temperature. Further investigations are needed on the consequences of damage due to defects at surfaces and interfaces [Ohs94]. In particular, oxide charge build-up may affect the coupling capacitor breakdown voltage and the interstrip capacitance and resistance.

Radiation-induced changes to leakage current and depletion voltage are estimated to be manageable

Further investigation is required in the area of radiation-induced damage at surfaces and interfaces, which can cause charge buildup 
Radiation-hard processes are available to fabricate the front-end electronics

The 4 ns bunch spacing of PEP-II mandates an asynchronous architecture

$A$ "data-pull" architecture provides better safety margin against backgrounds
Ionizing radiation also produces significant changes in the characteristics of MOS devices and integrated circuits. Radiation-hard technologies are available and have been characterized for three foundries: UTMC, Harris and Honeywell, with feature sizes of 1.2 and $0.8 \mu \mathrm{m}$. For these processes, radiation effects saturate after about $100 \mathrm{krad}$ and the devices are stable up to at least $100 \mathrm{Mrad}$.

\subsection{Front-End Electronics and Data Transmission}

The front-end electronic readout of the vertex detector will be implemented as monolithic integrated circuits, mounted directly on the detector. The very small bunch spacing at PEP-II (4 ns) requires an asynchronous architecture similar to front-end designs developed for the SSC. Because of space constraints in the region near the B1 magnets, the size of the electronic readout hybrid must be minimized, limiting the size of the readout ICs, the number and size of external components (including optical drivers/receivers), and the space available for cabling. Three types of readout systems are being evaluated: digital, analog, and time-over-threshold. The decision on which system to use will depend on issues such as impact on position resoiution, IC size, and power consumption.

The readout architecture and circuit implementation are dominated by background considerations. A recent report [Lev94] shows that occupancy in the worst readout sections of the first two layers is $0.5 \%$ and $0.2 \%$ (for $1 \mu \mathrm{s}$ time intervals and $50 \mu \mathrm{m}$ readout pitch). Although an architecture in which all data are transmitted to the data acquisition system ("data-push") could operate with an acceptable inefficiency $(<2 \%)$, it would be difficult to accommodate the safety margin for increased backgrounds that seems prudent at this time. Therefore, a "data-pull" architecture is being designed to transmit data from the front-end ICs on the silicon detector to the data acquisition system when requested by a fast trigger signal generated by the trigger electronics after $\_$fixed latency. However, a prompt output formed by a logical OR of all strips monitored by a single front-end IC will be provided in a "data-push" manner to be used in the formation of the trigger, as discussed in more detail in Chapter 11. 
A triggered readout requires some form of extended buffering to accommodate the hits occurring during the trigger latency time. One possibility is to use a linear pipeline, the length of which is the latency time divided by the time resolution. An alternative is to use a common buffer for all channels on a chip, for example a RAM or CAM structure. The decision will depend on whether analog information is transmitted, on expected background rates, and on the impact of the buffer and control logic on the chip size. The number of cells in the data buffer depends on the required time resolution. This will be the result of a compromise between noise considerations, which imply a preference for a long shaping time, and the need to keep the strip occupancy low.

The achievable noise levels depend on the lengths of the strips to be read out and the leakage current, as well as the pulse shaping scheme and time constant. Since the choice of time constant is driven by the hit rate per strip, the optimum value is likely to be different for inner and outer layers. A system with an adjustable time constant is being considered. For a 100 ns peaking time, noise levels that have been achieved with existing amplifiers fabricated by a $1.2 \mu \mathrm{m}$ CMOS process are 490 electrons equivalent noise charge for a $7 \mathrm{~cm}$ long detector and 940 electrons for a $14 \mathrm{~cm}$ detector. The major power contributions in the analog stage are from the preamplifier $(0.6 \mathrm{~mW})$, the shaper $(0.25 \mathrm{~mW})$ and the comparator $(0.1$ to 0.25 $\mathrm{mW}$ ). The data buffer and readout circuitry will add about $0.5 \mathrm{~mW}$ per channel, so the total power could be about $1.5 \mathrm{~mW}$ per channel. The 150k readout channels in the layout shown in Figure 5-3 would generate less than $250 \mathrm{~W}$.

Data from the silicon vertex detector must be transported from the front-end ICs mounted on the detector to the trigger and data acquisition electronics. The design of the data transmission system is driven by three requirements: to provide the required bandwidth to avoid deadtime; to minimize the amount of material in the detector fiducial volume; and to minimize the power dissipation and cooling.

After receiving the fast strobe from the trigger system, the data in each front-end IC will be compressed and merged with data from the other ICs on the same silicon detector module to form one data packet from each module. This data packet will be transmitted with a trigger tag and necessary error detection codes to the data
A final decision on the pipeline structure has not yet been made

Time-stamping to $\sim 100$ ns can be achieved with adequate noise performance 
Data transmission using either kapton/Al traces or optical fibers is being considered
A $\mu$ TPC could provide tracking information at small forward polar angles acquisition system. Its actual format has not yet been determined. The details of its design depend critically upon the decision whether to "ansmit analog or digital data from each strip, but estimates have been made of the data rates expected for each alternative.

Two technologies are being considered to transmit the data: electronic transmission over low mass cables made of kapton and aluminum, or optical transmission over fibers.

Both of these options are based on existing commercial technologies but require some customization in order to meet the detector needs. A likely choice will be that the front-end ICs transmit data directly over low mass cables to the area just outside the active detector volume (near the beam line magnets) where an optical system transmits the signals the remainder of the distance to the data acquisition and trigger electronics.

\section{$5.10 \quad$ Forward $\mu$ TPC Option}

The $\mu \mathrm{TPC}$ is a technology being explored to provide tracking in the forward region close to the beampipe. It consists of a cylindrical Time Projection Chamber with a drift distance of about $10 \mathrm{~cm}$. The $\mu \mathrm{TPC}$ would replace some of the silicon detectors in the forward region and would potentially provide tracking at smaller polar angles, more space-point measurements per track and less material than a silicon system in this region. The motivation for a $\mu \mathrm{TPC}$ is the observation that the unequal beam energies at PEP-II increase the number of particles produced at angles less than $300 \mathrm{mr}$ with respect to the beamline, a region in which there is no drift chamber tracking due to the shadow of the B1 dipole magnet. Although the region between 200 and $300 \mathrm{mr}$ from the beamline accounts for only $2.5 \%$ of the solid angle in the forward hemisphere, $6 \%$ of charged particles from $B$ decays fall in this region.

\subsection{1 $\mu$ TPC Setup and Performance}

The $\mu$ TPC [Wor93] design, shown in Figure 5-9, consists of a cylindrical drift volume with an electric drift field parallel to the magnetic field of the detector solenoid. The gas mixture is $80 \% \mathrm{Ar}, 20 \%$ 


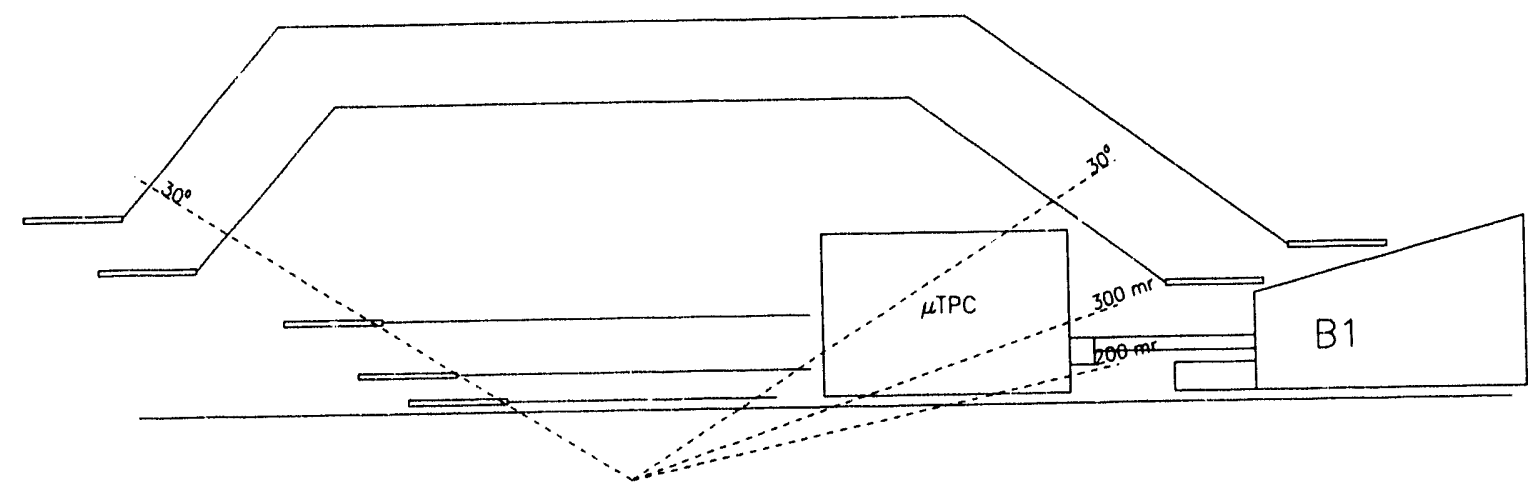

Figure 5-9. A vertex detector setup with five layers of silicon and a $\mu T P C$ in the forward region.

$\mathrm{CH}_{4}$. The ionization electrons drift up to $10 \mathrm{~cm}$ toward a microstrip gas chamber (MSGC) [Sau93] located at $z=18 \mathrm{~cm}$. The readout endplate is a glass substrate with thin metal anode and cathode strips. The $\mu \mathrm{TPC}$ extends from 3 to $9 \mathrm{~cm}$ in radius. The entrance windows consist of two thin epoxy foils (typically $70 \mu \mathrm{m}$ thick), giued to a light honeycomb structure for stiffness. The fraction of a radiation length due to the $\mu \mathrm{TPC}$ traversed by a particle is $0.1 \%$ before and $2 \%$ after the $\mu \mathrm{TPC}$ measurements. The silicon detectors in the three inner layers would have to be read out entirely in the backward region to minimize the amount of material in front of the $\mu \mathrm{TPC}$.

The measurement of the $z$ coordinate of the track is provided by a timing measurement. The $r$ and $\phi$ coordinates are provided by an array of crossing strips on the MSGC endplate. The cathodes and anodes are formed by semicircular strips with $200 \mu \mathrm{m}$ pitch. Two thousand radial "petals" for $\phi$ readout are separated from the anode strips by a $2 \mu \mathrm{m}$-thick layer of $\mathrm{SiO}_{2}$. With this array of strips, the number of space-point measurements along a track is 100 at $300 \mathrm{mr}$ and 22 at $200 \mathrm{mr}$.

The $z$ impact parameter resolution expected for this combined silicon$\mu \mathrm{TPC}$ system is shown in Fig 5-10. The solid line represents the combined system and the dashed line corresponds to the all-silicon setup described in Section 5.3. For the $\mu \mathrm{TPC}$, the spatial resolution is assumed to be $30 \mu \mathrm{m}$ in the $r-\phi$ plane and $\approx 350 \mu \mathrm{m}$ in $z$. The calculated resolution is up to $30 \%$ better for the $\mu \mathrm{TPC}$ system
The measurement of the $z$ coordinate of the track is provided by a timing measurement. The $r$ and $\phi$ coordinates are provided by an array of crossing strips on the MSGC endplate 


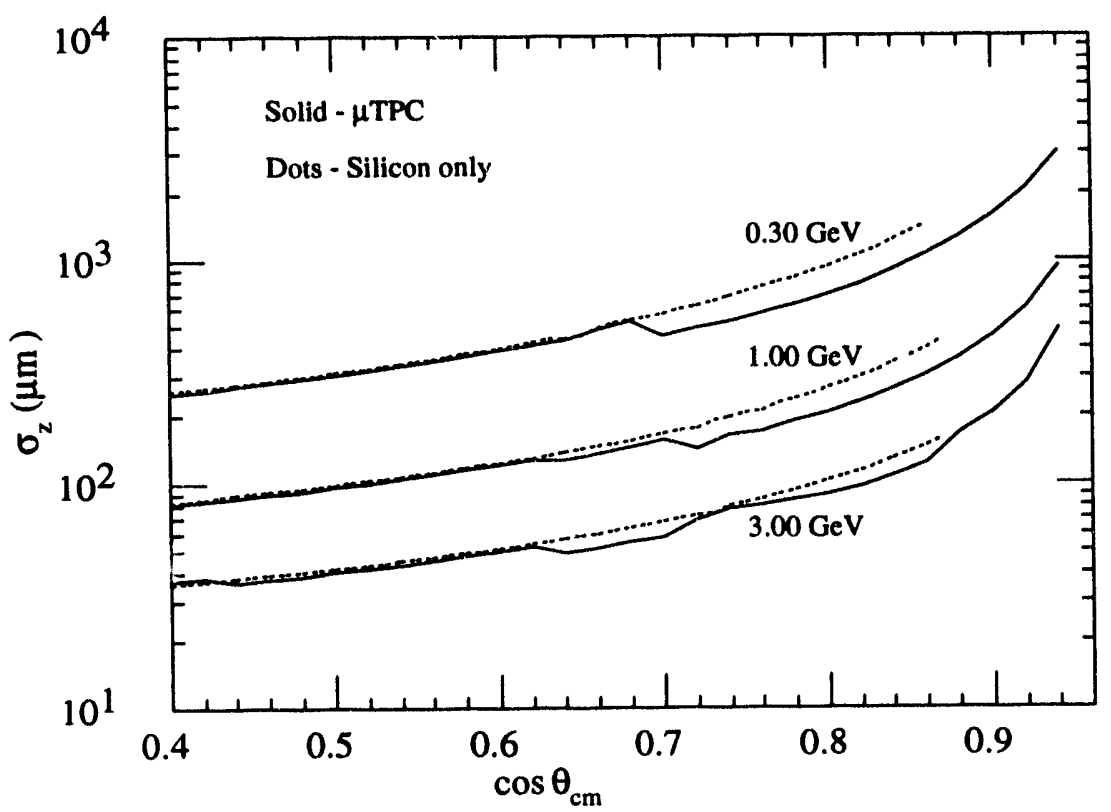

Figure 5-10. Resolution on the $z$ impact parameter for a combined silicon/ $\mu$ TPC system (solid line) and an all-silicon system (dashed line).

The potential increase in reconstruction efficiency provided by the $\mu$ TPC has been studied for $\cos \theta_{c m}>0.7\left(\theta\right.$ less than $30^{\circ}$ in the laboratory frame). A similar result is obtained for resolution on $\tan \lambda$ [OSh94]. The momentum resolution for tracks below $300 \mathrm{mr}$ is modest: $\sigma_{p} / p \approx$ $0.1 p$ with a beam constraint of $30 \mu \mathrm{m}$ in the plane perpendicular to the beamline.

The potential improvement in reconstruction efficiency provided by the increase in acceptance has been studied for the decay modes $B^{0} \rightarrow J / \psi K_{S}^{0}$ and $B^{0} \rightarrow D^{*} D^{*}$ with simulations based on parametric track-resolution functions using the ASLUND Monte Carlo. Mass constraints on intermediate resonances $\left(J / \psi, K_{S}^{0}, D^{*}\right)$ are used to compensate for the poor momentum resolution for tracks reconstructed with the $\mu$ TPC only. The $B$ mass resolution can be recovered to a large extent by applying these constraints. The simulations show a net gain in acceptance of $20 \%$ for $B^{0} \rightarrow J / \psi K_{S}^{0}$ and $25 \%$ for $B^{0} \rightarrow D^{*} D^{*}$ compared to the silicon-only design, due to the increased angular coverage. There is no indication of a significant increase in background from $B$ decays. The increased charged-particle coverage provided by the $\mu$ TPC may also help in reducing background in the decay $B^{-} \rightarrow \tau^{-} \nu_{\tau}$. 


\subsubsection{R\&D Issues and Prototype Schedule}

The operation of a $\mu \mathrm{TPC}$ with a $10 \mathrm{~cm}$ drift volume read out by a one-dimensional MSGC has recently been demonstrated with a laser beam and in a low-intensity proton test beam. A test with a stray magnetic field similar to the one produced by the B1 magnet was done, showing a track displacement of the order of $40 \mu \mathrm{m}$, which in principle can be corrected.

Two potentially serious background issues for the $\mu$ TPC must be addressed: space charge effects and occupancy. On average, the integrated radiation load to the MSGC is expected to be small compared to what has already been achieved with such chambers [Sau93]. However, space charge in the dift volume could cause track distortions at the $100 \mu \mathrm{m}$ level in the $r-\phi$ and $r-z$ planes. A static blocking grid could be considered to prevent the ions from drifting back into the drift volume. It is not clear whether the latency of the Level 1 strobe trigger would be adequate for this purpose. The occupancy, and the resulting track visibility, are currently under study using a full GEANT simulation and local pattern recognition. The precise design of the strips and pad segmentation will follow from this study. To address some of the operational questions, an extensive set of tests with two-dimensional readout, including a high-intensity beam test in a magnetic field, is scheduled for the summer of 1994.

\subsection{Pixel Detector Option}

Silicon pixel detectors have several properties that make them potentially very attractive for use at PEP-II. The relatively small capacitance of the charge collection structures leads to high signalto-noise ratios $(\sim 100)$ permitting the use of very thin detectors and resulting in an intrinsic spatial resolution of a few microns for both normally incident and angled tracks. The two-dimensional segmentation of pixel detectors would also provide high tolerance to backgrounds and efficient pattern recognition.

Both hybrid [Sha90] and monolithic [Ken92] pixel arrays have been developed, fabricated and studied in the laboratory and in test
Remaining questions concerning the viability of the $\mu$ TPC in a highbackground environment are being addressed

Pixel detectors could provide better tolerance to high backgrounds, improved spatial resolution and threedimensional space points 
$A$ uew low-mass beam pipe design would be required to take advantage of the improved spatial resolution of pixel devices beams. Recent improvements in design have resulted in arrays featuring a data-push architecture, which allows the passage of the particle itself to initiate the read cycle [Sha93]. Prototypes of these data-push arrays have been successfully tested in the laboratory, demonstrating low noise ( $<100$ electrons $\mathrm{rms}$ ) and adequate band width to record a particle's passage in less than $200 \mathrm{~ns}$. A detector based on $30 \times 135 \mu \mathrm{m}^{2}$ pixels with a hybrid thickness of $200 \mu \mathrm{m}$ ( $150 \mu \mathrm{m}$ detector and $50 \mu \mathrm{m}$ electronics) is described eisewhere [At193] along with mounting and cooling designs.

To take full advantage of the precision of a pixel detector, the multiple scattering in the beam pipe must be reduced below the levels currently being contemplated. If the challenges of building a very thin beam pipe can be overcome and if pixel detector systems (including readout and cooling) can be built with minimum material, then pixel detectors could provide the high resolution necessary for extracting $C P$-violating asymmetries in the $B_{s}^{0}$ system produced at the $\Upsilon(5 S)$, where the oscillations are expected to have very high frequency. Therefore, a pixel detector is considered as a potential future upgrade for the $B_{A} B_{A R}$ detector. 


\section{References}

[At193] E. Atlas et al., "Proposed Use of Hybrid Pixel Arrays Using the Data-Push Architectoure for a Vertex Detector at a SLAC Asymmetric B Factory", BABAR Note \#105, June 1993.

[Bat91] G. Batignani et al., "Development and performance of double sided silicon strip detectors", Nucl. Instr. Meth. A310, 160 (1991).

[Inn93] W. Innes, "Trackerr, a Program for Calculating Tracking Errors", BABAR Note \#121, Nov. 1993.

[Kem93] J. Kemmer and G. Lutz, "Concepts for simplification of strip detector design and production", Nucl. Instr. Meth. A326, 209 (1993).

[Ken92] W. Snoeys et al., "First Beam Test Rresults from a Monolithic Silicon Pixel Detector", Nucl. Instr. Meth. A326 144 (1993).

[LeD90a] F. Le Diberder, "Precision on CP Violation Measurements and Requirements on the Vertex Resolution", BABAR Note \#34, April 1990.

[LeD90b] F. Le Diberder, W. Toki, M. Witherell, "The Effect of Beam Pipe Radius on the Measurement of CP Asymmetries", BABAR Note \#45, June 1990.

[Lev94] M. Levi, "Impact of Backgrounds on the Silicon Vertex Architecture and Detector Trigger", BABAR Note \#136, April 1994.

[Ohs94] T. Ohsugi et al., "Microdischarges of AC-coupled silicon strip sensors", Nucl. Instr. Meth. A342, 22 (1994).

[Sau93] F. Sauli, "Development of micro-strip gas chambers for radiation detection and tracking at high rates", CERN/DRDC/93-34, Aug. 1993.

[Sha90] S.L. Shapiro, "Silicon PIN Diode Array Hybrids as Building Blocks for a Vertex Detector at an Asymmetric $B$ Factory", SLAC-PUB-5353, Sept. 1990. 
[Sha93] S. Shapiro et al., "Progress on the Design of a Data Push Architecture for an Array of Optimized Time Tagging Pixels", SLAC-PUB-6249, Sept. 1993.

[OSh94] K.O'Shaughnessy, "Design of the Vertex Region of the PEP-II Detector using Alternative Technologies", BABAR Note \#147, June 1994.

[SLA93] Status Report on the Design of a Detector for the Study of CP Violation at PEP-II at SLAC, SL $A$ C Report SLAC419, June 1993.

[Wor93] G .Wormser et al., "The $\mu$ TPCproject", BABAR Note \#122, Nov. 1993.

[Zio94] H.-J. Ziock et al., "Temperature dependence of the radiation induced change of depletion voltage in silicon PIN detectors", Nucl. Instr. Meth. A342, 96 (1994). 


\section{Main Tracking Chamber}

$\mathbf{T}$ he objectives of the $B A B A R$ experiment place a strong emphasis on the reconstruction of exclusive finai states from $B$ decays. This in turn requires efficient detection and identification of both charged and neutral particles, together with precise measurements of their kinematic properties. The main tracking chamber provides a precision $p_{\perp}$ measurement for charged particles above $60 \mathrm{MeV} / c$ along with a determination of the mean ionization deposition $(d E / d x)$ for particle identification. In fulfilling these functions, design optimization for tracking is constrained by the cost and performance of particle identification and calorimeter systems located behind the chamber. These factors effectively limit the size of the tracking volume and require that the structural material of the chamber be minimized.

The principal function of determining transverse momentum in the tracking chamber requires precision measurements of the curvature of charged-particle trajectories in the detector magnetic field. However, the chamber also provides measurements of track angles, locates secondary vertices that are outside the silicon vertex detector, supplies input for the reconstruction in the vertex and the particle identification systems, contributes (as a veto) to neutral particle identification, and augments the dedicated particle identification device with ionization loss measurements. Finally, fast chargedparticle tracking information is an essential part of the detector trigger design.

The full tracking system will consist of a silicon vertex detector and a $\sim 280 \mathrm{~cm}$ long cylindrical drift chamber in a $1.5 \mathrm{~T}$ axial magnetic field. The drift chamber is located radially between the support tube on the inside and the particle identification system on the outside. For charged particles of transverse momentum greater than $180 \mathrm{MeV} / c$, up to 40 spatial and ionization loss measurements are provided by layers of small cells arranged between radii of $22.5 \mathrm{~cm}$ The main tracking chamber should provide good spatial and $d E / d x$ resolution

The chamber provides a maximum of 40 measurements per track 
material, the effect of multiple scattering on $p_{\perp}$ resolution will be significantly reduced.

\subsection{Requirements}

Acceptance requirements for $B$ decays produced from a boosted $\Upsilon(4 S)$ define the geometry of the experiment as a whole, and the tracking chamber in particular. Figure 6-1 shows the integrated efficiency for a variety of all-charged $B$ decay modes as a function of the momentum and polar angle acceptance. The channels $B^{0} \rightarrow$ $\pi^{+} \pi^{-}, B^{0} \rightarrow J / \psi K_{S}^{0}$ with $K_{S}^{0} \rightarrow \pi^{+} \pi^{-}$and $B^{0} \rightarrow D^{+} D^{-}$with $D^{+} \rightarrow K^{-} \pi^{+} \pi^{-}$have been used in the upper two plots in Figures 6-1 to demonstrate the importance of the forward angle cutoff. For example, a factor of 6.5 would be lost in the $D^{+} D^{-}$efficiency if good momentum and particle identification information were not available beyond $\cos \theta=0.8$. The backward angle requirement is considerably less stringent. For the highest and lowest momentum requirements illustrated in the lower two plots of Figure 6-1, the channel $B^{0} \rightarrow D^{*+} D^{*-}$, with its subsequent cascade decay $D^{*+} \rightarrow$ $\pi^{+} D^{0}$ producing a soft pion, is the most stringent requirement. A minimum momentum cutoff of $200 \mathrm{MeV} / c$ would have zero acceptance for this mode. The ability to find and reconstruct charged particles down to $\sim 60 \mathrm{MeV} / c$ is clearly required for good efficiency in this mode and in other channels containing one or more $D^{*+}$ mesons.

These considerations, along with the ability to operate in the environment of the PEP-II collider, lead to the following principal design considerations for the main tracking chamber:

- The tracking system must cover the polar-angle range allowed by the beam-line components, namely down to $300 \mathrm{mr}$ in the forward direction, or about $\left|\cos \theta_{c m}\right|<0.9$, with maximum track reconstruction efficiency for charged particles with $p_{\perp}$ as low as $60 \mathrm{MeV} / c$ in a $1.5 \mathrm{~T}$ magnetic field.

- Excellent transverse momentun resolution must be provided in order to reconstruct exclusive final states with high precision for good background rejection. A resolution of $\sigma p_{\perp} / p_{\perp} \simeq$ $0.3 \% \times p_{\perp}$ for $p$ above $1 \mathrm{GeV} / c$ (e.g., for $B^{0} \rightarrow \pi^{+} \pi^{-}$), and 

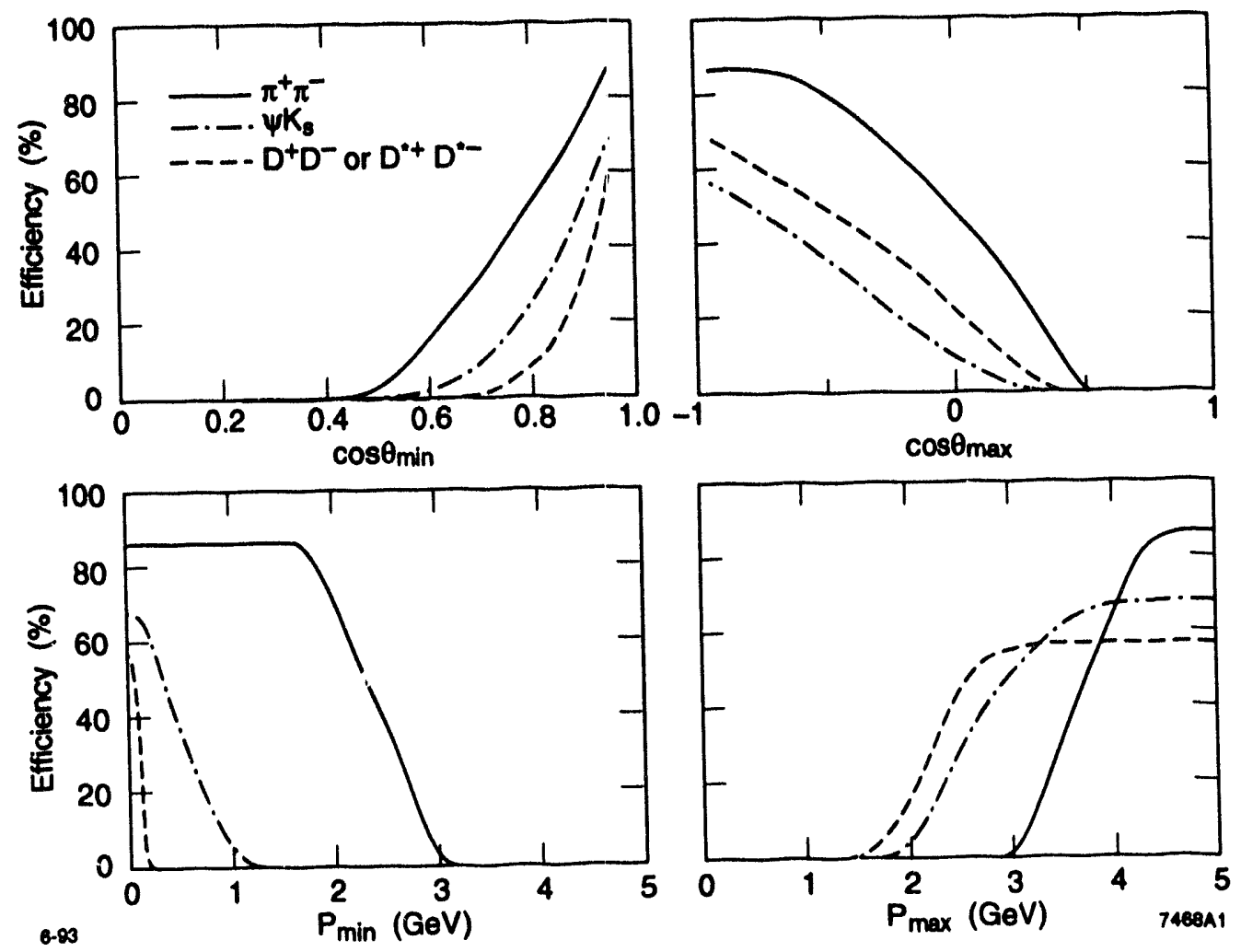

Figure 6-1. Starting clockwise from upper left, dependence of $B$ reconstruction efficiency on minimum detectable $\theta$, maximum detectable $\theta$, maximum detectable momentum and minimum detectable momentum for charged particle detection from $B^{0} \rightarrow$ $\pi^{+} \pi^{-}$(solid), $B^{0} \rightarrow J / \psi K_{S}^{0}, J / \psi \rightarrow \ell^{+} \ell^{-}, K_{S}^{0} \rightarrow \pi^{+} \pi^{-}$(dashdot), and the dashed curves representing $B^{0} \rightarrow D^{+} D^{-}, D^{+} \rightarrow$ $K^{-} \pi^{+} \pi^{+}$for the angular distributions and $B^{0} \rightarrow D^{*+} D^{*-}$, $D^{*+} \rightarrow \pi^{+} D^{0}, D^{0} \rightarrow K^{-} \pi^{+}$for the momentum distributions.

$\sigma p_{\perp} / p_{\perp} \simeq 0.3 \%$ for momenta between 0.1 and $1 \mathrm{GeV} / c$ (e.g. for $B^{0} \rightarrow D^{+} D^{-}$) should be achievable.

- The main tracking chamber should provide a $z$ resolution of $\sim 1 \mathrm{~mm}$ at its inner radius for linking to the silicon vertex detector measurements and for reconstruction of $K_{S}^{0}$ mesons with vertices outside the silicon detector. At the outer radius, the requirement on $z$ resolution for extrapolation to the particle identification system and the calorimeter depends upon the choice of PID technology, and ranges from 0.5 to $2.0 \mathrm{~mm}$. 
- Measurements of $d E / d x$ with sufficient precision to provide good $K / \pi$ separation at momenta up to $750 \mathrm{MeV} / c$ should bo available. This is especially important for the extreme backward direction, where there is no other particle identification device.

- The above specifications must be satisfied in the presence of expected charged-particle backgrounds of $1 \mathrm{MHz} /$ layer at the inner radius. The drift chamber should remain operab!o at much higher rates.

- The material in the cylinder walls, endplates, electronics, and cabling must be minimized to prevent degraded performance of the particle identification and calorimeter systems; less than $10 \%$ of a radiation length for the forward endplate should be possible, and only a few percent for the outer cylinder wall.

- The layer arrangement of the drift chamber should permit a fast Level 1 trigger determination.

\subsection{Design Considerations}

The material within the drift chamber can be kept to $0.12 \% X_{0}$
Neglec'ing material, the transverse momentum resolution is directly proportional to the single-cell spatial resolution, and inversely proportional to the square root of the number of measurements, to the magnetic field strength, and to the square of the measured arc length in the transverse plane. However, for most momenta encountered in this experiment, multiple scattering in the material within the tracking volume is also an important factor, dominating the resolution below $0.5 \mathrm{GeV} / c$.

Given these considerations, the most critical parameter is the magnitude of the radial tracking length which is uninterrupted by significant material. In the present design, the vertex detector and beam pipe together constitute about $3.5 \%$ of a radiation length at normal incidence. In contrast, the material within the drift chamber can, with care, be kept to around $0.12 \%$ of a radiation length for its entire $56 \mathrm{~cm}$ (interior) radial thickness. If there were no support tube between the silicon and the main tracking chamber, the effective track length for the $p_{\perp}$ measurement would extend from the outermost layer of the silicon through the entire tracking 
chamber. With a support tube representing $0.5 \% X_{0}$ of material and located at a radius of $21.5 \mathrm{~cm}$, the effectiveness of the vertex detector measurement is degraded, as may be concluded from Table 6-1. The outer radius of the drift chamber is restricted by the cost of the calorimeter and, depending on the particle identification device, will be about 80 to $96 \mathrm{~cm}$. Thus, the external factors of support tube and calorimeter radius severely limit the design choice for tracking length.

Another parameter which is available to improve resolution at high momentum is magnetic field strength. Table 6-1 shows that increasing the magnetic field from 1.0 to $1.5 \mathrm{~T}$ improves $B$ mass rcsolution by about $30 \%$. There are, however, drawbacks to using a strong magnetic field. It compromises the low momentum acceptance of the drift chamber as a stand-alone tracking device. Higher magnetic fields restrict the gas choice to those with sufficiently small Lorentz angle, so that excessively slow drift velocities or celldefinition problems do not arise. Trigger and pattern recognition efficiency may be degraded by the larger track curvature or by tracks which loop within the tracking volume. Nevertheless, given the restricted tracking length, the detector should be designed for a $1.5 \mathrm{~T}$ field.

With this magnetic field, only tracks with $p_{\perp}$ above $180 \mathrm{MeV} / c$ can reach the drift chamber outer wall. Those with $p_{\perp}<60 \mathrm{MeV} / c$ do not enter the drift chamber and must be tracked in the vertex detector alone. The vertex detector should be capable of providing independent pattern recognition and reconstruction for such tracks. For tracks which traverse both measurement systems, the role of the drift chamber is primarily to measure transverse momentum, while that of the vertex detector is to determine track direction and vertex location.

The effect of multiple scattering on $p_{\perp}$ resolution is discussed in Section 6.5. By comparing the last two entries in Table 6-1, it can be seen that minimizing the number of radiation lengths of material in the detector is at least as important as achieving the best possible position measurement resolution, particularly for lower momentum tracks. To this end, the proposed chamber uses low-density wires and a helium-based gas.

Finally, the average single-cell resolution depends on the amount of primary ionization, on the diffusion properties of the gas, and on
A $1.5 \mathrm{~T}$ field is desirable for momentum resolution in a necessarily small tracking chamber

A high magnetic field may present problems for pattern recognition and triggering

Tracks must have $p_{\perp}>$ $180 \mathrm{MeV} / \mathrm{c}$ to reach the outer layer of the drift chamber. Tracks with $p_{\perp}<60 \mathrm{MeV} / \mathrm{c}$ must be measured in the vertex detector alone 
Resolution of $110-150 \mu \mathrm{m}$ can be achieved using a helium-based gas the cell size. Having selected a low-mass gas, and recognizing that a larger cell size implies a smaller number of measurements per track, no large gains can be made over previous detectors. Prototype studies and experience elsewhere indicate that a spatial resolution of $110-150 \mu \mathrm{m}$ can be achieved using a helium-based gas.

Table 6-1 shows the mass resolution of reconstructed $B$ mesons in the $B^{0} \rightarrow \pi^{+} \pi^{-}$and $B^{0} \rightarrow D^{+} D^{-}$channels for a variety of detector configurations. These data are obtained from a parametrized simulation [Inn93] and, though they provide a relative comparison, the absolute values must still be verified with a detailed Monte Carlo simulation.

\begin{tabular}{|c|c|c|c|c|c|c|}
\hline \multirow{2}{*}{$\begin{array}{c}\text { Outer } \\
\text { Radius }[\mathrm{cm}]\end{array}$} & $\begin{array}{c}\text { Support- } \\
\text { Tube }\end{array}$ & $\begin{array}{c}\text { Gas- } \\
\text { Wires }\end{array}$ & $\begin{array}{c}\text { Resolution } \\
{[\mu \mathrm{m}]}\end{array}$ & $\begin{array}{c}\text { B-Field } \\
{[\text { Tesla }]}\end{array}$ & \multicolumn{2}{|c|}{ Mass Resolution $\left[\mathrm{MeV} / \mathrm{c}^{2}\right]$} \\
\cline { 7 - 8 } & $B^{0} \rightarrow \pi^{+} \pi^{-}$ & $B^{0} \rightarrow D^{+} D^{-}$ \\
\hline \hline 80 & Yes & He-Al & 140 & $\underline{1.0}$ & $30.7 \pm 0.3$ & $5.69 \pm 0.09$ \\
80 & Yes & He-Al & $\mathbf{1 4 0}$ & $\mathbf{1 . 5}$ & $\mathbf{2 0 . 8} \pm \mathbf{0 . 2}$ & $\mathbf{4 . 7 0} \pm \mathbf{0 . 0 8}$ \\
80 & $\underline{\text { No }}$ & He-Al & 140 & 1.5 & $17.1 \pm 0.1$ & $4.37 \pm 0.08$ \\
95 & Yes & He-Al & 140 & 1.5 & $14.0 \pm 0.1$ & $4.07 \pm 0.07$ \\
95 & $\underline{\text { No }}$ & He-Al & 140 & 1.5 & $12.5 \pm 0.1$ & $3.73 \pm 0.06$ \\
80 & Yes & Ar-Cu & 140 & 1.5 & $25.2 \pm 0.2$ & $5.76 \pm 0.09$ \\
80 & Yes & He-Al & $\underline{210}$ & 1.5 & $26.0 \pm 0.2$ & $5.09 \pm 0.09$ \\
\hline
\end{tabular}

Table 6-1. The effect of chamber parameters on mass resolution for $B \rightarrow \pi^{+} \pi^{-}$and $B \rightarrow D^{+} D^{-}$. The bold-face entries represent the parameters of the baseline design. The underlined values denote a variation with respect to the baseline.

\subsection{Conceptual Chamber Design}

The drift chamber is a cylindrical annulus of outer radius $80 \mathrm{~cm}$, inner radius $22.5 \mathrm{~cm}$, and length $280 \mathrm{~cm}$. Two internal geometries are being investigated: an axial/stereo chamber consisting of 4 axial superlayers and 6 stereo superlayers, and an all-stereo design in which the sign of the stereo angle alternates either layer-by-layer or by superlayer. There are approximately 7000 sense wires and 37,000 field wires strung between the endplates, each of which carries an axial load of $\sim 2400 \mathrm{~kg}$. 


\subsubsection{Geometrical Shape and Dimensions}

The ideal chamber would provide equal momentum resolution at all polar angles in the center of mass. This requirement leads to a rather long chamber of around $350 \mathrm{~cm}$, which raises problems of wire tension and stereo angle; it also leads to a prohibitively expensive calorimeter. On the other hand, making the chamber too short degrades the mass resolution and introduces non-Gaussian tails into invariant mass distributions. A study of the $C P$ channels $B^{0} \rightarrow$ $\pi^{+} \pi^{-}$nd $B^{0} \rightarrow D^{+} D^{-}$(see Figure 6-2) shows that the loss of mass resolution is small as long as at least half the layers of the chamber are traversed by particles at the $300 \mathrm{mr}$ acceptance limit. This results in a chamber with a forward length of around $167 \mathrm{~cm}$ from the interaction point. The length in the backward direction is set to $111 \mathrm{~cm}$ to provide adequate coverage for $d E / d x$ purposes. A particle at $\sim 430 \mathrm{mr}$ in the backward direction will pass through half the layers in the chamber before exiting. These choices lead to a maximum wire length of $278 \mathrm{~cm}$ at a radius of $51.25 \mathrm{~cm}$.

The current endplate design uses truncated cones with the outer part of the chamber endplates inclined at $20^{\circ}$ to the vertical, as shown in Figure 6-3. In the forward direction, the tip of the cone lies on the $300 \mathrm{mr}$ line.

The primary motivation for the conical shape at large radii is to allow particles to enter the particle identification device at close-toperpendicular incidence. This reduces the CsI volume and. improves the low-energy performance of the calorimeter by reducing the amount of material traversed before reaching the crystals. The reduction comes both from the near-normal angle of incidence, and from the structural strength of the conical shape, which permits a thinner endplate.

These arguments only apply to the outer region of the chamber; at smaller radii outside the acceptance region, the endplate surface either could be made conical or kept nearly flat. The doubleconical endplate design reduces the wire length at large and small radii. This allows a larger stereo angle at these points, and hence better $z$ resolution in an all-stereo design. The disadvantage is a slight loss of momentum resolution at certain angles because of the reduced tracking length; this is an issue which is coupled to the overall chamber length. Nearly flat endplates may ease access to the
The length of the chamber represents a compromise between coverage and performance

The endplates are sloped at a $20^{\circ}$ angle

The conical endplates improve the performance of the particle identification and electromagnetic calorimeter systems

The conical endplates slightly compromise the momentum resolution at certain angles 


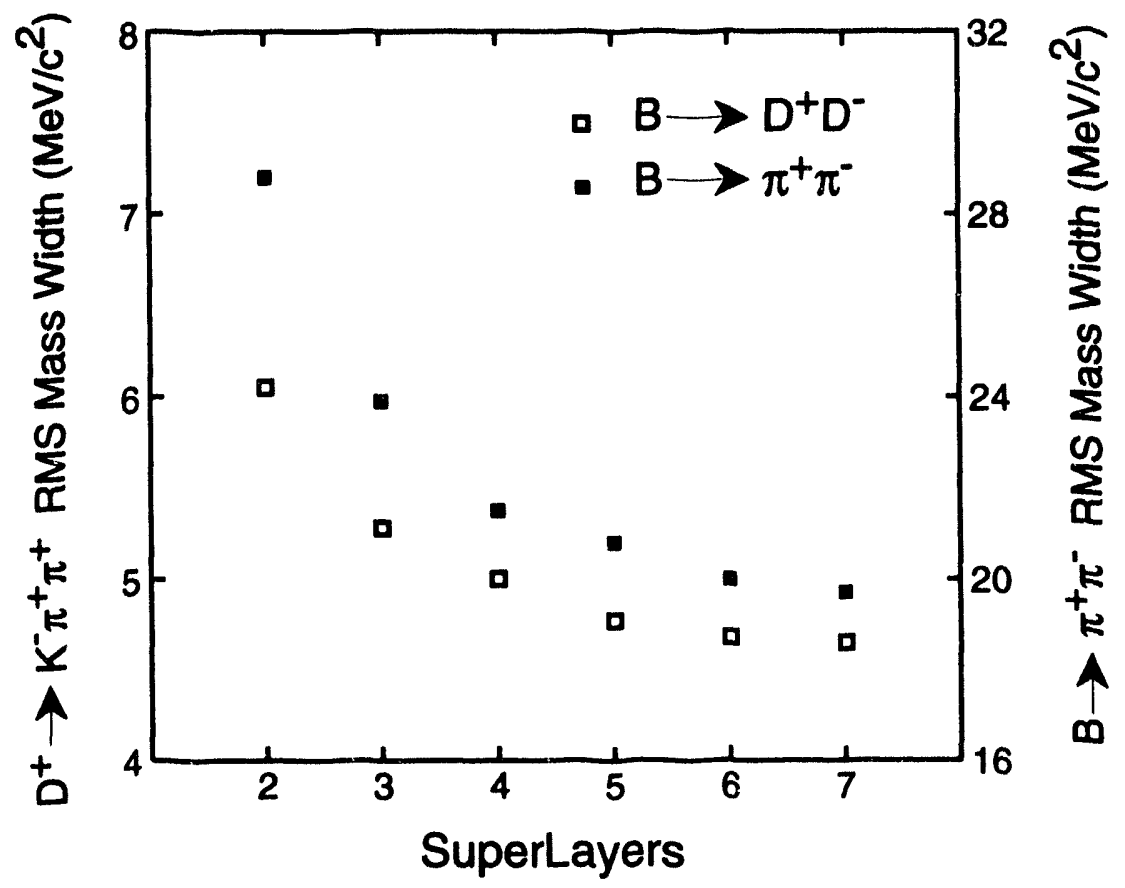

Figure 6-2. Invariant mass resolution for $D^{+}$mesons in the decay $B^{0} \rightarrow D^{+} D^{-}$and for $B^{0} \rightarrow \pi^{+} \pi^{-}$as a function of chamber forward length, defined by the number of superlayers (4 cells) crossed prior to exit from the drift chamber for tracks produced at an angle of $300 \mathrm{mr}$ in the forward direction. The resolution is observed to reach a plateau when half the chamber is traversed at this acceptance limit.

The two endplates are identical to reduce tooling costs

The axial wire load is $\sim 2400 \mathrm{~kg}$ chamber once it is installed. To simplify design and construction, the forward and rear endplates should be of identical shape.

\subsubsection{Inner and Outer Shells and the Endplates}

The chamber endplates must be made sufficiently strong to support an axial wire load of $\sim 2400 \mathrm{~kg}$ while minimizing the material in front of the calorimeter. Carbon fiber composites and aluminum have both been considered as possible endplate materials. Table 6-2 summarizes preliminary engineering estimates of endplate thicknesses required for both flat and conical geometries. The longer radiation length and higher structural strength of carbon fiber make it an attractive choice, and in this scenario, the feedthroughs and connec- 


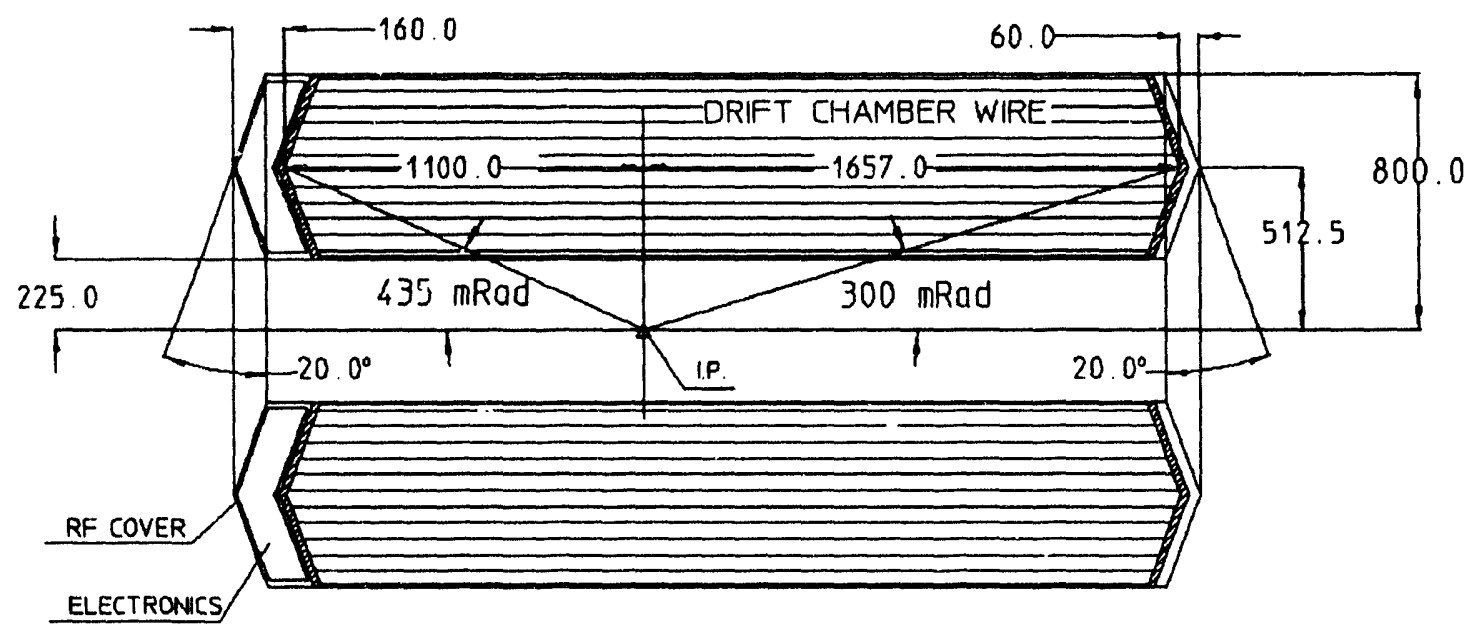

Figure 6-3. The tracking chamber geometry showing a possible concept for truncated cone endplates. The chamber is offset in $z$ from the interaction point and extends $1.67 \mathrm{~m}$ in the forward direction and $1.11 \mathrm{~m}$ in the backward direction. A ten-superlayer structure is indicated.

tors are likely to dominate the amount o: suacerial present. While high-precision drilling of flat surfaces is easily accomplished, the drilling accuracy for a conical surface still needs to be investigated. Solutions for conductivity and RF-shielding are being studied.

The force between the two endplates is carried almost entirely by the outer shell of the chamber. Engineering calculations indicate that this could be accommodated by a $1.6 \mathrm{~mm}$ thick, $0.7 \% X_{0}$, carbon-fiber shell. It is anticipated that the chamber will operate at constant gas pressure resulting in a pressure differential (3040 mbar) between the chamber and atmosphere. The inner wall is designed to act as a gas seal, but it must be able to support this differential pressure. A $0.5 \mathrm{~mm}$ beryllium wall is more than adequate for this purpose; thinner options are under investigation.
The outer drift chamber wall is a $1.6 \mathrm{~mm}$ thick, $0.7 \% X_{0}$, carbon-fiber shell 


\begin{tabular}{|l|c|c|c|}
\hline \multicolumn{1}{|c|}{ Endplate Model } & $\begin{array}{c}\text { Thickness } \\
{[\mathrm{cm}]}\end{array}$ & $\begin{array}{c}\text { Radiation } \\
\text { Length [\%] }\end{array}$ & $\begin{array}{c}\text { Deflection } \\
{[\mathrm{cm}]}\end{array}$ \\
\hline \hline Flat Aluminum & 2.4 & 27 & 0.46 \\
Flat Carbon Fiber & 1.6 & 7.2 & 1.73 \\
Flat Carbon Fiber + core & 0.15 & 1.4 & 1.73 \\
Aluminum, double cone & 0.61 & 6.8 & 0.07 \\
Carbon Fiber, double cone & 0.35 & 1.6 & 0.08 \\
\hline
\end{tabular}

Table 6-2. Endplate thicknesses and calculated deflections at the inner radius for a variety of structural materials and shapes.

\subsubsection{Cell Design}

Small cells of $\sim 14 \mathrm{~mm}$ width will be employed

Hexagonal and square cell geometries have been studied with a detailed simulation

The small-cell design of the drift chamber features a $20 \mu \mathrm{m}$ diameter gold-plated tungsten-rhenium anode wire, surrounded by $55 \mu \mathrm{m}$ diameter low-density cathode wires. The cathode wires, which could be either gold-plated aluminum or gold-plated silicon carbide, are arranged to form either a hexagonal or square cell. The cells are approximately $14 \mathrm{~mm}$ wide, and there are 5-6 cathode wires for each anode wire.

The surface field on the cathode wires is $<20 \mathrm{kV} / \mathrm{cm}$. The electrostatic forces have been calculated for tensions of $53 \mathrm{~g}$ on the sense wires and $50 \mathrm{~g}$ on the field wires. The wires are stable, with a maximum electrostatic deflection of $\sim 160 \mu \mathrm{m}$, and a gravitational sag of $120 \mu \mathrm{m}$.

The cell resolution, time-to-distance relationship and pulse shapes over the cell volume have been studied for the hexagonal and square cells. A program simulating the drifting of electrons in the chamber gas in a 1.5 T magnetic field has been used to map out arrival times of ions from tracks in the cells. The simulation has become a valuable tool for understanding the basic characteristics of drift cells; further investigations will include a comparison of these predictions with experience at presently operating chambers.

Cell resolution has been studied by simulating tracks at various angles to, and distances from, the sense wire. One such analysis determines the regions of a cell that has good resolution by mapping 
out tracks that have at least $1.3 \mathrm{~mm}$ of track segment contributing to the first $10 \mathrm{~ns}$ of pulse. It is found that $75 \%$ of the hexagonal cell satisfies this criterion, while square or rectangular cells are good over $69 \%$ to $71 \%$ of their cross-sectional area, depending on how many field wires are used. These efficiencies are for near normal-incidence tracks and do not explore the full range of cell performance.

Time-to-distance relationships have been studied for tracks at various angles to and distances from the sense wire. For each simulated track, the time was taken from the first arriving electron. There is very little difference in the time-to-distance function for tracks within approximately $6 \mathrm{~mm}$ of the sense wire. At the far edges of the cell, i.e. at a distance of 8 to $9 \mathrm{~mm}$, the time-to-distance relationship changes rapidly with track angle and distance for both hexagonal and square cells. The time-to-distance function is also simulated for cells at the interface between axial and stereo superlayers, where the radial size varies along the wire. The conclusion is that there is little difference in the time-to-distance relationship for hexagonal and square cells, and that the varying cell size at superlayer brundaries does not degrade resolution in a significant way.

Pulse shapes have also been simulated in the two cell types. The corners of the cells are regions of low electric field, and therefore low drift velocity. This leads to long electron-collection times for tracks passing near the corners. The simulated pulses are lengthened for square cells, but the effect is small when averaged over all radii and angles of incidence.

\subsubsection{Layer Arrangement}

Two arrarigements of layers within the drift chamber are under consideration. In the first, the axial/stereo design, the wires are arranged in 4 axial and 6 stereo superlayers (three with positive stereo angle, three with negative), each superlayer comprising four individual layers. If hexagonal cells are used, cells in the two layers bounding a superlayer become pentagonal to accommodate the superlayer structure, as illustrated in Figure 6-4a. Measurements of the $z$ coordinate are provided by stereo wires. The current design employs stereo angles of approximately $60 \mathrm{mr}$. A larger angle, which would improve $z$ resolution, is precluded due to the increase of the
There is little difference in the time-to-distance relationship for hexagonal and square cells

The corners of square cells yield somewhat longer pulses due to low electric fields and, therefore, low drift velocity in a nonsaturated gas

There are 4 axial and 6 stereo superlayers 
An all-stereo design is also being considered
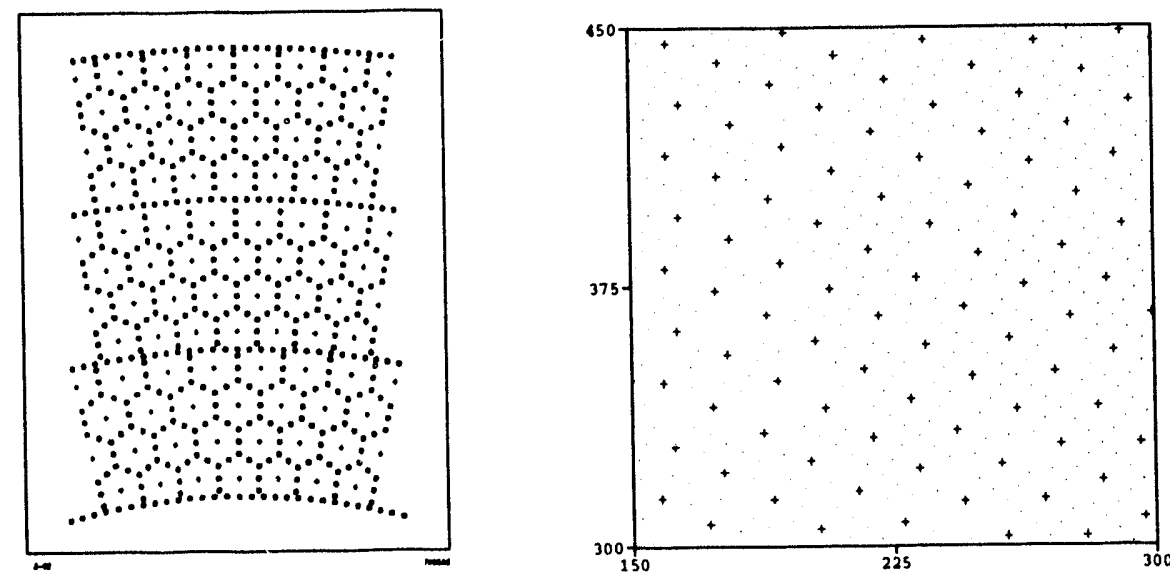

Figure 6-4. Two possible cell designs: A segment of three superlayers from an axial/stereo design (left). The hexagonal cells of $1 \mathrm{~cm}$ side are truncated at the layer boundaries. A segment of an all-stereo design incorporating $1.6 \mathrm{~cm}$ square cells (right).

(z-dependent) displacement of sense wires in the pentagonal cells. A quadrant of the chamber is shown in Figure 6-5.

The second option is the all-stereo chamber design [HEL92]. This option has approximately the same number of layers as the first, but incorporates only layers with positive and negative stereo angles. The layers may be arranged either in superlayers or in an alternating pattern; trigger requirements favor the former, while low momentum acceptance favors the latter. Another possibility would be to use alternating layers for the inner radii and superlayers for the outer radii. The absence of axial layers allows the stereo layers to nest together, since adjacent layers have nearly the same sagitta. With no axial/stereo boundaries the size and shape variations of cells across the chamber are minimized. Allowing the stereo sag to be at most the size of one cell, stereo angles of 90-150 mr can be achieved.

The all-stereo arrangement features more uniform cell size and shape, which may facilitate calibration, and provides better $z$ resolution for tracks that do not reach the outer radius of the drift chamber. In addition, the larger stereo angles provide the required $z$ resolution without requiring the use of cathode strips, thereby reducing the material in the chamber, and eliminating the complication and expense associated with such a system. 

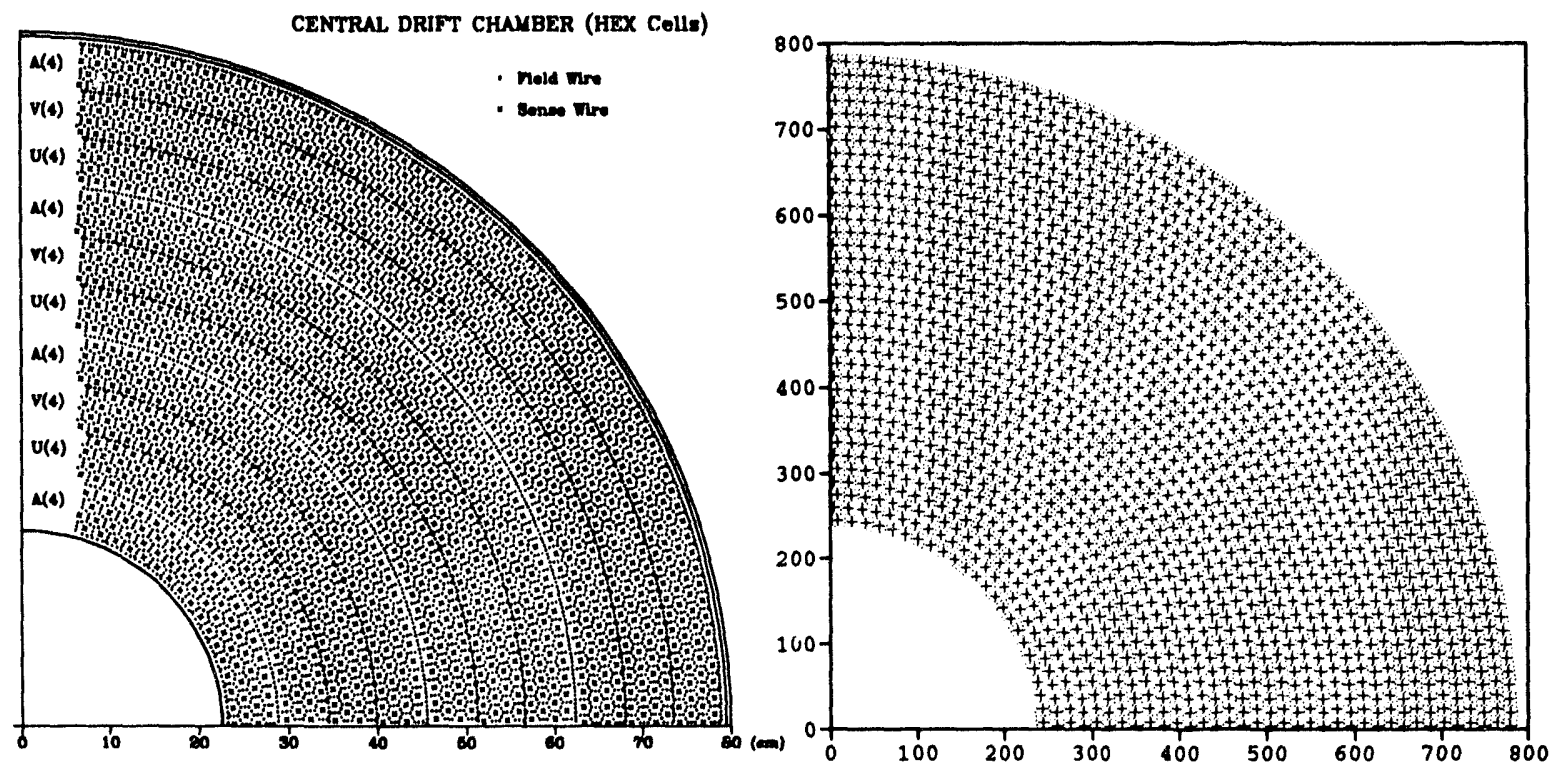

Figure 6-5. On the left, a quadrant of the axial/stereo chamber, and on the right, a quadrant of the all-stereo chamber.

The principal disadvantage of an all-stereo chamber is that it eliminates one of the three track views provided by an axial/stereo design, and therefore reduces the redundancy available for pattern recognition; another complication in pattern recognition is that tracks in an all-stereo chamber do not project to form arcs of circles in either view, although they do still form locally-smooth track segments. In addition, concerns about triggering must be addressed, although these are not expected to be insurmountable. The question of pattern recognition efficiency in the all-stereo case have been studied [Bri93], but a detailed simulation must be carried out to verify that the potential loss of track-finding efficiency is acceptable, even in the presence of high backgrounds. Neither the all-stereo nor the axial/stereo chamber design poses major construction difficulties.

\subsubsection{Wires and Feedthroughs}

In order to minimize multiple scattering in the drift chamber, material in the gas and in the wires of the chamber must be minimized. Aluminum, which has a long radiation length compared to other
The pattern recognition and triggering characteristics of axial/stereo and all-stereo designs are being compared in detail 
Field wires will be made of aluminum or silicon carbide

Wires will be crimped inside a brass pin within a Celanex feedthrough metals, is therefore a good candidate. However, bare aluminum is undesirable because it oxidizes, forming an insulating layer on the wire surface, which can lead to electrical discharge. A gold coating solves this problem, but thin layers of gold on aluminum have been difficult to produce. A thicker layer of gold becomes a non-negligible contribution to the radiation length. Aluminum wires are also known to suffer 'creep', whereby wires gradually lose tension over time, although this can be minimized by a careful choice of tension and type of wire. The other material being considered is a high-strength fiber wire such as silicon carbide $(\mathrm{SiC})$, coated with aluminum and gold to provide good conductivity and surface quality. The radiation length of $\mathrm{SiC}$ is slightly larger than that of aluminum, but the thickness of the gold-plating may be the dominant factor differentiating the two choices. SiC has an elastic modulus which is almost ten times that of aluminum, which may reduce the incidence of broken wires. Another advantage is the good match of thermal expansion coefficients between $\mathrm{SiC}$ and the carbon fibre structural cylinder. However, $\mathrm{SiC}$ is brittle and the feasibility of crimping and its use in a large chamber need investigation.

A modified version of a feedthrough pin used in two TRIUMF-built chambers, both of which have aluminum wires, may be employed here as well. In this design, the wires are held by crimping. The pins are made of brass with an inner diameter of $100 \mu \mathrm{m}$ and can be molded into a Celanex sleeve. The entire piece can then be press-fitted into a precision-drilled hole in the endplate.

\subsection{Gas}

For the $B A B A R$ experiment, helium provides an excellent alternative to argon or other conventional chamber gases, because its radiation length is 50 times longer. Helium must be used in combination with other gases for quenching and to increase the number of primary ion pairs produced. Test data is already available for the following combinations: $\mathrm{He}: \mathrm{CO}_{2}: \mathrm{C}_{4} \mathrm{H}_{10}$ [Boy92, Pla92]; He:DME [Pla92, Cin91]; and $\mathrm{He}: \mathrm{C}_{2} \mathrm{H}_{6}$ [Uno93].

The properties of various helium-based gas mixtures are shown in Table 6-3. The drift velocities and Lorentz angles are determined with the Boltzmann integration code MAGBOLTZ [Bia89]. The 


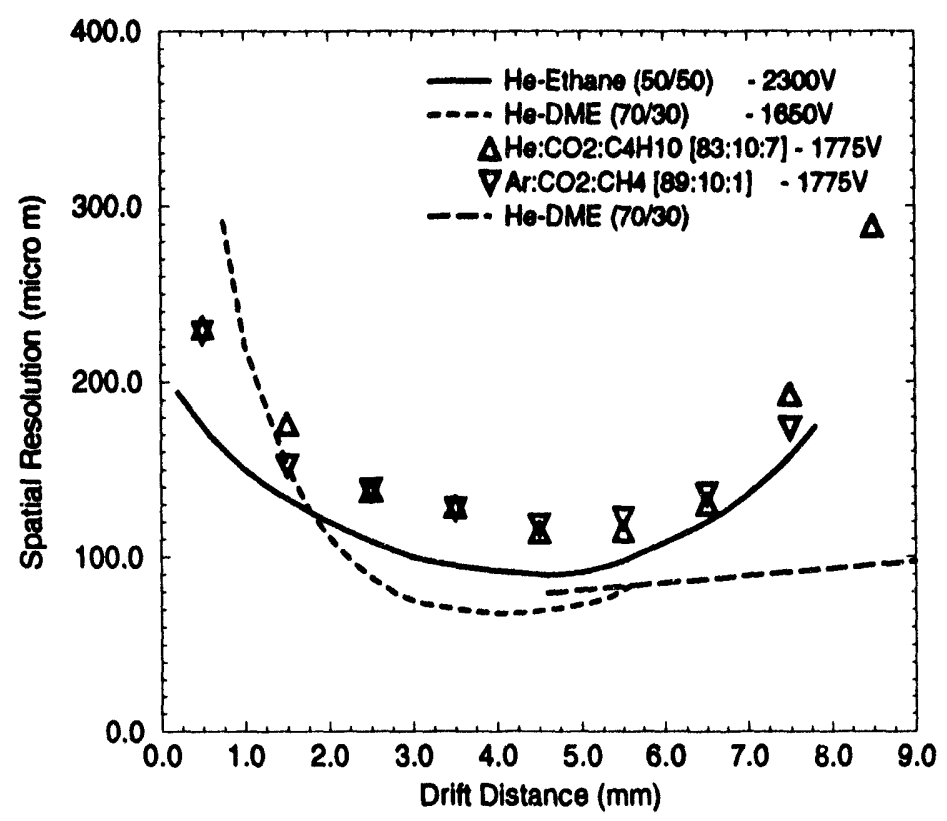

Figure 6-6. The prototype drift chamber results for HRS gas and $\mathrm{He}: \mathrm{C0}_{2}: \mathrm{C}_{4} \mathrm{H}_{10}$ [Pit94], (shown as trangles) with smooth linefit results from previously published He:DME [Pla92, Cin91], and $\mathrm{He}: \mathrm{C}_{2} \mathrm{H}_{6}$ [Uno93] studies.

$d E / d x$ calculations were performed with a modified version of code from Va'vra [Vav82]. The table shows that most helium mixtures under consideration have a radiation length five or more times larger than that of HRS gas, a commonly used argon-based mixture. On the other hand, since helium in a mixture produces only $\sim 6$ primary ions $/ \mathrm{cm}$, some helium-based mixtures are expected to provide fewer primary ion pairs than the HRS mixture. This should lead to a degraded spatial resolution close to the sense wire. However, recent measurements shown in Figure 6-6 seem to refute this argument.

The drift velocities in helium-based gases are relatively low. Figure 6-7 shows the drift velocities for some of the gas mixtures. For drift fields of about $600 \mathrm{~V} / \mathrm{cm}$, drift velocities range from 8$30 \mu \mathrm{m} / \mathrm{ns}$; none of the gas mixtures has a saturated drift velocity. Although the He:DME mix would provide the best spatial resolution, its low drift velocity could prove problematic in a high background environment.
Helium-based gases have a spatial resolution comparable to argonbased gases, despite a lower yield of primary ions

Drift velocities in heliumbased gases are relatively low 


\begin{tabular}{|c|c|c|c|c|c|c|c|c|}
\hline \multirow[b]{2}{*}{ Gas mixture } & \multirow[b]{2}{*}{ Ratio } & \multirow[b]{2}{*}{$\begin{array}{l}X_{0} \\
{[\mathrm{~m}]}\end{array}$} & \multirow[b]{2}{*}{$\begin{array}{l}\text { Primary } \\
\text { Ions/cm }\end{array}$} & \multirow[b]{2}{*}{$\begin{array}{c}v_{d} \\
{\left[\frac{\mu \mathrm{m}}{n s}\right]}\end{array}$} & \multirow[b]{2}{*}{$\begin{array}{l}\theta_{L} \\
\text { [deg] }\end{array}$} & \multirow{2}{*}{$\begin{array}{c}d E / d x \\
\text { resol } \\
{[\%]}\end{array}$} & \multicolumn{2}{|c|}{$K / \pi$ separation } \\
\hline & & & & & & & $\begin{array}{c}p \text { for } 3 \sigma \\
{\left[\frac{\mathrm{MeV}}{c}\right]}\end{array}$ & $\begin{array}{c}\# \sigma \text { at } \\
2.6 \mathrm{GeV} / c\end{array}$ \\
\hline$\overline{\mathrm{Ar}: \mathrm{CO}_{2}: \mathrm{CH}_{4}}$ & $89: 10: 1$ & $\overline{124}$ & 23.6 & 49 & $\overline{52}$ & $\overline{7.3}$ & 665 & $\overline{2.4}$ \\
\hline $\mathrm{He}: \mathrm{C}_{2} \mathrm{H}_{6}$ & $50: 50$ & 686 & 23.1 & 31 & 54 & 6.6 & 720 & 2.1 \\
\hline He:DMi & $70: 30$ & 723 & 22.4 & 6 & 8 & 6.7 & 720 & 2.1 \\
\hline $\mathrm{He}: \mathrm{C}_{3} \mathrm{H}_{8}$ & $70: 30$ & 733 & 24.1 & 24 & 36 & 6.5 & 730 & 2.2 \\
\hline $\mathrm{He}: \mathrm{C}_{4} \mathrm{H}_{10}$ & $80: 20$ & 807 & 21.2 & 22 & 32 & 6.9 & 710 & 2.1 \\
\hline $\mathrm{He}: \mathrm{CO}_{2}: \mathrm{C}_{4} \mathrm{H}_{10}$ & $83: 10: 7$ & 963 & 14.9 & 19 & 26 & 8.5 & 660 & 1.7 \\
\hline
\end{tabular}

Table 6-3. Properties of various gas mixtures at atmospheric pressure and $20^{\circ} \mathrm{C}$. The drift velocity $\left(v_{d}\right)$ and Lorentz angle $\left(\theta_{L}\right)$ are given for an electric field of $600 \mathrm{~V} / \mathrm{cm}$ with no magnetic field and with $1.5 T$, respectively. The $d E / d x$ resolution is calculated for a minimum-ionizing particle. Also listed are the momenta below which there is at least $3 \sigma K / \pi$ separation, and the $K / \pi$ separation at $2.6 \mathrm{GeV} / \mathrm{c}$.

Using a small-cell prototype chamber, the behavior of several helium mixtures in terms of resolution and operability is presently under investigation. Tests will be performed to study the ageing characteristics of these mixtures. Drift velocities will also be determined and studies will be made of whether small amounts of $\mathrm{CF}_{4}$ additive would be useful in extending the chamber lifetime.

\subsection{Performance}

The specified momentum resolution of $\sim 0.3 \%$ is attainable with a heliumbased gas
Figures 6-8 and 6-9 show the expected momentum resolution dependence on momentum and center-of-mass angle, as calculated by TRACKERR [Inn93] using the Kalman-filter technique to analytically propagate the error matrix and add appropriate multiple scattering contributions [Bil84]. A resolution of $\sim 0.3 \%$ is clearly attainable for radiation lengths corresponding to helium-based gas mixtures.

This calculation represents the performance of the baseline design tracking system, comprised of the five-layer silicon detector and the 40-layer drift chamber, including scattering material (beam pipe, silicon, support tube, and drift chamber), in a $1.5 \mathrm{~T}$ magnetic field, 


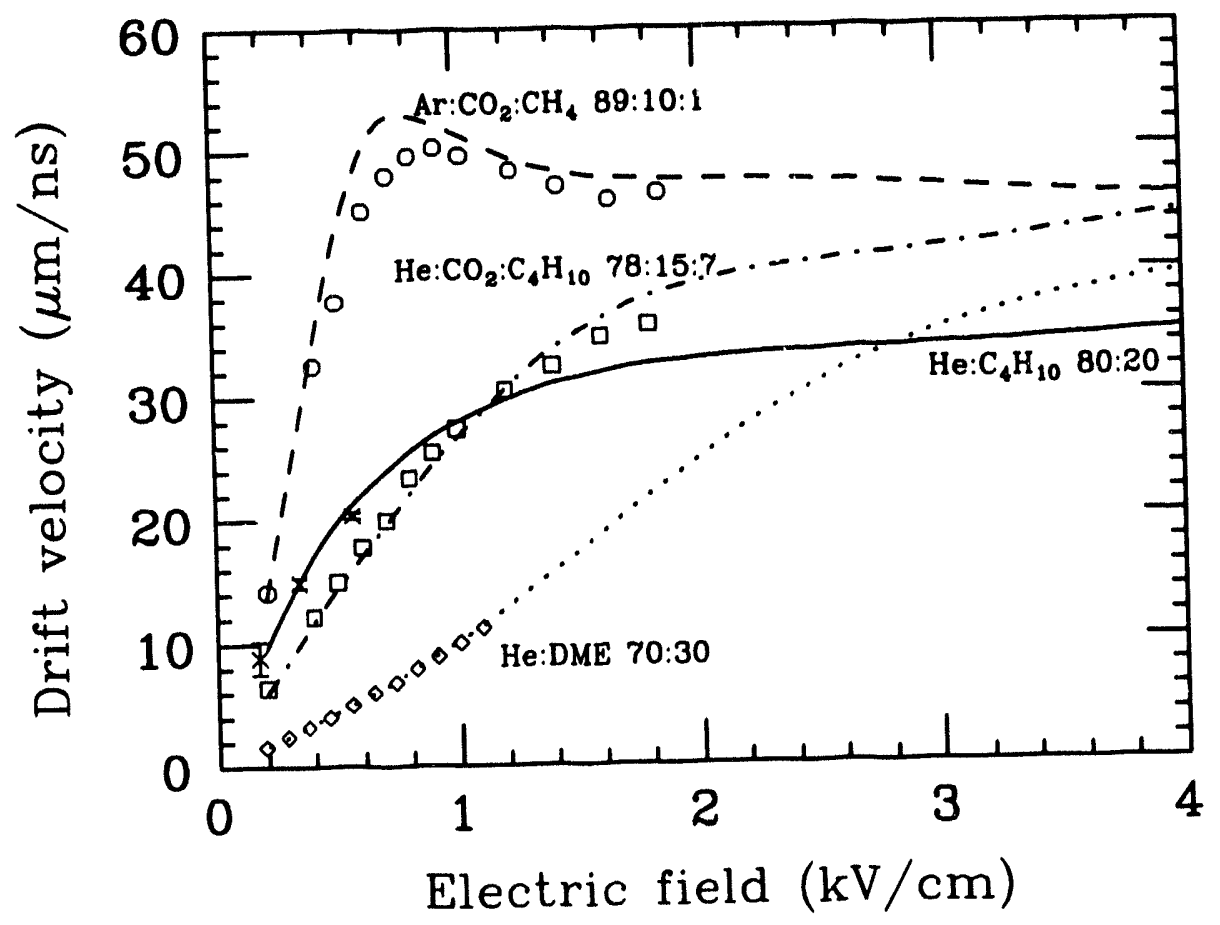

Figure 6-7. Calculated and measured drift velocities as a function of electric field for zero magnetic field. The calculations use the code of Biagi (see text). The solid curve and crosses [Pla92] are for the $\mathrm{He}: \mathrm{C}_{4} \mathrm{H}_{10}$ 80:20 mixture; dot-dashed curve and squares [Boy92] are for $\mathrm{He}: \mathrm{CO}_{2}: \mathrm{C}_{4} \mathrm{H}_{10}$ 78:15:7; the dotted curve and diamonds [Cin91] are for He:DME 70:30; and the dashed curve and circles [Boy92] are for argon: $\mathrm{CO}_{2}: \mathrm{CH}_{4}$ 89:10:1 (HRS gas).

and assuming the particle is a pion. For simplicity, the material of the drift chamber wires is taken to be uniformly distributed over the tracking volume. In this approximation, the wires add nearly the same number of radiation lengths to the chamber as a gas with an $800 \mathrm{~m}$ radiation length. The effect of discrete wires will be studied.

Figure 6-9 shows both the transverse and total momentum resolution expected for the full tracking system. At low momenta, the total momentum resolution is dominated by the uncertainty in the polar angle determination due to multiple scattering effects, especially in the silicon. At higher momenta, the error in the transverse component is seen to dominate.
The gas and the wires contribute approximately equally to the number of radiation lengths 


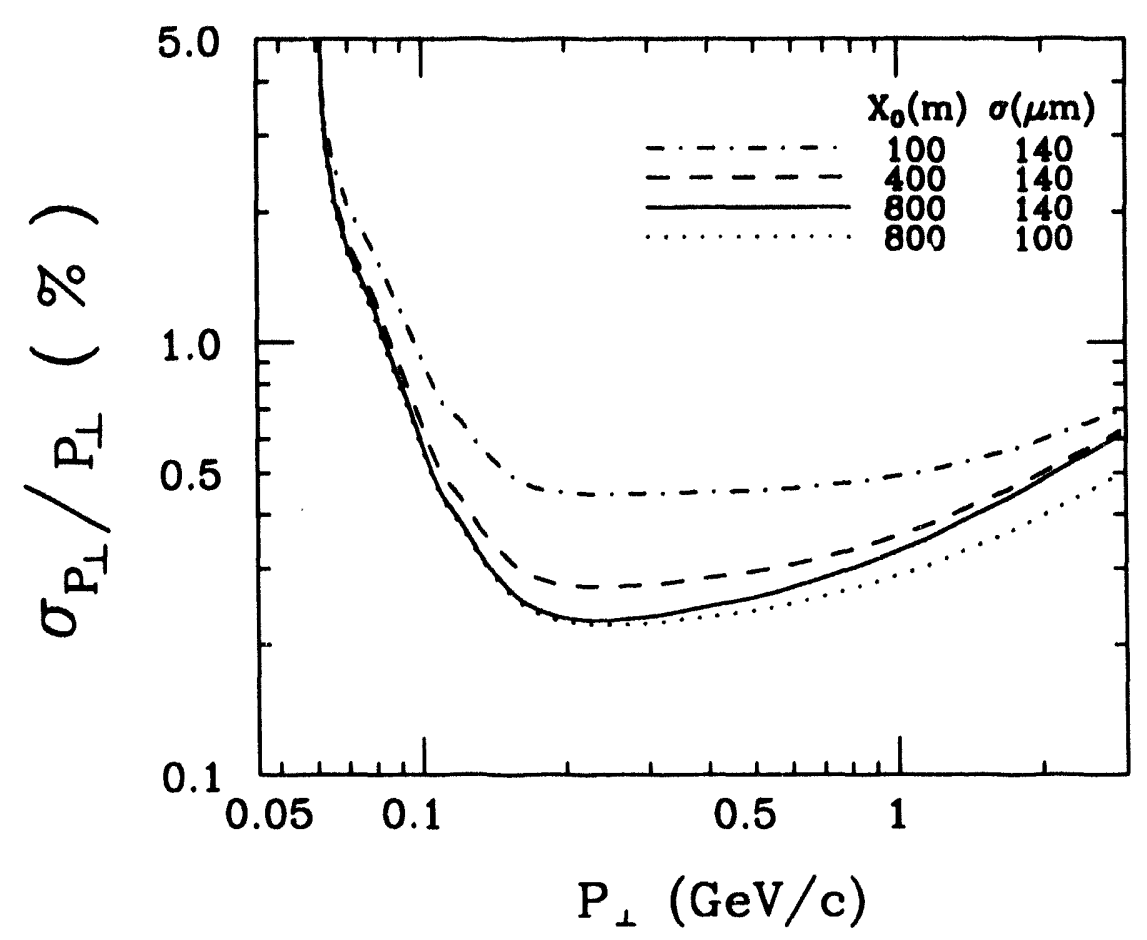

Figure 6-8. Momentum resolution expected for pions at $\theta_{\text {lab }}=$ $90^{\circ}$ in a drift chamber with 40 cylindrical layers from 22.5 to $80 \mathrm{~cm}$ in radius and a five-layer silicon detector in a $1.5 \mathrm{~T}$ field. The assumptions for the radiation lengths of the gas and the average single-cell resolutions are listed for each curve.

The estimated $d E / d x$ resolution with an 80:20 helium: $\mathrm{C}_{4} \mathrm{H}_{1} \mathrm{O}$ mixture is $6.9 \%$
The $d E / d x$ performance in the drift chamber is estimated by using a modified version of the simulation programs of Va'vra [Vav82]. For 40 samples, each $1.35 \mathrm{~cm}$ in length, the predicted $d E / d x$ resolution

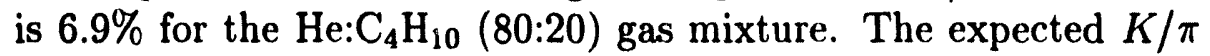
and $K / p$ separations are shown in Figure 6-10. The $K / \pi$ separation is better than $3 \sigma$ for tracks of momentum less than $710 \mathrm{MeV} / c$. For momenta of $3 \mathrm{GeV} / c$, the $K / \pi$ separation is at best $2 \sigma$.

The $z$ resolutions at the particle identification device as obtained by the full tracking system including the silicon vertex detector in the axial/stereo and all-stereo designs are shown in Figure 6-11. The approach of providing the $z$ information solely from the stereo layers allows a more homogeneous response to all particles regardless of momentum or origin. If better $z$ resolution is required in the barrel section, the use of cathode strips may be necessary in an axial/stereo design. 


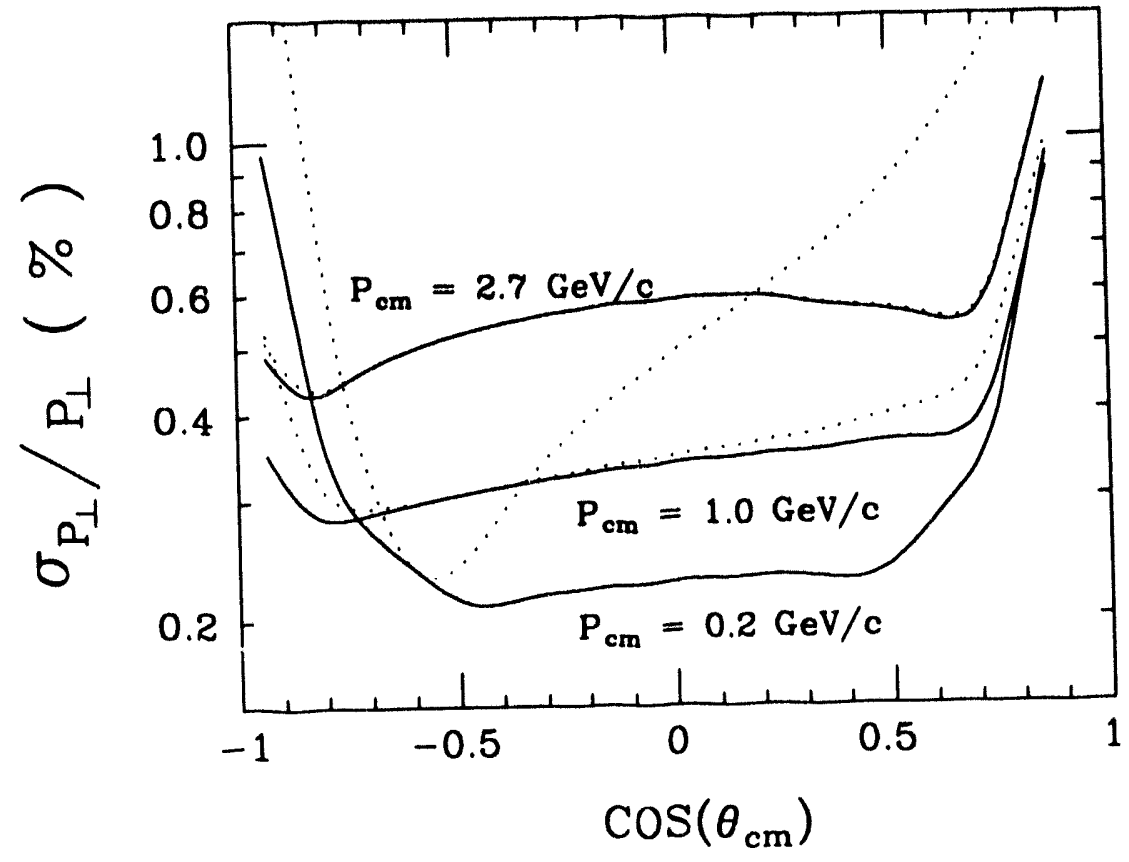

Figure 6-9. Transverse momentum resolution vs. center-ofmass angle for pions produced with three different center-ofmass momenta. The dotted curves show the total momentum resolution, $\sigma_{p} / p$. The gas model used a radiation length of $800 \mathrm{~m}$ with $140 \mu \mathrm{m}$ single cell average spatial resolution.

\subsection{Effect of Backgrounds}

Wire ageing and chamber occupancy due to beam-related backgrounds are two issues of concern for the operation of wire chambers. Ageing refers to changes in gain or increases in noise due to material deposited on the wires from the gas-avalanche process. Chamber occupancy is the fraction of cells that contain random hits during the event resolving time, i.e. the full drift time. If the occupancy exceeds a few percent, pattern recognition becomes difficult.

Bremsstrahlung radiation and scattering on residual gas in the accelerator upstream of the detector causes beam particles and photons to strike machine and detector components, producing electromagnetic showers. Debris from these showers is detected by the drift chamber. Simulations have been performed to estimate the background rates as a function of radius and $z$ in the drift chamber [Pit93]. For the innermost layers, a background rate of
Beam-related backgrounds raise concerns about chamber ageing and pattern recognition

Chamber backgrounds due to lost beam particles and synchrotron radiation photons have been simulated in detail 


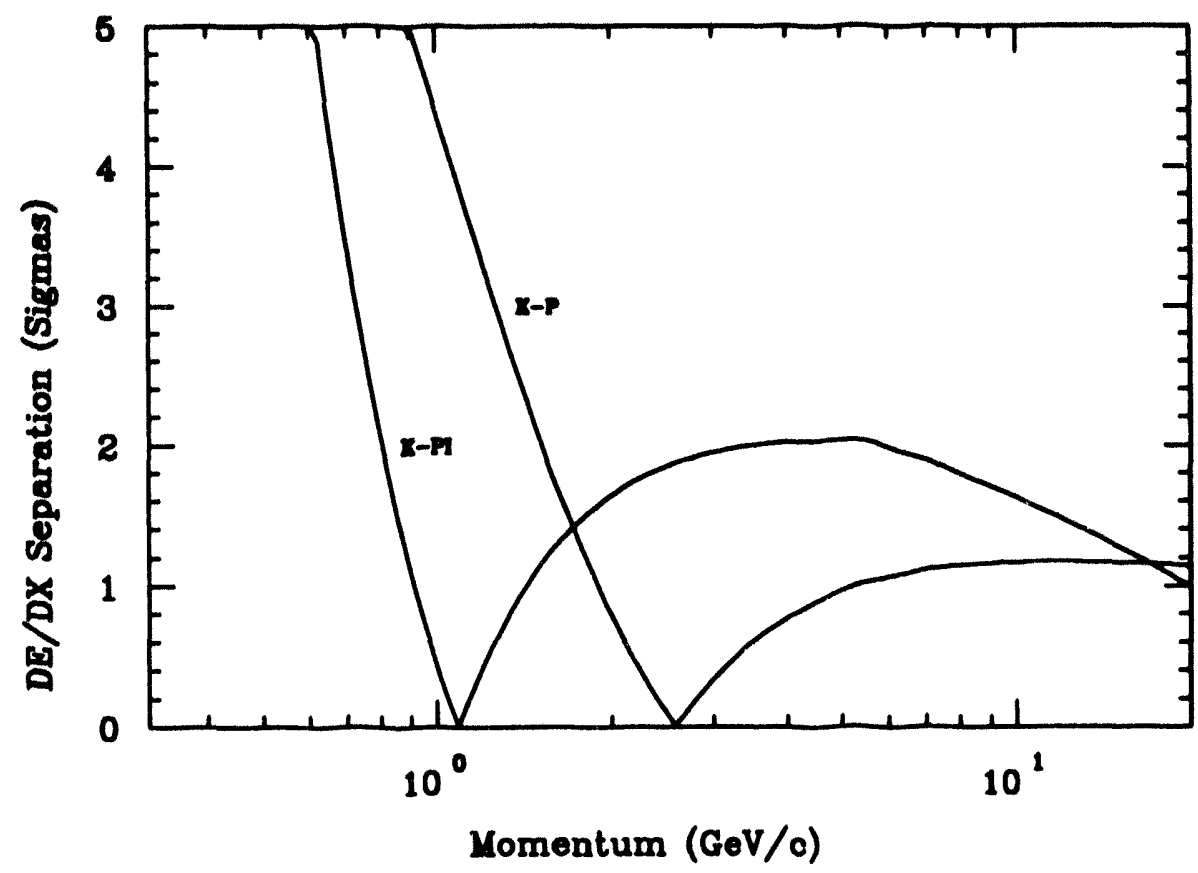

Figure 6-10. The predicted $d E / d x$ separation as a function of momentum for pions and kaons, and for protons and kaons.

$0.013 / \mu \mathrm{s} /$ wire is predicted. The distribution of this background is shown in Figure 6-12. These simulations are ongoing, and more detailed studies are underway.

Using the above background rate, the accumulated charge on a sense wire over 5 years of operation would be about $0.02 \mathrm{C} / \mathrm{cm}$. This assumes a worst case scenario where the background is concentrated over only $20 \mathrm{~cm}$ of the wire length. An avalanche gain of $5 \times 10^{4}$ is used. Azimuthal variation of background around the beam line could possibly increase this value by a factor of 2-3. Preliminary investigations of ageing [Kad94] in several helium-based gases look promising even for an integrated charge of $1 \mathrm{C} / \mathrm{cm}$.

For a signal collection time of $500 \mathrm{~ns}$, the occupancy for the predicted background rate above is only $0.6 \%$. A factor of ten increase can be tolerated before there would be any impact on pattern recognition. 


\subsection{Front-End Electronics}

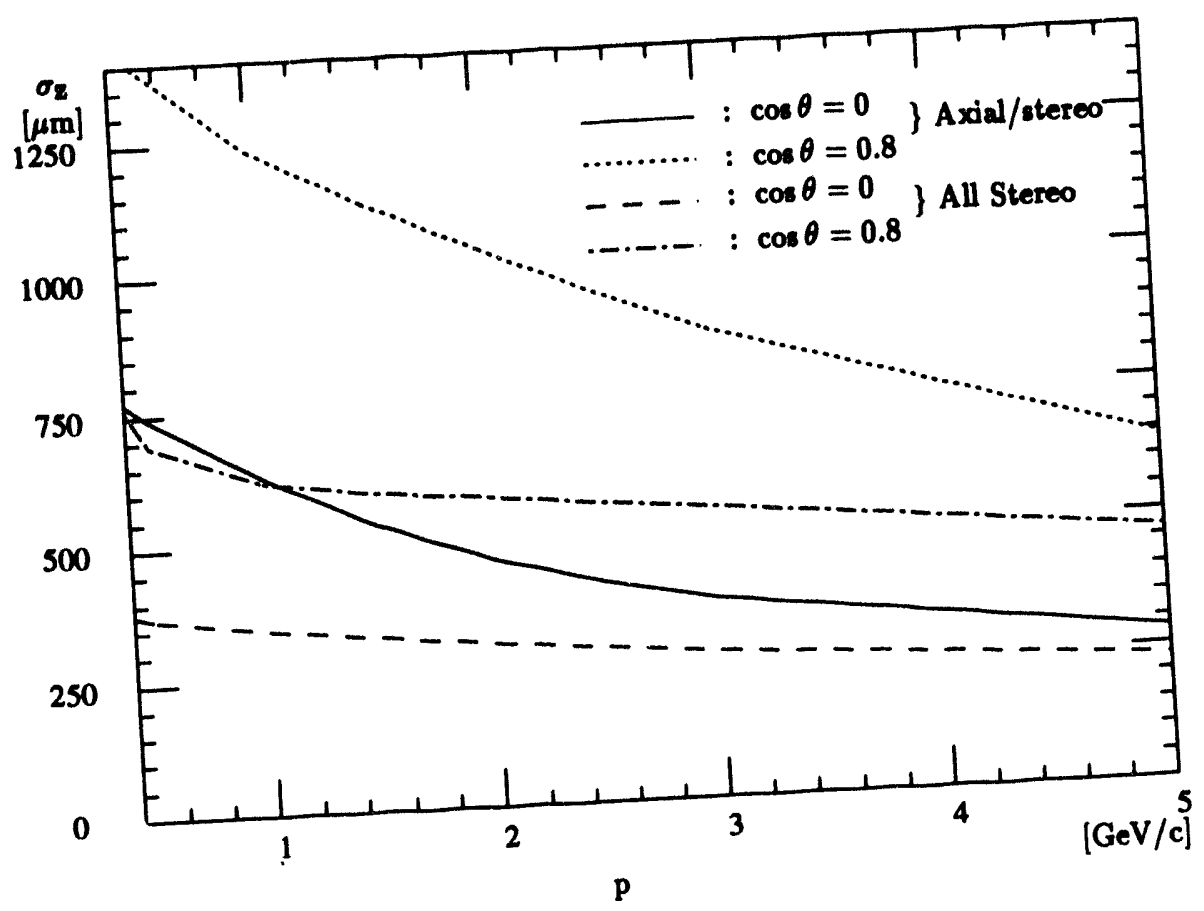

Figure 6-11. The $z$ resolution as a function of momentum for two center-of-mass angles for the axial/stereo and all-stereo chamber configurations. The calculations include the $z$ resolution provided by the silicon vertex detector.

\subsection{Front-End Electronics}

The front-end electronics provides amplification and shaping of the signals for optimal timing and pulse height $(d E / d x)$ resolution. The shaping time and required dynamic range depends on the drift gas selected and the mode of drift time measurement (TDC, FADC) and are the subject of simulations. In order to minimize power, the preamplifier consists of a bipolar ASIC chip serving several channels.

The preamplifiers will be bipolar ASIC's The front-end electronics are mounted on PC boards which connect directly to the feedthrough pins on the backward endplate. The same boards distribute high-voltage to the sense wires. 

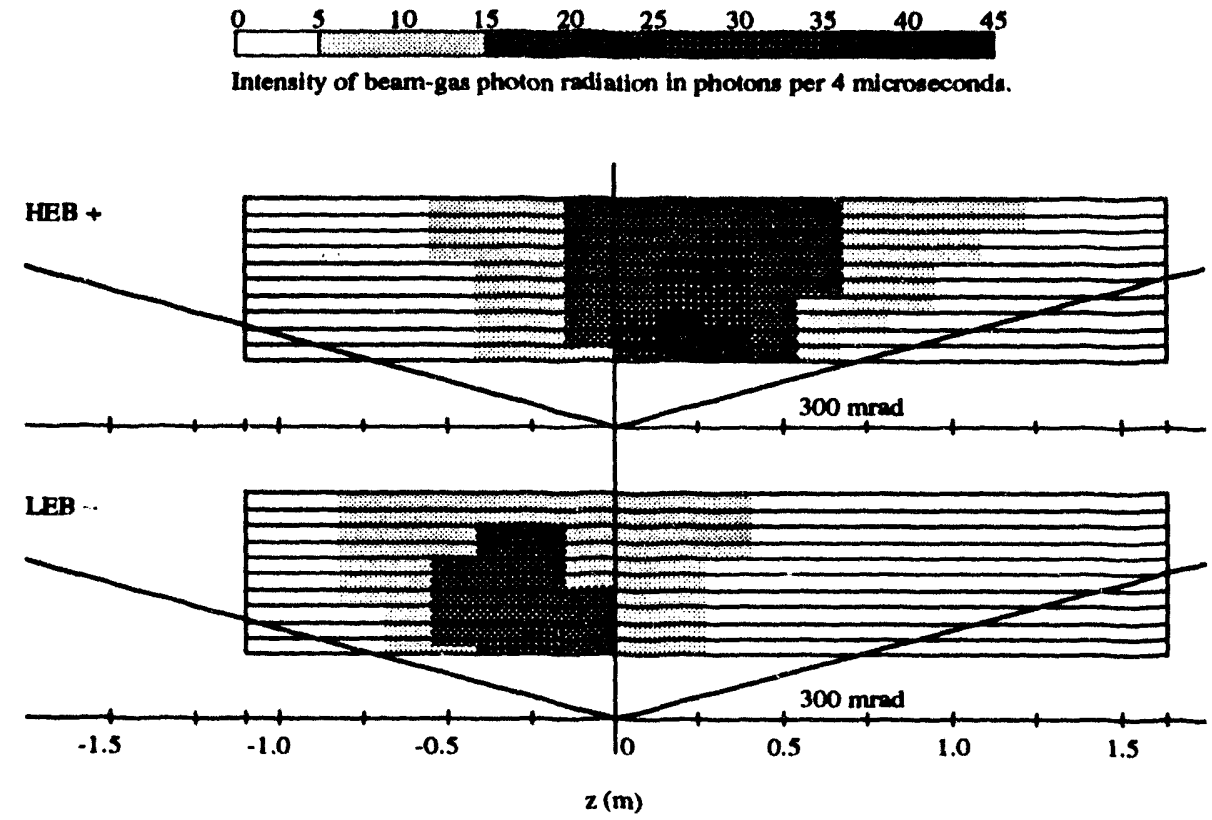

Figure 6-12. The expected background rate in the drift chamber due to converted photons from showers produced by upstream beam-gas interactions.

\subsection{High Voltage and Control System}

The sense wires are at $\sim 2 \mathrm{kV}$, the field wires at ground
An automated control system is required to monitor chamber voltages and currents, pressure, temperature, and gas properties. Commercially available electronics will be used for these functions.

The drift chamber operates with its approximately 7000 sense wires at $\sim 2 \mathrm{kV}$, while the field wires are at ground potential. Groups of sense wires are combined into $20-40$ zones, each with a separate HV supply. The HV supplies must be capable of providing about $3 \mathrm{kV}$ and $1 \mathrm{~mA}$, with controls for fast ramp-up and ramp-down. The high voltages is controlled by the online system.

The slow control system is also used to monitor the voltages and currents in the preamplifiers, using slow ADCs. There are approximately 40 low-voltage supplies. Slow ADCs are used to monitor ambient temperatures and pressures at various locations, and gas gain. Gas flow switches are controlled using parallel registers. 


\subsection{R\&D Program}

Although the overall design concept of the drift chamber is rapidly converging, many details of the actual implementation remain to be determined. Simulation and prototyping efforts underway in many of these areas, including cell geometries, orientation, and layer packing, will continue, as will the study of different pattern recognition and track reconstruction techniques. Development of a full simulation of the drift chamber is surrently underway.

A small-cell prototype chamber has been built and is being used to study electrostatic stability, feedthrough design, front-end electronics, cell crosstalk, and wire and gas properties. The prototype is $2.5 \mathrm{~m}$ in length, with 72 anode wires of $20 \mu \mathrm{m}$ diameter goldcoated tungsten, and 512 cathode wires of $55 \mu \mathrm{m}$ diameter unplated aluminum 5056 arranged into three superlayers of hexagonal celis. The center superlayer is positioned at a $50 \mathrm{mr}$ stereo angle.

Siudies of suitable drift gases are continuing with the prototype chamber using cosmic ray particles. To date, the three component gas mixture $\mathrm{He}: \mathrm{CO}_{2}: \mathrm{C}_{4} \mathrm{H}_{10}(83: 10: 7)$ has been investigated. Preliminary results are included in Figure 6-6, along with measurements from previous gas studies. Plans are underway to study other gas mixtures including $\mathrm{He}: \mathrm{C}_{3} \mathrm{H}_{8}, \mathrm{He}: \mathrm{C}_{4} \mathrm{H}_{10}$, and He:DME. The possibility of including $\mathrm{CF}_{4}$ as an additive to counter ageing will be investigated. With the availability of a test beam, a prototype chamber could be used for $d E / d x$ studies of these gas mixtures. After a gas has been chosen, the front-end electronics will be prototyped and the entire readout chain will be tested with the prototype chamber.

The candidate wire materials, aluminum, tungsten, and silicon carbide, are currently being evaluated. The prototype will be at least partially strung with gold-plated $\mathrm{SiC}$ wire. Present plans include evaluation of the successful TRIUMF crimped-pin design with silicon carbide wires. The present endplate design also requires extensive tests of composite materials and their properties, and of the accuracy of locating feedthrough holes at an angle relative to the surface or, alternatively, the redirection of the wire through the chosen stereo angle at the feedthrough. Engineering and prototyping work in this area is underway.
A $2.5 \mathrm{~m}$ long small-cell prototype chamber is currently under test

Additional low-mass gas mixtures will be studied

$\mathrm{SiC}$ wire will be studied in the prototype chamber; techniques to accurately locate drilled holes in composite materials will be studied as well 
The performance of the gas will have to be monitored continuously during operation. Studies of candidate monitoring systems will be undertaken. 


\section{References}

[Alb92] H.Albrecht et al., "HELENA: A Beauty Factory in Hamburg", DESY Report DESY PRC-92-041, 1992.

[Bia89] The MAGBOLTZ simulation code was kindly supplied by S.F. Biagi of the University of Liverpool. Some results from this code are discussed in S.F. Biagi, Nucl. Instr. Meth. A283, 716 (1989).

[Bil84] P. Billoir, Nucl. Instr. Meth. 225, 352 (1984).

[Bri93] D.Britton, E.Hyatt, "Pattern Recognition in an all Stereo Drift Chamber", BABAR Note \#102, Feb. 1993.

[Boy92] A. Boyarski, D. Briggs, P. Burchat, Nucl. Instr. Meth. A323, 267 (1992).

[Cin91] V. Cindro et al., Nucl. Instr. Meth. A309, 411 (1991).

[Inn93] W. Innes, "TRACKERR, A program for calculating tracking errors", BABAR Note \#121, Nov. 1993.

[Kad94] J. Kadyk, presentation to the BABAR Collaboration, May 21, 1994.

[Pit93] D. Pitman, C. Goodenough, "Drift Chamber Backgrounds Revisited", BaBar Note \#114, Sept. 1993.

[Pit94] D. Pitman, presentation to the BABAR Collaboration, May 1994.

[Pla92] S. Playfer et al., Nucl. Instr. Meth. A315, 494 (1992).

[Uno93] S. Uno et al., Nucl. Instr. Meth. A330, 55 (1993).

[Vav82] J. Va'vra, L. Roberts, D. Freytag and P. Clancey, Nucl. Instr. Meth. A203, 109 (1982). The code has been modified to use the number of primary ions in the calculations. 


\section{Particle Identification}

$\mathbf{E}$ xcellent particle identification for hadrons and leptons over a large momentum range is an essential design element in reaching the physics objectives of the experiment. In particular, measurements of $C P$ violation require particle identification, both to reconstruct exclusive final states and to tag the quark content of the other $B$ in the event. Information from the central tracker, calorimeter and instrumented flux return can be used to identify most particles. However, these systems are not sufficient to distinguish pions and kaons with momenta greater than approximately $0.7 \mathrm{GeV} / c$ or protons above $1.3 \mathrm{GeV} / c$. The goal of a dedicated particle identification (PID) system is to identify high momentum charged hadrons within the geometrical and cost constraints of the detector without significantly degrading the ability of the calorimeter to measure soft photons.

\subsection{Physics Requirements}

\subsubsection{Inclusive Spectra and $B$-Flavor Tagging}

The momentum spectrum of charged kaons for $B$ decays are shown in Figure 7-1; the correlation with polar angle due to the boost $(\beta \gamma=0.56)$ is evident. The relative abundances of pions, kaons and protons in inclusive $B$ decays is approximately $7: 1: 0.2$. The average $\pi(K)$ momentum depends on the polar angle, ranging from about $0.3(0.45) \mathrm{GeV} / c$ in the backward direction to about $0.75(1.1) \mathrm{GeV} / c$ in the forward direction. Because the forward region subtends a larger fraction of the center-of-mass solid angle, the forward region is both proportionally more important for acceptance and more difficult for particle identification.
A dedicated particle identification system is required to distinguish high momentum pions and kaons. The system must have minimal impact on the detection and measurement of photons consistent with good performance

Due to the boost of $\beta \gamma=0.56$, the mean momentum of hadrons is a factor of $\sim 2.5$ higher in the forward direction than the backward direction 
Since the effective efficiency of tagging for $C P$ violation studies is $\epsilon_{e f f}=\epsilon_{t a g} \times(1-2 w)^{2}$, where $w$ is the fraction of incorrectly tagged events, high purity particle identification is important

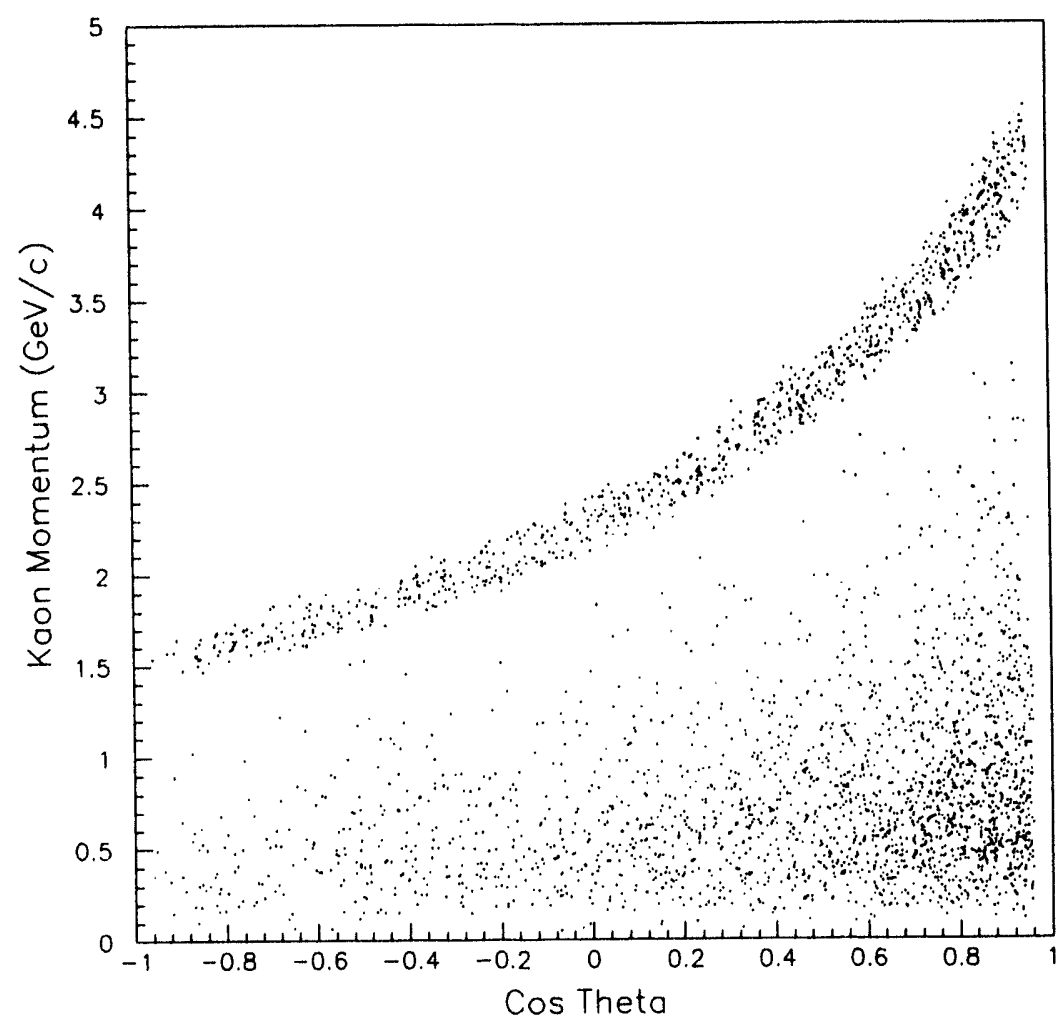

Figure 7-1. Inclusive momentum spectrum in the laboratory frame for kaons from $B$ decays as a function of polar angle. The banded region is an enhanced population of kaons from the decay $B^{0} \rightarrow \pi^{+} K^{-}$.

Charged kaons will be used to tag the flavor of $B$ mesons: $a$ quark decay leads to a $K^{-}$while a $\bar{b}$-quark decay leads to a $K^{+}$. For this purpose, identification is needed up to momenta of about $1.6 \mathrm{GeV} / c$; both high identification efficiency and high purity are required. The effective efficiency is $\epsilon_{e f f}=\epsilon_{t a g} \times(1-2 w)^{2}$, where $w$ is the fraction of incorrectly tagged events. Wrong-sign contamination from Cabibbo-suppressed decays and from kaon pairs leads to a minimum of about $10 \%$ for $w$. For good efficiency, the contamination introduced by the PID system must be small in comparison. Given the relative abundances noted above, a small misidentification rate for pions is particularly crucial. Misidentifying $1 \%$ of the pions as kaons would reduce $\epsilon_{\text {eff }}$ from $22 \%$ to $19 \%$.

In terms of the required particle identification coverage, it should be noted that only $3 \%$ of all kaons will be found in the solid angle 
subtended by the backward endcap, and most of these will have momenta less than $0.6 \mathrm{GeV} / c$. Therefore, no dedicated PID system is needed in the backward endcap; coverage will be required only between $300 \mathrm{mr}$ in the forward direction and $785 \mathrm{mr}$ in the rear.

The primary design criteria for the PID system rest on the requirements for $C P$ violation in $B$ decays. There are however many studies of inclusive and exclusive decays of charm and tau physics which would directly benefit from high-momentum hadron identification. One example is the inclusive decays of $\tau$ leptons into $\pi X$ and $K X$. In general, the study of these channels requires the separation of pions and kaons at considerably higher momenta than for $B$ tagging; indeed if the full acceptance is used, particle identification up to $8 \mathrm{GeV} / c$ is needed in the forward direction.

\subsubsection{Exclusive $B$ Decays}

High-momentum particle identification is also an inportant element in separating many exclusive final states. Examples of particularly challenging modes for the PID system are distinguishing $B^{0} \rightarrow$ $\pi^{+} \pi^{-}$from $B^{0} \rightarrow \pi^{+} K^{-}$and $B^{0} \rightarrow \rho^{ \pm} \pi^{\mp}$ from $B^{0} \rightarrow \rho^{ \pm} K^{\mp}$. The banded area in Figure 7-1 illustrates the momentum range populated by kaons in the mode $B^{0} \rightarrow \pi^{+} K^{-}$. A clean sample of $B^{0} \rightarrow \pi^{+} \pi^{-}$decays is critical to the measurement of $\mathrm{V}_{u b}$ and, if the other $B$ is tagged, of $C P$ violation; the $B^{0} \rightarrow \pi^{+} K^{-}$mode is of interest in its own right, since the decay mechanism is a penguin diagram which is sensitive to the presence of heavy particles in the loop. All these processes are rare, with branching ratios of the order of $10^{-5}$, and their relative rates are unknown. The $\pi^{+} \pi^{-}$final state produces pions ranging from $1.5 \mathrm{GeV} / c$ to $4.5 \mathrm{GeV} / c$ in the laboratory frame, with $50 \%$ having a polar angle above $60^{\circ}$.

\subsubsection{Summary of Requirements}

For exclusive channels, kinematic separation is not likely to be sufficient to distinguish different final states. Some additional separation can be obtained using the $d E / d x$ measurements provided by the central tracker, except for momenta near $1 \mathrm{GeV} / c$. A very
No particle identification system beyond $d E / d x$ is required in the rear endcap region

Charmed meson and $\tau$ lepton studies also benefit from hadron identification

$B^{0} \rightarrow \pi^{+} \pi^{-}$is a benchmark decay mode producing highmomentum hadrons 
A Cherenkov-based PID system is needed to provide the required performance $d E / d x$ gives excellent scparation at low momentum, but is marginal elsewhere good TOF counter system could be adequate for $K$-tagging of $B$ decays. However, the combination of $d E / d x$ and TOF is unlikely to provide the redundancy and robustness desirable for the high rates and potentially large backgrounds at PEP-II. More importantly, such a system would not match the full range of physics requirements noted above. The development of a dedicated PID device based on Cherenkov techniques is considered the most likely route to achieving the required performance. Options under development at this time include aerogel threshold counters and ringimaging devices. The system that is finally chosen must provide high-momentum hadron separation with fast time response, the lowest possible mass and robust operation. These requirements represent a large extrapolation from existing techniques and their implementations. At the moment there is no fully-proven solution, although intensive research and development is underway on several promising alternatives.

\subsection{Options for Particle Identification}

\subsection{1 $d E / d x$ and Kinematic Separation}

Ionization information $(d E / d x)$ will be available from the main tracking chamber, with as many as 40 samples per track (see Chapter 6$)$. In the low momentum $\left(1 / \beta^{2}\right)$ region, this will provide excellent separation of all species; for momenta above about $2 \mathrm{GeV} / c$ there also may be $\pi$ and $K$ separation at the 1.5-2.0 $\sigma$ level from the relativistic rise. Simulations predict a resolution of $6.9 \%$ for 40 samples with a relativistic rise factor of 1.43 from minimum to plateau for a 80:20 He:isobutane mixture. Tests are required to demonstrate that these predictions can actually be realized with the final gas choice and cell design adopted for the central tracker.

On the basis of the predicted resolution, pions and kaons are distinguished at about the $1.5 \sigma$ level for normal incidence outside the momentum range 0.8 to $2.0 \mathrm{GeV} / c$ (Figure $7-2$ ). This region of ambiguity is somewhat less of a problem fo- $K / p$ identification where the $\left(1 / \beta^{2}\right)$ region extends to considerably larger momenta. In addition to considerations for $B$-tagging, the region of ambiguity is also rather serious for modest multiplicity decay modes. For 


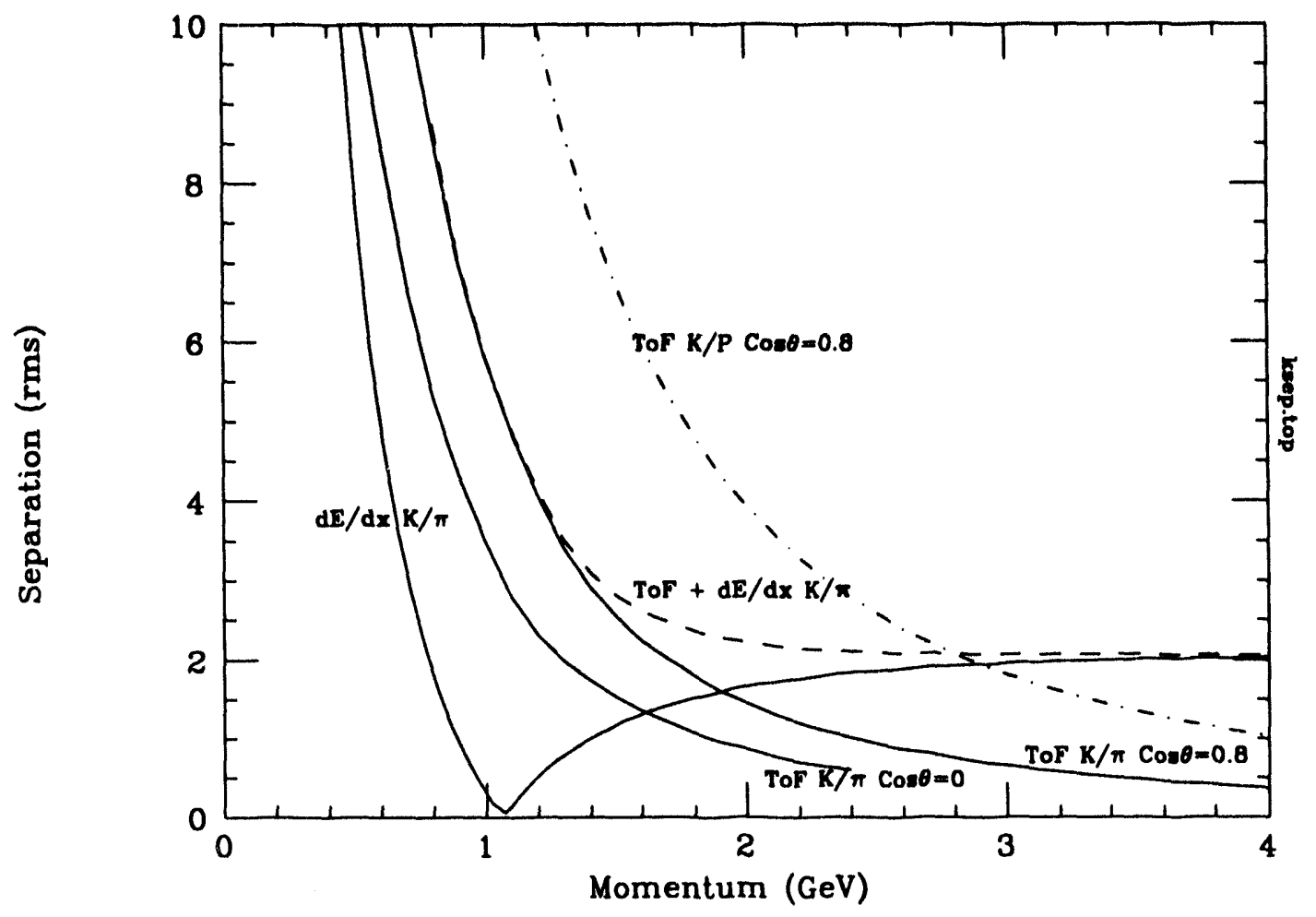

Figure 7-2. $K / \pi$ separation in $\mathrm{rms}$ as a function of momentum for time-of-fight system alone at $\cos \theta=0$ and 0.8 (solid lines) and $K /$ proton separation at $\cos \theta=0.8$ (dot-dash). Also shown is the $K / \pi$ separation for $d E / d x$ alone and the quadratic sum of the $d E / d x$ curve and the $\cos \theta=0.8$ TOF curve.

example, in the decay $B^{0} \rightarrow D^{+} D^{-}$where $D^{+} \rightarrow K^{-} \pi^{+} \pi^{+}, 45 \%$ of the tracks have less than $1.6 \sigma$ separation and $60 \%$ have less than $3 \sigma$. Coverage of the $\pi / K$ overlap region for kaon tagging alone requires that a separate PID system be included as part of the detector.

If the design values for momentum and $d E / d x$ resolutions can be achieved in practice, the combination of kinematic separation and $d E / d x$ could be used for distinguishing some exclusive final states, e.g. $B^{0} \rightarrow \pi^{+} \pi^{-}$and $B^{0} \rightarrow \pi^{+} K^{-}$, assuming comparable branching ratios. Using Gaussian resolution functions, the difference, $\Delta \chi^{2}$, between the $\pi^{+} \pi^{-}$hypothesis and either of $K^{+} \pi^{-}$or $\pi^{+} K^{-}$alternatives has been calculated for the decay $B^{0} \rightarrow \pi^{+} \pi^{-}$. Two cases have been considered, with two different radial requirements for the central tracker reflecting the different radial requirements of the Fast RICH, 
aerogel and DIRC systems. Both scenarios have the calorimeter starting at a radius of $105 \mathrm{~cm}$ in a magnetic field of $1.5 \mathrm{~T}$.

\begin{tabular}{|c|ccc|ccc|}
\hline \multirow{2}{*}{ Information used } & \multicolumn{3}{|c|}{$\mathrm{R}_{\max }^{C D C}=80 \mathrm{~cm}$} & \multicolumn{3}{|c|}{$\mathrm{R}_{\max }^{C D C}=96 \mathrm{~cm}$} \\
\cline { 2 - 7 } & $\Delta \chi^{2}=4$ & 10 & 16 & $\Delta \chi^{2}=4$ & 10 & 16 \\
\hline \hline Kinematics only & 39 & 5 & 0 & 69 & 36 & 7 \\
$d E / d x$ only & 34 & 6 & 0 & 44 & 11 & 0 \\
Kinematics $+d E / d x$ & 72 & 59 & 9 & 89 & 72 & 40 \\
\hline$\pi^{+} \pi^{-}$mass resolution & \multicolumn{2}{|c|}{$21.5 \mathrm{MeV} / c^{2}$} & \multicolumn{2}{|c|}{$14.6 \mathrm{MeV} / c^{2}$} \\
\hline
\end{tabular}

Table 7-1. Probability (\%) for accepting the correct assignment in the decay $B^{0} \rightarrow \pi^{+} \pi^{-}$as a function of the $\chi^{2}$ difference between the $\pi^{+} \pi^{-}, K^{+} \pi^{-}$and $\pi^{+} K^{-}$hypotheses using kinematics and $d E / d x$. Gaussian errors, nominal means and resolutions assumed.

A realistic analysis would require some reasonable separation, possibly 2-4 standard deviations, depending on the background rejection requirements. This would also remove sensitivity to systematic errors, including spatial variation, in the mean values of PID system responses to different particles, or to non-Gaussian tails in the resolution functions. In a common implementation, unique PID assignments would be made if the relative likelihoods for all competing hypotheses were less than some reasonable value, e.g. $5 \%$, corresponding roughly to $\Delta \chi^{2} \simeq 6$.

As shown in Table 7-1, this kind of approach would lead to acceptances of between $60 \%$ and $80 \%$ (depending on chamber radius) for the combination of kinematics and $d E / d x$; the acceptance would fall precipitously for a more stringent requirement on hypothesis separation. A conversion of the $\Delta \chi^{2}$ difference to a separation probability would require a detailed understanding of the PID system response functions. Thus, the combination of kinematic separation and $d E / d x$ information appears to be marginally effective for this particular channel. Further study is required to address questions such as a corresponding prediction for misidentification rates, as well as the impact of overlaps, possible saturation effects in $d E / d x$ at large angles, or imperfections in calibration. For most channels, such as $B^{0} \rightarrow \rho^{ \pm} \pi^{\mp}$, which involve a neutral pion in the decay chain, kinematic separation would not be nearly as effective. 


\subsubsection{Time-of-Flight Counter System}

A time-of-flight (TOF) system would be a relatively simple choice for a particle identification system. Such a system could be assembled using new "fine mesh" photomultiplier tubes (PMTs) and fast plastic scintillators. These PMTs may be able to operate with good time resolution inside the $1.5 \mathrm{~T}$ magnetic field of the detector, which would allow them to be mounted directly on the plastic scintillation counters without the need for light guides. This geometry provides excellent time resolution, as has been demonstrated by beam tests at KEK. Resolutions of $\leq 60 \mathrm{ps}$ have been achieved in these studies for geometries similar to those required here. Allowance for additional time jitter from the bunch length and electronics results in an expected system resolution of about $80 \mathrm{ps}$.

Assuming that a system resolution of 100 ps could be achieved, such a TOF system would be able to distinguish pions from kaons at the $3 \sigma$ level up to a momentum of at least $1.1-1.3 \mathrm{GeV} / c$, depending upon the polar angle (Figure 7-2). This is inadequate for high momentum hadron identification, but simulations show that kaons would be useful for tagging in at least $80 \%$ of events having tracks within the fiducial volume. Thus, TOF is considered to be a reasonable backup choice as a PID system which satisfies most of the tagging requirements; it may also be considered as part of a hybrid system in combination with aerogel threshold counters. Further development will be pursued only in this context, while we continue to evaluate the potential of the primary Cherenkov-based alternatives.

\section{Conceptual Design}

The geometric coverage of a TOF system would be divided into two regions: a barrel section between $(-0.75<\cos \theta<0.85)$ and a forward endcap over the region $(0.85<\cos \theta<0.96)$. In the barrel region, there would be about 90 counters of $3 \mathrm{~m}$ length, $5 \mathrm{~cm}$ thickness, and about $6.6 \mathrm{~cm}$ width. The exact segmentation would require a careful examination of occupancy and background issues, as well as timing performance. The amount of scintillator material (PVT) in the TOF system corresponds to about $12 \%$ of a radiation length at normal incidence. As the scintillator is fully active, it may
A TOF system can provide tagging, but not high momentum particle identification

A conceptual design of a TOF system is being developed as a backup 
A pipelined system with time resolution adequate for TOF has not been designed be possible to correct for the energy loss due to conversions in the counter material so as not to degrade significantly the calorimeter resolution. In the endcap region, three designs are being considered; a reference layout consists of 16 sectors, each with five scintillators $5 \mathrm{~cm}$ thick and about $10 \mathrm{~cm}$ wide, with the long dimension lying along the azimuthal direction. Each endcap scintillator is read out with two PMTs attached by a beveled mirror to allow the alignment of the tube axes at the appropriate angle to the magnetic field. Alternative designs use fewer PMTs, but may not have adequate timing resolution.

Optimization of the counter designs, including the scintillator thickness and width, would be based upon an existing Monte Carlo program. Included in this program are light flux, decay and rise times for the scintillator, the PMT time response, light propagation, effect of finite beam bunch size, PMT jitter, and effect of the TDC threshold. This program has given a predicted resolution for the KEK counters which agrees very well with results from beam tests.

The electronics would consist of both a TDC and an ADC for each PMT. With an ADC in the TOF readout electronics, an energy loss measurement would be obtained. The ADC measurement would also be useful to determine whether or not there are single hits in the counter, and to compensate the calorimeter measurement for the energy losses in the TOF counter. The fast TOF signal may be useful in the trigger system. A method for pipelining the TOF information with adequate time resolution is not in hand at this time. A monitoring system would be needed to ensure long-term stability of the time and $d E / d x$ resolutions. This could be supplied by a nitrogen laser system using fiber optics.

\section{Proof-of-Principle and R \& D Plans}

Much preliminary investigation of the possible TOF systems has already been accomplished by the KEK group, including important bcam test results. Their studies also included the effects of a magnetic field of up to $1.2 \mathrm{~T}$. Although requiring confirmation, these measurements and a long history of TOF systems in general, constitute a basic "proof-of-principle" for this option. Plans are underway to perform cosmic ray and beam tests with a fine mesh PMT and a scintillation counter with dimensions appropriate to 
the barrel in order to understand the performance in detail and to confirm the basic features of the angular and length dependence of the timing resolution at $1.5 \mathrm{~T}$.

\subsubsection{Dedicated Cherenkov Devices}

As demonstrated in Table 7-1 above, kinematic separation in combination with $d E / d x$ is of marginal benefit in rejecting correlated backgrounds to $B^{0} \rightarrow \pi^{+} \pi^{-}$; for $B^{0} \rightarrow \rho^{ \pm} \pi^{\mp}$ it is considerably less effective. This limitation holds generally for inclusive and exclusive decays involving hadrons with momenta above about $1.5 \mathrm{GeV} / c$; as such it represents a powerful argument for including in the detector baseline design a dedicated PID system based on a Cherenkov technique. Three options are under active consideration at this time:

ATC Aerogel Cherenkov counters with two indices of refraction chosen to allow $\pi / K$ separation up to $4.2 \mathrm{GeV} / c$; read out with fine mesh phototubes, micro-channel plates or Hybrid APDs;

DIRC An internal reflection imaging device using quartz bars to generate a Cherenkov image observed by an external array of phototubes;

Fast RICH A ring-imaging system using a freon radiator and a solid CsI photocathode in a fast proportional counter.

Technical descriptions, R\&D progress, and predicted performance for these systems are given in the succeeding sections. However, the improved separation in $B^{0} \rightarrow \pi^{+} \pi^{-}$brought about by such dedicated systems is instructive. Shown in Table 7-2 are the acceptances for identification of $B^{0} \rightarrow \pi^{+} \pi^{-}$after requiring $\Delta \chi^{2}$ to range from 4 to 16. Kinematic and $d E / d x$ errors are assumed to be Gaussian; for the dedicated PID systems, the number of photoelectrons is chosen from a Poisson distribution with either Gaussian measurement errors (Fast RICH and DIRC) or an exponential model of phototube responses (ATC). Misidentification rates are much more difficult to calculate, and require further detailed study. Dedicated Cherenkov systems can be seen to improve identification acceptances to the range of $84-96 \%$ for $\Delta \chi^{2}=10$; the performance of such systems is 
also much more robust with respect to the exact value chosen for this cutoff. Thus, even with this rather simple model which neglects complications of systematic problems and realistic resolution functions, dedicated PID systems can be seen to be an essential addition to the physics performance of the detector.

\begin{tabular}{|c|ccc|ccc|}
\hline & \multicolumn{3}{|c|}{$\mathrm{R}_{\max }^{C D C}=80 \mathrm{~cm}$} & \multicolumn{4}{c|}{$\mathrm{R}_{\max }^{C D C}=96 \mathrm{~cm}$} \\
\cline { 2 - 7 } Information used & $\Delta \chi^{2}=4$ & 10 & 16 & $\Delta \chi^{2}=4$ & 10 & 16 \\
\hline \hline Kinematics, $d E / d x+$ ATC & 97 & 89 & 72 & & & \\
Kinematics, $d E / d x+$ DIRC & 99 & 96 & 90 & 99 & 98 & 94 \\
Kinematics, $d E / d x+$ RICH & 95 & 84 & 66 & & & \\
\hline
\end{tabular}

Table 7-2. Probability (\%) for accepting the correct assignment in the decay $B^{0} \rightarrow \pi^{+} \pi^{-}$as a function of the $\chi^{2}$ difference between the $\pi^{+} \pi^{-}, K^{+} \pi^{-}$and $\pi^{+} K^{-}$hypotheses using kinematics, $d E / d x$ and a dedicated Cherenkov device. In the case of the DIRC, the solid angle coverage in the forward endcap is covered with a Fast RICH device.

\subsection{Aerogel Threshold Counters}

An aerogel system provides $\pi / K$ separation over the full momentum range
Aerogel Threshold Cherenkov (ATC) counters based on low-density silica aerogel may provide an attractive low-mass PID system. Such a detector could cover both barrel and endcap regions without major breaks or discontinuities. The expected performance does not appear to be sensitive to the angle of incidence, although some consideration may be required to the relative orientation of the photodetection system and the detector magnetic field. The system adds $\leq 8 \%$ of a radiation length in front of the calorimeter, except when a photodetector is struck. Together with $d E / d x$ measurements in the drift chamber, an aerogel PID system with refractive indices of $\mathrm{n} \simeq 1.008$ and $\mathrm{n} \simeq 1.06$ could provide $\pi / K$ separation over a momentum range up to $\simeq 4.2 \mathrm{GeV} / c$ (Figure $7-3$ ) as required for $C P$ violation studies in the PEP-II environment. $K / p$ identification would not be possible below $1.5 \mathrm{GeV} / c$ and again between 2.9 $4.3 \mathrm{GeV} / c$, leaving a gap in coverage for $B$-tagging from the end of the $\left(1 / \beta^{2}\right)$ region in the $d E / d x$ measurement. 

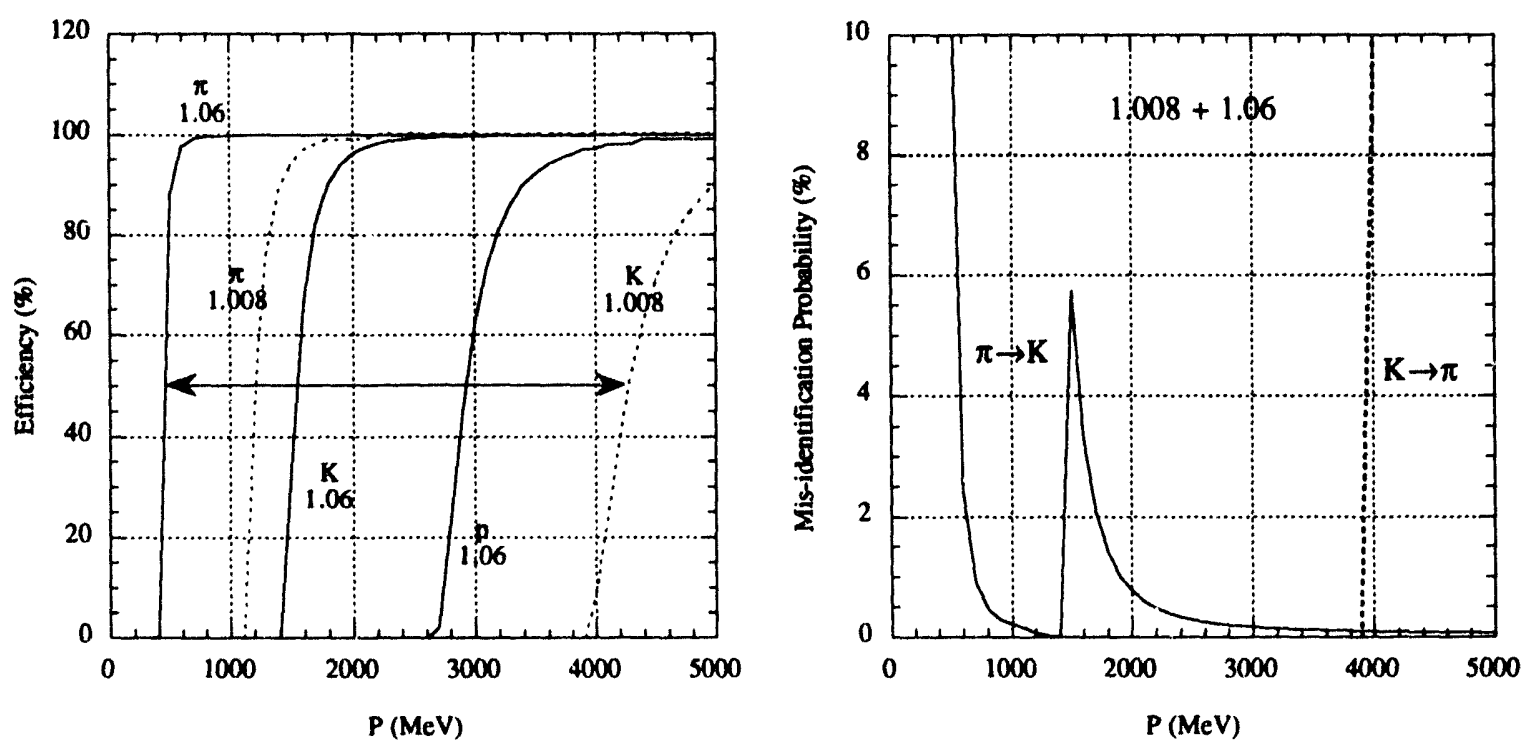

Figure 7-3. Calculation of the threshold efficiencies and misidentification probability for an ATC system with layers of $\mathrm{n}=1.008$ and $\mathrm{n}=1.06$, assuming 10 photoelectrons in each aerogel block.

The passage of a particle of charge $Z$ and velocity $\beta$ through a dielectric medium of thickness $d x$ with refractive index $n$ can produce photons according to the Cherenkov formula:

$$
\frac{d^{2} N}{d x d \lambda}=\frac{2 \pi}{\lambda^{2}} \alpha_{e m} Z^{2}\left(1-\frac{1}{n^{2} \beta^{2}}\right)
$$

The number of Cherenkov photons in the wavelengtu region $\lambda=$ 200-800 nm produced by a $Z=1$ and $\beta=1$ charged particle traversing $10 \mathrm{~cm}$ of $\mathrm{n}=1.008$ aerogel is thus about 270 . The key issue of the aerogel PID system is efficient collection of these Cherenkov photons. Several criteria need to be met: (1) the aerogel itself must have a long optical absorption length; $(2)$ the material used to wrap the aerogel must have high reflectance; (3) the photon detector must have a high quantum efficiency which matches the output photon spectrum.

In order to collect Cherenkov photons efficiently inside the solenoid, the photon detector must work in magnetic fields up to $1.5 \mathrm{~T}$, have a large sensitive area with high quantum efficiency extending as far as possible into the UV region, and have low noise. At the present 
Photomultipliers using both fine mesh and microchannel plate dynode structures are being considered time, consideration is being given to fine mesh photomultiplier tubes (FM PMT) from Hamamatsu and newly developed GaAsP microchannel plate photomultiplier tubes (MCP PMT) from Intevac. The FM PMT has a conventional bialkali photocathode; the MCP PMT has quantum efficiencies of $\simeq 10 \%$ at $\lambda=350 \mathrm{~nm}$ and $\simeq 30 \%$ at $\lambda=500-600 \mathrm{~nm}$, providing a better average efficiency for Cherenkov light. Hybrid photodiodes are a third alternative. The fast timing response of all these devices helps background rejection.

\subsubsection{Conceptual Design}

Two options are being considered for the ATC system layout. Figure 7-4 illustrates a two-layer design of an aerogel PID system, while Figure 7-5 depicts a two super-layer (multi-layer) design.
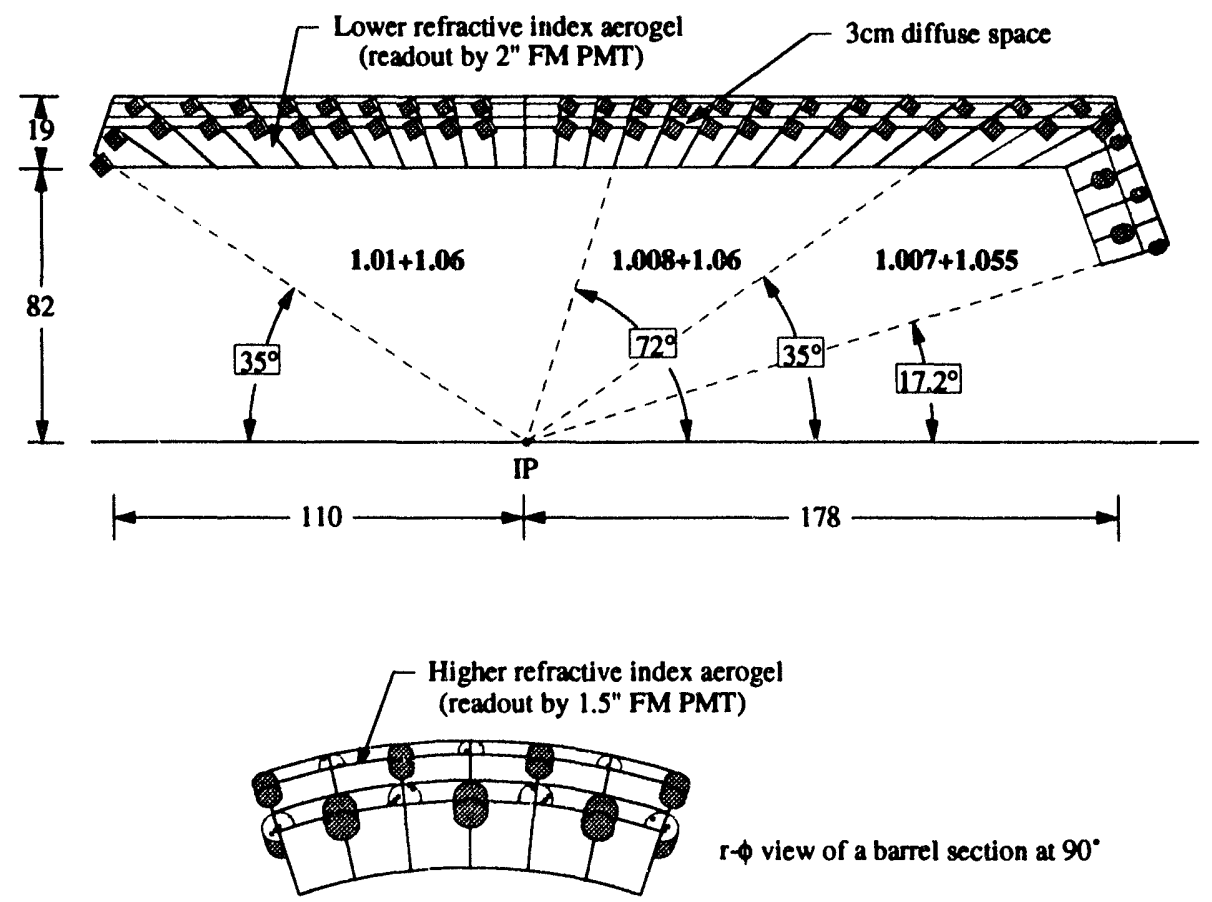

Figure 7-4. Conceptual design of a two-layer aerogel PID system with graded refractive indices to optimize $\pi / K$ separation vs. polar angle. 


\section{Errata for SLAC Report 443}

On page iii under Budker Institute of Nuclear Physics, E.A. Undrus should appear as A.E. Undrus.

On page vii under Stanford Linear Accelerator Center, J.L. Hewitt should appear as J.L. Hewett.

On page viii, L. Bosisio should be attributed to INFN, Sezione di Trieste and Università di Trie: 'e, Trieste, Italy.

In Figure 6-1 on page 95, the dashed and the dot-dashed lines are interchanged for the plot showing acceptance as a function of $\cos \theta_{\text {max }}$.

In Table 7-1 on page 124, the column labeled $\Delta \chi^{2}=10$ should read $\Delta \chi^{2}=9$.

In Table 13-7, the reconstructable fraction for the $J / \psi K_{S}^{0}$ channel should read 0.124 . 


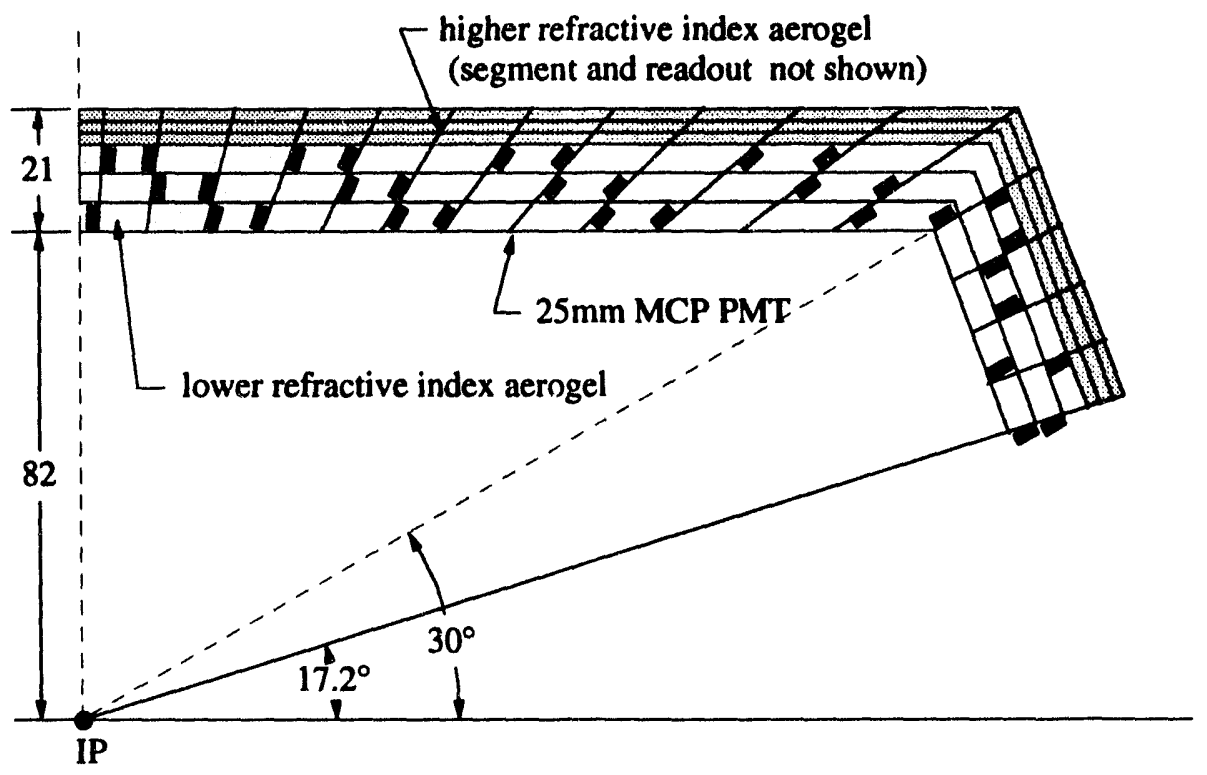

Figure 7-5. Conceptual design of a multi-layer aerogel PID system.

\section{Two-Layer Design}

The two-layer design employs about $\sim 3380$ aerogel blocks. In both barrel and forward endcap regions, aerogel cells with lower and higher refractive indices are appropriately tapered to provide projective geometry, with the lower refractive index cell being placed in front of the higher refractive index cell. The indices of refraction are varied with polar angle to optimize performance. Cell size at $90^{\circ}$ is $\simeq 10 \times 10 \mathrm{~cm}$; cell depths are $\simeq 10+3 \mathrm{~cm}$ (aerogel + diffusion space) for the lower refractive index aerogel and $\simeq 4+2 \mathrm{~cm}$ (aerogel + diffusion - space) for the higher refractive index aerogel, respectively. The diffusion space in both cells improves the photon collection efficiency, and reduces $\delta$-ray and calorimeter back-splash background. The $\delta$-ray contamination in the lower refractive index aerogel is reduced by placing the ATC about $2 \mathrm{~cm}$ away from the drift chamber.

The aerogel blocks are wrapped in water-resistant PTFE teflon and housed in light-tight containers made of a low-density material, such as aluminized mylar. The PTFE teflon is a highly efficient diffuse reflector, having a reflectance of about $95 \%$ over a wavelength region of $300-700 \mathrm{~nm}$. The possibility of coating the PTFE with
Aerogel blocks of two indices of refractive $(\sim 3380$ in total) are arranged in projective geometry

The blocks are wrapped in a PTFE diffuse reflector 
The superlayer design has a total of six thinner layers of aerogel, with larger transverse dimensions
There are a number of suppliers of high quality aerogel with low refractive index wavelength shifter (WLS) to increase photon collection efficiency is under study.

Each aerogel block is read out by two photon detectors embedded in neighboring cells. This arrangement allows for the observation of an adequate number of Cherenkov photons for an affordable cost. The redundant readout allows $\geq 99 \%$ detection efficiency, even in the case in which a charged particle passes through one of the photon sensors. The lower refractive index aerogel is read out by either $2^{\prime \prime}$ FM PMTs or the newly developed $25 \mathrm{~mm}$ MCP PMTs, while the higher refractive index aerogel is read out by $1.5^{\prime \prime}$ FM PMTs or the MCP PMTs.

\section{Super-Layer Design}

The multi-layer option has two projective superlayers. The lower refractive index superlayer consists of three layers of $4.6 \mathrm{~cm}$ aerogel counters, while the higher refractive index superlayer consists of three layers of $2.0 \mathrm{~cm}$ aerogel counters (Figure 7-5). The transverse dimensions of an aerogel block at $90^{\circ}$ is about $35 \times 20 \mathrm{~cm}^{2}$. Each aerogel cell has a WLS plate embedded and is read out by four photon detectors. The aerogel blocks and photon detectors are arranged in such a way that a particle from the interaction point crosses at least two aerogel counters of the same index of refraction without striking photon detectors. If a coincidence between two counters is required, this arrangement can substantially suppress background due to $\delta$-ray production and calorimeter back-splash, at the expense of detection efficiency.

\section{Detector Components}

Aerogel with good optical quality can be produced by Airglass, Lockheed, Jet Propulsion Laboratory (JPL), Lawrence Livermore National Laboratory (LLNL), Aerojet and the Institute of Catalysis in Novosibirsk. Caltech's Space Radiation Laboratory regularly uses large aerogel blocks from Airglass in balloon-based Cherenkov detectors. Lockheed's production facility can, in principle, produce enough high quality aerogel for the $B A B A R$ detector in 10 months [Men94]. The aerogel produced by JPL is hydrophobic and has good optical transmittance [Tso94]. A new two-step process technique 
for aerogel production has been developed by LLNL; the process is being transferred to a commercial company, Aerojet [Hru94].

The diffuse reflector for the aerogel wrapping must be of low density, highly reflective and stable over a long period. Candidate materials are PTFE teflon, millipore paper, and Kodak paint. All are hydrophobic and commercially available.

The characteristics of FM PMTs, MCP PMTs and hybrid photodiodes (HPDs) in magnetic fields have been extensively studied at KEK, DESY [Kic93, Suz86], and at CERN [Des94]. Hamamatsu FM PMTs have been widely used in high energy experiments [Gil88] for many years. Novosibirsk Institute of Semiconductors has successfully produced an InGaAsP MCP PMT with a quantum efficiency of $\sim 50 \%$ at $\lambda=600 \mathrm{~nm}$ [Alp94]; Intevac's GaAsP MCP PMTs, used for night vision, have $\sim 50 \%$ quantum efficiency in the visible region [Edg92]. Recent studies show that the FM PMT can provide a gain of $4 \times 10^{5}$ at $1.5 \mathrm{~T}$ if tilted by $45^{\circ}$ with respect to the field [Kic93, Suz86, Ham94].

\section{Calibration and Mechanical Support}

An embedded blue LED or fiber optics driven by a flasher will provide pulsed light to each aerogel block for on-line electronics test and calibration. During data-taking, Bhabha events, dimuon events and pions from $K_{S}^{0}$ decay will be utilized for calibration and to monitor the performance of the system.

Mechanical engineering for a conceptual design is under way. The ATC system is very light; the only significant weight is in the photon detectors, each of which weighs $100-200 \mathrm{~g}$. Each aerogel layer is surmounted by a light multi-layer PC board that supports the readout photon detectors, and distributes high voltage to the PMTs and pre-amplified signals to ADC's outside the detector. A similar structure, in planar geometry, is also appropriate for the endcap.
There are a number of high gain photosensor candidates 


\begin{tabular}{|llcccc|}
\hline Index & $\begin{array}{l}\text { Size } \\
\mathrm{cm}^{3}\end{array}$ & $\begin{array}{c}\text { Readout } \\
\text { Device }\end{array}$ & $\begin{array}{c}\text { Photon Yield } \\
\text { Cosmic Ray }\end{array}$ & $\begin{array}{c}\text { Monte Carlo } \\
\text { Prediction }\end{array}$ & $\begin{array}{c}\text { Photon Yield } \\
\beta=1\end{array}$ \\
\hline \hline 1.067 & $(4.3) \times 4.5 \times 4.5$ & $\begin{array}{c}\text { PMT } \\
45 \mathrm{~mm}\end{array}$ & $34.3 p e$ & $37.4 p e$ & $11 p e / \mathrm{cm}$ \\
& & & & \\
\hline 1.067 & $(2.2) \times 4.5 \times 4.5$ & $\begin{array}{c}\text { PMT } \\
25 \mathrm{~mm}\end{array}$ & $15.6 p e$ & $14.6 p e$ & $10 p e / \mathrm{cm}$ \\
& & & & \\
\hline 1.032 & $(8) \times 6 \times 3$ & $\begin{array}{c}\text { PMT } \\
2^{\prime \prime}\end{array}$ & $14.8 p e$ & $15.7 p e$ & $2.6 p e / \mathrm{cm}$ \\
& & & & \\
\hline 1.008 & $(\mathrm{~d}=4.4), 5.8$ & $\begin{array}{c}\text { PMT } \\
44 \mathrm{~mm}\end{array}$ & $4.4 p e$ & $4.8 p e$ & $1.4 p e / \mathrm{cm}$ \\
\hline
\end{tabular}

Table 7-3. Aerogel photon yield measurements. The numbers in parentheses indicate the aerogel thickness traversed by the cosmic ray muon. The predicted photon yields for $\beta=1$ charged particles have been corrected for the cosmic-ray muon inefficiency.

\subsubsection{Expected Physics Performance}

Experimental results on light collection in lowdensity aerogels are well reproduced by Monte Carlo calculations
An aerogel system providing 10 photoelectrons per block meets the required particle identification requirements
A series of photon yield measurements have been performed with small blocks of low-density aerogel. Photoelectrons have been successfully detected with a $2^{\prime \prime}$ bialkali PMT (RCA C31000M) from Cherenkov radiation in $\mathrm{n}=1.067,1.032$ and 1.008 silica aerogels. The observed results listed in Table 7-3 are well reproduced by a detailed Monte Carlo simulation of the response of individual aerogel blocks. A signal of 6 photoelectrons ( $p e$ ) shown in Figure 7-6 has been obtained in a recent measurement using cosmic ray muons with a $9 \times 9 \times 7 \mathrm{~cm}^{3}$ (formed by seven $9 \times 9 \times 1 \mathrm{~cm}^{3}$ pieces) aerogel block $(\mathrm{n}=1.0095)$ provided by JPL. On average, cosmic ray muons in low-density aerogel give about $70 \%$ of the Cherenkov photon yield that would be predicted for $\beta=1$ particles. While these studies look promising, full-size monolithic aerogel blocks have yet to be tested. In addition, FM PMTs have not yet been used for the readout nor have the effects of a $1.5 \mathrm{~T}$ detector magnetic field been investigated.

For the two-layer design, the detailed Monte Carlo shows that a $\beta=1$ charged particle passing through the aerogel should produce a signal with $\geq 10$ photoelectrons in both lower- and higher-refractive index aerogel blocks. Studies based on the $10 \mathrm{pe}$ signal [Oya94] indicate that the aerogel PID system will give $\pi / K$ separation up to $\simeq 4.2 \mathrm{GeV} / c$ and $K / p$ separation between $1.5 \mathrm{GeV} / c$ and $2.9 \mathrm{GeV} / c$, 


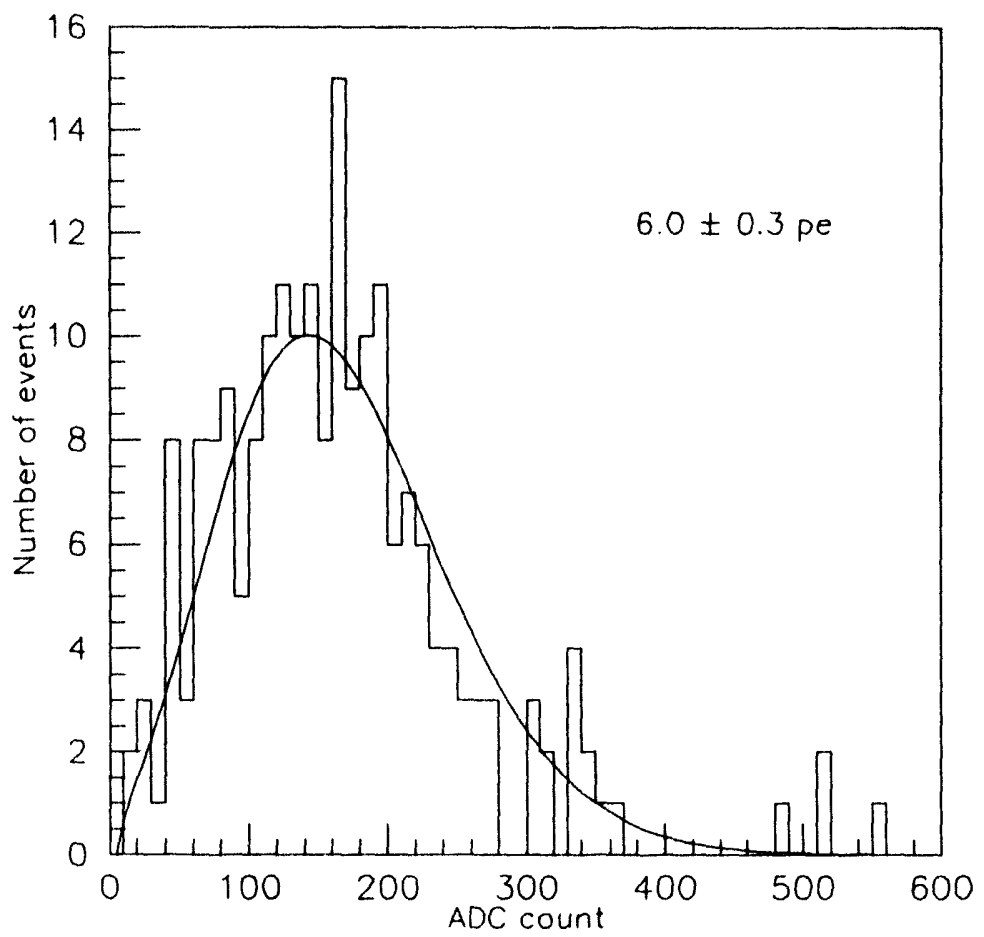

Figure 7-6. Recent measurement of photoelectron yield in a $9 \times 9 \times 7 \mathrm{~cm}^{3}$ aerogel block with $\mathrm{n}=1.0095$.

as shown on Figure 7-7a. As an example of the physics impact of such a system, c simple statistical example shows that an aerogel PID system could provide identification of $B^{0} \rightarrow \pi^{+} \pi^{-}$and $B^{0} \rightarrow$ $\pi^{+} K^{-}$at a confidence level $\sim 99 \%$ over $95 \%$ of the tracking volume, as shown in Figure 7-7b. In the transition region $(1.5<p<$ $2.0 \mathrm{GeV} / c$ ), where pions have not reached full efficiency in $n=1.008$ aerogel while kaons can already produce Cherenkov radiation in the $\mathrm{n}=1.06$ aerogel, the system may misidentify a pion as a kaon. The misidentification probability in this transition region starts at about $5.7 \%$ at $1.5 \mathrm{GeV} / c$ and decreases exponentially to $0.8 \%$ at $2.0 \mathrm{GeV} / c$. This results in a total inefficiency $\ll 1 \%$ for detecting $B^{0} \rightarrow \pi^{+} \pi^{-}$.

For the multi-layer design, the Monte Carlo simulation predicts that the passage of a $\beta=1$ charged particle yields 2.8 photoelectrons in each layer. This leads to a $99 \%$ detection efficiency if one takes the sum of two same refractive index aerogel layers and a $88 \%$ detection efficiency if one requires a coincidence of two layers.
The multilayer design has lower efficiency, but fewer problems with charged particles passing through photodetectors 


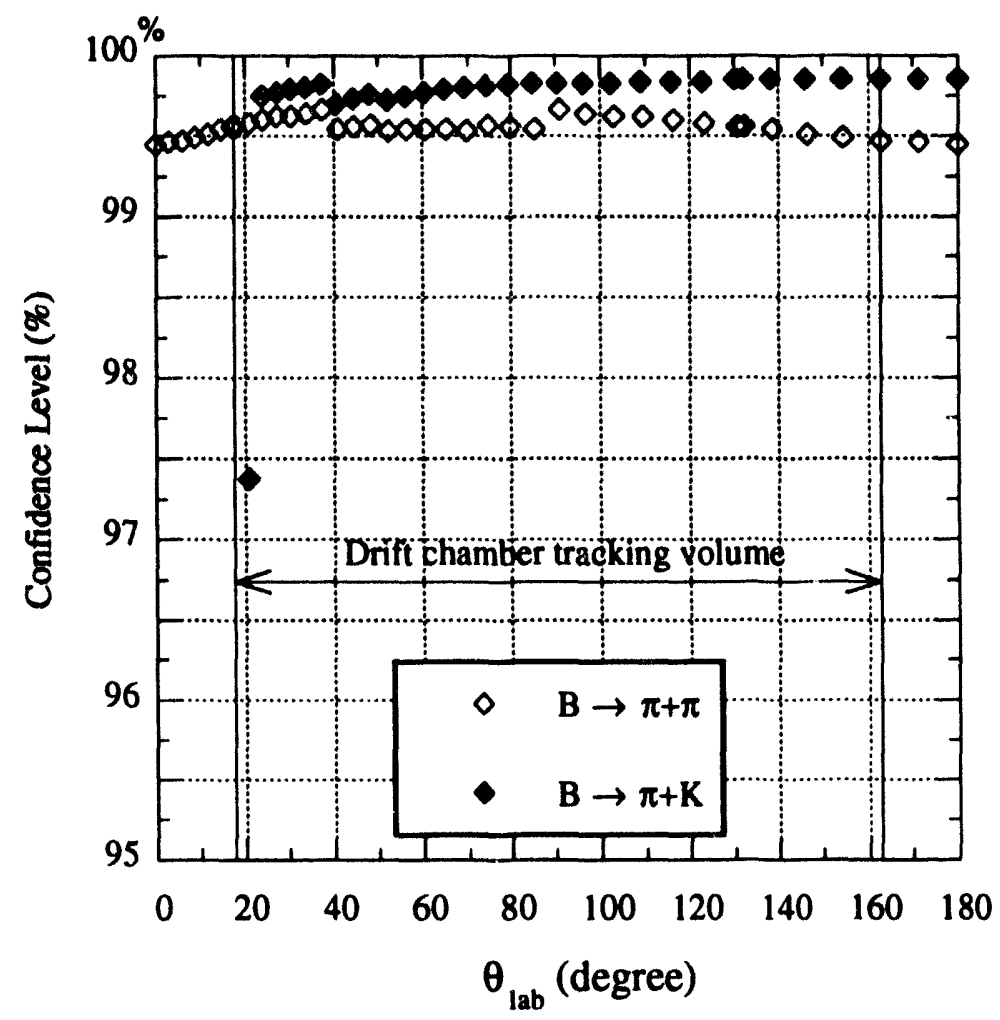

Figure 7-7. Calculated efficiencies of identifying the $\pi^{+} \pi^{-}$and $\pi^{+} K^{-}$decay modes.

Each cell is readout by at least two phototubes

$\delta$-ray backgrounds have been simulated in detail
Since each cell is read out by more than one PMT, charged particles passing through a PMT pose little problem for particle identification. One background source consists of shower leakage from the calorimeter. For incident photons, this is generally not a problem, as no track will point to that cell; one can actually use the photons to measure the size of the effect. Back-splash from electromagnetic showers consists mostly of photons or of particles below Cherenkov threshold. The photons cause a problem only to the extent that they convert in the material of the PID system.

$\delta$-ray production is a potential problem for an aerogel threshold Cherenkov system, since a kaon below threshold can knock out an electron with momentum above Cherenkov threshold and can therefore be misidentified as a $\pi$. A detailed study of this effect has been performed by Grove and Mewaldt [Gro92]. Both an analytic calculation [Oya94] and a detailed GEANT simulation [Shi94] have been used to study the effect. In the forward direction, the study shows that $\sim 2 \%$ of $4 \mathrm{GeV} / c$ kaons may be misidentified as pions, 
due to $\delta$-ray production in the drift chamber wall and back-splash from the calorimeter. With $\simeq 99 \%$ detection efficiency for pions, the system still provides better than $4 \sigma \pi / K$ separation at $4 \mathrm{GeV} / c$. Though $\delta$-ray production and back-splash cause $\sim 2-3 \%$ probability for misidentifying a low momentum $(p \leq 1.2 \mathrm{GeV} / c)$ kaon as a pion, it does not affect $B \rightarrow \pi \pi$ and $B \rightarrow \pi K$ separation since both daughters from these decays have $p \geq 1.5 \mathrm{GeV} / c$. In the worst case, this $K \rightarrow \pi$ misidentification results in a $B$-tagging inefficiency of $\leq 3 \%$.

The misidentification probability due to $\delta$-ray production and calorimeter back-splash can be substantially reduced with the multilayer design. Requiring the coincidence of two aerogel counters, one can suppress the kaon contamination down to $\sim 0.5 \%$ while still retaining $\sim 88 \%$ detection efficiency. This again corresponds to $\sim 4 \sigma$ $\pi / K$ separation.

\subsubsection{R\&D Program}

\section{Proof-of-Principle}

In the near term, tests will be conducted with full-size aerogel blocks of the design density using the primary choices for optical readout. UV sensitive 2" FM PMTs, three HPDs and two $18 \mathrm{~mm}$ MCP PMTs have already been obtained. Large blocks of aerogel from JPL, LLNL and Lockheed are on order. Development of high quality aerogel will be pursued with these suppliers. Improvements to the Cherenkov photon detection efficiency will also be investigated. Tests of the readout system will include measurements of gain, dark current, quantum efficiency and uniformity of response, as well as the effect on these parameters of operation in a $1.5 \mathrm{~T}$ magnetic field. Finally, light yield below the Cherenkov threshold will be studied.

\section{Long-Term Issues}

Once the characteristics of aerogel and photon detectors have been well understood, a beam test of a prototype would proceed at SLAC or CERN by Spring, 1995. This test will allow the investigation of a number of important issues in system development or design, includ-
Tests with full size aerogel blocks and the actual photodetectors are being prepared for a $1.5 \mathrm{~T}$ field 
A beam test is necessary to map out the threshold behavior and to study system issues
The DIRC images Cherenkov light transported down a quartz bar by total internal reflection ing aerogel housing, wrapping, potential use of wavelength shifters, readout electronics and mechanical support. Electron beams at SLAC can be used to examine directly the photon yield for a $\beta=1$ charged particle. Pion and electron beams will be needed to map out the threshold behavior and study the response of the system for charged particles below threshold Beam tests of the prototype will require serious consideration of mechanical support issues and data acquisition electronics to finalize the design of the aerogel particle identification system.

\section{Other Photodetector Options}

Another candidate for a readout photosensor is the hybrid avalanche photodiode (HAPD). A measurement with a pulsed green LED demonstrates that one can clearly see at least three individual photoelectron peaks above the pedestal. The separation between the single photoelectron peak and the ADC pedestal is about $6.5 \sigma$. The HAPD has been used to observe Cherenkov photons produced by electrons from a $R u^{106} \beta$ source in an $n=1.032$ aerogel. The spectrum, shown in Figure 7-8, looks promising, and is consistent with that observed with a masked PMT of the same effective area [SLA93]. Improvement in light yield may also be possible through (1) use of newly developed GaAsP or green-extended bialkali photocathodes; (2) coating aerogel blocks with appropriate wavelength shifters (for example, $3 \mathrm{HF}$ ) on a diffuse reflector, and/or (3) using a focusing mirror.

\subsection{DIRC}

The DIRC (Detection of Internally Reflected Cherenkov light) is a ring-imaging device which utilizes the total internal reflection of light in long thin quartz radiator bars to form a velocity-dependent image. The light is observed by an array of photomultiplier tubes displaced from the end of the bar to allow the pattern to expand to form a proximity focussed image. Thus, it uses only conventional components to provide a fast signal with a large number of photoelectrons for high quality particle identification. The principle of operation is illustrated schematically in Figure 7-9. The thinness 


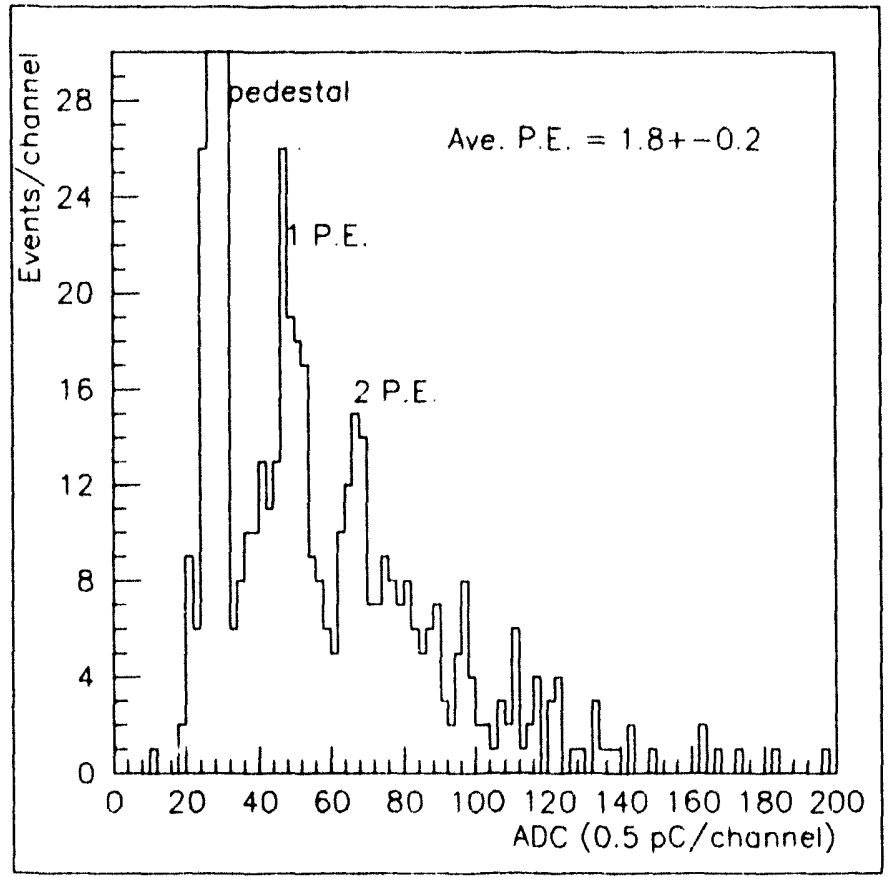

Figure 7-8. Light yield distribution obtained by using an $H A P D$ for $R u^{106}$ electrons passing through an $n=1.032$ aerogel.

of the bar allows for either a more compact detector geometry or a larger tracking volume. The fast timing contributes to very good background rejection. Recent studies on a prototype bar provide results on the number of photoelectrons collected and on the angular resolution. Both agree closely with predictions. The properties of the DIRC and design issues associated with it are discussed in more detail elsewhere [Rat92].

\subsubsection{Conceptual Design}

The basic unit of the DIRC radiator is a $1.5 \times 3 \times 460 \mathrm{~cm}^{3}\left(\mathrm{t}_{\mathrm{x}}, \mathrm{t}_{\mathrm{y}}, \mathrm{t}_{\mathrm{z}}\right)$ quartz bar. When supports are included the radiator module is $14 \%$ of a radiation length thick radially, and occupies $4 \mathrm{~cm}$ (plus $4 \mathrm{~cm}$ for clearance) of radial space. The bars are grouped into modules which then form a 20-sided polygonal surface, as viewed from the end of the detector, and cover about $95 \%$ of the azimuth. The detectors are closely packed arrays of $1.125^{\prime \prime}$ diameter conventional PMTs
Prototype results agree well with Monte Carlo predictions

The quartz bars are observed by PMTs attached to a large waterfilled tank 


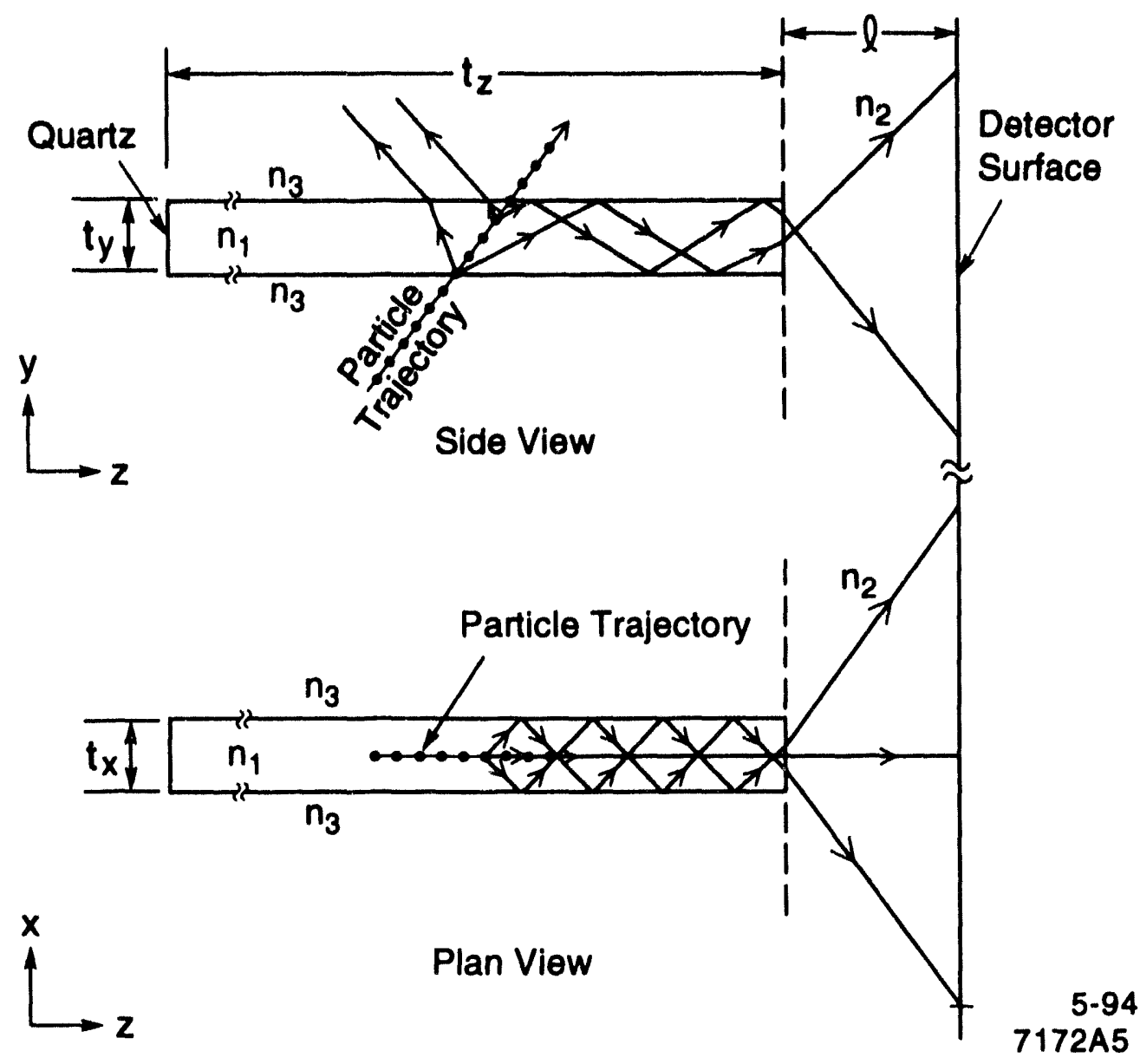

Figure 7-9. Schematic of a single radiator bar of a DIRC counter; the particle trajectory is shown as a line connected by dots; representative trajectories of Cherenkov photons are shown by the lines with arrows.

(16,000 in the reference design), which are placed at a standoff distance of $120 \mathrm{~cm}$. The photo-detection surface approximates a partial cylindrical section in elevation and a toroid when viewed from the end. The standoff region has a reflecting surface along the inner radius and an outer reflecting surface at a 1 radian angle in the same plane to reduce the number of phototubes required. To maintain good photon transmission for all track dip angles, the standoff region is filled with water. The large photodetector structure presents difficult engineering issues of shielding of the photomultipliers from the fringe field of the magnet and providing ready access to inner detector components that remain to be solved. 
The DIRC detector has several important advantages for the detector geometry. These include a thin homogeneous radiator and a readout outside the sensitive detection region. The thin radiator allows a compact design in the detector volume. An overall optimization of the experiment may take advantage of this by designing either a smaller and cheaper calorimeter or by allowing a larger drift chamber for better momentum resolution. The quartz bar provides a modest amount of material situated near the calorimeter, with a homogeneous distribution in polar angle and a simple, well-defined distribution in azimuth. The absence of electronics and cables in the sensitive region will minimize the impact of this detector on the calorimeter resolution and allow straightforward modeling of the detector material for Monte Carlo simulation and reconstruction.

\subsubsection{Geometric Layouts}

In the following sections we present a description of two geometrical configurations of the DIRC. The first is motivated by the desire to maintain a continuous and uniform calorimeter response in the forward direction; it fits into the basic geometry described in other sections of the Letter of Intent. The second represents a more complete optimization of the capabilities of the DIRC, at the expense of overall electromagnetic calorimeter performance. It provides significantly more robust particle identification, particularly in the forward direction where the separation between particle species actually improves at steeper angles, and allows the DIRC to be made thinner. At the same time it allows for a more compact, hence cheaper, calorimeter.

\section{Short Barrel}

Figure 7-10 shows a schematic of the short barrel design. The configuration of the calorimeter and tracking systems is essentially the same as described in other chapters of this Letter of Intent. One exception is that the main tracking chamber has been increased in radius to take advantage of the approximately $17 \mathrm{~cm}$ of radial space made available by the DIRC option. The forward endcap requires a PID system with a different teclinology.
The detector is thin and compact

Impact on the calorimeter is minimized in the barrel region 


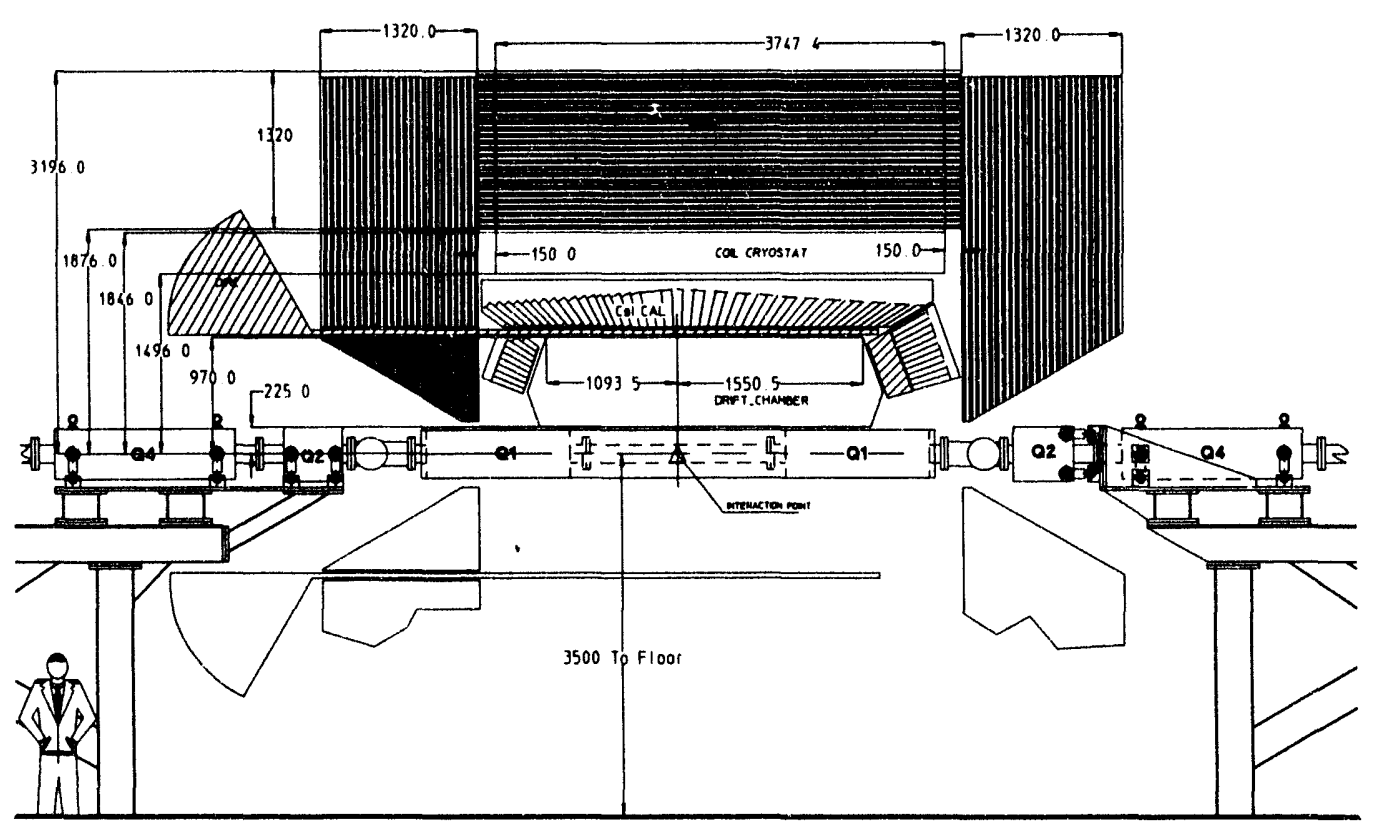

$A$ DIRC read out in the backwards direction is essentially compatible with the geometry required for the other two candidate particle identification systems
Figure 7-10. Schematic view of a detector incorporating a DIRC readout at the back.

The readout of the DIRC is in the backward direction to avoid penetrating the forward calorimeter. This increases the path length for Cherenkov photons from forward tracks which are reflected from the far end of the quartz bar. This is not expected to cause insurmountable difficulties, but some loss of photons down the bars is unavoidable, precisely for the high-momentum particles which are most difficult to identify. Measurements of the size of this effect are discussed below. The loss of Cherenkov photons has been partially compensated in this design by increasing the radiator thickness. A more favorable configuration would be to read out the DIRC in the forward direction so that the Cherenkov photons produced by the majority of high-momentum particles are imaged after a shorter path length in the quartz. This would also improve the access to the tracking chamber electronics located on the backward end of the chamber. However, it requires separating the forward calorimeter into distinct barrel and endcap regions. These can be arranged to have a high degree of overlap but the effect on energy resolution of the calorimeter in this region has not yet been established. The large radius tracking chamber provides excellent $d E / d x$ and momentum 
resolution, but at significant cost. If the tracking radius were to be kept at the nominal value of $80 \mathrm{~cm}$ described earlier, the cost for the DIRC itself would be substantially reduced, as would that of the calorimeter.

\section{Long Barrel}

The long barrel design covers the entire fiducial region down to $300 \mathrm{mr}$ forward without the need for endcaps. It allows for a smaller inner radius (about $88 \mathrm{~cm}$ ) for the calorimeter (due to the thin DIRC) while preserving the full tracking volume. The readout for this system is ideally in the forward direction, providing the benefits noted above without the drawback of splitting the calorimeter. The increased tracking length in the forward direction improves the $d E / d x$ measurements, augmenting the robustness of the system.

One drawback of this design is the need to provide a superconducting magnetic shield around the PEP-II quadrupole Q2. Conceptual designs for this shield exist, albeit with some access difficulties and cost increments. Another consideration, which has been studied to sorne extent [Atw92, Spi94], is the effect on the calorimeter performance of an elongated barrel section, although the advantages of eliminating the traditionally problematical barrel-endcap transition region are quite evident. For these reasons the long-barrel option has not been adopted as a proposed geometry in this Letter of Intent.

\subsubsection{Existing Experimental Measurements}

Since the DIRC is a new concept, it is important to understand and prototype it as thoroughly as possible to avoid surprises. The design concept is quite straightforward and makes use of well-understood conventional components (quartz radiators and PMTs); therefore its performance should be predictable based upon known properties of these materials and devices. A major technical issue is the construction of long quartz radiator bars which meet appropriate specifications. Eight moderate length ( 1/4 full length) bars have been produced to date and a number of lab studies have verified that these bars meet or exceed the required performance parame-
Long barrel designs accommodate the DIRC in a natural way, but are problematic in other areas and have therefore not been adopted
Quartz bars of $\sim 1 / 4$ full length have been shown to meet requirements 
ters [DIRC93]. For example, the absorption length of the material exceeds $30 \mathrm{~m}$ over the entire wavelength region $(300-600 \mathrm{~nm})$. The internal reflection coefficients substantially exceed 0.999 , and the edges are sharp and free of chips. Direct observation of the imaging properties of the bars with an artificial, laser-produced "Cherenkov" cone has demonstrated that the bar surface figure exceeds the requirenents. The conceptual prototype, Prototype I, including one of these bars imaged by a small array of phototubes, is under test with cosmic rays, and has demonstrated the proof-of-principle in preliminary tests. A substantially larger device to image the entire Cherenkov pattern is now being designed.

\section{Conceptual Prototype}

The goals of the conceptual prototype are (a) to demonstrate that the number of photoelectrons observed with Cherenkov light is adequate by measuring this number as a function of angle and length along the bar, and (b) to measure the single photoelectron resolution. The apparatus uses a hardened cosmic ray beam as shown schematically in Figure 7-11.

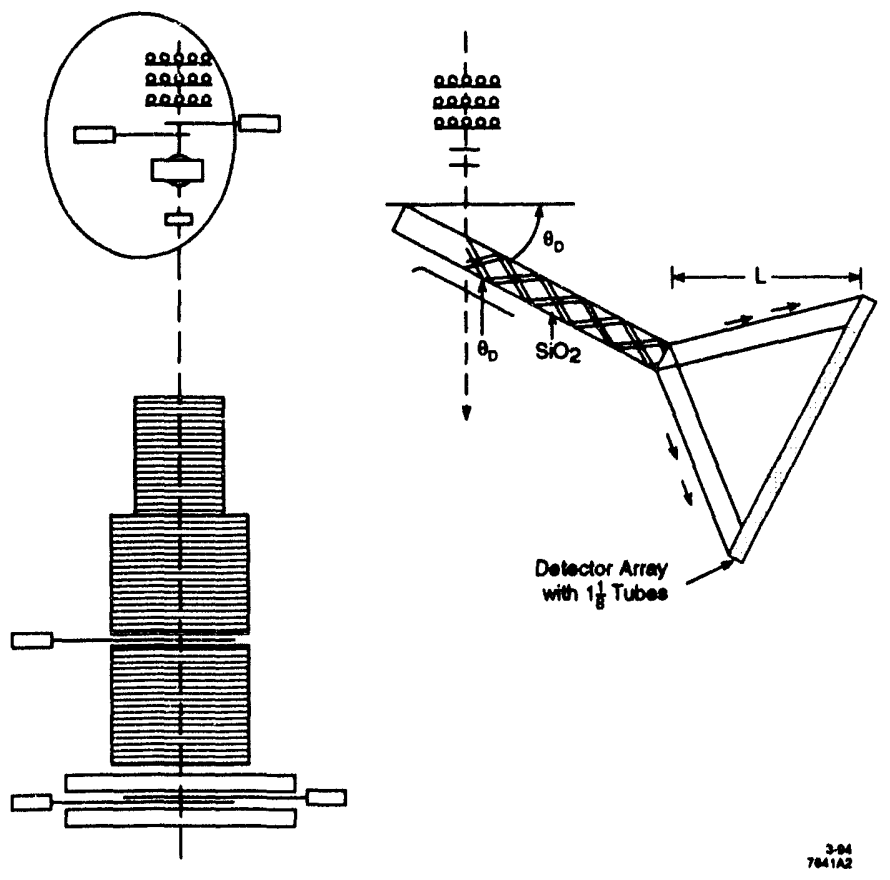

Figure 7-11. Schematic drawing of the conceptual prototype device. 


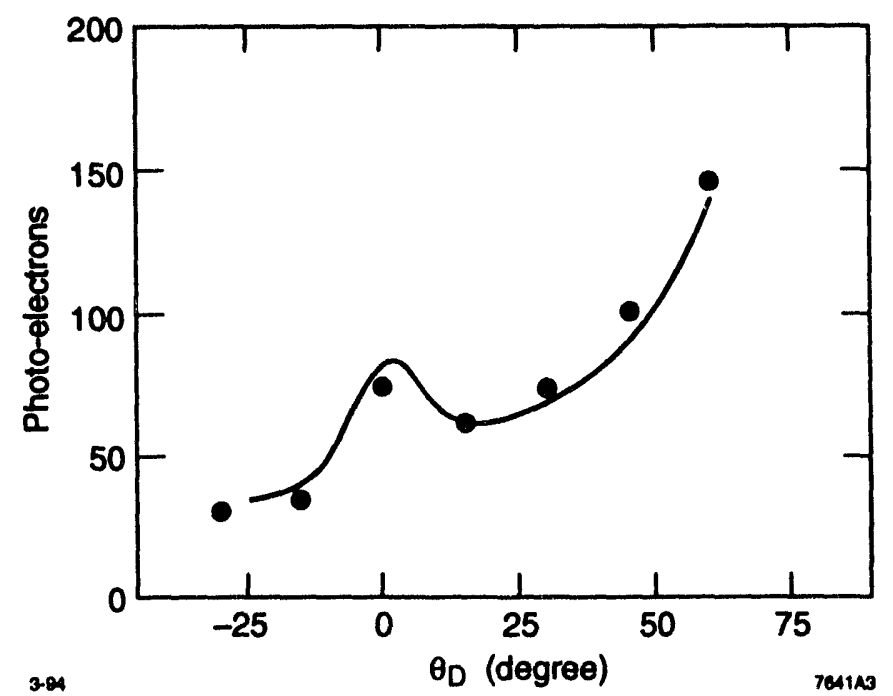

Figure 7-12. The observed photoelectron yield as a function of dip angle $\theta_{D}$, measured $60 \mathrm{~cm}$ from the phototube.

The cosmic ray telescope includes five scintillation counters which require the triggering particle to pass through the DIRC bar and then through a $700 \mathrm{MeV}$ energy-loss stack of iron plates within $7.5^{\circ}$ of the vertical. For the cosmic ray muon spectrum with this momentum cutoff, the variation of the Cherenkov angle has a standard deviation of $2.4 \mathrm{mr}$. The prototype bar can be rotated about the horizontal axis and displaced in length so that the performance can be fully studied as a function of angle and distance along the bar.

In one set of studies, a $120 \mathrm{~cm}$ long, $4.6 \mathrm{~cm}$ wide, and $1.67 \mathrm{~cm}$ thick quartz bar (Vitreosil 055) was directly coupled to a $2^{\prime \prime}$ diameter RCA-8850 PMT. The gain of the PMT was calibrated using thermal electrons from the photocathode.

Figure 7-12 shows the dip angle dependence of the photoelectron yield. This measurement was done at the middle of the bar. The solid line is a Monte Carlo simulation with $N_{0}=124 \mathrm{~cm}^{-1}$ (corrected for length dependence). The number of photoelectrons, $N_{P E}$, can be written as

$$
N_{P E}=\frac{\epsilon d N_{0} \sin ^{2} \theta_{c}}{\cos \theta_{D i p}},
$$

where $\epsilon$ is the collection efficiency of the bar as determined by Monte Carlo simulation, $d$ is the thickness of the bar, $\theta_{c}$ is the Cherenkov angle, and $\theta_{D i p}$ is the dip angle of the track. The quantum efficiency of the PMT and other factors are lumped to- 
There is an enhancement in the number of photons at large dip angles where the fastest particles are produced gether in the "Cherenkov Quality factor" $N_{0}$. A substantial number of measurements of this factor have been made in a variety of experiments for many of PMTs [Eig79]. For a good-quality glasswindowed PMT, it is expected to be around $100 \mathrm{~cm}^{-1}$. The Monte Carlo simulation with $N_{0}=124 \mathrm{~cm}^{-1}$ reproduces the measurements rather well. There is an enhancement at large dip angles due primarily to the track length within the radiator and to a lesser extent, to improved collection efficiency. More photons are seen near $0^{\circ}$ because the collection efficiency for these particles is increased by reflections from the other end of the bar. Small deviations from the Monte Carlo as a function of angle can arise from a number of sources, including variations in calibration and tube performance as a function of photon angle and polarization.

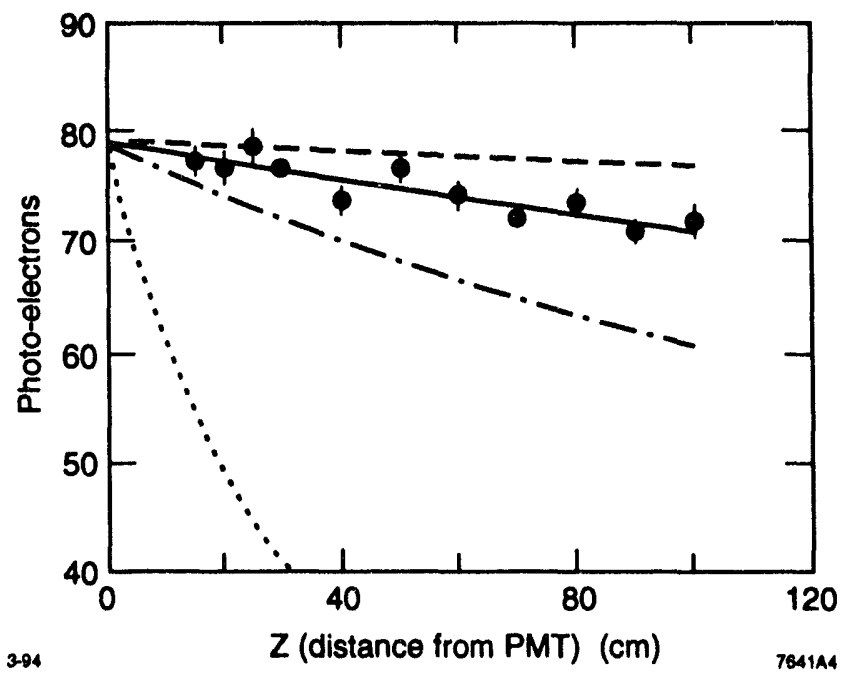

Figure 7-13. The observed photoelectron yield at a dip angle $\theta_{D}=30^{\circ}$ as a function of length: the lines are a Monte Carlo simulation with $N_{0}=135 \mathrm{~cm}^{-1}$; dashed for absorption length $\lambda=100 \mathrm{~m}$ and surface reflectivity $\eta=0.9999$; dot-dashed for $\lambda=10 \mathrm{~m}$ and $\eta=0.999$; and dotted for $\lambda=1 \mathrm{~m}$ and $\eta=0.99$.

Figure 7-13 shows the position dependence measured at $\theta_{D i p}=30^{\circ}$. A small position dependent effect can be seen along the bar. In general, the losses are a convolution of photons with different paths in the bar which will therefore have different characteristic behaviors as a function of the bar length. When viewed against bar length, the integrated curve is expected to have a steeper drop at short lengths and to flatten out as the length increases. Nonetheless, if losses are small, the loss rate should fit an exponential (or linear) 
hypothesis reasonably well over modest lengths. The results of a simple exponential fit are shown by the solid line. The effective loss corresponds to $11 \pm 3 \%$ per meter of bar length.

The conceptual prototype is now running with an array of fortyseven phototubes ( $1 \frac{1}{8} "$ EMI $\left.9124 \mathrm{~A}\right)$ to demonstrate the imaging properties of the DIRC. The coupling between the array and the bar is air, which allows for easy variation of the standoff distance.

Initial measurements have been made at a standoff distance of $66 \mathrm{~cm}$ and a dip angle of $60^{\circ}$. The preliminary results are shown in Figure 7-14. The measured resolution is about $12.5 \mathrm{mr} /$ photon as expected, given the known geometrical and chromatic aberrations and the relatively modest standoff distance. The observed number of photoelectrons is consistent with the measurements described above (see Figure 7-12), after correcting for simple geometrical factors.

The program for Prototype I is nearing completion. Measurements to date are consistent with initial expectations, and if attained in a full-scale device, would allow the DIRC to provide the performance shown in Figure 7-16. This conceptual prototype program is expected to be completed by about the end of the second quarter of 1994.

\subsubsection{Expected Physics Performance}

The performance of an imaging Cherenkov device is a strong function of momentum, because the separation (in Cherenkov angle) between particle species is a rapidly varying function of momentum. The response also depends on the angular accuracy of the photon measurement by the detector, which in the DIRC is a function of the phototube size, the standoff distance, the bar thickness, and thus the number of phototubes. It is also a function of the dip angle of the incident track, primarily because the production and transport of photons is angle dependent, and the readout direction because of light losses and, perhaps, emittance growth down the bar.

There is a natural enhancement in the separation power of the DIRC at the larger forward dip angles due mainly to the increased amount of radiator traversed. This is, of course, where the fastest particles
The effective loss rate is $11 \pm 3 \%$ per meter of bar length

Initial measurements made on a conceptual prototype confirm feasibility 
The long light path in a backward readout scheme results in somewhat degraded performance

The DIRC meets the performance requirements at all angles

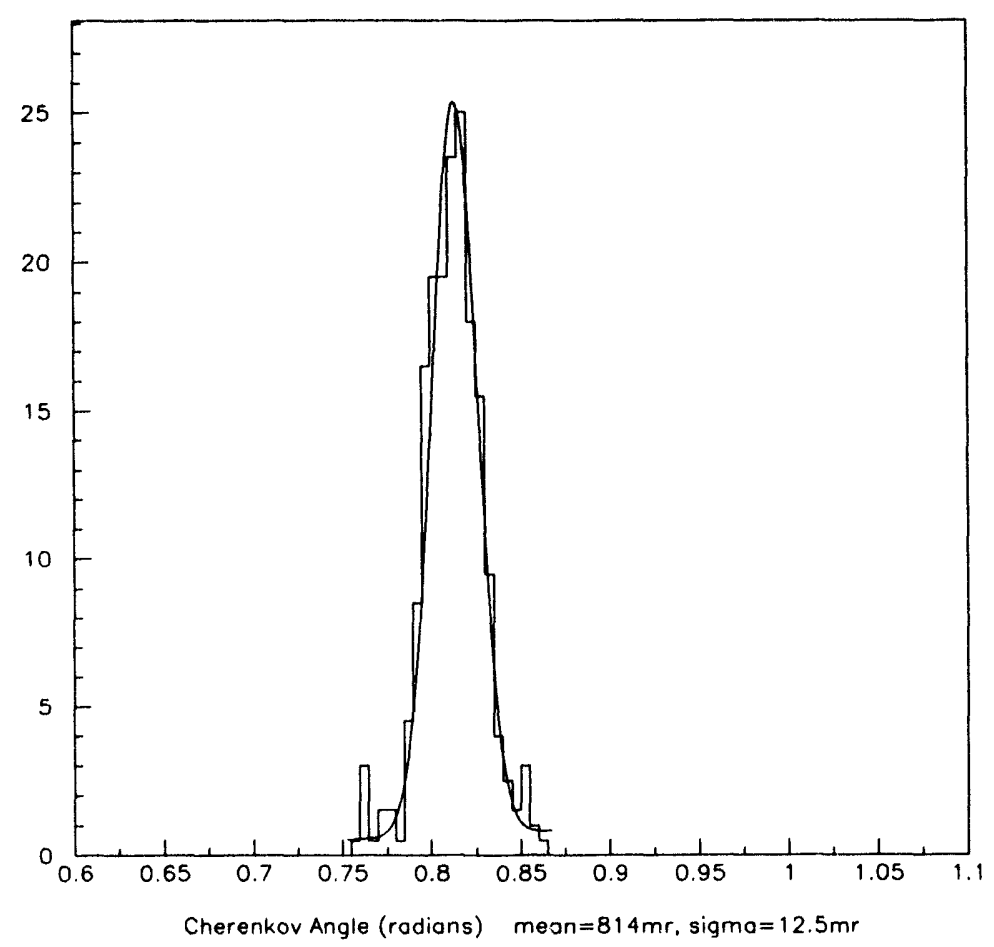

Figure 7-14. The distribution of Cherenkov angles from cosmic ray muons measured in Prototype $I$ at a dip angle $\theta_{D}=60^{\circ}$ and standoff distance of $66 \mathrm{~cm}$.

at an asymmetric machine are produced. With backward readout this effect is somewhat diminished because of light transmission loss due to the longer path length.

The predicted performance has been calculated by Monte Carlo using parameters for the number of photoelectrons and the length dependence of the bars as determined in the Prototype I studies discussed above. The angular dependence of the device matches very well the requirements imposed by the $B$ decays. For example, for the $B$ decay channel with the highest momentum particles, $B^{0} \rightarrow \pi^{+} \pi^{-}$, the discrimination is excellent over the whole region, as shown by the solid line in Figure 7-15. The very forward angles are not covered by a barrel DIRC in this geometry. For comparison, the dotted line shows the response of a long barrel DIRC which is read out in the forward direction. The readout direction is responsible for the overall performance difference-the long barrel geometry shows the effect of the endcap on the acceptance. 


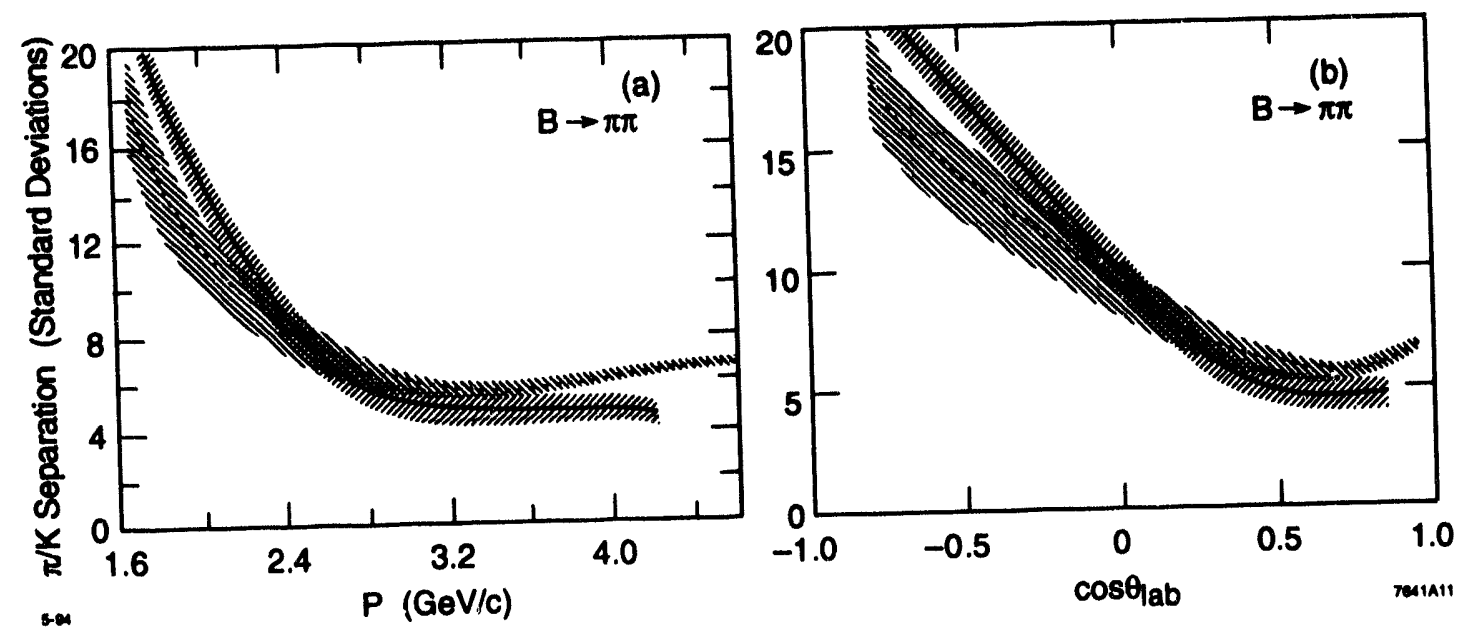

Figure 7-15. The predicted $\pi / K$ separation for pions from the reaction $B \rightarrow \pi^{+} \pi^{-}$as a function of (a) momentum and (b) $\cos \theta_{l a b}$ for this geometry with backward readout (solid line); for comparison, a long barrel DIRC with forward readout (dotted line) is also shown. The bands demonstrate the effect due to different assumptions concerning the loss rate (5\% to $17 \% /$ meter).

The difference in performance is driven by the observed number of photoelectrons. Figure 7-16 shows the number of photoelectrons expected for a backward or forward readout system for the reaction $B^{0} \rightarrow \pi^{+} \pi^{-}$. To provide an indication of the robustness of the device and its dependence on readout directions, bands are provided for photon loss rates which differ by $\pm 2 \sigma$ from those measured with the prototype.

\subsubsection{R\&D Program}

\section{Large-Scale Prototype}

Planning is now underway to scale up to a much larger system, Prototype II, so that the full Cherenkov image can be observed at a set of track angles, and the resolution (in mass or $d \beta$ ) can be measured on a track-by-track basis. This prototype will contain approximately 500 channels of photon detection and readout, a

A 500 channel DIRC prototype is being planned 


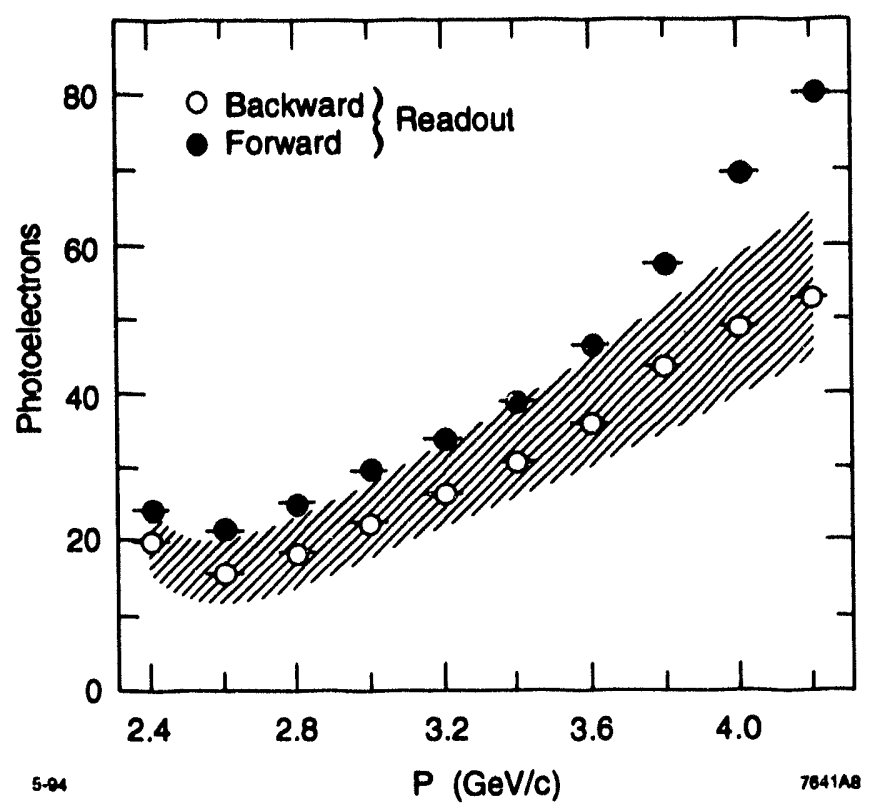

Figure 7-16. The expected number of photoelectrons for forward-going pions in the short barrel geometry from the reaction $B^{0} \rightarrow \pi^{+} \pi^{-}$as a function of momentum for different readout directions with the photon loss rate as measured in Prototype I (11\%/meter). The bands show the effect due to different assumptions on the loss rate (5\% to $17 \% /$ meter); backward readout (open circles); forward readout (solid circles).

mechanical support equipment. The device will also allow us to test engineering concepts for the support of the quartz bars, and for the large water-filled standoff region with its windows, mirrors, and couplings to the bar and phototube array. Since it is clearly important to demonstrate directly as many of the features of the detector as feasible before the CDR, there is time urgency to this project. Data taking is planned for the fall.

\section{Engineering Issues}

For the longer term, there are a number of other studies underway as briefly outlined below. These studies aim to optimize the performance of the DIRC and ensure its compatibility with the rest of the detector, develop the required engineering designs, and minimize the costs. 
For example, there is a program underway to explore techniques for the production of very long bars either by gluing or, preferably, by direct production. The optical properties of the bars that are produced will be examined, and issues such as temperature stability, radiation damage, stress relief, and moisture penetration will be addressed. In addition, an overall system design, at full conceptual engineering detail, will be developed for the mechanical, optical, electrical, and readout portions of the system. As input to this design, detailed studies of the performance of the DIRC in the presence of backgrounds, and further exploration of the effects of DIRC system mass and geometry on the calorimeter are also required. The options for the photodetection system will also be examined more thoroughly in order to optimize performance, detector access and cost. Finally, if pixelated photodetectors with appropriate performance and cost become available, it might become possible to make a focused standoff region which is much more compact. Thus, such devices will be obtained for further study if they become available.

\subsection{Fast $\mathrm{RICH}$}

The Fast RICH was originally proposed in connection with the design of a detector for the high-luminosity symmetric $B$ Factory at PSI [Eic86], and is an attractive possibility for operation at an asymmetric collider. The distinguishing feature of a Fast $\mathrm{RICH}$ is the use of fast photon detectors with pad readout to provide the position of the imaged Cherenkov photons. This device couples a liquid $\mathrm{C}_{6} \mathrm{~F}_{14}$ radiator via a proximity gap to a pad readout chamber based on a solid CsI photocathode [Seg90, Ale93]. The principle is schematically shown in Figures 7-17 and 7-18. The detector has fast response with good granularity, runs at room temperature and does not require the handling of difficult and corrosive gases such as TMAE. It has the extra advantages of providing tracking information for the calorimeter and a fast trigger signal.
The Fast RICH images Cherenkov rings from a liquid radiator onto a proportional chamber read out with CsI-coated cathode pads 


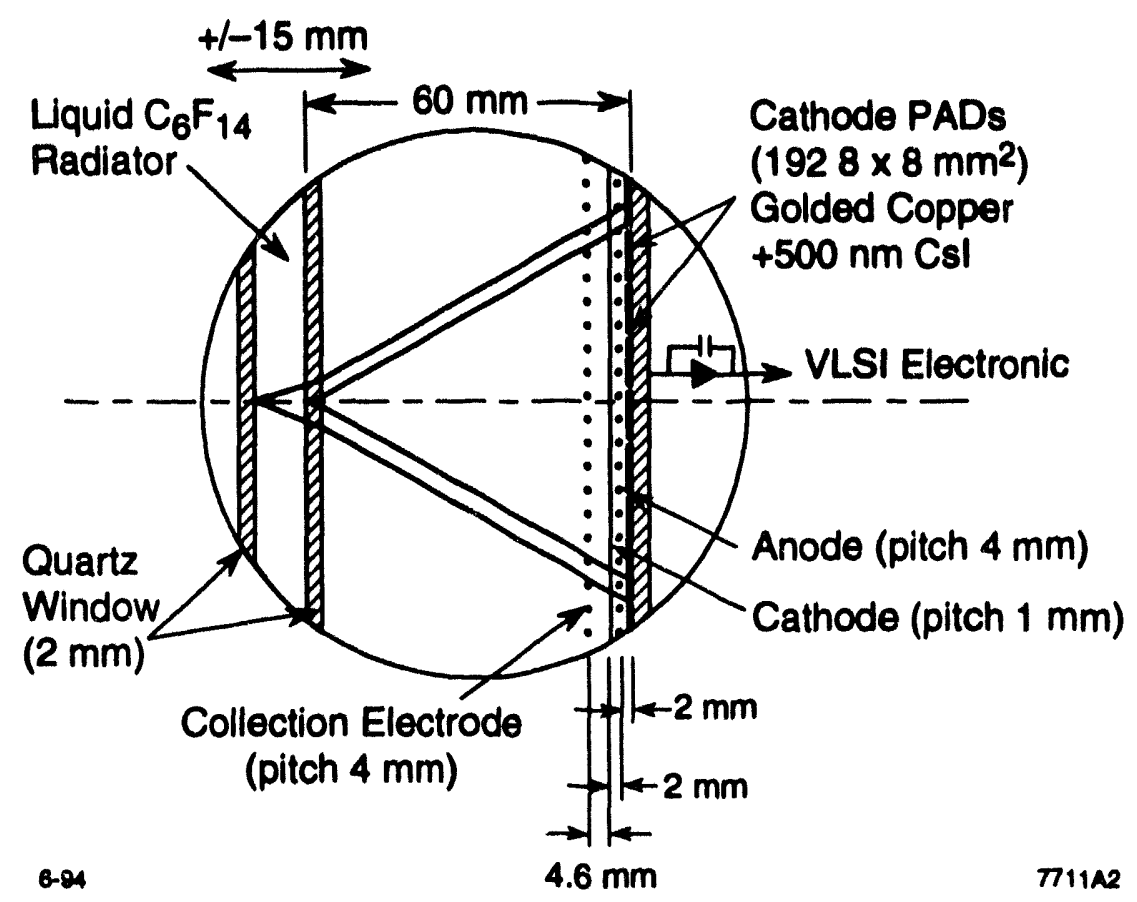

Figure 7-17. The Fast RICH conceptual prototype

\subsubsection{Conceptual Design}

Barrel and endcap detectors are divided into 24 azimuthal sectors

\section{System Overview}

The Fast RICH system, as embedded in the overall detector, is shown in Figure 2.3. The inner and outer radii, and length of the barrel RICH detector are 82 and 103, and $270 \mathrm{~cm}$, respectively. Similarly, the endcap disk RICH detector is situated at $+172 \mathrm{~cm}$ and is $21 \mathrm{~cm}$ thick with inner and outer radii of 38 and $103 \mathrm{~cm}$. The endcap and barrel detectors are segmented in 24 sectors of $15^{\circ}$ each. The barrel is divided in two longitudinally for the radiators and likewise for the chambers. It has an internal cylinder of $3 \mathrm{~mm}$ of carbon fiber which supports the liquid radiator and the glued quartz window, and an external cylinder made with hexcell which supports the chambers with their pad structure and electronics. Figure 7-19 shows an isometric view of the RICH where the radiator, detector and support structure are indicated. The amount of material at 


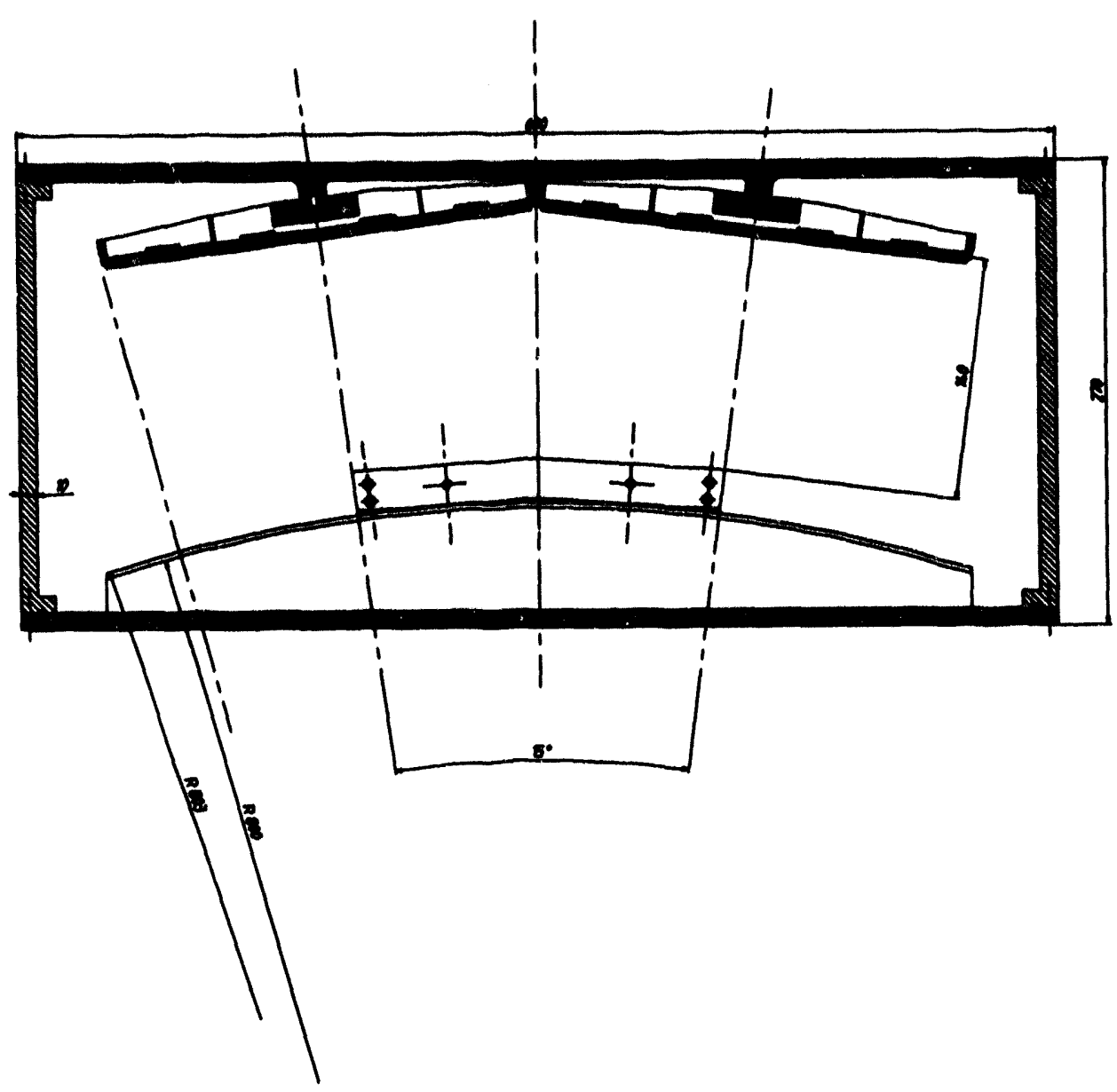

Figure 7-18. The Fast RICH engineering prototype

$90^{\circ}$ is estimated to be $13 \%$ of a radiation length, with about $9 \%$ embodied in the liquid radiator plus its support.

\section{Radiator}

Liquid freon $\left(\mathrm{C}_{6} \mathrm{~F}_{14}\right)$ is the present candidate for the adiator material. The liquid is circulated in a $1 \mathrm{~cm}$ thick container made of two thin membranes of composite material (kevlar/glass fiber) covered with a kapton foil and separated by foam. Similar containers have been used in the DELPHI RICH for four years and have proven The liquid freon radiator is contained in quartz boxes satisfactory. A $3 \mathrm{~mm}$ thick quartz window closes the container on with $3 \mathrm{~mm}$ thick walls the outward side. A layer of $100 \mu \mathrm{m}$ wires with $5 \mathrm{~mm}$ pitch are 


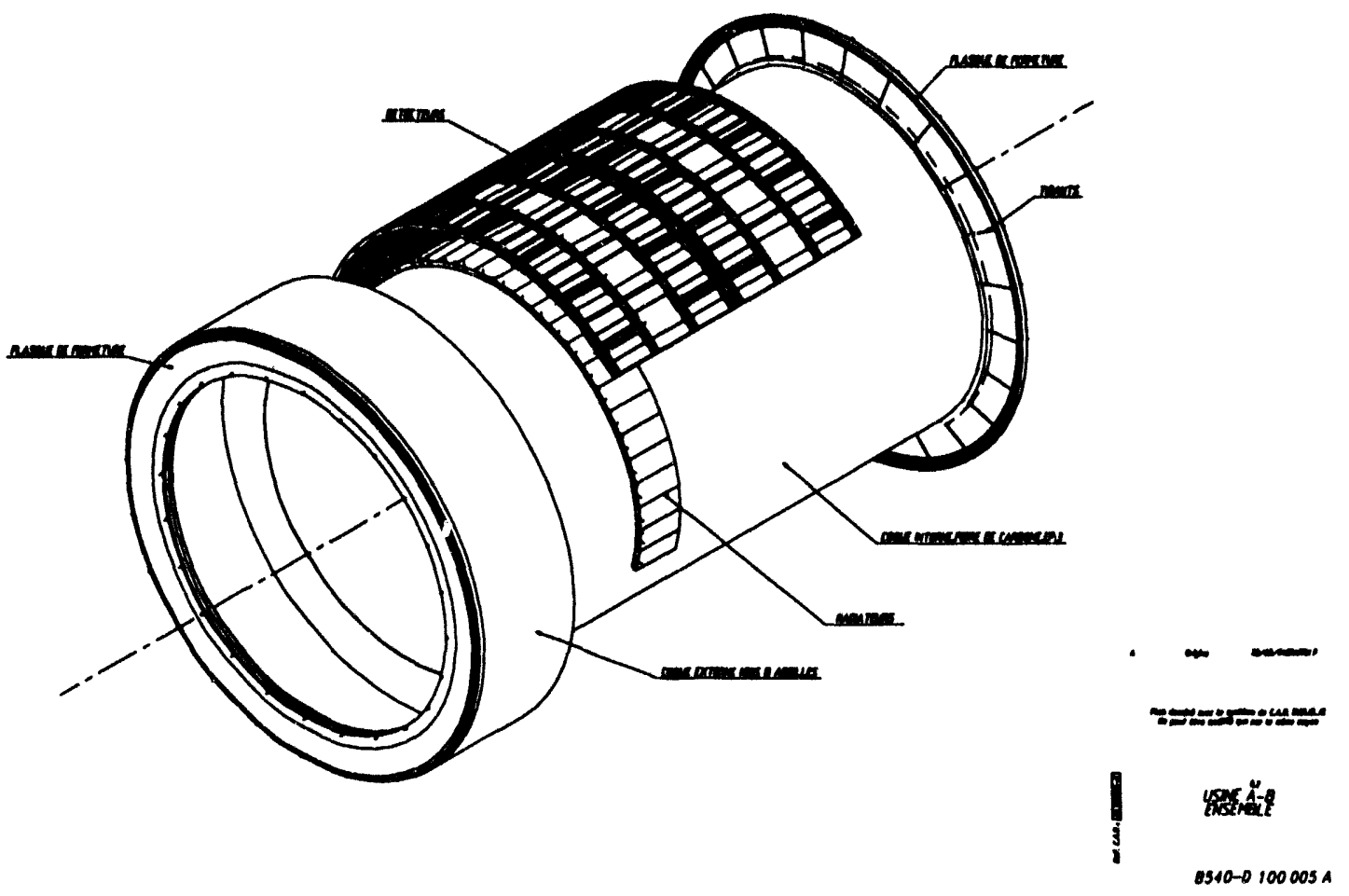

Figure 7-19. Isometric view of the Fast RICH

$A$ vacuum-deposited $500 \mathrm{~nm}$ layer of CsI is the photon detector placed on the window for the collection of the electrons from the ionization in the $14 \mathrm{~cm}$ proximity gap.

\section{Photon Detection}

The chambers are made of G10 printed circuit boards with $8 \times 8 \mathrm{~mm}^{2}$ pads on which a $500 \mathrm{~nm}$ CsI layer is deposited by vacuum evaporation. An anode plane made from $20 \mu \mathrm{m}$ gold-plated tungsten wires, strung in the azimuthal direction with a $2 \mathrm{~mm}$ pitch, is positioned at a distance of $2 \mathrm{~mm}$ from the pads located on the mechanical structure. The cathode plane consists of a layer of $100 \mu \mathrm{m}$ diameter gold-plated $\mathrm{Cu} / \mathrm{Be}$ wires strung in the longitudinal direction and positioned at a distance of $2 \mathrm{~mm}$ inside of the anodes. 


\section{Electronics}

Possible front-end electronic architectures are currently being reviewed. Both analog and digital solutions are being explored; the former has been more thoroughly examined at this time. In such a solution, each pad would be connected to an onboard ASIC chip including a low-noise preamplifier, followed by either a track-and-hold circuit or an analog pipeline memory. Multiplexing would occur downstream either on the detector (if power dissipation permits) or, more remotely, before digitization.

\subsubsection{Existing Experimental Measurements}

The primary concerns in the Fast $\mathrm{RICH}$ design relate to the properties of the new CsI photocathode material. In particular, there are uncertainties about (a) the quantum efficiency of the photocathodes, (b) the resolution which can be obtained, and (c) the robustness of the material and performance under operational conditions. Detailed descriptions of existing measurements with UV photons from lamp sources can be found in the references [Ale94, Mal93a, Bra93, Mal93b]. An extensive $R \& D$ program is underway worldwide to address these concerns, carried out by several groups for different applications [Seg90, Piu94].

Figure 7-20 shows the quantum efficiency as a function of wavelength as measured with UV lamps by many laboratories. The efficiencies vary over a wide range, possibly due to different calibration or photocathode production methods. The discrepancy is still not understood at this time and awaits definitive beam tests to clarify the situation.

Efforts have been focussed on studies of solid photocathodes and, in particular, on CsI photocathodes. As information on such photocathodes is still new, studies of fundamental properties are being undertaken both with bench and beam tests, including the quantum efficiency, number of photons and resolution, time stability and pollution problems.

Photocathodes have been produced by installing an evaporator which deposits thin layers of Cesium Iodide with good control of homogeneity and thickness. A six-month long study of the ageing of
Both analog and digital pipeline solutions are being studied

The quantum efficiency and robustness of the CsI photocathodes are primary concerns

An extensive worldwide $R \& D$ program is underway

CsI ageing studies have been performed 


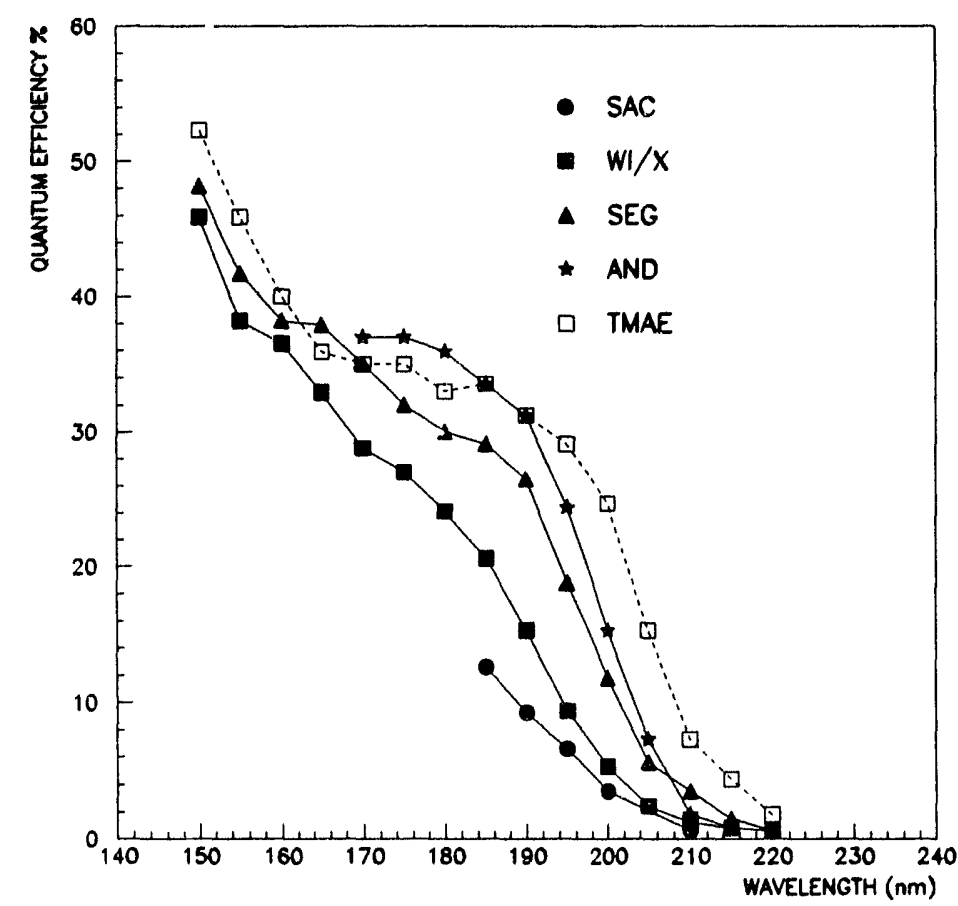

Figure 7-20. Quantum efficiency of CsI photocathodes vs. wavelength as measured by different laboratories and methods. The curves correspond to SAC: ref. [Ale94], WI/X: ref. [Mal93b], SEG: ref. [Seg90], AND: ref. [And92], TMAE: ref. [Hol87].

a CsI photocathode has been performed (Figure 7-21). A slight degradation of the quantum efficiency is observed, reaching a value of $7.5 \%$ at $190 \mathrm{~nm}$ at the end of the full study period. This small effect appears to be due to the presence of water which can be reduced or controlled.

A first beam test was conducted in April 1993 [Ale93] using a radiator made with a $\mathrm{NaF}$ crystal positioned at $7 \mathrm{~mm}$ from the photocathode (see [Ale93] for more detail). The observed signal from the CsI photocathode was seen to be about 1.9 times higher than that expected from a full Monte Carlo simulation of the experimental setup, assuming a low $(10 \%$ at $190 \mathrm{~nm})$ quantum efficiency for the CsI.

In order to confirm this result, another beam test was conducted in June 1993 where the setup was modified to have better control 


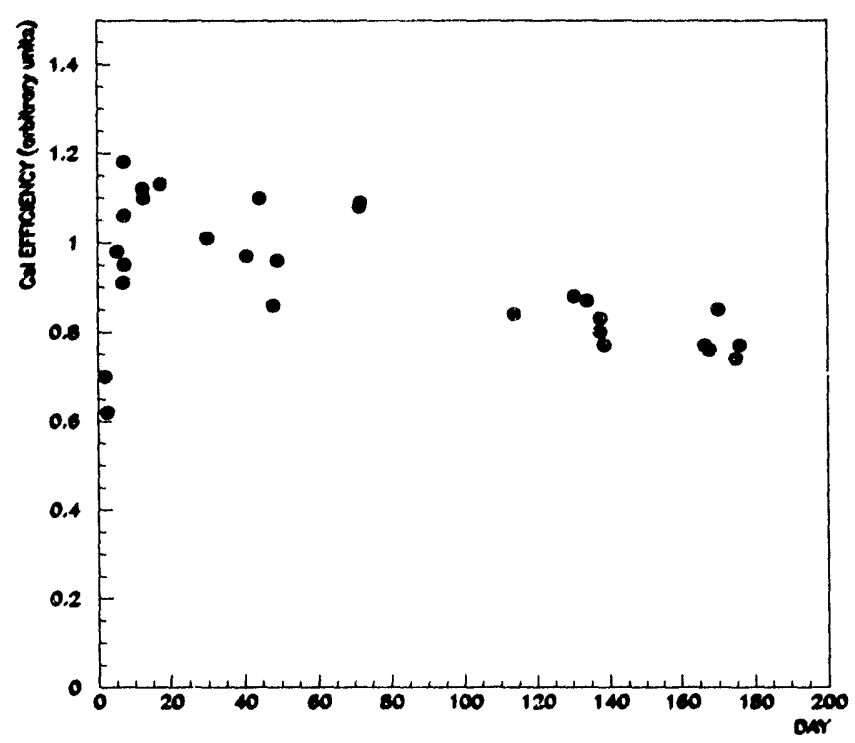

Figure 7-21. Ageing study of one photocathode over a period of 6 months

of the measurements. In particular, the capabilities of moving the position of the radiator with respect to the CsI photocathode and of using different radiator crystals $\left(\mathrm{NaF}, \mathrm{LiF}, \mathrm{CaF}_{2}\right)$ was introduced. Furthermore, the photocathode was divided into 5 parts (a central region with no CsI and 4 petals read out individually).

Figure 7-22 shows the measured number of photoelectrons as a function of the radiator-photocathode distance for the $\mathrm{NaF}$ crystal. The solid line shows the expected number of photoelectrons using our full GEANT Monte Carlo simulation. The expected photoelectron yields were obtained using the quantum efficiency curve with $o=10 \%$ at $190 \mathrm{~nm}$. Although the shape of the measured distribution is in reasonable agreement, the data points are about 1.7 times higher than the prediction. The results obtained with the April test are therefore confirmed by this later measurement. All indications from our tests with beam particles point to a CsI quantum efficiency of about $18 \%$ at $190 \mathrm{~nm}$, rather than the $10 \%$ obtained with a UV lamp.

Experimental information on the angular resolution expected per photon is also available. A $3 \mathrm{GeV} / c \pi$ beam test of a detector [Piu94] similar to that proposed here, in this case using a $13 \mathrm{~mm}$ thick radiator, a CsI photocathode segmented into $14408 \times 8 \mathrm{~mm}^{2}$ pads and a readout with the AMPLEX chip [Beu90], resulted in a single
Several beam tests have provided data on CsI quantum efficiency and on angular resolution for single photon detection 


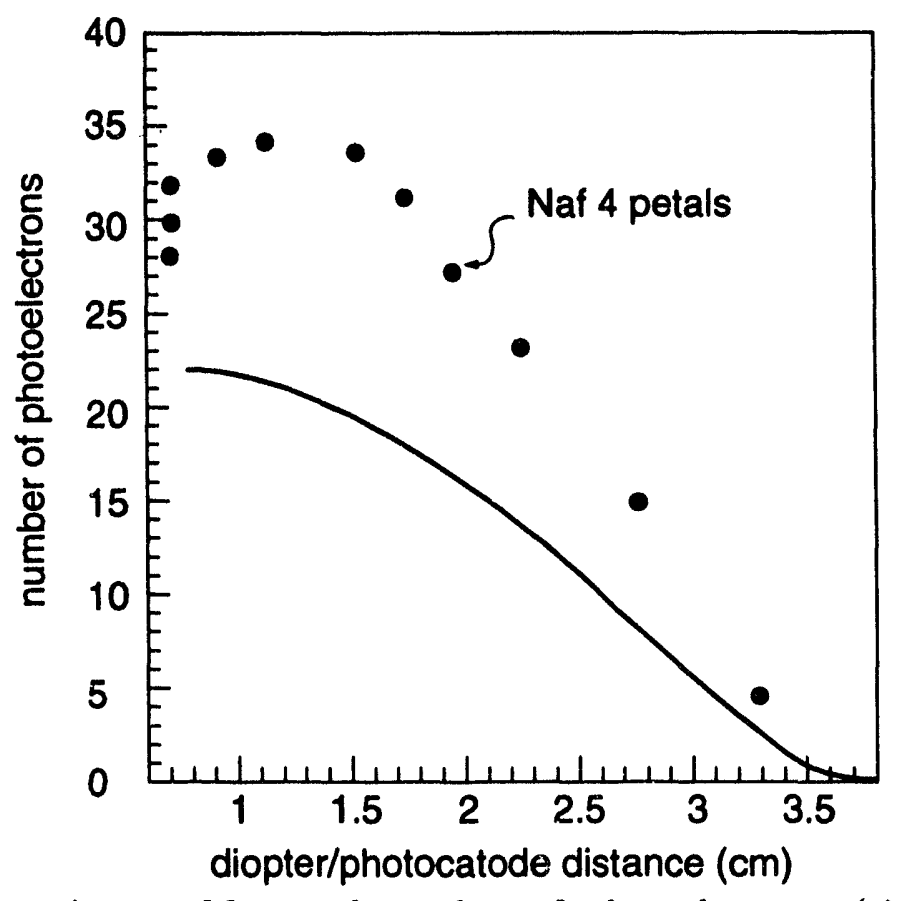

Figure 7-22. Measured number of photoelectrons (circles) compared with a simulation using a low quantum efficiency for CsI (solid line). Cherenkov light was generated with a $\mathrm{NaF}$ radiator.

photoelectron resolution of $8.6 \mathrm{mr}$ for the Cherenkov angle (Figure 7-23). This is quite encouraging and reasonably well reproduced with our simulation program. Pollution of the detector is thought to be the cause of the observed low photoelectron yield.

\subsubsection{Expected Physics Performance}

The test results indicating a quantum efficiency of $18 \%$ at $190 \mathrm{~nm}$ have been put into a full GEANT simulation which produces cathode pad and sense wire hits. Studies are in progress to simulate the resolution as a function of the geometry (radiator thickness, pad size), the magnetic field, multiple scattering, and on the precision of the track parameters as given by the central detector. Different pattern recognition methods have been tried; the best method appears to be that which assumes the five possible mass hypotheses and then scans the expected Cherenkov angle. Figure 724 shows the predicted $\pi / K$ separation as a function of momentum. 


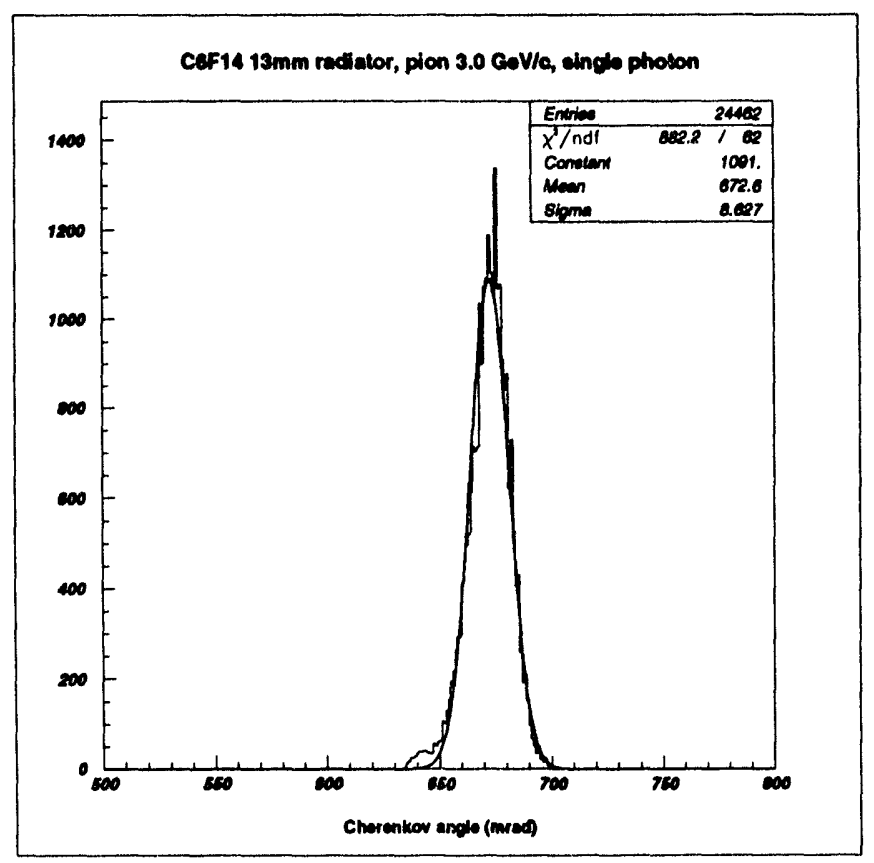

Figure 7-23. Single photoelectron resolution measured in $3 \mathrm{GeV} / \mathrm{c}$ pion beam. The proximity gap was $7 \mathrm{~cm}$ and the radiator thickness, $13 \mathrm{~mm}$.

Table 7-4 summarizes the predictions for the average resolution per photoelectron, the average number of photoelectrons, and the maximum momentum at which $3 \sigma \pi / K$ separation occurs as a function of the dip angle.

\subsubsection{R \& D Plans}

\section{Quantum Efficiency Measurements}

A conceptional prototype has been built (Figure 7-17) and will be tested in a beam during runs extending from March through June 1994. The device has design properties which are analogous to those of the final detector: $1928 \times 8 \mathrm{~mm}^{2}$ cathode pads, individual VLSI analog electronics on each pad [Beu90], CsI deposition and $\mathrm{C}_{6} \mathrm{~F}_{14}$ liquid radiator in closed cell or $\mathrm{NaF}$ solid radiator. The yielu of Cherenkov photons will be measured for particles traversing

The maximum momentum at which $3 \sigma \pi / K$ separation occurs is a function of the dip angle
A conceptual prototype is under test 


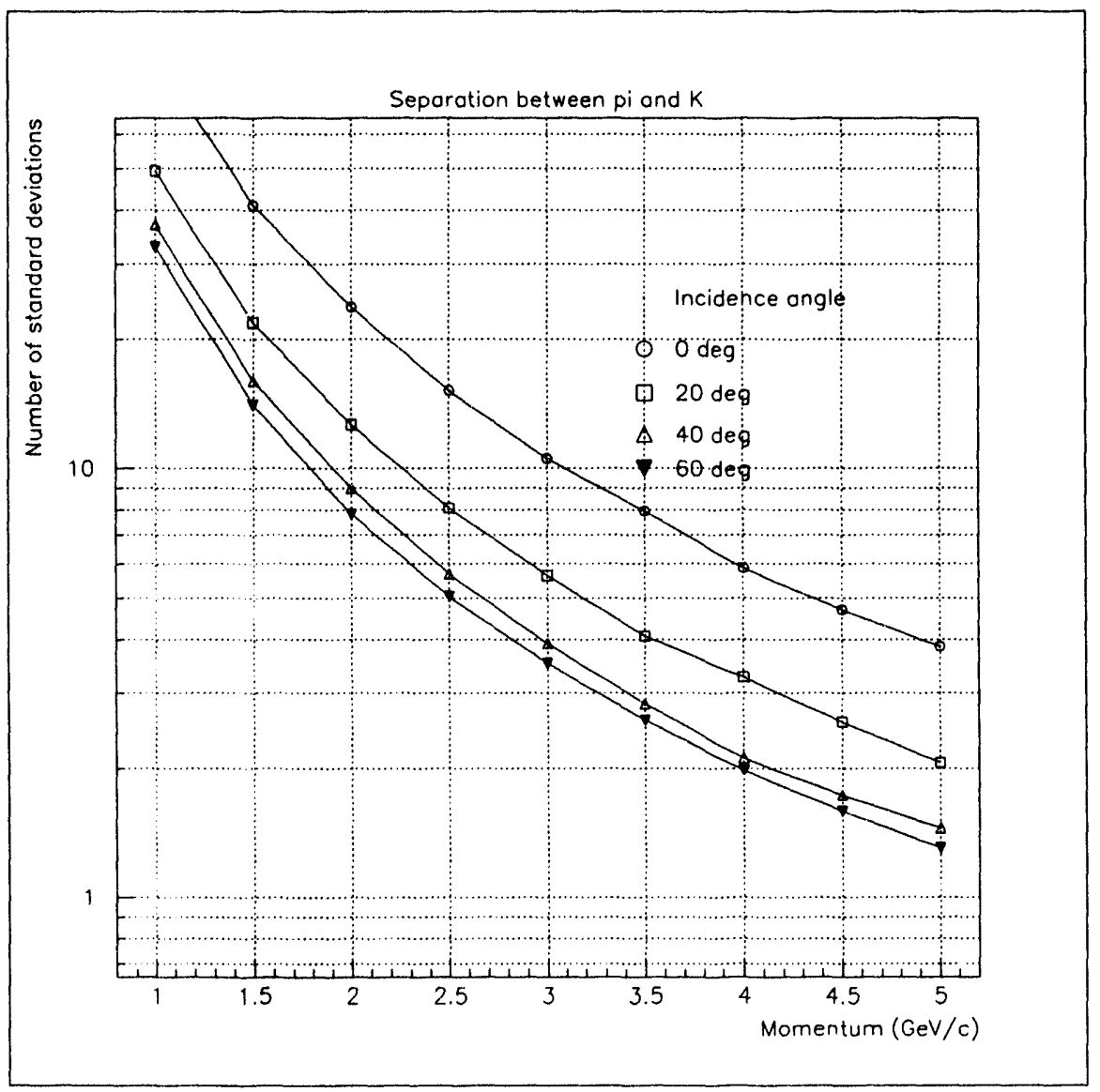

Figure 7-24. The predicted $\pi / K$ separation as a function of momentum in a Fast RICH counter for a quantum efficiency $=18 \%$ at $190 \mathrm{~nm}$.

\begin{tabular}{|c|c|c|c|}
\hline $\begin{array}{c}\theta \\
\left({ }^{\circ}\right)\end{array}$ & $\begin{array}{c}\sigma_{\theta} \\
(\mathrm{mr})\end{array}$ & $N_{p e}$ & $\begin{array}{c}p_{M A X}(3 \sigma) \\
(\mathrm{GeV} / c)\end{array}$ \\
\hline \hline 0 & 6.2 & 17.3 & $\geq 5.5$ \\
20 & 8.9 & 10.8 & 4.2 \\
40 & 12.3 & 9.3 & 3.4 \\
60 & 13.9 & 10.1 & 3.3 \\
\hline
\end{tabular}

Table 7-4. Predictions for the average resolution and the average number of photoelectrons for $4 \mathrm{GeV} / \mathrm{c}$ pion, and the maximum momentum at which $3 \sigma \pi / K$ separation occurs as a function of the dip angle, for a quantum efficiency $=18 \%$ at $190 \mathrm{~nm}$. 
the radiator, and more information collected on the resolution and robustness of the material under operational conditions.

\section{Large-Scale Prototype}

An engineering prototype (Figure 7-18), a one twelfth segment of the final full barrel detector, is being designed, and could be built and mounted for beam tests by early 1995 assuming encouraging results of the previous round of measurements. The detector will have two $300 \times 1500 \mathrm{~mm}^{2}$ sectors $2 \times 15^{\circ}$ (full length), two $250 \times$ $700 \mathrm{~mm}^{2}$ mobile chambers $(1 / 2$ length $)$, one $200 \times 200 \mathrm{~mm}^{2}$ mobile radiator (1/7 length), and 5500 cathode pads of $8 \times 8 \mathrm{~mm}^{2}$, with minimal material and dead space between two chambers. Studies will be made of the choice of materials, the deposition of CsI on large surfaces, and the importance of the dead space. Measurements of the separation of $\pi / K$ up to $4.5 \mathrm{GeV} / c$ will be made. The full prototype will be equipped with electronics currently used by the RD26 group at CERN.

$\mathrm{R} \& \mathrm{D}$ on the electronics to be used in the final detector is also proceeding. Specifications for the ASIC front-end are currently being defined. The best design should provide (a) an adequate dynamic range allowing the detection of single photoelectrons with high efficiency and the discrimination of charged particles crossing the MWPC, (b) low noise with a sensitive (peaking) time of less than $1 \mu \mathrm{s}$ and (c) negligible deadtime for a conservative high-background rate.

\subsection{Strategy for choosing a Particle ID System}

\subsubsection{Proof-of-principle Requirements for Cherenkov PID systems}

The development and use of a Cherenkov-based particle identification system is considered to be of central importance to the physics performance of the $B_{A} B A R$ detector. The specifications for 
Proof-of-principle demonstrations and tests of engineering prototypes will be required

Criteria have been established for what constitutes "proof-ofprinciple" for all PID systems such a dedicated PID device require either large extrapolations from the performance of existing devices or the development of new ones. Thus, extensive R\&D efforts are necessary to explore performance and feasibility aspects for all potential techniques: the aerogel threshold counter (ATC) and the two ring imaging techniques, the DIRC and the Fast RICH. The main thrust of this work can conveniently be divided into two categories: (1) proof-ofprinciple tests on a relatively small-scale device to demonstrate that the basic technique meets the requirements for particle identification at PEP-II, and (2) engineering prototypes to demonstrate that important issues of design, construction, and performance in a fullscale device are under control.

As a summary, the requirements developed by the PID group as proof-of-principle tests are as follows:

Aerogel Cherenkov Counters: Tests with the ATC should measure a threshold curve for an $n=1.008$ block which has the dimensions expected in the actual device. The sensitivity to the 1.5 $\mathrm{T}$ magnetic field and orientation should be determined. The minimum number of photoelectrons observed for a $\beta=1$ particle should be about 10 , and the number observed below Cherenkov threshold should be consistent with Monte Carlo calculations.

Ring-Imaging Systems: For the DIRC and Fast RICH, the minimum number of photoelectrons observed for a $\beta=1$ particle at any angle should also be about 10 , motivated by pattern recognition and robustness considerations. The angular resolution should be measured at two or more polar angles.

\subsubsection{Outlook}

It is very difficult at this time to select a single technology as the prime candidate for the PID system. Prototypes to demonstrate the properties of each of these systems at the "conceptual" level are under development or test, but not yet finished. Moreover, to develop confidence that a large system would succeed, the schedule has to allow for the construction and extensive tests of a larger prototype sufficient to demonstrate performance and operational feasibility at the appropriate scale. 
The optimal PID system may be a hybrid. One example could be a DIRC in the barrel and a RICH or aerogel system in the endcap. This combination would solve some of the geometric problem: associated with the DIRC. A hybrid with aerogel in the barrel and a RICH in the endcap would address possible $\delta$-ray problems with an endcap aerogel device and the low photoelectron yield of the RICH at steeper incident angles in the barrel. Another hybrid design would be to replace the high refractive index aerogel layer with a time-of-flight system in order to reduce the total system cost. A decision to use a hybrid device would have to confront the higher cost of designing, constructing and maintaining several systems, but optimum physics performance may dictate such a configuration.

The collaboration has attempted to address at an early stage the question of identification and development of a suitable strategy for particle identification. An ad hoc committee was formed in April 1994 to examine the present state of knowledge of the proposed systems. The report of this committee endorsed the continued exploration of the three options presented in this Letter of Intent, with a focus on R\&D directed towards provided proof-of-principle and conceptual engineering designs for a review in the early fall. At that time, a decision should be taken as to which systems should continue to the stage of a large-scale engineering prototype. The choice between the Aerogel/Fast RICH technologies on the one hand, and the DIRC technology on the other has a major impact on the overall detector layout. There are also significant cost implications embodied in the PID system choice, including those driven by variabie requirements for radial space. At a minimum, the outer geometric envelope of the PID system must be defined as early as possible so that engineering studies can concentrate on critical path items such as the solenoid and calorimeter.

It is obviously advantageous to come to a resolution of the PID question as soon as possible, so that the necessary engineering studies for the entire detector can proceed on a time scale compatible with the development of the Conceptual Design Report.
It may be necessary to consider a hybrid system, but such a choice would have to confront the additional costs involved

Attempts will be made to choose a particle identification system on a time scale that poses minimal interference with the critical path 


\section{References}

[Ale93] R. Aleksan et al., DAPNIA/SED 93-03, 1993.

[Ale94] R. Aleksan et al., Nucl. Instr. Meth. A340 293 (1994).

[Alp94] V. Alperovich et al., Nucl. Instr. Meth. A340, 429 (1994).

[And92] D.F. Anderson et al., Fermilab 92/285, 1992.

[Ast89] D. Aston et al., Nucl. Instr. Meth. A283, 590 (1989).

[Atw92] W.B. Atwood, transparencies presented at the $B_{A} B_{A R}$ Geometry Meeting (1992).

[Bra93] H. Brauning, A. Breskin, R. Chechik, P. Mine and D. Vartsky, Nucl. Instr. Meth. A327, 369 (1993).

[Beu90] E. de Beuville et al., Nucl. Instr. Meth. A288, 157 (1990).

[DEL83] DELPHI Technical Proposal, CERN/LEPC 83-3, 1983; P. Aarnio et al., Nucl. Instr. Meth. A303, 233 (1991).

[Des94] R. DeSalvo et al., CERN-LAA/HC/94-04, 1994; CERNPPE/93-101, 1993; DEP Scientific Technical Information (1993).

[DIRC93] See for example, Proceedings of the Particle Identifcation Parallel Sessions for the $B$ Factory Collaboration Meetings, SLAC, Dec. 1993 and Feb. 1994; Paris, March 1994; DIRC R\&D Plans, Internal Note.

[Edg92] J.P. Edgecumbe et al., Proceedings of SPIE, Feb. 1992.

[Eic86] R. Eichler et al., Motivation and Design Study for a $B$ Meson Factory with High Luminosity, PSI Report SINPR-86-13, 1986.

[Eig79] See for example, G. Eigen and E. Lorenz, Nucl. Instr. Meth. 165, 166 (1979).

[Gil88] T.T. Giles et al., CLEO II Collaboration, Nucl. Instr. Meth. A252, 41 (1988); S. Ahmad et al., STAR Collaboration, Nucl. Instr. Meth. A330, 416 (1993). 
[Gro92] J.E. Grove et al., Nucl. Instr. Meth. A314, 495 (1992).

[Ham94] Hamamatsu Corporation (private communication).

[Hol87] A. Holroyd et al., Nucl. Instr. Meth. A261, 440 (1987).

[Hru94] L. Hrubesh, Lawrence Livermore National Laboratory (private communication).

[Kic93] H. Kichimi et al., Nucl. Instr. Meth. A325, 451 (1993).

[Mal93a] G. Malamud, P. Mine, D. Vartsky, A. Breskin and R. Chechik, Nucl. Instr. Meth. A335, 136 (1993).

[Mal93b] G. Malamud, Ph. Mine, D. Vartsky, B. Equer, P. Besson, Ph. Bourgeois, A. Breskin and R. Chechik, Report XLPNHE/93-13 (submitted to Nucl. Instr. Meth. ).

[Men94] D. Mendez, Lockheed (private communication).

[Oya94] J. Oyang, BABAR Note \#137, April 1994.

[Piu94] F. Piuz, CERN/RD26, CsI and Fast RICH (private communication, 1993). Note that the RICH subdetector group also participates in this program.

[Rat92] B. Ratcliff, BABAR Note \#92, Dec. 1992; P. Coyle et al., SLAC Report SLAC-PUB-6371, 1993.

[Seg90] J. Séguinot et al., Nucl. Instr. Meth. A297, 133 (1990).

[Shi94] X. Shi, BABAR Note \#142, May 1994.

[SLA93] Status Report on the Design of a Detector for the Study of CP Violation at PEP-II at SLAC, SLAC Report SLAC419, June 1993.

[Sji94] A. Spitkovsky and F.C Porter, "Study of Light Transport in Different CsI Crystal Geometries", BABAR Note \#146, May 1994.

[Suz86] S. Suzuki et al., IEEE Trans. Nucl. Sci. NS-33, 377 (1986); H. Saito et al., Nucl. Instr. Meth. A270, 319 (1988); J. Janoth et al., DESY Report DESY 93-119, 1993.

[Tso94] P. Tsou, Jet Propulsion Laboratory (private communication). 


\section{Electromagnetic Calorimeter}

$\mathbf{E}$ xcellent electromagnetic calorimetry is essential to the physics goals of the BABAR experiment at PEP-II. The reconstruction of as many $C P$ eigenstate decays as possible, especially those involving $\pi^{0}$ decays, requires us to measure photons with the best achievable energy and angular resolution, thereby providing the best possible $\pi^{0}$ and $B$ mass resolution. Similarly, high detection efficiency for low-energy photons is crucial, in particular for final states involving several $\pi^{0}$ 's. Achieving both of these design goals increases measurement sensitivity by providing high signal-to-buckground ratios for decay modes with small branching fractions, which are typical for $C P$ eigenstates. The calorimeter also contributes to particle identification, particularly to $e / \pi$ and $e / \mu$ separation.

Several choices for an electromagnetic calorimeter have been considered, including a fully-absorbing liquid-krypton calorimeter, a scintillating-fiber calorimeter and a thallium-doped cesium iodide crystal calorimeter. After detailed comparison of these options, as discussed in Section 8.2, the CsI(Tl) crystal calorimeter has been selected as the $B_{A} B_{A R}$ calorimeter technology.

\subsection{Calorimeter Requirements}

\subsubsection{Physics Requirements}

The average multiplicity in $B$ decays for both charged particles and photons is 5.5 , where the latter originate mostly from $\pi^{0}$ 's. Figure 8-1a shows the photon energy distribution from generic $B$ decays at PEP-II, where the boost of the $B \bar{B}$ system is $\beta \gamma=$ 0.56 . More than $50 \%$ of the photons have energies below $0.2 \mathrm{GeV}$. For most decays the $\pi^{0}$ momentum is low and thus the $\pi^{0}$ mass

Excellent photon energy and angular resolution, coupled with good low-energy detection efficiency, is important for reconstructing $C P$ eigenstates with high signal-to-background ratios

The average multiplicity in $B$ decays for both charged particles and photons is 5.5 
$A$ measurement of the $B^{0} \rightarrow \pi^{0} \pi^{0}$ branching ratio is important for evaluating possible penguin contributions to $\sin 2 \alpha$ as measured in the decay $B^{0} \rightarrow \pi^{+} \pi^{-}$

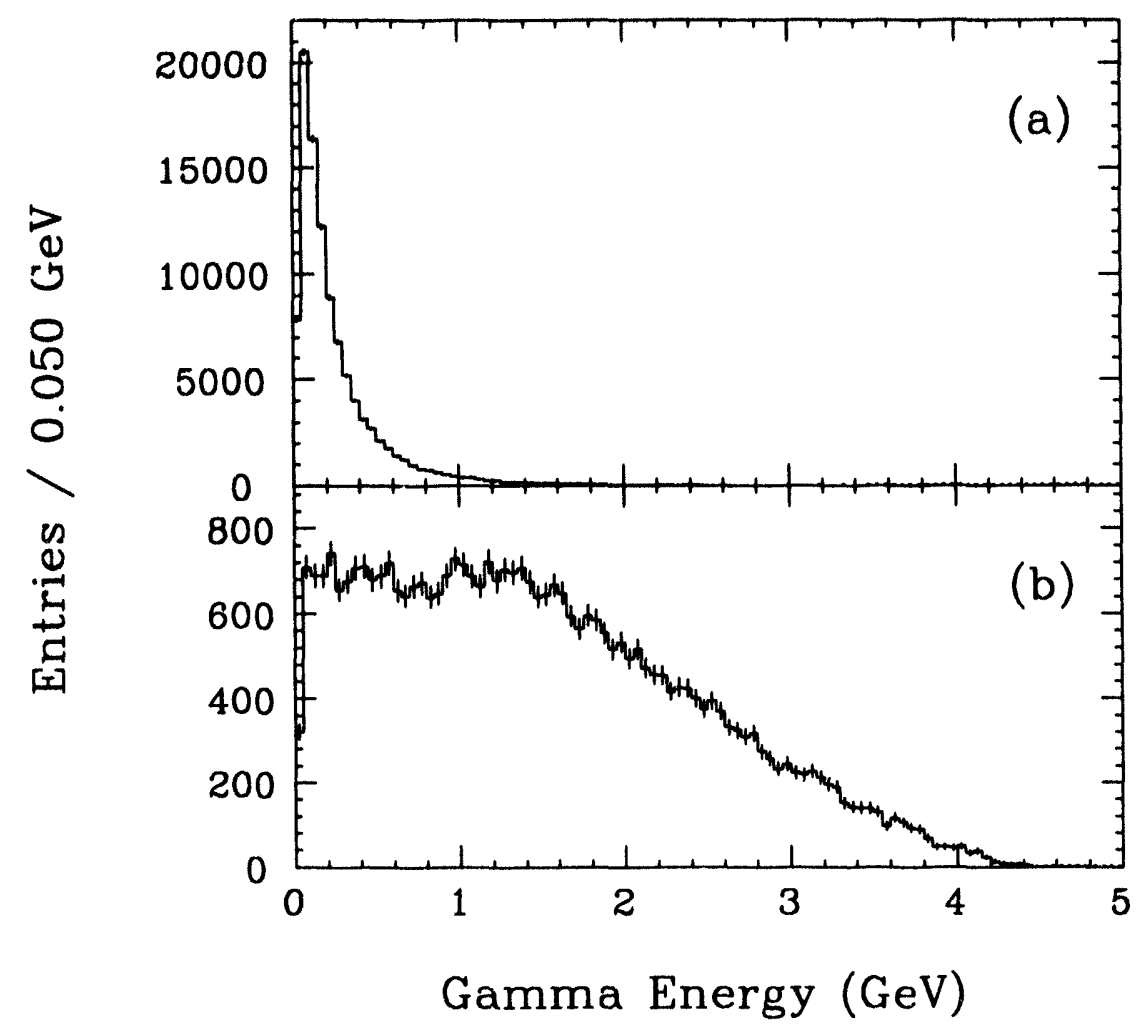

Figure 8-1. Photon energy spectrum in (a) generic $B$ decays and (b) $B^{0} \rightarrow \pi^{0} \pi^{0}$ events.

resolution is dominated by the photon energy resolution. In contrast, the photon energy spectrum from $B^{0} \rightarrow \pi^{0} \pi^{0}$ (which has a branching fraction $<10^{-6}$ [Dea93]) extends above $4 \mathrm{GeV}$ as shown in Figure 8-1b. In this case all $\pi^{0}$ 's have momenta above $1 \mathrm{GeV}$; the $B^{0}$ mass resolution is nonetheless still dominated by the photon energy resolution. A measurement of the branching ratio for $B^{0} \rightarrow$ $\pi^{0} \pi^{0}$ is important to determine penguin contributions to $B^{0} \rightarrow$ $\pi^{+} \pi^{-}$, one of the leading channels for measuring $\sin 2 \alpha$. In channels involving both charged particles and $\pi^{0}$ 's, such as $B^{0} \rightarrow J / \psi K^{* 0}$ and $B^{0} \rightarrow \rho^{ \pm} \pi^{\mp}$, where the $\pi^{0}$ 's have lower momenta, it is again the photon energy resolution that primarily determines the $B^{0}$ mass resolution [Ale94].

The importance of detecting very low-energy photons is demonstrated in Figure 8-2, which shows $\pi^{0}$ and $B$ meson reconstruction efficiency for generic $B$ decays versus minimum detectable photon energy. $B$ meson reconstruction efficiency for modes involv- 


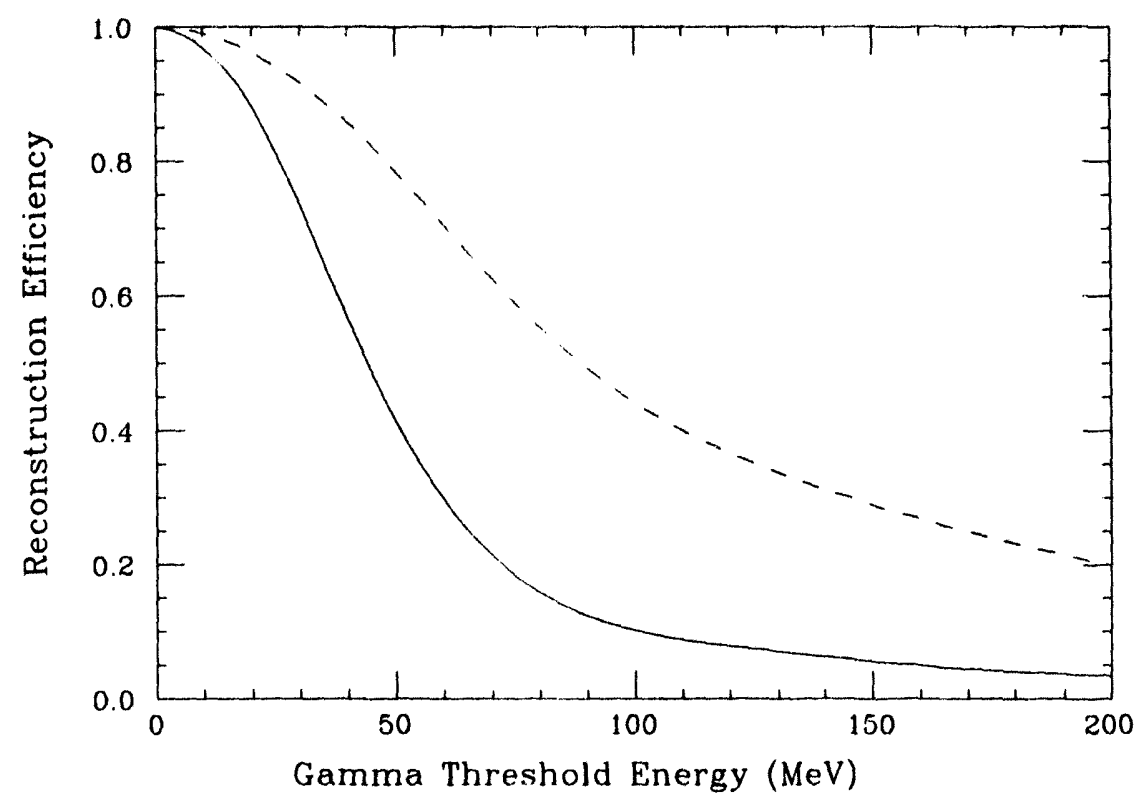

Figure 8-2. $\pi^{0}$ (dashed line) and $B$ meson reconstruction efficiency (solid line) vs. photon energy threshold for generic $B$ decays. (Taken from [HEL92]. Efficiencies exclude geometric acceptance.)

ing photons has been studied, both as a function of the minimum and maximum measured photon energy and as a function of the minimum and maximum detectable polar angle, using the ASLUND Monte Carlo. The channels considered are (i) $B^{0} \rightarrow \pi^{0} \pi^{0}$, producing four relatively high-energy photons, (ii) $B^{0} \rightarrow J / \psi K_{S}^{0}$, where $K_{S}^{0} \rightarrow \pi^{0} \pi^{0}$, producing four relatively soft photons, and (iii) $B^{0} \rightarrow \rho^{ \pm} \pi^{\mp}$, producing only two photons. Figure 8-3 shows the results. Generally, for these three modes the losses in the forward direction (upper left plot) are not as severe as for low-multiplicity all-charged modes, though $\pi^{0} \pi^{0}$ is somewhat worse than $\pi^{+} \pi^{-}$. In the backward direction (upper right plot) the demands on solid angle coverage are moderate.

Sensitivity to low-energy photons is the critical parameter for efficient $\pi^{0}$ detection. For example, if the photon energy were restricted to $E_{\gamma}>0.2 \mathrm{GeV}$, almost half the $J / \psi K_{S}^{0}$ decays would be lost (lower left plot). Similar losses can be expected for most modes with two $\pi^{0}$ 's. The exceptions are those few channels, such as $B^{0} \rightarrow \pi^{0} \pi^{0}$, which have high-momentum pions. In this case, the
For modes involving $\pi^{0}$ 's, losses in the forward direction are not as severe as for all-charged modes

Sensitivity to low-energy photons is critical for efficient $\pi^{0}$ detection 

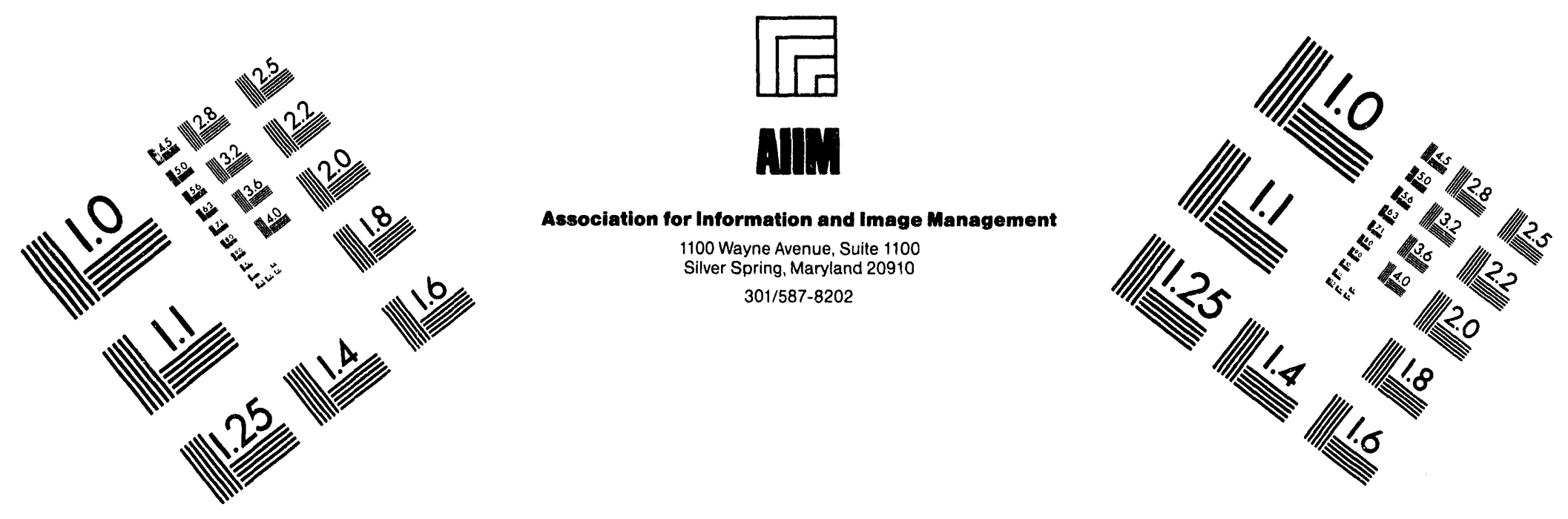

\section{Centimeter}

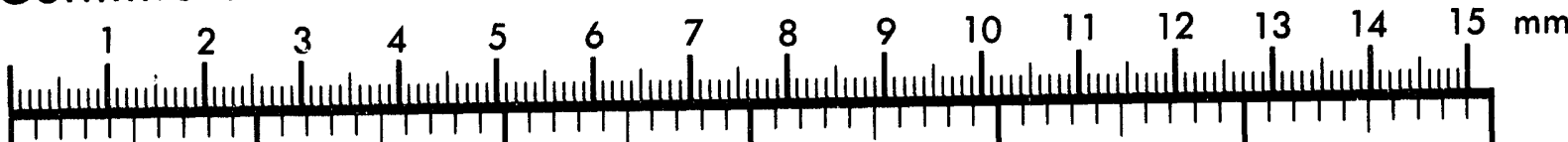

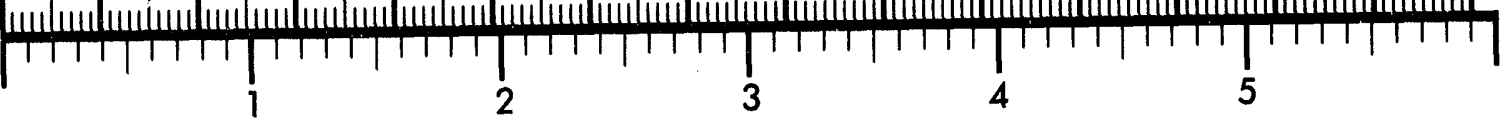
Inches
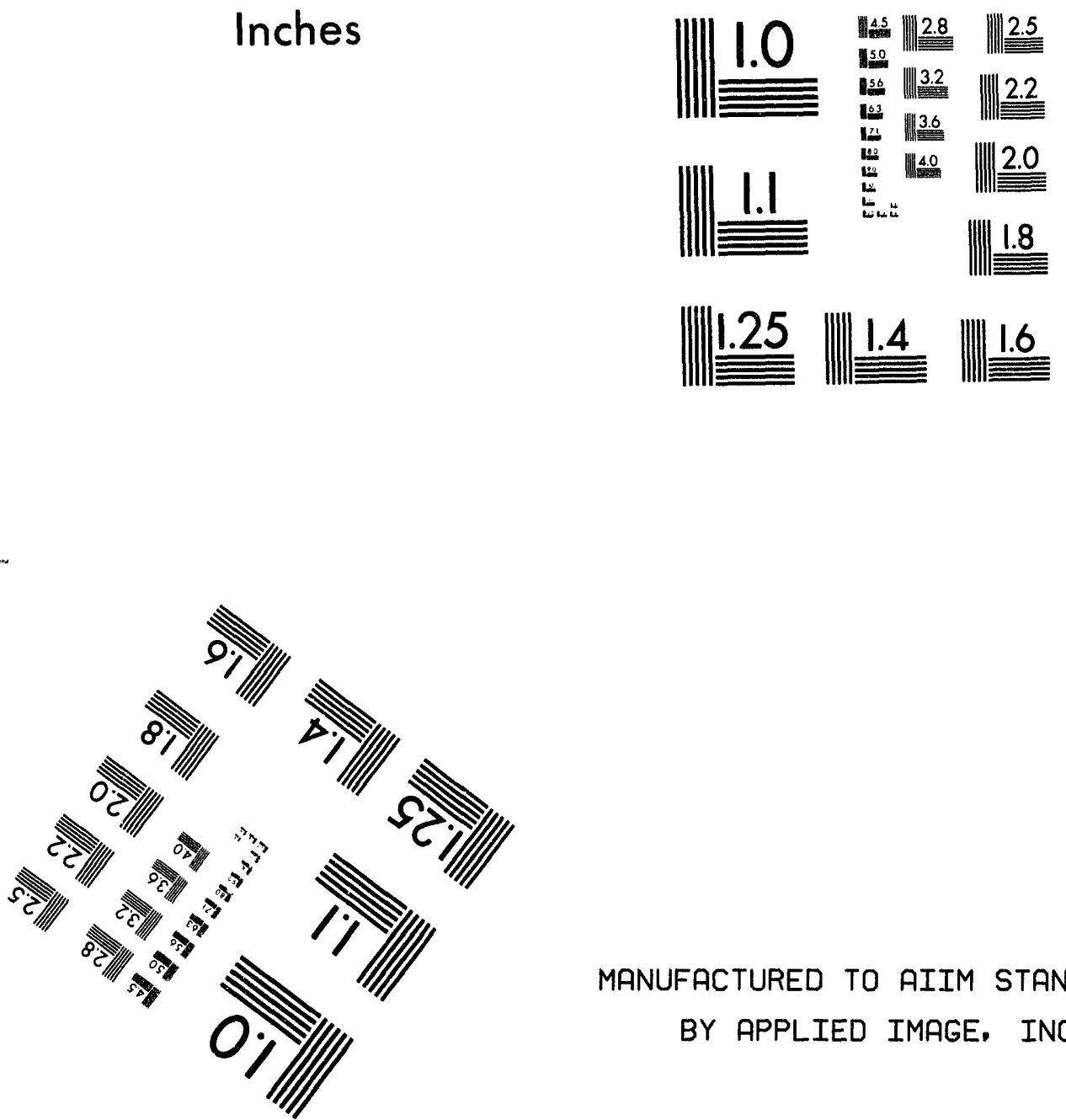

MANUFACTURED TO AIIM STANDARDS

BY APPLIED IMAGE, INC.

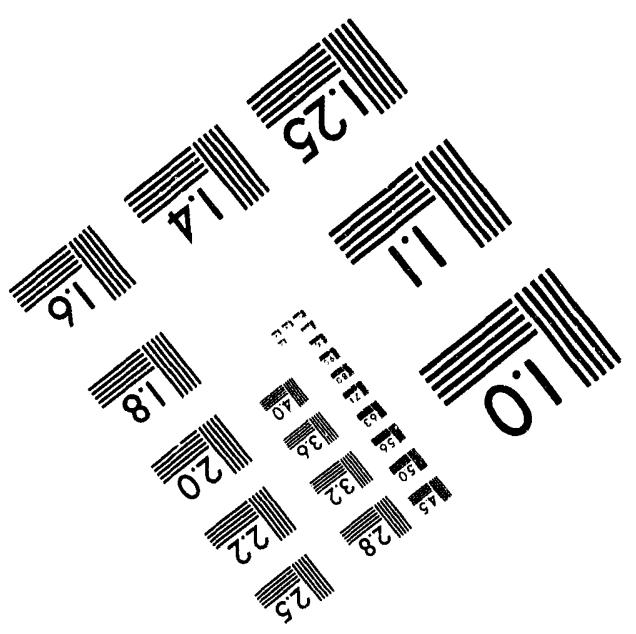



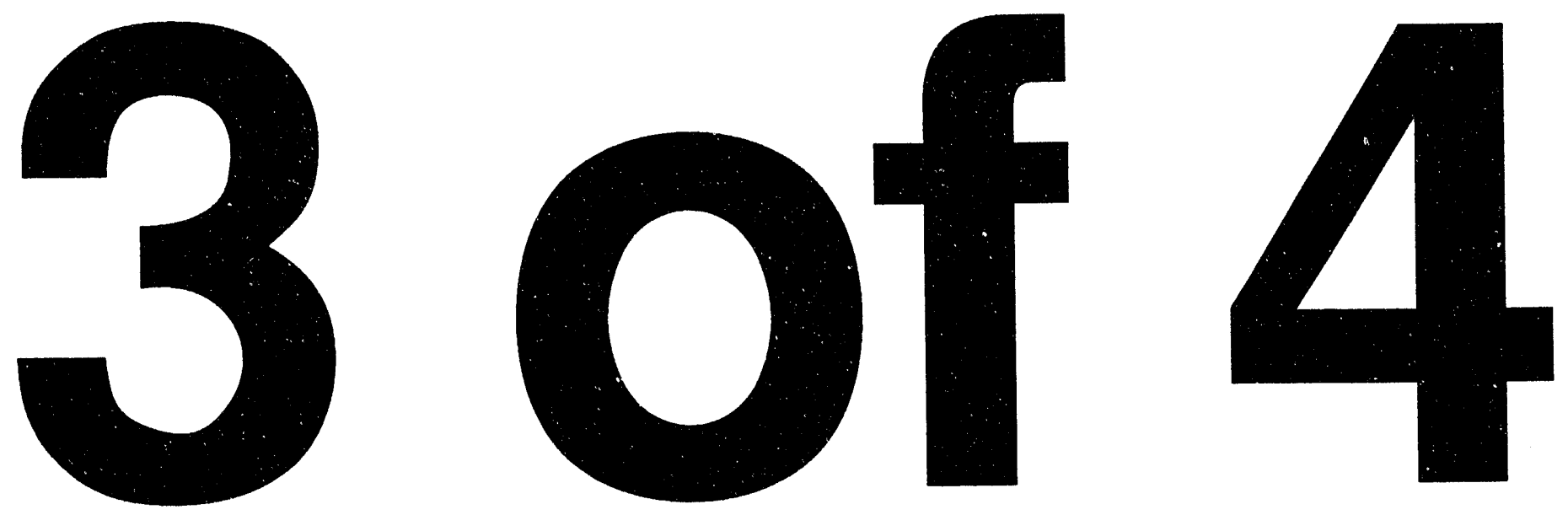

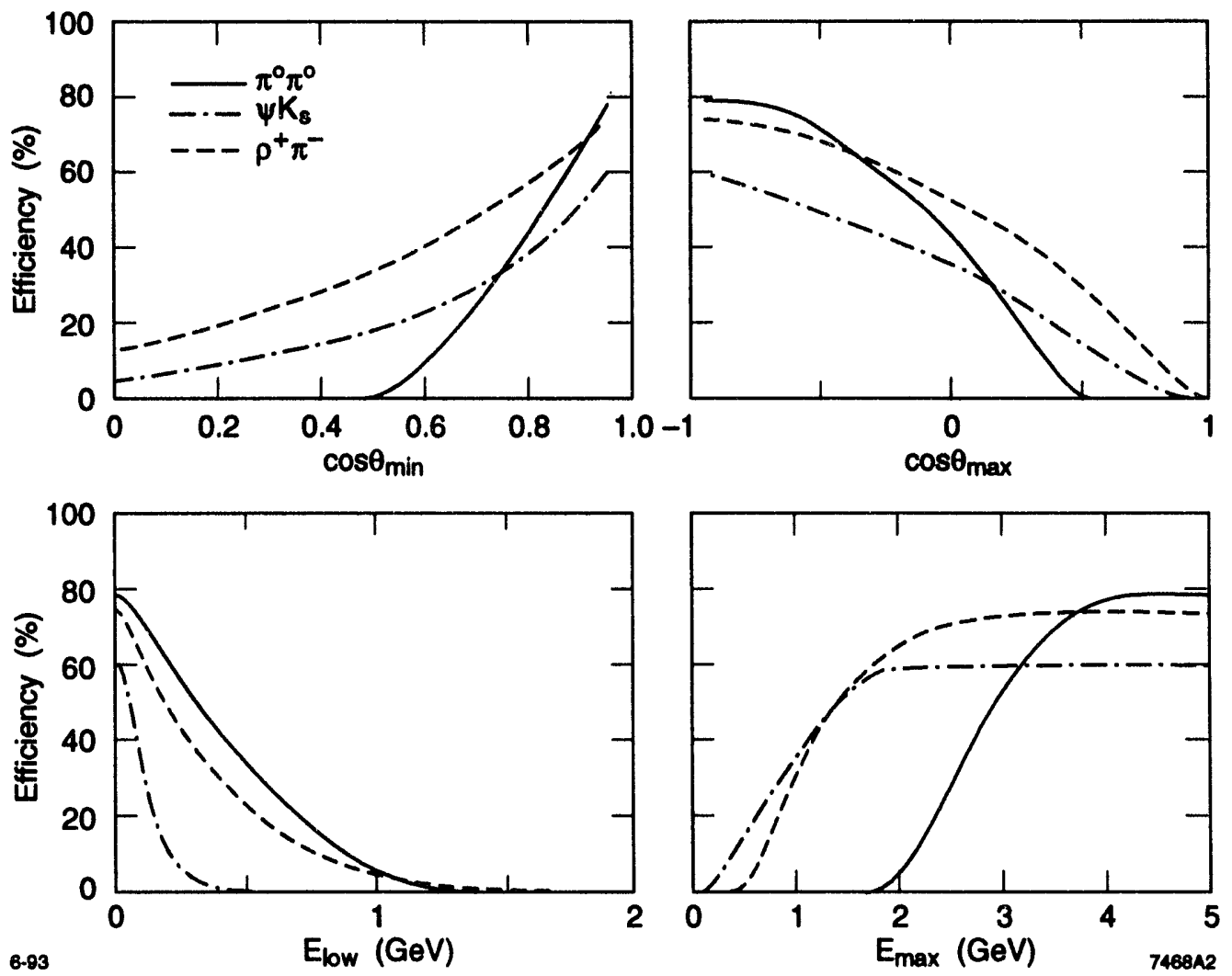

Figure 8-3. Starting clockwise from upper left, dependence of $B$ reconstruction efficiency on minimum detectable $\theta$, maximum detectable $\theta$, maximum detectable energy and minimum detectable energy for decay photons from $B^{0} \rightarrow \pi^{0} \pi^{0}$ (solid), $B^{0} \rightarrow J / \psi K_{S}^{0}, J / \psi \rightarrow \ell^{+} \ell^{-}, K_{S}^{0} \rightarrow \pi^{0} \pi^{0}$ (dash-dot) and $B^{0} \rightarrow \rho^{ \pm} \pi^{\mp}, \rho^{ \pm} \rightarrow \pi^{ \pm} \pi^{0}$ (dash).

$\pi^{0}$ 's are produced at or near the kinematic limit; therefore photons with energies up to $\sim 5 \mathrm{GeV}$ can be expected (lower right plot).

For $B$ meson reconstruction, it is important to achieve efficient photon detection in the energy range $0.02-5.0 \mathrm{GeV}$, along with good energy and angular resolution over the entire active solid angie. For other physics processes with low multiplicity final states, e.g. Bhabha scattering, $\tau$ decays, or tagged two-photon events, the energy range extends even higher, reaching values near that of the high-energy beam. In addition to reconstructing $B \bar{B}$ events, it is important to recognize rare decays of individual heavy mesons containing $b$ and $c$ quarks, and rare $\tau$ decays. Rejection of backgrounds 
in searches for decays with small branching fractions requires excellent lepton-hadron separation as well.

These requirements, along with the desire to achieve resolutions matching those of other detector subsystems, lead us to a design based on $\mathrm{CsI}(\mathrm{Tl})$ crystals. Taking CLEO-II as a baseline, the aim is to improve the energy resolution while minimizing the inactive material in front of, and within, the calorimeter and maximizing the solid-angle coverage. The energy resolution target of our design is:

$$
\frac{\sigma_{E}}{E}=\frac{1 \%}{\sqrt[4]{E(\mathrm{GeV})}} \oplus 1 \%
$$

The constant term is determined by front and rear leakage as well as by inter-calibration errors and light collection non-uniformity. The expression does not include electronic noise, because this contribution has been demonstrated to be negligible with the proposed readout system, even at low energies (see Section 8.3.3).

\subsubsection{Radiation and Backgrounds}

The radiation environment at PEP-II is more severe than that at existing $e^{+} e^{-}$colliders because of much higher beam currents. The calculated beam-related background rates shown in Chapter 4 provide a wide safety margin. However, in actual storage ring operations experience shows that most of the radiation exposure will come from injection and machine studies. Scaling typical CESR operation to the beam currents expected at PEP-II gives about $1.5 \mathrm{krad} /$ year at a radius of $45 \mathrm{~cm}$ and $0.5 \mathrm{krad} /$ year at $100 \mathrm{~cm}$ [Blu86]. The PEP-II masking system has been designed with graded apertures to prevent the deposition of large amounts of radiation in the interaction region due to accidental beam loss. It is still desirable, however, to provide a large safety margin by using detector components that are intrinsically radiation hard and can survive doses of tens of krads over the lifetime of the experiment.

The calorimeter is sensitive to cosmic ray and beam-related background. Simulations indicate that the latter is almost entirely caused by showers from beam-gas interactions or from off-axis beam par-
The excellent performance of the CLEO-II CsI(Tl) calorimeter can be improved upon by having better shower containment, less inactive material, lower electronic noise, and finer segmentation
The estimated radiation dose is $1.5 \mathrm{krad} /$ year at a radius of $\mathrm{r}=45 \mathrm{~cm}$ and $0.5 \mathrm{krad} /$ year at $\mathrm{r}=1 \mathrm{~m}$ 
Most beam-related background is caused by lost particles. Synchrotron radiation photons are unimportant

There are about 0.8 fake photons per $\mu$ s above $0.01 \mathrm{GeV}$
Three calorimeter options, $\mathrm{CsI}(T \mathrm{~T})$ as in CLEOII, liquid krypton as in $K E D R$, and scintillating fibers as in KLOE, were considered in detail ticles striking machine elements at or near the interaction region. Synchrotron radiation photons are a negligible source of background for the calorimeter [Zis91]. Shower particles have an energy distribution with a median around $500 \mathrm{keV}$ and are expected to cause abovt 120 depositions per $\mu$ s in the barrel, with a total energy of less than $0.15 \mathrm{GeV}$. There are on average 0.8 depositions per $\mu$ s above $0.01 \mathrm{GeV}$ that can produce fake photons in reconstructed physics events. Due to this background the shaping time for the calorimeter readout may need to be lowered from the optimal value for maximizing the signal-to-electronic-noise ratio.

\subsection{Choice of Technology}

The choice of calorimeter technology has an impact on many aspects of the detector design beyond the calorimeter itself. An ad hoc committee was formed to evaluate three proposed calorimeter technologies, a CsI(Tl) scintillating crystal device, a fully-absorbing liquid-krypton (LKr) device, and a fine-grained lead scintillatingfiber device. The committee considered relative physics performance, technology risk, relative costs and impact on the construction schedule.

The Committee's recommendation [BAB94], adopted by the Collaboration, was that a down-sized version of an earlier design of the $\mathrm{CsI}(\mathrm{Tl})$ calorimeter [SLA93] be chosen as the sole option for the Letter of Intent. A discussion follows of the issues upon which the decision was based.

\subsubsection{General Performance}

By increasing crystal length, decreasing electronic noise and improving light collection, $\mathrm{CsI}(\mathrm{Tl})$ as proposed for $B_{A} B_{A R}$ will have somewhat better intrinsic energy resolution than CLEO-II at low energies; a LKr calorimeter would likely achieve only comparable energy resolution to CLEO-II, and a scintillating fiber device will be significantly worse than either. Both scintillating fiber and $\mathrm{LKr}$ devices have, however, better angular resolution than $\mathrm{CsI}(\mathrm{Tl})$ by virtue of finer transverse and longitudinal segmentation. 
The CsI(Tl) device and the scintillating fiber devices have comparable detection efficiencies for low energy photons. The LKr device is poorer because tracks must traverse about $0.1 X_{0}$ of additional material in the dewars (and potentially convert therein) before reaching the active medium.

\subsubsection{Physics Considerations}

Performance was compared on two benchmark physics reactions $\left(B^{0} \rightarrow \pi^{0} \pi^{0}\right.$ and $\left.B^{0} \rightarrow \rho^{ \pm} \pi^{\mp}\right)$. As the $B$ mesons are nearly at rest in the center-of-mass system, it was found that energy resolution has a greater impact on $B$ mass resolution for final states containing $\pi^{0}$ 's than does angular resolution. Monte Carlo simulations show that while $\mathrm{CsI}(\mathrm{Tl})$ has the best mass mass resolution, $\mathrm{LKr}$ is only $\sim 5-10 \%$ poorer for these processes. A scintillating fiber device is found to be two to three times worse than either.

The main distinction between CsI and LKr for physics performance is, however, the $\sim 10-16 \%$ lower detection efficiency for $B$ meson final states with a single $\pi^{0}$ [Hea94]. This reflects a smaller detection efficiency for low energy photons in LKr.

The Committee was unable to evaluate some of the other more distinctive features of each calorimeter, including the added segmentation in the LKr and scintillation devices which allow better $\pi / e$ and $\pi / \mu$ separation, and improved beam background and hadron splitoff rejection.

\subsubsection{Technology Risk}

The $\mathrm{CsI}(\mathrm{Tl})$ calorimetry and scintillating fiber calorimetry were considered to pose little or no technological risks. If adequately radiation-hard CsI, required for the endcap region, is not available, several alternatives (longitudinal segmentation or pure CsI) are available without performance loss (Section 8.7.2). LKr calorimetry does not suffer from this problem, but does require the development of very thin state-of-the-art dewars to minimize inactive material; this was not considered a serious concern, as many cryogenic devices have been built and the Collaboration has expertise in this area.
CsI provides the best $B$ mass resolution, while scintillating fiber is distinctly worse

Soft-photon detection efficiency in $L K r$ is lower 


\subsubsection{Impact on the Detector}

LKr calorimetry dramatically increases the detector solenoid length and radius
Net cost of a LKr system, including increased magnet costs, is within $\sim 20 \%$ of the CsI option
The intrinsic differences in radiation length of CsI, LKr and scintillating fiber devices, as well as their different containment requirements result in dramatically different dimensions for the final engineered detector.

While CsI and scintillating fiber devices have a radial thickness of $35-45 \mathrm{~cm}$, the LKr device would require about $85 \mathrm{~cm}$ of radial space for the active medium and the dewars. The additional thickness of LKr implies that the detector length must also increase in proportion, requiring a superconducting magnetic shield for Q2.

LKr geometries having a conventional barrel/endcap and an endcapless design were considered. Both were found to have a serious impact on access to inner detector components, as well as beamline elements. These restrictions were judged to be unacceptable.

\subsubsection{Cost and Schedule Impact}

The baseline $\mathrm{CsI}(\mathrm{Tl})$ calorimeter is the most expensive of the three options. The cost risks arise from uncertainties in CsI raw material and production costs. The cost of the scintillating fiber device is driven by the photodetector cost. It is likely to be about half as expensive as CsI. The $\mathrm{LKr}$ costs are, in isolation, substantially less than those of CsI, and are driven primarily by mechanical and cryogenic components. While the baseline $\mathrm{LKr}$ device is about onehalf the cost of the CsI system, the cost impact on the rest of the detector was found to be significant. When the incremental costs for the large solenoid, flux return steel, Q2 shielding and muon detectors are included, the net difference between the CsI and LKr systems is reduced to $\sim 20 \%$. Furthermore, the $\mathrm{LKr}$ option requires a significantly larger commitment of funding to the solenoid and flux return in the initial two years of the project. 


\subsubsection{Conclusion}

While the CsI and LKr options both appeared adequate in physics performance, matching or exceeding that of CLEO-II; the scintillating fiber option was found to be inadequate. The serious impact of a LKr device on access to the inner detector and to PEP-II machine elements, and the likely need to redesign Q2 made a compelling case for CsI. The influence on the detector schedule resulting from the large increase in the solenoid and flux return costs associated with the LKr option was viewed as a serious problern.

The modest cost savings of the LKr were therefore not judged to be sufficient to outweigh other considerations and hence the CsI crystal calorimeter was chosen in preference to $\mathrm{LKr}$.

\subsection{Calorimeter Description}

\subsubsection{Cesium Iodide Properties}

CsI has a radiation length of $1.86 \mathrm{~cm}$, a density of $4.5 \mathrm{~g} / \mathrm{cm}^{3}$, and a Molière radius of $3.8 \mathrm{~cm}$. It has good mechanical and thermal stability, is only weakly hygroscopic, and is adequately radiation hard up to tens of krads. The light output of thallium-doped CsI is high (65000 photons/ $\mathrm{MeV}$ at room temperature). The photon spectrum peaks at $560 \mathrm{~nm}$, making $\mathrm{CsI}(\mathrm{Tl})$ suitable for silicon photodiode readout. The decay time of the scintillation light is about $1 \mu \mathrm{s}$.

\subsubsection{Geometry and Crystal Dimensions}

The geometrical configuration of the calorimeter shown in Figure 8-4 consists of a cylindrical barrel with an inner radius of $105 \mathrm{~cm}$ and a length of $288 \mathrm{~cm}$ along the beam line at the inner surface of the calorimeter. Forward (backward) endcaps extend the angular coverage for fully contained showers to $300(400) \mathrm{mr}$ in the laboratory. In order to preserve optimal performance for the Fast RICH particle identification option, the endcaps are tilted at $20^{\circ}$. This tilt is also
CsI is the preferred option for calorimetry

CsI(Tl) has high light output and a spectrum suitable for readout with silicon photodiodes
The CsI calorimeter covers the angular range between $300 \mathrm{mr}$ forward and $400 \mathrm{mr}$ backward, with endcaps tilted at $20^{\circ}$ for the benefit of the particle identification system 


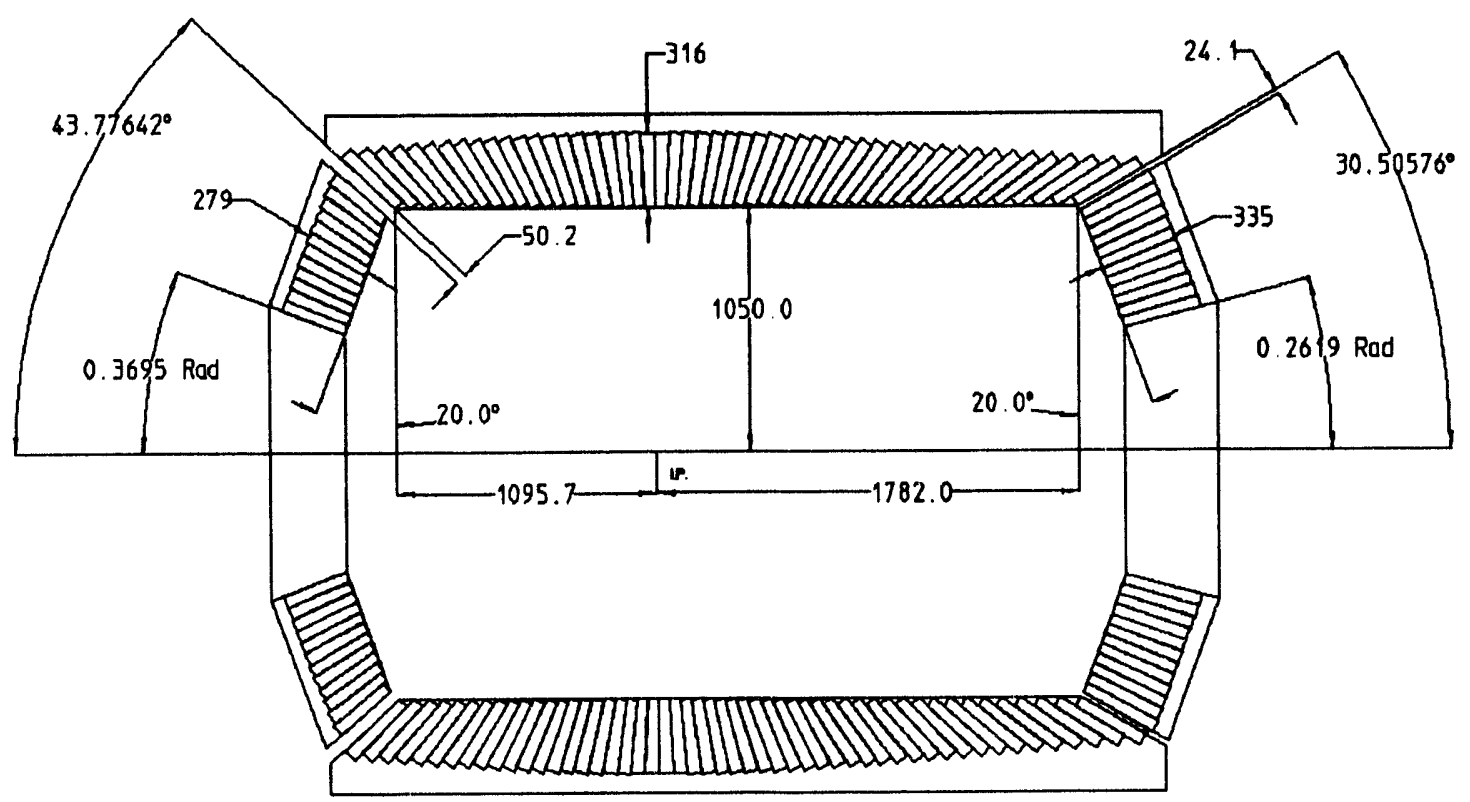

Figure 8-4. Configuration of the barrel and endcaps of the CsI (Tl) calorimeter.

Projective geometry is used in both the barrel and endcaps

There are a total of $\sim 10,000$ crystals

The crystal length is varied as a function of polar angle to match the average energy of the photons convenient for optimizing the gain of the fine mesh photomultiplier tubes used in the aerogel particle identification option. In addition, the amount of material in front of the endcap crystals is reduced. The calorimeter is mounted entirely inside the magnet solenoid.

The crystals in the barrel and endcaps are arranged in projective geometry, i.e. each crystal has the shape of a truncated pyramid with its longitudinal axis pointing to the interaction point. A typical barrel transverse crystal size is about $4.5 \times 4.5 \mathrm{~cm}^{2}$ at the front face, flaring out to about $5.5 \times 5.5 \mathrm{~cm}^{2}$ at the rear. Endcap crystals are smaller. There are a total of $\sim 10,000$ crystals, with $\sim 7,300$ crystals in the barrel and $\sim 2,700$ crystals in the endcaps.

The crystal length varies with polar angle to optimize cost while retaining good physics performance. In the forward endcap and forward part of the barrel 18 radiation lengths $\left(X_{0}\right)$ are used. The crystal length is reduced to $17 X_{0}$ in the central part of the barrel, $16 X_{0}$ in the backward region of the barrel, and $15 X_{0}$ in the backward endcap. This results in a total of 54 different crystal shapes and a total active volume of $8.1 \mathrm{~m}^{3}$. 


\subsubsection{Light Collection and Readout}

The crystals are read out at their rear face by silicon photodiodes. Optimal energy resolution is achievable only if (a) a high light collection is obtained, (b) the light collection is uniform, and (c) the electronic noise contribution of the readout chain is minimal. In addition, the readout system should be reliable, stable, compact, affordable and operate efficiently in a high magnetic field. These criteria can be met by a readout system using silicon PIN photodiodes coupled to plastic wavelength shifter plates and low-noise charge-sensitive preamplifiers [Hol88].

Because $\mathrm{CsI}(\mathrm{Tl})$ is slightly hygroscopic, the entire calorimeter is kept in a low-humidity atmosphere. In addition, the crystals are sealed with a thin coating that does not affect their optical properties. A candidate for such a coating is Teflon AF with a thickness of about $50 \mu \mathrm{m}$. Long term aging properties of this material are under study.

Excellent energy resolution is correlated with high light output from $\mathrm{CsI}(\mathrm{Tl})$. Studies have shown that the light yield is considerably improved by surrounding the crystals with an optimized white diffuse reflecting material. Choices that have been investigated are multilayers of PTFE teflon, Tyvek, aluminum foil, and $\mathrm{TiO}_{2}$.

Because the crystals have a trapezoidal shape, light collection efficiency in a fully polished crystal is not uniform along the length. The common method for minimizing this effect consists of roughening the surface at different places until the light collection is uniform. An alternative method is to vary the reflectivity of the wrapping material.

Figure 8-5 shows the crystal-readout assembly utilizing a wavelength shifter plate or "fluorescent flux concentrator" (FFC) [Lor86] and two silicon PIN diodes. The FFC, which covers the entire rear face, consists of a square acrylic plate doped with a fluorescent dye. The FFC combines two functions: wavelength shifting and light concentration. A large fraction of the CsI(Tl) scintillation light is absorbed by the FFC and shifted towards longer wavelengths where the photodiodes have higher quantum efficiency. The FFC also acts as a wave guide; the re-emitted light is concentrated along the FFC side faces. This allows the use of small-area $\left(\sim 1 \mathrm{~cm}^{2}\right)$ rectangular
The crystals are read out with plastic wavelength shifters and small photodiodes

The crystals are sealed with a thin coating of Teflon $A F$

The magnitude and uniformity of the light output are governed by surface treatment and choice of wrapping material

The fluorescent flux concentrator/small photodiode readout system gives excellent performance at a lower cost than direct photodiode readout 


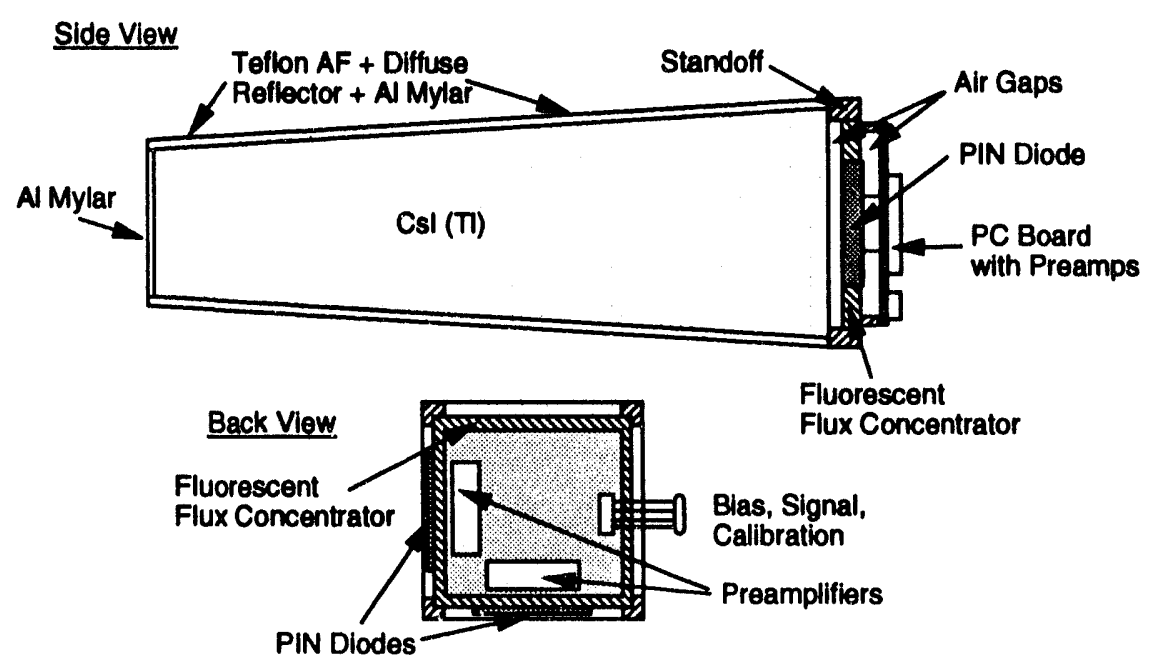

Figure 8-5. A CsI (Tl) readout assembly (not to scale)

Direct readout of the CsI crystal with large photodiodes is also an option

Careful electromagnetic shielding of the readout system is vital for good noise performance photodiodes, which have low noise and are less expensive than largearea devices. For redundancy, two photodiodes are glued to two adjacent sicle faces of the FFC.

The photodiodes can, alternatively, be placed directly on the back of the FFC. Botls the shifted light and a portion of the primary light can be detected, thus increasing the detection efficiency. This design is particularly favorable if the FFC spectral absorption range does not extend over the entire $\mathrm{CsI}(\mathrm{Tl})$ emission wavelength region. The option of gluing two large-area $\left(18 \times 18 \mathrm{~mm}^{2}\right)$ photodiodes directly on the crystal is also being considered. This technique is somewhat more expensive, but can potentially achieve the best noise performance. Direct readout with four small-area photodiodes is used in the CLEO-II calorimeter [Kub92].

The Hamamatsu S3588 diode (or an equivalent type with similar geometry) is a candidate for the FFC readout system. The diode has an active area of $3.4 \times 30 \mathrm{~mm}^{2}$. Each photodiode is coupled to a low-noise, charge-sensitive preamplifier, which incorporates the photodiode bias, and a network consisting of a $100 \mathrm{M} \Omega$ resistor and a filter.

Shielding is needed for protection against coherent pickup noise. The photodiode is shielded by a metallic cap and the length of the connection between the photodiode and the preamplifier is minimized and fully shielded. The preamplifier has its own EMI shield- 
ing and microphonic protection. The entire calorimeter is itself shielded by virtue of the metallic support structure.

Full-size crystals have been tested both with FFC/photodiode readout and with direct photodiode readout. Figure 8-6 shows the ${ }^{22} \mathrm{Na}$ energy spectrum measured with $\mathrm{FFC} /$ photodiode readout. An $18 X_{0}$ crystal with a front cross section of $3.4 \times 3.4 \mathrm{~cm}^{2}$ and a rear cross section of $4.5 \times 4.5 \mathrm{~cm}^{2}$ was used. A $4 \times 4 \mathrm{~cm}^{2} \mathrm{FFC}$ containing a red BASF laser dye (\#339), read out with a single photodiode (Hamamatsu S2575), yields 5200 photoelectrons ( $p e$ ) per $\mathrm{MeV}$, and an equivalent noise charge (ENC) of $280 e^{-}(\mathrm{rms})$ for a $3 \mu$ s shaping time [Hol88]. This corresponds to an equivalent noise energy of $55 \mathrm{keV}$. For comparison, the Crystal Barrel Collaboration achieves a noise energy of $200 \mathrm{keV}$ with an FFC/photodiode system [Ake92], while the CLEO-II direct photodiode readout yields a noise energy of $500 \mathrm{keV}$ [Kub92].

Using direct photodiode readout of full-size crystals, a noise level identical to that of the FFC/photodiode readout has been achieved. Coupling a single Hamamatsu photodiode (model S3584-05 with an active area of $28 \times 28 \mathrm{~mm}^{2}$ and a $500 \mu \mathrm{m}$ thick depletion layer) via a silastic cookie to a $\mathrm{CsI}(\mathrm{Tl})$ crystal with the same dimensions as for the FFC-photodiode readout, a yield of $12000 \mathrm{pe} / \mathrm{MeV}$ and an equivalent noise energy of $55 \mathrm{keV}$ [Eig94] has been measured. For purposes of calculating the electronic noise contribution to the low-energy photon resolution, these bench test values have been increased to $100 \mathrm{keV} /$ crystal for the full-size calorimeter system. This increase is intended to conservatively account for coherent noise, component parameter spreads and variations in crystal light output, wrapping, and the possible need for using shorter shaping times to minimize background effects.

\subsubsection{Mechanical Support Structure}

The CsI crystal support structure design must combine mechanical strength with minimal mass in front of and between the crystals. The calorimeter support structure must contain the individual crystal elements with good mechanical integrity for positioning crystals, without long-term creep and sag of the crystals or the loaded structure. It may be necessary to provide mounting and attachment
Both FFC/photodiode and direct photodiode readout of large $C s I(T l)$ crystals have achieved an equivalent noise energy of $55 \mathrm{keV}$
The crystal support structure must not use the crystals as structural elements, as CsI is a plastic material 


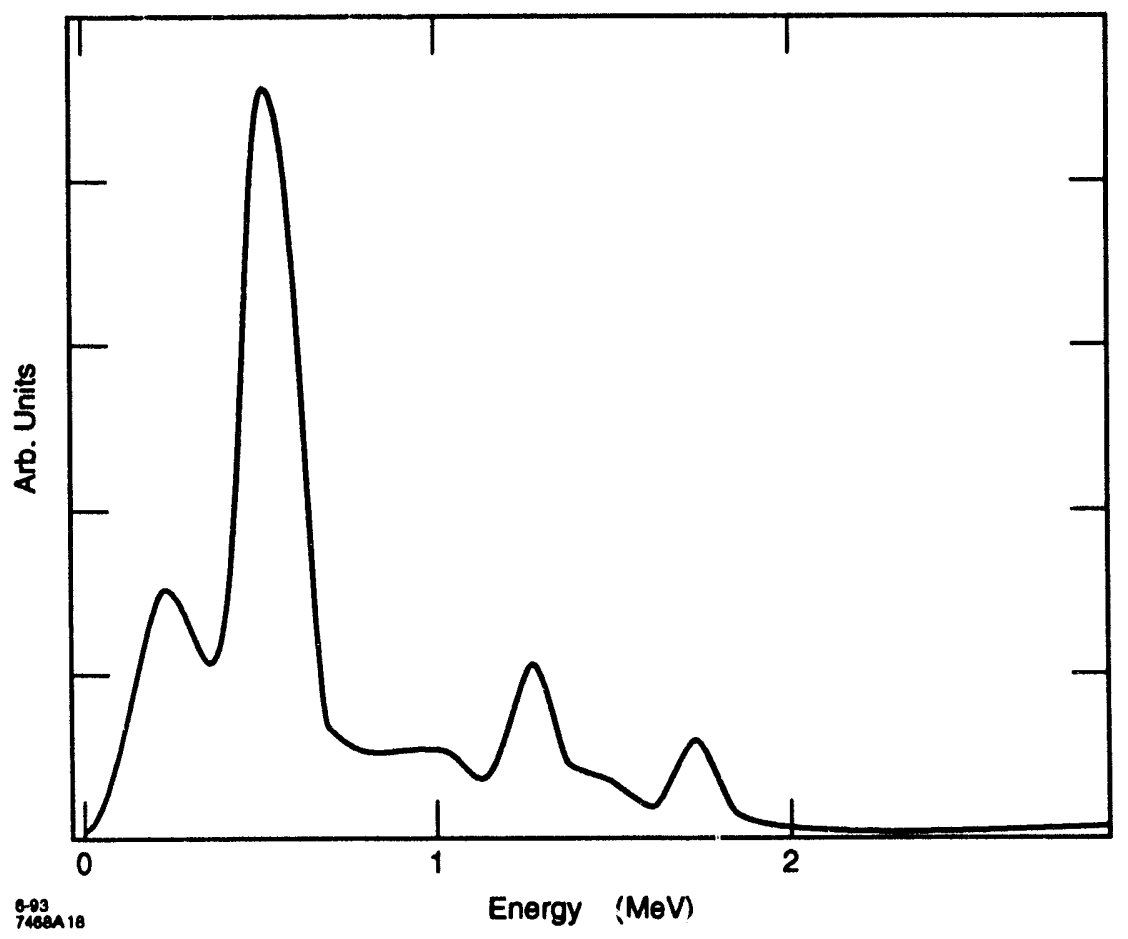

Figure 8-6. Energy spectrum of a ${ }^{22} \mathrm{Na}$ source measured with an $18 X_{0} C s I(T l)$ crystal with $F F C$-photodiode readout

Using finite-element analysis, a barrel mounting structure with individual $75 \mu \mathrm{m} \mathrm{Al}$ compartments has been shown to be of adequate strength points for inner detector elements such as the particle identification system and tracking drift chamber. It must also allow for temperature and humidity control as well as electrical shielding and grounding for the photodiodes. Cesium iodide is a plastic material. The crystals therefore cannot be used as structural elements or be placed under significant loading. The support structure must also meet safety and seismic standards for the SLAC environment.

These design requirements have been met with a calorimeter support structure that consists of crystal compartments with $75 \mu \mathrm{m}$ thick aluminum walls attached to aluminum or composite fiber inner and outer cylinders and end-plates. Individual compartments are spot-welded or epoxied together into a number of subsections reflecting the symmetry of the structure. For final assembly, the subsections are attached to an inner and outer cylinder with the necessary strength to support the barrel structure from the magnet coil cryostat. The endcap structures, of a similar design, are mounted onto the movable flux return doors, which are split vertically to allow access to the inner parts of the detector. To validate the 


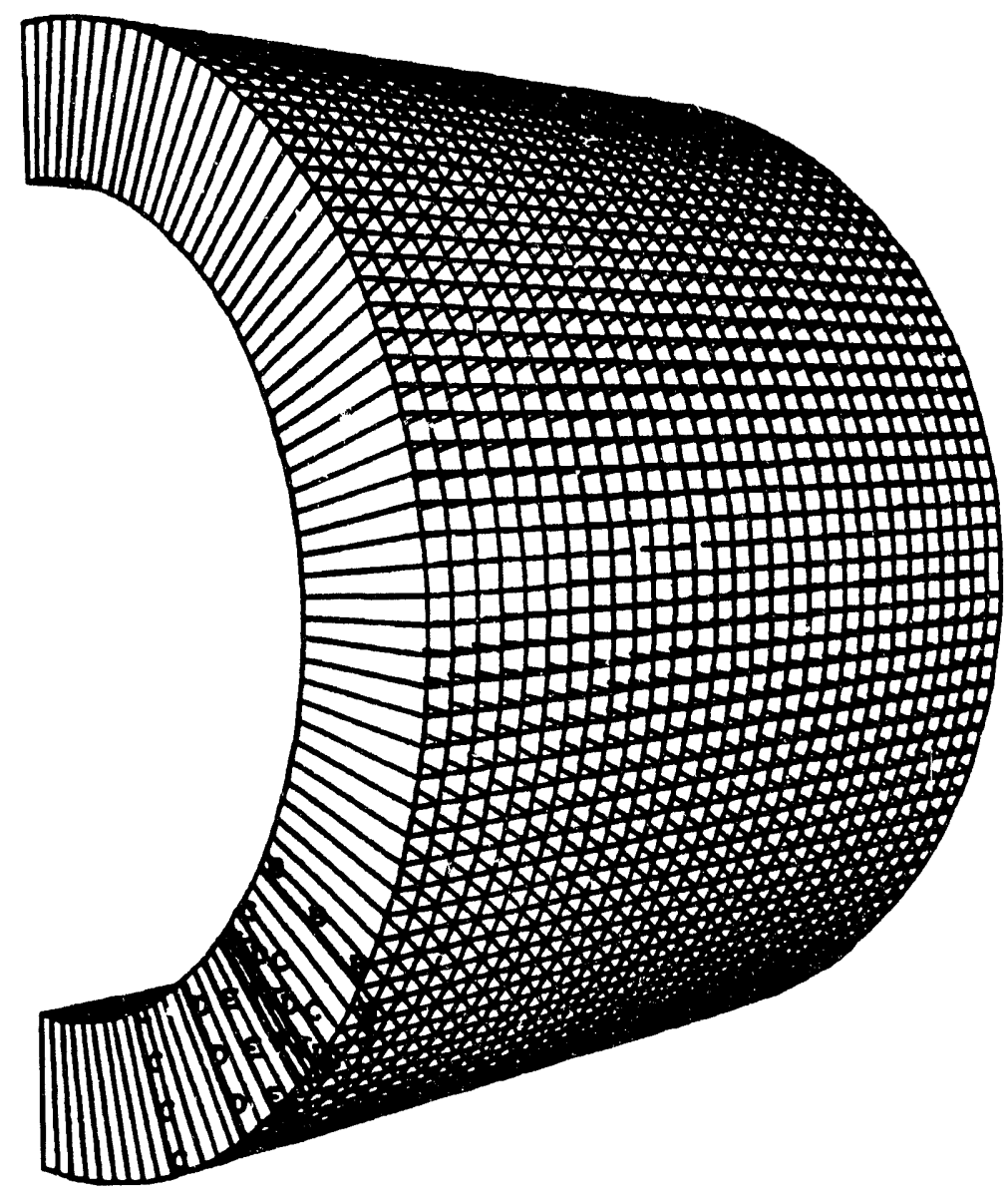

Figure 8-7. Finite-element model of the mechanical support structure for the barrel.

conceptual design, a finite-element analysis of the barrel structure has been performed; the results, an example of which is shown in Figure 8-7, indicate that the structure is quite rigid and is free of significant sag or deformation.

The impact on physics performance for this design is currently being assessed. Preliminary GEANT modeling with $150 \mu \mathrm{m} \mathrm{Al}$ between crystals indicates that the $\pi^{0}$ mass resolution is not significantly affected. Alternative crystal compartment schemes have not been modeled and analysed for strength and performance; however it is clear that the compartment material thickness will be greater for schemes involving multiple crystals per compartment.
The material in the compartments has a negligible effect on $\pi^{0}$ mass resolution 
Composite structures are more expensive and provide little performance improvement
A dynamic range of 1718 bits, along with an effective resolution of 10 bits, is required

Two independent photodiode/amplifier channels per crystal are provided to the digitizer

The amplitude and timing of a shaped $\mathrm{CsI}(\mathrm{Tl})$ signal are extracted from waveform sampling and digitization
Composite structures of carbon and glass fiber composite such as the L3 BGO structure [Leb90] and the Tau/Charm structure [Per91] have also been examined in terms of performance and cost, and are conservatively estimated to be at least twice the cost of a simple metal structure for a minimal improvement in performance.

\subsubsection{Electronics}

The prime requirement for the calorimeter data acquisition system is that it not significantly impact the calorimeter resolution for energy depositions from the lowest photon energy of interest $(\sim 0.02 \mathrm{GeV})$ to the maximum Bhabha energy. Taking into account the transverse spread of electromagnetic showers and resolution requirements for calibration signals from radioactive sources (Section 8.5), this requirement mandates a dynamic range of 17 to 18 bits, which covers the range from at least $100 \mathrm{keV}$ to $12 \mathrm{GeV}$, along with an effective resolution of 10 bits.

Optimal shaping times for signals from PIN diodes are in the range of 1-4 $\mathrm{s}$. The required noise characteristics of the crystal preamplifier are an rms intrinsic noise of no more than $150-200 e^{-}$for the unloaded preamplifier and a gradient of $\leq 2 e^{-} / \mathrm{pF}$. The charge will be integrated by a local shaping amplifier and then digitized. Alternatively, digitization could occur outside the calorimeter. To insure against signal loss due to the failure of the diode and/or preamplifier, two readout channels per crystal are kept independent at least up to the digitizer.

The proposed data acquisition system (see Chapter 11) is based on untriggered clocked digitization using fast sampling ADCs. A digital pipeline is used both for the development of trigger decisions and for storage of the data until those decisions have been made. Within this context, a shaped detector signal is sampled and digitized several times in order to determine its amplitude. The fit to several contiguous time bins, required to ascertain the total charge in the pulse, also yields an event time with good resolution, well below $100 \mathrm{~ns}$. This, in turn, allows the efficient rejection of uncorrelated signals from beam background and delayed neutron capture. The 1$4 \mu$ s shaping time implies a digitization rate between 1 and $10 \mathrm{MHz}$. 
Section 11.5.1 describes the electronics design proposed to achieve the required dynamic range, resolution, and sampling rate.

\subsection{Calorimeter Performance}

\subsubsection{Factors Influencing Energy Resolution}

A number of effects beyond shower statistics contribute to the calorimeter resolution; different effects dominate in different energy regions. Reducing the effect of one contribution may facilitate doing better with another. Most of these lead to design decisions which have little direct effect on cost.

The main contributions to the energy resolution, beginning with those related to shower development and ending with effects external to the calorimeter, are:

a) Fluctuations in energy loss caused by leakage out of the front and the rear of the crystals, losses due to the staggered crystal geometry, and energy deposited in the material between crystals.

b) Transverse energy spread, which requires optimization of clustering algorithms to minimize the combined effects of energy spread, noise, and backgrounds.

c) Photon statistics of collected scintillation light.

d) Non-uniformity of light collection.

e) Incoherent electronic noise associated with the readout device.

f) Pickup and other coherent noise, which should be kept well below the level of incoherent noise.

g) Digitizer resolution, which can be made negligible.

h) Calibration of the energy scale, including crystal inter-calibration and the effects of time or temperature dependence.

i) Material en route to the calorimeter, which affects efficiency as well as resolution.
A number of effects other than shower statistics contribute to the energy resolution 
Electronic noise dominates the resolution at low photon energies, while pattern recognition and shower leakage are the most important effects at higher energies
The random contribution of energy from lost particles can have a significant effect on the resolution for lowenergy photons. This may motivate the use of shorter shaping times in the $C s I(T I)$ readout system j) Beam-related backgrounds.

Noise (listed in items (e) and (f)) affects the low-energy range, while rear leakage (item (a)) dominates at the highest energies. Fluctuations in energy loss (item (a)), along with contributions from non-uniform light collection and calibration effects (items (d) and (h)), are expected to yield a constant term of the size shown in Equation 8.1. Transverse shower spread and choice of reconstruction algorithms (as mentioned in item (b)) are important to the energy resolution over the intermediate range. Finally, other effects like material in front of the calorimeter and beam-related backgrounds $(\mathbf{i})$ and $(\mathbf{j}))$ can impact the energy resolution, mainly at low energies.

The effect of machine-related backgrounds on pileup and resolution for low-energy photons has been studied [Sny92, Lev94]. Using EGS, $0.1 \mathrm{GeV}$ photons at normal incidence have been simulated together with a randomly distributed background from lost beam particles; both are integrated over a $2.0 \mu$ s shaping time. This simulation does not include material in front of and between crystals. Simple energy sums over rectangular crystal clusters are formed.

To estimate the effect of the background pileup, the rms energy spread for a range of cluster sizes has been computed with and without pileup. With 16 crystals summed in a cluster, the rms spread including background is $2.5 \pm 0.2 \mathrm{MeV}$, to be compared with an rms spread of $2.1 \pm 0.2 \mathrm{MeV}$ without background. This background thus exceeds the expected contribution from electronic noise of $0.4 \mathrm{MeV}$ $(100 \mathrm{keV} /$ crystal). For the case of 25 (36) crystals, the calculation shows an increase in rms energy spread from 1.8 (1.6) MeV without background pileup to 2.6 (2.5) $\mathrm{MeV}$ with background. Thus, amplifier noise, shaping time and pileup all must be considered simultaneously.

\subsubsection{Expected Performance for Photons}

The expected calorimeter performance has been studied using a GEANT Monte Carlo simulation of the full detector. Previous studies have shown that $18 X_{0}$ crystals are necessary to keep the resolution, $\sigma_{E} / E$, below $1 \%$ at energies above $1 \mathrm{GeV}$, whereas shorter 
crystals give satisfactory performance at lower energies [SLA93]. Taking into account the correlation between (average) photon energy and polar angle caused by the boost of the center-of-mass system, crystal lengths are varied as a function of polar angle. The GEANT model of the calorimeter contains a description of the barrel with $17 X_{0}$ crystals and the forward and backward endcaps with $18 X_{0}$ and $15 X_{0}$ crystals, respectively. The model includes $150 \mu \mathrm{m}$ of aluminum as structural material between the crystals.

In the study described in this section, showers are reconstructed in the barrel part only. Their energies are determined by summing the energy deposited in a $5 \times 5$ crystal cluster, including an incoherent electronic noise contribution of $100 \mathrm{keV}$ per crystal. Since the distributions typically show a non-Gaussian tail on the low energy side, the resolution is defined as $\sigma=\mathrm{FWHM} / 2.36$. In order to account for effects not included in the GEANT simulation, particularly non-uniform light collection and calibration uncertainties, a constant term of $0.5 \%$ is added in quadrature to $\sigma_{E} / E$. "Truncated acceptance" $(\epsilon)$ is defined as the fraction of entries under a Gaussian with $\sigma$ defined above and with the observed height. Equivalently, $1-\epsilon$ is equal to the percentage of events lying in the non-Gaussian tail.

Initially, the performance of the barrel calorimeter has been studied without any material in front of it. Figure 8-8 shows the energy resolution and truncated acceptance vs. energy, for photons with polar angles near $90^{\circ}$. Also shown (solid line) is the target energy resolution, listed in Equation 7.1. The Monte Carlo prediction, based on $17 X_{0}$ crystals, is just below the target resolution at all energies except the very highest.

The sawtooth pattern at the inner radius of the barrel calorimeter causes some performance degradation towards small polar angles. Table 8-1 summarizes the Monte Carlo results for energy resolution and truncated acceptance vs. polar angle for $0.1 \mathrm{GeV}$ and $1 \mathrm{GeV}$ photons. One can see a $40 \%(20 \%)$ increase in resolution for $0.1 \mathrm{GeV}$ $(1 \mathrm{GeV})$ photons at the largest $|\cos \theta|$, where the break between barrel and endcap occurs. This increase is due to an increased leakage contribution, mostly out the uncovered crystal side faces.

The leakage problem can be somewhat alleviated, at the cost of a substantial increase in the number of electronics channels, by decreasing the crystal transverse dimensions, thereby reducing the
The calorimeter resolution has been modeled with a GEANT simulation

The simulated energy resolution is consistent with the target resolution 


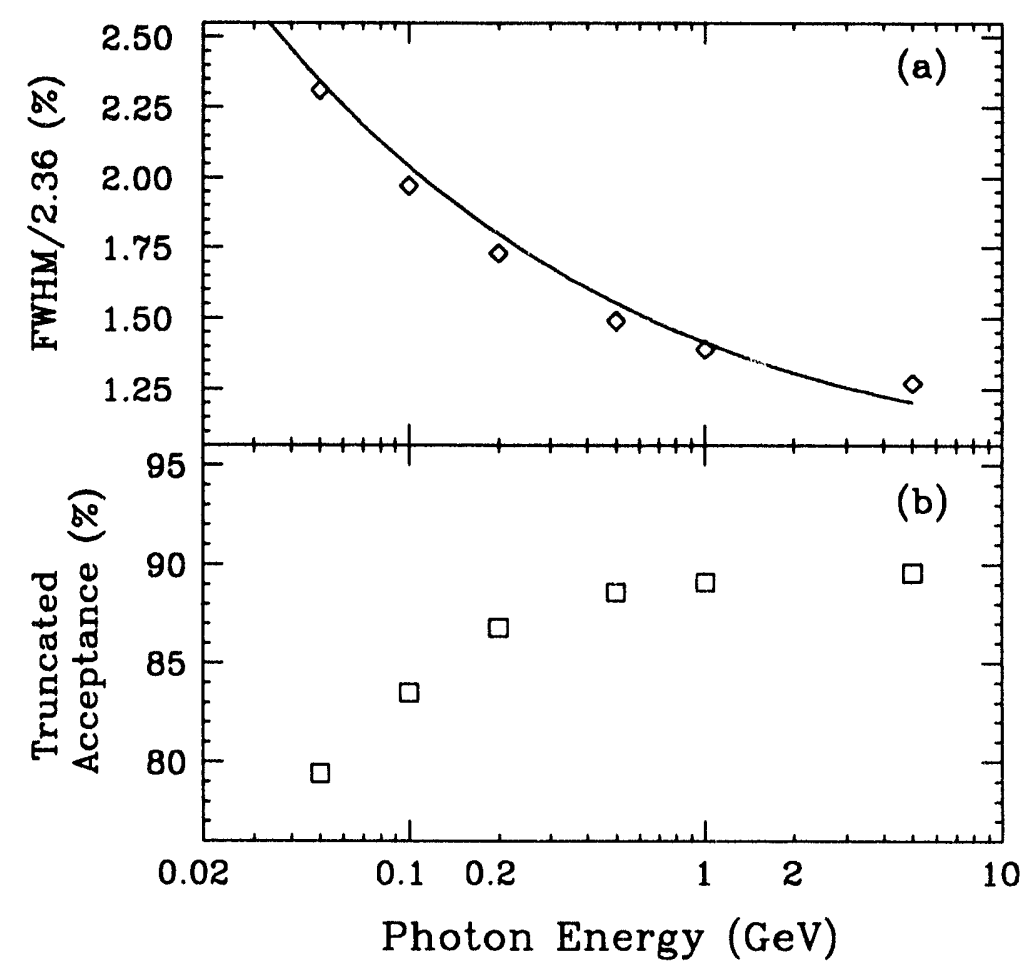

Figure 8-8. (a) Expected energy resolution, $\sigma_{E} / E$, and (b) truncated acceptance vs, energy for photons with polar angles near $90^{\circ}$. The solid line shows the target energy resolution, Equation 8.1. The plateau in truncated acceptance at $90 \%$ is a consequence of the low-energy tail of the energy distributions.

sawtooth effect. Lengthening the crystals at large polar angles can cure the rear leakage problem, although the required lengths are impractical. Studies have shown that simply filling in the sawtooth pattern degrades efficiency and uniformity of light collection significantly [Spi94].

Finally, the effect on energy resolution and truncated acceptance from inactive material between crystals is shown in Table 8-2 for $0.1 \mathrm{GeV}$ and $1 \mathrm{GeV}$ photons for polar angles near $90^{\circ}$.

A photon which converts in the material between the main tracking volume and the calorimeter can either be lost entirely, or have its energy so degraded that it will fail to lead to a satisfactory $\pi^{0}$ mass. While energy loss by the conversion electrons is a contributing factor, even more important is their deflection by the solenoidal field: one or both of the leptons might not reach the barrel calorime- 


\begin{tabular}{|c|c|c|c|c|}
\hline \multirow[t]{2}{*}{$|\cos \theta|$} & \multicolumn{2}{|c|}{$\sigma_{E} / E(\%)$} & \multicolumn{2}{|c|}{$\begin{array}{c}\text { Truncated } \\
\text { Acceptance }(\%)\end{array}$} \\
\hline & $0.1 \mathrm{GeV}$ & $1 \mathrm{GeV}$ & $0.1 \mathrm{GeV}$ & $1 \mathrm{GeV}$ \\
\hline$\overline{0}$ & 2.0 & 1.4 & 84 & 90 \\
\hline 0.5 & 2.1 & 1.4 & 84 & 90 \\
\hline 0.7 & 2.2 & 1.5 & 84 & 87 \\
\hline 0.8 & 2.5 & 1.6 & 84 & 89 \\
\hline 0.85 & 2.8 & 1.7 & 82 & 90 \\
\hline
\end{tabular}

Table 8-1. Energy resolution and truncated acceptance for $0.1 \mathrm{GeV}$ and $1 \mathrm{GeV}$ photons vs. polar angle.

\begin{tabular}{|c|c|c|c|c|}
\hline $\begin{array}{c}\text { Material } \\
\text { Thickness }(\mu \mathrm{m})\end{array}$ & \multicolumn{2}{|c|}{$\sigma_{E} / E(\%)$} & \multicolumn{2}{c|}{$\begin{array}{c}\text { Truncated } \\
\text { Acceptance }(\%)\end{array}$} \\
\hline \hline & $0.1 \mathrm{GeV}$ & $1 \mathrm{GeV}$ & $0.1 \mathrm{GeV}$ & $1 \mathrm{GeV}$ \\
\hline 0 & 1.8 & 1.3 & 84 & 87 \\
150 & 2.0 & 1.4 & 84 & 89 \\
300 & 2.1 & 1.4 & 83 & 86 \\
\hline
\end{tabular}

Table 8-2. Energy resolution and truncated acceptance for $0.1 \mathrm{GeV}$ and $1 \mathrm{GeV}$ photons at polar angles around $90^{\circ}$ for different thicknesses of inactive material between crystals.

ter. For the endcaps, in contrast, the conversion leptons will spiral around the field lines and eventually reach the calorimeter not too far off course.

Important considerations besides the amount of material traversed include the proximity of that material to the calorimeter (the closer the better) and whether the particle identification detector can "flag" the conversion. The effect of a Fast RICH system on photons near polar angles of $90^{\circ}$ has been simulated; at other angles, to lowest order, the effect scales with the amount of material traversed, i.e. as $1 / \sin \theta$.

The Fast RICH is modeled after the device described in Section 7.5 with $0.09 X_{0}$ and $0.04 X_{0}$ of material located $23 \mathrm{~cm}$ and $3 \mathrm{~cm}$, respectively, in front of the calorimeter. The results for energy resolution and truncated acceptance are given in Table 8-3. Hardly any effect on resolution is visible, since most photons pass through

The amount and distribution of material in the particle identification system in front of the calorimeter affects the resolution and detection efficiency 
the material without converting. Those which do interact, however, lose a significant amount of energy, resulting in an efficiency loss ranging from $8 \%$ at $0.05 \mathrm{GeV}$ to $2 \%$ at $1 \mathrm{GeV}$. More details can be found in reference [Eis93]; effects of $\pi^{0}$ reconstruction will be presented below. Note that aerogel and DIRC particle identification options have similar or somewhat less material than the Fast RICH.

\begin{tabular}{|c|c|c|c|}
\hline Energy (GeV) & $\sigma_{E} / E(\%)$ & $\begin{array}{c}\text { Truncated } \\
\text { Acceptance (\%) }\end{array}$ & $\begin{array}{c}\text { Acceptance } \\
\text { Ratio } R(\%)\end{array}$ \\
\hline \hline 0.05 & 2.3 & 74 & 92 \\
0.1 & 2.0 & 78 & 93 \\
0.2 & 1.7 & 82 & 95 \\
0.5 & 1.5 & 84 & 94 \\
1.0 & 1.4 & 87 & 98 \\
\hline
\end{tabular}

Table 8-3. Energy resolution and truncated acceptance for photons at polar angles around $90^{\circ}$ with a Fast RICH detector in front of the calorimeter. Also given is the ratio, $R$, of truncated acceptance with and without material in front.

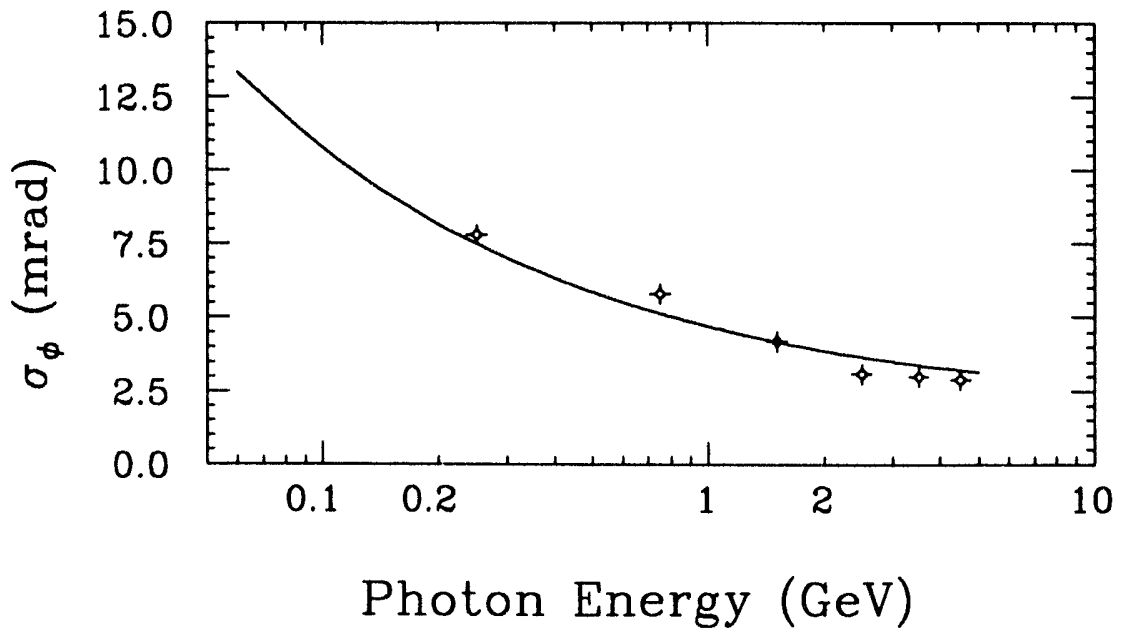

Figure 8-9. Expected resolution in azimuthal angle vs. energy. The solid line shows the measured CLEO-II resolution.

Optimization is continuing on the detailed reconstruction algorithms needed to determine the position of showers. One algorithm being tried is based on the energy-weighted center-of-gravity. Due to the finite granularity of the calorimeter, the resulting shower positions are biased towards the crystal centers. A correction factor, derived 
from Monte Carlo, is applied in an attempt to remove this bias. The resulting resolution in azimuthal angle, $\phi, v s$. energy is shown in Figure 8-9, together with a parametrization of the CLEO-II angular resolution [Kub92]. The result is slightly worse than the CLEO-II resolution for energies below $1.5 \mathrm{GeV}$ and slightly better for higher values. Given the anticipated low electronic noise levels, smaller transverse crystal sizes and longer distances to many of the crystals, somewhat better performance than CLEO-II is expected. This discrepancy is still under investigation. Nevertheless the algorithm has been used in some of the $\pi^{0}$ mass resolution studies in $C P$ benchmark processes described below. For studies of $\pi^{0}$ 's reconstructed from isolated showers in the barrel part of the calorimeter, a parametrization derived from CLEO-II [Kub92] has been used instead: $\sigma_{\phi}=0.9 \times(2.8 / \sqrt{E(G e V)}+1.9) \mathrm{mr}$ and $\sigma_{\theta}=\sin \theta \times \sigma_{\phi}$. Similar angular resolution is expected for showers in the endcaps since, in contrast to CLEO-II, the crystals there are arranged in projective geometry.

\subsubsection{Expected Perfornance for $\pi^{0}$ 's}

Most photons detected in the calorimeter arise from $\pi^{0}$ 's; $\pi^{0}$ reconstruction is therefore the primary task of the calorimeter. To study $\pi^{0}$ mass resolution and efficiency, and the effect of material in front of the calorimeter, single, mono-energetic $\pi^{0}$ 's have been used initially. As in our single-photon studies, only isolated showers in the barrel are reconstructed, and the CLEO-based parametrizations for the angular resolutions are applied; mass resolution and truncated acceptance are defined the same way as in the previous section.

Figure 8-10 shows $\pi^{0}$ mass resolution and truncated acceptance $v s$. $\pi^{0}$ momentum. In this case, the model includes not only the material from a Fast RICH but also from the beam pipe, the silicon vertex detector, and the main tracking chamber, representing a total of $0.2 X_{0}$ at normal incidence. Also indicated is the performance with no material in front of the calorimeter for $\pi^{0} \mathrm{~s}$ with a momentum of $0.1 \mathrm{GeV}$ and $1 \mathrm{GeV}$. As with single photons, the inactive material has very little effect on the resolution, but significantly degrades the efficien:y.
Single $\pi^{0}$ 's and several benchmark reactions have been used to study $\pi^{0}$ resolution and reconstruction efficiency

Material in front of the calorimeter has a small effect on $\pi^{0}$ mass resolution, but a significant effect on reconstruction efficiency 


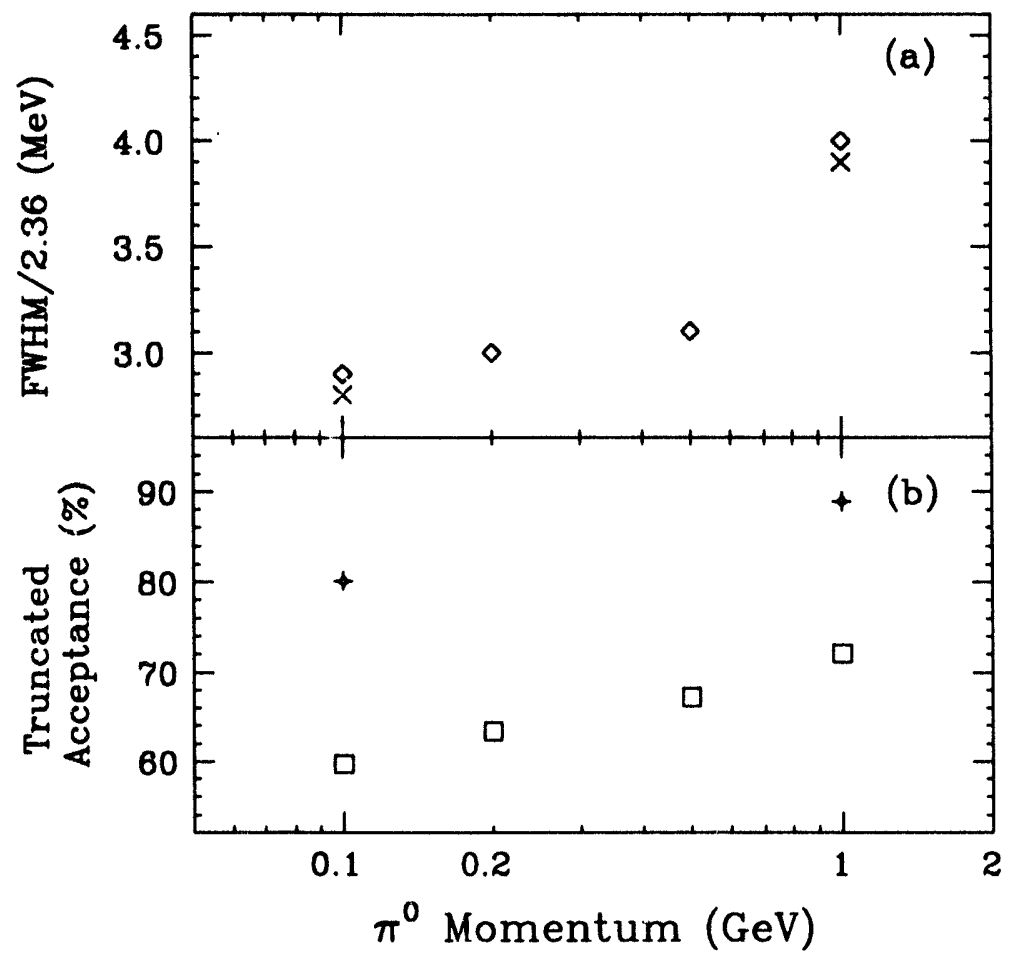

Figure 8-10. (a) Expected mass resolution and (b) truncated acceptance for single, mono-energetic $\pi^{0}$ 's vs. $\pi^{0}$ momentum for $0.2 X_{0}$ in front of the calorimeter. Only $\pi^{0}$ 's with both photons entering the barrel calorimeter were considered. The crosses indicate the performance for $0.1 \mathrm{GeV}$ and $1 \mathrm{GeV} \pi^{0}$ 's with no material in front of the calorimeter.

In addition, several benchmark processes, relevant for measuring $C P$ asymmetries, have been used to study $\pi^{0}$ mass resolution and efficiency: (1) $B^{0} \rightarrow J / \psi K^{* 0}$ where $K^{* 0} \rightarrow K_{S}^{0} \pi^{0}$, (2) $B^{0} \rightarrow \rho^{ \pm} \pi^{\mp}$ where $\rho^{ \pm} \rightarrow \pi^{ \pm} \pi^{0}$, and (3) $B^{0} \rightarrow \pi^{0} \pi^{0}$. The $\pi^{0}$ 's from those processes have a wide range of momentum, with $B^{0} \rightarrow J / \psi K^{* 0}$ yielding the softest $\pi^{0}$ 's and $B^{0} \rightarrow \pi^{0} \pi^{0}$ the hardest.

In one study, only isolated photon showers in the barrel are reconstructed. For this case, Figure 8-11a shows the $\gamma \gamma$ invariant mass spectrum in the decay $B^{0} \rightarrow J / \psi K^{* 0}$ with no material in front of the calorimeter. (Note that $J / \psi$ and $K_{S}^{0}$ decays are not simulated in this study.) The $\pi^{0}$ mass resolution and truncated acceptance are $\sigma_{m_{\pi^{0}}}=3.2 \mathrm{MeV} / c^{2}$ and $82 \%$ respectively. Also shown (Figure 8-11b) is the mass spectrum with the material of the nominal design in front. Again, the additional material leads to 
a relatively small degradation in resolution, to $\sigma_{m_{z^{0}}}=3.3 \mathrm{MeV} / c^{2}$, but causes a very significant low-mass tail which results in a drop in $\pi^{0}$ truncated acceptance to $60 \%$. The results for all three channels are summarized in Table 8-4.

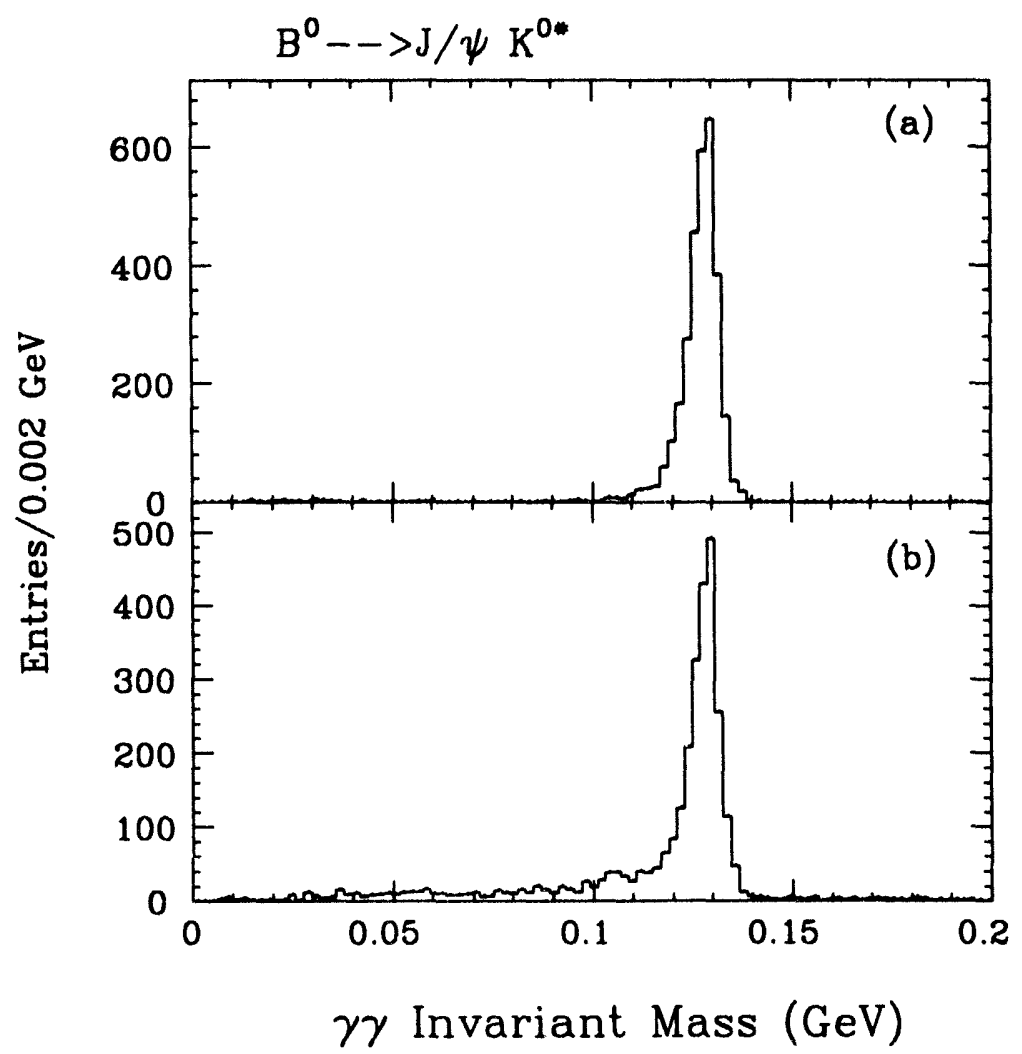

Figure 8-11. $\quad \gamma \gamma$ invariant mass spectrum from the process $B^{0} \rightarrow J / \psi K^{* 0}$ with $K^{* 0} \rightarrow K_{S}^{0} \pi^{0}$ for (a) no material in front of the calorimeter and (b) $0.2 X_{0}$ in front of the calorimeter at normal incidence.

In a second study, an attempt is made to reconstruct $\pi^{0}$ 's without requiring isolated showers [Ale94]. Shower energies are determined using a contiguous cluster algorithm and positions are measured with the center-of-gravity method described above. Clusters are reconstructed in both the barrel and endcap. The results are given in parentheses in Table 8-4. One can see that this yields a substantially better $\pi^{0}$ efficiency in the channels $B^{0} \rightarrow \rho^{ \pm} \pi^{\mp}$ and $B^{0} \rightarrow \pi^{0} \pi^{0}$, at the expense of significantly worse $\pi^{0}$ mass resolution. This is mainly caused by position errors for overlapping showers. It is expected, however, that the mass resolution can be much improved in this case by performing a mass-constraint fit; this will be the subject of 


\begin{tabular}{|c|c|c|c|}
\hline$B$ decay channel & $\begin{array}{c}\sigma\left(m_{\pi^{0}}\right) \\
(\mathrm{MeV})\end{array}$ & $\begin{array}{c}\pi^{0} \text { Truncated } \\
\text { Acceptance (\%) }\end{array}$ & $\begin{array}{c}\text { Full } \pi^{0} \\
\text { Acceptance (\%) }\end{array}$ \\
\hline \hline$B^{0} \rightarrow J / \psi K^{* 0}$ & $3.3(4.0)$ & 60 & \\
$B^{0} \rightarrow \rho^{ \pm} \pi^{\mp}$ & $4.7(6.3)$ & $57(67)$ & $(60)$ \\
$B^{0} \rightarrow \pi^{0} \pi^{0}$ & $6.2(7.6)$ & $30(73)$ & $(68)$ \\
\hline
\end{tabular}

Table 8-4. $\quad \pi^{0}$ mass resolution and efficiency in various $B$ decay channels, modeled with all the material in front. Truncated acceptance is quoted for the barrei region only, or barrel plus endcaps in parentheses. Full acceptance includes the loss due to the solid angle coverage of the calorimeter.

further studies. The present technique yields a $B^{0}$ mass resolution of $\sigma=43 \mathrm{MeV} / \mathrm{c}^{2}$ and an overall reconstruction efficiency of $50 \%$ for $B^{0} \rightarrow \pi^{0} \pi^{0}$.

\subsection{Calibration, Monitoring and Sta- bility}

Overall calorimeter performance can be monitored using high energy physics processes

The performance of individual calorimeter channels is best monitored with an electronic pulser and radioactive sources
The performance of the $\mathrm{CsI}(\mathrm{Tl})$ crystals, photodiodes and readout electronics must be monitored periodically to maintain the required resolutions. Charge injection into the preamplifier frontend is expected to yield a $1 \%$ relative calibration of the gains of the system beyond the photodiodes. A number of physics processes provide photons and electrons that are suitable for crystal and photodetector calibration purposes. Reconstructed $\pi^{0}$ masses, energy depositions from minimum-ionizing particles (typically $0.23 \mathrm{GeV}$ ), and the reconstruction of kinematically constrained final states, such as $e^{+} e^{-}, e^{+} e^{-} \gamma, \gamma \gamma$, and $\gamma \gamma \gamma$, permit an absolute calibration accuracy of better than $1 \%$ over the energy range relevant for physics events.

High energy processes are, however, not well-suited for tracking changes in the light-collection properties of individual crystals, since it takes 1 to 2 weeks to accumulate adequate statistics except with Bhabha events. An independent system to monitor the light output of each crystal on demand is therefore highly desirable. For this purpose, one can utilize any of a set of long-lived gamma sources, such as $(\gamma$ energies in $\mathrm{MeV}):{ }^{137} \mathrm{Cs}(0.66),{ }^{60} \mathrm{Co}(1.17,1.33),{ }^{207} \mathrm{Bi}(0.57$, 
1.06 , and a weak 1.33$),{ }^{214} \mathrm{Bi}(1.67)$, or ${ }^{208} \mathrm{Bi}(2.6)$. These sources would be moved mechanically into place during a calibration run through a system of tubes.

Higher photon energy sources with short half-lives can provide calibration signals with greater noise margin. These can be produced externally in a liquid and pumped through the detector. An exsple is the $6.13 \mathrm{MeV}$ line from ${ }^{16} \mathrm{O}$, which can be produced using a low energy neutron source via the ${ }^{19} \mathrm{~F}(\mathrm{n}, \alpha){ }^{16} \mathrm{~N}$ followed by the ${ }^{16} \mathrm{~N} \rightarrow{ }^{16} \mathrm{O}^{*} e^{-} \bar{\nu}_{e}$ reaction in freon. The intermediate ${ }^{16} \mathrm{~N}$ state has a half-life of seven seconds. The activated liquid can therefore safely be pumped through the detector for a calibration run. The final choice of a calibration source depends on practicality, cost and radiation safety.

The humidity in the vicinity of the crystals must be kept low, as the CsI(Tl) crystals are mildly hygroscopic. The temperature of the calorimeter must be kept stable, as the light output of the $\mathrm{CsI}(\mathrm{Tl})$ as well as the photodiode dark current vary slightly with temperature [Val93]. Humidity control is straightforward to achieve by circulating dry nitrogen through the crystal calorimeter. Studies from CLEO-II show that the best stability is obtained at a temperature of $(22 \pm 1)^{\circ} \mathrm{C}$. Thermocouples will constantly monitor temperature at several positions in the calorimeter and will be used to control distributed heating and cooling elements.

\subsection{Procurement and Assembly}

The detector construction schedule places stringent requirements on the procurement of the calorimeter crystals and their assembly into the mounting structure; the complete barrel section must be installed inside the solenoid coil during the early phases of detector assembly in the interaction region. The most critical item in terms of cost and schedule is the production of the $\mathrm{CsI}(\mathrm{Tl})$ crystals. In order to better understand the process of crystal growth, a detailed model that includes all phases of crystal production and incorporates input from industry has been developed. The cost of finished $\mathrm{CsI}(\mathrm{Tl})$ crystals is driven primarily by the cost of the CsI salt and by the yield of the growing, cutting and polishing processes. Most crystal producers have initial overall yields of $50 \%$, rising perhaps
Radioactive sources with $\gamma$ lines from 0.5 to $6 \mathrm{MeV}$ can provide low energy calibration

The humidity in the calorimeter must be kept low and the temperature must be kept constant

The barrel CsI crystals must be produced in time to allow them to be mounted inside the solenoid coil during the early phases of detector assembly

The cost of the CsI crystals is driven by the cost of the raw material and by the overall production yield 
There will be adequate supplies of high purity CsI salt

Crystal production facilities can be expanded to the required level in a number of countries

Large scale cutting and polishing techniques are under development to about $65 \%$ over a production run. Two crystal growth pilot projects have been started in an effort to understand and optimize the various stages of the production process: new crystal growth techniques, machining and polishing, and the CsI salt recycling process. The recycling of CsI material from aborted growing cycles, and from the machining of crystals, is an important determinant of the overall price.

Large numbers of CsI crystals have already been produced for the CLEO-II, Crystal Barrel, KTeV and BNL E852 experiments. Building on this experience, the techniques of crystal growth have been explored in detail with a variety of manufacturers. Production of the crystals begins with acquisition of the $\mathrm{CsI}(\mathrm{Tl})$ salt. The major producers of high-purity CsI salt have indicated their intention to increase their CsI production and recycling capacity to meet the projected world demand for CsI. This increased capacity will be available beginning in 1995 .

Several producers with sizable $\mathrm{CsI}(\mathrm{Tl})$ crystal production capacity are available in China, the former Soviet Union, Europe, Japan, and the US. A "make-or-buy" study is currently underway to ascertain whether to grow the crystals using the facilities of several of our collaborating laboratories.

Special tooling has been developed for finishing multiple copies of crystals to the proper tapered shape required for the calorimeter. Up to 5 crystals at a time can be ground and polished with dimensional accuracy of about $25 \mu \mathrm{m}$. Multiple crystal processing is especially important in mass production because of the labor and time-intensive nature of these manufacturing steps. Alternative sawing methods, such as diamond wire cutting of CsI, are also being investigated to substantially reduce the time involved in rough cutting CsI crystal out of the boule, and in fine cutting prior to grinding and polishing.

The schedule foresees production of a modest number of crystals (perhaps $15 \%$ of the total) in 1995, as manufacturing capacity is ramped up. The main emphasis in 1995 will be to optimize surface treatment and to gain experience with handling large numbers of crystals. Production will increase in 1996, with a plateau reached in the third quarter of 1996, continuing throughout 1997 into the first quarter of 1998 . The production for the endcap crystals will 
begin in 1996 to allow us to understand and perhaps improve the radiation hardness of the material closer to the beamline.

The second important item is the readout. In the case of FFC/photodiode readout, wavelength-shifter-doped acrylic is acquired, machined and polished. The photodiodes, along with the frontend electronics package, all of which have been pre-tested, are attached to the acrylic. The readout plate assembly is then tested and mounted on the crystal. This work is most naturally performed at a number of collaborating institutions.

The production of both the photodiodes and the FFCs can proceed rather quickly, as industry has a large capacity available and can produce the required quantities within 6 months. The production of the charge-sensitive amplifiers and support elements such as the bias network, shielding, thermal isolation, etc., is again not on the critical path because industrial production can be achieved in relatively short time. The final decisions on design details are required before the end of 1995 . The final assembly, which is rather labor intensive, will be done at the collaborating institutions.

The third important element is the support structure. The final assembly of the calorimeter barrel will start in late 1997 and extend until spring 1998. System tests will start when the barrel is mounted in a transfer fixture. When tests are completed, the barrel will be inserted into the magnet. The endcap assembly schedule is not yet fully worked out, but is less intimately connected with the assembly of other system components.

\subsection{Optimization Studies}

\subsubsection{Light Collection and Readout}

Studies are under way to optimize the crystal size based upon considerations of energy resolution, angular resolution, and cost. Light collection and readout schemes used by other experiments, such as Crystal Barrel [Ake92] and CLEO-II [Kub92], have been studied. Several improvements are possible and are proposed to be tested in the $R \& D$ program: comparison of various crystal wrapping schemes,
The production of the FFC/photodiode assemblies and the readout electronics is not a critical path item

Final assembly and testing of the CsI barrel system must commence a year before detector completion
Studies are continuing to optimize crystal sizes and to improve wrapping and readout techniques 
both for optimal overall light collection and for compensation of light collection differences due to the trapezoidal crystal shapes; development of wavelength-shifter materials with high refractive index for enhanced light trapping; studies of new wavelength-shifting dyes with higher efficiency; tests of different wavelength-shifter geometries to increase collection efficiency; development of photodiodes with increased signal-to-noise performance, especially with enhanced quantum efficiency between 600 and $1000 \mathrm{~nm}$; and extensive reliability studies of all readout components to minimize failures.

The Tau/Charm design, using wavelength shifters and photodiodes to separately read out crystals that have been split longitudinally at 4 radiation lengths, will be studied in more detail. This may improve radiation resistance (see Section 8.7.2), light output, and crystal cost at the expense of doubling the number of readout channels. It also provides more pattern recognition capability for rejection of background and better $\pi / \mu$ and $e / \pi$ separation.

\subsubsection{Radiation Hardness Studies}

Large crystal scintillators are affected by radiation damage [Daf92]. The main effects come from absorption bands, caused by color center formation, which lead to a reduction of light transmission. Investigations on radiation hardness of CsI crystals [Zhu94] indicate

Reduction of light output with radiation dose appears to be due primarily to changes in absorption length for visible light that: (a) there are performance changes above 1 to $10 \mathrm{krad}$ of $\gamma$ radiation, which continue to worsen at high doses; (b) there is essentially no difference between Tl- or Na-doped CsI, while undoped CsI appears to have better radiation hardness; (c) there is little reduction in the emission of scintillation light with dose, i.e. changes in pulse height result primarily from changes in transmission; (d) the amount of damage is a function of the dose rate as well as the integrated dose; (e) there exist slow self-recovery mechanisms that restore transparency over long time scales.

A compilation of previous radiation damage studies in CsI shows inconsistent results [SLA91]. A systematic R\&D program has therefore been undertaken to better understand radiation damage in $\mathrm{CsI}(\mathrm{Tl})$ [Hit92]. Figure 8-12 shows the relative light output of $2.5 \mathrm{~cm}$ diameter, $2.5 \mathrm{~cm}$ long $\mathrm{CsI}(\mathrm{Tl})$ crystals grown by Quartz \& Silice, using the $1.275 \mathrm{MeV} \gamma$ line of ${ }^{22} \mathrm{Na}$ measured with an avalanche 


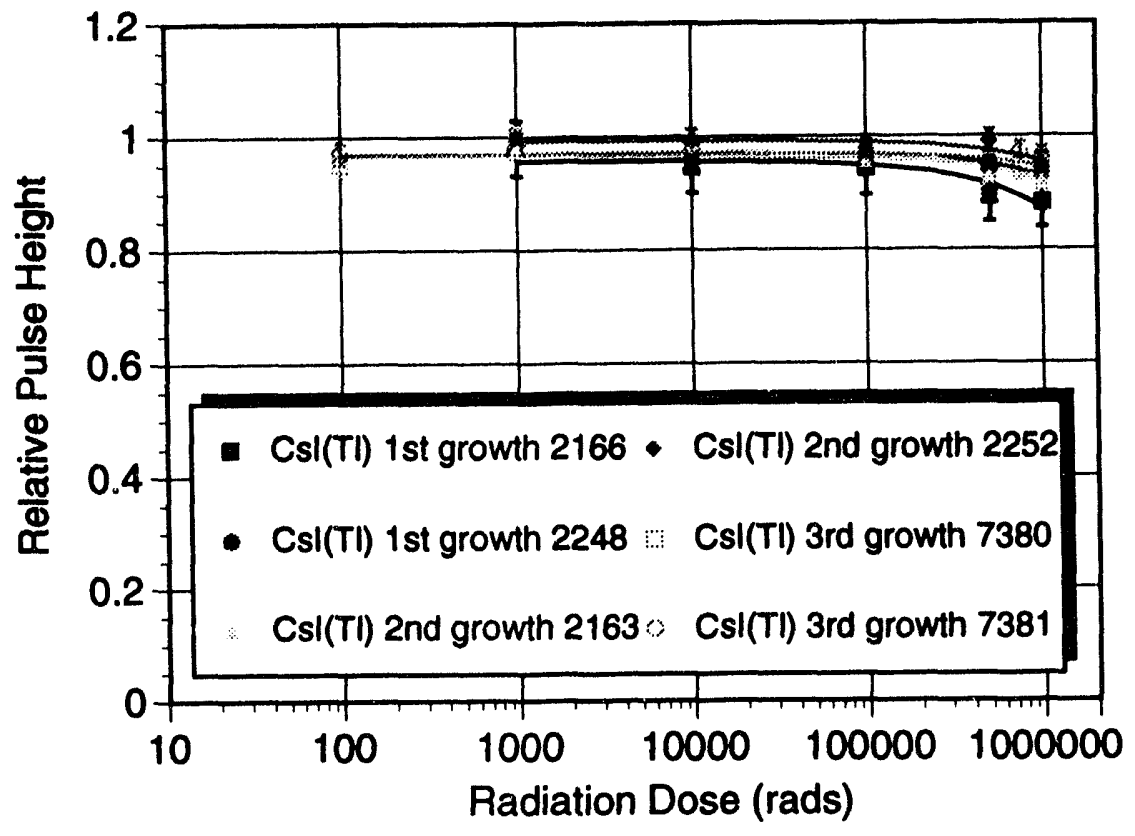

Figure 8-12. Relative light yield vs, radiation dose of $2.5 \mathrm{~cm}$ diameter, $2.5 \mathrm{~cm}$ long $C s I(T)$ crystals made by $Q \& S$; measured using $1.275 \mathrm{MeV} \gamma$-rays from a ${ }^{22} \mathrm{Na}$ source and avalanche photodiode readout. [Hit92].

photodiode. These results on small crystals show that small impurities and/or structural defects are the main sources of radiationinduced color center formation [Gra94]. Measurements on crystals from different raw materials of well-defined purity have not yet been performed in a systematic way.

A more focused R\&D effort is now underway. Transmission measurements between 300 and $1000 \mathrm{~nm}$ wavelengths after intense irradiation with ${ }^{60} \mathrm{Co}$ sources will be correlated with the purity of the raw material, the $\mathrm{Tl}$ concentration, and the growing techniques, using small-, and ultimately, full-size crystals. There is some evidence that pure CsI is sufficiently radiation hard to be considered for use in the endcap region [Ari92]. While its light output is lower than that of $\mathrm{CsI}(\mathrm{Tl})$, its emission spectrum is a reasonable match to a conventional photocathode, in which case readout with fine mesh photomultipliers could be considered for an endcap implementation.
A program to improve our understanding of the mechanism of radiation damage in $\mathrm{CsI}(\mathrm{Tl})$ crystals is underway 


\subsubsection{A Prototype Calorimeter}

A prototype calorimeter will be built to demonstrate system performance
After completing the optimization of crystal sizes, the wrapping and readout system design, and the design of the mechanical support structure of the calorimeter, a small prototype will be built with 36 to 49 crystals of final dimensions in a realistic support structure. With this prototype the energy and angular resolutions of highenergy electrons will be measured under various crystal conditions and tests of both the readout electronics and the calibration scheme will be made. The availability of test beams at SLAC and elsewhere is under investigation. 


\section{References}

[Ale94] R. Aleksan et al., "Resolution Studies using GEANT", $B_{A} B_{A R}$ Note \#152 (in preparation), 1994.

[Ake92] E. Aker et al., Nucl. Instr. Meth. A321, 69 (1992).

[Ari92] K. Arisaka et al., "KTEV Design Report: Physics Goals, Technical Components, and Detector Costs", Fermilab Report FN-580, January 1992.

[BAB94] "Report of the ad hoc Calorimeter Committee", May 1994.

[Blu86] E. Blucher et al., Nucl. Instr. Meth. A249, 201 (1986).

[Dea93] A. Deandrea et al., Phys. Lett. B318, 549 (1993).

[Daf92] See papers by Y. Dafinei, D. Hitlin, H. Newman, B. Winstein, C. Woody, C. Wuest and R.Y. Zhu, Proceedings of International Workshop on Heavy Scintillators for Scientific and Industrial Applications, ed. by F. Nataristefani et al.,(Editions Frontieres, 1992).

[Eig94] G. Eigen and D.G. Hitlin, "Light Collection and Readout Studies of CsI(Tl) Crystals", BABAR Note \#150 (in preparation), 1994.

[Eis93] A.M. Eisner, "The Effect of Material en route to the EM Calorimeter", BABAR Note \#110, July 1993.

[Gra94] G. Gratta, H. Newman and R.Y. Zhu, Caltech Report68-1922, to be published in Ann. Rev. Nucl. Part. Sci. 44 (1994).

[Hea94] C. Hearty, "Performance Comparision between CsI and LKr Calorimeters for PEP-II", BABAR Note \#141, May 1994.

[HEL92] HELENA, A Beauty Factory in Hamburg, DESY Report DESY-92-041, 1992. 
[Hit92] D. Hitlin and G. Eigen, Proceedings of International Workshop on Heavy Scintillators for Scientific and Industrial Applications, ed. by F. Nataristefani et al., p. 467 (Editions Frontieres, 1992).

[Hol88] I. Holl et al., IEEE Trans. Nucl. Sci. 35, 105 (1988).

[Kub92] Y. Kubota et al., Nucl. Instr. Meth. A320, 66 (1992).

[Leb90] M. Lebeau, KEK Internal Report 89-19, Feb. 1990.

[Lev94] M. Levi, "Impact of Backgrounds on the Calorimeter Resolution", BABAR Note \#151 (in preparation), 1994.

[Lor86] E. Lorenz et al., Nucl. Instr. Meth. A239, 235 (1986).

[Per91] J.L. Perez, K. Skarpaas, private communication, 1991.

[SLA91] See references in Workshop on Physics and Detector Issues for a High Luminosity Asymmetric B Factory at SLAC, ed. by D. Hitlin, SLAC-373, 1991; also see [Hit92].

[SLA93] Status Report on the Design of a Detector for the Study of $C P$ violation at PEP-II at SLAC, SLAC-419, June 1993.

[Sny92] A. Snyder, "Backgrounds in the BABAR CsI Calorimeters", BABAR Note \#83, May 1992.

[Spi94] A. Spitkovsky and F.C. Porter, "Study of Light Transport in Different CsI Crystal Geometries", BABAR Note \#146 (in preparation), 1994.

[Val93] J. Valentine et al., "Temperature dependence of $\mathrm{CsI}(\mathrm{Tl})$ gamma-ray excited scintillation characteristics", Nucl. Instr. Meth. A325, 147 (1993).

[Zhu94] R.Y. Zhu et al., "CsI(TI) Crystal Study for PEP-II Asymmetric B-Factory", Cal Tech Report CALT-68-1909, January 1994.

[Zis01] An Asymmetric $B$ Factory Based on PEP, ed. by M. Zisman, SLAC-372, 1991. 


\section{9}

\section{Muon and Neutral Hadron Detector}

$\mathbf{T}$ he iron structure that constitutes the return yoke for the magnetic field is fully instrumented in order to provide muon identification and $K_{L}^{0}$ detection. This Instrumented Flux Return (IFR) consists of a barrel, enclosing the superconducting coil, and two endcaps, and is subdivided into slabs with active detectors inserted into the intervening gaps. The total steel thickness required to contain the magnetic flux from the solenoid coil is about $60 \mathrm{~cm}$; this is a good match to the absorber thickness required for a muon filter. Particles reaching the iron will have passed through the inner detectors and the coil (a total of 0.9 interaction lengths); to emerge from the iron and strike the outermost chamber they must pass through an additional 3.6 interaction lengths. This is enough material to reduce the pion punch-through misidentification probability to the percent level in the momentum range between 1 and $3 \mathrm{GeV} / c$, comparable to the probability for hadron decay to muons.

Since the measurement of $C P$-violating asymmetries and the experimental program at PEP-II in general are based on exclusive-state reconstruction, the measurement of the hadron energy deposition is not of primary importance; the IFR is not used as a calorimeter for total energy measurement, but as a neutral hadron (primarily $K_{L}^{0}$ ) and muon identifier. Thus, a strip type detector with digital readout is envisioned; strips can run along the $x$ or $z$ direction in the barrel and in the $x$ or $y$ direction in the endcaps. Bi-dimensional information is possible, either by using strips in both directions or by measuring the difference in arrival time of signals at the ends of the detector.

Solid angle coverage is complete down to the region occupied by beamline components. The precise shape of the end cap yoke in the region near the beam line is not yet defined; it is possible that muon identification could be extended to angles below $300 \mathrm{mrad}$. This would not affect the cost since the number of readout channels
The CsI calorimeter, coil, cryostat, and iron flux return represent more than $4.5 \lambda_{\text {int }}$

The IFR is primarily a $K_{L}^{0}$ and muon identifier 
Hermeticity is important for the measurement of the branching ratio for $B \rightarrow \tau \nu_{\tau}$
It should be possible to identify with good efficiency muons with momenta as low as $500 \mathrm{MeV} / \mathrm{c}$ and $K_{L}^{0}$ 's in the range $1-3 \mathrm{GeV} / \mathrm{c}$

Most Monte Carlo hadron simulations are not well benchmarked in the relevant energy region would sot change significantly, but could allow an increase in $B$ tagging efficiency and make the IFR more hermetic. Maximum solid angle coverage for hadrons is important, for example, in the measurement of the branching ratio of $B \rightarrow \tau \nu_{\tau}$. A detailed Monte Carlo is needed to evaluate the background, and in general to verify the benefits of the IFR covering a larger solid angle than the rest of the detector.

The IFR could also allow us to veto cosmic rays at the trigger level by determining the direction (inward or outward) of a track. This could be done by adding a small number of TDCs (one per chamber in a few layers) to measure the time of the first hit in the chambers. Cosmic rays could be well separated from events originating at the interaction point, even with a modest time resolution (in the range of a few nanoseconds).

\subsection{Design Considerations}

The physics objectives driving the performance required of the IFR detector are: $B$ (and $D$ ) tagging with muons, study of semimuonic decays, reconstruction of $J / \psi \rightarrow \mu^{+} \mu^{-}$, and $K_{L}^{0}$ identification in the $B$ decay products. To reach these goals, the IFR should deliver $\mu-\pi$ separation from $\sim 4 \mathrm{GeV} / c$ down to as far below $1 \mathrm{GeV} / c$ as possible (muons from the charm quark cascade peak at $\sim 500 \mathrm{MeV} / c$ ), with high efficiency and little misidentification; and $K_{L}^{0}$ identification, with directional information, in the energy range of $1-3 \mathrm{GeV}$. The momentum spectrum for all muons in $\Upsilon(4 S)$ to $B^{0} \bar{B}^{0}$ decays is shown in Figure 9-1. The light quark (uds) contribution is almost entirely from pion and kaon decay.

The total detector surface and the number of readout channels are the key factors in the cost estimate of this subsystem. Detailed Monte Carlo simulation studies of the full detector with single particle and $\Upsilon(4 S)$ event inputs have begun to address the issues of IFR design. The hadronic shower Monte Carlos in most sommon use are known not to simulate low momentum particles correctly; different generators use different cross sections and models. This difficulty is compounded by the fact that there is little data against which to test the simulations. This issue will be addressed by researching available data [PS202] and carefully comparing results 


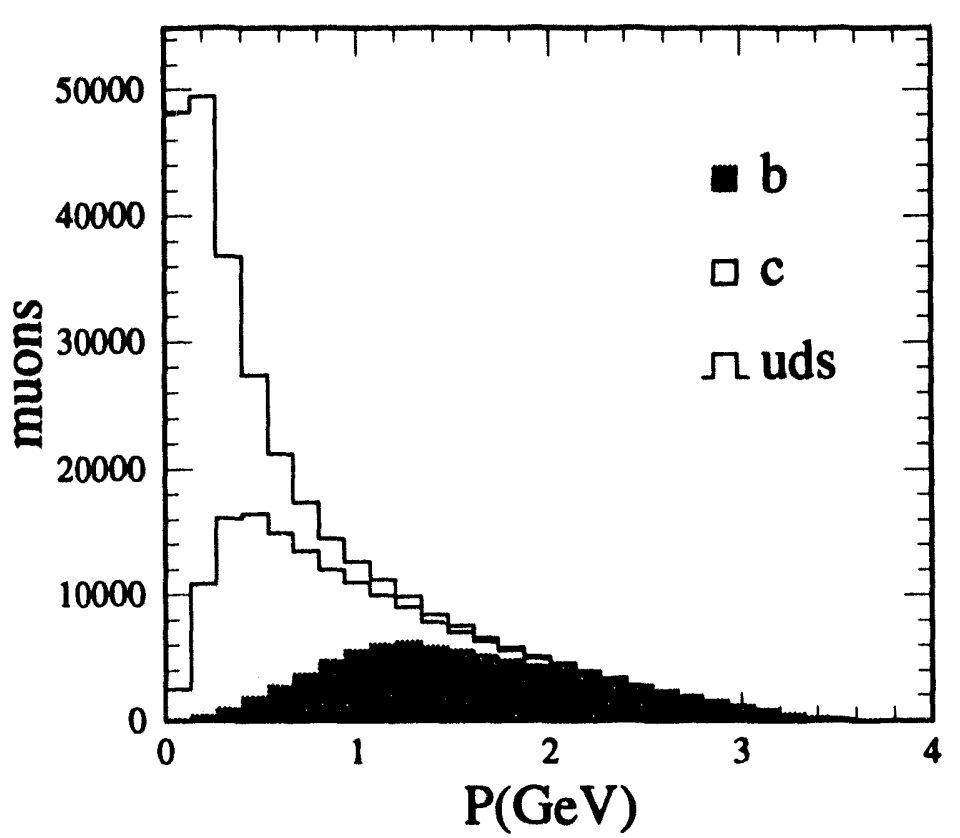

Figure 9-1. Momentum spectrum for muons in $\Upsilon(4 S) \rightarrow$ $B^{0} \bar{B}^{0}$ events. Muons from pion and kaon decay after the central tracking chamber were excluded. The contributions from heavy quark $(b, c)$ and light quark $(u, d, s)$ decay are highlighted.

obtained with different programs. The results reported here were obtained using the package FLUKA, which is considered to provide the worst case scenario for pion misidentification.

The optimization process will proceed as more work is performed in preparation for the CDR. The main points on which activity will be focused on are the iron segmentation, both in the barrel and in the two endcaps, the design of the active detector components, and the effect of the solenoid coil thickness.

The impact of detector inefficiencies (mostly due to inactive areas) on performance will be investigated, as well as the merits of using double layers. The number of readout channels, determined by the size of the strips and the number of layers to be equipped with bidimensional readout, must also be optimized. The possible benefit of inserting an additional detector layer between the CsI calorimeter and the coil is being investigated. The energy asymmetry of PEP-II leads to a momentum distribution that is a function of polar angle; the design of the endcap detector system should reflect this, to the
The number of layers and the design of the active detector elements remain to be optimized 
The IFR system under study uses 24 layers of $2.5 \mathrm{~cm}$ steel
Below $1 \mathrm{GeV} / \mathrm{c}$ the $\pi / \mu$ range difference is approximately $2-3 \mathrm{~cm}$ degree permitted by magnetic considerations, in order to be cost effective without compromising performance.

This optimization process has begun with a detailed study of muon identification efficiency $v s$. hadron contamination, as a function of momentum and angle of incidence; $B$ tagging efficiency, in particular for benchmark channels. and $K_{L}^{0}$ identification and angular resolution in $B^{0} \bar{B}^{0}$ events.

For the purpose of the budget estimate, a system with 24 single layers, each $2.5 \mathrm{~cm}$ thick through the entire solid angle, and a strip pitch of $2 \mathrm{~cm}$ has been considered. The estimate will be refined when the design optimization is complete.

\subsection{Muon Identification}

Since the interaction region is enclosed by the CsI calorimeter and magnet solenoid, muons with momenta below $400 \mathrm{MeV} / c$ do not reach the IFR (see Figure 9-2). Those muons that do reach the IFR must be distinguished from backgrounds resulting from primary charged hadrons (mostly pions) and secondaries from hadronic showers and decays.

Considering their mass difference and ignoring hadronic interactions, pions and muons differ in their most probable penetration depth (range) in iron by approximately $2-3 \mathrm{~cm}$ for momenta below $1 \mathrm{GeV} / c$. This effect is overshadowed by the high pion-nuclear interaction cross-section. The momentum dependence of the penetration depth for pions and muons is shown in Figure 9-3. Muons with momenta greater than $1.5 \mathrm{GeV} / c$ completely penetrate the IFR iron.

To exploit the range difference between pions and muons for pion rejection, the momentum of the charged particle must be measured. Furthermore, the charge of the muon must be determined for $B^{0}$ tagging. The central tracking chamber provides this information; its geometrical acceptance is less than that of the flux return, thereby restricting the solid angle for muon identification. The fraction of muons reaching the IFR that have at least 20 hits in the central tracking chamber is indicated in Figure 9-2. 


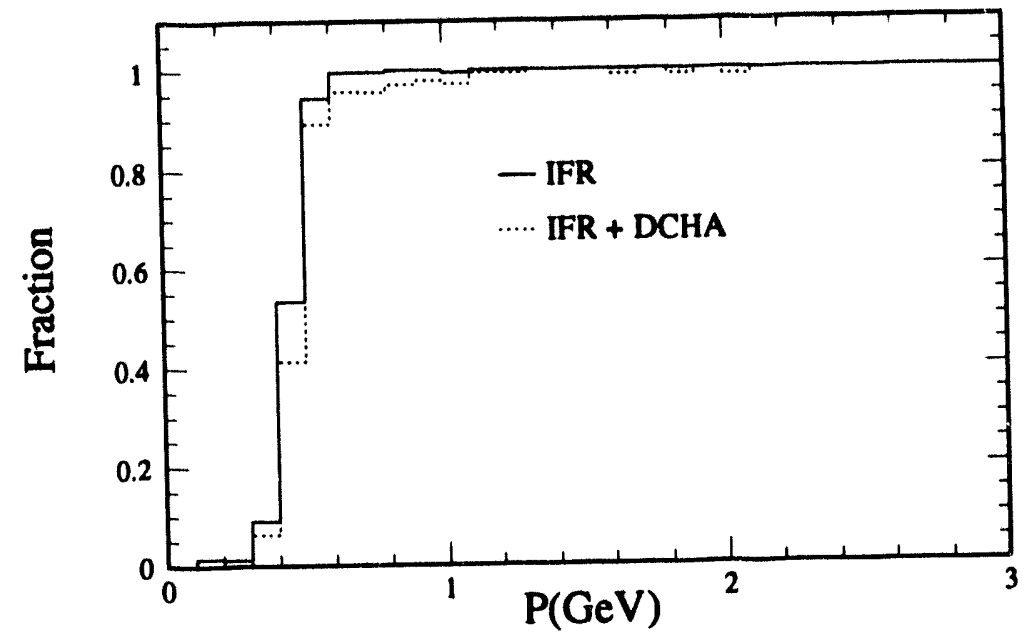

Figure 9-2. Fraction of muons that reach the IFR (solid line) and the fraction that reach the IFR and give at least 20 hits in the central tracking chamber (detted line). The data is from Monte Carlo simulation of the full detector for muons in $\Upsilon(4 S) \rightarrow B^{0} \bar{B}^{0}$ decays.

Depending on how much loss of muon efficiency one can tolerate, a range cut reduces the pion misidentification probability to a few percent. Monte Carlo studies have indicated that reducing the iron plate thickness to $2.5 \mathrm{~cm}$ from $0.5 \mathrm{~cm}$ did not significantly improve the pion rejection. Pion background can be further reduced by requiring a high degree of continuity in the pattern of hits in the IFR. Muons tend to give a hit in every layer they go through; pions initiate hadronic showers that can 'skip' detection layers. Examples of this are shown in Figure 9-4. In the figure, single pions or muons are simulated in a simplified geometry at $90^{\circ}$ incidence and the IFR is represented as 60 plates of $1 \mathrm{~cm}$ thickness. For each momentum the total number of layers hit is plotted as a function of the last layer hit. The muon data lie on a straight line at $45^{\circ}$, while the distribution for pions is scattered.

Preliminary Monte Carlo studies indicate that muon identification can be done with high efficiency (above 90\%) and low contamination (a few \%) from 500 to $1000 \mathrm{MeV} / \mathrm{c}$ momentum, using a total thickness of $40 \mathrm{~cm}$ of iron divided into plates of approximately $2 \mathrm{~cm}$. The remaining portion of the IFR could be divided into thicker plates without compromising performance. Monte Carlo studies are
Requiring a large number of hits on a track in the IFR can further improve $\pi / \mu$ separation 

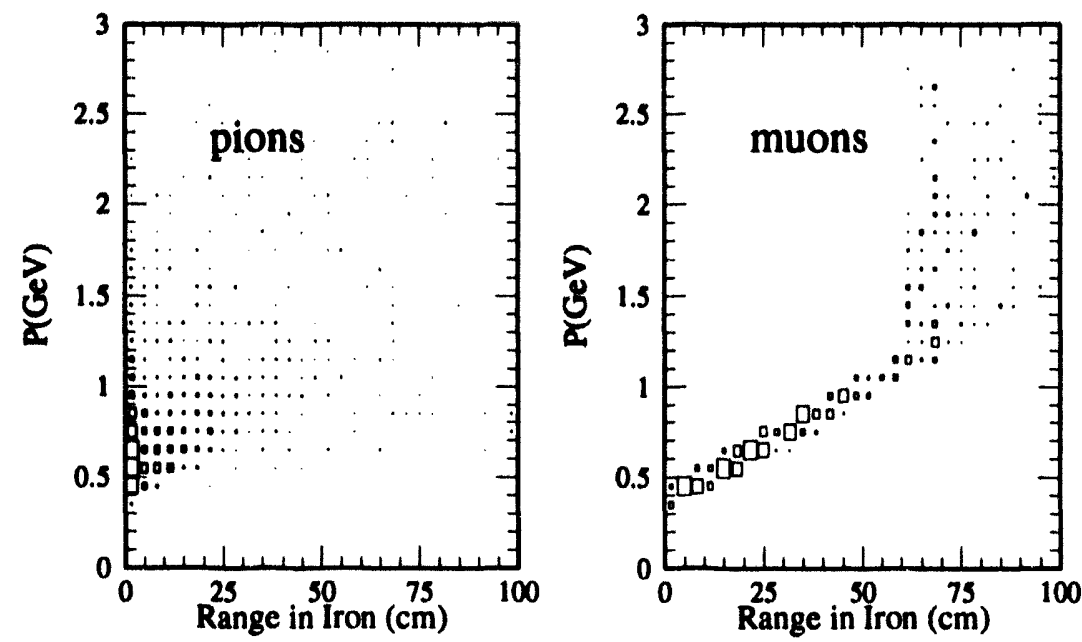

Figure 9-3. Penetration depth in IFR versus momentum for muons and pions. The data is from Monte Carlo simulation of the full detector for muons and pions in $\Upsilon(4 S) \rightarrow B^{0} \bar{B}^{0}$ decays.

under way to optimize the choice of plate thickness and to verify the impact of segmentation on $K_{L}^{0}$ detection.

\section{$9.3 \quad K_{L}^{0}$ Detection}

The $K_{L}^{0}$ detection efficiency is 50 to $70 \%$, depending on the thickness of the steel plates
The granularity of the IFR detector is also driven by the need to identify the $K_{L}^{0}$ 's produced in $B$ decays. A sizeable fraction of $K_{L}^{0}$ produced in $B^{0} \bar{B}^{0}$ interact in the CsI calorimeter. Preliminary Monte Carlo studies have shown that the fraction of all $K_{L}^{0}$ which reach the IFR varies according to the hadron simulation package used; the only experimental data available [Say68] seem to indicate that $K_{L}^{0}$ cross sections on nuclei are overestimated in the GHEISHA package and agree with those used in FLUKA. This is consistent with our findings and encourages us to use FLUKA for these studies.

Some preliminary results from a simulation employing FLUKA indicate that the probability of identifying a $2 \mathrm{GeV} / c K_{L}^{0}$ requiring at least 5 layers hit is about $70 \%$ for iron plates $1 \mathrm{~cm}$ thick (as seen in Figure 9-5), dropping to $\sim 50 \%$ if the plates are $2 \mathrm{~cm}$ thick. Showers produced by photons of the same momentum in the CsI calorimeter do not penetrate past the first $1-2 \mathrm{~cm}$ of iron. Requiring no energy to be deposited in the CsI calorimeter (i.e. selecting those $K_{L}^{0}$ 's that 

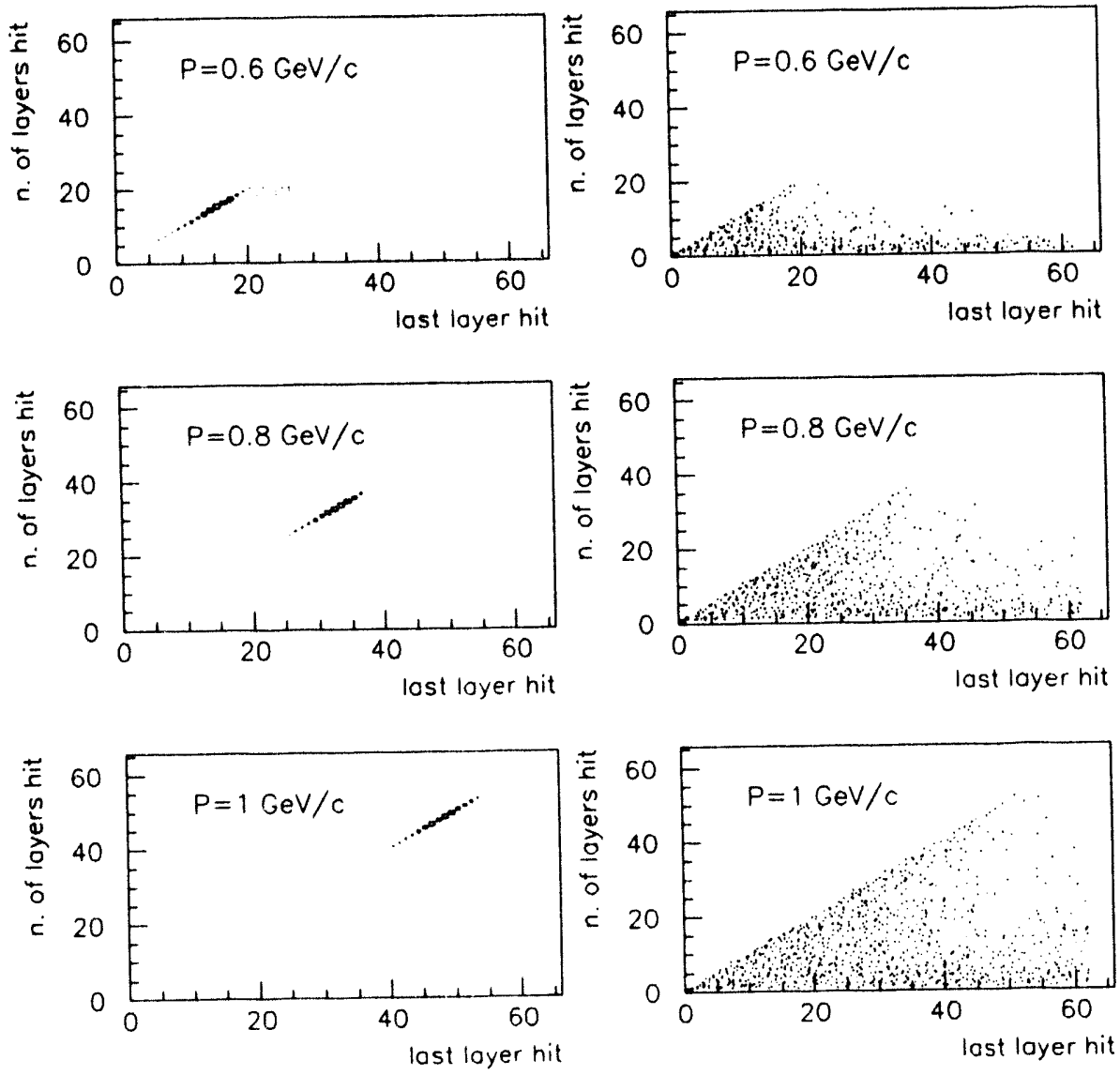

Figure 9-4. Number of planes hit versus last plane hit for muons (left) and pions (right) at different momenta

interact directly in the IFR) reduces the identification probability to $20-25 \%$. The angular distribution of the IFR hits relative to the incident $K_{L}^{0}$ direction is given in Figure 9-6. The angular resolution is typically a few degrees, which is adequate for the kinematic cuts used to reconstruct $B^{0} \rightarrow J / \psi K_{L}^{0}$ final states. A detector layer inserted before the coil may be of real benefit here and is being investigated. 


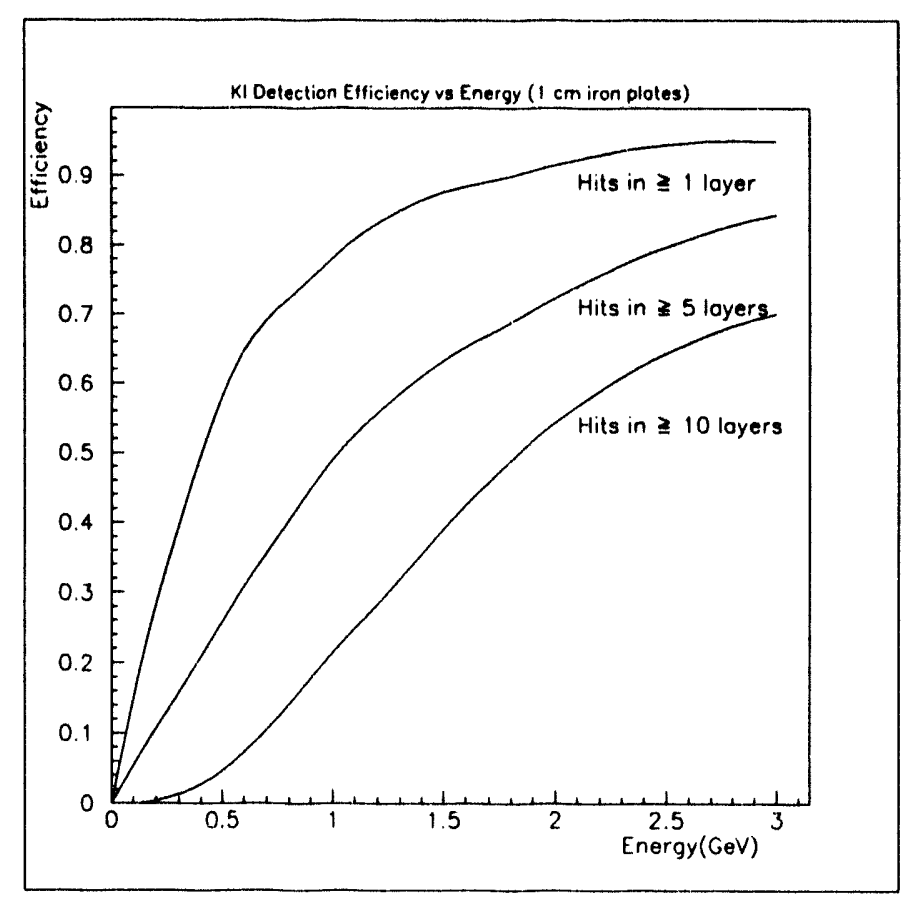

Figure 9-5. Fraction of $K_{L}^{0}$ that produce hits in an IFR with $1 \mathrm{~cm}$ steel plates.

\subsection{The Active Detectors}

The sensitive area is $\sim 4000 \mathrm{~m}^{2}$, with $\sim 50,000$ readout channels
The instrumentation of the flux return covers a surface of $\sim 4000 \mathrm{~m}^{2}$, has a total of $\sim 50,000$ readout channels and is inserted into the gaps between the steel plates. Access for maintenance and/or repair will be very difficult at best. The main requirements for the detector technology are therefore the following: long term reliability, sturdiness, simplicity of constructicn and operation, and low cost of the raw materials and readout electronics.

Two well-known techniques that satisfy these requirements have been considered: Plastic Tubes operated in the limited streamer mode (PSTs) and Resistive Plate Chambers (RPCs). Both are gas detectors in which a sizeable pulse is induced on external electrodes by the charge produced by a particle ionizing the gas. In principle, RPCs have better time resolution and less dead space and the lack of wires makes them less likely to have breakdowns. PSTs are a more mature technique; both have been proven to work reliably in large detectors. 


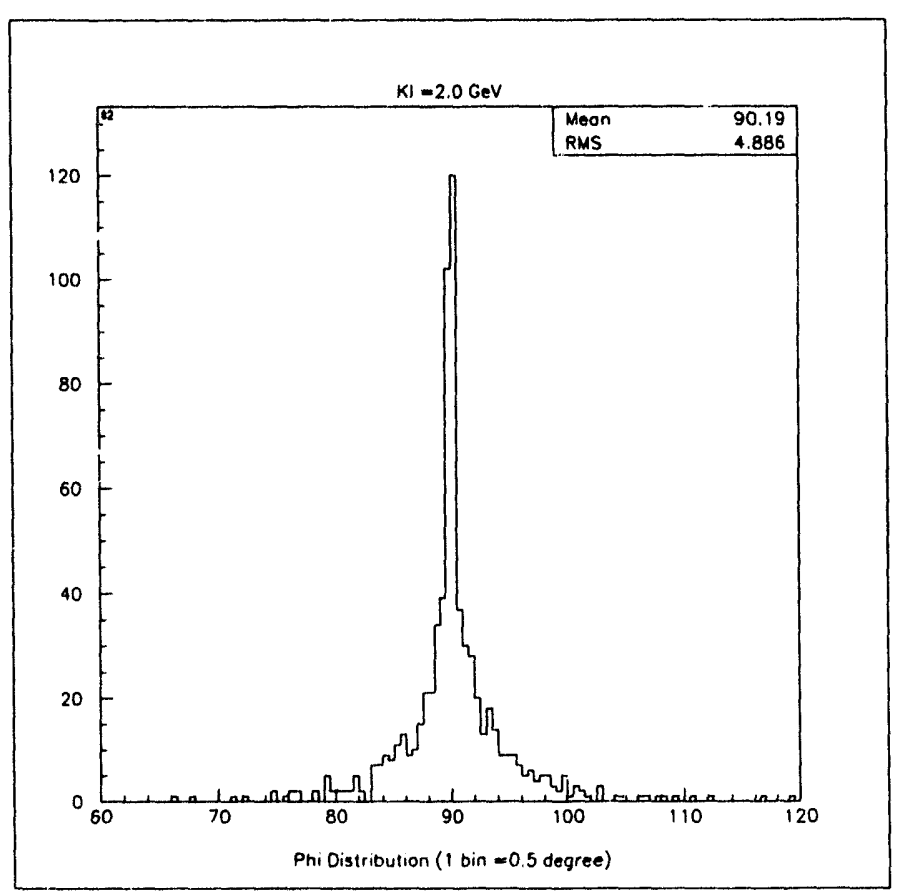

Figure 9-6. Angular difference between the incident $K_{L}^{0}$ direction and the IFR hits.

The collaborating institutions on BABAR have expertise in both detector technologies; the group is confident that either technology will work. The cost estimate is based on an RPC system, which is currently the preferred choice of the majority of the IFR group. The final choice will be made after a careful evaluation of the relative costs and merits of the two technologies.

\subsubsection{Resistive Plate Chambers}

Resistive Plate Chambers (RPCs) are large area, parallel plate detectors operated in DC mode. They are under consideration for the IFR because of their low cost, high efficiency, good timing, and simple electronics requirements. RPCs are constructed of two parallel plate electrodes (traditionally bakelite) of high electrical resistivity held apart by spacer buttons and filled with a mixture of argon, isobutane, and freon. With an electric field of $4 \mathrm{kV} / \mathrm{mm}$ between the electrodes, a charged particle crossing the gap will initiate a sparklike discharge between the electrodes which termi-
An RPC system is the basis for the cost estimate 
Bakelite RPCs have been successfully used in a variety of experiments

ABS-based RPCs have also been developed nates when the electric field collapses, due to the resistance of the plates. The spark is of sufficient amplitude to induce large puises on external striplines, which are then read out to determine the particle's position.

This technique, originally developed by $\mathrm{R}$. Santonico and collaborators [San81] [San88], has been proposed for muon detectors at future hadron collider experiments, because of the superior timing performance and suitability to detectors with very large surface area. This technique also looks attractive for $B A B A R$ because the assembly of wireless chambers is straightforward; they can be readily adapted to the endcap design, where the geometry is not longitudinal as in the barrel and the chambers will not have a rectangular shape. Another advantage of this technique is that the production process has already been adapted to industry and factories with the necessary tooling exist.

RPCs have been used successfully in the NADIR experiment at Grenoble, FENICE at Adone (Frascati), E771 at FNAL and WA92 at CERN, and in several cosmic ray detectors. A recent larger scale application of this technique is the trigger system built by members of the group [Nap93] for the L3 muon endcaps, consisting of $600 \mathrm{~m}^{2}$ of double gap chambers. This design has an independent high voltage supply to each gap, resulting in more than $99 \%$ efficiency and providing a backup capability in case of fairure. This feature seems particularly attractive for a system that is difficult to access, such as the IFR. Both sides of the high voltage plane are active gaps, with the readout strips in the middle, as shown in Figure 97. A two-dimensional scheme, with two planes of strips running in orthogonal directions, has also been proposed [Bac93].

Other members of the group have prototyped several large RPCs that utilize a doped ABS plastic sheet as the resistive electrode. The doped $A B S$ is manufactured and sold as a static dispersive packaging material for electronics components; it is manufactured with strict quality control on resistivity and produced in easy-to-handle plastic rolls. Like their traditional bakelite counterparts, the doped ABS RPCs exhibit high efficiency and time resolutions of 1 to 3 ns. Figure 9-8 shows a cross section of this design. The two resistive electrodes are made of sheets of $0.030^{\prime \prime}$ thick doped ABS plastic. They are held apart by $2 \mathrm{~mm}$ high plastic buttons. High voltage $(8500 \mathrm{~V})$ is applied to the top electrode through a $0.004^{\prime \prime}$ graphite 


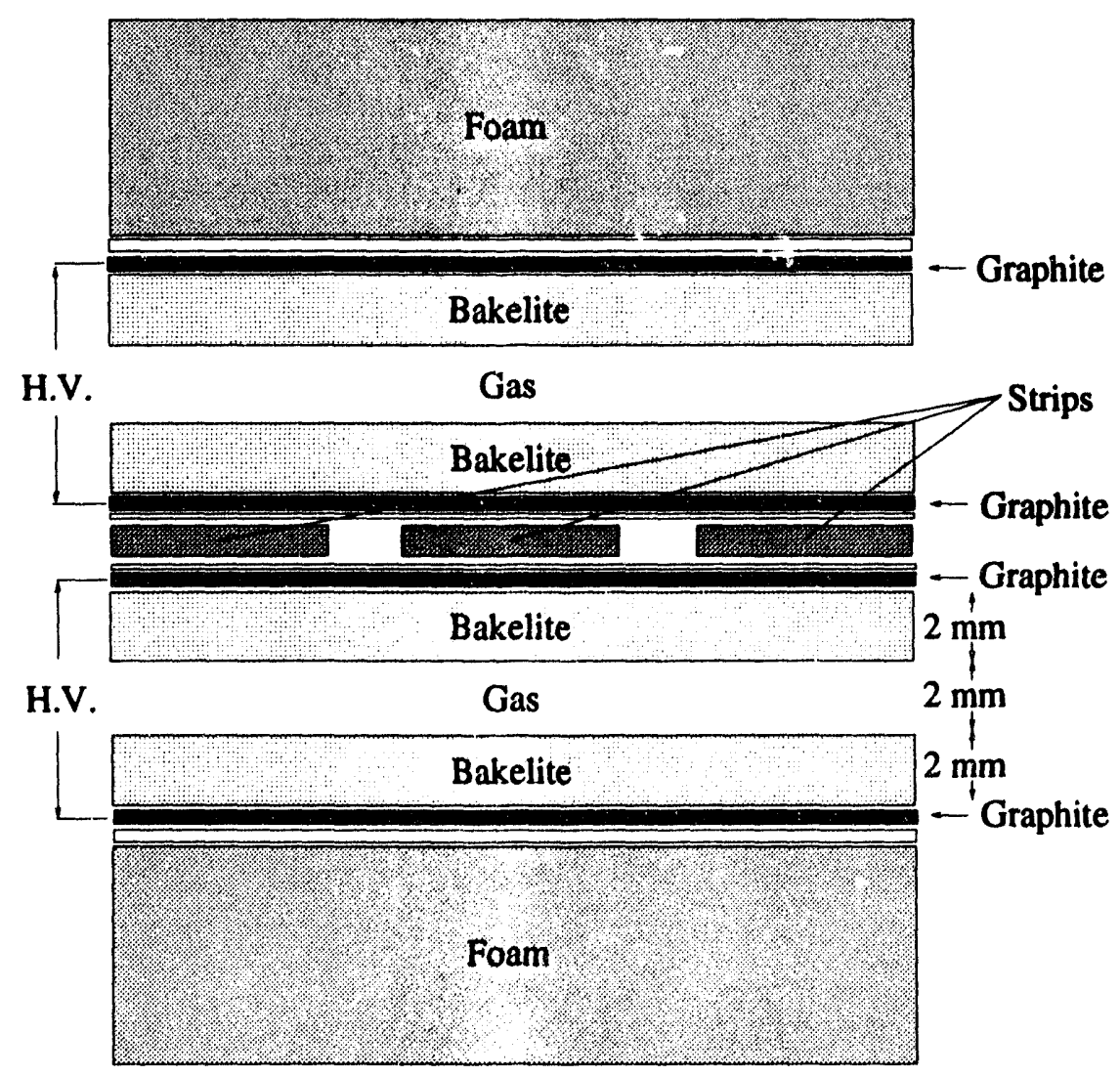

Figure 9-7. Cross section of the L3 RPCs.

sheet (Velostat) laminated directly to the ABS plastic and protected by a $0.005^{\prime \prime}$ mylar sheet. The bottom electrode is grounded through an array of copper readout strips deposited directly on the ABS plastic. A thicker spacer grid holds a $0.0007^{\prime \prime}$ thick sheet of $\mathrm{Al}$ foil at the proper distance to form the ground plane of a $50 \Omega$ transmission line for propagating the induced pulses.

$\mathrm{R} \& \mathrm{D}$ activity at the various institutions in the coming months will focus on alternative gas mixtures, with emphasis on eliminating freon and reducing flammability, and on mass production techniques with stringent quality control.

\subsubsection{Plastic Streamer Tubes}

Plastic Streamer Tubes (PSTs) are modular chambers made of extruded PVC, $1 \mathrm{~mm}$ thick, coated inside with graphite-loaded sol- 


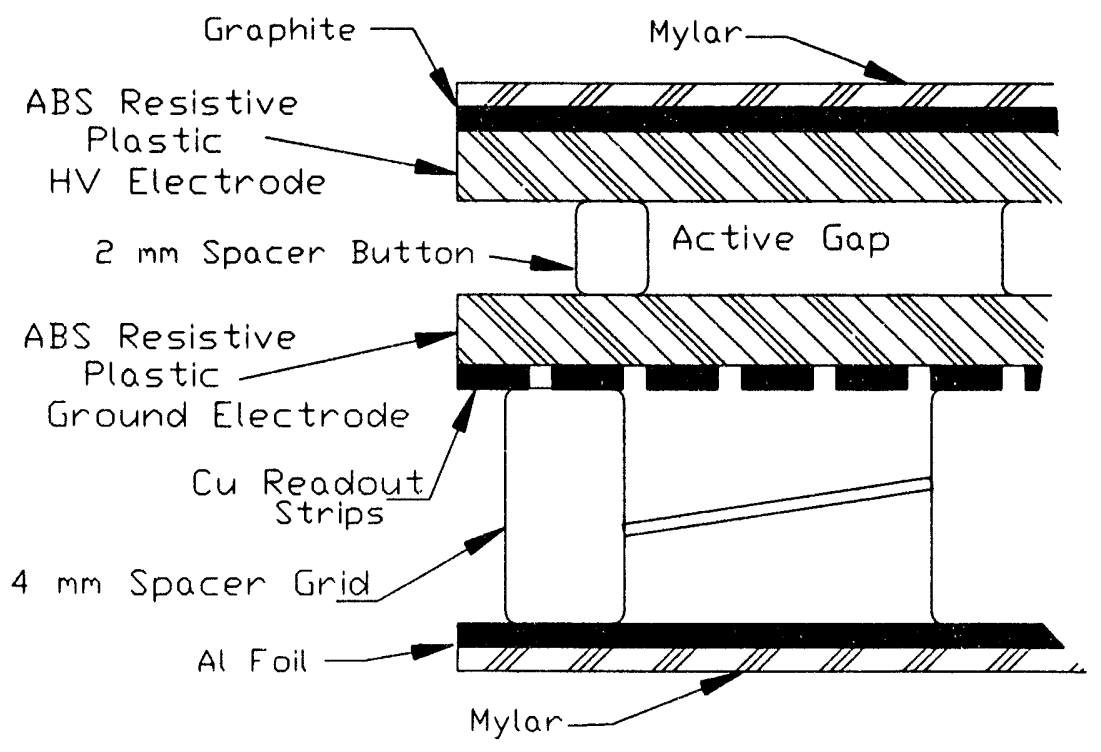

Figure 9-8. Cross section of the prototype ABS RPC.

PSTs, mostly of similar design, are in wide use in many HEP experiments vent. The resistivity of the coating is typicaliy 0.2 to $2.0 \mathrm{M} \Omega$ per square, so that the outer wall of the tube is transparent to fast voltage transients.

This technique was first used in the NUSEX Proton Decay Experiment at Mont Blanc [Iar83], in the early 1980's. It is presently employed in ALEPH, OPAL and DELPHI at LEP, SLD at SLC, ZEUS at HERA, MACRO and LVD at Gran Sasso, and several other experiments.

Most of these systems are based on the same design: a comb-shaped extruded PVC profile, coated with graphite, forms 8 square open cells, $1 \times 1 \mathrm{~cm}^{2}$ in size. The cathode is three sided, with an active cross section of $0.9 \times 0.9 \mathrm{~cm}^{2}$. The anode wire, typically $100 \mu \mathrm{m}$ thick, is strung in the middle and kept in position by $\mathrm{V}$-shaped grooves in a plastic holder at both ends. The wires are soldered onto a PC board which is supported by a board holder. Plastic bridges, placed about every $50 \mathrm{~cm}$ along the chamber, keep the wire in a fixed position. The 8-cell profiles are inserted in a plastic envelope which is sealed at both ends with plastic plugs which include connectors for $\mathrm{HV}$ and gas. A cutaway view of one module is shown in Figure 9-9. 
Several modules can be assembled together in large chambers with either one or two planes of readout strips.

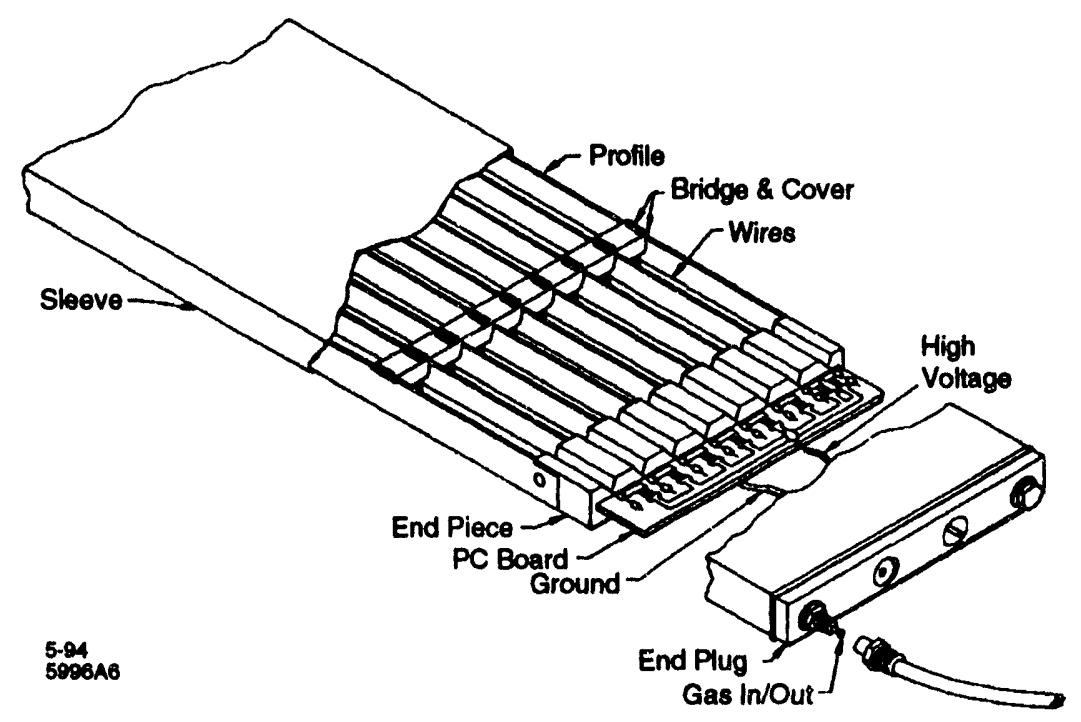

Figure 9-9. Cutaway view of one end of a PST module.

The standard gas mixture used to operate PSTs in limited streamer mode is argon-isobutane 1:3, but non-flammable mixtures have also been found to be suitable. The SLD tubes operate with a mixture of $\mathrm{CO}_{2}$, Argon and less than $10 \%$ isobutane, which is non-flammable and satisfies SLAC safety standards.

As a result of design standardization, highly automated machines have been developed for mass production. Within the collaborating institutions there are facilities at IHEP in Beijing, JINR in Dubna and at the University of Houston. PSTs are also produced by industry which sells the final tested assemblies complete with readout electrodes.

\subsection{Collaborating Institutions}

Several institutions, from different countries, have expressed interest in contributing to the Instrumented Flux Return detectors; all teams have previous experience in building large systems of either PSTs or RPCs and/or have done extensive R\&D on these detectors.
Several collaborating institutions have PST production facilities. There are also commercial sources 


\section{References}

[Bac93] Bacci et al., Sezione di Roma dell'INFN Report 1024, Dec. 1993 (submitted to Nucl. Instr. Meth. ).

[Iar83] E. Iarocci et al., Nucl. Instr. Meth. A217, 30 (1983).

[Nap93] Naples group, L3 Internal Note, Dec. 1993.

[PS202] Experiment PS202, preliminary data (private communication).

[San81] R. Santonico and R. Cardarelli, Nucl. Instr. Meth. A187, 377 (1981).

[San88] R. Santonico and R. Cardarelli, Nucl. Instr. Meth. A263, 20 (1988).

[Say68] G.A. Sayer, E.F. Beall, Phys. Rev. 169, 1045 (1968). 


\section{0}

\section{Magnet Coil and Flux Return}

$\mathbf{T}$ he $B A B_{A R}$ magnet is a $1.5 \mathrm{~T}$ thin superconducting solenoid design within a hexagonal flux return, as shown in Figure 10-1. The design satisfies requirements identified during detector performance simulations, geometry studies, and cost evaluations. The magnet inner radius and length will be optimized in conjunction with other detector subsystems. The anticipated inner radius is $1.5 \mathrm{~m}$ with a cryostat length of $3.8 \mathrm{~m}$.

This magnet, though specifically tailored to the requirements of PEP-II physics, is similar to many operating detector magnets. In developing this design, physics criteria, including central field magnitude and uniformity, size, and overall geometry were examined. The range of feasible magnet options narrowed as principal technologies of detector elements were selected. Features of the proposed magnet are now reasonably well characterized. Similar coils operate in many detectors throughout the world in both resistive (SLD) and superconducting (ALEPH, CDF, CLEO-II, DELPHI, H1, TOPAZ, ZEUS) configurations. The state-of-the-art is well understood [Des85, And82, Coils] and a strongly competitive industrial base exists.

Objectives for the detector, including the fabrication schedule, can be achieved [And82]. A good understanding exists of the influence of detector physics requirements on the major cost drivers (central field, size, and type; superconducting or resistive). Final specification of magnet parameters will balance detector performance with operating and construction cost objectives.

Fifteen collaborating institutions have expressed interest in magnet system design. This team is composed of personnel with expertise necessary to complete this effort. Many detector magnets and hundreds of other large magnets have been fabricated by these institutions. Delegation of responsibilities among the team has not been completed.

The BABAR magnet uses a superconducting coil with an inner radius of $1.5 \mathrm{~m}$ and a cryostat length of $3.8 \mathrm{~m}$ 

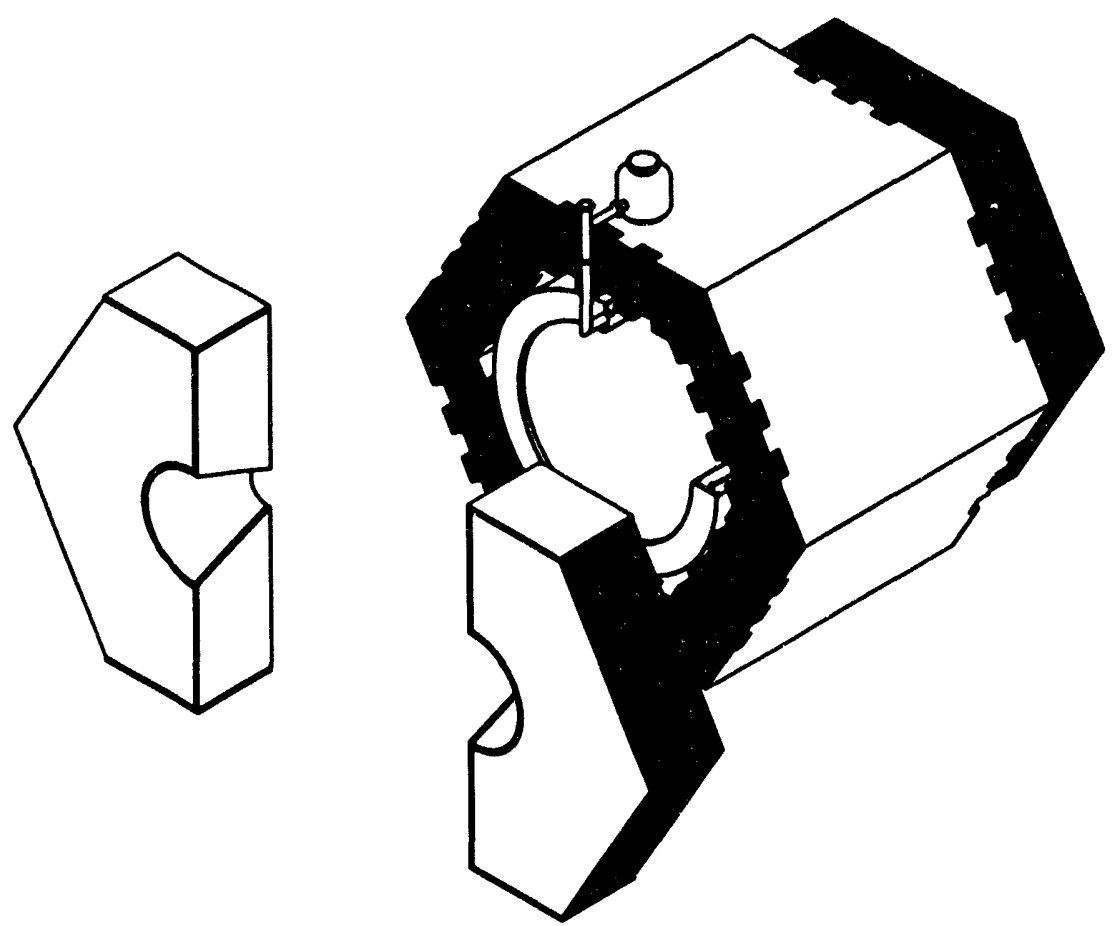

Figure 10-1. Geometry of the magnet system. The thin solenoid is shown within the barrel of the hexagonal flux return. The endcaps are separable about the vertical center to clear nearby fixed beamline components. The storage dewar and service stack is shown above the barrel flux return. Slots are provided on the periphery of each end of the barrel for detector services.

\subsection{Physics Motivation}

Fundamental detector performance and geometry considerations determine the design of both the solenoid and the flux return. Charged particle tracking resolution is a sensitive function of magnetic field and of the radius and length of the tracking volume. The required field uniformity is a function of main tracker volume and performance objectives. Studies of detector resol tion for $B^{0} \rightarrow \pi^{+} \pi^{-}$ suggest that a magnetic field of $1.5 \mathrm{~T}$ is necessary to achieve the desired resolution $\left(<25 \mathrm{MeV} / c^{2}\right)$ in the presence of the support tube for interaction region beam line components [Ale94]. The combined radii of the vertex detector, tracking chamber, particle identification system, electromagnetic calorimeter and appropriate 
clearances specify the minimum solenoid inner bore. Solenoid length is determined by the length of the nested subsystems within. The desire to lower the momentum threshold for identifying muon detection and to increase the efficiency of $K_{L}^{0}$ detection within the instrumented flux return limits solenoid thickness, and drives the design towards a superconducting solution.

The segmented geometry of the flux return allows the tracking of muons and provides for detection of $K_{L}^{0}$ 's with adequate angular resolution (see Chapter 9 ). Overall steel thickness is determined by magnetic considerations and the requirements of $\pi / \mu$ separation. Individual plate thicknesses are specified to allow identification of low momentum muons in a background of penetrating pions by virtue of their range and scattering angle in iron for a given momentum, and to efficiently identify $K_{L}^{0}$ 's.

The overall thickness of the flux return is the sum of both the steel thickness and the number and thickness of the IFR layers. The number of iron/detector segments in the flux return defines the lowmomentum muon and neutral hadron detection performance for a given cost. Separation and movement of the endcaps is constrained by beam line components and by the need to provide ready access to detector subsystems.

\subsection{Magnet System Requirements}

The magnet system consists of the solenoid, the flux return and supporting systems including cryogenics. Magnet requirements are derived from detector and accelcrator performance objectives, engineering considerations, operational and facility requirements and project constraints (cost, schedule, risk, and ES\&H). Identification of these requirements and constraints began during the development of the Letter of Intent design and will continue during conceptual design.
The segmented flux return serves for $\pi / \mu$ identification and for $K_{L}^{0}$ detection 


\subsubsection{Solenoid Requirements}

Magnetic fields in the range from 1 to $1.5 \mathrm{~T}$ have been considered. Magnets with lower fields are less expensive, but a $1.5 \mathrm{~T}$ field provides better mass resolution in the presence of the interaction region support tube.

The range of warm bore diameters considered varied between 2.8 and $4.2 \mathrm{~m}$, encompassing the range of sizes required by the CsI and liquid krypton calorimeter options. With the elimination of the LKr electromagnetic calorimeter option, the range now under consideration has narrowed to $2.8-3.1 \mathrm{~m}$. The aerogel and Fast RICH particle identification options require more radial space than does the DIRC option. If a choice of particle identification system can be made at an early enough date, the coil diameter can be reoptimized.

As the coil length scales in proportion to the radius, cryostat lengths varying from $3.8-5.8 \mathrm{~m}$ have been considered. The range of cryostat lengths now under consideration is $3.8-4.1 \mathrm{~m}$.

The magnet has been modelled with the $2 \mathrm{D}$ magnetostatic analysis code, PE-2D, part of the Tosca-3D family of magnetic analysis codes [VecF]. The code verified a simple Ampere's Law estimate that $1.23 \mathrm{MAT} / \mathrm{m}$ is required to produce a central induction of $1.5 \mathrm{~T}$. A plot of central field along the detector axis is shown in Figure 10-2.

The cost of cryogenics is included

The thickness of the coil in interaction lengths limits low momentum muon identification
Facility constraints limited some of the options considered. The IR2 site can provide a maximum of $3 \mathrm{MW}$ of low conductivity water (LCW) cooling for a resistive magnet. LHe cryogenics is not currently available at IR2. The cost of the superconducting magnet includes the cost of associated cryogenics.

The desired thickness of the solenoid and cryostat is $\sim 25-40 \%$ of a nuclear interaction length $\left(\lambda_{\text {int }}\right)$ to assure low momentum muon identification. Initial simulations predict that an increase in thickness of $5 \%$ will increase the muon identification threshold by $10 \mathrm{MeV}$. More detailed analysis of these effects is currently underway. 
Magnetic Field on the Beam Axis

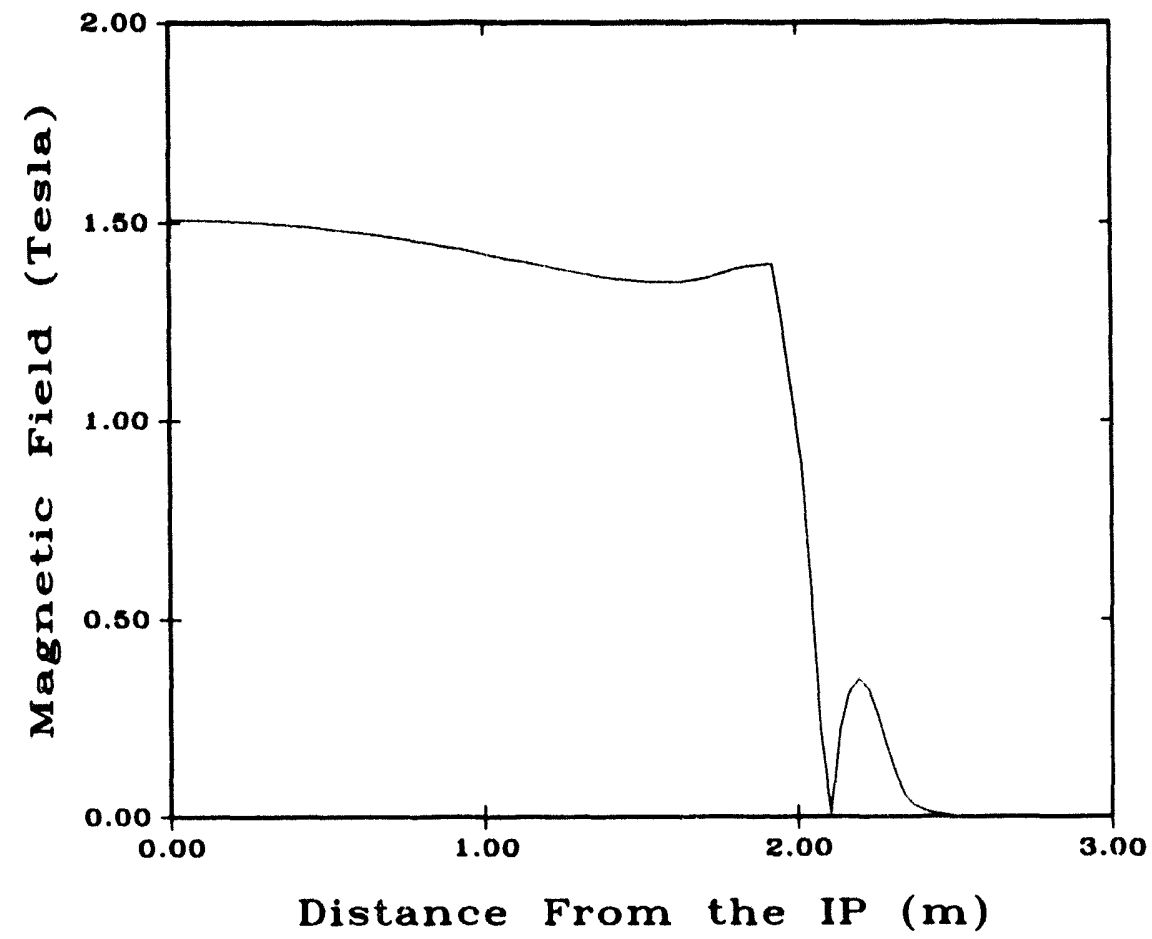

Figure 10-2. Central induction along the axis. The region near the beamline $Q 2$ quadrupole $(2.5 \mathrm{~m}<r<3.0 \mathrm{~m})$ is shielded for proper septum quadrupole performance. No optimization of field quality has yet been made.

\subsubsection{Flux Return Requirements}

The flux return has several functions. It supports detector components, provides a low reluctance flux return path and acts as a $\pi / \mu$ identifier and $K_{L}^{0}$ detector. The spacing between steel plates for hadron calorimeter measurement layers is tentatively specified as $3 \mathrm{~cm}, 0.8 \mathrm{~cm}$ larger than provided in the SLD flux return, to allow inclusion of double layers of detectors, if required.

The flux return plate thickness is currently $2.5 \mathrm{~cm}$, spaced at regular $3 \mathrm{~cm}$ intervals. Simulations are underway to optimize the plate segmentation for a low momentum threshold for $\pi / \mu$ separation and efficiency for $K_{L}^{0}$ detection.

The total thickness of the stecl layers in the barrel and endcap is determined both by the minimum steel required to avoid magnetic 


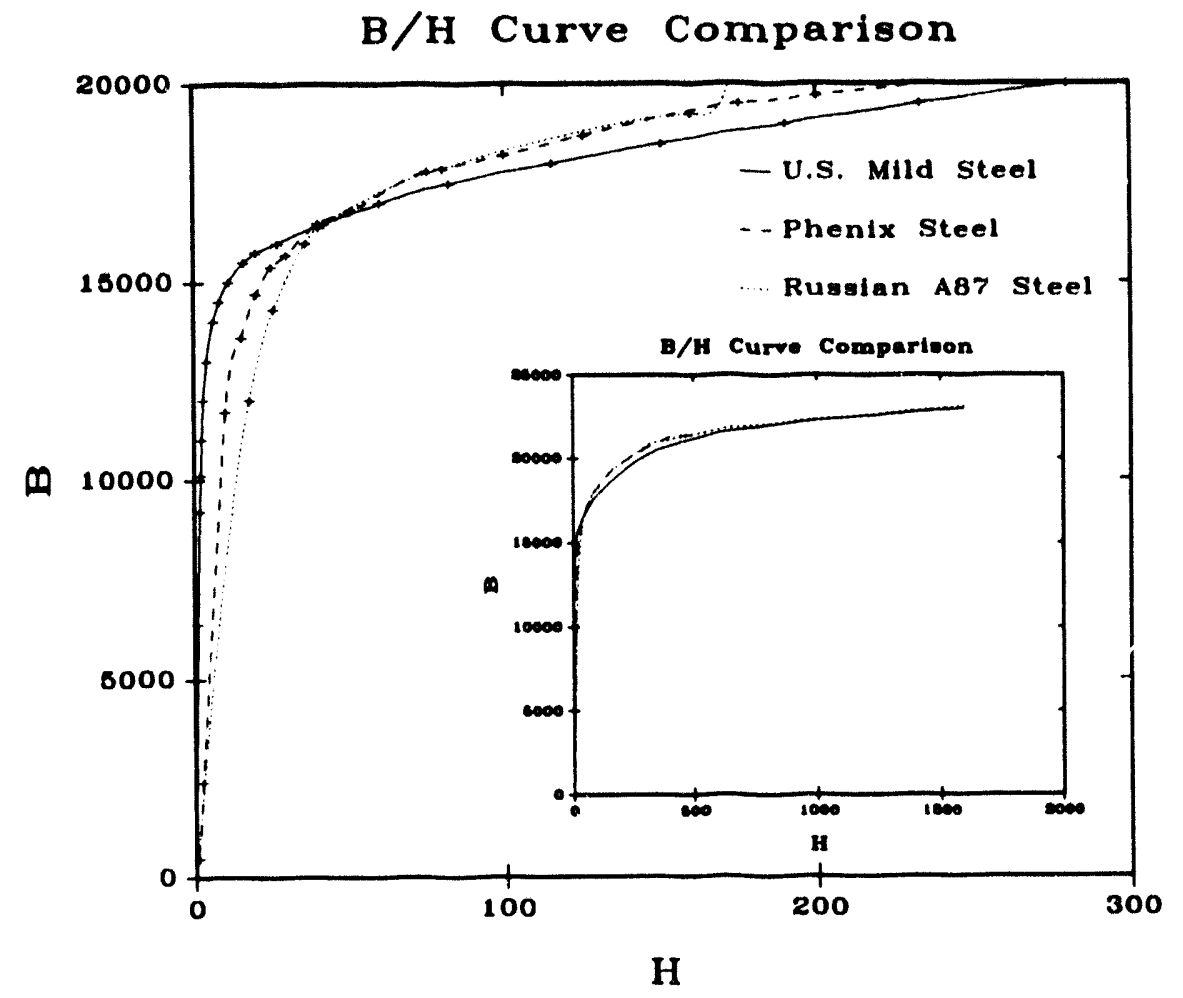

Figure 10-3. Comparison of magnetic properties for typical US and Russian low-carbon steels reflecting differences in processing techniques.

The total thickness of steel required for magnetic reasons and by the pion punch-through requirement is roughly identical
The beam intersection point is $3.5 \mathrm{~m}$ above the floor of IR2 saturation and by the need for sufficient thickness to ensure that most of the pions interact in the steel. The initial estimate of the amount of steel necessary to prevent pion punch-through is $60 \mathrm{~cm}\left(\sim 3.6 \lambda_{\text {int }}\right)$. This estimate will be refined during detector simulations. The thickness of steel required to contain magnetic flux will depend on the specific magnetic properties of the steel employed. With typical US low-carbon steel, $62 \mathrm{~cm}$ is estimated for the barrel region. This could increase if less expensive steel provided by foreign sources [Fac94] were used. A comparison of the magnetic properties of typical US and Russian low carbon steels is shown in Figure 10-3, and reflects differences in the processing. Thus the magnetic and punch-through requirements for total steel thickness are quite compatible.

The radial distance from the outer surface of the flux return to the detector center line cannot exceed $3.5 \mathrm{~m}$ in the downward direction, 
the distance from the interaction point to the IR2 Experimental Hall floor.

Operational access requirements of detector components and nearby accelerator beam line components make it desirable that the hexagonal endcaps be split on a vertical center line. The doors can retract at an angle of $\sim 45^{\circ}$ to the beam line to allow both clearance of CsI crystal endcaps attached to the doors and clear passage by the nearby components [Bow92, p317]. Clearance must be provided for beam line components, detector services and cabling, and the support tube [Bow94].

The endcap field-shaping pole pieces must accommodate beam line components and provide adequate magnetic shielding (down to less than a few hundred gauss) for the enclosed septum quadrupole (Q2). The pole piece also contributes to the axial field uniformity within the main tracking chamber; uniformity is specified as better than $1-2 \%$. This optimization has not yet been completed.

The four year $B_{A} B_{A R}$ project requires challenging but achievable construction schedules for the solenoid and flux return. Both must be ready for assembly at the IR2 hall in the Spring of 1997 in order to accommodate the detector assembly, installation, and commissioning tasks that follow magnetic field mapping.

\subsection{Trade Studies}

Magnet trade studies were carried out as part of detector performance and geometry studies. The three principal magnet option considered were the TOPAZ superconducting solenoid, a new thin superconducting solenoid and a new thick resistive solenoid.

Magnet conceptual designs and cost models were evaluated for compatibility with the principal magnet requirements as part of the study of detector performance. Resistive coil options are limited to $1 \mathrm{~T}$ by cost, risk and local LCW limitations. A thin, $1.5 \mathrm{~T}, 3 \mathrm{~m}$ diameter, $4 \mathrm{~m}$ long superconducting magnet has been selected as the best balance of performance, cost and risk. The advantage of a new superconducting solenoid is that it precisely matches detector requirements. The principal disadvantage is that, especially for
The magnet doors are split vertically and retract at an angle
Superconducting and resistive coils were evaluated, as was the reuse of existing solenoids

The practical limit for a resistive coil of this size is $1 T$ 
The use of the TOPAZ magnet was investigated

A normal magnet has a lower capital cost and a higher operating cost

$A$ layered and instrumented resistive coil was also investigated larger fields and volumes, the capital cost is higher than that for other options.

The 1.2 $\mathrm{T}$ superconducting magnet now in use in the TOPAZ experiment at KEK was considered as a possible low-cost alternative. It is likely to be available on a reasonable timescale and provides magnetic fields of $1-1.2 \mathrm{~T}$ in a warm bore of $2.72 \mathrm{~m}$ for something over half the cost of a new magnet. It was rejected because, at $5.4 \mathrm{~m}$, it is too long. The extra length immerses the Q2 septum quadrupole deeper into the detector magnetic volume, requiring heavy, unwieldy pole pieces and a superconducting shielding solenoid for proper quadrupole operation. These extra beam line components make access to the forward region of the drift chamber a difficult and time-consuming endeavor.

A resistive coil must be thicker than a superconducting coil of equivalent performance. A resistive coil has 3-5 times the interaction lengths of a thin superconducting coil. To develop a $1 \mathrm{~T}$ central field in a $3 \mathrm{~m}$ diameter by $4 \mathrm{~m}$ long volume with $2 \mathrm{MW}$, would require an aluminum coil of $60 \mathrm{~cm}\left(1.5 \lambda_{\text {int }}\right)$ thickness. Hadron calorimetry simulations suggests significant loss of performance for such designs. The resistive solenoid option has a capital cost savings of $\$ 1-2 \mathrm{M}$ over a superconducting coil of $1 \mathrm{~T}$, but has a larger operating cost $(\$ 1.1 \mathrm{M} /$ year vs. $\$ 250 \mathrm{k} /$ year). Operation at $1.5 \mathrm{~T}$ is not practical even if the thickness were to be acceptable, due to an estimated annual operating cost of $\$ 2.5 \mathrm{M} /$ year.

An interesting design alternative is to instrument a layered resistive coil. An instrumented coil would consist of nested cylinders wound in layers as in the SLD design. With ten $3 \mathrm{~cm}$ thick layers and five $6 \mathrm{~cm}$ thick outer layers, an aluminum $1 \mathrm{~T}$ solenoid could satisfy the $3 \mathrm{MW}$ cooling water limitation. While the total thickness remains $1.5 \lambda_{\text {int }}$, it is now subdivided for muon and $K_{L}^{0}$ identification, with detectors placed between the self-supporting coils. A further benefit is that aluminum better differentiates by range between muons and hadrons than iron. Unlike a superconducting coil, an instrumented resistive coil has fewer dead regions where muons can range out and $K_{L}^{0}$ 's can first interact. The cost of an $80 \mathrm{t}$ instrumented solenoid would be somewhat lower than a comparable superconducting coil, but operating costs would be higher, as would the technical risk of this untried approach. 


\begin{tabular}{|l|c|c|}
\hline Item & Value & Unit \\
\hline \hline Central Induction & 1.5 & $\mathrm{~T}$ \\
Mean Winding Radius & 1.676 & $\mathrm{~m}$ \\
Inner Radius of Cryostat & 1.496 & $\mathrm{~m}$ \\
Outer Radius of Cryostat & 1.846 & $\mathrm{~m}$ \\
Cryostat Length & 3.75 & $\mathrm{~m}$ \\
Axis Above Floor & 3.5 & $\mathrm{~m}$ \\
Magnetic Field (5\% margin) & 5.0 & $\mathrm{MAT}$ \\
Number of Turns & 1000 & \\
Operating Current & 5.0 & $\mathrm{kA}$ \\
Stored Energy & 30.2 & $\mathrm{MJ}$ \\
Inductance & 2.4 & $\mathrm{H}$ \\
Coil Weight & 13 & $\mathrm{t}$ \\
Cold Mass Weight & 7 & $\mathrm{t}$ \\
He Refrigeration Load & 40 & $\mathrm{~W}$ \\
Current lead He Consumption & 18 & $\mathrm{l} / \mathrm{h}$ \\
Nuclear Interaction Length & $0.25-0.4$ & $\lambda_{\text {int }}$ \\
Adiabatic Hot Spot Temperature & 80 & ${ }^{\circ} \mathrm{K}$ \\
Discharge Time Constant (t) & 21 & $\mathrm{~s}$ \\
Emergency Discharge Time (5t) & 100 & $\mathrm{~s}$ \\
Maximum Discharge Voltage & 500 & $\mathrm{~V}$ \\
\hline
\end{tabular}

Table 10-1. Preliminary solenoid parameters.

\subsection{Solenoid Description}

The major elements of the magnet are the thin superconducting solenoid, the flux return and the cryogenic system. The superconducting solenoid consists of the coil in a cryostat; the vacuum system to provide the insulating vacuum; the power supply, quench detection and protection system; and the control system. The flux return is composed of a barrel and endcaps, support/translation/alignment stages for each movable section and a restraint to provide structural stability. The cryogenic system supplies LHe for the cold mass and vapor-cooled leads and $\mathrm{LN}$ for the thermal shields and heat intercepts.

The superconducting solenoid design will benefit from experience gained producing similar systems for other detectors over the past 


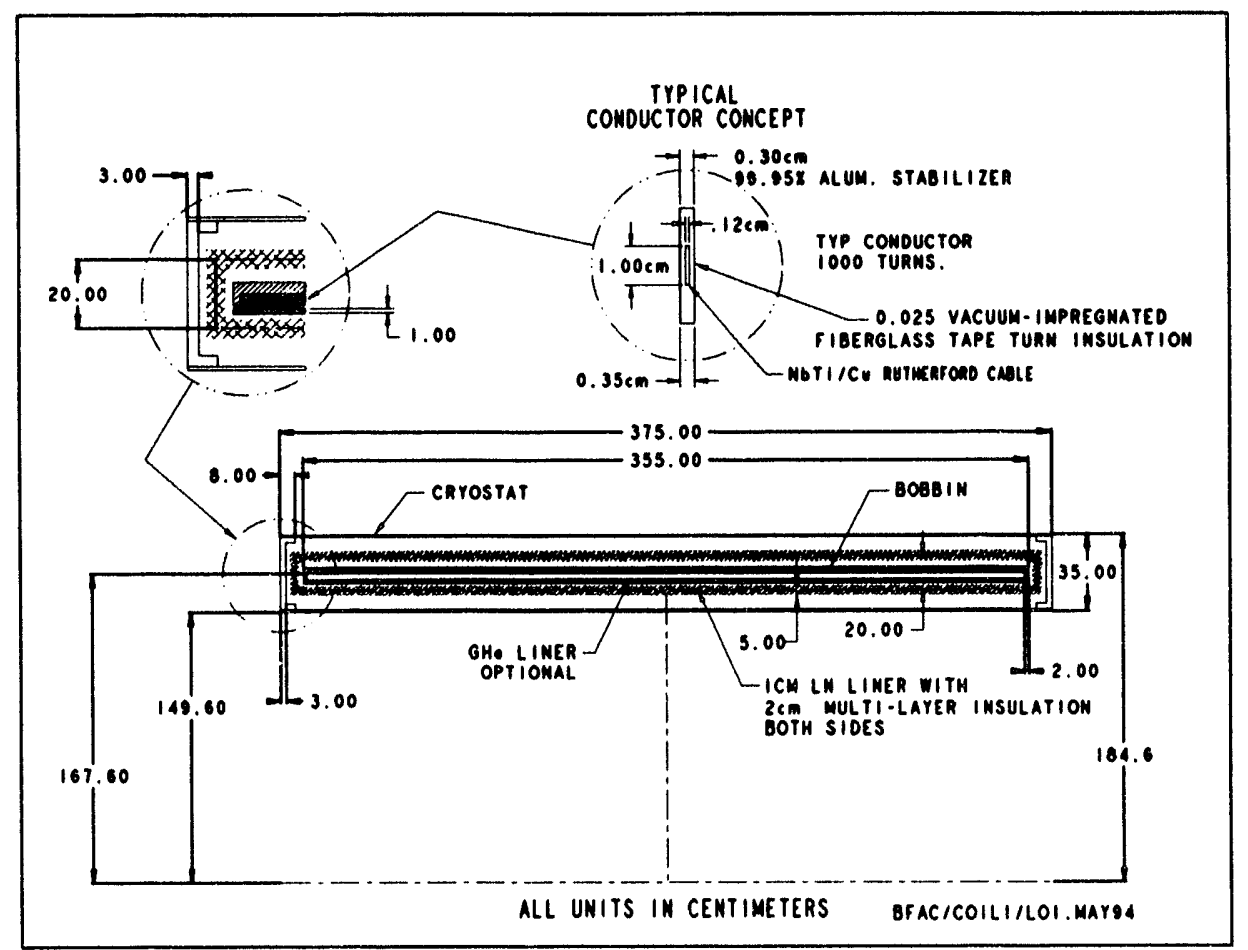

Figure 10-4. Cross-section of the superconducting magnet concept. Aluminum stabilized $\mathrm{NbTi} / \mathrm{Cu}$ superconductor is wrapped with insulation and wound into an external highstrength aluminum restraining hoop. The conductor is vacuum impregnated for stability and ridgidity. The resulting cold mass is suspended from the enclosing thin aluminum cryostat by radia! and axial supports that are optimized for strength and low heat input. Cryogen consumption is reduced by superinsulationcovered, LN-cooled radiation shields surrounding the cold mass within the insulating vacuum.

The technology of thin superconducting solenoids is well developed
15 years. The technology of modern thin superconducting solenoids has been described by Desportes [Des85] and Hirabayashi [Hir88]. High purity ( $>99.95 \%$ ) aluminum-stabilized $\mathrm{NbTi} / \mathrm{Cu}$ superconductor is employed to assure conductor stability with minimum radiation thickness. The inner bobbin is replaced by a simple radiation shield. The conductor is restrained only by an outer high-strength aluminum hoop that is the primary structural member of the cold mass. Fiberglass-tape insulated conductor is often wound directly into the bore of this cylinder and then vacuum impregnated for stable, effective electrical insulation. Coolant is supplied through tubes attached to the outer surface of the restraining hoop. This 


\begin{tabular}{|l|c|c|}
\hline Item & Value & Unit \\
\hline \hline Conductor Type: NbTi/Cu/Al & $(1: 1: 20)$ & \\
Superconductor Area & 10 & $\mathrm{~mm}^{2}$ \\
Conductor length & 11 & $\mathrm{~km}$ \\
Stabilizer Type:High purity Al & $(>99.95 \%)$ & \\
Stabilizer Size & $3.0 \times 30$ & $\mathrm{~mm}^{2}$ \\
Method of Stabilizer Fabrication & Co-extrusion & \\
Cooling Method & $\mathrm{TBD}$ & \\
Maximum Field on Conductor & 1.8 & $\mathrm{~T}$ \\
Maximum Operating Temperature & 5.0 & ${ }^{\circ} \mathrm{K}$ \\
Joint Type & $\mathrm{TBD}$ & \\
Joint Resistance (each) & $<5 \times 10^{-10}$ & $\mathrm{ohms}$ \\
Number of Joints & $<12$ & \\
$j_{o p}$ (NbTi/Cu) & $<1000$ & $\mathrm{~A} / \mathrm{mm}^{2}$ \\
$j_{c}$ (1.8 T, 5.2K) & 2,700 & $\mathrm{~A} / \mathrm{mm}^{2}$ \\
Temperature Margin & 3.2 & ${ }^{\circ} \mathrm{K}$ \\
Current Margin & 1700 & $\mathrm{~A} / \mathrm{mm}^{2}$ \\
\hline
\end{tabular}

Table 10-2. Preliminary superconductor parameters.

eliminates the large cryogen inventory and thick cryostat that is necessary for bath-cooled coils. The conductor is cooled by thermal conduction through the thickness of the hoop restraint. Coolant paths are usually designed to operate by thermosiphon (driven by the LHe density gradient), a technique pioneered on the ALEPH solenoid, to simplify operation and to somewhat decouple solenoid availability from He liquefier availability. In applications where it is important to keep the number of nuclear interaction lengths small, special techniques or materials (aluminum honeycomb-SDC; or carbon fiber reinforced plastic-VENUS) have been employed for the cryostat. These are not required in this application.

A preliminary design study is underway to characterize the important parameters of the superconducting solenoid conceptual design. Tables 10.1 to 10.5 show the current parameters of the coil and flux return. Figure 10-4 shows a cross-section of the solenoid concept.
Thermosiphon cooling, as pioneered by the ALEPH coil, will be employed 


\begin{tabular}{|l|c|c|}
\hline Item & Value & Unit \\
\hline \hline Winding Method & Internal & \\
External Hoop Restraint Material & High Strength Aluminum & \\
External Hoop Restraint Thickness & 3 & $\mathrm{~cm}$ \\
External Hoop Restraint Length & 3.54 & $\mathrm{~m}$ \\
Insulation Thickness (Turn to Turn) & 0.22 & $\mathrm{~mm}$ \\
Ground Insulation Thickness & 3 & $\mathrm{~mm}$ \\
Insulation System & TBD & \\
Radial Support system & Compressive (H1) or & \\
& Tangential (VENUS, TOPAZ) & \\
\hline
\end{tabular}

Table 10-3. Preliminary cold mass properties.

\begin{tabular}{|l|c|c|}
\hline Item & Value & Unit \\
\hline \hline Endcap Weight (each) & 100 & $\mathrm{t}$ \\
Barrel Weight & 310 & $\mathrm{t}$ \\
Restraint Structure Weight & 75 & $\mathrm{t}$ \\
Restraint Structure Method & TBD & \\
Total Steel Thickness & $>60$ & $\mathrm{~cm}$ \\
(Pion Criteria) & $>50$ & $\mathrm{~cm}$ \\
Total Steel Thickness & & \\
(Saturation Criteria) & 2.5 & $\mathrm{~cm}$ \\
Plate Thickness & 3 & $\mathrm{~cm}$ \\
Plate Spacing & TBD & \\
Thickness Grading & 15 & $\mathrm{~cm}$ \\
Barrel Slot for Services & Hydraulic cylinders on & \\
Transporter Type & Hillman rollers & \\
&
\end{tabular}

Table 10-4. Preliminary flux return parameters. 


\begin{tabular}{|l|c|c|}
\hline Item & $\begin{array}{c}\text { Thickness } \\
{[\mathrm{mm}]}\end{array}$ & $\begin{array}{c}\text { Interaction } \\
\text { Lengths }\end{array}$ \\
\hline \hline Al Inner Cryostat & 20 & 0.051 \\
Al 90K LN Shield & 2 & 0.005 \\
NbTi/Cu Conductor & 10 & 0.066 \\
Al Stabilizer & 20 & 0.051 \\
External Hoop Restraint & 30 & 0.076 \\
Al 20K He Shield (optional) & 2 & 0.005 \\
Al 90K LN Shield & 2 & 0.005 \\
Al Outer Cryostat & 20 & 0.051 \\
Insulation & N/A & \\
\hline Total & & $0.31 \lambda_{\text {int }}$ \\
\hline
\end{tabular}

Table 10-5. Estimate of coil and cyrostat thickness in nuclear interaction lengths.

\subsection{Cryogen Supply System}

Operation of the superconducting solenoid requires liquid helium and liquid nitrogen. Similar systems cool magnets throughout the HEP community [Lot86, Doi86].

Liquid nitrogen, for cold mass radiation shielding and the initial stages of coil cooldown, is supplied from a 5,000 gallon tank located on the apron above the experimental hall. The tank, which is an existing SLAC unit, will be refurbished to meet cryogenic system requirements. A second, similar vessel is available if a reserve LN2 supply is desired. Vacuum-insulated transfer lines supply LN2 to the thermal shields and the helium liquefaction plant.

The helium plant, consisting of a helium liquefier/refrigerator, a 4,000 1 supply dewar and a distribution valve box (DVB) is located adjacent to the experimental hall, approximately $60 \mathrm{~m}$ from the magnet.

The liquefier/refrigerator supplies a number of PEP-II systems. It is sized to meet the requirements of the BABAR superconducting solenoid, the two potentially superconducting beam line quadrupole magnets (Q1) and an auxiliary dewar/trailer for other SLAC ex-

The LN system will employ a refurbished existing tank

The steady state liquid helium requirement of the coil is $100 \mathrm{l} / \mathrm{h}$ 


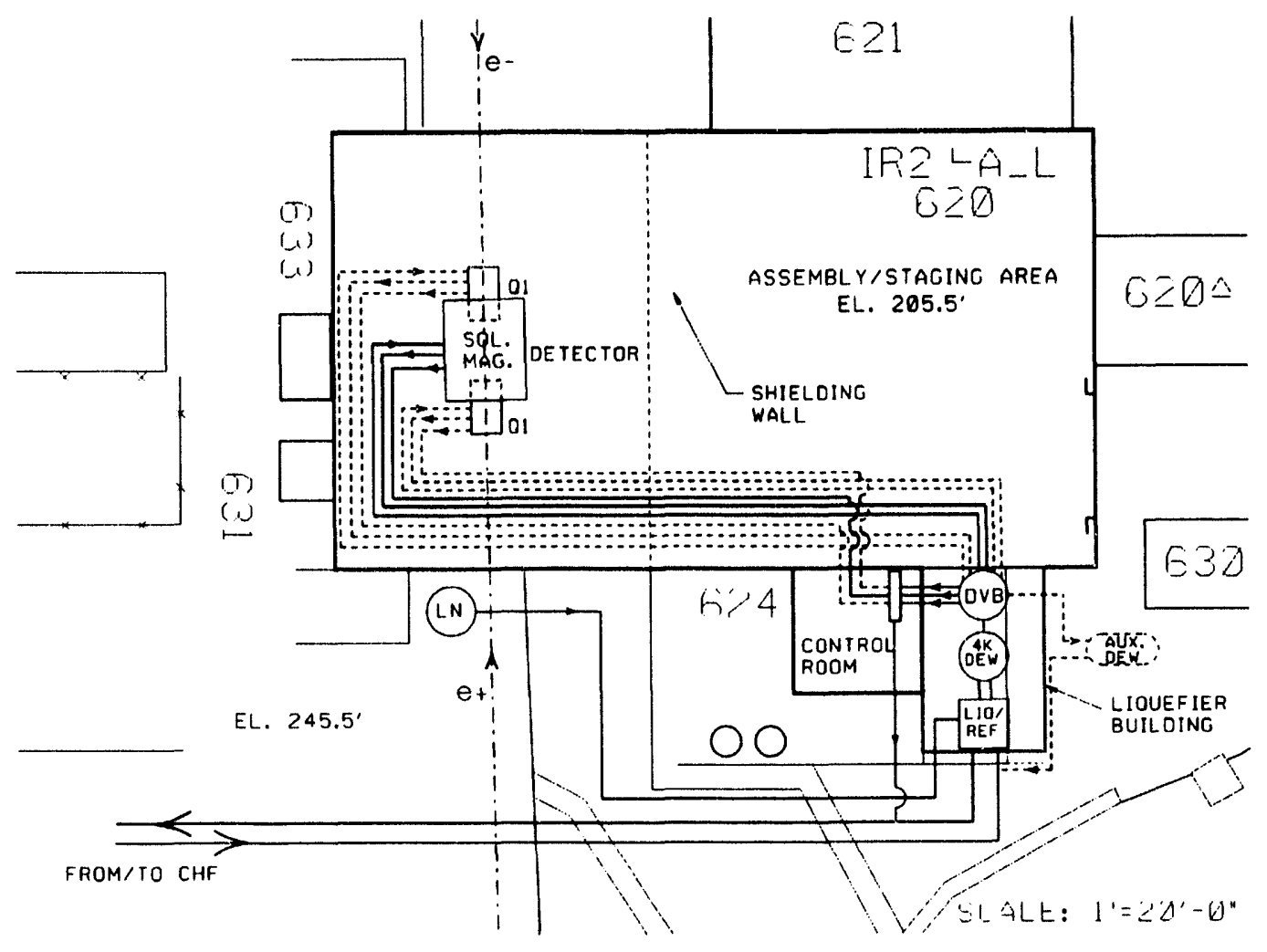

Figure 10-5. Layout of the cryogenic supply system in the Experimental Hall (IR2).

A refurbished 40001 liquid helium storage tank, fed by the central liquifier, provides a buffer against malfunctions periments. This is conservatively estimated at $150-200 \mathrm{l} / \mathrm{h}$, with solenoid steady-state consumption less than $100 \mathrm{l} / \mathrm{h}$.

High pressure (16 bar) helium gas is supplied to the liquefier from an existing compressor and supply/return lines installed between the Cryogenic Group compressor facility and IR2. The 40001 liquid helium dewar is a refurbished SLAC unit, fed directly from the liquefier. This volume allows approximately 30 hours of autonomous operation of the solenoid and quadrupole magnets in the event of a minor liquefier/compressor malfunction. LHe from the dewar is supplied at $\sim 1.25$ bar, via proportional control valves in the DVB, to the respective magnet/auxiliary dewar system. These electro/pneumatic operated valves are actuated by process controllers responding to superconducting LHe level gauges. 
The $60 \mathrm{~m}$ LHe supply transfer line is a low-loss, vacuum-insulated, gas screened, coaxial line, tested and commissioned prior to installation. Magnet cooldown to $\sim 20^{\circ} \mathrm{K}$ is achieved with cold helium gas from the liquefier/refrigerator via the DVB.

Control and monitoring of the cryogenic plant and the magnet, together with remote control and monitoring of the compressor room, is carried out from a control room adjacent to the plant room and IR2. Main operating parameters are interfaced with the $B_{A} B_{A R}$ and PEP-II data acquisition and monitoring systems.

Tables 10.6 and 10.7 summarize the cryogenic requirements of the superconducting solenoid system.

\begin{tabular}{|l|c|c|}
\hline Item & Value & Unit \\
\hline \hline Cooling Method (Forced or Thermosiphon) & TBD & \\
Cold Mass weight & 7 & $\mathrm{t}$ \\
Cold Mass Surface Area & 100 & $\mathrm{~m}^{2}$ \\
Design Conductor Temperature & 5.0 & ${ }^{\circ} \mathrm{K}$ \\
Cool-Down Time & TBD & \\
\hline
\end{tabular}

Table 10-6. Preliminary refrigeration requirements.

\begin{tabular}{|l|c|c|}
\hline Item & $\begin{array}{c}\text { Refrigeration } \\
\text { [watts] }\end{array}$ & $\begin{array}{c}\text { Liquefaction } \\
{[\mathrm{l} / \mathrm{h}]}\end{array}$ \\
\hline \hline Solenoid & 40 & \\
Current leads & 3 & 20 \\
Transfer Line (Liq/Dewar) & & 3 \\
4000 1 Dewar (1\%/day) & 3 & \\
Transfer Line (Dewar/DVB) & 8 & \\
DVB and Valves & 3 & \\
Transfer Lines (DVB/magnet) & 57 & 23 \\
\hline Total & & \\
\hline
\end{tabular}

Table 10-7. Steady-state heat loads for the solenoid, dewars and transfer lines. Transient coolant requirements during cool down and charging must be added to the steady-state requirements to specify the required cryoplant capacity. 


\subsection{Procurement Plan}

The design and procurement of the superconducting solenoid must be expedited to meet the schedule
While the overall detector schedule is still being developed, the preliminary detector schedule suggests that the $B_{A} B A R$ Collaboration must be poised to award the fabrication contract for the superconducting solenoid in the Spring of 1995 , as soon as possible after SLAC/DOE approval of the project. Thus, as in past detector collaborations, this magnet must be "fast-tracked" if it is to arrive on schedule. Most of the engineering to support the purchase specifications of the solenoid and flux return must be completed during the conceptual design phase.

The integrated detector schedule identifies the magnet as a critical procurement item. The four-year-long critical path is formed by solenoid design and procurement; assembly with the flux return, verification testing and mapping, followed by detector subsystem installation and commissioning. While some of these task durations may be trimmed, such reductions expose the project to budget and schedule risk. The design and fabrication duration of 24-36 months is ambitious, but not unprecedented.

We propose to "jump start" the solenoid so that the contract can be awarded soon after the detector is approved in the Spring of 1995 . A similar plan was followed for early approval of the GEM magnet in parallel with the formation of the GEM Collaboration. We have therefore initiated preliminary contacts with qualified vendors. Interest is strong; there are six to ten potential suppliers worldwide.

Table 10.8 is an example of the type of schedule that will be required to place the order for the superconducting solenoid.

\subsection{Summary}

We propose a magnet with sufficient margin to meet current and anticipated physics requirements. It is a low risk option, within the state-of-the-art of magnet fabrication. Managing cost and schedule are the principal challenges. In order to place the magnet contract in a timely manner, the Collaboration must select the final magnet parameters rapidly. In parallel with this effort, the magnet will 


\begin{tabular}{|l|r|}
\hline Task & Date \\
\hline \hline Identify Critical Procurements & Jun 1994 \\
(Solenoid, IFR, Cryoplant, Power) & \\
Draft Acquisition Plan & Jun 1994 \\
SLAC/DOE Conditional Approve Plan & Jul 1994 \\
Draft Preliminary Specification & Aug 1994 \\
Magnet Workshop & Sep 1994 \\
First Vendor Briefing & Sep 1994 \\
Magnet Preliminary Requirements Review & Oct 1994 \\
Collaboration Council Fixes Parameters & Oct 1994 \\
Magnet Technical Review & Dec 1994 \\
Complete Procurement Specification & Dec 1994 \\
Draft Request for Proposal (RFP) & Dec 1994 \\
Magnet Cost Review & Jan 1995 \\
SLAC/DOE Approve Acquisition Plan & Jan 1995 \\
Source Selection Authority (SSA) Selected & Jan 1995 \\
Issue RFP & Jan 1995 \\
Second Vendor Briefing & Jan 1995 \\
Proposals Due/SSA Convenes & Mar 1995 \\
SSA Recommendation to DOE/SLAC & Apr 1995 \\
Order Placed with Supplier & May 1995 \\
\hline
\end{tabular}

Table 10-8. Plan for early start of magnet procurement.

be designed and critical procurement actions taken. This requires commitment of resources soon after approval of the Letter of Intent to expedite the schedule. 


\section{References}

[Ale94] R. Aleksan, Minutes of the Geometry Committee Meeting of April 26, 1994.

[And82] D. Andrews et al., "A Superconducting Solenoid for Colliding Beam Experiments", Adv. Cry. Eng. 27 (1982).

[Bow92] G. Bowden, "IR Mechanical Design for $B$ Factories", $B$ Factories: The State of the Art in Accelerators, Detectors and Physics, SLAC Report SLAC-400, p. 3171992.

[Bow94] G. Bowden, "Cantilever Support of Q1", SLAC Memorandum, April 4, 1994.

[Coils] A. Bonito Oliva et al., "Zeus Magnets Construction Status Report", Proceedings of 11th International Conference on Magnet Technology (MT-11), p. 229, 1989.

P. Clee et al., "Towards the Realization of two 1.2 T Superconducting Solenoids for Particle Physics Experiments", Proceedings of 11th International Conference on Magnet Technology (MT-11), p. 206, 1989.

H. Desportes et al., "Construction and Test of the CELLO Thin-Wall Solenoid", Advances in Cryogenics Engineering 25, 175 (1980).

H. Desportes et al., "General Design and Conductor Study for the "ALEPH" Superconducting Solenoid", Journal de Physique, C1-341 S1 T45 (1984).

Y. Doi et al., "A 3T Superconducting Magnet for the AMY Detector", Nucl. Instr. Meth. A274, 95 (1989).

M.A. Green et al., "Construction and Testing of the Two-Meter-Diameter TPC Thin Superconducting Solenoid", Adv. Cry. Eng. 25, 194 (1980).

M.A. Green et al., "A Large Superconducting Thin Solenoid for the STAR Experiment at RHIC", IEEE Trans. Appl. Supercond. 104 (1993).

H. Hirabayashi, "Detector Magnet Technology for High Energy Accelerators", Proceedings of the 11th International Cryongenic Engineering Conference (ICEC-11), p. $115,1986$.

H. Minemura et al., "Fabrication of a $3 \mathrm{~m} \mathrm{dia.} \times 5 \mathrm{~m} \mathrm{Su}$ perconducting Solenoid for the Fermilab Collider Dctector 
Facility", Journal de Physique, C1-333, S1 T45 (1984).

C.M. Monroe et al., "The CLEO-II Magnet - Design, Manufacture and Tests", Proceedings of the 12th International Cryongenic Engineering Conference (ICEC-12) p. $773,1988$.

M. Wake et al., "Excitation of a Superconducting Large Thin Solenoid Magnet", MAG-23, 1236 (1987).

F. Wittgenstein et al., "Construction of the L3 Magnet", 11th International Conference on Magnet Technology (MT-11), p. 131, 1989.

A. Yamamoto et al., "Thin Superconducting Solenoid Wound with the Internal Winding Method for Colliding Beam Experiments", Journal de Physique, C1-337, S1 T45 (1984).

[Des85] H. Desportes, "Recent Progress in the Design and Construction of Beam and Detector Magnets", International Symposium on Magnet Technology (MT-9), p. 149, 1985.

[Doi86] Y. Doi et al., "Cryogenic System of the TOPAZ Thin Superconducting Solenoid", Proceedings of the 11th International Cryongenic Engineering Conference (ICEC11), p.424, 1986.

[Fac94] O. Fackler, private communication.

[Hir88] H. Hirabayashi, "Detector Magnets in High Energy Physics", IEEE Trans. on Magnets 24, 1256 (1988).

[Lot86] J.C. Lottin et al., "Aleph Solenoid Cryogenic System", Adv. Cry. Eng. 31, 175 (1986).

[VecF] Vector Fields Ltd, 24 Bankside, Kidlington, Oxford OX5 1JE, England. 
LETTER OF INTENT FOR THE BABAR Detector 


\section{Electronics, Trigger, and Data Acquisition}

$\mathbf{T}$ he electronics subsystem encompasses all detector electronics exclusive of workstations. In this chapter, triggering and data acquisition are discussed. Monitoring and control will be implemented in a consistent manner throughout the experiment, but a specific electronic scheme is not discussed in this Letter of Intent.

The primary criteria guiding the design of the trigger and data acquisition subsystem are that these systems will: (1) not cause substantial dead time as long as the data are useful for physics; (2) not compromise the performance of the individual detector subsystems; (3) accept with high efficiency the physics events of interest. Furthermore, (4) even when backgrounds are such that the data are not useful, the performance will degrade gracefully, allowing the background and detector performance to be studied. Since the behavior of an asymmetric $e^{+} e^{-}$machine may hold some surprises, the trigger must be flexible and the number of data paths and processors must be scalable, i.e. readily expandable.

\subsection{Environment}

From the point of view of the data acquisition system designer, $B_{A} B_{A R}$ resembles a fixed target or cosmic ray experiment more than it does one at any current $e^{+} e^{-}$storage ring. The bunch crossing period ( $4.2 \mathrm{~ns}$ ) is much smaller than any possible trigger decision time; interactions may be considered continuous. The design luminosity of $3 \times 10^{33} \mathrm{~cm}^{-2} s^{-1}$ yields a physics event rate of $30 \mathrm{~Hz}$. The rate of Bhabha events with both tracks in the calorimeter exceeds $90 \mathrm{~Hz}$. This combination of high event rate and short crossing interval make data acquisition designs used in conventional $e^{+} e^{-}$machines
The performance of the data acquisition system will degrade gracefully in the presence of high backgrounds 
The drift chamber is the first system to become unusable with higher than expected backgrounds, at a rate thirty times higher than nominal unsuitable for $B A B A R$. To avoid overburdening the data path and the mass storage, trigger decisions must be accurate. Such trigger decisions take time. Thus the data must be extensively buffered to avoid dead time while trigger decisions are made and while the data is read out. In addition, the trigger logic must be constructed so that trigger decisions on multiple events can be processed in an overlapping manner; i.e. the trigger logic must be either pipelined or made parallel.

The usefulness of data in the presence of backgrounds is limited by the tracking system; the specification for the trigger rate capability is derived from this system. The background sources expected in PEP-II include synchrotron radiation, particles lost near the IP, beam-gas, and cosmic rays. Simulations predict isolated hit rates of several $\mathrm{MHz}$ per layer in the silicon vertex detector, $1 \mathrm{MHz}$ per layer in the inner layers of the drift chamber, and $0.3 \mathrm{MHz}$ per layer in the outer layers of the drift chamber. The background rate in the calorimeter is approximately $1 \mathrm{MHz}$ for energy deposits $>10 \mathrm{MeV}$.

At higher background rates $(30 \times$ nominal $)$, the first device to become unussable is the drift chamber. At this singles hit rate, the occupancy at a point one third of the way through the chamber is greater than $10 \%$, making track reconstruction unreliable. The background rate indicated by simulations is scaled up by this factor of 30 to specify the rate capability of the trigger and data acquisition system.

Nearly all spurious drift chamber hits are due to converted photons from lost particle showers that have energies of a few $\mathrm{MeV}$. The trigger rates, however, will be dominated by tracks from deep inelastic scattering interactions. Tracks which traverse at least half of the drift chamber are expected to occur at a rate less than $300 \mathrm{~Hz}$. These tracks come (non-uniformly) from the entire length and width of the exposed beam pipe, with rates which are assumed to increase no faster than the single hit rates as backgrounds become worse.

\subsection{Trigger Requirements}

Trigger efficiencies should ideally approach unity for the physics processes of interest, including $B$ physics (especially $C P$ violation), 
$\tau$ physics, and some subset of $2 \gamma$ physics. The trigger must be redundant and verifiable. Efficiencies must be well-measured and stable. The goal of high efficiency for the physics processes of interest and the desire for redundancy motivate the trigger criteria.

There will be two orthogonal primary triggers: a tracking trigger and a calorimetric trigger. The tracking trigger requires at least one charged particle emerging from the interaction point to reach the outer radius of the drift chamber, in coincidence with at least one charged particle reaching the middle radius (" $1 \frac{1}{2}$ track trigger"). The calorimetric trigger requires two isolated clusters of energy deposition, at a level which is efficient for minimum ionizing particles that reach the calorimeter, i.e. with transverse momenta greater than about $250 \mathrm{MeV}$ for a magnetic field of $1.5 \mathrm{~T}$ [Kra94]. The efficiency of such a cluster requirement for a charged particle is shown in Figure 11-1, and the motivation for these choices is further explicated in reference [SLA91].

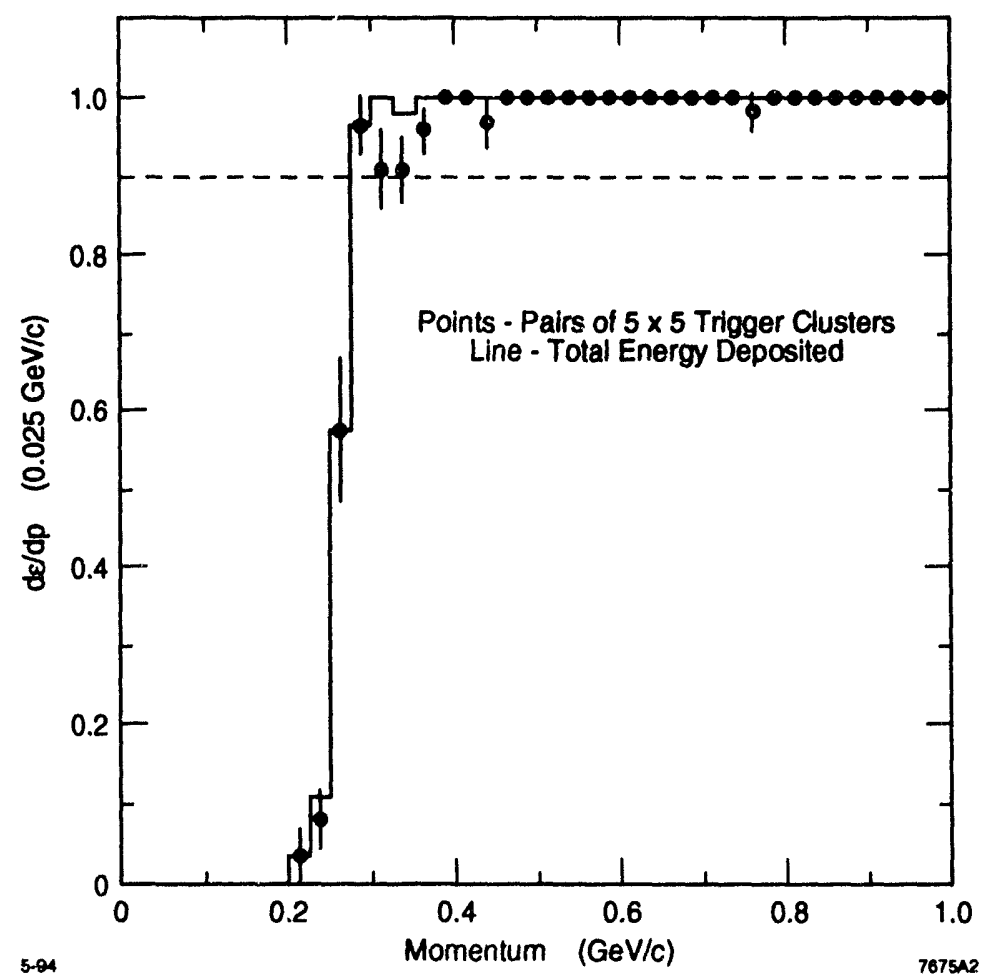

Figure 11-1. Calculated single-muon trigger efficiency for a $150 \mathrm{MeV}$ cut. The muons were generated with $|\cos \theta|<0.7$ in a magnetic field of $1.5 \mathrm{~T}$.
There are two orthogonal primary triggers: a tracking trigger and a calorimetric trigger 
The trigger system is flexible; most plausible triggers can be implemented without hardware modifications
The implementation of these triggers should be flexible enough that a wide range of plausible triggers based on the drift chamber and the calorimeter system may be implemented. This implies that the algorithms are executed by processors or programmable logic hardware. The orthogonality of these triggers should be maintained until the results of at least partial reconstruction of the events, performed using full digitized data, can be brought bear on the trigger decision. All the detector subsystems may be used in these latter stages of the decision process. The final rate of events written to mass storage should be less than twice the physics rate.

\subsection{System Organization}

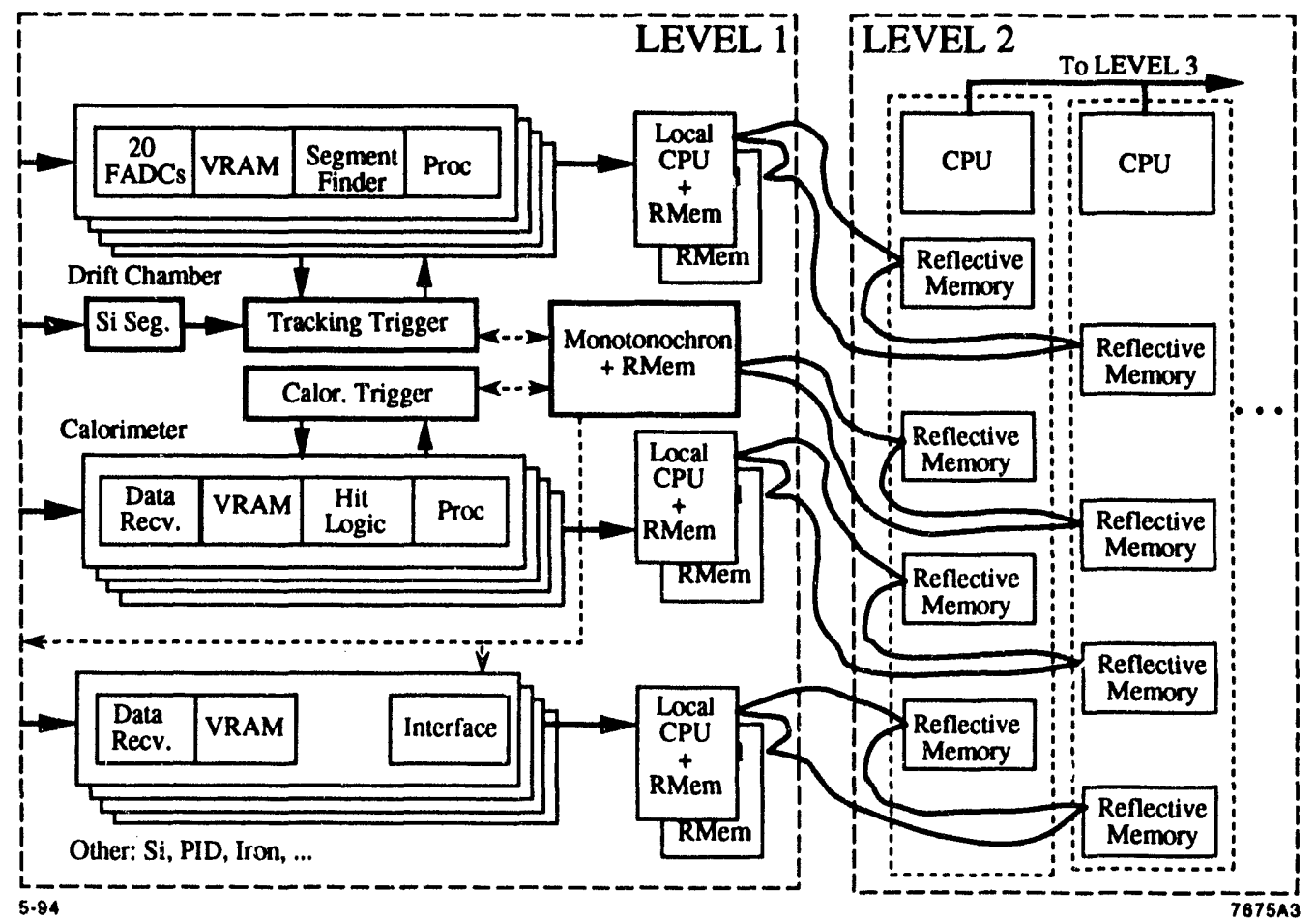

Figure 11-2. Example of one implementation of a data acquisition system.

The trigger and data acquisition system comprises three levels. Figure 11-2 shows one possible implementation, which is a slightly modified version of the design described in reference [SLA93b]. Here, 
the system is discussed in more general terms. More detail can be found in references [SLA93b, Wun94].

Level 1 digitizes signals, buffers them, and makes initial trigger decisions. These trigger decisions are based independently on information from the vertex detector and the drift chamber, and from the calorimeter. Level 1 handles events at a few $\mathrm{kHz}$, and up to about $10 \mathrm{kHz}$ in adverse running conditions, with minimal dead time. Level 1 also generates a strobe for the silicon vertex detector with a latency less than 10 microseconds [Lev94b]. Other highchannel-count $\left(10^{5}\right)$ subdetectors may also use this strobe, which may or may not be the same as the Level 1 trigger.

Level 2 verifies the trigger decision using full digitized data from the relevant sections of the detector to discard background induced triggers and then builds complete events. If the Level 1 trigger rate is low enough the previous step may be skipped. Level 2 then uses the complete event information to further eliminate background and reduce the event rate to near the final value.

Level 3 comprises a farm of workstations to analyze, filter, and store the events. If the Level 1 trigger rates are near the final trigger rate, it may be possible to combine Levels 2 and 3 .

\subsection{Trigger Rates}

Under normal conditions the Level 1 charged trigger rate is expected to be less than $300 \mathrm{~Hz}$. The total Level 1 rate is dominated by cosmic rays, and is expected to be less than $1.5 \mathrm{kHz}$. The system is designed to operate at a peak capacity of $10 \mathrm{kHz}$ with minimal dead-time (note that only the rate of lost particle induced triggers increases with increasing backgrounds). If the rate is this high, Level 2 verification will be used to reduce this by a factor of at least 10 , so that the rate of event building is at most $1 \mathrm{kHz}$.

If necessary, a final Level 2 decision based on the reconstructed event will reduce the rate at which events are sent to Level 3 to no more than a factor of two greater than the rate to tape, i.e., less than $200 \mathrm{~Hz}$.
The Level 1 trigger uses vertex detector and drift chamber information, generating events at rates up to $\sim 10 \mathrm{kHz}$ with minimal deadtime

The Level 2 trigger discards backgroundinduced triggers and builds events

Level 3 analyzes and stores events

The expected Level 1 trigger rate is less than $1.5 \mathrm{kHz}$ 


\begin{tabular}{|ll|c|c|l|l|}
\hline Source & & Type & $\begin{array}{c}\text { Rate } \\
(\mathrm{Hz})\end{array}$ & Assumptions & Section \\
\hline \hline Cosmics & & $\mathrm{T}$ & 1 & & 11.4 .1 \\
& $\mathrm{C}$ & $<1000$ & & 11.4 .1 \\
\hline Lost particle & Electro- & $\mathrm{T}$ & $\sim 0$ & & 11.4 .2 \\
(beam-gas) & magnetic & $\mathrm{C}$ & $<2300$ & $E>50 \mathrm{MeV}$ & 11.4 .2 \\
& Hadronic & $\mathrm{C}+\mathrm{T}$ & $200-500$ & $P_{\mathrm{IP}}=10 \mathrm{nT}$ Torr & 11.4 .3 \\
& & & $20-50$ & $P_{\mathrm{IP}}=1$ nTorr & \\
\hline Physics & Bhabha & $\mathrm{C}+\mathrm{T}$ & 100 & & 11.4 .4 \\
& $1 / 2$ Bhabha & $\mathrm{C}$ & $<10$ & prescaled & 11.4 .4 \\
& $\tau, \mu$ & $\mathrm{C}+\mathrm{T}$ & 5 & & 11.4 .4 \\
& $\Upsilon(4 S)$ & $\mathrm{C}+\mathrm{T}$ & 12 & & 11.4 .4 \\
& $2 \gamma$ physics & $\mathrm{C}+\mathrm{T}$ & $\sim 3$ & & 11.4 .4 \\
& $2 \gamma$ background & $\mathrm{C}+\mathrm{T}$ & $\sim 50$ & & 11.4 .4 \\
\hline
\end{tabular}

Table 11-1. Components of the Level 1 trigger output rate

The event size is about 25 kBytes
To produce a tracking trigger, a cosmic ray must cross the beam pipe near the vertex detector
The size of a good event is approximately $25 \mathrm{kBytes}$. At an event building rate of $1 \mathrm{kHz}$ this is $25 \mathrm{MBytes}$ per second. The total rate between Level 1 and Level 2 under peak conditions is 35 MBytes per second.

Table 11-1 summarizes the trigger rates in Level 1. For each component of the rate, the contribution to the tracking $(\mathrm{T})$ and calorimetric (C) trigger, the expected output rate, and the assumptions used to calculate the rate, if applicable, are shown. The last column refers to the section below in which the specific rate is discussed. Level 1 has negligible acceptance for synchrotron radiation background.

The following subsections review the basis for these trigger rate estimates in more detail.

\subsubsection{Cosmic Rays}

The cosmic ray trigger rate is estimated using the rate of $1 / \mathrm{cm}^{2} / \mathrm{min}$ for single muons with momenta above $0.3 \mathrm{GeV}$ crossing a horizontal area. The tracking trigger acceptance for cosmics presents an area of about $0.05 \times 0.15 \mathrm{~m}^{2}$, determined from requiring a track to pass through the beam pipe somewhere along the vertex detector. This 
estimate ignores the contribution of secondary delta electrons in the chamber.

The calorimetric trigger acceptance for cosmics presents an area of $5 \times 1.6 \mathrm{~m}^{2}$, the overall length of the calorimeter times the diameter of the drift chamber. The flux return and the calorimeter provide an energy cutoff at $0.8 \mathrm{GeV}$. Tracks which simply graze the calorimeter without passing through the inner radius at some point do not leave two separate clusters, and so do not trigger [Sny92a].

\subsubsection{Lost Particles (Beam-Gas Scattering)}

Lost particles are those beam particles which scatter from residual gas in the beam pipe upstream of the IP and are steered by the magnets into the masks and beam pipe near the IP. There they interact, generating electromagnetic showers and inelastic scattering events. The rate of such events is proportional to the beam current and to the pressure in the beam pipe in a region from 1.5 to $30 \mathrm{~m}$ from the IP. Because this is a major source of background, the PEP-II design calls for the aggressively low pressure of $0.2 \mathrm{nTorr}$ in this region. Occupancy resulting from this background is expected to limit detector operation. This is expected to occur at a rate $30 \times$ this design value, driven by the ability to reconstruct tracks in the drift chamber data, and at $20 \times$ this value, on the basis of the long-term survival of the vertex detector and/or the drift chamber [SL.493a]. The operability of the drift chamber under these extreme conditions has not been considered as a factor. This section deals exclusively with the electromagnetic showers of these background events [Sny92a, Sny92b, Lev94a, Lev94b]. Hadronic interactions will be discussed in Section 11.4.3.

\section{Tracking Triggers}

Tracking simulations [Sny92b] indicate that electromagnetic interactions of lost particles striking the beam pipe do not generate a significant rate of $1 \frac{1}{2}$ track triggers. This calculation examined the rate of triggers, varying the definition of the " $A$ " trigger (the " $B$ " trigger is set at $1 / 2$ the drift chamber). The rate drops roughly exponentially from $5 \mathrm{kHz}$ when the " $\mathrm{A}$ " track is required to travel
To produce a calorimetric trigger, a cosmic ray must deposit energy in two separate clusters in the CsI calorimeter
Lost particle background is expected to limit detector operation at 20 to 30 times the nominal design rate 
Electromagnetic interactions of lost particles contribute to the tracking trigger at a rate of $\sim 150 \mathrm{~Hz}$

Electromagnetic interactions of lost particles contribute $<2.3 \mathrm{kHz}$ to the calorimetric trigger rate at $90 \%$ confidence level
The hadronic interaction trigger rate for lost particles is estimated by scaling from Mark II data at PEP-I
$1 / 5^{\text {th }}$ of the way through chamber, to $200 \mathrm{~Hz}$ when required to travel through $1 / 2$. Our " $A$ " track definition is the whole chamber, but this calculation is for nominal beam pipe pressure. Assuming a continued exponential falloff and that the rate is due to tracks and not accidental coincidences, a trigger rate of $150 \mathrm{~Hz}$ is estimated at the higher beam pipe pressure. Further background simulation will be done to confirm this calculation.

\section{Calorimetric Triggers}

The rate of two $22 \times 22 \mathrm{~cm}^{2}$ calorimeter towers was estimated to be $6 \pm 2.4 \mathrm{kHz}$ with a tower threshold of $40 \mathrm{MeV}$. No events were observed above $50 \mathrm{MeV}$, resulting in a limit of $2.3 \mathrm{kHz}$ at the $90 \%$ confidence level [Lev94a]. A substantially higher operating threshold is anticipated. This calculation is for the design vacuum of $0.2 \mathrm{nTorr}$ in the beam pipe near the IP. More background simulation is required to estimate the trigger rate for the combination of higher thresholds and backgrounds $30 \times$ this nominal level.

\subsubsection{Hadronic Interactions from Lost Particles}

The hadronic component of the beam-gas contribution to the trigger rate is estimated by scaling from Mark II trigger rates measured at PEP-I. There, the two-track machine background rate was 1$2 \mathrm{~Hz}$ [MK-II]. To be conservative, this is assumed to have been exclusively due to hadronic beam-gas interactions, which would scale as the ratio of beam currents, a factor of $75=3 \mathrm{~A} / 40 \mathrm{~mA}$, yielding a predicted $75-150 \mathrm{~Hz}$. The Mark II chamber size $(1.5 \mathrm{~m}$ outer radius) and magnetic field $(0.45 \mathrm{~T})$ s`rength roughly scale to the $B A B A R$ detector $(0.8 \mathrm{~m}$ outer radius at $1 \mathrm{~T})$ : the Mark II track threshold was about $100 \mathrm{MeV} / c$, compared to $120 \mathrm{MeV} / c$ for $B_{A} B A R$ at $1 \mathrm{~T}$. This rate is for a two-track trigger requirement; the rate increased by roughly a factor of three in going to a $1 \frac{1}{2}$ track trigger [MK-II]. This yields a scaled rate of $200-500 \mathrm{~Hz}$ for a $B_{A} B_{A R} 1 \frac{1}{2}$ track trigger at $P E P$ vacuum. Since this rate is mostly due to tracks which do not come from the origin, the rate will by lowered by the inclusion of a vertex detector requirement in Level 1 


\begin{tabular}{|l|c|c|}
\hline Trigger & $\tau^{+} \tau^{-}$ & $B^{0}, J / \psi K_{S}^{0}$ \\
\hline \hline Two central tracks (T2) & 0.530 & 0.997 \\
One and 'one half' tracks (T1.5) & 0.730 & 1.000 \\
Two tower-pair clusters $>100 \mathrm{MeV}(\mathrm{C} 2)$ & 0.944 & 1.000 \\
\hline
\end{tabular}

Table 11-2. Efficiencies of triggers for two physics processes [Kra94] [Bol91].

and/or track pointing cuts in Level 2. The PEP vacuum was about $10 \mathrm{nTorr}$ at the IP [MK-II]. The PEP-II design vacuum is $1 \mathrm{nTorr}$ at the IP and $0.2 \mathrm{nTorr}$ in the nearby beam pipe. If this is achieved, the background trigger rate becomes $<20-50 \mathrm{~Hz}$. However, even if the vacuum is $10 \mathrm{nTorr}$, the rate is still not serious. Note that the BABAR detector is better shielded from the beams of PEP-II, by masks and the permanent magnets, than Mark II was from PEP. On the other hand, the $B_{A} B_{A R}$ detector has bending magnets very near the IP.

\subsubsection{Physics Triggers}

The following discussion always assumes $\mathcal{L}=3 \times 10^{33} \mathrm{~cm}^{-2} \mathrm{~s}^{-1}$, or $3 \mathrm{~Hz}$ for each $\mathrm{nb}$ of cross section. The cross sections for hadronic production at the $\Upsilon(4 S)$ are $4 \mathrm{nb}$, and for $\mu$ - and $\tau$-pairs $0.8 \mathrm{nb}$ each.

Efficiencies for $\Upsilon(4 S)$ and $\tau^{+} \tau^{-}$events

Estimated efficiencies for $\Upsilon(4 S)$ and $\tau^{+} \tau^{-}$events are summarized in Table 11-2.

\section{Bhabha Scattering}

The acceptance in the forward direction determines the rate of Bhabhas events into Level 1: $\cos \theta_{l a b}=0.955$ implies $\cos \theta_{c m}=0.86$. This results in a Bhabha rate of $90 \mathrm{~Hz}$ [Eis90]. Bhabha events are, however, very easy to recognize (two clusters of energy, opposed in $\phi$, each with energy appropriate to its $\cos \theta_{l a b}$, and just two stiff 
Bhabha triggers are prescaled to flatten the $\cos \theta_{\text {lab }}$ distribution, reducing the rate to $\sim 10 \mathrm{~Hz}$

The $2 \gamma$ rate will be $3 \mathrm{~Hz}$ with our trigger cuts

More detailed simulations of trigger performance are required tracks, each pointing to a cluster). The trigger prescales Bhabha events to a flatter distribution in $\cos \theta_{l a b}$, and reduces this rate to about $10 \mathrm{~Hz}$; the exact prescaling algorithm is determined such that sufficient Bhabha events are collected for short-term calibration of the detector. For a more detailed discussion of Bhabha scattering see reference [Eis90].

\section{Asymmetric Bhabhas in the Backward Calorimeter}

Note that Level 1 is insensitive to a high rate (order $1 \mathrm{kHz}$ ) of 1-prong Bhabhas, where the forward prong is lost due to the PEP-II energy asymmetry. Some fraction of this type of event will be retained for calibration purposes by a prescaled one-cluster trigger.

\section{Two-Photon Interactions}

The rate for $2 \gamma$ triggers is scaled from the experience of the TPC/TwoGamma experiment at PEP. The $2 \gamma$ physics-event rate at PEP-II will be $3 \mathrm{~Hz}$ with our trigger cuts [TPC]. The rate of backgroundtriggers caused by $2 \gamma$ interactions can be scaled from an estimated trigger contribution of $0.5 \mathrm{~Hz}$ at PEP [TPC]. Scaling by luminosity $\left(3 \times 10^{31}\right.$ versus $\left.3 \times 10^{33}\right)$ gives the $50 \mathrm{~Hz}$ shown in Table 11-1.

\subsubsection{Further Work on Trigger Rates}

The following issues need further investigation to firm up these estimates:

- studies with much larger beam-background data samples,

- a study of triggers caused by hadronic interactions in beamgas and beam-wall events,

- a study of the trigger rate from radiative Bhabhas,

- a detailed simulation of triggers caused by small-angle Bhabhas showering in the material around the IP,

- a study of the rate of background triggers caused by $2 \gamma$ events without a tag. 


\begin{tabular}{|l|r|}
\hline Subsystem & Channel Count \\
\hline \hline Vertex Detector & 150,000 \\
Drift Chamber & 7000 \\
Time of Flight & 340 \\
Aerogel & 3400 \\
DIRC & 16,000 \\
Fast RICH & 300,000 \\
Calorimeter & 10,000 \\
Muon & 50,000 \\
\hline
\end{tabular}

Table 11-3. Approximate subsystem channel counts. Note that only one of Time of Flight, Aerogel, DIRC, and Fast RICH will be implemented.

\subsection{Implementation}

The trigger and data acquisition system design departs from existing systems in $e^{+} e^{-}$colliders in several ways. It is pipelined, scalable and asynchronously triggered. The system's capacity largely scales with the number of data links and processors installed. The design uses commercial processors and clata links for pattern recognition and data flow wherever practical, and minimizes custom hardware. The price/performance ratio of such commercially available modules is constantly improving.

\subsubsection{Level 1 Electronics}

The frontend electronics designs may be put into two categories. The central drift chamber, the calorimeter and the aerogel and DIRC particle identification options, each with the order of $10^{4}$ channels, and the silicon vertex detector, Fast RICH particle identification option, and the muon detector, each with of the order of $10^{5}$ channels (See Table 11-3).

The former subsystems operate in a full sampling mode while the latter use extensive multiplexing and/or some type of sparsification.

There are two classes of frontend electronics: fully sampling, and multiplexed and/or sparsified 
In each case, the data are acquired continuously and a long time history is kept in time-addressable meniory.

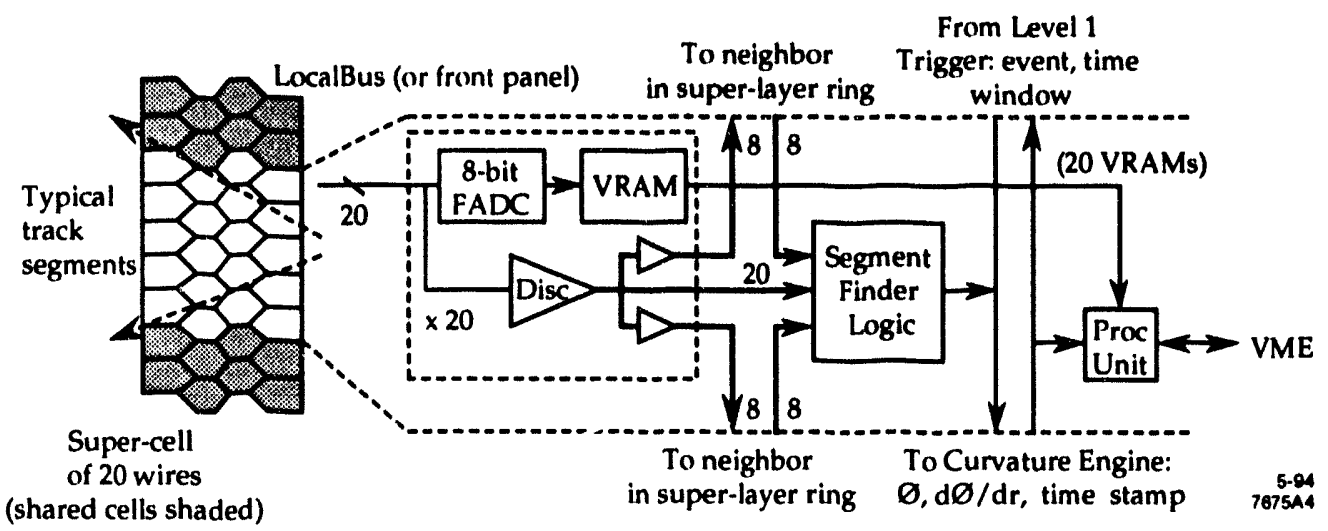

Figure 11-3. Drift chamber $D A Q$ module.

A 256 kByte VRAM stores 4 to $8 \mathrm{~ms}$ of digitized drift chamber data

A segment finder at Level 1 is the first step in track reconstruction

\section{Drift Chamber}

The analog signals pass through chamber-mounted amplifiers and into DAQ modules. Each DAQ module handles the signals for 20 chamnels which come from a block of five adjacent cells in each of four adjacent layers. The inputs are digitized every two to four bunch crossings ( 60 to $120 \mathrm{MHz}$ ) by an 8-bit Flash ADC, and the result is loaded into a Video Random Access Memory (VRAM). The sampling rate will be chosen after further studies. A VRAM has a fast sequential port and a random access parallel port. As used here, they yield a multi-ms pipeline which is randomly accessible by time. For example, a $256 \mathrm{kByte}$ VRAM could store 4 to $8 \mathrm{~ms}$ of data from a single channel. To form the Level 1 trigger, each signal is also connected to a discriminator. Its output is fed to the segment finder. The segment finder uses the signals from the 20 channels on its own module, together with those from 8 channels on each of the adjacent modules, to search for track segments. The segment finder output is passed to the Level 1 trigger logic and used in conjunction with similar information from the silicon vertex detector to find tracks. 


\section{Calorimeter}

The CsI(Tl) calorimeter electronics is similar in concept, but differs in implementation because a dynamic range of 17 to 18 bits is required. An effective resolution of 10 bits is needed, but reconstruction of waveform-sampled pulses is likely to allow a lower resolution on individual digitizations.

The signal from the photodiode is amplified and split into multiple gain stages by a custom integrated circuit amplifier. This amplifier chip is mounted physically close to the detector elements in order to minimize noise problems. The outputs of the gain stages are transmitted with a dynamic range of 10 to 14 bits to the digitization logic. This logic can be located either at the end of the calorimeter barrel, but within the clectrostatic enclosure of the subdetector, or on the DAQ boards, or it can be split between the two locations.

The signals are fed to a second custom integrated circuit containing range-selection logic which determines the appropriate gain range to be digitized and connects the sclected analog signal to the output, of the device. The analog signal is then digitized by a commercial converter every 25 to 250 bunch crossings ( 1 to $10 \mathrm{MHz}$ ) in order to determine its amplitude. The ADC output forms the ( 8 to 12 bit) mantissa of a floating-point number with the range bits from the range-selection $\mathrm{IC}$ as the exponent. The total dynamic range (e.g. 18 bits) is given by the dynamic range of the $\mathrm{ADC}$ (e.g. 10 bits) multiplied by the highest gain (e.g. 256). If the ADC is on the end of the subdetector, the floating-point numbers of several channels may be multiplexed onto a scrial fibcr-optic link and transmitted to external data-acquisition crates. Transmitting the data to the outside of the detector in digital form on fibers would minimize electromagnetic interference and noise injection into the frontend.

The data stream is continnously recorded in VRAM. Logic on the calorimeter DAQ board also extracts information from the stream for use by the Level 1 calorimeter trigger. Some particular proposals for calorimeter electronics are described in references [SLA93b, Dow94, Hal94].
The 17 to 18 bit dynamic range required for the calorimeter is achieved using a multi-range floating point $A D C$ 


\section{Vertex Detector}

The veltex detector electronics is discussed in Chapter 5.

\section{Particle Identification}

Since the particle identification technology has not been selected, the effort on electronics for this subsystem has been limited. Nevertheless, possible solutions for each candidate technology have been identified.

For the Acrogel and the DIRC some variation on the PHENIXdetector Fast-RICH electronics [PHE92] will likely meet the requirements. In this system the incoming discriminated signal starts the charging of a capacitor. The next occurrence of a $100 \mathrm{MHz}$ clock stops the charging and the charge is transferred to a switched capacitor array. The array stores more than $5 \mu$ s of hits. Upon receiving a trigger an ADC digitizes hits at the appropriate times from several channels. These data are sent over a fiber optic link to the buffer modules. The pulse height measurement is handled in a similar manner by substituting an integrator for the time to charge convertor.

A TOF system could also be done this way, but the requirements are more stringent. While the construction costs would be modest (the channel count is low), the engineering effort might be substantial.

The Fast-RICH system electronics can take advantage of the relatively modest time requirements $(\sim 400 \mathrm{~ns})$ to achieve a high degree of multiplexing. A single inexpensive detector mounted FADC could service many channels. Adding some buffering and using the silicon strobe could increase the multiplexing level.

\section{Muon Detector}

The muon detector electronics in the baseline design contains about $50 \mathrm{~K}$ channels of digital readout. Due to the low occupancy, a multiplexing scheme is envisioned, with a large reduction in DAQ modules and cables. In the present scheme, a 256:1 reduction will be accomplished by taking the outputs of 256 discriminators and encoding them into an 8 bit address. This address is connected to 
the VRAM input of a DAQ module. If the RPC option is selected for the IFR, their fast timing properties may be exploited for low momentum particle identification and for cosmic-ray rejection. A TDC system can be implemented, whose design and dimension will result from optimization studies currently in progress. Otherwise, no timing is required outside of the DAQ module.

\section{All}

All the DAQ modules have a processor and some conventional (nontime addressable) RAM. The processor extracts the physical quantities from the data in the VRAM and also selects data for requested time intervals and passes it to Level 2. Details of the data flow are still under investigation[Wun94].

Decisions in this area depend on the trigger rates and latencies at both Level 1 and Level 2. At one extreme is the case when both the rates and latencies are low in Level 1. In this case the data can be moved directly from the VRAM to the Level 3 workstations. At the other extreme the Level 1 trigger rates and latencies are both high. Then a time-consuming trigger verification by Level 2 may be required before event building. To allow time for this the data would need to be moved from the the VRAM to DRAM in the DAQ board upon the occurrence of a Level 1 trigger. Portions of the data are then processed and moved to Level 2 to be used in the verification process. Verified events are then built by transferring all the data in the trigger's time window to Level 2 . Level 2 would then make a further trigger decision based on complete events before passing them on to the workstations. Many intermediate data flow models are possible.

\subsubsection{Level 1 Trigger}

The implementation of the Level 1 trigger algorithms is under study. The best solution could be a one-step all-hardware implementation, or a simple hardware step followed by a processor-based trigger. The optimum algorithms will depend on which route is chosen.

The calorimeter trigger problem is relatively straightforward. A hardware solution would first sim fixed groups of crystals, then
All DAQ modules have a processor that extracts physical quantities from the data

A variety of data flow schemes are under consideration 
The Level 1 calorimeter trigger would require two separated energy clusters

The Level 1 tracking trigger will link locally. found track segments form sums of all pairs of groups, and finally look for two such spatially-separated sums above a threshold. A software implementation might possibly gain by summing groups of varying size and varying boundaries.

The tracking trigger solution will depend on the geometries of the vertex detector and drift chamber. Current designs first use hardware to find local track segments as described in subsection 11.5.1. These would then be linked into tracks by either a linking algorithm or a Hough transform technique. The latter has the advantage of taking a fixed length of time and could be implemented in either hardware or software. If the track finding is done in a processor, then some simple hardware-implemented occupancy criteria might be used generate the strobe for the vertex detector.

\subsubsection{Connecting Level 1 to Level 2}

The network technology which connects Levels 1 and 2 has not been chosen. This decision awaits the outcome of decisions on the Level 1 trigger, and further background studies. While the reflective memory solution shown in Figure 11-2 will work, it may not be the most cost-effective choice. Other solutions being considered include the Scalable Coherent Interface [IE1596] and a commercial Gigabit fiber-channel data switch [Lev93]. Which solution is best depends in part the data flow model selected.

\subsubsection{Level 2}

Level 2 consists of commercial processor boards. It is responsible for making a trigger decision based on information from the Level 1 trigger and (possibly a subset of) the full digitized data stored in the Level 1 buffers. Level 2 builds the events by requesting the data for the relevant time period from Level 1 . If the built event is still deemed good, it is sent to a Level 3 workstation via a Local Area Network. These Level 2 functions might actually be carried out by the workstations themselves. 


\subsection{Research and Development}

Progress in completing the DAQ design resuires the generation of substantially more simulated background data. These are needed to evaluate trigger designs, and to decide on an optimum trigger strategy. They are also needed in conjunction with the trigger design to evaluate data flow requirements and finalize a data flow model. This in turn will enable the final design of the data acquisition architecture and the choice of hardware.

While the requisite studies are being carried out, studies of the DAQ problem have been made in abstract terms in order to identify the critical design issues. The current state of this work is illustrated in Figure 11-4.

The detailed design of some of the subsystems awaits the choice of a specific detector technology.

\subsection{Summary}

The $B A B A R$ trigger and data acquisition system is fully buffered or pipelined in both the trigger and the data flow paths. As a result the system is nearly deadtime-free at background levels up to $30 \times$ the rates given by machine simulations. It uses commercial equipment wherever suitable modules are available, with all components beyond the frontend electronics being off-the-shelf. The architecture is such that the capacity of the system is easily expandable by the addition of data paths and processors.

Further progress on the $D A Q$ system design will require additional simulations of backgrounds

A choice of detector technology must be made in the particle identification system before detailed design of that data acquisition system can be done 


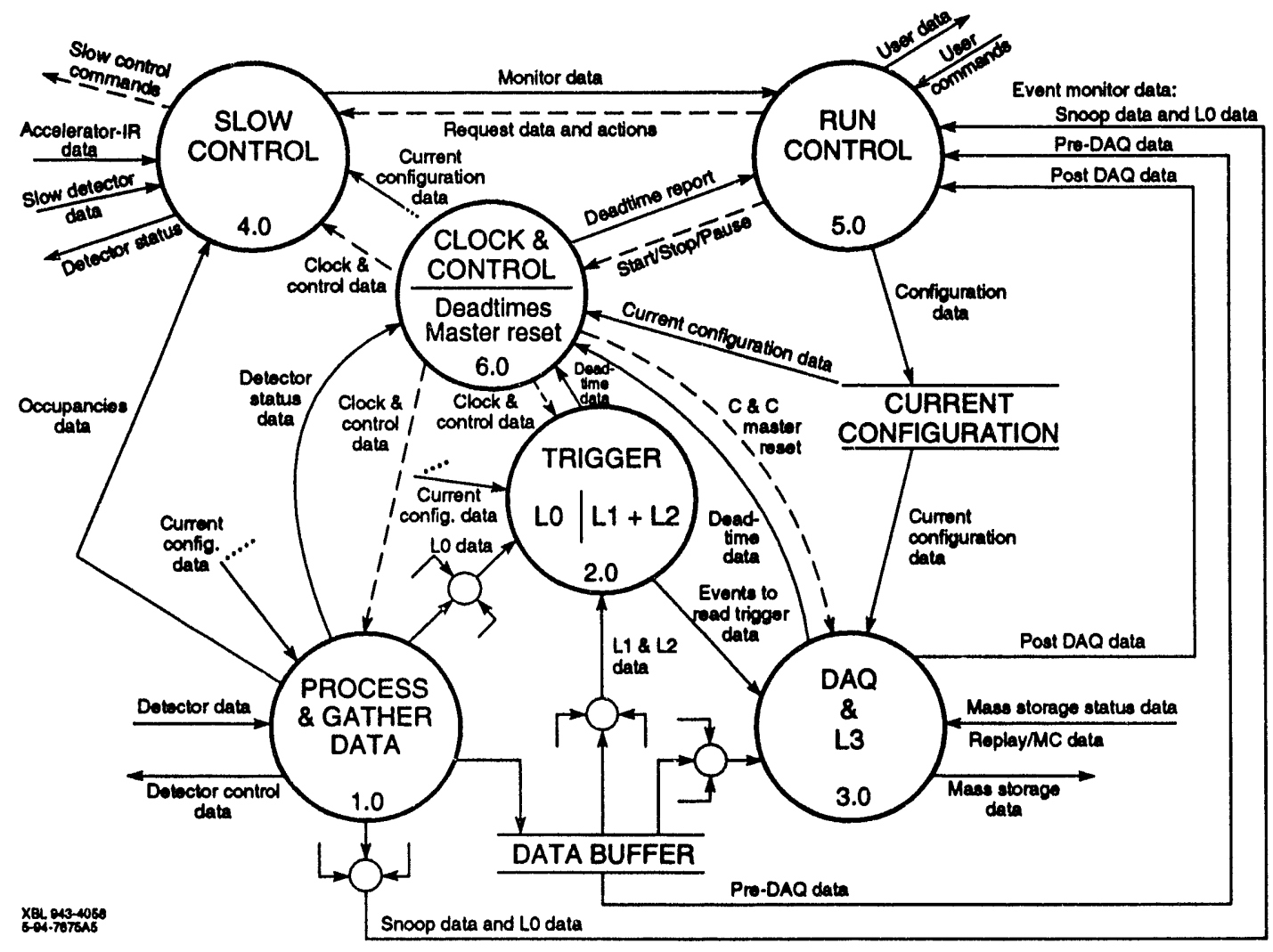

Figure 11-4. Schematic diagram of the $D A Q$ and online computing system. Each bubble represents a transformation, and the small bubbles with multiple input arrows represent collection points for partial information from multiple locations. Detector data from the frontend electronics are received in Process and Gather Data (1.0). The Data Buffer holds data during trigger decisions and consists of many buffers. The Trigger (2.0) function is independent of the number of levels. Data acquisition and Level 3 Trigger (3.0) operate on complete events and write data to Mass Storage. Clock and Control (6.0) is separate from but related to Trigger decisions. Slow Control (4.0) involves monitoring the detector and the accelerator. Run Control (5.0) provides the user interface of the online system. The user can monitor data from all stages of the DAQ. The Current Configuration data repository is a logical entity (not necessarily in one place) that holds the calibration constants, trigger configuration, etc. 


\section{References}

[Bol91] T. Bolton et al., in Reference [SLA91], p. 597.

[Dow94] S. Dow, I. Kipnis, F. Kral, R. Jared, F. Kirsten, M. Levi, G. Lauer, R. Minor and S. Wunduke, "A CMOS Front End for the CsI Calorimeter", BABAR Note \#139, April 1994.

[Eis90] A. Eisner, "Bhabhas at an Asymmetric B-Factory", , BABAR Note \#32, April 1990.

[Hal94] G. Haller, talk given to the Calorimeter Working Group at the Paris meeting of this collaboration, March 1994.

[IE1596] IEEE Standard for Scalable Coherent Interface, IEEE STD 1596-1992 (Institute of Electrical and Electronics Engineers, New York, 1993); also D. Gustavson, et al, "Overview of the Scalable Coherent Interface, IEEE STD 1596", SLAC Report SLAC-PUB-5967, Oct. 1992. The Scalable Coherent Interface is a ring-style local-area network protocol which implements a shared memory model.

[Kra94] J.F. Kral, "GEANT Simulation Results for a Fast Calorimeter Trigger", BABAR Note \#133, April 1994.

[Lev93] M. Levi, "A Commercial Gigabit Data Switch for Event Building after Level 1 Accept", talk given at PEP-II Detector Collaboration meeting, December 3, 1993; W.H. Greiman, S.C. Loken, and C.P. McFarland, "Use of Commercial Gigabit Data Switches for SSC and LHC Event Builders", Proceedings of the International Conference on Computing in High Energy Physics '92, December 21, 1992, CERN Report CERN-92-07, p. 184.

[Lev94a] M. Levi and F. Kral, "Simulated Background Rates in Calorimeter Trigger Towers", BABAR Note \#134, April 1994.

[Lev94b] M. Levi, "Impact of Backgrounds on the Silicon Vertex Detector Architecture and Detector Trigger", $B_{A} B_{A R}$ Note \#136, April 1994. 
[MK-II] Operating experience with the Mark II Detector at PEP I. The beam current at PEP-I was 3 bunches of $7 \mathrm{~mA}$ each in either direction. The PEP-I vacuum measured at the IP used to be between 5 and $20 \mathrm{nTorr}$. See R. Becker, D. Briggs and F. Porter, "Level 1 Trigger Rate Estimates", BABAR Note \#112, Sept. 1993.

[PHE92] "PHENIX Preliminary Conceptual Design Report", BNLProposal-R2, June 1992.

[SLA91] Workshop on Physics and Detector Issues for a HighLuminosity Asymmetric B Factory at SLAC, SLAC-373, March 1991.

[SLA93a] PEP-II Conceptual Design Report, SLAC-418, June 1993.

[SLA93b] Status Report on the Design of a Detector for the Study of CP Violation at PEP-II at SLAC, SLAC-419, June 1993.

[Sny92a] A. Snyder, "Backgrounds in the BABAR CsI Calorimeters", BABAR Note \#83, May 1992.

[Sny92b] A. Snyder, "Backgrounds in the BABAR Drift Chamber", BABAR Note \#88, July 1992.

[TPC] Operating experience with the TPC/Two-Gamma Detector at PEP I. See R. Becker, D. Briggs and F. Porter, "Level 1 Trigger Rate Estimates", BABAR Note \#112, Sept. 1993.

[Wun94] S. Wunduke, M. Levi and F. Kral, "Architecture for Readout Electronics", BABAR Note \#138, April 1994. 


\section{Computing}

$\mathbf{I}$ $\mathrm{n}$ this chapter the present understanding of computing issues for the proposed $B A B A R$ detector are summarized. The computing requirements are assessed, and a feasible hardware implementation is developed, based on UNIX processors. The general software issues of architecture, data model, and code development are discussed followed by an outline of the on-line computing environment. Support issues and manpower requirements are summarized.

\subsection{Functional Requirements}

In this section the basic hardware requirements for $B_{A} B_{A R}$ computing are considered: CPU power, bandwidth, and storage space. Early estimates of the requirements were made and discussed in reference [Boy90].

\subsubsection{CPU Requirements}

The computing tasks include the on-line system operation (Level 3 trigger \& data acquisition, event sampling, detector monitoring, calibration and control), the off-line reconstruction, creation of DSTs, Monte Carlo simulation, and analysis. The estimates of both CPU power and bandwidth are established by requiring that the corresponding tasks are completed in an acceptable time. For example, the goal is that the reconstruction should be able to keep up with data acquisition. The estimated CPU power required for these tasks is summarized in Table 12-1.

"MIPS" is a somewhat poorly defined term (1 MIPS corresponds to the power of a VAX 11/780), however, it has become a customary unit. For conversions, an IBM RS/6000 model 550 is approximately

A total of $2 \times 10^{4}$ MIPS is required for on-line and off-line computing 
The SLAC-installed capacity requirement is envisioned as $10^{4}$ MIPS

The Monte Carlo computing requirement is based on CLEO-II experience

\begin{tabular}{|l||l|c|}
\hline On-line (Level 3) & MIPS & 2500 \\
\hline Reconstruction & MIPS-s/evt & 25 \\
& Events/yr & $10^{9}$ \\
& Passes/yr & 2 \\
& MIPS & 5000 \\
\hline Monte Carlo & MIPS-s/evt & 500 \\
& Events/yr & $10^{8}$ \\
& MIPS & 5000 \\
\hline DST creation & MIPS & 500 \\
\hline DST analysis & MIPS & 2000 \\
\hline Interactive analysis & MIPS & 5000 \\
\hline
\end{tabular}

Table 12-1. Estimated CPU power requirements

a 60 MIPS machine. The usual cautions apply concerning dependence of performance on type of code, etc. Thus, our numbers are necessarily approximations, but are regarded as adequate for planning.

The reconstruction requirement is actually a peak value (as is that for the on-line), obtained from CLEO-II reconstruction program performance and assuming that the events are reconstructed at the same rate they are recorded on tape. Hence, the total installed MIPS may be less than the sum of the above requirements, depending on the model (see the next section). If, nonetheless, the numbers are simply summed, the total is 20,000 MIPS. At least 10,000 MIPS is required at the SLAC site for data acquisition, reconstruction, and other tasks.

The Monte Carlo estimate is based on the CLEO-II experience with a complete simulation [CLE94]. It is estimated that $10^{8}$ Monte Carlo events per year is an appropriate level for comfortable statistical precision within the feasible systematic limitations of the simulation.

"DST creation" means a major pass through the data to create a selected dataset (in which the output data may or may not be compressed):

$$
\text { MIPS }=0.25(\text { MIPS-s } / \text { evt }) \times 10^{9}(\text { events }) \times 2(\text { users }) / 10^{6}(\mathrm{~s})
$$


This task must take less than one month, hence the $10^{6}$ second time period. The two users allows for up to two such major passes through the data to be simultaneous. This estimate, of course, is a particular model for how an analysis progresses into selected subsets of the data. Our model is rather conventional; however, alternatives will be investigated, such as using a direct access database technology $[\operatorname{Bad} 90]$.

"DST analysis" is a substantial processing of a selected DST dataset for physics analysis:

$$
\text { MIPS }=1(\text { MIPS-s } / \text { evt }) \times 10^{8}(\text { events }) \times 20(\text { users }) / 10^{6}(\mathrm{~s})
$$

Such a process is assumed to occur once, when a user has completed the design and debugging of the analysis and is ready to apply it to the full data set of interest.

Interactive analysis involves debugging, exploratory analysis, graphics, and program development. Our assumption is that there will be 100 active users, each employing a 50 MIPS workstation.

\subsubsection{Bandwidth}

Transformation of the raw data to the final reconstructed event information, and subsequent physics analysis, requires several data transfer steps. The most significant steps (in terms of required bandwidth) include:

- transfer of data between the Level 2 trigger and the Level 3 trigger,

- transfer of data from the Level 3 farm to mass storage (tape),

- reading and writing the data in the bulk reconstruction,

- writing of Monte Carlo events to tape,

- reading through the data to create DSTs,

- reads through the DSTs for physics results,

- interactive analysis on small datasets.

The estimated requirements are summarized in Table 12-2.

An upper limit requirement of 1000 events/s is assumed to the Level 3 data acquisition farm. This is sufficient for a comfortable
A single pass suffices for a given DST per analysis 


\begin{tabular}{|c|c|}
\hline Data acquisition: & \\
\hline Events/s to Level 3 & 1000 \\
Mbytes/s to Level 3 & 25 \\
Events/s to tape & 100 \\
Mbytes/s to tape & 2.5 \\
\hline \hline Reconstruction: & \\
\hline Events/s/reconstruction analysis & 100 \\
Mbytes/s/reconstruction analysis & 7.5 \\
\hline \hline Monte Carlo: & \\
\hline Events/s & 10 \\
Mbytes/s & 0.5 \\
\hline \hline DST creation: & \\
\hline Mbytes/s/DST & $50(1+f)$ \\
\hline \hline DST analysis: & \\
\hline Mbytes/s/task & 5 \\
\hline \hline Interactive analysis: & \\
\hline Mbytes/s/user: & 0.2 \\
\hline
\end{tabular}

Table 12-2. Estimated bandwidth requirements

The event size will be 25 kbytes. The addition of reconstruction information will double the event size on tape

DST creation is heavily I/O bound

DST analysis requires a network bandwidth of 100 Mbytes/s margin over the expected rates from the Level 2 trigger (see Chapter 11), where the event size is assumed to be 25 kbytes [Por93]. This is assumed to double to 50 kbytes once the reconstruction information is added.

DST creation and analysis represent a large network load. In DST creation, $f$ is the product of the sample fraction times the average sampled event size divided by 50 kbytes. If $f$ is small, and two such tasks are running, $100 \mathrm{Mbytes} / \mathrm{s}$ is required. It should be noted that the task of DST creation will typically be heavily I/O bound.

DST analysis, with twenty user analysis tasks simultaneously running, requires $100 \mathrm{Mbytes} / \mathrm{s}$. The interactive analysis number is estimated assuming a 2 kbyte/event micro-DST, and a throughput of 1000 events in 10 seconds, appropriate for small datasets, which are imagined to be analyzed at a desktop workstation. There may be occasional need for interactive analyses on larger DSTs, with larger bandwidth requirements. It is anticipated that such analyses 
would take place on the same machines as the "DST analysis" tasks, supported with higher bandwidth.

Aggregate network capacity for off-line tasks in excess of $200 \mathrm{Mbyte} / \mathrm{s}$ is required, dominated by the reconstruction and the multiple reads through the data required for DST creation and analysis.

\subsubsection{Storage}

Table 12-3 summarizes the estimated storage requirements for the $B A B A R$ experiment.

\begin{tabular}{|c|c|}
\hline Tape storage: & \\
\hline Raw data (Tbytes/yr) & 25 \\
Reconstruction output (Tbytes/yr) & 50 \\
Monte Carlo (Tbytes/yr) & 5 \\
DSTs (Tbytes/yr) & 5 \\
\hline \hline Disk storage: & \\
\hline DSTs (Tbytes) & 2 \\
Databases (Tbytes/yr) & 0.03 \\
\hline
\end{tabular}

Table 12-3. Estimated storage requirement

Approximately 100 Tbytes/yr of tape storage is required. If necessary, the 25 Tbytes of raw data could be moved off-line after reconstruction. The 5 Tbytes/yr for DST storage is estimated by assuming $10 \mathrm{DSTs}$ produced/yr, with $10^{7}$ events in each (sampling fraction of 0.01 ) and 50 kbytes/event.

The more disk space, the better - a reasonable minimum appears to be around two Tbytes. Current experience at SLAC is that a (disk) staging space of $2 \%$ of the active tape storage is required, and this is approximately consistent with the estimate here. Alternatively, 2 Tbytes may be regarded as sufficient for $40 \%$ of the DSTs produced to be on disk. However, it is probably appropriate to regard this space as staging space, with the details of whether a particular job finds its data already staged to disk or not transparent to the user. The database space requirement is implementation dependent, but should be small compared with the event data requirement.
The total network bandwidth must be in excess of $200 \mathrm{Mbyte} / \mathrm{s}$
A total of 100 Tbytes/yr of tape storage is needed

A disk storage capacity of 2 Tbytes is required 


\subsection{Computing Model}

The BABAR computing model is based on ?rorkstations running UNIX

Real-time extensions to UNIX allow uniform online and off-line computing environments
The design of a comprehensive computing model must satisfy the functional requirements of the previous section. In addition, such a model must address practical considerations such as, for example: the "abilities" (usability, flexibility, scalability, reliability, maintainability, affordability); smoothly integrate all hardware and software components; and be able to integrate SLAC on-site computing with that of off-site collaborators. Options for such a model, based upon present and near future technologies, are presented in the following subsections. An example of a schematic model which could be implemented within the next one year concludes this section.

\subsubsection{Computing Hardware and Operating System}

High performance microprocessor based workstations are a natural choice for the experiment. A large number of vendors competitively offer such workstations running similar and largely interoperable UNIX environments. This ensures the high degree of vendor independence needed to support the evolving set of platforms used throughout the collaboration. The availability of real-time extensions in some UNIX systems and of UNIX-like real-time systems (e.g. VxWorks) makes possible, for the first time, a uniform working environment both on-line and off-line.

Use of UNIX is increasing at many high energy physics labs such as CERN, Fermilab, DESY, and in many university groups. This is a crucial element for successfully integrating the computing for the entire collaboration and the rest of the HEP community.

The current industry trend is to unify the various flavors of UNIX (e.g. the X/OPEN consortium), based on the use of standards. It is our plan to adopt and enforce the use of the relevant standards. This, in turn, may make a possible future evolution away from UNIX more manageable. 


\subsubsection{Networking}

There are two levels in which networking is crucial to this experiment: local networking, required by the large number of machines and high data rates involved both on-line and off-line; and wide area networking, required by the wide-spread international nature of the collaboration.

Local area networking refers to the links between the various components of the experiment, including those to desktop machines and the central computing facility. Such networkin; can be designed with today's technology using, for example, ethernet hubs, and FDDI (Fiber Distributed Data Interface) switches.

The wide area networking situation is not as clear, given that the actual links to some European sites and Asia are neither satisfactory for substantial data transmission nor even for remote logins. Improvements to the wide-area network to some sites will certainly be necessary.

\subsubsection{Integration of Remote Sites and Data Sharing}

The problem of integrating remote sites into the computing model may be split into two separate categories. The first category, remote login, code development and distribution, has relatively modest demands and will be treated in a later section. The second category, data access, is more complex in that one can foresee various models for the data access by remote collaborators, such as: access to real data only at SLAC; access to data over the wide-area network; or, local access via data transferred on tape.

In the last two models, it is foreseen that mass storage compatible with that at SLAC will be present at remote regional centers, while lower-cost tape drives with stackers may suffice for smaller institutions. In all models remote farms could be used both for event reconstruction and Monte Carlo generation. The alternatives must be considered very carefully since costs associated with data access vary significantly depending upon the required rate of access and total quantity of data involved.
Both local and wide-area networking is required

In some cases an upgrade of existing WAN capability is' seen as necessary

The integration of remote sites is a basic precept of the computing model

There may be remote regional centers with tape silo capability. Smaller sites will use low cost tape drives with stackers 
Large-scale parallel processing systems, based on existing models, will be used for event reconstruction and Monte Carlo generation

Scalable parallel systems may eventually be costcompetitive with simple cluster farms

\subsubsection{Event Processing Systems}

Much of the large scale computing need is due to reconstruction and Monte Carlo simulation, both of which are event oriented. Therefore, developing large scale event-parallel processing systems will be of great importance for implementing an effective computing model. There is already expertise available within $B_{A} B_{A R}$ collaborating institutions. SLAC is currently conducting UNIX-based cluster development for centralized computing[Boe94]. This work is being performed in a series of phases and focuses upon both CPU-bound and I/O-bound tasks. Elsewhere, a simple multiprocessor management system for event-parallel computing, for example, has been developed at Mississippi[Gou94] and is being used for reconstruction of the E791 data set - the largest data set so far in particle physics (50 Tbytes). This simple multiprocessor system (farm) can be adapted at remote locations and operated effectively. Finally, ordinary desktop workstations clustered together can serve, for some computing tasks, a secondary purpose as farm nodes when not being utilized as a personal workstation.

Further investigations into both hardware and software are necessary before deciding upon a suitable model. Various commercial options are available now, such as scalable parallel systems. Some offer significant advantages, such as operating with a single system image for simpler management. The price/performance ratio currently favors the simple cluster of independent workstations, although this difference may decrease over the time-scale of interest.

\subsubsection{Schematic Computing Model}

A specific computing model based upon the functional requirements and current and near-future technologies is presented in Figure 12-1. Specific technologies are assigned for the purposes of estimating the budget. Over the construction period these technologies are expected to evolve or to become obsolete, being replaced by something better and/or cheaper. The goal of this design is to be flexible and to anticipate such improvements.

An understanding of the data flow is necessary to follow the various links in the figure. Event data originates in the frontend electronics, 


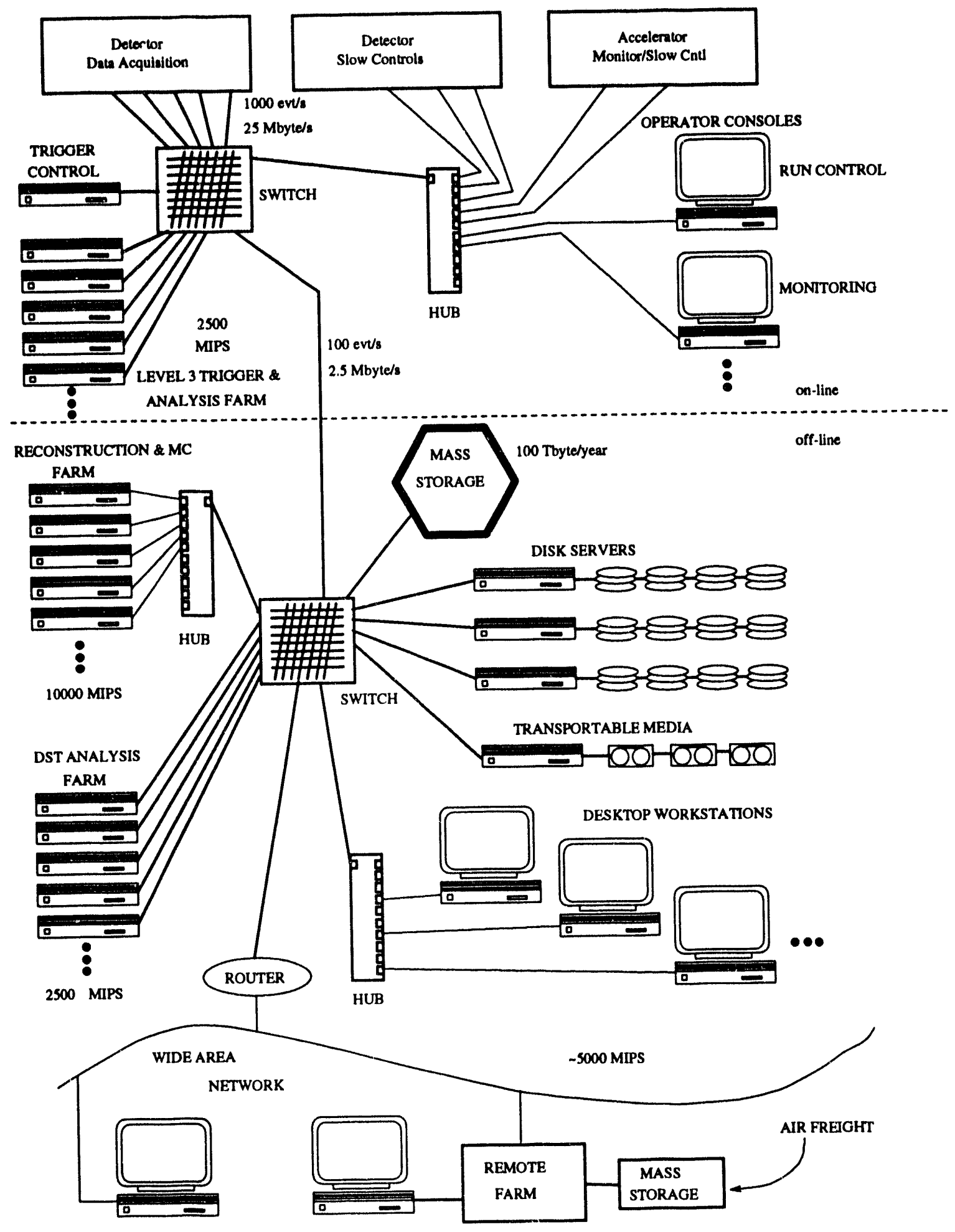

Figure 12-1. Conceptual computing architecture 
The computing model is based on FDDI links, crossbar switches and network-attached mass storage

Event reconstruction is done from staged tapes

Both reconstruction and Monte Carlo generation tasks are CPU bound

DST creation and analysis tend to be I/O bound

Interactive analysis, envisioned as an important requirement, can also be $I / O$ intensive buffers and triggers, then enters the diagram along multiple FDDI (100 Mbits/s) links through a non-blocking cross-bar switch into the Level 3 trigger (L3) processing farm. The events are already built at Level 2 (L2); the switch routes only whole events. A special trigger controller workstation is used to keep track of the event queues in the L3 machines, and to direct the L2 processors as to where to send each new event. Events satisfying the L3 trigger criteria are then sent back through the switch and into the network-attached mass storage device. Remaining sources of data from the experiment connect via slower and less-expensive ethernet $(10 \mathrm{Mbits} / \mathrm{s})$ links through network hubs which connect ten ethernet segments to one FDDI branch. These hubs are also used for connecting the various operator consoles that control and monitor the experiment.

Event reconstruction is done in the off-line reconstruction and Monte Carlo farm. Data from tape is first staged to a series of disk servers via FDDI connections through an off-line switch (identical to the on-line switch). Each reconstruction farm machine is directly connected to a network hub, which in turn connects to the disk server. Both reconstruction and Monte Carlo tasks tend to be CPU bound rather than $\mathrm{I} / \mathrm{O}$ bound, thus allowing the use of slower and less-expensive ethernet network links to these machines.

Creation and analysis of data summary tapes (DSTs) occurs in a second farm of machines with direct FDDI connections to the off-line switch. These jobs are $\mathrm{I} / \mathrm{O}$ intensive, thus requiring the higher performance and more costly FDDI network links. Data flow is similar to that for the reconstruction and Monte Carlo, from the mass storage to disk servers and finally to/from the DST farm. About 100 machines are needed for the combined DST, reconstruction and Monte Carlo tasks.

Interactive analysis involves the manipulation (and sometimes creation) of n-tuple datasets, and other event data in the highly interactive manner of PAW or HippoDraw. This is also an I/O intensive task, but, in general, involves data sets of modest size. This type of exploratory analysis occurs on desktop workstations. The data flow is identical to that of the farm machines. At some point, a given analysis may cross a threshold requiring more aggregate CPU and I/O bandwidth to investigate larger and larger datasets. This will mean moving that analysis onto a DST farm machine. 

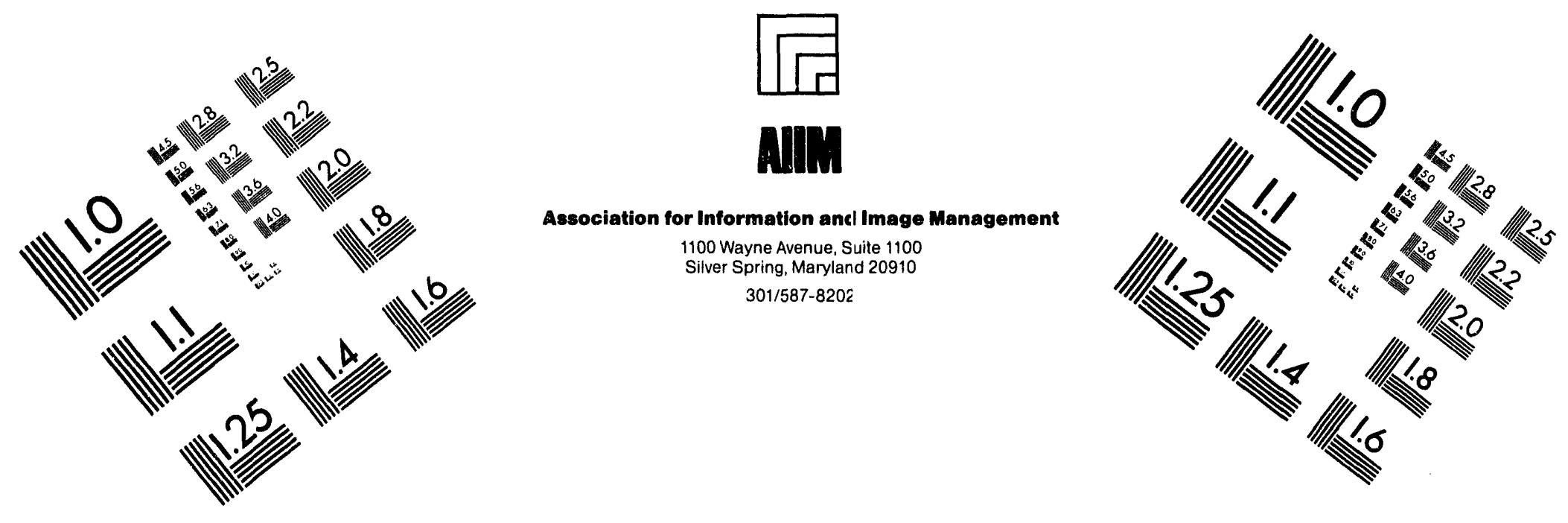

\section{Centimeter}

$\begin{array}{llllllllllllllll}1 & 2 & 3 & 4 & 5 & 6 & 7 & 8 & 9 & 10 & 11 & 12 & 13 & 14 & 15 & \mathrm{~mm}\end{array}$ | Inches
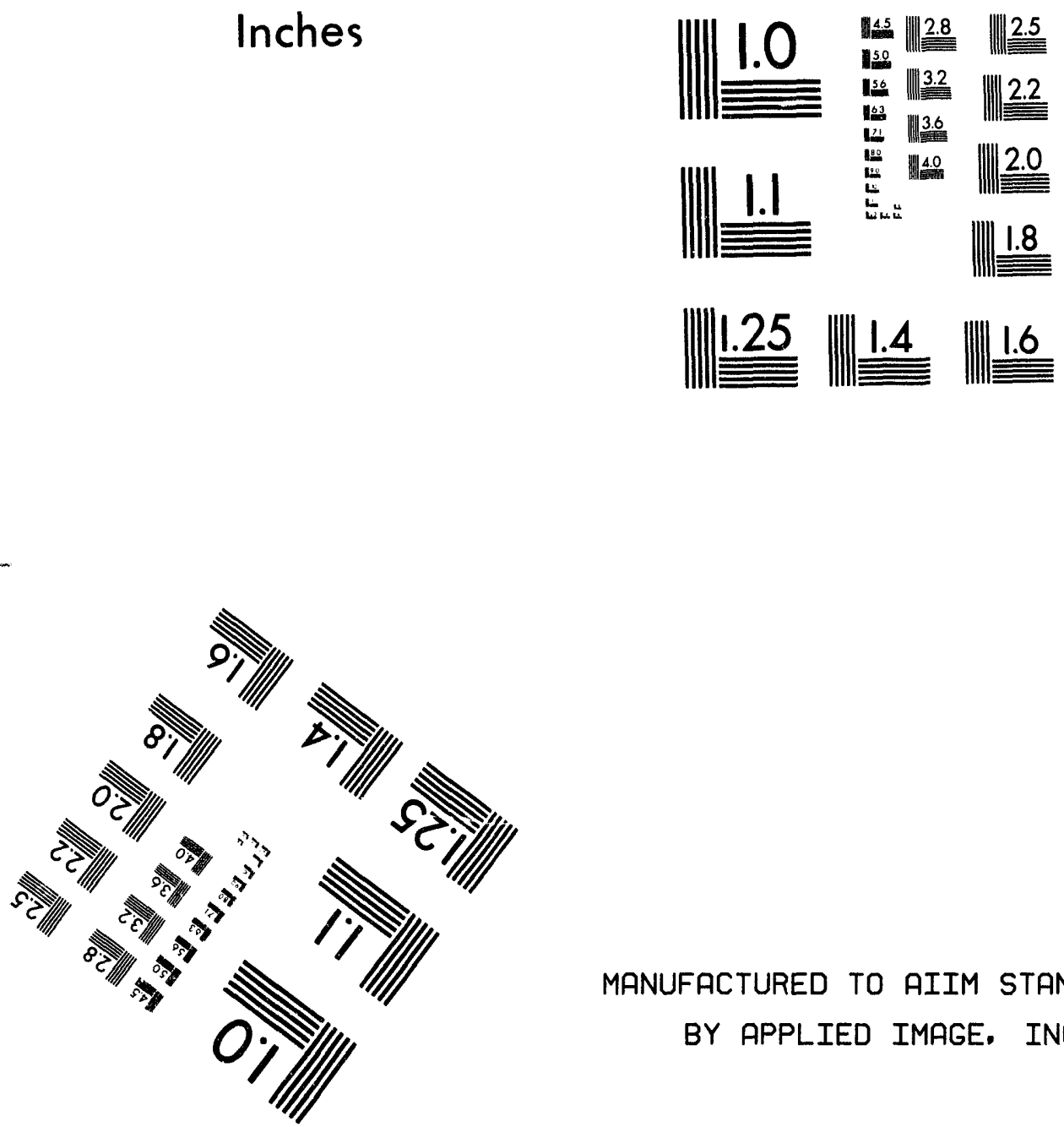

MANUFACTURED TO AIIM STANDARDS

BY APPLIED IMAGE, INC.

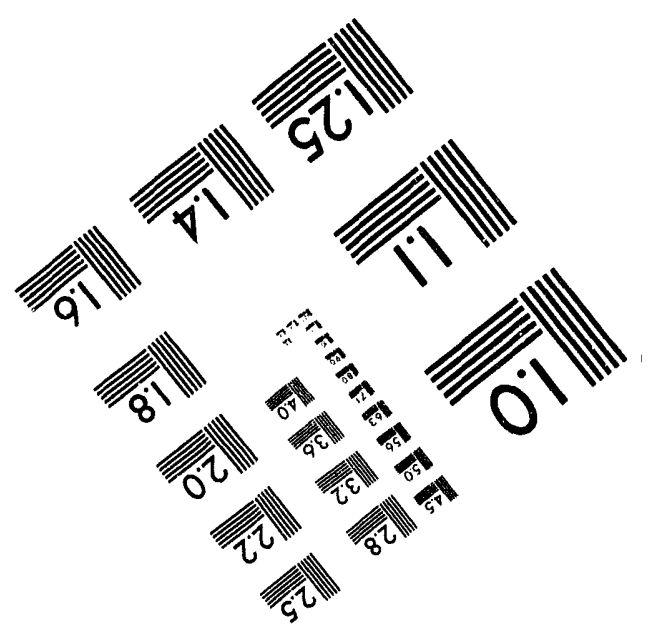



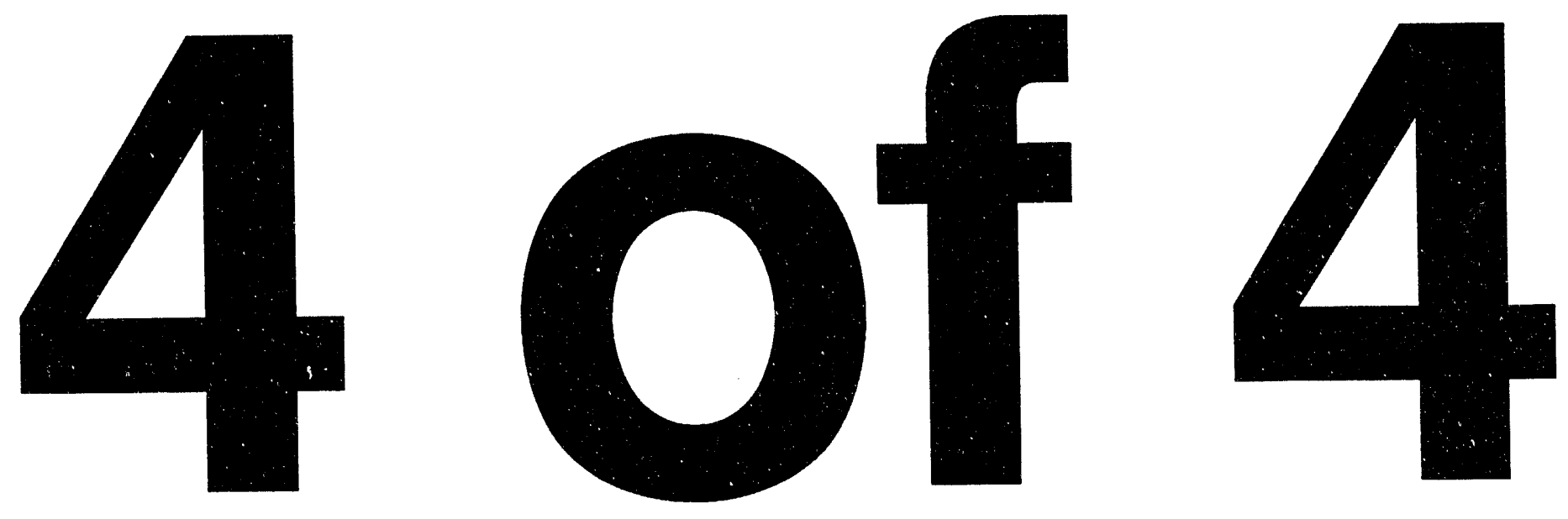
All of the computing tasks (other than data acquisition) can occur at the sites of remote collaborators. The degree to which this may be done is regulated by the computing investment at the remote sites and by the development of the WAN connectivity between SLAC and the collaborating institutes in the following years. The WAN will probably be used to transfer small samples of data; it is presently not feasible to have a complete real-time distribution of event data through the WAN.

It is expected that some form of affordable, high-density, portable storage medium will be used for exchanging moderate amounts of data, such as DSTs, with non-SLAC sites. Such media can then be transferred by air freight. At minimum, all collaborators will need reasonable WAN connectivity to SLAC for the purposes of transferring code, and performing interactive work using the $\mathrm{X}$ window system. This type of link should have a bandwidth of at least $60 \mathrm{k}$ bits/s per interactive session.

Present CPU technology is already adequate to meet the functional requirements. Portions of the model illustrated in Figure 12-1 are currently being prototyped at SLAC. Our model is that the central farm machines are workstations (without monitors or keyboards), with 100 or $200 \mathrm{MIPS} / \mathrm{CPU}$ (which is anticipated to be available by 1998), while the one hundred required desktop workstations consist of 50-MIPS computers.

The leading mass storage technology is an upgrade of the existing STK robotic silos with helical scan technology. This is expected to be available by 1995 , at a capacity of 40 GBytes/tape The transfer rate per drive is $15 \mathrm{Mbytes} / \mathrm{s}$; there are eight drives per silo. There are presently four silos at SLAC each with $\sim 6000$ cartridges, yielding a total capacity of approximately 0.96 petabytes. It is expected that data access will be via staged disk files.

\subsection{Software Environment}

There is much to gain by establishing a common environment across all areas of software development, including the trigger/data acquisition, on-line and off-line. Activities that traditionally were considered purely off-line are fully integrated into the triggering,
!

All computing tasks, other than data acquisition, may be done at remote sites

The effective bandwidth of tape shipment is unlikely to be approached in the near future by realistic wide-area networks

Tapes in robotic silos are envisioned as the primary mass storage medium

A common software environment will be used in all areas 
Code management and distribution standards will be established
The on-line and off-line system will share common stindards

A graphical user interface can hide the complexities of the system from the average user
Gains in hardware performance have not been accompanied by gains in software development productivity monitoring and analysis activities. Similarly, entities that were traditionally based on hardware will now have a significant software component (e.g. the Level 1 trigger system).

Much duplication of effort can be avoided by utilizing common facilities such as the code management and distribution environment, and techniques such as coding guidelines and standards. However, there must be sufficient flexibility to permit specialized solutions (e.g. DSP software development) where required. The goal of the software development environment is therefore to take advantage of natural commonalities, without burdening the developer with significant overhead. The development of robust, efficient and reliable software within the on-line and trigger/data acquisition environment is crucial to the correct functioning of the experiment, since data will be lost as a result of failures.

The overall goal is to create software that hides the complexities of the system from the user as far as possible, allowing both novices and experts to interact with it in a manner that maximizes their productivity. The user interface is crucial to this, since it represents the visible face of the system and must accommodate a wide range of abilities and biases. Again the widespread adoption of common standards between the on-line and off-line environments is envisaged, including a scripting language for repetitive operations in both the on-line and off-line environments and a graphical user interface that provides an iconic style of interaction. A well-defined graphical user interface can minimize the learning curve for casual or infrequent users, maximizing their productivity, while simultaneously providing short-cuts for experienced users.

\subsubsection{Development Environment}

The efficient development of software within a collaboration comprised of physicists from a widely dispersed community requires both good management and the use of adequate support tools. While computer system performance has increased rapidly over the last decade, these increases have not been accompanied by similar increases in software development productivity. However, new techniques, especially when supported by appropriate development and management tools, hold the promise of significant improvements in 
both the software development process and the robustness of the resulting software. This implies that the ease with which changes to the software system may be made in response to changes in requirements caused, for example, either by new and unforeseen physics processes or the addition of new detector components, is considerably improved. The basis for these new techniques is the object oriented paradigm.

A further gain can be achieved by the identification of different types of software developers, ranging from individual physicists writing code for their own "quick and dirty" exploratory analyses, to developers working on the algorithms for the real-time trigger filters. In the latter case a trivial mistake in the code may result in data being irretrievably lost. It is sensible to accommodate efficient program development in these different environments by adjusting the administrative overhead appropriately. This also implies that a mechanism must exist whereby code may be migrated up through the various levels from exploratory, through production analysis and reconstruction, to on-line and trigger filters.

\subsubsection{Software Methodology}

Based upon experience within both the HEP and industrial communities, adherence to the object oriented paradigm, appropriately applied and managed, appears to be the optimum software development environment for the BABAR software effort. This implies the selection of an analysis and design methodology and of a programming language or languages. Several detailed analysis and design methodologies exist, for example, Booch, OMT, Fusion, and Objecteering, etc. [OOM94]. Their most important roles are in aiding the exploratory thought process that analysis and design entails and in providing a notation whereby information may be disseminated throughout the developer community in a standardized fashion. Choice of a specific design methodology will depend on the availability of support tools and the automatic binding to the programming language of choice. Indeed, different tools might be appropriate for the different software levels identified earlier; sophisticated CASE tools may support the real-time environment and other critical areas.
Appropriate levels of code standards will be enforced

Object oriented methodology will be employed 
C++ appears to be the language of choice

Older FORTRAN code will be incorporated

Some new code may be written in Fortran-90
Given the object oriented methodology, it is most natural to use an object oriented programming language for the implementation of the system. This need not necessarily be the case, but this combination affords the most effective solution. However, such an approach must recognize the wide-spread availability of software that already exists, mainly written in FORTRAN-77, and the widespread expertise that exists within the HEP user community in the use of this language. Conversely, most new entrants to the HEP field have exposure to the $\mathrm{C}$ programming language rather than FORTRAN, and it is on their shoulders that much of the implementation will fall. Taking into account the technical merits of the language, the availability of support tools such as interactive debuggers and development environments and the degree of support within the scientific programming community, $\mathrm{C}++$ appears to be the language of choice. Several projects both inside and outside of HEP have validated this choice, while indicating that there is a significant learning curve and that a policy for its use (e.g. code guidelines and templates) must be adopted.

Therefore, it is intended that much of the new software development within the experiment be based on $\mathrm{C}++$, whilst ensuring that existing FORTRAN-based software can efficiently be integrated. It is recognized that this implies a mixed language environment and is an issue that people have traditionally felt very strongly about. It is our recommendation therefore that $\mathrm{C}++$ be used, but it is also our realization that some fraction of the new code will be created using FORTRAN because of the wealth of experience already within the HEP community. For example, R\&D is presently underway on the creation of a formalized mixed-language environment through the use of an Interface Definition Languagr (IDL) that compiles into both $\mathrm{C}++$ and Fortran-90.

\subsubsection{Data Model}

The use of an object oriented methodology for the software development environment leads naturally to the description of the data model for the experiment in terms of objects. This bypasses many of the ad-hoc solutions that have arisen throughout the FORTRAN era such as BOS or ZEBRA and integrates access to the data naturally into the programming language.
The data model is also object oriented 
A uniform data model should be applied throughout all aspects of the software. Thus the same model should apply to the access of raw data, reconstructed data, calibration, and geometrical constants. This model must also allow for a smooth schema evolution. Advantages of this approach include access to the new generation of database management systems that manage objects and the decoupling of the implementation of the data from its access as seen by the programmer. Thus the data might be highly compressed, but this compression would be invisible from the point of view of the programmer accessing it. This affords great flexibility and also an added degree of protection against inadvertent data corruption.

\subsubsection{Code Management}

The software system for the $B A B A R$ Collaboration will be the result of the efforts of many people, many of whom will combine the roles of software designer and implementer. Achieving a stable development environment will require the use of a code management system and a management policy. The CVS code management system has been selected for use by this experiment, and will use the rCVS distributed code management system for distribution of code throughout the collaboration. During the lifetime of the experiment distributed file systems such as AFS and DFS are expected to become widely available; these will probably remove the need for rCVS.

CVS is a mechanism for managing evolving software modules; an additional management layer is needed. This layer implements policies, such as determining who has access to which modules, the availability of a stable "production" version as well as less stable public and development versions and setting up of a directory hierarchy that reflects the natural clustering of code files into modules. The ASLUND and GEANT Monte Carlo packages are being used as a testbeds for validating these concepts.
$A$ uniform data model will be applied to the management of data, calibration constants and geometrical constants

Code management and distribution will be done using CVS and rCVS 


\subsection{Analysis Framework}

Both the "toolkit" and "framework" approaches are being explored
The analysis framework has to cope with operation in several different environments; within Level 3, within the on-line system for monitoring, within the bulk reconstruction phase of the experiment, and finally in physics analysis. In addition it should provide an object oriented environment for access to event data whilst still supporting modules written in non object oriented languages. Customization to suit these applications should require a minimum of changes to the code. Furthermore, the framework should promote exploratory analyses where the focus is in rapid turn-around and "what if" analyses as well as supplying a stable production platform for the reconstruction of data.

Two basic approaches to these requirements are under active investigation. The first is a toolkit that is implemented as a subroutine or class library, where the thread of control is provided by the application programmer. Specialization is achieved with the object oriented technique of inheritance, with no need to modify the already existing code base. The second approach is a framework or skeleton that provides a mechanism whereby modules that adhere to a well-defined protocol are called on an event-by-event basis under the overall control of the framework rather than by application programmer directly. The sequencing is determined externally from the application program using a scripting language or iconic interface. This latter approach reduces the required level of expertise and allows rapid changes to be made with no need for recompilation or relinking. The two approaches are not incompatible; careful design can base the second on the foundation of the first, supporting both styles of programming - conventional and icoric.

\subsection{On-line computing}

The function of the on-line system includes data acquisition, control and monitoring of the detector and its environs. It also provides the Level 3 trigger, comprising both a workstation farm and embedded processors at intermediate levels. The user interface to experimental 
components is the basic mechanism for the operator or experimenter to interact with the detector and its surrounding systems.

A central distinguishing characteristic of the proposed system is an effort to push high level distributed computing components (e.g. embedded processors running UNIX-like real-time kernels) to as early in the trigger/data acquisition streams as possible without an undue incursion of dead-time. Such a system can provide sophisticated tools for software development and problem diagnosis an essential element for the detector system to run as reliably "as a factory". It is also flexible and scalable for adapting to future changes.

\subsubsection{General Requirements}

The choice of operating systems for the on-line computers must balance the performance requirements of the data acquisition system against the other goals of the on-line system. Hard real-time operations require a deterministic behavior, so that trigger response and deadtime can both be accurately modelled and reliably predicted. The Level 1 trigger is envisioned to be such a real-time system, while the Level 3 trigger will be implemented on conventional UNIX systems (perhaps with POSIX-compliant real-time extensions). The appropriate system for intermediate levels is under investigation. A real-time kernel such as VxWorks provides, at some level, both deterministic real-time response and the advantages of a full-featured UNIX-like operating system.

The distributed computers and processors of the on-line system require a cooperating set of tasks to properly sequence the processing of event data (fast control), and of detector/environment states (slow control). The finite state machine model will be used to effect this coordination.

A goal of the on-line system is to provide the operator with an interface which hides the complexity of the system at a level appropriate to the task at hand. The interface will draw upon the commonality within sub-systems and within tasks to present a uniform environment in which to interact, borrowing utilities and interfaces from the off-line computing environment to enhance the uniformity. This system must be inherently flexible to address potential changes of
Reliability of the online system is assured by using high-level tools for program development and problem diagnosis

The Level 3 trigger will depend on conventional UivIX processors. 
requirements over time due, for example, to changes in the physics emphasis or in background conditions.

\subsubsection{Monitor and Control}

EPICS may form the basis of the monitor and control system
Autonomous partitions will service individual detector systems
Monitoring and control are needed for all relevant aspects of the detector system. The Experimental Physics and Industrial Control System (EPICS[EPI92]), developed by a consortium of US national laboratories, provides a tool-kit for building accelerator and detector control systems. The basic model for the controlling intelligence is one of embedded processors running under VxWorks within VME crates and UNIX workstations as the operator interface. This model closely parallels that proposed for the $B_{A B A R}$ detector; $R \& D$ is being pursued to evaluate the applicability of EPICS in the role of slow control system. It is expected that the on-line software will be augmented with an expert system to assist with problem diagnosis and repair. Note that the actual transport of event data through the data acquisition will occur at a software level beneath EPICS.

\subsubsection{Partitioning and Configuration}

One important goal of the computing configuration is to minimize the duration of the commissioning phase of the experiment, during which new detector subsystems and their associated electronics are being installed. Another is to minimize the data-taking time that is lost due to having to recalibrate portions of the detector. The usual solution is to segment the system into partitions that operate autonomously as independent experiments. This approach has implications for the frontend electronics, the trigger system, its dataflow control mechanisms and the software infrastructure. It must be incorporated into the basic design of the experiment, since it cannot be easily retrofitted. The concept of multiple partitions also leads naturally to the creation of distinct mini-systems for beam and bench tests, as well as enabling components to be taken off-line for diagnostics without inhibiting the operation of the remainder of the system. 
Each partition can contain multiple components, most of which correspond to physical hardware (e.g. readout controllers or trigger processors), although others might be purely logical in nature. Some hardware components will have to be shared between partitions (e.g. level 1 segment finder). Each component is managed by a software server process that controls its status. Each partition has associated with it a single broker process that is responsible for the overall control of the partition. This broker issues commands to the servers within the partition and maintains a consistent partition status. Finally, each partition may have multiple consoles, each console providing an independent (graphical) user interface. If a partition uses multiple consoles, only one of them is allowed mastership at a given time, that is, only the master console can modify the state of any component within the partition, although other slave consoles may perform monitoring. At any time, mastership may be passed from one console to another. Whether all communication between the consoles and the servers will be mediated via the broker as shown, or whether direct links will exist, has yet to be decided.

A global configuration or partition manager controls overall access to all hardware components within the experiment, identifying the partition to which they belong and which console currently has mastership of each partition. This manager will be based on a database (e.g. the internal EPICS daiabase or an object-oriented database system). This database will contain all configuration and status information relevant to a given device.

\subsubsection{Accelerator/Detector Interface}

There will be periodic transfers of data between the $B A B A R$ detector and the accelerator control system. Such data transfers will occur on several timescales. On the slowest timescale, the accelerator data will include the beam currents, energies and lifetimes, pressure gauge readings from the ring vacuum system, etc. From the detector these data will include background rates in various detector components, status and performance of the data acquisition system, status of the magnet, etc. Slow exchanges of data will occur between the machine and detector software control systems across a standard network link.
Within a partition there can be master and slave consoles

A partition manager provides overall coordination

Slow data exchange will be via a standard network link 
Certain signals, such as permissives and beam aborts, will be exchanged using specialized hardware
SCS will be a central provider of computing support
Physicists will write analysis and reconstruction code

Software engineers will be used to develop the software framework and the database technology
On a shorter timescale, a variety of data and controls (e.g. permissives, inhibits and aborts) will be exchanged. For example, PEP-II will have a permissive to enable energizing the detector solenoid. Similarly, the detector will have an inhibit to prevent beam injection. A beam dump (abort) signal will be needed to protect the detector from radiation damage. Fast, time-stamped signals from detector elements may be needed by the accelerator control system to assist in beam tuning. Specialized hardware may be necessary to accommodate these fast exchanges of data.

\subsection{Computing Support}

The support of the BaBar computing environment will be split between the collaboration, which will support experiment-specific tools and the on-line system, and SLAC Computing Services (SCS) which will provide support for "infrastructure" aspects. An active discussion with SCS has been underway to identify the support which they will provide to the BABAR Collaboration [SCS94].

SCS will support the computing hardware, including CPUs, networking, and storage. This includes the SLAC off-line environment, and the network components at the detector. It may also include certain aspects of the Level 3 farm itself, which is envisioned to consist of general-purpose workstations.

Software aspects supported by SCS include system management of the UNIX workstations and the X/Motif graphical environment. In addition, a number of general programming and database tools will be managed by SCS, including compilers, editors, graphics, and Oracle.

The collaboration must design, maintain and document both the on-line and off-line software framework, data model, analysis environment, and user interface to the various tasks. Our personnel model is similar to recent typical approaches in HEP, where physicists write most of the analysis and reconstruction code including many of the peripheral tools and scripts, but software engineers will address other aspects, in some instances using appropriate commercial design tools. Examples of such aspects include the database technology and the design of much of the software framework. This 
model has the benefit that the physicists will have close contact with the "physics" aspects of the code, while the "computer science" and systems aspects are handled by people with the requisite expertise.

It must be stressed that the software for the $B A B A R$ experiment represents a very large system. This will require considerable manpower. Most of this manpower will be physicists and students, and each collaborating institution should expect to contribute a significant portion of their manpower to software development. As is typical, the use of software engineers is limited to those tasks for which they are essential, as this minimizes costs. To carry out these tasks, which include infrastructure development, database technology, and coordination, professional software engineers will be required. 


\section{References}

[Ast92] D. Aston, A. Boyarski, A. Breakstone, D. Cords, T. Glanzman, F. Harris, T. Hung, P. Kunz, and F. Porter, "Computing for a B Factory", BABAR Note \#82, June 1992.

[Bad90] A. Baden and R. Grossman, "A Model for Computing at the SSC", Superconducting Super Collider Laboratory Technical Report No. 288, 1990; C. T. Day et al.,, "Database Computing in HEP - Progress Report", In Proceedings of Computing in High Energy Physics, 1992; C. T. Day et al.," "The PASS Project Architecturai Model", In Proceedings of Computing in High Energy Physics, 1994.

[Boe94] C. Boeheim, "SLAC's prototype computing farm", private communication, 1994.

[Boy90] A. Boyarski, T. Glanzman, F. A. Harris, and F. C. Porter, "Report of the Computing Group", in Proceedings of the Workshop on Physics and Detector Issues for a HighLuminosity Asymmetric B Factory at SLAC, JanuaryJune, 1990, ed. by D. Hitlin, SLAC-373, LBL-30097, CALT-68-1697, pp. 575-595.

[CLE94] We thank M. Ogg, J. Urheim, and A. Weinstein for providing information concerning CLEO-II computing.

[EPI92] "Experimental Physics and Industrial Control System (EPICS)", Argonne National Laboratory and Los Alamos National Laboratory, 1992.

[Gou94] K. Gounder, S. Bracker, K. Hendrix and D. Summers, "A Simple Multiprocessor Management System for EventParallel Computing", BABAR Note \#128, March 1994.

[OOM94] G. Booch, "Object Oriented Analysis and Design with Applications" 2nd edition (Benjamin Cummings, 1994), ISBN 0-8053-5340-2; D. Coleman, "Object Oriented Development, the Fusion Method", 1994; P. Desfray, "Object Oriented Modeling" (Addison Wesley, 1994). 
[Por93] F. Porter, "BABAR Event Size Estimate", BABAR Note \#108, June 1993.

[SCS94] "SCS Support of the B Factory", BABAR Note \#145, May 1994. 
LETTER OF INTENT FOR THE BABAR DETECTOR 


\section{3}

\section{CP Asymmetry Simulations}

\subsection{Simulation Tools}

$\mathbf{S}$ imulation studies to optimize the detector design have been undertaken with the programs ASLUND and BBSIM. ASLUND is a fast parametric Monte Carlo, while BBSIM is a detailed simulation based on the GEANT package. Both are designed to be flexible, so that variations in detector design can be investigated. Brief descriptions of each are given below.

\subsubsection{ASLUND}

ASLUND consists of two major components: the JETSET 7.3 event generator configured for the PEP II environment, and a parametric simulation of the detector. Other generators (e.g. KORALB) are available using the interface routine BEGET. The parametric simulation includes charged tracking, particle identification and calorimetry.

Charged particle tracking is simulated using the TRACKERR package [Inne93] to estimate the full correlated error matrix, which is then used to smear the track parameters. Both the main drift chamber, the silicon vertex detector, and any intervening material are modeled. The TRACKERR input format is used to specify detector geometry, measurement precision and material distribution. The combination of ASLUND and TRACKERR has been compared to BBSIM for the mode $B^{0} \rightarrow \pi^{+} \pi^{-}$and found to agree within $10 \%$ in the predicted $B^{0}$ mass width.

Simulation of particle identification $(\pi / K / p)$ information is available for $d E / d x$, TOF, Fast RICH and DIRC; an aerogel model is nearly ready. The geometrical configuration of these devices can
ASLUND is a fast parametrized Monte Carlo 
A more detailed simulation of calorimeter response is being developed
BBSIM is a detailed GEANT-based simulation also be specified using the TRACKERR format and performance parameters can be varied to assess the sensitivity of an analysis to detector capabilities.

The geometry of the calorimeter and any material in front of it, can also be specified in the TRACKERR format. Photons converted into $e^{+} e^{-}$pairs in the material in front of the calorimeter are tracked to determine whether or not they will deposit any energy in the calorimeter. Energy loss in material and acceptance losses due to the curlup in the magnetic field are accounted for in determining the calorimeter signal. A detailed parametrization of the calorimeter response based on GEANT simulations is being developed; at present, a simple Gaussian smearing is used. Analogous to the other devices discussed above, energy and angular resolutions can be varied by modifying simple parameters.

\subsubsection{GEANT Simulation - BBSIM}

A simulation package for event generation, BEGET, and detector response and subsequent analysis, BBSIM, has been written to aid in the design and optimization of the $B A B A R$ detector.

The generation of an event from an $e^{+} e^{-}$collision and the subsequent loading of decay products into GEANT banks is handled by the BEGET package. The generators currently available include $\Upsilon(4 S)$ decays and hadronic continuum events via JETSET [Sjo93], and $\tau^{+} \tau^{-}$decays via KORALB [Jad90]. In the near future, BEGET will be enhanced with the capability to overlay background hits originating from beam-gas collisions.

The BBSIM package uses the CERN detector description and simulation tool, GEANT [CER93]. GEANT consists of packages (1) to construct the detector geometry using a set of 15 different volume types, (2) to step charged and neutral particles through the detector simulating the full variety of interactions with the detector, (3) to define, register and digitize the Monte Carlo track "hits" (typically track positions and directions), and (4) to display the detector components, particle trajectories and track hits. The BBSIM framework consists of a driver routine to invoke GEANT, a database facility, DBIN, to define detector geometry, materials and media, and a set of subpackages, one per detector subsystem, to define the subde- 


\begin{tabular}{|l|l|}
\hline Subdetector & Simulation level \\
\hline \hline beam line, beam pipe and support tube & geometry \\
Silicon vertex detector & hits \\
Forward $\mu$ TPC & hits, clusters \\
Drift chamber & hits \\
RICH & hits \\
Time of flight & geometry \\
DIRC & geometry \\
CsI calorimeter & hits \\
Coil and instrumented flux return & hits \\
\hline
\end{tabular}

Table 13-1. B Factory subdetectors modeled in BBSIM.

tector geometry, register track hits, simulate the detector response, store the results and perform subsequent analysis. The subsystems included in BBSIM, and the corresponding levels of simulation, are shown in Table 13-1. In addition, an aerogel simulation package is under development.

\section{2 $C P$ Reach Calculations}

The expectations for the level of sensitivity to $C P$-violating asymmetries ( $C P$-reach) which are presented in Table 2.1 result from preliminary simulation work using ASLUND and BBSIM for the $B_{A} B A R$ detector design proposed in this Letter of Intent. In this Chapter, a more detailed presentation of this analysis is made, in order to better elucidate the assumptions behind the calculation. Significant improvements in these results are possible; estimates of the sensitivity discussed here are conservative within the physics assumptions stated below for each mode.

The error on $\sin 2 \phi(\phi=\alpha, \beta, \gamma)$ is calculated from:

$$
\sigma(\sin 2 \phi)=\frac{\sqrt{S+B}}{S} \frac{1}{d(1-2 w)}
$$

where $S$ and $B$ are the estimated sizes of the signal and background, $d$ is a factor that accounts for dilution effects of mixing and the experimental determination of the time separation, and $w$ is the

The tagging efficiency is assumed to be independent of the decay mode of the secord $B^{0}$ 
The vertex resolution is characterized by two Gaussians
The effective efficiency must be corrected for tagging errors due to misidentification wrong-sign fraction in the recoil $B^{0}$ tags. In order to estimate values for the parameters that enter this equation, for each $C P$ channel it is assumed that efficiencies and resolutions can be obtained independently for the tag and for the $C P$ state. For some channels (e.g. $\pi^{+} \pi^{-}$) this assumption may not, in the end, be completely vaiid, as more stringent criteria for tagging might need to be imposed to reduce background.

For both tagging studies and for the reconstructed $C P$ eigenstate, vertex resolutions are expressed in terms of a fit using two Gaussian distributions, with free parameters being the two widths $\left(\sigma_{n}\right.$ and $\left.\sigma_{w}\right)$ and the fraction of the events associated with the narrow Gaussian $\left(f_{n}\right)$. The fact that the wrong-sign background will have a different vertex resolution than the signal is ignored. This approximation causes us to underestimate the $C P$ sensitivity. The vertex resolutions for the tag and the $C P$ mode are combined in quadrature to estimate the resolution for the distance between the two $B^{0}$ decays and, ultimately, for the difference between the decay times for the two $B^{0}$ mesons. The program DILUTE [LeDi91] is used to calculate the contribution of these vertex errors to the dilution factor $d$.

For the $C P$ states under study, the background is given in terms of the number of events per $B^{0}$ decay and the signal-to-background ratio is calculated accordingly. For tagging, the background is expressed in terms of the wrong-sign fraction. The quality of a tag is characterized by two factors:

- Particle detection efficiency $\epsilon$, including the contribution of possible wrong-sign tags;

- Fraction of wrong-sign tags, $w$.

A useful figure-of-merit for tagging is the effective efficiency $\epsilon_{e f f}$ given by:

$$
\epsilon_{e f f}=\epsilon(1-2 w)^{2}
$$

The effective efficiencies for different tags can be summed to give the overall effective efficiency. A detailed description of the kaon tagging and lepton tagging procedures in $B A B A R$ are presented in the next two sections. A summary of these results is shown in Table 13-2. 


\begin{tabular}{|l|c|c|c|c|c|c|c|}
\hline $\begin{array}{l}\text { Tag } \\
\text { Type }\end{array}$ & $\begin{array}{c}\text { Branching } \\
\text { Fraction }(b)\end{array}$ & $\begin{array}{c}\text { Efficiency } \\
(b \epsilon)\end{array}$ & $\begin{array}{c}\text { Wrong-sign } \\
\text { Fraction }(w)\end{array}$ & $\epsilon(1-2 w)^{2}$ & \multicolumn{3}{|c|}{ Vertex Parameters } \\
\cline { 6 - 8 } & {$[\%]$} & {$[\%]$} & {$[\%]$} & {$[\%]$} & $\begin{array}{c}f_{n} \\
{[\%]}\end{array}$ & $\begin{array}{c}\sigma_{n} \\
{[\mu \mathrm{m}]}\end{array}$ & $\begin{array}{c}\sigma_{w} \\
{[\mu \mathrm{m}]}\end{array}$ \\
\hline \hline Kaons & 37 & 30 & 10 & 19 & 75 & 48 & 174 \\
Muons & 11 & 8.8 & 11.7 & 5.2 & 69 & 56 & 193 \\
Electrons & 11 & 8.5 & 9.9 & 5.5 & 69 & 56 & 193 \\
\hline
\end{tabular}

Table 13-2. Summary of tagging efficiencies and resolution for the tagging vertex.

\subsubsection{Kaon Tags}

All $B^{0}$ decay modes with one and only one detected charged final state kaon, typically coming from $D$-decays, are included in the tag sample.

The particle identification confidence level is calculated using the probability that observed values in the various PID devices is consistent with the $K$ or $\pi$ mass hypothesis. The overall probability, $P_{i=\pi, K}$ is computed as the sum of $\chi^{2}$ s for a particle of type $i$ to produce the simulated signal in the available PID systems. A particle is deemed to be a kaon if the following requirement is met:

$$
P_{K}>P_{\min }(K), \quad P_{\pi}<P_{\max }(\pi)
$$

where $P_{\min }(K)$ is the minimum allowed probability for the kaon mass hypothesis and $P_{\max }(\pi)$ is maximum allowed probability for the pion mass hypothesis. The effective efficiency is insensitive to the exact value chosen for these variables; the analysis described here uses $P_{\min }(K)=5 \%$ and $P_{\max }(\pi)=10 \%$.

For the $d E / d x$ simulation, the resolution is taken to be $6.9 \%$ and the Sternheimer parametrization of the mean $d E / d x$ response is used as described in Chapter 7. In the presence of an additional particle identification system, the overall results obtained are insensitive to exact value of these parameters.

For the dedicated Cherenkov imaging devices, it is assumed that a minimum of five photons must be detected in order reconstruct the cone angle. The results quoted here are based on the combination of $d E / d x$ and a Fast RICH system; $d E / d x$ along with
An overall $\chi^{2}$ is formed from the several types of particle identification information available
A minimum of five detected photons is assumed necessary to define a Cherenkov ring 
The effect of $\pi$ and $K$ decay is included either TOF or the DIRC has a similar capability for tagęing. The quantum efficiency of the Fast RICH photocathodes is assumed to be $18 \%$. The tagging efficiencies are quite sensitive to these assumptions concerning Fast RICH performance; if more photons are required to efficiently find rings or the quantum efficiency is lower, the effectiveness of this device for kaon tagging degrades rapidly. Other possible choices for dedicated particle identification (DIRC and aerogel) are also under investigation as described in Chapter 7. A final decision on the particle identification system awaits further prototype results.

The effect of $K$ and $\pi$ decay-in-flight on the tagging capability is modeled by correcting the value of the mean $d E / d x$ and the central value of the measured momentum by a factor proportional to the fraction of the track contained in the drift chamber before the particle decays. Only two-body decays of $K$ and $\pi$ are simulated. The net effect is a loss of identification efficiency for kaons that decay before or inside the drift chamber. Decays-in-flight reduce the effective tagging efficiency by $22 \%$, of which $12 \%$ is directly due to loss of kaons and the remainder occurs because of an increase in the wrong-sign background, since more events with two kaons are accepted. Substantial effort will be directed towards the recovery of decaying kaons by proper pattern recognition and fitting of track segments from kinks

For the purpose of this study, the worst case has been assumed for tagging when a particle decays in flight, namely that (1) none of the lost kaons can be recovered, and (2) all particle identification information from the Fast RICH is lost.

In order to properly account for the overlap of events which have both a lepton and a kaon tag, the overall kaon tagging efficiency has been reduced by $18 \%$. This correction assumes the fraction of identified leptons in all $B \bar{B}$ events is similar to that in events with a kaon. The increased purity of such overlapping tags has not been used in this analysis.

The position of the tagging $B^{0}$ decay is estimated by forming a vertex from all the charged tracks not associated with the $C P$ state under study. The bias caused by tracks from $D$ decays is corrected using the Monte Carlo simulation. These results are summarized in Table 13-2. 


\subsubsection{Lepton Tags}

The lepton tag sample includes high momentum electrons and muons produced directly from the decay of a $B^{0}$. Some improvement can be made by including the low-momentum indirect tags of leptons from $B \rightarrow D \rightarrow e$ or $\mu$ decays; this is still under investigation and has not been included here.

Muon identification efficiencies and pion misidentification probabilities were determined using a GEANT simulation. These results were encoded as a look-up table in ASLUND. Because of the variation of the wrong-sign fraction $(w)$ with muon momentum, the muon analysis is divided into discrete momentum regions. The results quoted here use only the regions above $1.0 \mathrm{GeV} / c$, which are dominated by leptons from direct $B$ decays. This yields an efficiency of $8.8 \%$, an overall wrong-sign fraction $w$ of $11.70 \%$, and thus an effective tagging efficiency of $5.2 \%$.

Electron identification uses the original ASLUND look-up table, which is based on the expected performance of a CsI calorimeter only; the potential gains from using $d E / d x$ and other particle identification devices are not yet included. Electrons below $1 \mathrm{GeV}$ are not used. This gives an efficiency of $8.5 \%$ with an overall wrong-sign fraction of $9.9 \%$ and an effective efficiency of $5.5 \%$.

For leptons the $z$ impact parameter is used to estimate the decay position of the tagging $B^{0}$ meson. The results for lepton tagging are summarized in Table 13-2.

\subsubsection{CP Asymmetry Channels}

The channels considered here are decays to $C P$ eigenstates, or modes that involve a mixture of different $C P$ states but with a self-conjugate final-state particle content. The assumptions made for each channel are outlined below. These modes are a representative but not exhaustive list of possible $C P$-Asymmetry channels. 


\begin{tabular}{|l|c|}
\hline Channel & Reconstructable Fraction \\
\hline \hline$J / \psi \rightarrow \mu^{+} \mu^{-}, e^{+} e^{-}$ & 0.123 \\
$K^{0} \rightarrow K_{S}^{0}$ & 0.500 \\
$K_{S}^{0} \rightarrow \pi^{+} \pi^{-}$ & 0.667 \\
$K_{S}^{0} \rightarrow \pi^{0} \pi^{0}$ & 0.333 \\
\hline Overall & 0.062 \\
\hline
\end{tabular}

Table 13-3. Reconstructable fractions for $J / \psi K_{S}^{0}$ decays.

\subsection{4 $\quad B^{0} \rightarrow J / \psi K^{0}$}

A branching fraction of $7.7 \times 10^{-4}$ is used for this channel, calculated from the observed $B^{+} \rightarrow J / \psi K^{+}$rate using isospin arguments. The backgrounds for this channel are insignificant. Table 13-3 shows the assumed reconstructable fraction of $J / \psi$ and $K_{S}^{0}$ decays.

The detection efficiencies are predicted (using the ASLUND simulation) to be 0.55 for $K_{S}^{0} \rightarrow \pi^{+} \pi^{-}$and 0.42 for $\pi^{0} \pi^{0}$, giving an average efficiency of 0.51 using both $K_{S}^{0}$ decay modes.

The vertex resolution in this channel is obtained by fitting two Gaussians to the distribution of the difference between the measured and the generated longitudinal vertex position. The values extracted from the fit for the free parameters are given in Table 13-6.

\subsection{5 $B^{0} \rightarrow J / \psi K^{* 0}$}

The measured branching fraction $1.3 \times 10^{-3}$ is used for this channel. The $K^{* 0}$ decays $1 / 3$ of the time to $K^{0} \pi^{0}$; the reconstructable fractions are then as given above for $J / \psi K_{S}^{0}$. This yields an overall reconstructable fraction of $2 \%$ for this mode. The calculated detection efficiency is $38 \%$ with an insignificant background contribution.

Since this mode is not a pure $C P$ eigenstate, in principle, one must use a partial wave analysis [Dun91] to separate the different $C P$ states. This could dilute the effectiveness of this mode for extracting the angle $\sin 2 \beta$. However, recent measurements [Ala94] indicate that the channel is highly polarized and thus dominated by a single 


\begin{tabular}{|l|c|c|}
\hline Mode & $\begin{array}{c}\text { Branching } \\
\text { Fraction }\end{array}$ & $\begin{array}{c}\text { Estimated } \\
\text { Efficiency }\end{array}$ \\
\hline \hline$K^{-} \pi^{+} \pi^{+}$ & 0.103 & 0.66 \\
$K^{-} \pi^{+} \pi^{+} \pi^{0}$ & 0.062 & 0.47 \\
$K_{S}^{0} \pi^{+} \pi^{0}$ & 0.028 & 0.47 \\
$K_{S}^{0} \pi^{+}$ & 0.011 & 0.66 \\
$K_{S}^{0} \pi^{-} \pi^{+} \pi^{+}$ & 0.026 & 0.50 \\
$K_{S}^{0} \pi^{-} \pi^{+} \pi^{+} \pi^{0}$ & 0.029 & 0.35 \\
\hline Overall & 0.26 & 0.54 \\
\hline
\end{tabular}

Table 13-4. Efficiency for $D^{+}$reconstruction.

$C P$ eigenstate. Therefore, full polarization is assumed in estimating the $C P$ reach provided by this channel.

In estimating the vertex resolution, the assumtion of a pure-polarization state is also taken into account. The result given in Table136 is slightly better than for $J / \psi K_{S}^{0}$.

\subsection{6 $\quad B^{0} \rightarrow D^{+} D^{-}$}

A branching fraction of $6 \times 10^{-4}$ is used for this channel, obtained by averaging the branching fractions for $B^{+}$and $B^{0}$ to decay to $D D_{S}$ and multiplying by $\sin ^{2} \theta_{C}$.

The reconstructable fractions are estimated as follows. All $D^{+}$ modes with no more than one $\pi^{0}$ and a branching fraction greater than about $1 \%$ are included, as are Cabibbo-suppressed channels with an additional kaon. All pion decay modes are not used. Thus $K^{-} K^{+} \pi^{+}$is included along witll $K^{--} \pi^{+} \pi^{+}$, but not $\pi^{-} \pi^{+} \pi^{+} . K_{S}^{-0}$ 's are considered only in their $\pi^{+} \pi^{-}$decay mode. These actual channels used are shown in Table13-4.

The efficiency $(\epsilon)$ given for each mode in Table 13-4 is based on scaling the acceptance using the following rule: $\epsilon=0.87^{n(c h)} \times$ $0.71^{n\left(\pi^{0}\right)}$. An additional factor of $88.5 \%$ is used to account for the $4 \%$ probability cut on each $D$ decay vertex from the $D \bar{D}$ pair forming the $B$ meson, and for the $B$ vertex itself. 
These efficiencies have been adjusted on the basis of an analysis using ASLUND in combination with the vertex and kinematic fitting package FITVER for the modes $D^{+} \rightarrow K^{-} \pi^{+} \pi^{+}$and $K^{-} \pi^{+} \pi^{+} \pi^{0}$. For the case where both $D$ mesons decay to $K^{-} \pi^{+} \pi^{+}$the full analysis yields an efficiency of $39 \%$, to be compared with $38 \%$ calculated with our simplified expression. Good agreement is also obtained for $K^{-} \pi^{+} \pi^{+} v s . K^{+} \pi^{-} \pi^{-} \pi^{0}$, and cases where both $D$ 's decay to $K^{-} \pi^{+} \pi^{+} \pi^{0}$.

Backgrounds have also been investigated for these channels. For all-charged modes, a background of $3.3 \times 10^{-8}$ events per $B^{0}$ decay is found. This corresponds to a background-to-signal ratio $(B / S)$ of $1 / 61$, which is quite negligible. For the decays with one or more neutral pions, a rate of $4.4 \times 10^{-7}$ is found, which gives a backgroundto-signal ratio in these channels of $1 / 4$. The overall $B / S$ ratio for the fully simulated channels is $1 / 9$. The same ratio is assumed to hold when all channels are considered; the net effect is to increase the error on $\sin 2 \beta$ by $\sim 5 \%$. The degradation would be even smaller if each channel were treated separately with its proper statistical weight.

The longitudinal vertex distribution for $D^{+} D^{-}$modes is found to be consistent with a single Gaussian in the FITVER analysis. The width for decays with with and without neutral pions is $50 \mu \mathrm{m}$ and $39 \mu \mathrm{m}$ respectively. A single Gaussian with a width of $50 \mu \mathrm{m}$ is assumed for all modes to estimate the dilution factor for the $C P$ reach calculation.

\subsection{7 $\quad B^{0} \rightarrow D^{*+} D^{*-}$}

A branching fraction of $1.5 \times 10^{-3}$ is used for this mode, which is a 2.5 times larger than that for $D^{+} D^{-}$. This factor is the typical increase for vector-vector decay modes over comparable scalar-scalar channels.

The only decay chain considered is that in which each $D^{* \pm}$ decays to $D^{0} \pi^{ \pm}$with a branching fraction of $68 \pm 1.6 \%$. Since the $D^{*+}$ signal is then extremely clean, due to the slow pion, many more modes of the $D^{0}$ can be reconstructed. All modes with up to two neutral pions and branching fractions greater than $1 \%$ are assumed to be usable, including Cabibbo-suppressed decays with an extra 


\begin{tabular}{|l|c|c|}
\hline Mode & $\begin{array}{c}\text { Branching } \\
\text { Fraction }\end{array}$ & $\begin{array}{c}\text { Estimated } \\
\text { Efficiency }\end{array}$ \\
\hline \hline$K^{-} \pi^{+}$ & 0.041 & 0.76 \\
$K^{-} \pi^{+} \pi^{0}$ & 0.113 & 0.54 \\
$K_{S}^{0} \pi^{+} \pi^{-}$ & 0.022 & 0.57 \\
$K^{-} \pi^{+} \pi^{+} \pi^{-}$ & 0.077 & 0.57 \\
$K_{S}^{0} \pi^{+} \pi^{-} \pi^{0}$ & 0.034 & 0.41 \\
$K^{-} \pi^{+} \pi^{0} \pi^{0}$ & 0.153 & 0.38 \\
$K^{-} \pi^{+} \pi^{+} \pi^{-} \pi^{0}$ & 0.036 & 0.41 \\
\hline Overall & 0.40 & 0.50 \\
\hline
\end{tabular}

Table 13-5. Efficiency for reconstruction of $D^{0}$ produced in $B \rightarrow D^{*+} D^{*-}$ decays.

kaon. The efficiency for finding both slow pions is estimated to be $76 \%$.

The reconstructable fraction and the efficiency for the reconstruction of various $D^{0}$ modes is given in Table 13-5. Efficiencies have been estimated using the same acceptance-scaling argument employed for $B^{0} \rightarrow D^{+} D^{-}$above.

In reconstructing a $B^{0}$ meson from a pair of $D^{*}$ mesons, additional efficiency factors must be included. These are $82 \%$ for the five vertex fits involved and the $76 \%$ to reconstruct the two slow pions, as noted earlier. For the channel

$$
B^{0} \rightarrow D^{*+} D^{*-} \rightarrow\left(\pi^{+} D^{0}\right)\left(\pi^{-} \bar{D}^{0}\right) \rightarrow \pi^{+}\left(K^{-} \pi^{+}\right) \pi^{-}\left(K^{+} \pi^{-}\right)
$$

this procedure yields an efficiency of $36 \%$, which is exactly the same as found with the full FITVER analysis. For the mode in which a $\pi^{0}$ is added to one of the decay chains, the full analysis and the scaling rule also agree. A substantial loss is incurred by the restriction to the $\pi^{+} D^{0}$ decay mode; it may be possible to use some of the $\pi^{0} D^{0}$ channels as well, depending on the level of background.

Background calculations for $D^{*+} D^{*-}$ are not yet complete. In the $C P$ reach table, the background level $(B / S)$ is taken to be the same as $D^{+} D^{-}$on assumption that the constraint provide by the $D^{*}$ decay will allow us to reconstruct channels with one more $\pi^{0}$ without an increasing background. This will be investigated with a more detailed simulation. 
In computing $C P$ reach, $D^{*+} D^{*-}$ channels are assumed to be dominated by a single $C P$ state. If this turns out not the case, a partialwave analysis will need to be performed to disentangle the $C P=+$ and $C P=-$ states. This will decrease the effectiveness of these channels by a factor that depends on the fraction of each $C P$ state present.

\subsection{8 $B^{0} \rightarrow \pi^{+} \pi^{-}$}

This mode, and the $\rho^{ \pm} \pi^{\mp}$ channel discussed below, are assumed to be dominated by ihe tree-diagram contributions, with negligible contamination from penguin amplitudes. This is expected theoretically, but must nonetheless be checked by a more complete analysis which includes other isospin-related channels [Gro91].

A branching fraction of $2 \times 10^{-5}$ is assumed for the $B^{0} \rightarrow \pi^{+} \pi^{-}$ mode. CLEO-II reports this value [Bat93] for the sum of $\pi^{+} \pi^{-}$and $K^{+} \pi^{-}$, but is unable to discriminate between the two modes at a significant level. Since the $\pi^{+} \pi^{-}$channel is expected to dominate, the entire two-body signal is optimistically assumed to be in this channel. The CLEO-II best fit is for equal contributions, but the evidence is weak. For this mode the reconstructable fraction is $100 \%$ and the detector acceptance is $86 \%$. However, other cuts are needed to suppress the combinatorial background from continuum events reducing the final efficiency to $35 \%$. This result is based on that quoted in reference [ST. $\Delta{ }^{\circ} 9$ ], although it has been confirmed that the $B A B A R$ detecror design has essentially the same acceptance. Details of the background suppression will require further investigation. One requirement in particular is that not all the charged tracks in an event be consistent with originating from a single vertex. This is very effective in reducing continumm background, but it also removes about $35 \%$ of the signal. However, most of the lost events are at small time separation and therefore do not contribute significantly to the $C P$ asymmetry determination. The degradation in $C P$ reach caused by this cut is, therefore, overestimated in the present analysis. The efficiency without this cut would be $54 \%$.

The background for $\rho^{ \pm} \pi^{\mp}$ mode is estimated to be $5.8 \times 10^{-7}$ per $B^{0}$ decay. Vertex resolution parameters can be found in Table 13-6. 


\begin{tabular}{|l|c|c|c|}
\hline Mode or tag & $f_{n}$ & $\sigma_{n}$ & $\sigma_{w}$ \\
\hline \hline$J / \psi K_{S}^{0}$ & 0.85 & $34 \mu \mathrm{m}$ & $144 \mu \mathrm{m}$ \\
$J / \psi K^{* 0}$ & 0.93 & $32 \mu \mathrm{m}$ & $116 \mu \mathrm{m}$ \\
$D^{+} D^{-}$and $D^{*+} D^{*-}$ & 1.0 & $50 \mu \mathrm{m}$ & - \\
$\pi^{+} \pi^{-}$ & 0.84 & $22 \mu \mathrm{m}$ & $77 \mu \mathrm{m}$ \\
$\rho^{ \pm} \pi^{\mp}$ & 0.74 & $32 \mu \mathrm{m}$ & $169 \mu \mathrm{m}$ \\
\hline
\end{tabular}

Table 13-6. Vertex resolutions for tags and CP study states.

\subsection{9 $\quad B^{0} \rightarrow \rho^{ \pm} \pi^{\mp}$}

Estimates provided for this mode are made on the basis of the work in reference [Ale91]. The branching fraction is assumed to be $8 \times 10^{-5}$, based on comparison with the $\pi^{+} \pi^{-}$channel. This estimate depends on the fraction of each contributing isospin amplitude; equal contribution from $\rho^{+} \pi^{-}$and $\rho^{-} \pi^{+}$are assumed here, so there is no additional dilution for not being a $C P$ eigenstate. The reconstructable fraction is $100 \%$ and the reconstruction efficiency is taken to be $48 \%$ [Ale91]. The vertex resolution parameters are given in Table 13-6.

\subsection{Summary of $C P$ Reach Analysis}

\begin{tabular}{|l|c|c|c|c|c|c|c|}
\hline Mode & $\begin{array}{c}\text { Br } \\
\left(10^{-4}\right)\end{array}$ & $\begin{array}{c}\text { Reconstructable } \\
\text { Fraction }\end{array}$ & Efficiency & $\begin{array}{c}\text { Dilution } \\
\text { Factor }\end{array}$ & Events & Bkd & $\delta[\sin 2 \phi]$ \\
\hline \hline$J / \psi K_{S}^{0}$ & 3.85 & 0.062 & 0.51 & 0.52 & 367 & 0 & 0.13 \\
$J / \psi K^{* 0}$ & 12.6 & 0.021 & 0.38 & 0.53 & 150 & 0 & 0.20 \\
$D^{+} D^{-}$ & 6.0 & 0.067 & 0.26 & 0.53 & 158 & 17 & 0.20 \\
$D^{*+} D^{*-}$ & 15.0 & 0.105 & 0.12 & 0.52 & 272 & 30 & 0.16 \\
\hline$\pi^{+} \pi^{-}$ & 0.2 & 1.000 & 0.35 & 0.54 & 106 & 9 & 0.24 \\
$\rho^{ \pm} \pi^{\mp}$ & 0.8 & 1.000 & 0.47 & 0.51 & 567 & 127 & 0.12 \\
\hline
\end{tabular}

Table 13-7. Summary of $C P$ reach for the BABAR detector for some major decay modes in a data sample of $30 \mathrm{fb}^{-1}$ (one "Snowmass year") accumulated on the $\Upsilon(4 S)$ resonance.

Table 13-7 summarizes the CP reach resulting from these analyses. The results, particularly those for the benchmark mode $J / \psi K_{S}^{0}$, are 
worse than those found in earlier analysis [SLA89]. The main causes of this deterioration are (1) a decrease in the assumed branching fraction and (2) a decrease in the effective kaon tagging efficiency. The latter results from including the effects of kaon decay-in-flight, accounting for overlaps with other tags, and employing more realistic simulations of the performance of particle identification systems. As discussed in the text, some of the loss will almost certainly be recovered by using other tagging procedures such as low momentum leptons, missing $D$ or slow pions, or by using a more sophisticated tagging analysis (see, e.g. [Jaff93]). 


\section{References}

[Ala94] M.S. Alam et al., Cornell preprint CLNS 94/1270

[Ale91] R. Aleksan et al., Nucl. Phys. B361 141, 1991.

[Bat93] M. Battle, et al., Phys. Rev. D71, 3922 (1993).

[Ber90] Brian Berliner, CVS II: Parallelizing Software Development, Proceedings of the Winter 1990 USENIX Conference, Washington, DC, January, 1990.

[Bru91] R. Brun, M. Goosens and J. Zoll, ZEBRA User's Guide, CERN Program Library Q100, CERN, 1991.

[CER93] GEANT Detector Description and Simulation Tool, version 3.15, CERN Program Library W5103, CERN, June 1993.

[Dun91] I. Dunietz et al., Phys. Rev. D43 2193, 1991.

[Gro91] M. Gronau and D. London, Phys. Rev. Lett. 65 3381, 1991.

[Inne93] W. Innes, BABAR note \#121.

[Jad90] S. Jadach and Z. Was, KORALB 2.1: An Upgrade with Tauola Library of Tau Decays, Comput. Phys. Comm. 64, 267 (1991). We currently use KORALB 2.2 and TAUOLA 2.4 .

[Jaff93] D.E. Jaffe, F. Le Diberder, M.H. Schune and G. Wormser, BABAR note\#132.

[Kun93] Paul Kunz and Terry Hung, Source Code Management System, BABAR Note \#109, July 1993.

[LeDi91] F. Le Diberder, Babar note \#34

[SLA89] For earlier studies see SLAC-353, 1989 SLAC-400, 1992 and SLAC-419, 1993.

[Sjo93] JETSET 7.4, T. Sjostrand, CERN-TH-7111-93, December 1993. 


\section{4}

\section{Collaboration Issues}

$\mathbf{T}$ he BABAR Collaboration had its inaugural meeting at SLAC in December, 1993. At that time an Interim Steering Committee was chosen to organize and direct the Collaboration efforts until a permanent organization structure could be set up. The major milestone dates set were June 3, 1994, for the submission of this Letter of Intent, and December of 1994 for the Conceptual Design Report.

The Steering Committee formed a subcommittee to develop the draft of a governance document for the collaboration. The full Steering Committee discussed the resulting document extensively with the laboratory management and with the collaboration before approving it at the Collabcration meeting in May of 1994. It was then amended slightly and then approved by the newly formed Collaboration Council at the same meeting. The procedures described in the governance document for selecting the officers and management of the collaboration are now occurring, and the full structure should be in place by August, 1994 .

In this chapter we summarize the collaboration organization as detailed in that document [BAB94].

\subsection{Membership}

Ph.D. physicists, engineers and Ph.D. thesis students who contribute significantly to the $B A B A R$ detector, as well as those who contribute significantly to the accelerator and plan to participate in the physics program, are eligible to be members of the collaboration. Until submission of the Conceptual Design Report (CDR), membership is open to all individuals who meet the above criteria, and their institutions. After submission of the CDR, new groups
The BABAR Collaboration formed in December 1993, with the first major milestone being this Letter of Intent

The governance rules for the Collaboration were initially drafted by the Interim Steering Committee, then amended and ratified by the newly formed Collaboration Council in May 1994
Membership is open until submission of the Conceptual Design Report; afterwards new groups may submit their proposals to the Collaboration Council 
The Collaboration Council is responsible for policies concerning membership, publications and conferences will join by submitting a proposal which will be voted on by the Collaboration Council.

\subsection{Collaboration Council}

Institutions with three or more collaborating members who are $\mathrm{Ph}$.D. physicists are represented directly on the Collaboration Council Members of the collaboration from institutions with less than three $\mathrm{Ph}$.D. physicists may affiliate with another institution for the purposes of representation on the Collaboration Council. Large institutions have one vote for every ten collaboration members. The Collaboration Council will have an elected Chairperson and ViceChairperson.

The Collaboration Council deals with issues related to the overall framework of the collaboration. It will be responsible for membership policy, publication policy, and the selection of speakers for conferences.

The Council also appoints a Nominating Committee to nominate the Spokesperson every three years, in consultation with SLAC management and with the collaboration at large. This nominee must be ratified by a two-thirds majority of the Collaboration Council. The Council may vote to remove the Executive Board, also by a two-thirds majority.

\subsection{Spokesperson}

The Spokesperson is the scientific representative of the collaboration, and is responsible for all scientific, technical, organizational and financial affairs. On financial matters, the Spokesperson's authority will be consistent with the requirements of the various funding agencies. The Spokesperson will consult with the Technical Board on technical matters. In addition, the Spokesperson is responsible for keeping SLAC management and the Collaboration Council informed about collaboration affairs. 
The Spokesperson, after broad consultation with the collaboration and SLAC management, will nominate a Deputy Spokesperson who must be ratified by the Collaboration Council. The term of appointment for both offices is three years, renewable.

\subsection{Executive and Technical Boards, Finance Review Committee}

The Executive Board will advise the Spokesperson on all scientific financial and organization matters. It will consist of memoers distinguished by their scientific judgment, technical expertise, and commitment to the experiment. The Executive Board may remove the Spokesperson with a two-thirds majority vote. The membership will reflect the national composition of the collaboration. Initially, the Executive Board will consist of one representative each from Canada, France, Germany, Italy, and the U.K., and five representatives from the U.S. This composition may change as the collaboration grows. The Spokesperson, Deputy Spokesperson, Technical Coordinator, and a PEP-II representative will serve as non-voting ex-officio members.

The Technical Board will advise the Spokesperson on technical and financial matters. It will consist of the Technical Coordinator as chairperson, the Project Engineer, a PEP-II representative, the Collaboration Safety Officer, the Spokesperson ex-officio, and the managers of the detector subsystems. The Spokesperson will nominate the subsystem managers in consultation with the Executive Board, and may nominate additional members of the Technical Board.

The Finance Review Committee will monitor the financial aspects of the experiment as set forth in the management plan for the detector and agreed to between SLAC and the collaboration. This will be detailed in Memoranda of Understanding between SLAC and each participating institution. The committee will be chaired by the SLAC Director of Research, and will include on representative of each of the funding agencies, or someone designated by the agency. The Spokesperson will normally also attend meetings of the committee.
The Spokesperson and Deputy Spokesperson have terms of three years, which may be renewed

The Executive and Technical Boards advise the Spokesperson on scientific, organization and technical matters. The Finance Review Committee monitors the financial affairs of the collaboration 


\section{References}

[BAB94] PEP-II Detector Collaboration Governance (1994). 


\section{Project Organization and Management}

$T$ he organization of the $B_{A} B_{A R}$ Collaboration involves several $\mathcal{1}$ aspects, among them the governance of the collaboration (see Chapter 14), the construction of the detector, and the production and analysis of scientific data.

A preliminary organization chart is shown in Figure 15-1. The final project organization chart will be worked out in conjunction with the SLAC management. The relationship of the Spokesperson, the Collaboration Council, the Executive Board and the Technical Board is spelled out in the Collaboration Governance document, and will not be further addressed here.

\subsection{Project Organization}

The focus of this chapter is the organization of the detector construction project. There will be a Spokesperson, Technical Coordinator who reports to the Spokesperson, a Project Engineer or Engineers and a support and administrative staff. The relationship between the Technical Coordinator and the Project Engineer(s) has not been worked out in detail at this point. The more detailed discussion which follows will assume for the sake of definiteness the existence of Project Engineers for mechanical, electrical, and software areas.

The Governance Rules also provide for a Technical Board that advises the Spokesperson. The composition of the Technical board is specified therein. 


\section{BABAR Collaboration Management}

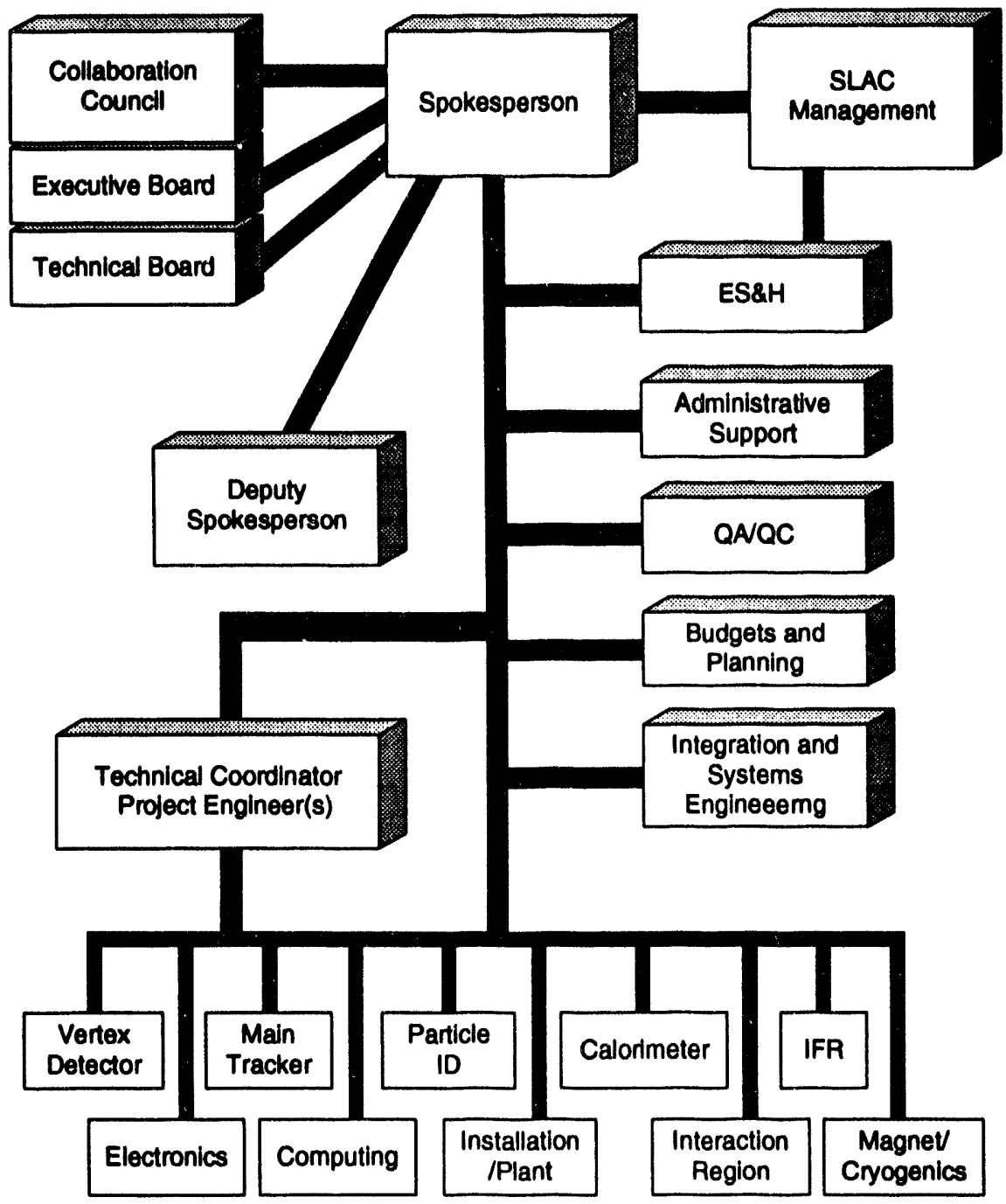

Figure 15-1. Preliminary organization chart for the BABAR Project 


\subsection{Management}

The day-to-day technical coordination of the BABAR detector design, construction and installation will be the responsibility of the Technical Coordinator, the Project Engineer(s), and the managers of the individual detector subsystems, reporting to the Spokesperson.

This organization will insure that the detector, as a whole, can be assembled, serviced and operated as envisioned by the collaboration. The organization will also support the Technical Board in assessing the engineering impact of future subsystem design changes and provide a clear path to experimental upgrades.

There will be a Quality Assurance/Quality Control (QA/QC) specialist and an Environmental Health and Safety (ES\&H) specialist responsible for issues that may arise in either of these areas.

There will also be an Administrative Assistant, a Budget Officer and a staff of designers, draftsmen and clerical assistants.

\subsection{Hall Facilities and Services}

\subsubsection{Facility Integration}

A preliminary study of detector facility requirements was made for the Letter of Intent. Figure 15-2 shows the detector located on the beam line. The IR hall has two cranes (50 and 10 ton) on a single bridge. Crane capacity and coverage are sufficient to assemble the detector.

The IR hall has steel plates inset into the floor to carry the weight of the detector. Since BABAR is almost twice the weight of the TPC detector which previously was located in IR2, a new rail system to distribute the detector load and guide the detector transport system will have to be designed.

A building to house the cryogenic plant will be located on an existing pad outside the southern wall of IR2. Cryogenic tanks will be located on another pad above the hill at the western end of the 


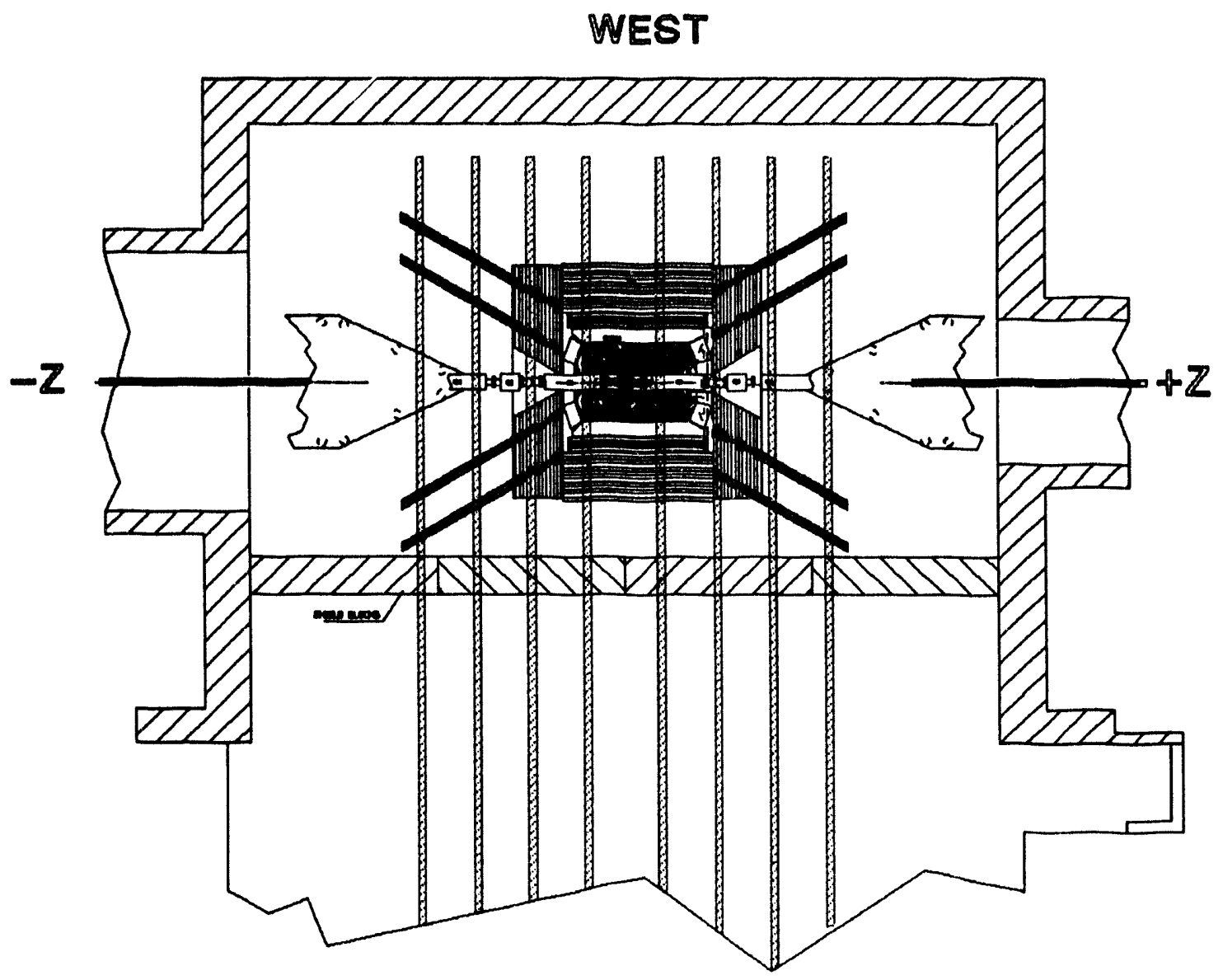

Figure 15-2. Plan view of the BABAR detector in operating position.

building. A gas mixing house and gas storage rack will also be erected near the building.

The existing detector control and data analysis room appears to have sufficient space for computers and workstations.

Existing shielding blocks from the TPC experiment will be used. Several new blocks will be cast to contain the cable labyrinth. A study of radiation skyshine will have to be performed to determine the adequacy of the existing concrete curtain that completes the shield wall from the crane hook to the building roof. 


\subsubsection{Conventional Systems}

Conventional systems consist of the mechanical and electrical utilities required to power and cool the detector subsystems. They also include the assembly and service fixtures and the permanent platforms that house racks and equipment that must be near the detector.

A Resources Requirement Report (RRR) detailing detector conventional system needs and defining the conventional system interfaces with the PEP-II facility will be developed prior to the Conceptual Design Report.

The electric power requirement for the detector consists of filtered electric power for the subsystems and facility power to run the cryogenic plant, pumps, and other equipment. Emergency power and uninterruptible power will be supplied to critical subsystems and controls. An integrated approach to common electrical design issues will be addressed by this group. This will include clean $\mathrm{AC}$ power distribution for all subsystems, an electrical grounding plan, cable and connector selection, rack and cableway design, and emergency power.

Chilled water distribution and control will be supplied to the detector subsystems for thermal control of heat dissipated by the electronics.

There will be an electronics platform to house racks for the data acquisition system crates and power supplies. In order to facilitate access to the racks when the detector is located on the beamline, a shield wall with cabl labyrinth will be erected between the detector and the electronics platform.

The electronics platform will be transported with the detector from the assembly area to the beamline location. This will eliminate the need to disconnect the cables running between the detector and the platform after subsystem testing is completed. The area of the two story platform will be determined based on the number of racks and other equipment that must be housed on it. 


\subsection{Mechanical Integration}

\subsubsection{Subsystem Dimension Control}

Overall subsystem envelope dimensions are specified to define the detector volume. Subsystem envelope dimensions describe the entire subsystem volume including space required for mechanical support, electronics, cables, machining tolerances and assembly clearance. Figure 15-3 shows a half section of the detector with some

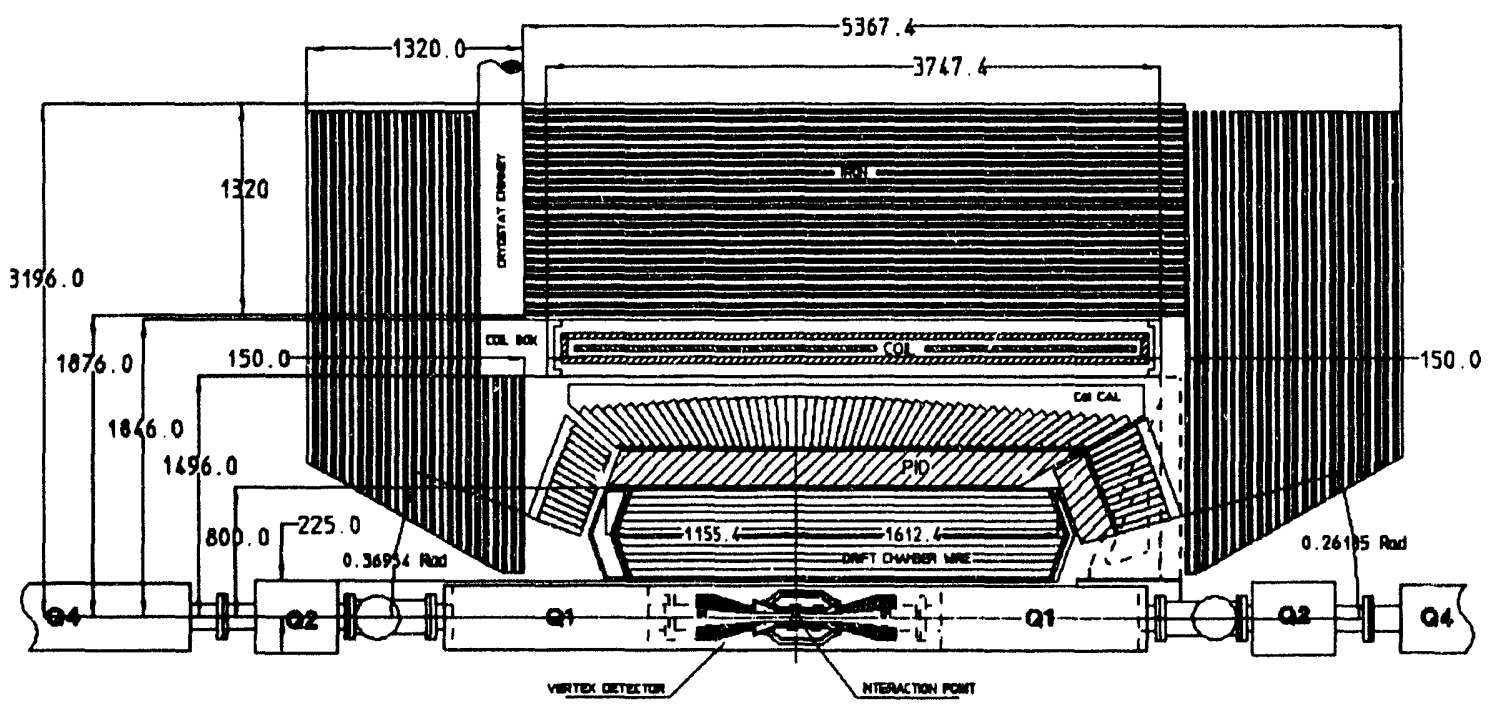

Figure 15-3. Subsystem envelope dimensions in $\mathrm{mm}$.

As the subsystem designs evolve, the envelope drawings will facilitate the subsystem dimension control procedure; changes to envelope dimensions will require the approval of the Technical Board. 


\subsubsection{Cables, Utilities Routing}

Plans for cable and utility routing will be incorporated early on into the design of the detector. A list of expected services (including allowance for future upgrades and packing factor) will be compiled to determine the required service space. Figures 15-4 show the preliminary routing plan for the detector. Services to and from the silicon vertex detector are routed inside the support tube along the beam line. Drift chamber electronics readout is located only at the backward end. The drift chamber and barrel particle identification services are routed along a $5 \mathrm{~cm}$ gap between the calorimeter barrel and end cap. These cables are first joined by the calorimeter services, then exit via a $15 \mathrm{~cm}$ gap between the magnet cryostat and the magnet return iron. All services then exit the detector through slots between the barrel return iron and the pole tips as indicated in Figure 15-4.

\subsection{Detector Assembly}

A conceptual detector assembly study has been made for the Letter of Intent. Installation of the detector should be possible in the space available in the IR hall on the outside of the shield wall, facilitating assembly of the detector while PEP-II commissioning activities are going on.

The instrumented iron section will be designed to facilitate handling with the available 50-ton crane. Figures 15-5 show a possible assembly scenario. The barrel return flux will be assembled into a self-supported structure which will house the transport mechanism. The detector transport system will be tested before completing the assembly of other subsystems. The magnet coil will be inserted into the iron with a C-lifting fixture.

The normal installation sequence would involve calorimeter, particle identification and then tracking subsystems after the solenoidal field is mapped. Depending on the installation schedule, the particle identification system could be installed inside the calorimeter before inserting the calorimeter into the coil with an insertion beam, although this is unlikely. 


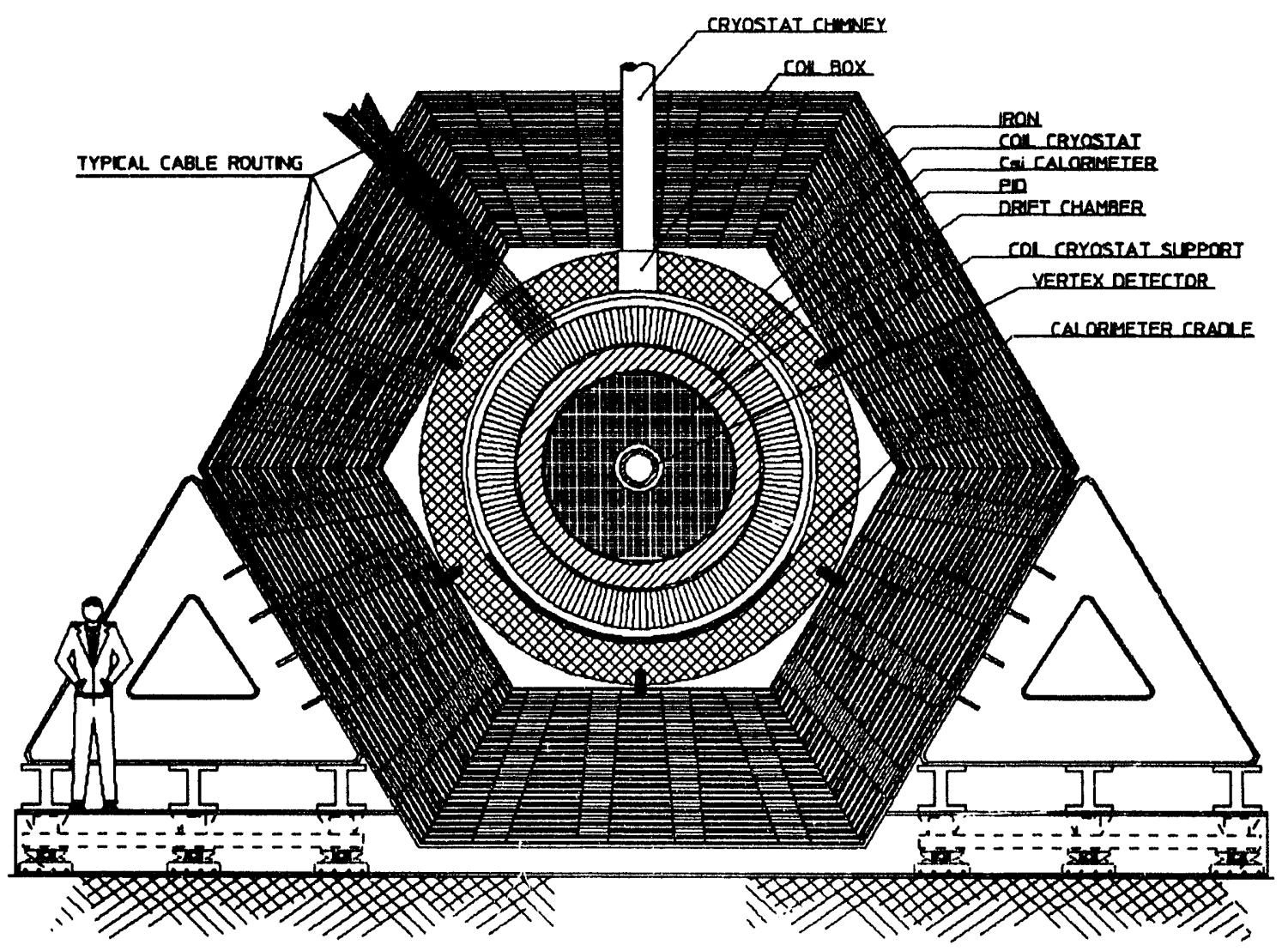

Figure 15-4. Cross-sectional view of preliminary cable/utility routing path.

The drift chamber will be installed using a small insertion beam and temporarily supported on the coil support ring. The vertex detector will be mounted inside the support tube at the same time as the beampipe, masks, Q1 an B1 magnets are assembled. The support tube will then be installed as one piece inside the detector.

The electronics platform will be partially assembled outside the IR hall and brought into the hall in large pieces for final assembly after completing the installation of the detector components.

When the detector has been commissioned on cosmic rays, the shield wall will be removed and the detector rolled onto the beam line. Detector alignment and final beam line installation will then take place. 


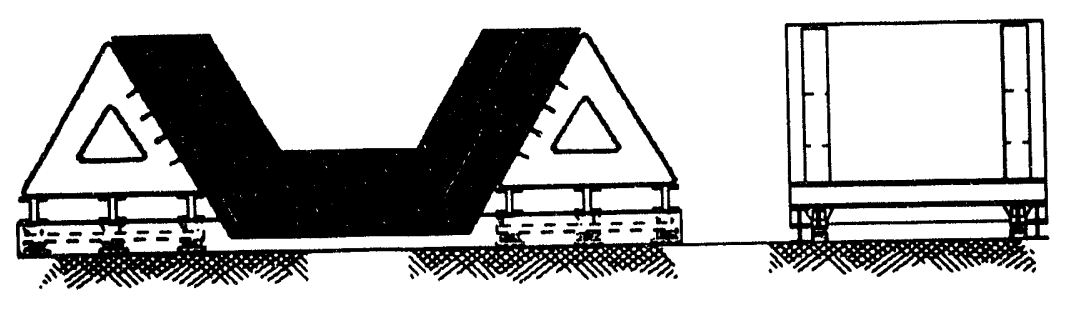

ASSEMEC Y SEOLENCE ?

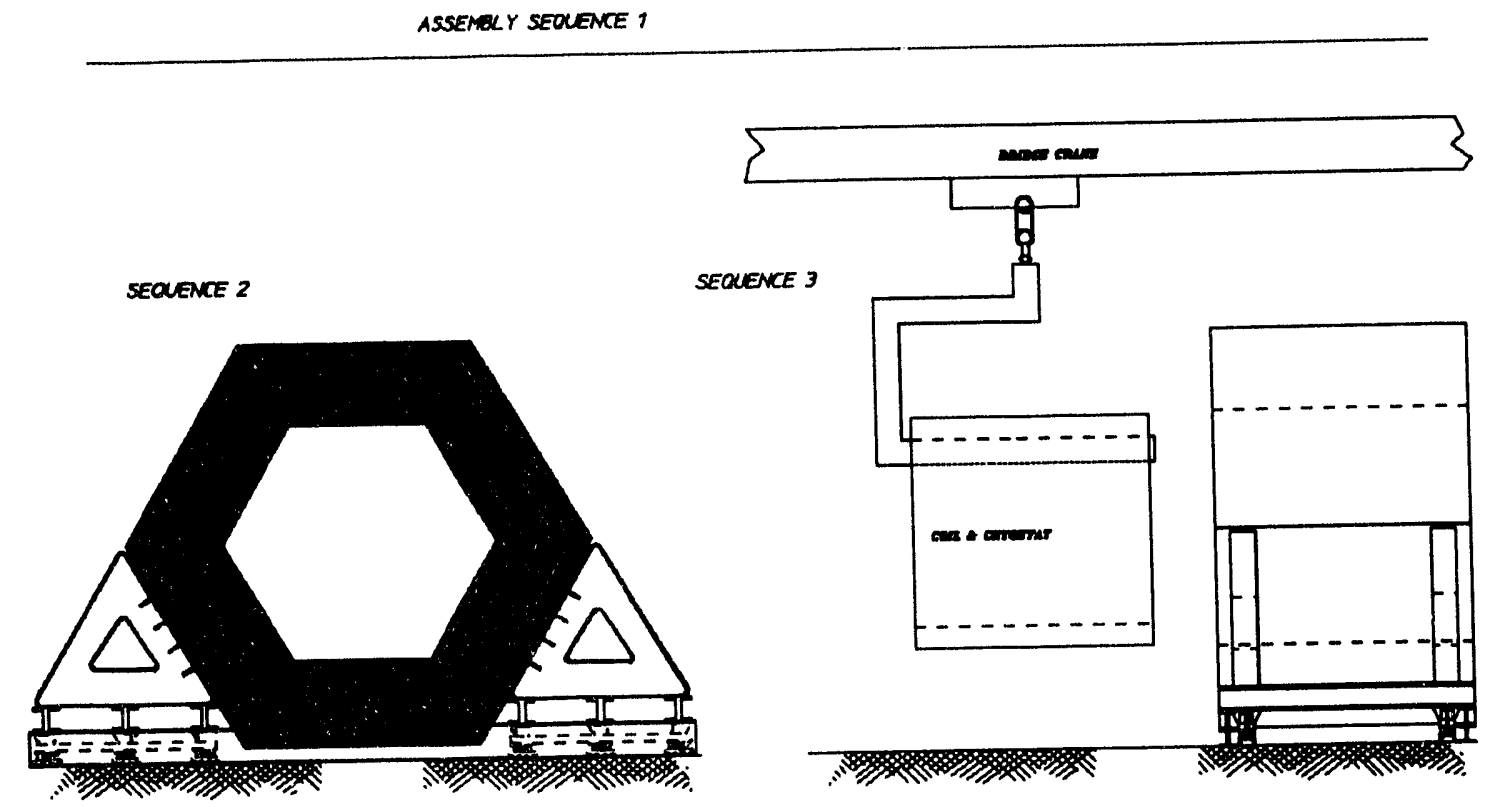

Figure 15-5. Preliminary assembly sequence.

Assembly and service fixtures required for common assembly work such as access platforms and general lifting fixtures will be procured and maintained by the engineers and support personnel of the installation group. Fixtures such as rails or beams required for specific elements of the detector will be designed and built by each subsystem group in consultation and under the review of the Technical Coordination group.

Access requirements for detector service are divided into two major categories, depending on the access frequency and duration. By retracting the end doors, rapid access to the inner detector will be possible at either end. Figure 15-6 shows the detector on beamline with an end door retracted. An access platform will be erected to facilitate reaching the drift chamber endplate. This procedure can be done without disrupting the beam line system, disconnecting 
cables, or removing mechanical support elements. It is expected that the door can be opened and closed in less than one shift allowing service to be performed during normal beam down periods. Access to the vertex detector is more involved because the beam line has to be disassembled. The work can be performed during longer shutdown periods without moving the detector.

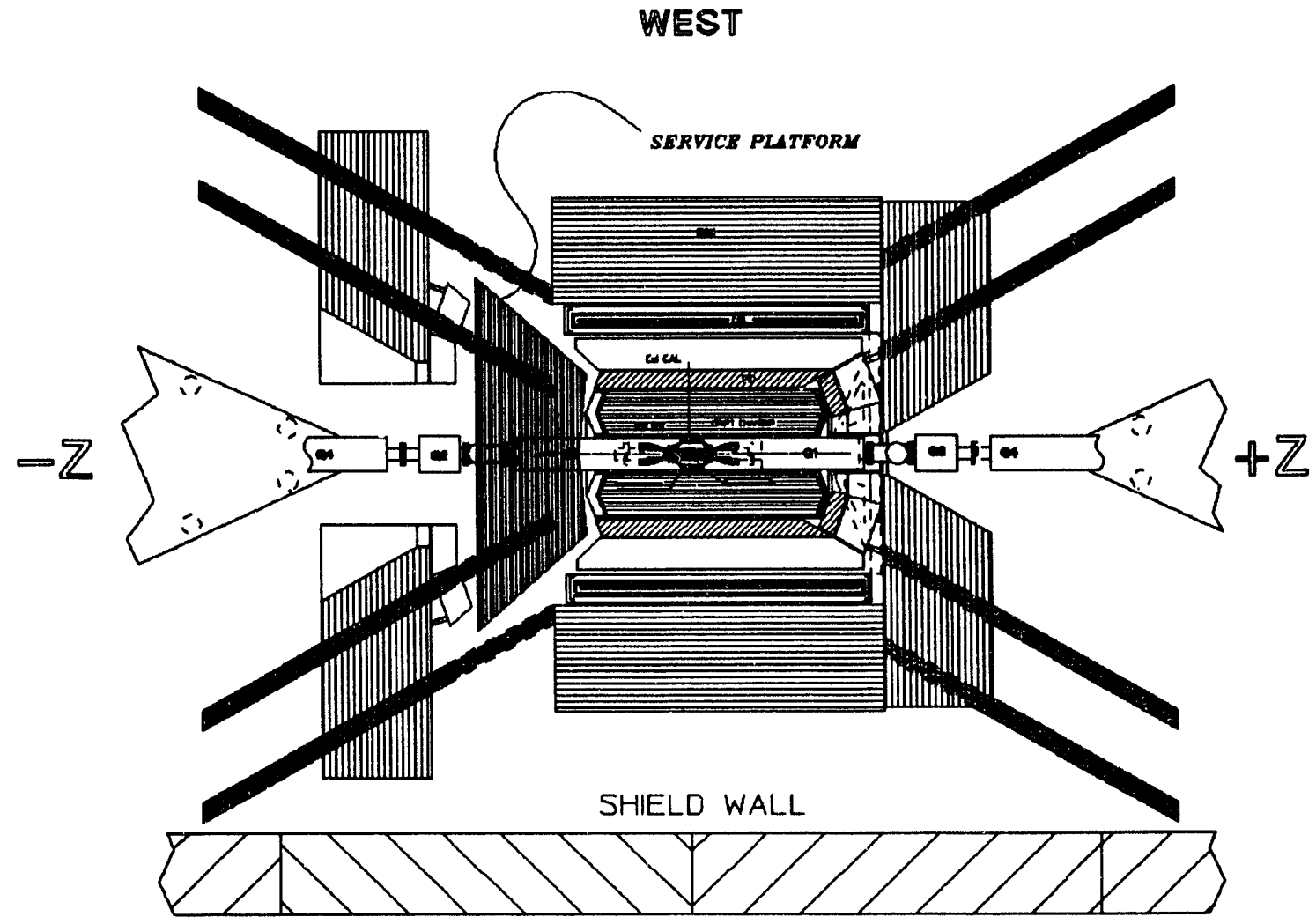

Figure 15-6. Plan view of BABAR detector with end doors retracted for access to inner detector components.

\subsection{Detector Interface with PEP-II}

The integration specialist will provide oversight on issues concerning the detector and machine interface. At a strictly hardware level, this includes integration of accelerator elements (optics, pumps, etc.) in the support tube that carries the vertex detector and the design of 
the vacuum chamber and its mating to the accelerator. At present, it is planned that the load from the support tube itself will be carried by vertical members which are themselves affixed to the magnet support ring.

Any necessary detector protection interlocks (e.g. radiation mnnitors for the silicon vertex detectors or the drift chamber, etc.) will be the responsibility of the Technical Coordinator, who will also be responsible for coordination with PEP-II in implementation and testing of the the Personnel Protection System (PPS).

There will be a specialist in QA/QC matters, who will actively monitor the fabrication and assembly of the coil and flux-return, the detector subsystems, and their installations. This person will work with system managers, engineers and physicists to advise on the writing of proper fabrication, assembly and inspection procedures and protocols to assure that the detector is built in an expeditious and efficient manner.

\subsection{Environmental Safety and Health}

There will be a specialist in ES\&H matters, who will actively advise and monitor safety issues surrounding choice and implementation of detector technologies, safety in the experimental hall, and appropriate operational issues.

\subsubsection{Safety Analysis Report}

There will be a Preliminary Safety Analysis Report (PSAR) on the timescale of the submission of the Conceptual Design Report. This report will be updated as the technology choices are established. A final Safety Analysis Report (SAR) must be approved by the SLAC Director and appropriate SLAC ES\&H staff before operation of any elements of the detector that require review in the SAR, is permitted. 


\subsubsection{Seismic Protection}

The SAR will address all seismic protection issues associated with the detector construction and operation. Emphasis will be given to reviewing safety during the installation period.

\subsubsection{Hazard Protection}

The SAR will address all issues of ES\&H and personnel protection. However, a guiding principle in the design of the detector will be an emphasis on passive safety - the a priori reduction of hazards through conscious technical choices.

For example, the use of passive fire protection is envisioned throughout the detector by stressing the employment of non-flammable materials for detector construction (cables etc.), and implementing sealed cable conduits to reduce fire spreading rates. Where necessary (for example, in areas with massed electronics). an appropriate active fire suppression system (sprinklers or a HALON equivalent) will be employed.

The detector as presently envisioned contains two subsystems (drift chamber and muon detectors) which may employ modest quantities of flammable or explosive gases. In both systems alternative gas mixtures are being sought (at a potential cost in performance) to eliminate the hazard. The SLD detector is notable for choosing this route and succeeding in eliminating all flammable gases from the detector. As appropriate, Hazardous Atmosphere Detectors (HAD) and Oxygen Deficiency Monitors (ODM) as well as active dilution could be used to address the problem if substitute gases are not found.

No element of the detector presently is envisioned to contain significant quantities of chemical substances posing a serious health or environmental hazard. 


\subsection{Electrical Engineering Integration}

To insure that the detector electronics and data acquisition system is designed and built in a coherent and cost-effective manner, there will be a chief electrical engineer. To the extent that components will be designed and fabricated in many institutions, integration and the imposition of common standards will be critical. Electronics for all subsystems in the detector includes data acquisition systems, monitoring systems, control systems, and cable plant.

\subsection{Software Engineering Integration}

To insure that the data acquisition system software, the offline software, the PEP-II interface, and the computing hardware is designed and put in place in a coherent and effective manner, there will be a chief software engineer. Elements of the software system will of necessity be written at many institutions. Hardware will to some extent be distributed. Integration and adherence to common standards will be important to the success of the project. 


\section{Budget and Schedule}

$\mathbf{C}$ onstruction of PEP-II was formally begun in October, 1993. Having a detector ready to confront $C P$ violation physics of PEP-II on a time scale commensurate with the collider completion date of October, 1998 is a formidable task.

The BABAR collaboration was initiated in December, 1993. The time allotted by the Laboratory's schedule for the development and submission of a Letter of Intent and a Conceptual Design Report amounts to $25 \%$ of a five-year detector construction schedule. It is therefore important that a detailed budget and critical path schedule be worked out during this period. It is particularly vital that critical path items be identified at a sufficiently early stage that available resources can be allocated to these areas.

The conceptual detector design discussed herein has been developed by the Collaboration over a six month period, based on the substantial effort that has gone into a variety of asymmetric $B$ Factory proposals over the last several years. In most areas, particularly those affecting overall geometry, it has been possible to arrive at specific technical choices for each subsystem. In certain instances, options are described, but only the principal choice has been included in the budget and schedule. The exception to this is the particle identification subsystem, where there is as yet no convincing demonstration of the three leading technologies, so that more than one primary alternative has been specified. So as not to impede engineering design of the coil, flux return and electromagnetic calorimeter, which are critical path items, the three particle identification options are designed to be compatible with a single geometry, with only minor modifications.

The $B_{A} B_{A R}$ design is conservative, in that in nearly all systems the technology chosen is well-developed and mature. Thus, while a certain amount of detailed development must go on over the next year, the basic issues involve engineering and procurement, not basic
The proposed LOI and CDR phases amount to $25 \%$ of a five-year project

A primary technology selection has been made for all subsystems, with the exception of particle identification

The three particle identification options are compatible with a single overall detector geometry

The subsystem technologies chosen do not, in the main, require extensive $R \& D$ 
There are three cost estimates, differing only in the choice of particle identification system and associated electronics
The "technically-limited schedule" is compatible with the projected PEP-II completion date
R\&D. Particle identification is, again, an exception. In this case, the process of choosing a terhnology and bringing it to maturity has been structured in such a way as to have minimal impact on the overall project.

The initial cost estimate and schedule have been based on this conceptual design. There are therefore, of necessity, three cost estimates, one for each of the particle identification options. These three estimates differ only in the cost of the particle identification system and associated electronics. Should it be possible to make a final choice of particle identification system at a sufficiently early date, it may be desirable to re-optimize the geometry, with concomitant cost savings.

In general, to this point there have been only preliminary engineering studies on individual detector subsystems. Cost estimation based on these designs is presented at "WBS Level 3", although the estimates have been done at a more detailed level. The schedules presented serve mainly to identify interactions between systems, critical path items and items that have a serious impact on yearly budgeting.

The technically-limited schedule allows completion and commissioning of $B_{A} B_{A R}$ at a date compatible with the PEP-II schedule. Accomplishing this will be a formidable task, requiring the cooperation of DOE and the funding agencies of a number of other countries.

A brief discussion of the individual detector systems follows.

\subsection{Vertex Detector}

The five-layer double-sided silicon strip vertex detector chosen for $B A B A R$ is a straighforward extrapolation of existing designs. The emphasis on precision in the $z$ direction, basic to the main physics thrust of the experiment, requires transporting signals from large numbers of transverse strips out of the fiducial volume. This will be done by one of two techniques, both of which are being employed in current experiments. Reduction of material to decrease multiple scattering is a primary design requirement. Performance with $300 \mu \mathrm{m}$ silicon is satisfactory; thinner devices will be explored. 
Radiation hard fabrication techniques developed for SSC and LHC are more than sufficient for the PEP-II environment.

Members of the vertex detector group have been active in the design and construction of similar devices for CLEO-II, SDC, ALEPH, D0, CDF and Mark II. There is substantial expertise within the group in detector and readout chip design, in trigger design and in mechanical structures. The cost estimate is based on the this recent experience. The project is of a familiar scale and can be completed within the required time.

\subsection{Main Tracking Chamber}

Several technical innovations in the main tracking chamber are motivated by the requirement of minimal multiple Coulomb scattering. Low-mass gases have been developed within the group over the past several years. Large chambers have already been constructed in several experiments using carbon composite technology. Lowmass field wires can be made of aluminum, although some existing chambers have had wire breakage problems. Thinner gold plating of the $\mathrm{Al}$ wires must be developed. The use of silicon carbide wires is an innovative possibility.

Construction of main tracking chamber on the needed timescale requires the early resolution of mechanical engineering issues involving endplate and feedthrough design and choice of field wire material. The electronics and data acquisition system is straightforward. The cost estimate has been developed using experience from the Mark II and SLD drift chambers among others.

\subsection{Particle Identification}

The three candidate systems present different budget and schedule issues. In addition, the fact that none of the technologies being considered has as yet had a convincing proof-of-principle demonstration is in itself a problem in the context of the overall detector schedule. The ensuing discussion assumes that a proof-of-principle in at least one system will be forthcoming in a timely fashion. It will 
therefore emphasize the schedule and budget issues for the system once chosen.

\subsubsection{Aerogel Threshold Cherenkov Detector}

Facilities exist for the production of the required quantities of low density aerogel in less than a year.

Fine mesh photomultipliers, the most conservative photodetector choice, are being produced in large quantity for the KLOE detector. Should the more innovative microchannel plate-based readout devices prove attractive, these would be produced with equipment currently used for large-scale production of very similar image intensifiers. Questions regarding large-scale production of hybrid avalanche photodiodes remain to be resolved.

The mechanical mounting structure, and the calibration and readout systems are relatively simple. The engineering effort involved here is not large.

Cost estimation is also straightforward, as the cost of the aerogel radiator and photosensitive devices dominate the total and both are well defined. The electronics is similarly uncomplicated.

\subsubsection{DIRC Detector}

Concerns here principally involve engineering of the readout structure and of modifications to the flux return to support endcap steel while providing access to inner detector components.

The production, assembly and characterization of the quartz Cherenkov radiators present a relatively well-characterized problem that should have no schedule impact.

The rearlout system uses large numbers of conventional photomultiplier tubes; system engineering is thus the principal concern. Design of a readout structure that works in the magnet fringe field and can be removed to allow access would be an important milestone.

The adoption of DIRC particle identification technology for the barrel would require the use of a different technology for the forward 
endcap system. A forward endcap of acrogel would cost an additional $\$ 2.8 \mathrm{M}$, while a Fast RICH endcap would add approximately $\$ 4.3 \mathrm{M}$, both including electronics and readout.

\subsubsection{Fast RICH Detector}

The Fast RICH system is the most complex of the particle identification options, involving extensive gas and liquid control systems, large-area proportional chambers with CsI-coated cathodes and very large numbers of electronics channels.

A large prototype with distinct but related technology has been tested by the European Fast RICH Collaboration, providing some real-world experience. The engineering tasks involved in this system appear solvable, but are certainly complex; a plan demonstrating that this system could be built on the required time scale would be an important milestone.

\subsection{CsI(Tl) Calorimeter}

Large CsI calorimeter systems have been built and work well. The problem here is thus one of ensuring that $\sim 10,000$ crystals of high quality can be produced on the required schedule. This involves having an adequate supply of raw material, which is available, and a sufficient number of crystal growing furnaces, which must be built over the first two years.

Extensive contacts with crystal growing manufacturers and research institutes have been established. It appears that the production capacity for CsI crystals can be expanded sufficiently rapidly to meet our schedule. Two pilot projects have also begun to explore new, more efficient growing techniques, which could have a considerable impact on costs. A time lag has been introduced into the endcap fabrication schedule to allow for the completion of $R \& D$ on radiation hardness.

The mechanical support structure chosen is simple and inexpensive and is not a critical path item. Large numbers of wavelength shifter/photodiode/preamplifier readout assemblies must be pro- 
duced; this task will be widely distributed. Photodiode production capacity is more than adequate to out needs.

The final assembly of the crystals equipped with readout assemblies into the support structure requires quite a bit of time. This is reflected in the three year crystal production schedule, allowing the last year for final assembly and test.

\subsection{Magnet}

The specified superconducting solenoid is similar in dimensions and other properties to several existing coils. There is serious interest from a number of experienced vendors. The solenoid is a critical path item; the order must be placed in FY95 to meet the schedule.

The segmented flux return is also similar to other recent designs. It must be completely specified rather rapidly, as it forms the basis for assembly of the other detector components. The magnet and solenoid must therefore be assembled and measured in time to allow the installation of the IFR chambers and systems internal to the coil in a timely way.

\subsection{Flux Return Instrumentation}

The construction of the required number of RPCs or PSTs presents a problem of a scale that has been met by nearly every major $4 \pi$ detector built in the last decade. Facilities with adequate capacity already exist, as do experienced collaborating institutions.

\subsection{Trigger and Data Acquisition}

The scale of the trigger and data acquisition system is familiar. Technical innovations relate mostly to the asynchronous nature of the system and its fully pipelined architecture. 
Given the worldwide nature of the collaboration, the major task in this area is the coordination of the system design to take advantage of economies of scale and to ensure a coherent system architecture.

\subsection{Computing}

This system will be based on networked processors running UNIX or its real-time derivatives. The primary question here is adherence to a coherent set of standards for programming and system activities in a worldwide collaboration. Intensive efforts to put such a wellcoordinated system in place appear to be proceeding well.

\subsection{Installation and Integration}

Substantial emphasis will be placed on system integration from the start of the project. This will involve coordination of the service and utility requirements of the various subsystems from the beginning, as well as detailed planning for the staged assembly of the detector in IR2 and its commissioning. This effort will be coordinated by a central staff which has been included in the budget estimate. 


\begin{tabular}{|c|c|c|c|c|c|c|c|}
\hline WaS & SUESYSTEN & $\begin{array}{l}\text { EDs } \\
\text { (KS) }\end{array}$ & $\begin{array}{c}\text { LABOR } \\
(\mathrm{K} \$)\end{array}$ & $\begin{array}{l}\text { ROVECT } \\
\text { ms } \\
\text { (KS) }\end{array}$ & $\begin{array}{l}\text { TOTALS } \\
\text { SUBTOTAL } \\
\text { (KS) }\end{array}$ & $\begin{array}{c}\text { CONT. } \\
\text { (\%) }\end{array}$ & $\begin{array}{c}\text { TOTAL } \\
\text { (KS) }\end{array}$ \\
\hline & AEROCEL OPTIOA & $\$ 4003$ & 5820 & 42948 & 82760 & 10.1 & 74785 \\
\hline & DIRC OPTON & 14602 & 6268 & 44846 & 85708 & 10.9 & 78785 \\
\hline & FAST RICH OPTION & 16499 & 7075 & 42471 & 66046 & 10.4 & 78829 \\
\hline 1.1 & VERTEX DETECTOR & 1152 & 900 & 1825 & 3877 & 24.2 & 4815 \\
\hline 1.1 .1 & MECHANCAL SUPPORT \& COOUNG & 268 & 147 & 156 & 572 & 22.0 & 698 \\
\hline 1.1 .2 & SULONCOMPONENTS & 395 & 399 & 1499 & 2203 & 26.6 & 2000 \\
\hline 1.1 .4 & POWERSUPPLY & 17 & 8 & 8 & 32 & 25.0 & 40 \\
\hline 1.1 .5 & ONLINE MONTTOA \& CONIROL & 20 & 23 & 13 & 56 & 20.0 & 67 \\
\hline 1.1 .6 & ASSEMELY \& WSTALATION & 239 & 324 & 146 & 700 & 20.2 & 853 \\
\hline 1.1 .7 & SYSTEM ENGNEEFING \& COOADNMTION & 212 & & 2 & 214 & 20.0 & 257 \\
\hline 1.2 & CENTRAL DAITT CHAMBEA & 900 & 470 & 2010 & 3398 & 24.2 & 4220 \\
\hline 1.2 .1 & CONCEPTUAL DESTEN & 50 & & 67 & 117 & 25.0 & 146 \\
\hline 1.2 .2 & MECHMNCN STRUCTUPE & 400 & 24 & 880 & 1404 & 25.0 & 1755 \\
\hline 1.2 .3 & FEEDTHFOUGHS DESIGN \& FABRICATE & 70 & 24 & 220 & 314 & 25.0 & 393 \\
\hline 1.2 .4 & WIRE & 40 & 24 & 485 & 549 & 20.0 & 658 \\
\hline 1.2 .5 & STRWNGNG \& TESTING & 100 & 247 & 110 & 457 & 25.0 & 572 \\
\hline 1.2 .6 & GAS SYSTEM & 80 & 48 & 50 & 178 & 25.0 & 223 \\
\hline 1.2 .7 & HGH VOLTAGE DISTRBUIION & 30 & 24 & 20 & 74 & 25.0 & 93 \\
\hline 1.2 .8 & FRONT END ELECTRONNCS MOUNTS & 20 & 16 & 12 & 48 & 25.0 & 60 \\
\hline 1.2 .9 & ONLLNE MONTTOR \& CONTROL. & 60 & 16 & 40 & 116 & 25.0 & 145 \\
\hline 1.2 .10 & NSTNLATION \& TESTING & 50 & 56 & 35 & 141 & 25.0 & 176 \\
\hline 1.3 & PARTICLE DD (AEROGEL OPTION) & 400 & 329 & 5335 & 6154 & 21.6 & 7482 \\
\hline 1.3 .1 & AEROGEL BLOOKS & 60 & 42 & 1084 & 1168 & 25.0 & 1458 \\
\hline 1.3 .2 & PHOTODETECTOAS & 200 & 126 & 3884 & 4210 & 20.0 & 5052 \\
\hline 1.3 .3 & CALIERATION SYSTEM & 70 & 21 & 102 & 193 & 25.0 & 241 \\
\hline 1.3 .4 & MECHUNCAL SUPPORT & 60 & 28 & 225 & 333 & 25.0 & 416 \\
\hline 1.3 .5 & MSTNLLATION \& TESTING & 60 & 112 & 60 & 252 & 25.0 & 315 \\
\hline 1.3 & PARTICLE DO (DIRC OPTION) & 1080 & 777 & 5440 & 7277 & 26.6 & 0215 \\
\hline 1.3.1 & QUARTZ & 180 & 70 & 2098 & 2348 & 28.8 & 3047 \\
\hline 1.3 .2 & PHOTODETECTOAS & 120 & 357 & 2660 & 3137 & 20.4 & 3777 \\
\hline 1.3 .3 & CALIBPATION \& TIMING & 60 & 7 & 27 & 04 & 20.0 & 113 \\
\hline 1.3 .4 & MECHUNCAL SUPPOAT & 460 & 168 & 580 & 1208 & 38.0 & 1667 \\
\hline 1.3 .5 & NSTALATION \& TESTING & 240 & 175 & 75 & 490 & 25.0 & 613 \\
\hline 1.3 & PARTICLE ID (FAST RICH OPTION) & 2691 & 1587 & 3068 & 7346 & 21.7 & 8941 \\
\hline 1.3 .1 & RADIATORS & 649 & 439 & 1010 & 2098 & 20.0 & 2537 \\
\hline 1.3 .2 & PROPOPIIONLL CHWMERS & 639 & 488 & 769 & 1896 & 20.1 & 2283 \\
\hline 1.3 .3 & CEI CATHODES & 348 & 94 & 227 & 669 & 29.2 & 865 \\
\hline 1.3 .4 & MECHUNCAN SUPPOAT & 390 & 109 & 586 & 1085 & 20.0 & 1302 \\
\hline 1.3 .5 & GAS CIRCULATION SYSTEM & 243 & 176 & 216 & 635 & 23.1 & 782 \\
\hline 1.3 .6 & ONLINE MONTOA \& CONTROL & 243 & 62 & 187 & 492 & 20.0 & 580 \\
\hline 1.3 .7 & NSTALLATION \& TESTING & 180 & 218 & 73 & 471 & 23.4 & 581 \\
\hline 1.4 & CAI CALORIMETER & 852 & 1483 & 18776 & 21110 & 11.0 & 23615 \\
\hline 1.4 .1 & CSI ACQUISTION, FABRICATION \& TESTING & 50 & 211 & 17358 & 17618 & 10.3 & 19428 \\
\hline 1.4 .2 & LIGHT COUECTIONELEMENTS \& TESTHG & 30 & 148 & 1025 & 1204 & 20.0 & 1445 \\
\hline 1.4 .3 & READOUT SUB-ASSEMBUES \& TESTING & 40 & 145 & 70 & 255 & 20.0 & 306 \\
\hline 1.4 .4 & MECHANCAL SUPPORTS & 164 & 343 & 70 & 577 & 20.0 & 682 \\
\hline 1.4 .5 & SOUPCE CNLERATION SYSTEM & 30 & 29 & 100 & 159 & 20.0 & 180 \\
\hline 1.4 .6 & MSTALATION \& TESTING & 60 & 375 & 153 & 588 & 20.0 & 705 \\
\hline 1.4 .7 & SYSTEM ENGNEEPUNG \& COORDNATION & 478 & 230 & & 708 & 20.0 & 850 \\
\hline 1.5 & MUON SYSTEM (RPC OPTION) & 232 & 698 & 664 & 1595 & 21.1 & 1031 \\
\hline 1.5.1 & APC FABRICATION & 37 & 384 & 300 & 721 & 23.1 & 887 \\
\hline 1.5 .2 & GAS SYSTEM & 48 & 81 & 80 & 210 & 10.0 & 231 \\
\hline 1.5 .3 & HGH VOLTAGE SYSTEM & 25 & 18 & 100 & 143 & 20.0 & 171 \\
\hline 1.5 .4 & OC\& TESTMG & 48 & 120 & 164 & 332 & 25.0 & 415 \\
\hline 1.5 .5 & MSTNLATION WTO FLUX RETURN & 74 & 85 & 20 & 189 & 20.0 & 227 \\
\hline
\end{tabular}




\begin{tabular}{|c|c|c|c|c|c|c|c|}
\hline \multirow[b]{2}{*}{ WBS } & \multirow[b]{2}{*}{ SUBSYSTEM } & \multicolumn{6}{|c|}{ PRONECT TOTALS } \\
\hline & & $\begin{array}{l}\text { EDS } \\
\text { (KS) }\end{array}$ & $\begin{array}{c}\text { LAOA } \\
\text { (Ks) }\end{array}$ & $\begin{array}{l}\text { Mns } \\
\text { (KS) }\end{array}$ & $\begin{array}{l}\text { SUBTOTAL } \\
\text { (KS) }\end{array}$ & $\begin{array}{c}\text { CONT. } \\
(\%)\end{array}$ & $\begin{array}{c}\text { TOTAL } \\
\text { (KS) }\end{array}$ \\
\hline 1.6 & SC SOLENON \& FLUX RETURN & 1440 & 740 & 5948 & 8120 & 21.8 & 9898 \\
\hline 1.6 .1 & PREL. DESIGN $1.5 T 3 \mathrm{~m}$ Diam. $+2 \mathrm{~m}$ Long & 166 & & 17 & 103 & 17.0 & 214 \\
\hline 1.6 .2 & SUPERCONDUCTMG CON (1.5T) & 218 & & 3472 & 3690 & 20.0 & 4426 \\
\hline 1.6 .3 & POWEPPPOTECTION & 83 & & 133 & 217 & 15.0 & 248 \\
\hline 1.6.4 & MACNET LOCAL CONTAOLS & 68 & & 47 & 114 & 14.0 & 130 \\
\hline 1.6 .5 & VACUUM SYSTEM & 62 & 32 & 61 & 146 & 14.0 & 166 \\
\hline 1.6 .6 & CPMOGENCS & 197 & 284 & 618 & 1100 & 25.0 & 1375 \\
\hline 1.6.7 & FLUXFETUFN & 480 & & 1362 & 1861 & 24.1 & 2309 \\
\hline 1.6 .8 & TEST HARDWARE & 17 & 24 & 25 & 66 & 33.0 & 88 \\
\hline 1.6 .9 & WSTALLATION \& TEST & 125 & 382 & 132 & 648 & 25.0 & 812 \\
\hline 1.6 .10 & MISC. EQUP. \& CONSUMMALES & 4 & 8 & 90 & 102 & 25.0 & 128 \\
\hline 1.7 & ELECTRONICS (AEROCEL OPTION) & 4114 & 73 & 6746 & 10933 & 24.8 & 13644 \\
\hline 1.7 & ELECTRONICS (DIAC OPTION) & 4133 & 73 & 8541 & 12747 & 24.8 & 15911 \\
\hline 1.7 & ELECTRONICS (RICH OPTION) & 4400 & 71 & 8538 & 13018 & 24.8 & 16250 \\
\hline 1.7 .1 & VERTEX DETECTOAPEADOUT & 487 & & 430 & 917 & 25.0 & 1146 \\
\hline 1.7 .2 & DFFT CHAMBEREECTRONCS & 768 & 7 & 2185 & 2960 & 25.0 & 3700 \\
\hline 1.7 .3 & PARTICLE ID (AEROGEL 3360 CHAN.) & 522 & 13 & 637 & 1172 & 25.0 & 1485 \\
\hline 1.7 .3 & PARTICLE ID (DIRC 1EK CHAN.) & 541 & 13 & 2432 & 2886 & 25.0 & 3733 \\
\hline 1.7 .3 & PARTICLE ID (FAST RICH 30OK CHAN.) & 817 & 11 & 2429 & 3267 & 25.0 & 4071 \\
\hline 1.7 .4 & CNLOPNETEREECTRONCS & 838 & 7 & 2271 & 3116 & 25.0 & 3885 \\
\hline 1.7 .5 & MUON IDELLECTRONICS & 723 & 9 & 739 & 1471 & 25.0 & 1839 \\
\hline 1.7 .6 & RESERIED WBS & & & & & & \\
\hline 1.7 .7 & LEVEL 1 TRIGGER & 324 & 6 & 224 & $\mathbf{5 5 3}$ & 25.0 & 691 \\
\hline 1.7 .6 & LEVEL 2 TAIGGEA \& DATA ACOUISTION & 144 & & 183 & 327 & 25.0 & 408 \\
\hline 1.7 .9 & CONIROLS \& ACCEE ERATOR NTERFACE & 63 & & 47 & 110 & 25.0 & 137 \\
\hline 1.7 .10 & CABLE PLANT INSTALLATION & 25 & 32 & 30 & 87 & 25.0 & 108 \\
\hline 1.7 .11 & ENGNEERING NTEGPATION \& COORONATION & 220 & & & 220 & 15.0 & 253 \\
\hline 1.8 & ONLINE COMP. \& SOFTW. DEV. & 1265 & 534 & 1220 & 3027 & 29.8 & 3930 \\
\hline 1.8 .1 & ONLNE WFRUSTRUCTURE & & 63 & 682 & 745 & 30.0 & 968 \\
\hline 1.8 .2 & SOFTWARE DEVELOPMENT & 1265 & 471 & 547 & 2282 & 29.8 & 2962 \\
\hline 1.9. & INTEGRATION, ES\&H \& INSTALLATION & 3558 & 584 & 405 & 4547 & 15.0 & 5220 \\
\hline 1.8. & TECHNCAL COOAOMNATION & 3264 & 432 & 355 & 4051 & 15.0 & 4658 \\
\hline 1.8 .2 & QA ANO ESSHOVERSKGHT & 150 & 80 & 30 & 260 & 15.0 & 298 \\
\hline 1.8 .3 & NSTANATIONENGINEERING SUPPORT & 144 & 72 & 20 & 238 & 15.0 & 271 \\
\hline
\end{tabular}

Figure 16-1. Detector budget in FY1994 dollars. 


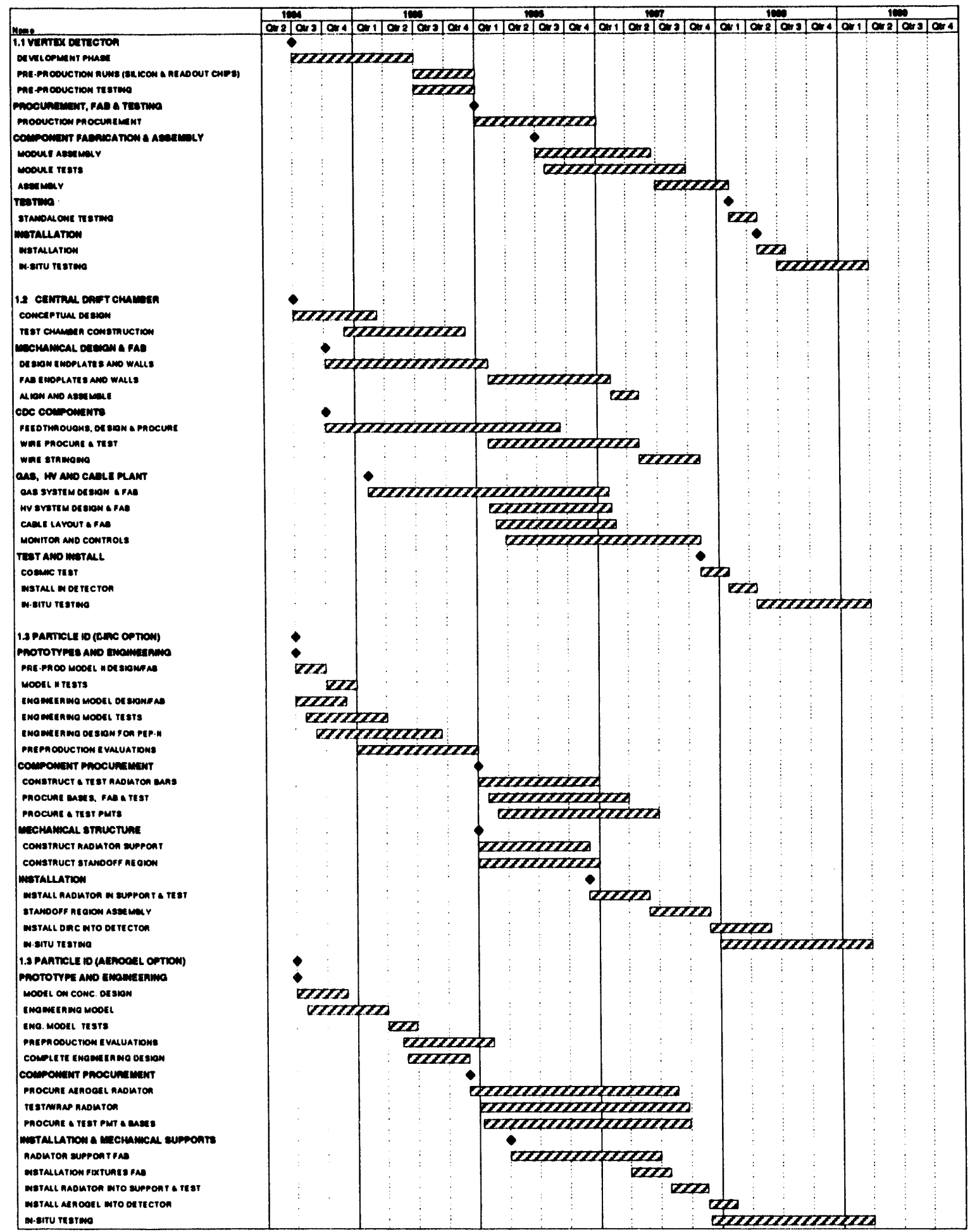




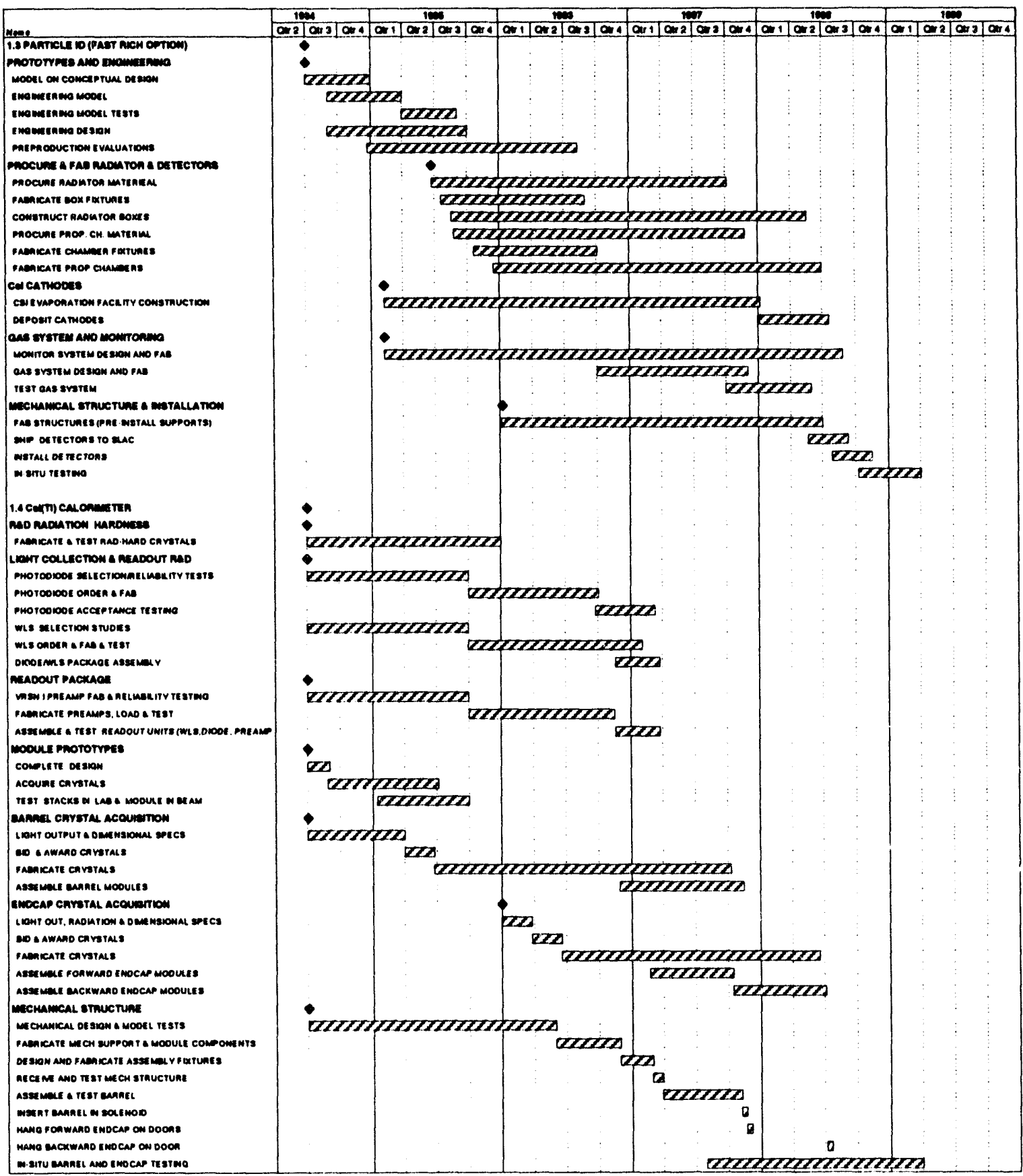




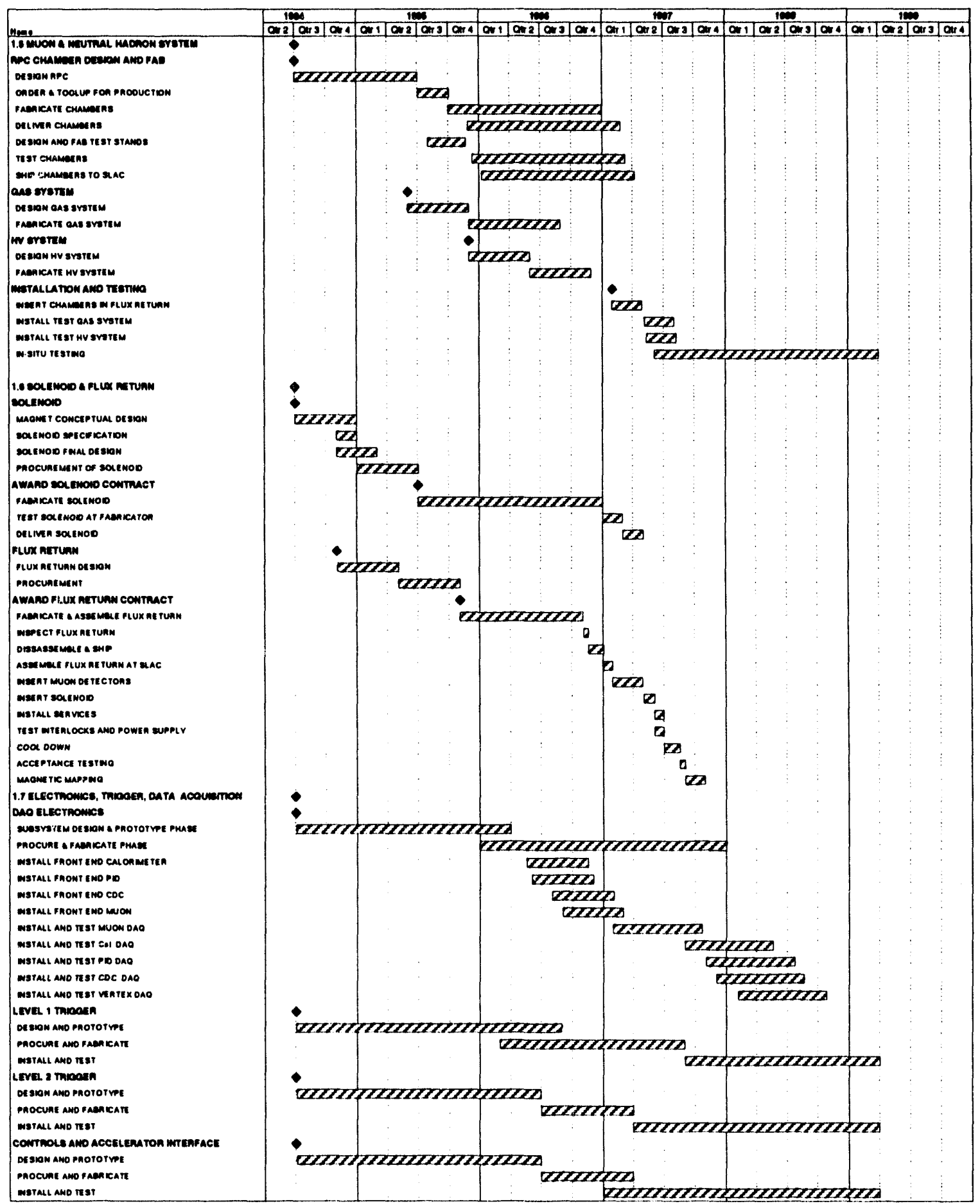




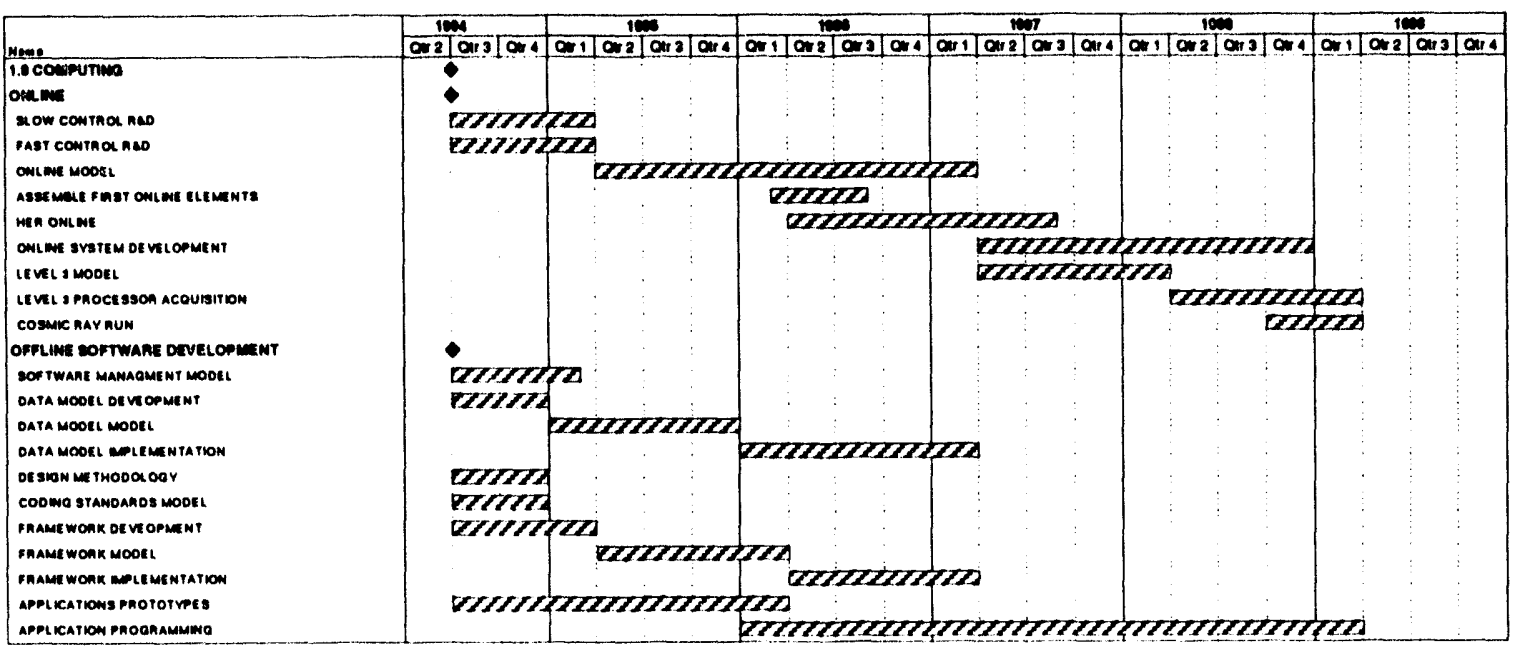

Figure 16-2. Detector construction schedule. 

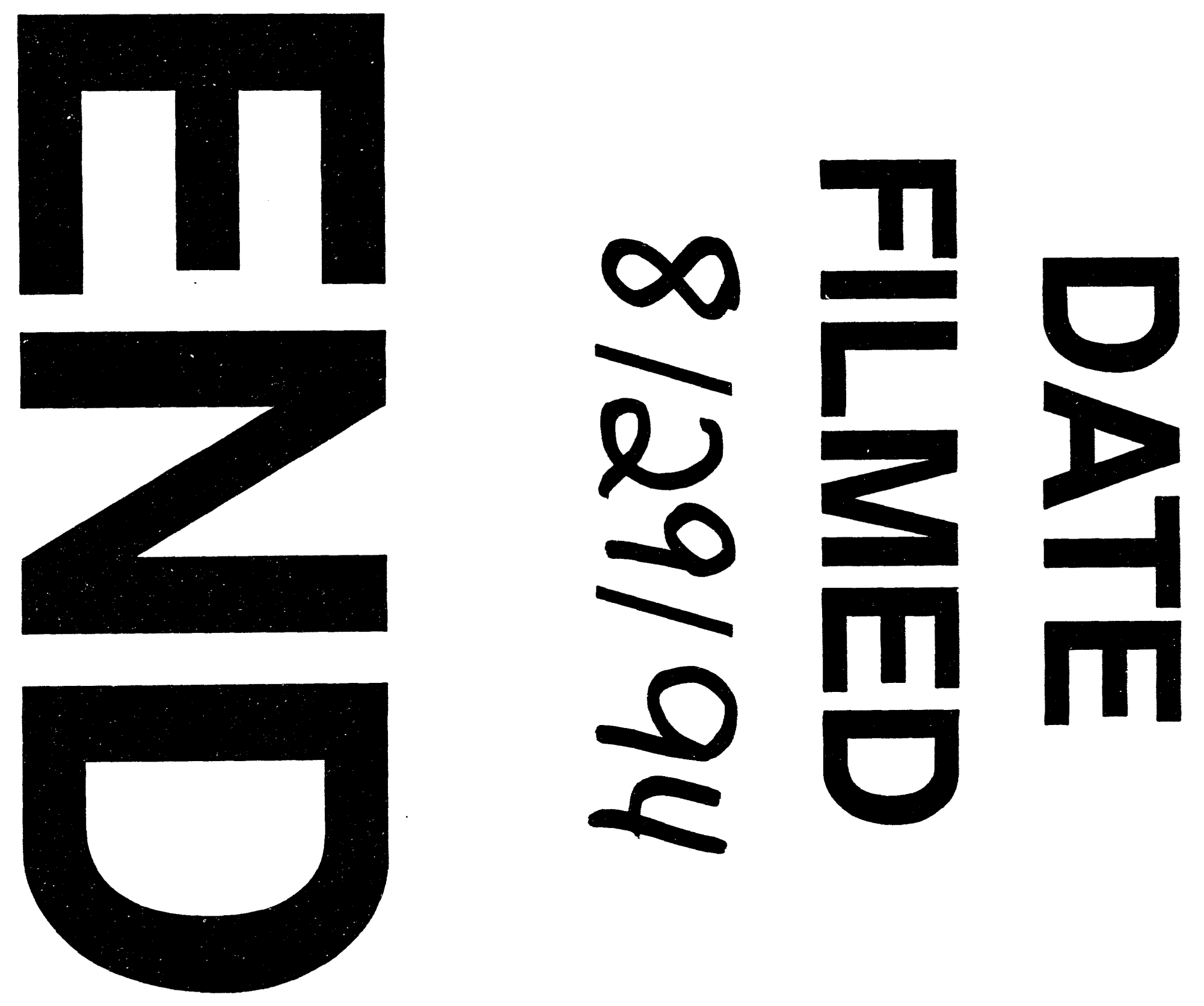


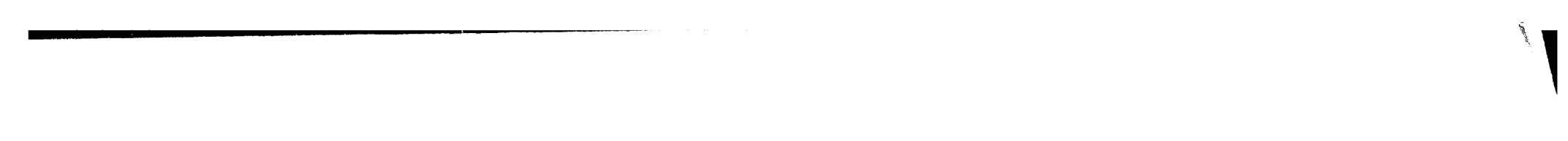

Plon
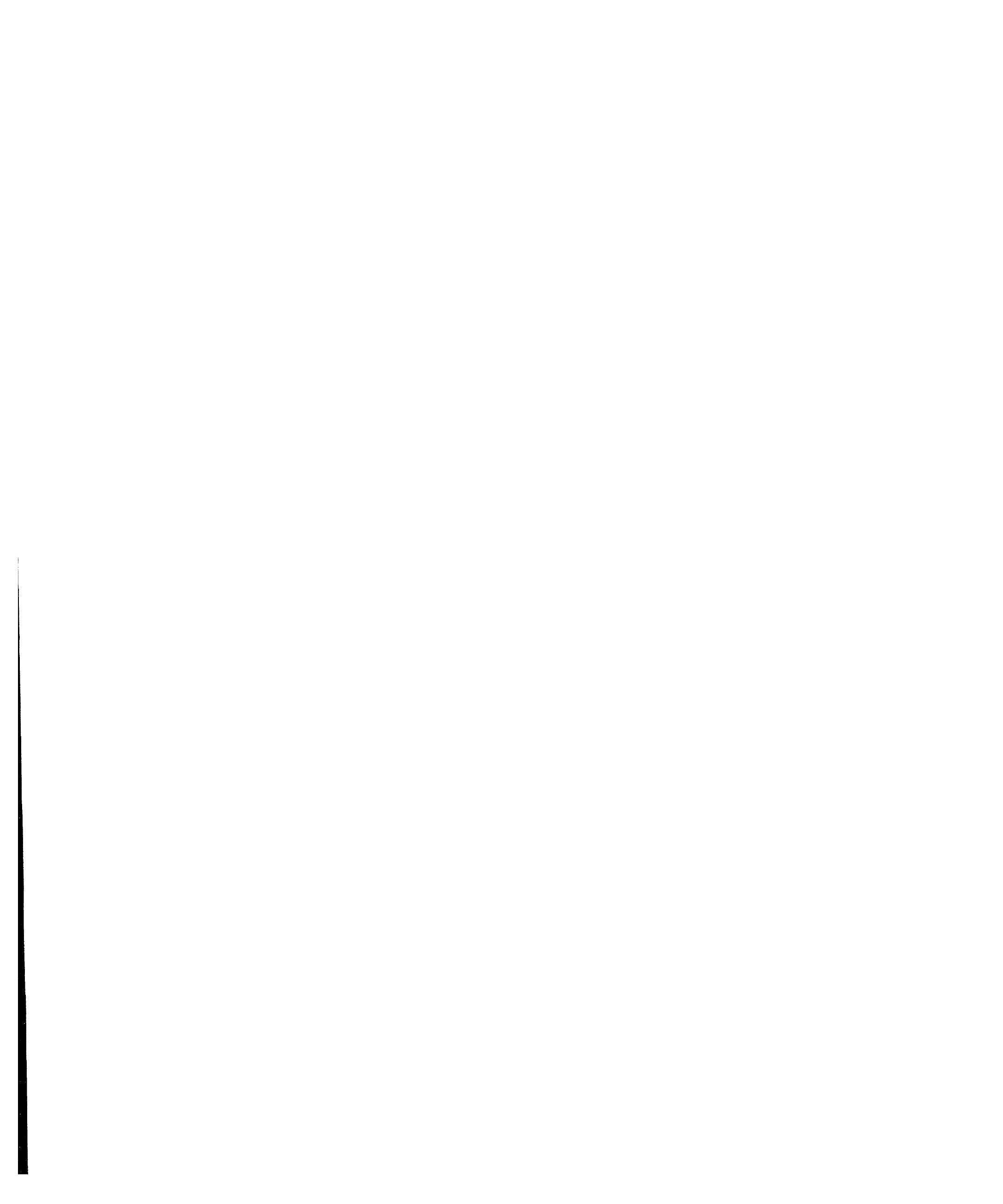